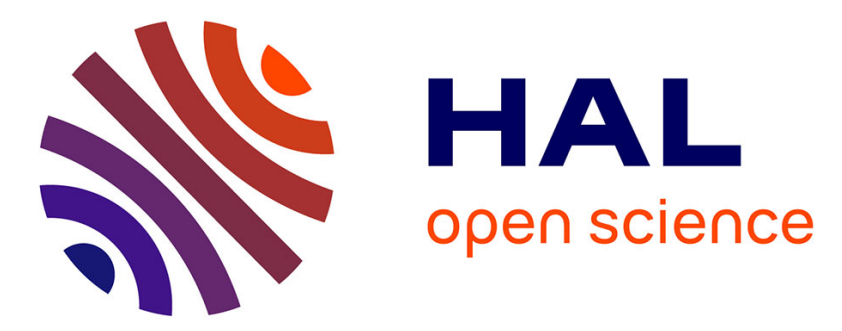

\title{
Simulating the density of organic species in the atmosphere of Titan with a coupled ion-neutral photochemical model
}

\author{
V. Vuitton, R.V. Yelle, S.J. Klippenstein, S.M. Hörst, P. Lavvas
}

\section{To cite this version:}

V. Vuitton, R.V. Yelle, S.J. Klippenstein, S.M. Hörst, P. Lavvas. Simulating the density of organic species in the atmosphere of Titan with a coupled ion-neutral photochemical model. Icarus, 2019, 324, pp.120-197. 10.1016/j.icarus.2018.06.013 . hal-03038393

\section{HAL Id: hal-03038393 https://hal.science/hal-03038393}

Submitted on 3 Dec 2020

HAL is a multi-disciplinary open access archive for the deposit and dissemination of scientific research documents, whether they are published or not. The documents may come from teaching and research institutions in France or abroad, or from public or private research centers.
L'archive ouverte pluridisciplinaire HAL, est destinée au dépôt et à la diffusion de documents scientifiques de niveau recherche, publiés ou non, émanant des établissements d'enseignement et de recherche français ou étrangers, des laboratoires publics ou privés. 


\title{
Simulating the density of organic species in the atmosphere of Titan with a coupled ion-neutral photochemical model
}

\author{
V. Vuitton ${ }^{\mathrm{a}}$, R. V. Yelle ${ }^{\mathrm{b}}$, S. J. Klippenstein ${ }^{\mathrm{c}}$, S. M. Hörst ${ }^{\mathrm{d}}$, P. Lavvas ${ }^{\mathrm{e}}$ \\ ${ }^{a}$ Institut de Planétologie et d'Astrophysique de Grenoble, Univ. Grenoble Alpes, CNRS, \\ Grenoble, 38000, France \\ ${ }^{b}$ Lunar and Planetary Laboratory, Univ. of Arizona, Tucson, AZ 85721, USA \\ ${ }^{c}$ Chemical Sciences and Engineering Division, Argonne National Lab., Argonne, IL 60439, \\ $U S A$ \\ ${ }^{d}$ Department of Earth and Planetary Sciences, Johns Hopkins Univ., Baltimore, MD 21218, \\ USA \\ ${ }^{e}$ Groupe de Spectrométrie Moléculaire et Atmosphérique, Univ. Reims \\ Champagne-Ardenne, CNRS, Reims, 51687, France
}

\begin{abstract}
We present a one-dimensional coupled ion-neutral photochemical kinetics and diffusion model to study the atmospheric composition of Titan in light of new theoretical kinetics calculations and scientific findings from the Cassini-Huygens mission. The model extends from the surface to the exobase. The atmospheric background, boundary conditions, vertical transport and aerosol opacity are all constrained by the Cassini-Huygens observations. The chemical network includes reactions between hydrocarbons, nitrogen and oxygen bearing species. It takes into account neutrals and both positive and negative ions with masses extending up to about $100 \mathrm{u}$. We incorporate high-resolution isotopic photoabsorption and photodissociation cross sections for $\mathrm{N}_{2}$ as well as new photodissociation branching ratios for $\mathrm{CH}_{4}$ and $\mathrm{C}_{2} \mathrm{H}_{2}$. Ab initio transition state theory calculations are performed in order to estimate the rate coefficients and products for critical reactions.

Main reactions of production and loss for neutrals and ions are quantitatively assessed and thoroughly discussed. The vertical distributions of neutrals and ions predicted by the model generally reproduce observational data, suggesting that for the small species most chemical processes in Titan's atmosphere and ionosphere are adequately described and understood; some differences are highlighted. Notable remaining issues include (i) the total positive ion density (essentially $\mathrm{HCNH}^{+}$) in the upper ionosphere, (ii) the low mass negative ion densities $\left(\mathrm{CN}^{-}, \mathrm{C}_{3} \mathrm{~N}^{-} / \mathrm{C}_{4} \mathrm{H}^{-}\right)$in the upper atmosphere, and (iii) the minor oxygen-bearing species $\left(\mathrm{CO}_{2}, \mathrm{H}_{2} \mathrm{O}\right)$ density in the stratosphere. Pathways towards complex molecules and the impact of aerosols (UV shielding, atomic and molecular hydrogen budget, nitriles heterogeneous chemistry and condensation) are evaluated in the model, along with lifetimes and solar cycle variations.
\end{abstract}


Keywords: Titan, atmosphere, Ionospheres, Atmospheres, chemistry, Photochemistry, Organic chemistry

\section{Introduction}

Titan is a fascinating world, with its dense (1.5 bars on the surface) and cold $(70-200 \mathrm{~K})$ atmosphere, and its layers of orange haze that extend to almost 1000 $\mathrm{km}$ above its surface. As on Earth, the main atmospheric molecule is nitrogen $\left(\mathrm{N}_{2}\right)$. The second most abundant molecule in Titan's atmosphere is methane $\left(\mathrm{CH}_{4}\right)$; Titan's surface conditions are near its triple point resulting in a cycle (evaporation, condensation, precipitation) similar to that of water on Earth. Consequently, Titan is the object of the solar system whose superficial structure is the closest to that of the Earth and it has many of the same physical processes as the Earth but they are based on a completely different chemistry. The few percent of methane and the low amount of oxygen (mainly carried by carbon monoxide, $\mathrm{CO}$ ) make the atmosphere more chemically reducing than present day Earth, where chemistry is based on oxidizing processes linked to the presence of oxygen. The study of Titan's chemistry thus provides the opportunity to determine how the organic nitrogen molecules involved in the chemistry of living organisms (e.g. nitrogen bases, amines ...) can be generated naturally in a planetary environment (Hörst et al., 2012).

Understanding the chemistry that takes place in Titan's atmosphere is an intimidating task because of the multiplicity of chemical and physical processes involved. Chemical processes begin with the dissociation and ionization of $\mathrm{N}_{2}$ and $\mathrm{CH}_{4}$ by solar ultraviolet radiation and energetic particles, which provide the $\mathrm{H}, \mathrm{C}$, and $\mathrm{N}$ atoms necessary for the synthesis of complex organic molecules. The species produced react with the ambient gas to generate a multitude of molecules which will gradually grow to become micron size solid particles, or aerosols. Thus, molecular growth is induced by gas phase reactions which involve radicals as well as positive and negative ions, possibly in excited states and also probably by heterogeneous chemistry on the surface of aerosols. The efficiency and the products of these reactions depend strongly on the physical characteristics of the atmosphere: pressure and temperature. In addition, the distribution of species is affected by molecular diffusion, vertical and horizontal winds, as well as the escape of the lightest compounds and the condensation of the heaviest compounds in the lower stratosphere. A diagram illustrating the atmospheric chemistry of Titan is shown in Figure 1 of Hörst (2017).

The Voyager 1 mission to Saturn in 1980 and subsequent Earth-based observations identified more than a dozen neutral hydrocarbons, nitriles and oxygenbearing species. From 2004 to 2017, the Cassini spacecraft has observed Titan in situ and by remote sensing and has made exceptional discoveries. In the upper atmosphere, it detected the presence of positively and negatively charged ions with $\mathrm{m} / \mathrm{z}$ reaching up to thousands of atomic mass units (Waite et al., 2007; Coates et al., 2009; Crary et al., 2009; Coates et al., 2010), revealing that Titan has the most compositionally complex ionosphere in the Solar System. It 
became clear that these extremely complex particles are the precursors of the aerosols known to be present at lower altitude. Also, energetic $\mathrm{OH}_{x}^{+}(x=0-3)$ ions have been observed in Saturn's magnetosphere in the vicinity of Titan (Hartle et al., 2006) and could be at the origin of the oxygen-bearing compounds. Finally, the ${ }^{14} \mathrm{~N} /{ }^{15} \mathrm{~N}$ ratios in $\mathrm{N}_{2}, \mathrm{HCN}$ and $\mathrm{CH}_{3} \mathrm{CN}$ have been retrieved and are one of the keys to furthering our understanding of the energy sources, primary processes and therefore the evolution of the atmospheric molecular complexity (Vuitton et al., 2014).

A large number of chemical models of the atmosphere were created prior to the Cassini mission, with main focus on the ionosphere (Keller et al., 1992; Fox and Yelle, 1997; Keller et al., 1998; Galand et al., 1999), the stratosphere (Yung et al., 1984; Toublanc et al., 1995; Lara et al., 1996), both (Banaszkiewicz et al., 2000; Wilson and Atreya, 2004) or the galactic cosmic rays (GCR) induced layer (Molina-Cuberos et al., 1999a,b, 2002). They mostly explained the neutral composition in the stratosphere but their predictions regarding the thermosphere turned out to be incomplete. For example, it was predicted that $\mathrm{HCNH}^{+}, \mathrm{C}_{2} \mathrm{H}_{5}{ }^{+}$and $\mathrm{CH}_{5}{ }^{+}$would be abundant but many of the ion species detected by Cassini were not anticipated. Also, the presence of negative ions was not predicted in the ionosphere. This triggered the re-examination of Titan's upper atmospheric chemistry and put a strong emphasis on ion chemistry.

Motivated by the return of the first in situ measurements of Titan's upper atmospheric composition, we developed a OD ionospheric model to constrain the density of minor neutral constituents (Vuitton et al., 2006b). The model assumed local chemical equilibrium and adopted atmospheric conditions appropriate for the $1100 \mathrm{~km}$ level. The densities of neutral species were fixed at values determined during the outbound portion of the T5 flyby, that was deep into the nightside. The relative abundance of the primary ions was calculated for a mixture of $\mathrm{N}_{2}$ and $\mathrm{CH}_{4}$ irradiated by $100 \mathrm{eV}$ magnetospheric electrons and the magnitude of the ion production was scaled to reproduce the electron density measured by the Langmuir probe. The chemical network included $\sim 40$ neutrals, $\sim 150$ ions and $\sim 1250$ reactions. We showed that the most abundant positive ions are closed-shell species because radical cations are not very stable and therefore react quickly. This implies that while odd mass channels are attributed to hydrocarbon ions, peaks at even $\mathrm{m} / \mathrm{z}$ are the signature of ions containing one nitrogen atom. All ion-molecule reactions do not necessarily lead to molecular growth because proton transfer to molecules having a higher proton affinity, typically nitrogen-bearing species, is very efficient. We determined that the density of ions depends strongly on the composition of the neutral atmosphere and inferred the presence of the most complex molecules identified so far on Titan. This includes $\mathrm{C}_{2} \mathrm{H}_{3} \mathrm{CN}$ and $\mathrm{C}_{2} \mathrm{H}_{5} \mathrm{CN}$, whose presence was recently confirmed by ALMA (Cordiner et al., 2015b; Palmer et al., 2017).

In order to study the distribution of benzene, this model was later extended to $1 \mathrm{D}$ and daytime conditions (Vuitton et al., 2008). The calculations allowed retrieval of the integrated vertical production rate of $\mathrm{C}_{6} \mathrm{H}_{7}{ }^{+}$. In parallel, we constructed an independent 1D neutral photochemical-diffusion model focused on benzene. The model included eddy and molecular diffusion. The solar irra- 
diation was based on a solar spectrum obtained with the EUV flux model for Aeronomic Calculations (EUVAC) and was representative of a global average, while the temperature and total density were representative of the equatorial region. The chemical network consisted of 40 hydrocarbon species, 130 neutralneutral reactions and $\sim 40$ photodissociation reactions. These models predicted that the globally averaged production rate of benzene by ion-molecule reactions is $\sim 10^{7} \mathrm{~cm}^{-2} \mathrm{~s}^{-1}$, of the same order of magnitude as the production rate by neutral reactions of $\sim 4 \times 10^{6} \mathrm{~cm}^{-2} \mathrm{~s}^{-1}$. We showed that benzene is quickly photolyzed in the thermosphere and that $\mathrm{C}_{6} \mathrm{H}_{5}$ radicals, the main photodissociation products, react primarily with other radicals, leading to the formation of complex aromatic species. These species, along with benzene, diffuse downward, eventually condensing near the tropopause. We found a total production rate of solid aromatics of $\sim 10^{-15} \mathrm{~g} \mathrm{~cm}^{-2} \mathrm{~s}^{-1}$, corresponding to an accumulated surface layer of $\sim 3 \mathrm{~m}$.

In order to investigate the chemistry of oxygen-bearing species, ten oxygenbearing species participating in 32 reactions have been added by Hörst et al. (2008) to the hydrocarbon network of Vuitton et al. (2008). The sources of oxygen in this model are the precipitation of $\mathrm{O}$ and $\mathrm{OH}$ or $\mathrm{H}_{2} \mathrm{O}$ into the upper atmosphere. We modeled these assuming a Chapman production function with a peak at $1100 \mathrm{~km}$ for $\mathrm{O}$ (from $\mathrm{O}^{+}$deposition calculations) and $750 \mathrm{~km}$ for $\mathrm{OH}$ and $\mathrm{H}_{2} \mathrm{O}$. We showed that using an oxygen flux consistent with the reported observations of the Ion Mass Spectrometer (IMS), one of the sensors of the Cassini Plasma Spectrometer (CAPS) and an $\mathrm{OH}$ flux consistent with predicted production from micrometeorite ablation allowed the model to reproduce the observed abundances of $\mathrm{CO}, \mathrm{CO}_{2}$ and $\mathrm{H}_{2} \mathrm{O}$ available at that time.

Independently, Lavvas et al. (2008a,b) presented a 1D photochemical-microphysical model, whose main objective was the understanding of the atmospheric processes that lead to haze formation. The model incorporated detailed radiation transfer calculations providing the vertical structure of the radiation field and temperature profile. Gaseous species photochemistry initiated the production of the haze precursors, while their evolution was described by the microphysical part of the model. The calculated aerosol and gas opacities were iteratively included in the radiation transfer calculations in order to investigate their effect on the resulting temperature profile and geometric albedo. 68 neutral hydrocarbon and nitrile species were included in the photochemical calculations, the evolution of which was controlled by about 520 reactions. Ions or ion chemistry were not included. Pathways providing the stronger contribution to the haze production supported the incorporation of nitrogen, with a major peak between 500 and $900 \mathrm{~km}$.

Following the detection of negative ions, their chemistry was taken into account in an ionospheric model for the first time (Vuitton et al., 2009). This lead to the identification of $\mathrm{CN}^{-}, \mathrm{C}_{3} \mathrm{~N}^{-} / \mathrm{C}_{4} \mathrm{H}^{-}$and $\mathrm{C}_{5} \mathrm{~N}^{-}$in the negative ion spectrum, which was recently confirmed by a reanalysis of the Electron Spectrometer (ELS) data, one of the sensors making up the Cassini Plasma Spectrometer (CAPS) (Desai et al., 2017). We found that these ions are essentially formed by dissociative attachment of electrons with energies of a few $\mathrm{eV}$ onto 
trace neutral species. Just as with positive ions, proton transfer reactions lead to the formation of heavier negative ions. Finally, ions are lost by associative detachment reactions with the most abundant radicals, typically $\mathrm{H}$ and $\mathrm{CH}_{3}$.

The ion and neutral models presented in Vuitton et al. (2008) were turned into a self-consistent ion-neutral chemistry model to investigate the formation of $\mathrm{NH}_{3}$ and $\mathrm{CH}_{2} \mathrm{NH}$ in the upper atmosphere (Yelle et al., 2010). Some aspects of the neutral photochemistry as well as the treatment of eddy and molecular diffusion were based on Hörst et al. (2008), while the N chemistry was adapted from Lavvas et al. (2008a). From the measured abundance of $\mathrm{NH}_{4}{ }^{+}$and $\mathrm{CH}_{2} \mathrm{NH}_{2}{ }^{+}$, we established that $\mathrm{NH}_{3}$ and $\mathrm{CH}_{2} \mathrm{NH}$ have a large abundance in the upper atmosphere and that it is the consequence of coupled ion and neutral chemistry.

Lavvas et al. (2011a) used high-resolution cross sections for the neutral photodissociation of $\mathrm{N}_{2}$ between 80 and $100 \mathrm{~nm}$ and provided a different picture of energy deposition compared to results based on low-resolution cross sections. Our results showed that the contributions of both photons and photoelectrons are required for the accurate representation of the chemical precursors in the upper atmosphere. The highly structured cross sections imply that photons experience a smaller absorption by $\mathrm{N}_{2}$ and are able to penetrate deeper in the atmosphere. The main consequence is that ionization of methane to $\mathrm{CH}_{4}{ }^{+}$takes place at lower altitudes, close to $800 \mathrm{~km}$, which affects significantly the $\mathrm{CH}_{4}{ }^{+}$ production.

Vuitton et al. (2012) included these detailed calculations for the energy deposition in the coupled ion-neutral photochemical model described in Yelle et al. (2010). The hydrocarbon chemistry was updated with new chemical parameters for association reactions based on ab initio TST calculations. These calculations indicate that association reactions are fast even at low pressure for adducts having as few as four $\mathrm{C}$ atoms. Although the column-integrated production and condensation rates of hydrocarbons change only by a factor of a few, the mole fractions can increase by as much as one order of magnitude in the upper atmosphere.

Lavvas et al. (2015) presented a detailed model for the vibrational population of all non pre-dissociating excited electronic states of $\mathrm{N}_{2}$, as well as for the ground and ionic states. The calculations demonstrate that a significant population of vibrationally excited ground state $\mathrm{N}_{2}$ survives in the upper atmosphere but the implications on the ionospheric densities are negligible.

In this paper, we present a globally representative picture of the understanding of Titan's atmospheric chemistry post Cassini-Huygens. We base our investigations on the comparison of the Cassini data with a one-dimensional coupled ion-neutral photochemical kinetics and diffusion model developed in our previous targeted studies (Vuitton et al., 2006b, 2007; Hörst et al., 2008; Vuitton et al., 2008, 2009; Yelle et al., 2010; Lavvas et al., 2011a; Vuitton et al., 2012; Lavvas et al., 2015). In section 2, we present a detailed description of the model, highlighting the new constraints brought by Cassini-Huygens for the model atmosphere, boundary conditions and vertical transport, as well as our improved chemical scheme. A unique aspect of the model is a detailed investigation of numerous reaction pathways including new theoretical calculations of 
reaction rate coefficients. The next two sections are dedicated to the main output of the model: the atmospheric chemical composition. In section 3 , we focus on the main reaction pathways for the production and loss of ions and neutrals. A review of available observations is given in section 4 before comparing them to the present and other model results. In section 5, we pinpoint several important aspects of Titan's chemistry, starting from molecular weight growth and the subsequent effect of aerosols, and further discussing species lifetimes and expected variations in density profiles induced over the course of a solar cycle. Finally, in section 6 , we present our conclusions before reviewing the issues that are still remaining.

\section{Photochemical Model}

\subsection{General Description}

The development of the photochemical model described herein and the associated investigations has been motivated by the tremendous harvest of compositional information from the Cassini mission. Cassini has operated for more than thirteen years, but despite its extended dataset covering daily (De La Haye et al., 2008; Cui et al., 2009a), seasonal (Lebonnois et al., 2001; Bampasidis et al., 2012; Coustenis et al., 2013) and latitudinal variations (Crespin et al., 2008; Teanby et al., 2006; Teanby et al., 2008a, 2009), sampling remains inadequate to study geophysical variations and therefore constrain the circulation patterns. We therefore aim here at establishing the chemistry that is applicable to low- and mid-latitudes, a required step before more sophisticated tools capturing diurnal/latitudinal/seasonal variations can be devised. Our approach to constrain the model atmosphere and vertical transport is to average all available measurements in order to reproduce the global-mean conditions. We subsequently compare the species density profiles to the dayside equatorial and mid-latitude observations.

Our model includes the region from the surface to $1500 \mathrm{~km}$, the nominal exobase, with a grid spacing of $5 \mathrm{~km}$. It solves the one-dimensional coupled continuity and diffusion equation for each active species,

$$
\frac{\partial N_{i}}{\partial t}=P_{i}-L_{i}-\frac{1}{r^{2}} \frac{\partial\left(r^{2} \Phi_{i}\right)}{\partial r},
$$

where $N_{i}$ is the density of species $i, P_{i}$ and $L_{i}$ are its chemical production and loss terms and $\Phi_{i}$ the vertical flux. The radius $r=\left(R_{0}+z\right)$, where $R_{0}$ is the radius of Titan and $\mathrm{z}$ is the altitude. Steady-state conditions are assumed such that $\partial N_{i} / \partial t=0$. The chemical production of the $i$ th species is given by:

$$
P_{i}=\sum_{j} \sum_{k} R^{i, j k} k_{j k} N_{j} N_{k}+\sum_{l} J^{i, l} N_{l}+\gamma_{e}^{i, m} N_{m}+\gamma_{G C R}^{i, n},
$$

where $k_{j k}$ is the rate coefficient of the reaction between species $j$ and $k$ producing species $i, R^{i, j k}$ is the branching ratio of the channel leading to $i, J^{i, l}$ is the photodissociation/photoionization rate coefficient of species $l$ leading to $i, \gamma_{e}^{i, m}$ 
is the production rate through electron impact on species $m$ and $\gamma_{G C R}^{i, n}$ is the production rate through galactic cosmic rays.

The chemical loss of the $i$ th species is given by:

$$
L_{i}=\sum_{j} k_{i j} N_{i} N_{j}+J_{i} N_{i} .
$$

Here, $k_{i j}$ is the rate coefficient of the reaction between species $i$ and $j$ and $J_{i}$ is the photolysis rate coefficient of species $i$.

We find solutions for the chemical abundances by integrating the coupled continuity equations forward in time until a steady-state is reached. We use a fully implicit Newton-Raphson technique to advance densities to the next time step. The stability of the implicit integration allows us to achieve long simulation times. To judge if a run is converged we examine both the change in density from one time step to the next and the balance of production, loss, and flux divergence in the continuity equation. Because some molecular species on Titan have extremely long chemical lifetimes (CO in particular) the time integration must be very long to reach steady-state. This is achieved by using increasing time steps, reaching eventually $10^{20} \mathrm{~s}$ or larger. We know from experience with the model that even time steps of $10^{8}-10^{10}$ seconds are inadequate to reach convergence. We consider that convergence is achieved when the balance of production, loss, and flux divergence is a small fraction of the largest of those terms. This relative balance is less than $10^{-6}$ for all species and reaches machine precision for many species.

\subsection{Background Structure}

\subsubsection{Model Atmosphere}

The main reservoirs of nitrogen, carbon, hydrogen and oxygen in Titan's atmosphere are $\mathrm{N}_{2}, \mathrm{CH}_{4}$ and $\mathrm{CO}$. Their detection poses the question of their origin. They could be a primordial remnant, they could be supplied to the atmosphere from the interior or surface, or they could be delivered to the atmosphere from an external source; their abundance has likely varied over the course of Titan's history. It is not our goal to address these questions here. Since their lifetime is extremely long compared to the time it takes to generate photochemically produced molecules (cf. section 5.4), we keep their vertical profiles fixed to their current values. However, we will see that although minor hydrocarbons and nitrogen-bearing species are the daughter species of nitrogen and methane photochemistry, the origin of minor oxygen-bearing species cannot be CO only and that an external source of oxygen is required. We will return to this in section 4.2.3.3 and focus here on the background atmosphere for our photochemical model.

The background atmosphere is based on Cassini observations of the main constituents, $\mathrm{N}_{2},{ }^{14} \mathrm{~N}^{15} \mathrm{~N}$, and $\mathrm{CH}_{4}$. To create a global/temporal mean we simply average all of the Ion Neutral Mass Spectrometer (INMS) measurements of these species. A similar average was presented and discussed in Cui et al. (2009b). The only difference between that average and the one used here is 
that we now include data through T71. The average density profiles for $\mathrm{N}_{2}$ and ${ }^{14} \mathrm{~N}^{15} \mathrm{~N}$ are shown in Figure 1a, the average $\mathrm{CH}_{4}$ profile in Figure $1 \mathrm{~b}$, and a temperature profile consistent with these averages is shown in Figure 3.

As discussed in Snowden et al. (2013), the temperature profile in Titan's upper atmosphere shows large pass-to-pass variations. When averaged over many passes though, the mean temperature in the upper atmosphere is $150 \mathrm{~K}$. Temperatures in the stratosphere do not exhibit this pass-to-pass variability but vary strongly with season. Temperatures in the lower atmosphere vary far less, by only a few degrees from equator to pole. Our model temperature profile uses the HASI measurements below $200 \mathrm{~km}$ (Colombatti et al., 2008), assumes an isothermal stratosphere at $180 \mathrm{~K}$ between 200 and $500 \mathrm{~km}$, an isothermal thermosphere at $150 \mathrm{~K}$ above $700 \mathrm{~km}$, and connects the stratosphere and thermosphere with a linear temperature gradient. This temperature profile predicts $\mathrm{N}_{2}$ and ${ }^{14} \mathrm{~N}^{15} \mathrm{~N}$ density profiles that agree well with the mean of the measured values in the thermosphere, as shown in Figure 1a.
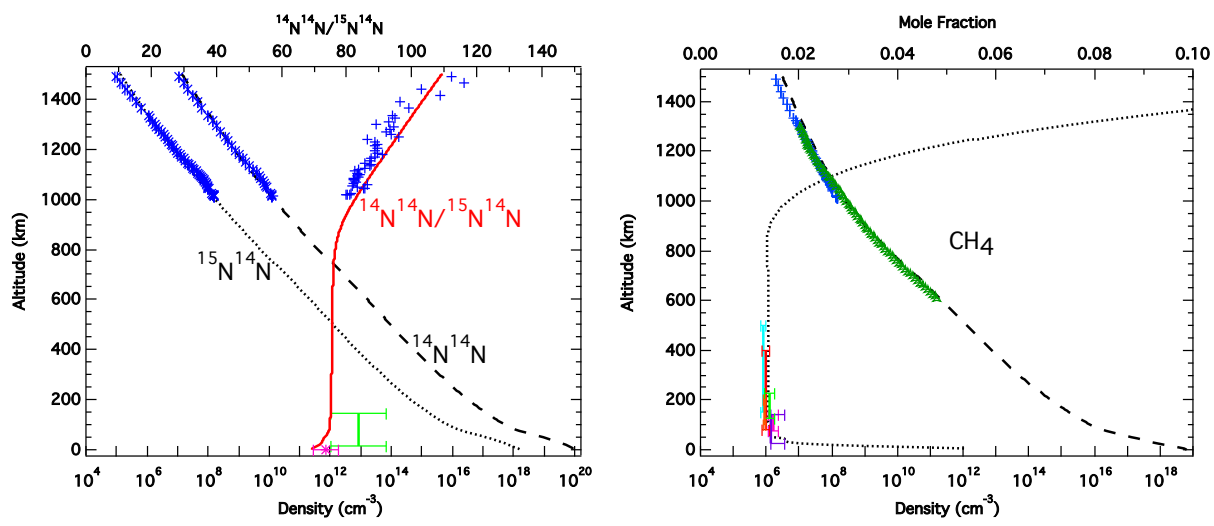

Figure 1: Density of the main constituents (model input and observations) versus altitude. Left panel: ${ }^{28} \mathrm{~N}_{2}$ and ${ }^{14} \mathrm{~N}^{15} \mathrm{~N}$ density, and ${ }^{14} \mathrm{~N}^{14} \mathrm{~N} /{ }^{14} \mathrm{~N}^{15} \mathrm{~N}$ ratio. The observations in the thermosphere are INMS data, while observations in the stratosphere are GCMS data from Niemann et al. (2010). Right Panel: Density (dashed line) and mole fraction (dotted line) of $\mathrm{CH}_{4}$. The observations are listed in Table 1.

The $\mathrm{CH}_{4}$ density model is based on the mixing ratios measured with GCMS, CIRS, and DISR in the lower atmosphere reaching a value of 0.014 above the cold trap near $70 \mathrm{~km}$. The mole fraction remains constant from that altitude to the lower thermosphere, near $800 \mathrm{~km}$, where it starts to increase with altitude due to diffusive separation. The strength of diffusive separation, i.e. the eddy diffusion coefficient, is determined from the ${ }^{40} \mathrm{Ar}$ profile (shown in Figure 2). It has been a puzzle that this eddy coefficient over predicts the speed of diffusive separation of $\mathrm{CH}_{4}$. Several authors have interpreted this as evidence for enhanced escape of $\mathrm{CH}_{4}$ (Yelle et al., 2008; Strobel, 2008; Cui et al., 2012), but the physical mechanisms for enhanced escape are not understood (Tucker et al., 2016). We do not further investigate this dilemma here but simply impose an enhanced escape velocity at the upper boundary in order to produce a $\mathrm{CH}_{4}$ distribution 
Table 1: Summary of the observational data on Titan's methane composition.

\begin{tabular}{llll}
\hline \hline Altitude $(\mathrm{km})$ & Mixing Ratio (\%) & Instrument & Reference \\
\hline \hline $600-1200$ & & UVIS & Koskinen et al. (2011) \\
$800-1300$ & & UVIS & Stevens et al. (2015) \\
$150-500$ & $1.28 \pm 0.06$ & VIMS & Maltagliati et al. (2015) \\
$\sim 80-400$ & $1.33 \pm 0.07$ & Herschel-SPIRE & Courtin et al. (2011) \\
$\sim 100-200$ & $1.29 \pm 0.03$ & Herschel-PACS & Rengel et al. (2014) \\
$125-225$ & $1.41 \pm 0.1^{(a)}$ & CIRS & Lellouch et al. (2014) \\
$75-140$ & $1.48 \pm 0.09$ & GCMS & Niemann et al. (2010) \\
$27-141$ & $1.44(+0.27 /-0.11)$ & DISR-ULIS & Bézard (2014) \\
\hline \hline
\end{tabular}

${ }^{(a)}$ At $30^{\circ} \mathrm{N}$.

that matches the observations.

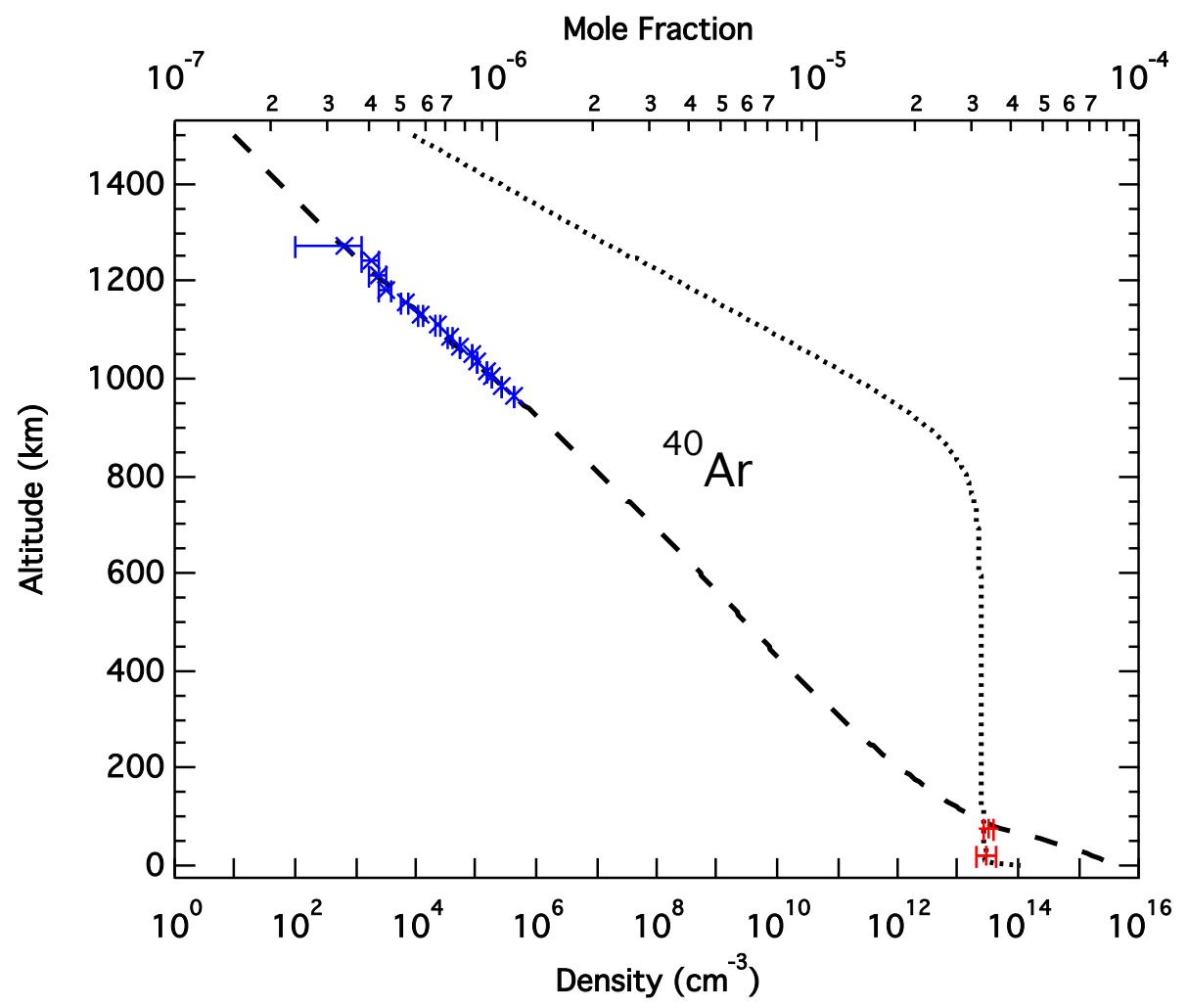

Figure 2: Density (dashed line) and mole fraction (dotted line) of ${ }^{40} \mathrm{Ar}$ (model input and observations) versus altitude. The observations in the thermosphere (blue) are INMS data, while observations in the stratosphere (red) are GCMS data from Niemann et al. (2010).

For some of the models discussed here we also hold the $\mathrm{CO}$ profile constant. 
As discussed in section 4.2.3.3, the lifetime of CO in Titan's atmosphere is much longer than the vertical mixing time. For the models with CO held constant, we fix the mole fraction at the surface to the observed value of $50 \mathrm{ppm}$ and calculate a diffusive equilibrium profile under the effects of molecular and eddy diffusion (cf. Figure 59).

\subsubsection{Electron Temperature}

The electron temperature profile also affects the chemistry on Titan, primarily through the temperature dependence of the electron-ion recombination rate coefficient. Electron temperatures on Titan have been measured with the Langmuir Probe (LP) channel of the Radio and Plasma Wave System (RPWS) instrument (Wahlund et al., 2005). As with the neutral temperature profile we construct an electron temperature profile that is smoothly varying and consistent with the general trends of the LP measurements. The electron temperature rises rapidly through the main ionosphere, reaching values of $\sim 1000 \mathrm{~K}$ near the exobase at $\sim 1400 \mathrm{~km}$. At lower altitudes, where the collision rate is large, the electron temperature should be equal to the neutral temperature (Richard et al., 2011). Langmuir probes are not able to measure temperatures this low. We therefore set the electron temperature equal to the neutral temperature at altitudes below $800 \mathrm{~km}$ and have it increase linearly with altitude throughout the thermosphere at a rate that is consistent with the LP measurements. Results are shown in Figure 3. 


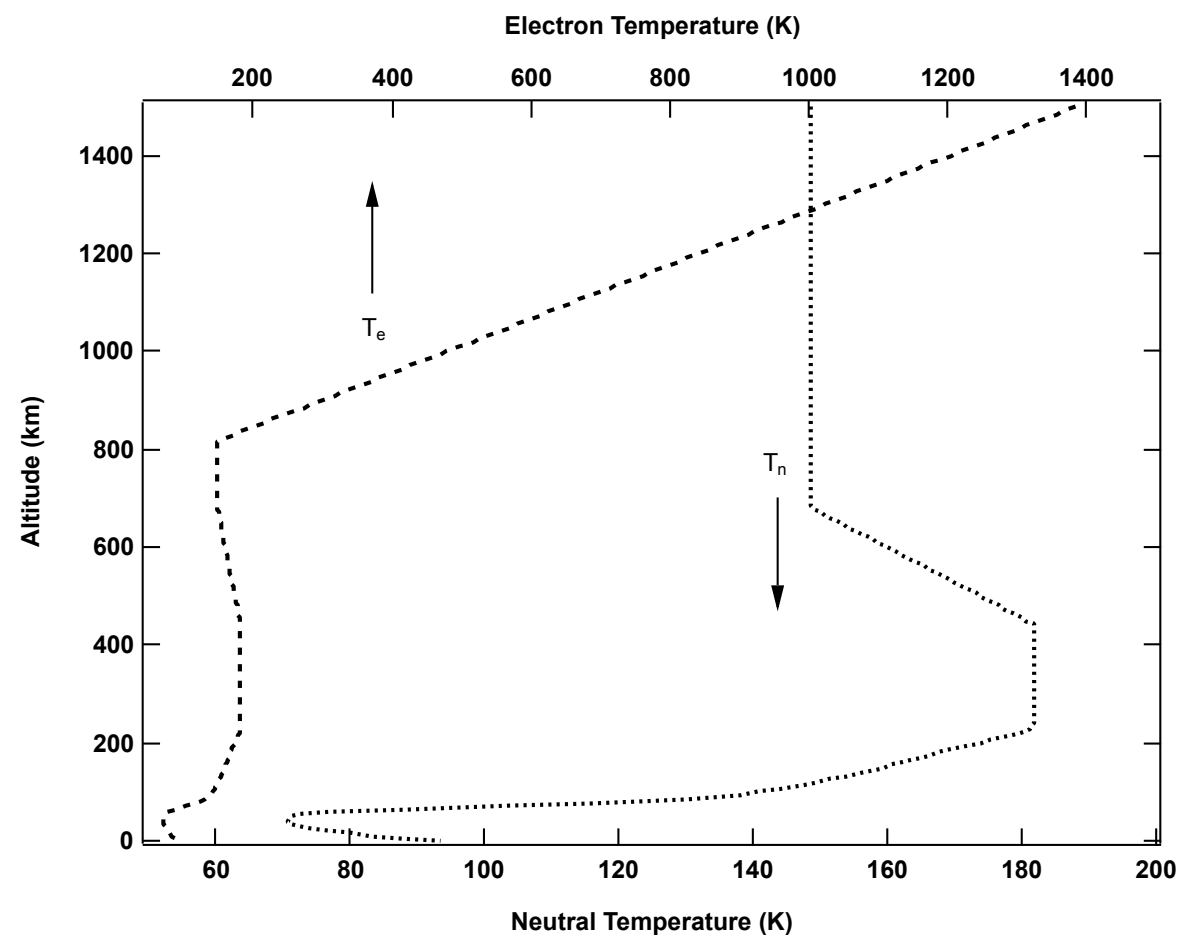

Figure 3: The neutral (lower axis) and electron temperatures (upper axis) used as model inputs. Note that the scales for $\mathrm{T}_{n}$ and $\mathrm{T}_{e}$ are different and that as a consequence, both temperatures do not overlap below $800 \mathrm{~km}$, even though they are set equal.

\subsection{Boundary Conditions}

Most of the photochemically active species in Titan's atmosphere are too heavy to escape from the top of the atmosphere. The exceptions are atomic and molecular hydrogen and for these species we set the velocity at the top of the model equal to the Jeans escape velocity, i.e. $1.5 \times 10^{4} \mathrm{~cm}^{-2} \mathrm{~s}^{-1}$ and $1.52 \times 10^{3}$ $\mathrm{cm}^{-2} \mathrm{~s}^{-1}$, respectively. We use a zero velocity upper boundary condition for all other species. As mentioned earlier, although $\mathrm{CH}_{4}$ may be escaping, we treat its distribution as fixed and it therefore requires no boundary condition.

Most of the photochemical species in Titan's atmosphere will condense near the tropopause (discussed in section 5.3.4) and the densities at lower altitudes become vanishingly small. Surface boundary conditions for these species are therefore not particularly important. We specify a downward velocity of $10^{3} \mathrm{~cm}$ $\mathrm{s}^{-1}$ to simulate condensation on the surface, but these species have all condensed before they reach the surface. The exceptions are $\mathrm{H}_{2}$ and $\mathrm{CO}$ which do not condense at Titan temperatures. For these species we specify zero velocity at the surface.

As discussed in section 4.2.3.3, the influx of oxygen species from the external environment into Titan's atmosphere has a significant affect on the chemistry. English et al. (1996) investigated the delivery of water into Titan's atmosphere 
by precipitation of micrometeorites and calculated that peak ablation occurs around $750 \mathrm{~km}$ resulting in an integrated $\mathrm{H}_{2} \mathrm{O}$ deposition rate of $2.5 \times 10^{6}$ $\mathrm{cm}^{-2} \mathrm{~s}^{-1}$. CAPS detected high energy $(\sim 1 \mathrm{keV}) \mathrm{O}^{+}$ions precipitating from Saturn's magnetosphere into Titan's atmosphere, with a flux of $\sim 10^{6} \mathrm{~cm}^{-2}$ $\mathrm{s}^{-1}$ during flyby TA (Hartle et al., 2006). They mostly charge transfer with neutrals near $1100 \mathrm{~km}$, forming a flux of thermal $\mathrm{O}$ atoms. In our previous work (Hörst et al., 2008) we modeled the $\mathrm{O}$ input to the atmosphere by specifying downward fluxes at the upper boundary. In the current model we specify an internal production profile for the incoming species, modeled as a Chapman function. The magnitude of the production rate is adjusted so that the columnintegrated rate is equal to the expected input fluxes of $\mathrm{O}$ and $\mathrm{OH}$ to try to reproduce the observed abundances of $\mathrm{CO}, \mathrm{CO}_{2}$ and $\mathrm{H}_{2} \mathrm{O}$.

\subsection{Vertical Transport}

The bulk of Titan's ionosphere is in chemical equilibrium and ambipolar diffusion can be ignored below 1200 to $1400 \mathrm{~km}$ (cf. Figure 6 in Cravens et al. (2009)). This includes the region with interesting ion chemistry and where ion chemistry produces neutrals. The vertical flux for neutral species is given by

$$
\begin{aligned}
\Phi_{i}=-D_{i}\left(\frac{\partial N_{i}}{\partial z}+\frac{N_{i}}{H_{i}}+\frac{N_{i}\left(1+\alpha_{i}\right)}{T} \frac{d T}{d z}\right) & \\
& -K\left(\frac{\partial N_{i}}{\partial z}+\frac{N_{i}}{H_{a}}+\frac{N_{i}}{T} \frac{d T}{d z}\right)
\end{aligned}
$$

where $D_{i}$ and $\alpha_{i}$ are the molecular and thermal diffusion coefficient of species $i$ through the background $\mathrm{N}_{2}$ atmosphere, $\mathrm{T}$ is the temperature, $K$ is the eddy mixing coefficient that parameterizes large-scale motions, and $H_{i}$ is the scale height of species $i$

$$
H_{i}=\frac{R T}{m_{i} g}
$$

where $R$ is the gas constant, $m_{i}$ the molecular weight of species $i$ and $g$ the gravity. $H_{a}$ is the scale height of the background atmosphere.

The binary molecular diffusion coefficient $D_{i}\left(\mathrm{~cm}^{2} \mathrm{~s}^{-1}\right)$ of a molecule $i$ in $\mathrm{N}_{2}$ is

$$
D_{i}=\frac{b_{i}}{N_{a}}
$$

with $N_{a}$ the density of $\mathrm{N}_{2}\left(\mathrm{~cm}^{3}\right) . \quad b_{i}$ are model input parameters that are estimated for each constituent from experimental and theoretical information (cf. Appendix B.1).

An important step in one-dimensional photochemical models is the choice of an eddy mixing coefficient, $K(z)$, for the inclusion of turbulent diffusion effects on the vertical distribution of long-lived species. This parameter varies with 
altitude and since it is difficult to predict from first principles, it is usually left as a free parameter that has to be adjusted to best match constituent observations.

It has long been recognized that $K(z)$ must be small in the lower stratosphere to reproduce the observed high levels of minor species above the condensation region. Essentially, just above the condensation region $K(z)$ must be very low so there is a barrier between the sources at high altitude and the sink at low altitude, resulting in a buildup of large densities. It is one of the main reasons for this complex chemistry on Titan. This requirement was implicit in the Yung et al. (1984) photochemical model and has been assumed in all subsequent models but has received little discussion.

Measurements of the mole fraction of ${ }^{40} \mathrm{Ar}$ by GCMS just above the surface and by INMS around $1000 \mathrm{~km}$ provided the required constraints to render the determination of $K(z)$ relatively robust. ${ }^{40} \mathrm{Ar}$ being an inert species, its vertical profile is entirely governed by dynamics without any spurious effects from chemistry and it is expected to be depleted as a heavy species above the homopause and uniformly mixed below (Yelle et al., 2008). Another species that has been measured by both GCMS and INMS is ${ }^{15} \mathrm{~N}^{14} \mathrm{~N}$ and it has also been used as an independent tracer species for constraining the homopause altitude and the eddy mixing profile (Bell et al., 2011; Mandt et al., 2012b). This determination however depends sensitively on the inter calibration of INMS and GCMS and, as a consequence, is far less precise than use of ${ }^{40} \mathrm{Ar}$. We note that the uncertainty in the ${ }^{40} \mathrm{Ar}$ densities shown in Figure 2 are far smaller than those displayed in Bell et al. (2011) and Mandt et al. (2012b). This is because the densities in Figure 2 are a mean of the measurements and the uncertainty on the mean is quite small. Bell et al. (2011) and Mandt et al. (2012b) confuse geographic variability and uncertainty. The assumption behind our approach is that by averaging over all INMS measurements we obtain and average over the spectrum of eddies in the atmosphere, which is the requirement for an eddy mixing approach to diffusion. In this view, the uncertainty in mean profile is far smaller than the geographic/temporal variability of the densities and the eddy diffusion coefficients in Titan's upper atmosphere is accurately constrained.

In order to find the best tracer(s) for determining the eddy mixing coefficient in the stratosphere, we calculate the chemical and transport time constants for $\mathrm{C}_{2} \mathrm{H}_{6}, \mathrm{C}_{2} \mathrm{H}_{2}, \mathrm{H}_{2} \mathrm{O}$ and $\mathrm{HCN}$. The chemical time constant is estimated from $t_{C}=N_{i} / L_{i}$ where $N_{i}$ is the species density $\left(\right.$ in $\mathrm{cm}^{-3}$ ) and $L_{i}$ is the chemical loss rate $\left(\right.$ in $\mathrm{cm}^{-3} \mathrm{~s}^{-1}$ ). The time constant characterizing transport (diffusion and eddy mixing) is estimated through $t_{K}=N_{i} /\left|\vec{\nabla} \cdot \vec{F}_{i}\right|$, where $\vec{\nabla} \cdot \vec{F}_{i}$ is the divergence of the vertical flux (in $\mathrm{cm}^{-3} \mathrm{~s}^{-1}$ ). Results are presented in Figure 4. We find that vertical transport dominates the $\mathrm{C}_{2} \mathrm{H}_{2}$ loss only in the 800$400 \mathrm{~km}$ altitude range, while it dominates the $\mathrm{C}_{2} \mathrm{H}_{6}$ loss from $800 \mathrm{~km}$ down to the tropopause. Moreover, $\mathrm{C}_{2} \mathrm{H}_{6}$ does not exhibit much latitudinal variation in the stratosphere (cf. upper left panel of Figure 44), making $\mathrm{C}_{2} \mathrm{H}_{6}$ a better tracer than $\mathrm{C}_{2} \mathrm{H}_{2} \cdot \mathrm{H}_{2} \mathrm{O}$ is another good tracer for vertical mixing in the lower atmosphere. 

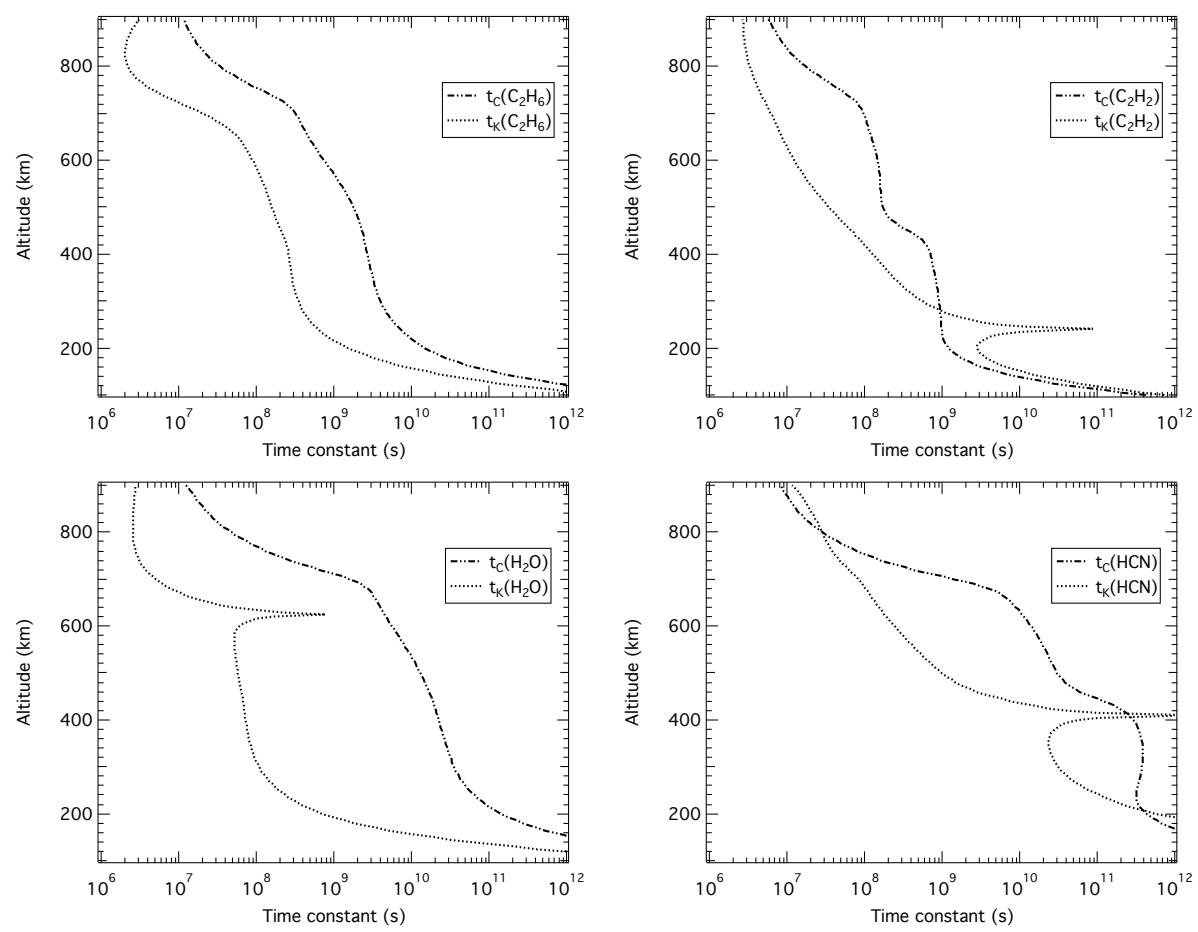

Figure 4: Chemical $\left(\mathrm{t}_{C}\right)$ and transport $\left(\mathrm{t}_{K}\right)$ time constant of $\mathrm{C}_{2} \mathrm{H}_{6}, \mathrm{C}_{2} \mathrm{H}_{2}, \mathrm{H}_{2} \mathrm{O}$ and $\mathrm{HCN}$ versus altitude. These are results of our nominal model that are used to illustrate our choice of $\mathrm{C}_{2} \mathrm{H}_{6}$ as the best tracer. The spikes visible for $\mathrm{t}_{K}$ correspond to changes of the sign of the divergence of the vertical flux, i.e. to switches from production to loss of the transport term.

We computed the vertical profiles of the main neutral species with several possible profiles of the eddy mixing coefficient, obtained by varying $K_{0}$, the lower atmosphere value, using as a starting point the values given in Yelle et al. (2008) and Hörst et al. (2008). We model the eddy mixing profile with:

$$
K(z)=\frac{K_{0}\left(p_{0} / p\right)^{\gamma} K_{\infty}}{K_{0}\left(p_{0} / p\right)^{\gamma}+K_{\infty}},
$$

where the subscript 0 refers to the value at $10^{5} \mathrm{~Pa}$ and the subscript $\infty$ to the asymptotic value in the upper atmosphere. We determine that $K_{0}=300 \mathrm{~cm}^{2}$ $\mathrm{s}^{-1}, K_{\infty}=3 \times 10^{7} \mathrm{~cm}^{2} \mathrm{~s}^{-1}, p_{0}=10^{5} \mathrm{~Pa}$ and $\gamma=1.5$ provide the best fit. The resulting nominal profile $\left(\mathrm{K}_{\text {nom }}\right)$, along with the methane molecular diffusion coefficient profile $\left(\mathrm{D}_{\mathrm{CH} 4}\right)$, is shown in Figure 5, corresponding to a homopause level of $875 \mathrm{~km}$. Results of the calculations with several possible $K_{0}$ are given for simple hydrocarbons $\left(\mathrm{C}_{2} \mathrm{H}_{2}, \mathrm{C}_{2} \mathrm{H}_{6}, \mathrm{C}_{3} \mathrm{H}_{8}\right)$ and $\mathrm{HCN}$, and compared with observational determinations in Figures 44, 45 and 51. 


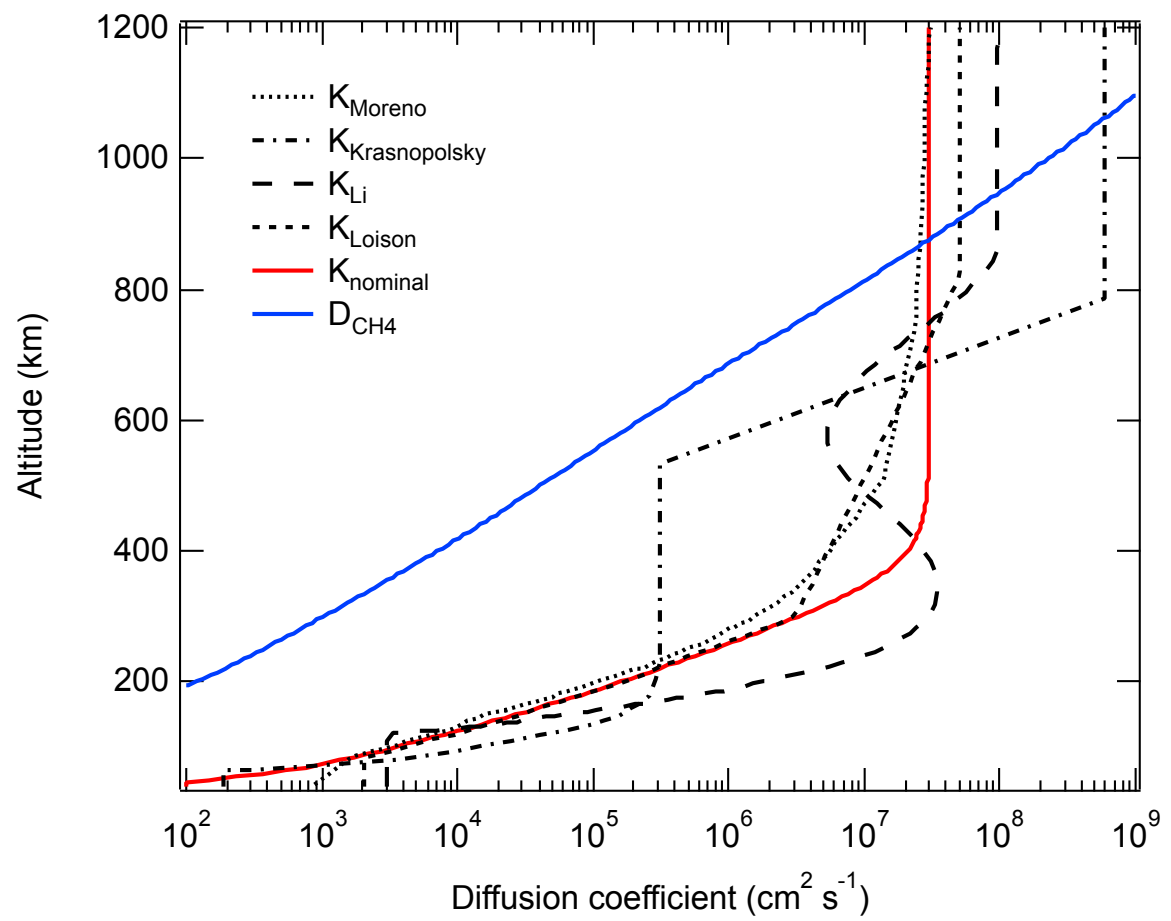

Figure 5: Plots of the nominal eddy mixing profile (solid red line) and methane molecular diffusion profile (solid blue line) used as model inputs. Also included are eddy mixing profiles from various photochemical models - Moreno et al. (2012) K2 (dotted line), Krasnopolsky (2012, 2014) (dot dashed line), Li et al. (2014) (long dashed line) and Loison et al. (2015) (short dashed line).

Before Cassini, a large variety of eddy mixing profiles were put forward (cf. Figure 3 of Lavvas et al. (2008b) for example). The methane vertical profile was used to retrieve the location of the homopause and the extent of turbulent mixing in the thermosphere, while hydrogen cyanide was used for the determination of mixing in the stratosphere. Nevertheless, the observations were equivocal and could not efficiently constrain $K(z)$ in the upper atmosphere. Vinatier et al. (2007a) suggested using the CIRS ethane values to constrain the eddy mixing coefficient in the stratosphere. As a saturated hydrocarbon, it has a low chemical reactivity and because its absorption cross-section in the UV region overlaps with that of methane, it is not efficiently photodissociated. The exact method is detailed in Lavvas et al. (2008b) and was used in most of the subsequent models, sometimes also adding $\mathrm{H}_{2} \mathrm{O}$ as a tracer species (Hörst et al., 2008; Moreno et al., 2012; Loison et al., 2015). Krasnopolsky (2009, 2012) used a different approach where the eddy mixing profile is described by six parameters (three breakpoints altitudes and values) that are chosen to minimize the mean difference between the model and 63 observational points. The resulting profile differs significantly from all the other profiles with the homopause located at 
$1000 \mathrm{~km}$ instead of $\sim 800 \mathrm{~km}$ (cf. Figure 5). Li et al. (2014) developed an optimization method to retrieve the eddy mixing profile, with acetylene as the tracer species. They base their choice of $\mathrm{C}_{2} \mathrm{H}_{2}$ on the low sensitivity of its abundance to the uncertainties in reaction rate coefficients, which is attributed to the dominant effect of vertical transport. The retrieved eddy profile features are similar to the other profiles at the exception of a low eddy diffusion zone near $550 \mathrm{~km}$ (cf. Figure 5), which the authors rationalize by aerosol heating in the detached haze layer. Another interpretation of this feature is that it is actually a wave (Koskinen et al., 2011), in which case the feature should not be interpreted in terms of $K(z)$. The retrieved eddy mixing profiles are presented in Figure 5.

\subsection{Condensation}

Because of the cold temperatures in Titan's lower stratosphere, many gases become saturated and condense according to their saturation vapor pressures. We treat condensation in an empirical manner by specifying a chemical production/loss rate that is proportional to the difference between the partial pressure of the constituent and its saturation vapor pressure at the local atmospheric temperature. We determine a proportionality constant of $10^{6} \mathrm{erg}^{-1} \mathrm{~s}^{-1}$ through trial and error. This value ensures that the partial pressure never significantly exceeds the saturation vapor pressure, nor does it caused numerical stability problems. The reader is referred to Lavvas et al. (2011b) for a discussion of the detailed physics of the condensation process and its effect on the constituent densities in the troposphere. The saturation expressions governing condensation are given for each neutral molecule in Table B.2.

\subsection{Energy Input}

Ionization and dissociation sources in the atmosphere of Titan include solar extreme ultraviolet (EUV) and ultraviolet (UV) radiation, energetic photoelectrons (produced by solar X-ray and EUV radiation) and Saturnian magnetospheric electrons and ions. On the dayside, the dominant energy source is solar UV radiation and associated photoelectrons (Lavvas et al., 2011a). Saturnian magnetospheric electrons are dominant on the nightside but their contribution is always much lower than solar radiation on the dayside ( $\AA$ gren et al., 2009; Galand et al., 2010). Cravens et al. (2008) demonstrate that energetic protons and oxygen ions can also ionize the neutral species between 500 and $1000 \mathrm{~km}$ altitude but their contribution is small under typical conditions. Molina-Cuberos et al. (2001) predict meteoritic ablation in the altitude range $600-800 \mathrm{~km}$ and creation of long-lived metallic ions but this represents a weak source of ionization overall. Galactic cosmic rays (GCR) generate some ionization in the lower atmosphere, with a peak contribution around $100 \mathrm{~km}$ and a magnitude comparable to ionization by solar photons in the upper atmosphere (Gronoff et al., 2009). Our model accounts for solar UV and EUV photons, photoelectrons and cosmic rays but neglects magnetospheric electrons and protons. Although oxygen ions and meteorite influx of $\mathrm{H}_{2} \mathrm{O}$ are considered as a source of oxygen (section 2.3), they do not contribute to the overall ionization. 


\subsubsection{Photons}

The model includes photolysis driven by absorption of solar photons between 0 and $300 \mathrm{~nm}$. We use the TIMED/SEE level 3 data to represent the solar flux at wavelengths from 0-80 $\mathrm{nm}$ and 100-200 $\mathrm{nm}$ (Woods et al., 2005). The solar flux and photoabsorption cross sections in these regions are tabulated on a 1 $\mathrm{nm}$ wavelength grid. When cross sections are available at higher spectral resolution they are averaged to a $1 \mathrm{~nm}$ resolution for consistency with the solar spectrum. This procedure is followed except between 80 and $100 \mathrm{~nm}$, where accurate representation of the high resolution structure of the $\mathrm{N}_{2}$ cross section is essential. In this region, we use the SOHO/SUMER whole disc spectra (Curdt et al., 2013) to represent the shape of the solar spectrum. The SOHO/SUMER spectrum is tabulated at a sampling of $0.0045 \mathrm{~nm}$. For consistency with lower and higher wavelengths, we normalize the net flux in the $80-100 \mathrm{~nm}$ region for the SOHO/SUMER spectrum to be equal to that in the lower resolution TIMED/SEE spectrum. We use the SOURCE SOLSTICE solar spectrum between 200 and $300 \mathrm{~nm}$ (Snow et al., 2005).

Cassini arrived at Saturn near the end of solar cycle 23 and the majority of the observations of Titan were made during the extended solar minimum between cycles 23 and 24. For most of the models presented here we use TIMED/SEE spectra for 5 January 2008, the date of the T40 encounter. The solar F10.7 index on this date was $75 \mathrm{sfu}$ (Solar Flux Unit), close to its lowest value. In section 5.5 we also consider the effects of higher solar activity levels on Titan photochemistry and for these studies we use the solar spectrum for 7 April 2014, the date of the T100 encounter, when the F10.7 index was 109 sfu (cf. Figure 6). 


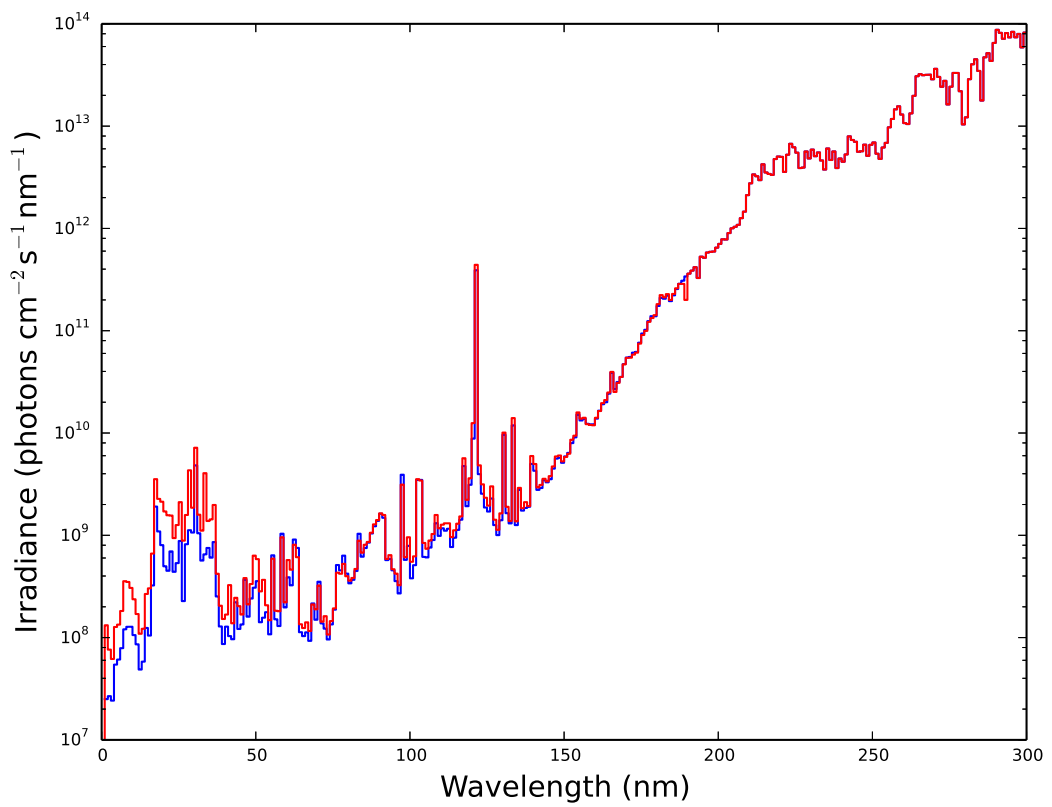

Figure 6: Solar irradiance at $1 \mathrm{AU}$ (photons $\mathrm{cm}^{-2} \mathrm{~s}^{-1} \mathrm{~nm}^{-1}$ ) versus wavelength (nm) used as model input. They were recorded on 5 January 2008, corresponding to solar minimum conditions (blue line) and 7 April 2014, corresponding to solar maximum conditions (red line).

Photolysis rate coefficients are given by

$$
J_{l}^{i}(z)=\int_{\lambda} R_{l}^{i}(\lambda) \sigma_{l}(\lambda) I(z, \lambda) d \lambda
$$

where $\lambda$ is the wavelength, $\sigma_{l}(\lambda)$ is the absorption/ionization cross-section of species $l$ as a function of wavelength, $R_{l}^{i}(\lambda)$ is the branching ratio of the channel leading to $i$ and $I(z, \lambda)$ is the solar flux at altitude $\mathrm{z}$ and wavelength $\lambda$. To reproduce global mean conditions, all photolysis rates are calculated for a solar zenith angle of $60^{\circ}$ and the solar flux at the top of the atmosphere is divided by 2 for diurnal averaging. This ensures that the column-integrated photolysis rates are $\frac{1}{4}$ the sub-solar values, as they should be for a global average.

\subsubsection{Photoelectrons}

The photoionization of a neutral species leads to the production of a positive ion along with a free electron (photoelectron). Suprathermal photoelectrons collide with neutral species, producing secondary ionization events and the cascade continues until the electrons have energies smaller than the ionization thresholds of the neutral species. Our calculations include photoelectron energy degradation using a local energy deposition model, which is described in Lavvas et al. 
(2011a). The model has been validated against the electron fluxes measured by CAPS and the far ultraviolet (FUV) emissions detected by UVIS. The resulting differential flux for electrons of less than $10 \mathrm{eV}$ deviates from the CAPS observations for altitudes above $1200 \mathrm{~km}$, where the effects of electron transport are no longer negligible (cf. Figure 5a in Lavvas et al. (2011a)). However, the secondary electron production rates, with and without suprathermal electron transport, only differ by a factor of a few up to $1400 \mathrm{~km}$ (cf. Figure 5b in Lavvas et al. (2011a)). The calculated differential electron fluxes used as input in the model are presented in Figure 7 at several altitudes.

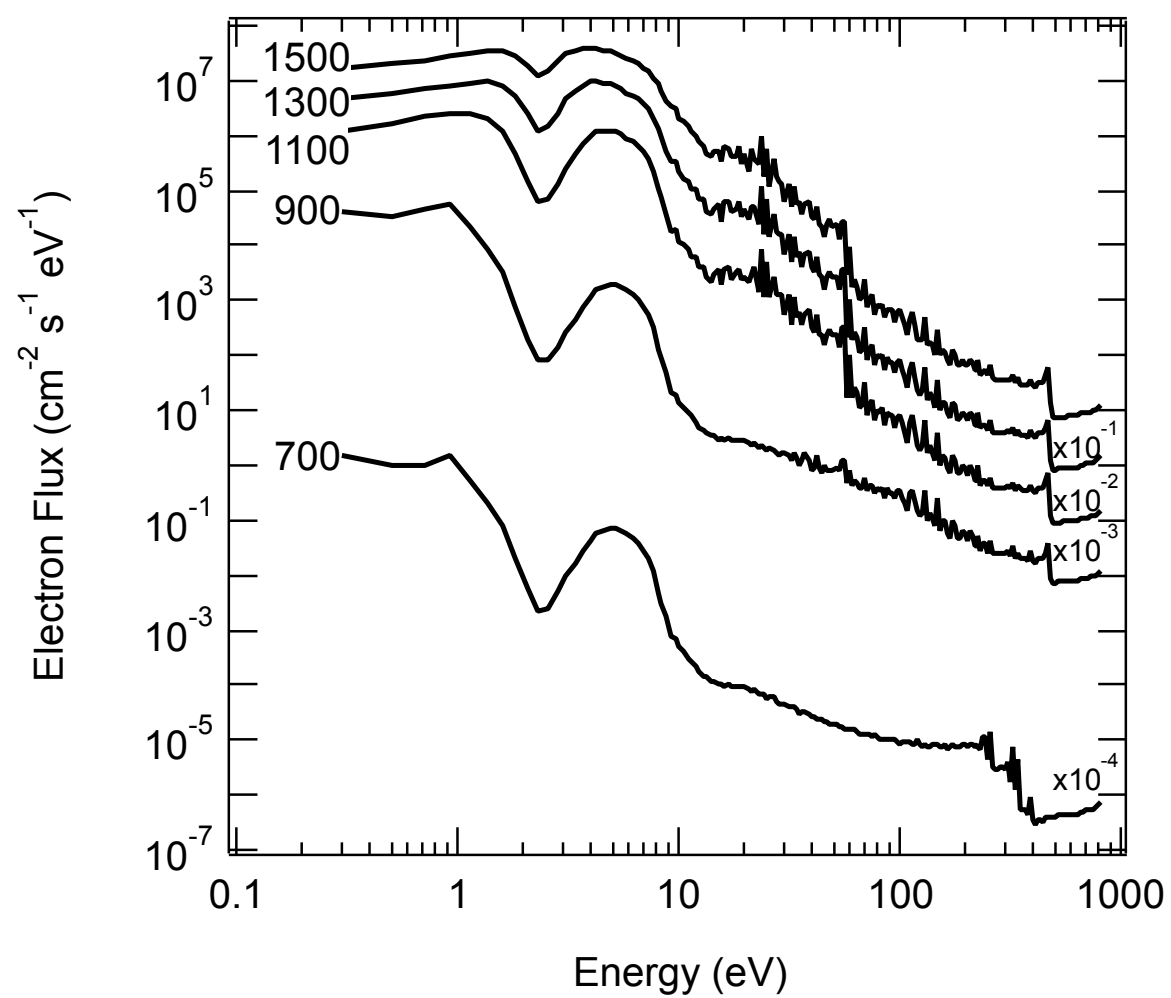

Figure 7: Calculated differential electron flux $\left(\mathrm{cm}^{-2} \mathrm{~s}^{-1} \mathrm{eV}^{-1}\right)$ versus energy $(\mathrm{eV})$ at 1500 , $1300,1100,900$ and $700 \mathrm{~km}$.

\subsubsection{Cross Sections and Branching Ratios}

2.6.3.1. Dissociation and Ionization. We include in our model the photolysis of 47 species. The calculated rates of photodissociation $J_{l}^{i}(z)$ (equation E8) rely on the knowledge of their absorption cross-sections and branching ratios. They are obtained through dedicated laboratory experiments and/or theoretical calculations. The cross-sections and branching ratios used are listed in Tables B.5, B.6, B.7, B.8 and B.9. We describe the dissociation and ionization of $\mathrm{N}_{2}$ and $\mathrm{CH}_{4}$ by both photons and photoelectrons from their dissociation threshold 
(100 and $160 \mathrm{~nm}$, respectively) to 1.5 and $11 \mathrm{~nm}$, respectively. CO is largely shielded from photolysis by $\mathrm{N}_{2}$ and $\mathrm{CH}_{4}$ but we do include photodissociation and photoionization below $100 \mathrm{~nm}$ while predissociation in the electronic band systems is not included. We also ignore $\mathrm{H}_{2}$ photodissociation. $\mathrm{H}_{2}$ photodissociation occurs through predissociation in highly structured electronic bands, like $\mathrm{N}_{2}$. Most of the photons will be absorbed in the more abundant $\mathrm{N}_{2}$ and $\mathrm{CH}_{4}$. Moreover, considering that $\mathrm{CH}_{4}$ photolysis will produce far more $\mathrm{H}$ than $\mathrm{H}_{2}$ photolysis we choose to neglect $\mathrm{H}_{2}$ photodissociation. Because its photolysis cross-section is essentially unknown, we do not include either photodissociation or photoisomerization of HNC in the model. HNC is highly reactive with all radicals and photolysis is not expected to be a major loss for this species (cf. discussion in Petrie (2001)). Hébrard et al. (2012) find that photolysis is a minor loss for HNC, assuming identical cross-sections and branching ratios as for HCN. Photodissociation of other stable neutral molecules is considered between their dissociation threshold and their ionization threshold. Again, nitrogen and methane largely dominate the photon absorption in the region where ionization of minor species becomes possible (typically below $145 \mathrm{~nm}$, cf. Figure 15) and the latter is therefore not included. In some cases $\left(\mathrm{HC}_{3} \mathrm{~N}, \mathrm{C}_{2} \mathrm{~N}_{2}, \mathrm{C}_{4} \mathrm{~N}_{2}\right)$, crosssections are not available for the entire wavelength range and we fill in the gap in the cross-sections by performing a linear interpolation between the available datasets.

The cross-sections and branching ratios are often uncertain for several reasons:

- Cross-sections are only known for a limited wavelength range, leading to some systematic underestimation of the rate of photodissociation. For example, Gladstone et al. (1996) points out that $\mathrm{CH}_{3}$ cross-sections are only available around 215 and $150 \mathrm{~nm}$ although photodissociation at shorter wavelengths is expected. Another case is the lack of data at longer wavelength for molecules that have a monotonically decreasing cross-section. The solar flux increases rapidly in this wavelength range and even small cross-sections can still substantially contribute to the total photodissociation. For example, Bénilan et al. (2000) showed that $25 \%$ of the rate of dissociation of $\mathrm{C}_{2} \mathrm{H}_{2}$ is due to absorption above $205 \mathrm{~nm}$, even though the $\mathrm{C}_{2} \mathrm{H}_{2}$ cross-section decreases by four orders of magnitude between 150 and $220 \mathrm{~nm}$.

- The temperature dependence of cross-sections is unavailable, leading to some uncertainty in the rate of photodissociation. Cross-sections at $150 \mathrm{~K}$ typically vary by tens of percents from the room temperature values, where the majority of the cross-section data are measured (Jolly and Bénilan (2008) and references therein). For our calculations, we utilize the values obtained at the available temperature the closest to $150 \mathrm{~K}$. The exact temperature is indicated for each dataset in Table B.6.

- The determination of photo fragments is complex and there often are huge discrepancies between branching ratios measured by different teams. A 
most illuminating example is that of $\mathrm{CH}_{4}$ for which the branching ratios at Lyman- $\alpha$ (121.6 nm) have been a subject of debate for more than 50 years, as discussed in the review of laboratory experiments by Smith and Raulin (1999) and further below. Although fragments are known to be wavelength dependent, branching ratios are in the best case scenario available at only a few specific wavelengths and have to be extrapolated over the entire wavelength range, adding some huge uncertainty. As an example of highly unconstrained branching ratios, see the discussion about $\mathrm{C}_{3} \mathrm{H}_{6}$ further below.

The photochemistry of stable molecules has been studied in previous works (Hörst et al., 2008; Lavvas et al., 2008a; Krasnopolsky, 2009; Hébrard et al., 2013) and will not be discussed in detail here, except where revision is needed either because newer data have become available or because species have recently been detected on Titan and deserve some more scrutiny. We specifically discuss below the cross-sections and branching ratios for nine species: $\mathrm{N}_{2},{ }^{14} \mathrm{~N}^{15} \mathrm{~N}, \mathrm{CH}_{4}$, $\mathrm{C}_{2} \mathrm{H}_{2}, \mathrm{C}_{3} \mathrm{H}_{6}, \mathrm{C}_{4} \mathrm{H}_{2}, \mathrm{HC}_{3} \mathrm{~N}, \mathrm{C}_{2} \mathrm{H}_{3} \mathrm{CN}$ and $\mathrm{C}_{2} \mathrm{H}_{5} \mathrm{CN}$.

$\mathbf{N}_{2}$ and ${ }^{14} \mathbf{N}^{15} \mathbf{N}$. The photodissociation and photoionization of ${ }^{28} \mathrm{~N}_{2}$ have already been extensively discussed in Lavvas et al. (2011a) and a similar scheme is being used here (cf. Figure 8a). Table B.5 lists the adiabatic energy thresholds for the $\mathrm{N}_{2}$ dissociation and ionization channels and the references used to establish their branching ratios and cross-sections.
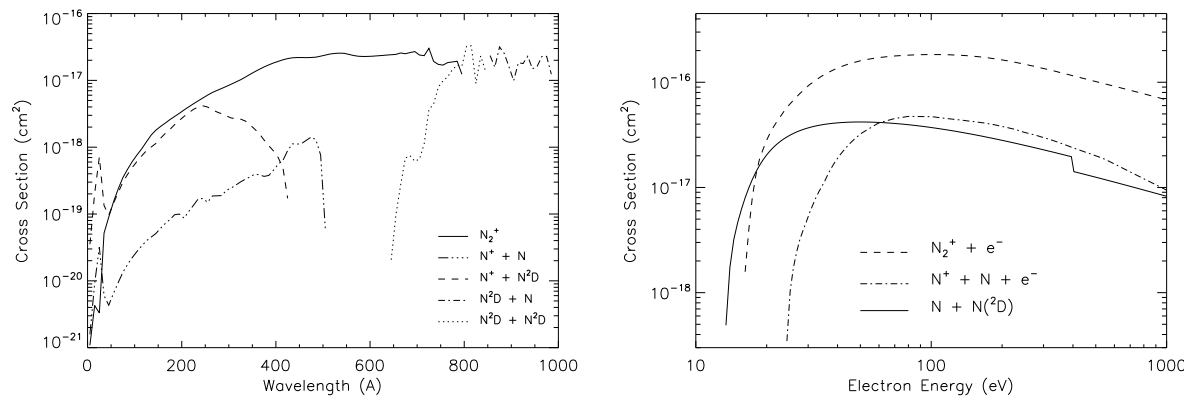

Figure 8: $\mathrm{N}_{2}$ cross-sections for ionization and dissociation used as model inputs. (a) photons, (b) electrons.

Briefly, although the dissociation energy of $\mathrm{N}_{2}$ is at $127 \mathrm{~nm}$, absorption becomes significant only near $100 \mathrm{~nm}$, mainly through predissociation transitions to Rydberg and valence states. These states have long enough lifetimes to display rotational and vibrational structures. The vibrational bands of the two isotopes ${ }^{28} \mathrm{~N}_{2}$ and ${ }^{29} \mathrm{~N}_{2}$ can be shifted by tens of wavenumbers and as the solar spectrum is also highly structured in this spectral region (cf. Figure 6), the radiative transfer calculation requires photoabsorption, photodissociation and photoionization cross-sections of high spectral resolution for both ${ }^{28} \mathrm{~N}_{2}$ and 
${ }^{29} \mathrm{~N}_{2}$. Isotope-selective shielding has been proposed to explain the ${ }^{15} \mathrm{~N}$ enrichment observed in $\mathrm{HCN}$ compared to $\mathrm{N}_{2}$ and to impact the vertical profiles of some minor species such as $\mathrm{C}_{2} \mathrm{H}_{6}$ and $\mathrm{HCN}$ (Liang et al., 2007; Luspay-Kuti et al., 2015; Dobrijevic et al., 2016).

Down to $79.5 \mathrm{~nm},{ }^{28} \mathrm{~N}_{2}$ and ${ }^{29} \mathrm{~N}_{2}$ cross-sections are computed at $150 \mathrm{~K}$ using a coupled-channel Schrödinger equation quantum-mechanical model (Heays et al., 2011) and are presented in Figure 9. However, their accuracy decreases at shorter wavelengths and lower resolution laboratory measurements (Samson et al., 1987), which represent a lower limit to the true cross-sections, are used between $83.5 \mathrm{~nm}$ and the ionization threshold at $79.5 \mathrm{~nm}$. Although formation of dissociative neutral Rydberg states can still occur below this wavelength, this process is largely dominated by the formation of $\mathrm{N}_{2}{ }^{+}$. Between 79.5 and $66 \mathrm{~nm}$, we improve on the Lavvas et al. (2011a) study by using the high resolution crosssections measured by Shaw et al. (1992) that demonstrate significant structure in this wavelength range. With $\mathrm{N}_{2}^{+}$being essentially formed by photoionization above $1000 \mathrm{~km}$, the convolution of the high resolution cross-sections with the solar flux could have some impact on the $\mathrm{N}_{2}{ }^{+}$production rates.

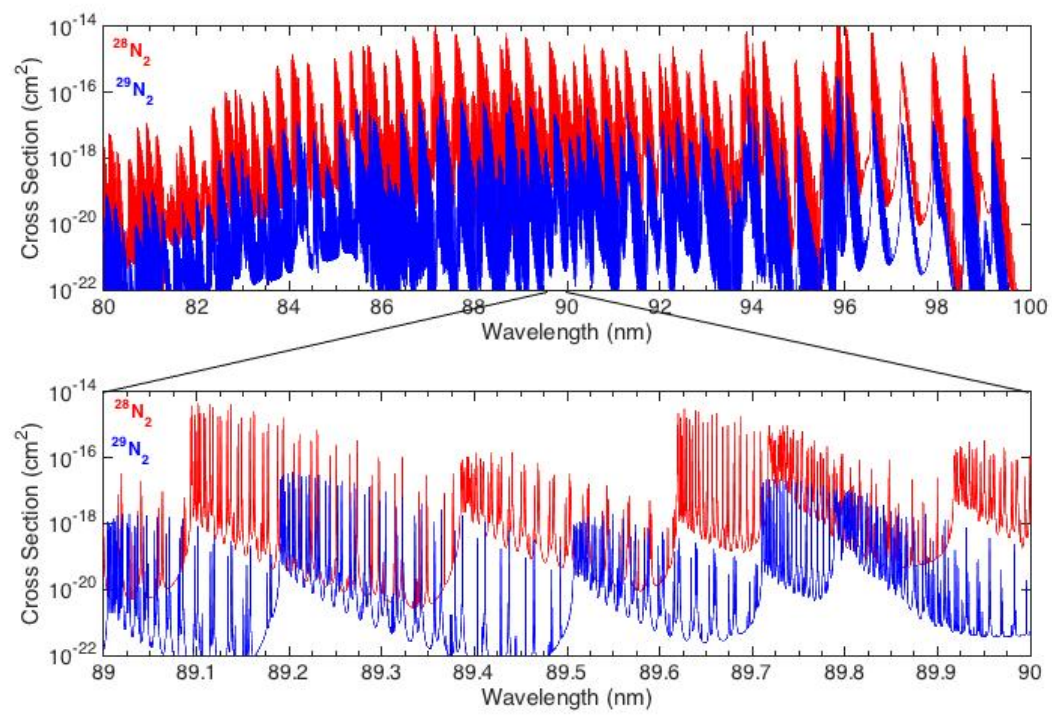

Figure 9: ${ }^{28} \mathrm{~N}_{2}$ and ${ }^{29} \mathrm{~N}_{2}$ photoabsorption cross-sections with a resolution of $3-5 \times 10^{-6} \mathrm{~nm}$ between 80 and $100 \mathrm{~nm}$ (upper panel) and in the narrow region between 89 and $90 \mathrm{~nm}$ (lower panel) used as model inputs. 


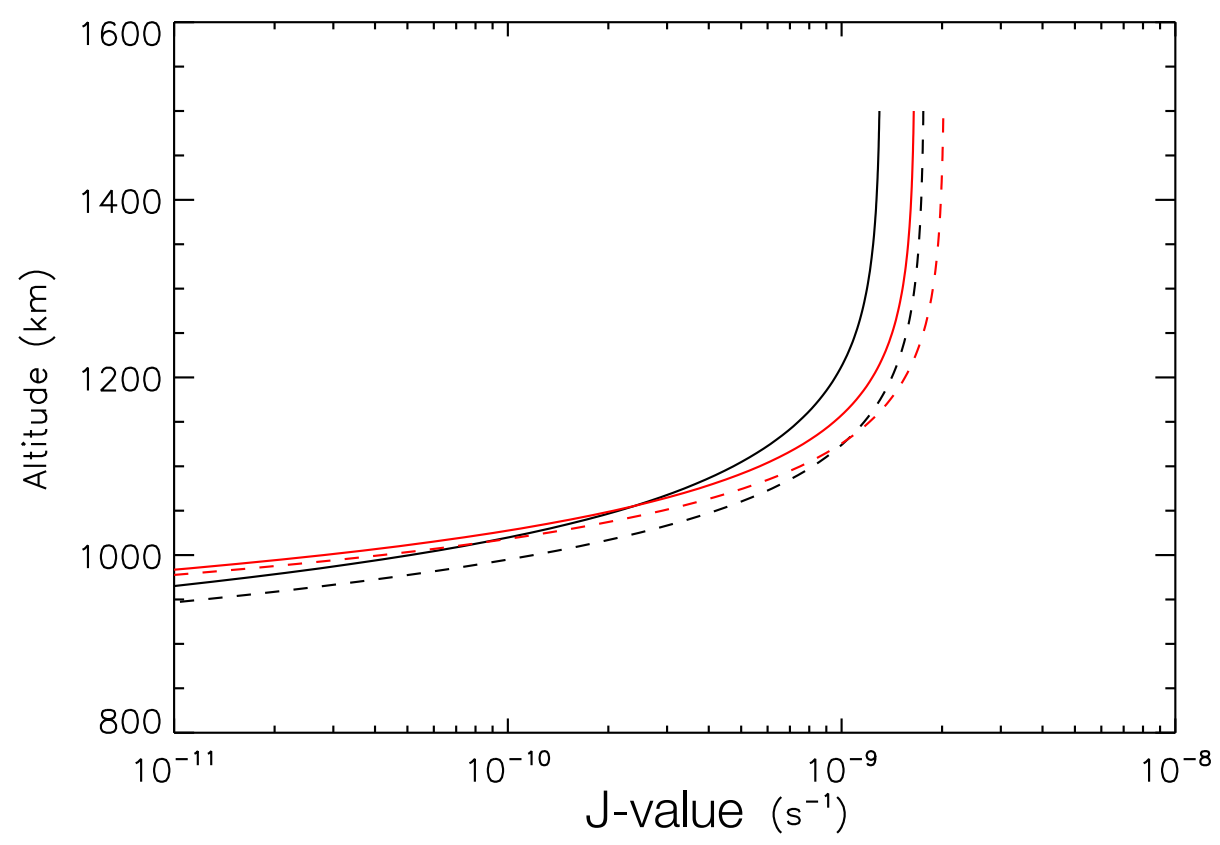

Figure 10: Photolysis rate coefficients ( $\mathrm{J}$ values) for $\mathrm{N}_{2}$ (solid lines) and $\mathrm{CH}_{4}$ (dashed lines) from photons between 80 and $66 \mathrm{~nm}$. Black lines correspond to calculations with the Shaw et al. (1992) cross-sections and red lines to the calculations with the Samson et al. (1987) cross sections.

Figure 10 compares the resulting photolysis rate coefficients (from photons in the range 80 to $66 \mathrm{~nm}$ ) with those derived with the model based on the lower resolution (Samson et al., 1987) cross-sections. We see that the $\mathrm{N}_{2}$ photolysis rate coefficient in this region is lower with the higher resolution (Shaw et al., 1992) cross-sections. This statement also holds for $\mathrm{CH}_{4}$, which is attributed to the increase of the $\mathrm{N}_{2}$ cross-sections in some regions of the spectrum. It is clear though that the highly structured $\mathrm{N}_{2}$ cross-section allows for more photons to interact with $\mathrm{CH}_{4}$ relative to the low resolution cross-sections. However, the cross-sections update has little effect on the $\mathrm{N}_{2}{ }^{+}$production rate, essentially because of the small wavelength range impacted by the high resolution crosssections.

The $\mathrm{N}\left({ }^{4} \mathrm{~S}\right), \mathrm{N}\left({ }^{2} \mathrm{D}\right)$ and $\mathrm{N}\left({ }^{2} \mathrm{P}\right)$ production yields display a complex behavior and are not well constrained. As explained in Lavvas et al. (2011a), a simplified scheme that matches the experimental measurements is put forward and given here:

$$
\begin{aligned}
\mathrm{N}_{2}+h \nu & \rightarrow \mathrm{N}\left({ }^{2} \mathrm{D}\right)+\mathrm{N}\left({ }^{4} \mathrm{~S}\right) \\
& \rightarrow \mathrm{N}\left({ }^{2} \mathrm{P}\right)+\mathrm{N}\left({ }^{4} \mathrm{~S}\right) \\
& \rightarrow \mathrm{N}\left({ }^{2} \mathrm{D}\right)+\mathrm{N}\left({ }^{2} \mathrm{D}\right)
\end{aligned}
$$$$
102.1 \mathrm{~nm}>\lambda>89.1 \mathrm{~nm}
$$$$
89.1 \mathrm{~nm}>\lambda>85.4 \mathrm{~nm}
$$

$$
\lambda<85.4 \mathrm{~nm}
$$


Because of the low chemical reactivity of $\left.\mathrm{N}^{2} \mathrm{P}\right)$ and because its relative production versus that of $\mathrm{N}\left({ }^{2} \mathrm{D}\right)$ is not well constrained, we assume that all $\mathrm{N}\left({ }^{2} \mathrm{P}\right)$ is converted to $\mathrm{N}\left({ }^{2} \mathrm{D}\right)$, as discussed in section 2.7.2.2.

Finally, the model includes electron-impact dissociation and ionization of $\mathrm{N}_{2}$ from 15 to $1000 \mathrm{eV}$ (cf. Table B.5 and Figure 8b). These cross sections are extensively reviewed in Lavvas et al. (2011a) and Lavvas et al. (2015) and the interested reader is referred to these paper for discussion of these processes.

$\mathbf{C H}_{4}$. Table B.5 lists the adiabatic energy thresholds for the $\mathrm{CH}_{4}$ dissociation and ionization channels and the references used to establish their branching ratios and cross-sections. With the exception of the dissociation branching ratios, the scheme used here is the same as in Lavvas et al. (2011a).
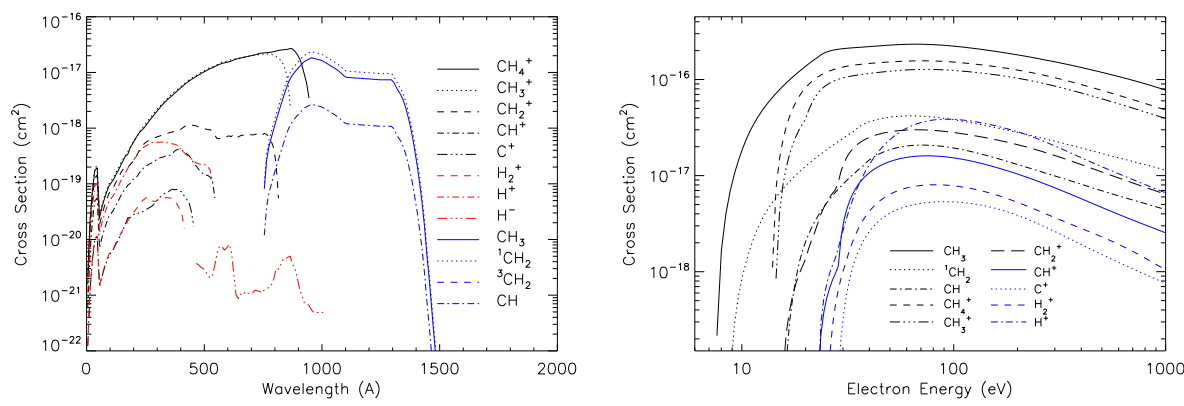

Figure 11: The $\mathrm{CH}_{4}$ cross-sections for ionization and dissociation by photons (left panel) and electrons (right panel) used as model inputs.

The dominant role of solar hydrogen Lyman- $\alpha$ in $\mathrm{CH}_{4}$ photolysis was recognized early (Strobel, 1974; Allen et al., 1980; Yung et al., 1984). But, despite the large number of studies performed, the photodissociation of methane has long provided serious challenges to theoreticians and experimentalists alike: the branching ratios at Lyman- $\alpha$ have been accurately constrained only recently (Gans et al., 2011). The major improvement of this study is accurate characterization of the molecular products of the various channels. A secondary finding of Gans et al. (2011) is that branching ratios are strongly wavelength-dependent. While Lyman- $\alpha$ contributes predominantly to the fragmentation of methane from the top of the atmosphere to $650 \mathrm{~km}$, non-Lyman- $\alpha$ wavelengths become important below $700 \mathrm{~km}$ and branching ratios for these wavelengths are required as well (cf. section 2.6.4). We use a wavelength-dependent model $(<121.6 \mathrm{~nm}$ / $121.6 \mathrm{~nm} />121.6 \mathrm{~nm}$ ) for $\mathrm{CH}_{4}$ photolysis branching ratios, based on the values measured experimentally by Gans et al. (2011) and the sensitivity study of Gans et al. (2013): 


$$
\begin{aligned}
& <121.6 \mathrm{~nm} \quad 121.6 \mathrm{~nm} \quad>121.6 \mathrm{~nm} \\
& \begin{array}{lllll}
\mathrm{CH}_{4}+h \nu \rightarrow \mathrm{CH}_{3}+\mathrm{H} & 0.26 & 0.42 & 0.50 & \left(\mathrm{~J}_{d} 2 \mathrm{a}\right)
\end{array} \\
& \rightarrow{ }^{1} \mathrm{CH}_{2}+\mathrm{H}_{2} \quad 0.17 \quad 0.48 \quad 0.50 \quad\left(\mathrm{~J}_{d} 2 \mathrm{~b}\right) \\
& \rightarrow \mathrm{CH}+\mathrm{H}_{2}+\mathrm{H} \quad 0.09 \quad 0.07 \quad 0.00 \quad\left(\mathrm{~J}_{d} 2 \mathrm{c}\right) \\
& \rightarrow{ }^{3} \mathrm{CH}_{2}+\mathrm{H}+\mathrm{H} \quad 0.48 \quad 0.03 \quad 0.00 \quad\left(\mathrm{~J}_{d} 2 \mathrm{~d}\right)
\end{aligned}
$$

Photoionization of $\mathrm{CH}_{4}$ into $\mathrm{CH}_{\mathrm{x}}^{+}(\mathrm{x}=0-4)$ and $\mathrm{H}_{\mathrm{y}}^{+}(\mathrm{y}=1-2)$ based on Samson et al. (1989) is also included in the model (cf. Table B.5 and left panel of Figure 11). The electron impact cross-sections for methane are presented in the right panel of Figure 11. They are based on the references listed in Table B.5, after Lavvas et al. (2011a).

$\mathbf{C}_{2} \mathbf{H}_{2}$. Acetylene plays an important role in the atmosphere of Titan, as it absorbs significantly up to $235 \mathrm{~nm}$ (Bénilan et al., 2000), a region where the intensity of solar radiation is significantly greater than in the regions of $\mathrm{CH}_{4}$ $(<160 \mathrm{~nm})$ or $\mathrm{N}_{2}(<100 \mathrm{~nm})$ absorption. It is in this way that $\mathrm{C}_{2} \mathrm{H}_{2}$ participates in the catalytic dissociation of $\mathrm{CH}_{4}$ in the stratosphere as first described in Allen et al. (1980).

A number of experiments were performed to determine the branching ratios of the primary products of $\mathrm{C}_{2} \mathrm{H}_{2}$ photodissociation at various UV energies. All the relevant studies have been summarized by Hébrard et al. (2006). Early experiments (mainly performed employing static sample photolysis with endproduct analysis) indicated that the branching ratio for $\mathrm{C}_{2} \mathrm{H}+\mathrm{H}$ was $0.10-0.30$ and for $\mathrm{C}_{2}+\mathrm{H}_{2}$ between 0.10 and 0.15 . The main photochemical process was believed to be the formation of an electronically excited metastable acetylene and/or vinylidene molecule (Seki and Okabe, 1993):

$$
\begin{aligned}
\mathrm{C}_{2} \mathrm{H}_{2}+h \nu(>217 \mathrm{~nm}) & \rightarrow \mathrm{C}_{2} \mathrm{H}+\mathrm{H} \\
(>203 \mathrm{~nm}) & \rightarrow \mathrm{C}_{2}+\mathrm{H}_{2} \\
& \rightarrow \mathrm{C}_{2} \mathrm{H}_{2}^{*}
\end{aligned}
$$

Therefore, those two $\mathrm{C}_{2} \mathrm{H}_{2}$ dissociation channels have been considered in all the photochemical models with comparable branching ratios. For instance, Lavvas et al. (2008a) use 0.1 for the first channel, and for the second one use 0.1 at $\lambda<$ $124 \mathrm{~nm}, 0.3$ at $124<\lambda<150 \mathrm{~nm}, 0.06$ at $150<\lambda<190 \mathrm{~nm}$ and 0.3 at 190 $<\lambda<225 \mathrm{~nm}$. Krasnopolsky (2009) use a branching ratio of 0.3 for $\mathrm{C}_{2} \mathrm{H}+\mathrm{H}$ and 0.1 for $\mathrm{C}_{2}+\mathrm{H}_{2}$ in the entire wavelength range.

More recent experiments, performed under single collision conditions, have questioned the validity of this assumption (cf., for instance, Boyé et al. (2002), and references therein). Finally, the acetylene dissociation dynamics after photoexcitation at $193 \mathrm{~nm}$ and at Lyman- $\alpha$ were studied under collision free conditions by Läuter et al. (2002) and Kovács et al. (2010), who obtained an absolute 
branching ratio for $\mathrm{H}$ atom formation close to unity, thus suggesting that the ethynyl $\left(\mathrm{C}_{2} \mathrm{H}\right)$ radical is the only molecular primary product for both energies. In other words, $\mathrm{C}_{2}$ is not formed directly by UV photolysis of $\mathrm{C}_{2} \mathrm{H}_{2}$ and we use a branching ratio of one for channel $\left(\mathrm{J}_{d} 5\right)$ from 100 to $225 \mathrm{~nm}$, as reported in Table B.6.

$\mathbf{C}_{\mathbf{3}} \mathbf{H}_{\mathbf{6}}$. Nixon et al. (2013) reported the presence of propene in CIRS spectra of Titan's stratosphere, completing the detection of the $\mathrm{C}_{3}$-hydrocarbons family, along with $\mathrm{C}_{3} \mathrm{H}_{8}$ and $\mathrm{CH}_{3} \mathrm{CCH} . \mathrm{C}_{3} \mathrm{H}_{6}$ absorbs up to $200 \mathrm{~nm}$ (Fahr and Nayak, 1996) and although a dozen early studies have focused on the determination of its stable photodissociation products at five different wavelengths ranging from 123.6 to $184.9 \mathrm{~nm}$, the major channels are still far from being well established.

The branching ratios used in previous photochemical models are presented in Table 2 and highlight some huge discrepancies in the interpretation of the published data. While Lavvas et al. (2008a) and Krasnopolsky (2009) use a roughly similar photodissociation scheme with $\mathrm{C}_{3} \mathrm{H}_{5}+\mathrm{H}\left(\mathrm{J}_{d} 10 \mathrm{~b}\right)$ being the major products at longer wavelength, $\mathrm{C}_{3} \mathrm{H}_{4}+\mathrm{H}_{2}\left(\mathrm{~J}_{d} 10 \mathrm{e}, \mathrm{f}\right)$ at shorter wavelength and $\mathrm{C}_{2} \mathrm{H}_{3}+\mathrm{CH}_{3}\left(\mathrm{~J}_{d} 10 \mathrm{c}\right)$ throughout, Li et al. (2015) consider wavelength independent branching ratios giving only $\mathrm{C}_{3} \mathrm{H}_{5}+\mathrm{H}$ and $\mathrm{C}_{2} \mathrm{H}_{3}+\mathrm{CH}_{3}$. Hébrard et al. (2013) utilized completely different channels with similar production of $\mathrm{C}_{2} \mathrm{H}_{2}+\mathrm{CH}_{4}\left(\mathrm{~J}_{d} 10 \mathrm{a}\right), \mathrm{C}_{3} \mathrm{H}_{5}+\mathrm{H}\left(\mathrm{J}_{d} 10 \mathrm{~b}\right), \mathrm{C}_{2} \mathrm{H}_{3}+\mathrm{CH}_{3}\left(\mathrm{~J}_{d} 10 \mathrm{c}\right)$ and $\mathrm{C}_{3} \mathrm{H}_{3}+\mathrm{H}_{2}+\mathrm{H}$ $\left(\mathrm{J}_{d} 10 \mathrm{~g}\right)$ at longer wavelength and a dominant production of $\mathrm{C}_{2} \mathrm{H}_{2}+\mathrm{CH}_{4}\left(\mathrm{~J}_{d} 10 \mathrm{a}\right)$ at shorter wavelength.

Considering the studies of Borrell et al. (1971) at $184.9 \mathrm{~nm}$, of Collin and coworkers (Collin et al., 1979; Collin, 1988) at 163.3, and of Niedzielski and coworkers (Niedzielski et al., 1982; Naroznik and Niedzielski, 1986; Gierczak et al., 1988) at 123.6 and $147.0 \mathrm{~nm}$, we independently come to the conclusion that $\mathrm{C}_{3} \mathrm{H}_{5}+\mathrm{H}\left(\mathrm{J}_{d} 10 \mathrm{~b}\right)$ and $\mathrm{C}_{2} \mathrm{H}_{3}+\mathrm{CH}_{3}\left(\mathrm{~J}_{d} 10 \mathrm{c}\right)$ as well as $\mathrm{C}_{3} \mathrm{H}_{4}+\mathrm{H}_{2}\left(\mathrm{~J}_{d} 10 \mathrm{e}, \mathrm{f}\right)$ at shorter wavelengths should be the dominant photodissociation products, as shown in Table B.6. This scheme is somewhat similar to that presented in Lavvas et al. (2008a) and Krasnopolsky (2009) but is nevertheless highly uncertain. Fortunately, we find that photodissociation only accounts for $\sim 15 \%$ of the total $\mathrm{C}_{3} \mathrm{H}_{6}$ loss, as explained in section 3.2.1. 
Table 2: Branching ratios for $\mathrm{C}_{3} \mathrm{H}_{6}$ photolysis in several photochemical models.

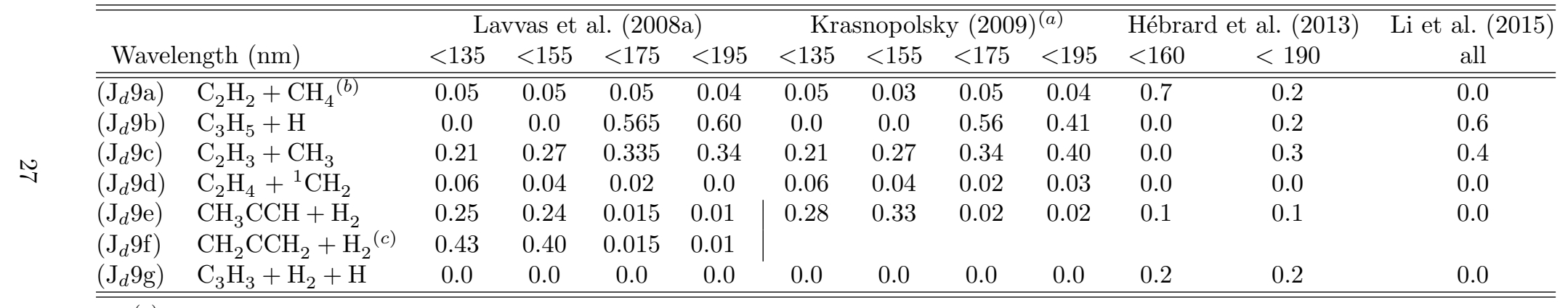

${ }^{(a)}$ The branching ratios do not sum to one in Krasnopolsky (2009).

${ }^{(b)} \mathrm{C}_{2} \mathrm{H}_{2}+\mathrm{CH}_{3}+\mathrm{H}$ instead of $\mathrm{C}_{2} \mathrm{H}_{2}+\mathrm{CH}_{4}$ in Hébrard et al. (2013).

${ }^{(c)} \mathrm{CH}_{3} \mathrm{CCH}+\mathrm{H}_{2}$ and $\mathrm{CH}_{2} \mathrm{CCH}_{2}+\mathrm{H}_{2}$ are undifferentiated in Krasnopolsky (2009), Hébrard et al. (2013) and Li et al. (2015). 
$\mathbf{C}_{4} \mathbf{H}_{2}$. Diacetylene can absorb at even longer wavelengths than acetylene, up to $250 \mathrm{~nm}$ (Smith et al., 1998), to produce reactive species that can instigate catalytic hydrogen atom abstraction cycles as well as leading to the formation of heavier species, such as longer-chain polyynes (cf. section 3.2).

The photochemistry of diacetylene has been studied at 147.0, 184.9, 228.8 and $253.7 \mathrm{~nm}$ in a pioneering study by Glicker and Okabe (1987). At 147 $\mathrm{nm}$, three dissociation channels were deduced $\left(\mathrm{J}_{d} 12 \mathrm{a}, \mathrm{b}, \mathrm{c}\right)$. At the three other wavelengths, the disappearance of diacetylene was ascribed to the formation of an electronically excited metastable state, $\mathrm{C}_{4} \mathrm{H}_{2}^{*}$, assumed to be the lowest triplet state. Numerous laser-based studies, principally at 231 and $243 \mathrm{~nm}$, investigated the reactivity of $\mathrm{C}_{4} \mathrm{H}_{2}^{*}$ and found no evidence for radical products (Robinson et al. (2002) and references therein).

Lavvas et al. (2008a) and Krasnopolsky (2009) relied heavily on the study of Glicker and Okabe (1987) and both considered the following branching ratios for the photolysis of $\mathrm{C}_{4} \mathrm{H}_{2}$ :

$$
\begin{aligned}
& <165 \quad 165-205 \quad>205 \\
& \begin{array}{llll}
\mathrm{C}_{4} \mathrm{H}_{2}+h \nu \rightarrow \mathrm{C}_{4} \mathrm{H}+\mathrm{H} & 0.20 & 0.00 & 0.00
\end{array} \\
& \rightarrow \mathrm{C}_{2} \mathrm{H}_{2}+\mathrm{C}_{2} \quad 0.03 \quad 0.01 \quad 0.00 \quad\left(\mathrm{~J}_{d} 12 \mathrm{~b}\right) \\
& \rightarrow \mathrm{C}_{2} \mathrm{H}+\mathrm{C}_{2} \mathrm{H} \quad 0.10 \quad 0.06 \quad 0.00 \quad\left(\mathrm{~J}_{d} 12 \mathrm{~d}\right) \\
& \begin{array}{lllll}
\rightarrow \mathrm{C}_{4} \mathrm{H}_{2}^{*} & 0.67 & 0.93 & 1.00 & \left(\mathrm{~J}_{d} 12^{\prime}\right)
\end{array}
\end{aligned}
$$

A recent experimental investigation of the photochemistry of diacetylene under collisionless conditions and after excitation at 121.6, 157.0, 193.3 and 212.0 $\mathrm{nm}$, coupled to statistical calculations suggests instead that radical formation, and in particular $\mathrm{C}_{4} \mathrm{H}$, is dominant at all wavelengths (Silva et al., 2008). We follow this study and adopt the branching ratio reported in Table B.6. As a consequence, there is no production reaction of $\mathrm{C}_{4} \mathrm{H}_{2}^{*}$ in the model and it is not considered.

$\mathbf{H C}_{\mathbf{3}} \mathbf{N}$. Cyanoacetylene is the nitrogen-bearing analog of diacetylene and the first member of the cyanopolyyne family. Like $\mathrm{C}_{4} \mathrm{H}_{2}$, it exhibits strong absorption bands in the far ultraviolet (Bénilan et al., 1994) and is easily photodissociated in Titan's atmosphere. There have been few investigations into the primary processes resulting from its UV irradiation. Halpern et al. (1988, 1990) identified two photodissociation channels and an excited state $\mathrm{HC}_{3} \mathrm{~N}^{*}$ :

$$
\begin{aligned}
\mathrm{HC}_{3} \mathrm{~N}+h \nu & \rightarrow \mathrm{C}_{3} \mathrm{~N}+\mathrm{H} \\
& \rightarrow \mathrm{C}_{2} \mathrm{H}+\mathrm{CN}, \\
& \rightarrow \mathrm{HC}_{3} \mathrm{~N}^{*}
\end{aligned}
$$

They determined a branching ratio of $0.05 \pm 0.02$ at $193 \mathrm{~nm}$ for channel $\left(\mathrm{J}_{d} 27 \mathrm{~b}\right)$. Later on, Seki et al. (1996) reported a branching ratio of $0.30 \pm 0.05$ for channel 
$\left(\mathrm{J}_{d} 27 \mathrm{a}\right)$ and an upper limit for channel $\left(\mathrm{J}_{d} 27 \mathrm{~b}\right)$ of 0.02 . These results confirmed that, at $193 \mathrm{~nm}, \mathrm{C}_{2} \mathrm{H}+\mathrm{CN}$ are minor products and the authors suggested that the major photochemical process is the formation of a metastable $\mathrm{HC}_{3} \mathrm{~N}^{*}$. At $185 \mathrm{~nm}$, Clarke and Ferris (1995) determined a branching ratio of $\sim 0.09$ for channel $\left(\mathrm{J}_{d} 27 \mathrm{a}\right)$.

These results led Lavvas et al. (2008a) and Krasnopolsky (2009) to use a branching ratio of 0.3 and 0.05 , respectively, for channel $\left(\mathrm{J}_{d} 27 \mathrm{~b}\right)$ and 0.09 for channel $\left(\mathrm{J}_{d} 27 \mathrm{a}\right)$, implying the formation of some excited state, whose reactivity is not considered.

More recently, the photodissociation of cyanoacetylene has been studied in a molecular beam under collisionless conditions and the experimental approach was augmented by statistical calculations (Silva et al., 2009). The results indicate that at 212 and $193 \mathrm{~nm}$, the only products are $\mathrm{C}_{3} \mathrm{~N}+\mathrm{H}$, while at 157 and $121.6 \mathrm{~nm}$, this same channel represents only $57 \%$ of the products, with channel $\left(\mathrm{J}_{d} 27 \mathrm{~b}\right)$ accounting for the difference. There is again no evidence for a metastable state up to the dissociation threshold of $\left(\mathrm{J}_{d} 27 \mathrm{a}\right)$, at $214 \mathrm{~nm}$. We use the results of this latter study to constrain our branching ratios, as shown in Table B.6.

$\mathbf{C}_{2} \mathbf{H}_{3} \mathbf{C N}$. Palmer et al. (2017) reported very recently the presence of acrylonitrile in ALMA spectra of Titan's stratosphere. Acrylonitrile exhibits a strong absorption in the far UV range (Eden et al., 2003), making it a particularly reactive species. There have been various experimental studies of the primary fragments, mostly at $193.0 \mathrm{~nm}$, but also at $157.6 \mathrm{~nm}$. They reported the detection of $\mathrm{CH}_{2} \mathrm{CCN}, \mathrm{HC}_{3} \mathrm{~N}, \mathrm{HCN}, \mathrm{C}_{2} \mathrm{H}_{2}$ and minor amounts of $\mathrm{CN}$, although few branching ratio measurements have been performed (Fahr and Laufer, 1992; North and Hall, 1996; Blank et al., 1998; Guo et al., 2001). The theoretical work of Derecskei-Kovacs and North (1999) led to quantitative branching ratios at $193.0 \mathrm{~nm}$. Wilhelm et al. (2009) observed with time-resolved infrared emission spectroscopy the appearance of $\mathrm{HNC}$ as well as $\mathrm{HCN}$ and spectral modeling allowed for the determination of the ratio $\mathrm{HCN} / \mathrm{HNC}$ again at $193 \mathrm{~nm}$.

Lavvas et al. (2008a) adopt a wavelength independent photodissociation scheme based on the results from Derecskei-Kovacs and North (1999). Krasnopolsky (2009) uses a single channel, $\mathrm{CN}+\mathrm{C}_{2} \mathrm{H}_{3}\left(\mathrm{~J}_{d} 28 \mathrm{e}\right)$, while Loison et al. (2015) assumes that $\mathrm{HCN}+\mathrm{C}_{2} \mathrm{H}_{2}\left(\mathrm{~J}_{d} 28 \mathrm{c}\right)$ are the major products with smaller amounts of $\mathrm{HC}_{3} \mathrm{~N}+\mathrm{H}_{2}\left(\mathrm{~J}_{d} 28 \mathrm{a}\right)$, $\mathrm{HNC}+\mathrm{C}_{2} \mathrm{H}_{2}\left(\mathrm{~J}_{d} 28 \mathrm{~b}\right)$ and $\mathrm{CH}_{2} \mathrm{CCN}+\mathrm{H}\left(\mathrm{J}_{d} 28 \mathrm{~d}\right)$. As indicated in Table B.6, we follow Lavvas et al. (2008a) and use throughout our wavelength range, the branching ratios calculated by Derecskei-Kovacs and North (1999) but with the HCN/HNC ratio measured by Wilhelm et al. (2009), which is supported by more recent theoretical calculations (Homayoon et al., 2011). 


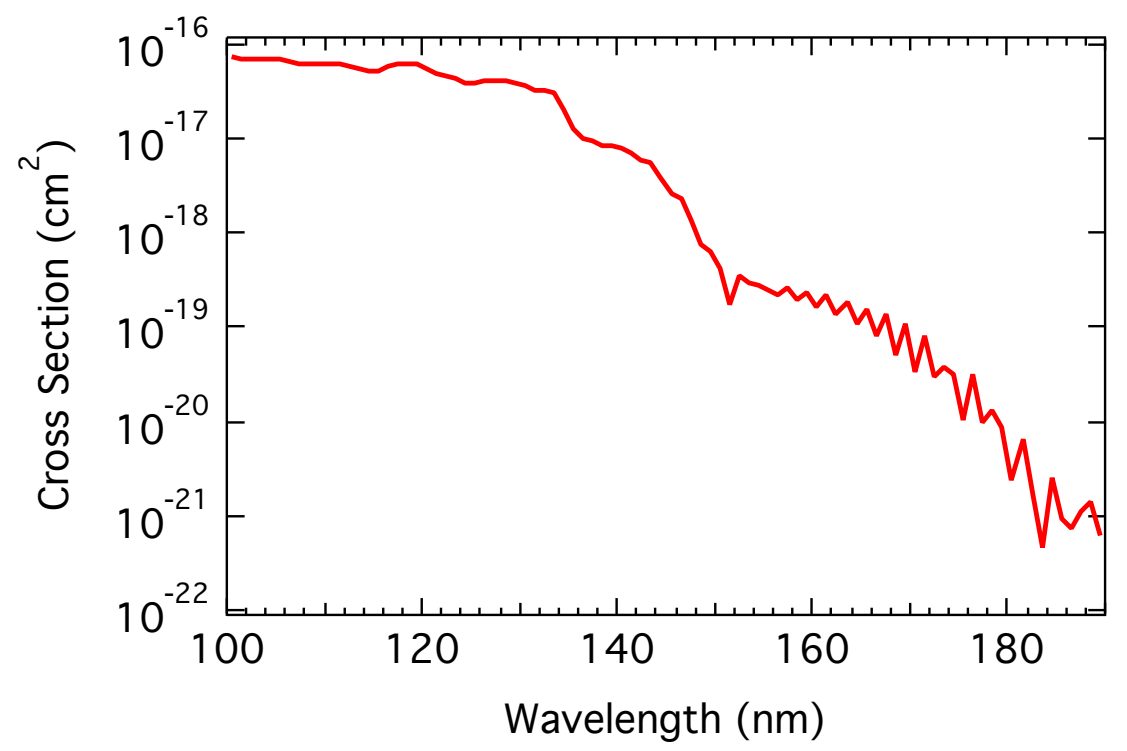

Figure 12: The cross-section of propionitrile $\left(\mathrm{C}_{2} \mathrm{H}_{5} \mathrm{CN}\right)$ versus wavelength used as model inputs (adapted from Kanda et al. (1999), see text).

$\mathbf{C}_{2} \mathbf{H}_{5} \mathbf{C N}$. Cordiner et al. (2015b) recently reported the presence of propionitrile in ALMA spectra of Titan's stratosphere. The absorption spectrum of $\mathrm{C}_{2} \mathrm{H}_{5} \mathrm{CN}$ has only been recorded up to $152 \mathrm{~nm}$ (Kanda et al., 1999) although it may extend beyond $200 \mathrm{~nm}$ (Y. Bénilan, private communication, 2015). As this extended absorption to longer wavelength may play a crucial role, we assume that in the missing range the $\mathrm{C}_{2} \mathrm{H}_{5} \mathrm{CN}$ cross sections are the same as for $\mathrm{C}_{2} \mathrm{H}_{2}$ (cf. Figure 12). We note that Krasnopolsky (2009) assumes the same J value as for $\mathrm{C}_{2} \mathrm{H}_{3} \mathrm{CN}$ and that Loison et al. (2015) do not consider any absorption beyond $150 \mathrm{~nm}$.

As far as we know, there are no branching ratios available for $\mathrm{C}_{2} \mathrm{H}_{5} \mathrm{CN}$. Krasnopolsky (2009) assumes that photodissociation leads to a single channel: $\mathrm{C}_{2} \mathrm{H}_{5}+\mathrm{CN}\left(\mathrm{J}_{d} 29 \mathrm{c}\right)$, while Loison et al. (2015) speculates that three other channels are open: $\mathrm{HCN}+\mathrm{C}_{2} \mathrm{H}_{4}\left(\mathrm{~J}_{d} 29 \mathrm{a}\right), \mathrm{C}_{2} \mathrm{H}_{3} \mathrm{CN}+\mathrm{H}_{2}\left(\mathrm{~J}_{d} 29 \mathrm{~b}\right)$ and $\mathrm{CH}_{2} \mathrm{CN}+\mathrm{CH}_{3}$. We base our photodissociation scheme on that of $\mathrm{C}_{2} \mathrm{H}_{3} \mathrm{CN}$ which translates into $\mathrm{C}_{2} \mathrm{H}_{3} \mathrm{CN}+\mathrm{H}_{2}$ being the main channel (cf. Table B.6).

Radicals. Radicals often absorb from the UV up to the visible part of the spectrum where the solar flux is highest. Nevertheless, these absorptions have been much less studied than for stable molecules and the subject is seldom discussed in the context of Titan.

In their pioneering work, Yung et al. (1984) state that photolysis of $\mathrm{CH}_{2}$ and $\mathrm{C}_{2} \mathrm{H}_{5}$ is relatively unimportant but do include the dissociation of $\mathrm{CH}_{3}, \mathrm{C}_{3} \mathrm{H}_{3}$ and $\mathrm{HCO}$ (cf. their Table 3). For the photolysis of $\mathrm{CH}_{3}$ they consider the band at $216 \mathrm{~nm}$ and assume a branching ratio of unity towards ${ }^{1} \mathrm{CH}_{2}+\mathrm{H}$, based on 
spin conservation. They however find that this process is marginally important compared with removal of $\mathrm{CH}_{3}$ by chemical reactions. Nevertheless, methyl photolysis has been included in all subsequent photochemical models for Titan's atmosphere. Both Wilson and Atreya (2000) and Lavvas et al. (2008b) note that it is a significant source of excited methylene radicals. ${ }^{1} \mathrm{CH}_{2}$, unlike $\mathrm{CH}_{3}$, can further react with $\mathrm{CH}_{4}$ to produce two $\mathrm{CH}_{3}$ radicals. Methyl photolysis therefore enhances methane's destruction and hence affects the production of all hydrocarbons.

Like Yung et al. (1984), subsequent models (Toublanc et al., 1995; Lara et al., 1996; Lebonnois et al., 2001) did consider a similar photolysis scheme for radicals. As models became more sophisticated, the photolysis of a couple more radical hydrocarbons beyond $\mathrm{CH}_{3}$ and $\mathrm{C}_{3} \mathrm{H}_{3}$ were considered: $\mathrm{C}_{2} \mathrm{H}_{3}, \mathrm{C}_{2} \mathrm{H}_{5}$ and $\mathrm{C}_{3} \mathrm{H}_{5}$ in Wilson and Atreya $(2004), \mathrm{C}_{2} \mathrm{H}, \mathrm{C}_{2} \mathrm{H}_{3}$ and $\mathrm{C}_{2} \mathrm{H}_{5}$ in Lavvas et al. (2008a,b) and $\mathrm{C}_{2} \mathrm{H}_{3}, \mathrm{C}_{2} \mathrm{H}_{5}, \mathrm{C}_{3} \mathrm{H}_{2}$ and $\mathrm{C}_{3} \mathrm{H}_{5}$ in Krasnopolsky (2009). The latter also considers the photolysis of two nitrogen-bearing species: $\mathrm{NH}_{2}$ and $\mathrm{CH}_{2} \mathrm{CN}$. Neither of these authors, however, discuss the importance of these reactions for the overall chemistry.

Dobrijevic et al. recently revisited their chemistry of $\mathrm{C}_{3} \mathrm{H}_{p}$ hydrocarbons, $\mathrm{HCN}$ and oxygen species in the stratosphere and included the photodissociation of $\mathrm{C}_{3}, \mathrm{C}_{3} \mathrm{H}$ (2 isomers), $\mathrm{C}_{3} \mathrm{H}_{2}$ (3 isomers), $\mathrm{C}_{3} \mathrm{H}_{3}, \mathrm{C}_{3} \mathrm{H}_{5}, \mathrm{C}_{3} \mathrm{H}_{7}, \mathrm{H}_{2} \mathrm{CN}, \mathrm{OH}$ and HCO (Hébrard et al., 2012, 2013; Dobrijevic et al., 2014). The crosssections, branching ratios and dissociation limits are extensively discussed in the appendices. They find that photolysis of $\mathrm{H}_{2} \mathrm{CN}$ is responsible for $7 \%$ of the HCN production at $1300 \mathrm{~km}$. They also report that because $\mathrm{HCO}$ is efficiently destroyed through visible-UV photolysis, its steady state abundance is low and that this process controls the $\mathrm{HCO} \rightleftharpoons \mathrm{H}+\mathrm{CO}$ equilibrium (Dobrijevic et al., 2014).

Our model includes all the radicals discussed above and, in addition, $\mathrm{C}_{6} \mathrm{H}_{5}$ and $\mathrm{C}_{7} \mathrm{H}_{7}$, as listed in Table B.9. Cross-sections are represented in Figure 13.
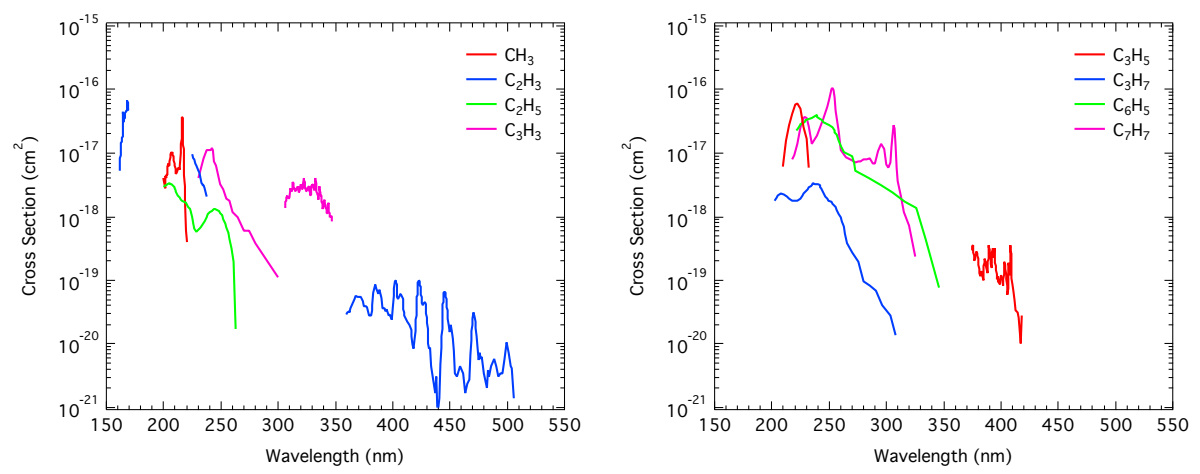

Figure 13: Cross-sections of radicals versus wavelength used as model inputs: $\mathrm{CH}_{3}, \mathrm{C}_{2} \mathrm{H}_{3}$, $\mathrm{C}_{2} \mathrm{H}_{5}, \mathrm{C}_{3} \mathrm{H}_{3}$ (left panel) and $\mathrm{C}_{3} \mathrm{H}_{5}, \mathrm{C}_{3} \mathrm{H}_{7}, \mathrm{C}_{6} \mathrm{H}_{5}, \mathrm{C}_{7} \mathrm{H}_{7}$ (right panel). References are given in Table B.9. 
2.6.3.2. Rayleigh Scattering. Most of the molecules considered here only absorb radiation but we have also considered scattering by $\mathrm{N}_{2}$ and aerosols. Aerosol scattering properties are based on the DISR observations (Tomasko et al., 2008; Lavvas et al., 2010) while $\mathrm{N}_{2}$ scattering properties are based on the Rayleigh scattering cross section (Dalgarno et al., 1967). We have modeled the effects of scattering with a two-stream radiative transfer code, but find that the resulting chemistry is similar to that obtained when scattering is neglected. This is due to the low single scattering albedo of the aerosols. For the runs presented here we do not include scattering because the effects of scattering on the radiation field are smaller than the uncertainties caused by our approximate knowledge of the aerosol distribution.

2.6.3.3. Aerosol Opacity. Aerosol opacities for fractal aggregates were taken from Lavvas et al. (2010), using aerosol single scattering albedos and extinction retrieved by DISR (Tomasko et al., 2008). A newer analysis of DISR data (Doose et al., 2016) implies a different aerosol scale height above $80 \mathrm{~km}$. The impact of this recent study on the aerosol opacity has not been investigated yet. A sensitivity study of the effect of aerosol opacity on the model results is presented in section 5.3. 


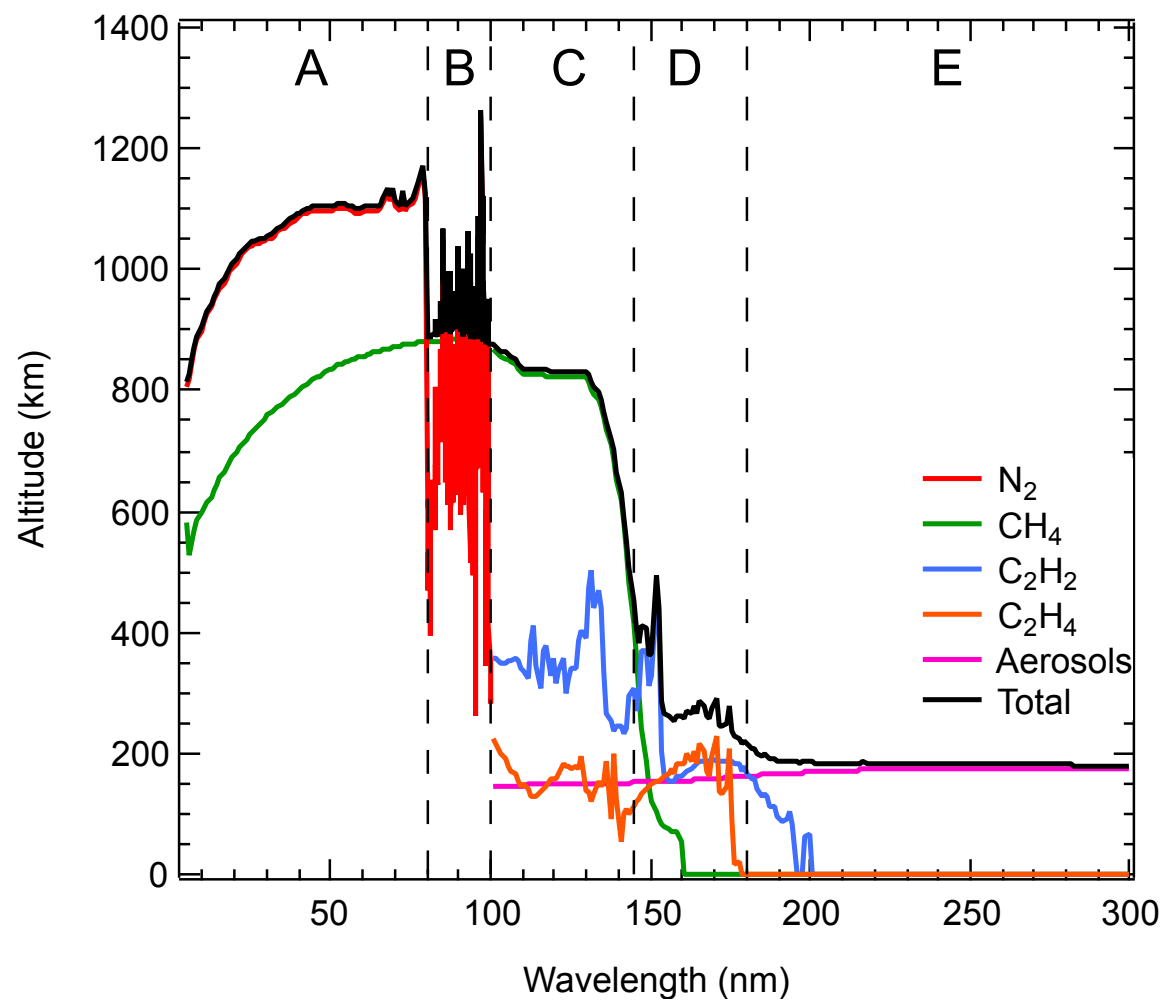

Figure 14: Photon penetration altitude as a function of wavelength.

Table 3: Main absorbers as a function of wavelength.

\begin{tabular}{lll}
\hline \hline Band & Wavelength range $(\mathrm{nm})$ & Main absorber \\
\hline \hline $\mathrm{A}$ & $0-80$ & $\mathrm{~N}_{2}$ (Ionization) \\
$\mathrm{B}$ & $80-100$ & $\mathrm{~N}_{2}$ (Dissociation) \\
$\mathrm{C}$ & $100-145$ & $\mathrm{CH}_{4}$ \\
$\mathrm{D}$ & $145-180$ & $\mathrm{C}_{2} \mathrm{H}_{2}-\mathrm{C}_{2} \mathrm{H}_{4}$ \\
$\mathrm{E}$ & $180-300$ & Aerosols \\
\hline \hline
\end{tabular}




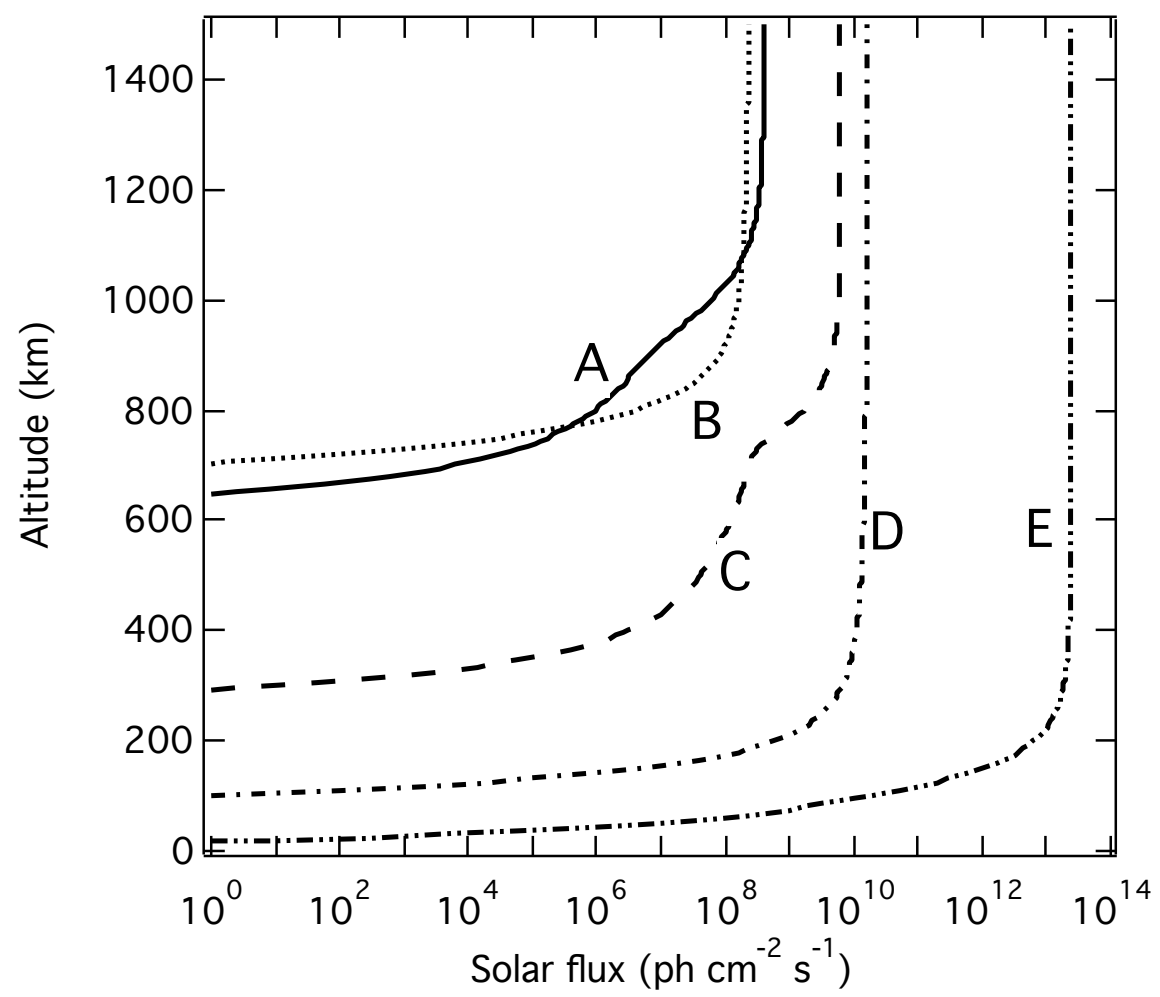

Figure 15: Solar flux in bands as a function of altitude.

Figure 14 displays the altitude at which the optical depth equals one for the main absorbers and for the total atmosphere. This graph can be separated into five distinct bands corresponding to wavelength regions where the dominant process differs, as shown in Table 3 and Figure 15. Plotted in this figure is the flux for an overhead sun for the 5 bands shown in Figure 14 and listed in Table 3. Below $100 \mathrm{~nm}$, the optical depth is dominated by $\mathrm{N}_{2}$ absorption, which occurs at altitudes from $800-1200 \mathrm{~km}$. The threshold region near 80 $\mathrm{nm}$ is not well described. The problem is that numerous high lying energy states contribute right near threshold and these high energy states are not well understood. Unlike Figure 15 that is accurate, Figure 14 only gives a very rough indication of the altitudes in the $\mathrm{N}_{2}$ dissociation region because the actual $\tau=1$ level varies strongly on very small wavelength scales in this region (Lavvas et al., 2011a, 2015). For this reason we should not expect a precise agreement between this plot and the altitude profile of $\mathrm{N}$ production, for example. Between 100 and $145 \mathrm{~nm}, \mathrm{CH}_{4}$ is the dominant photon absorber, with the unit optical depth level decreasing from 800 to $400 \mathrm{~km}$. Between 145 and $180 \mathrm{~nm}, \mathrm{C}_{2} \mathrm{H}_{2}$ and $\mathrm{C}_{2} \mathrm{H}_{4}$ contribute mostly to the optical depth, with a maximum absorption located between 400 and $200 \mathrm{~km}$. Finally, above $180 \mathrm{~nm}$, the opacity is entirely due to 
aerosols with $\tau=1$ at $200 \mathrm{~km}$. As a consequence, the entire solar ultraviolet flux is essentially absorbed before it can reach the tropopause/ surface (cf. Figure $16)$.

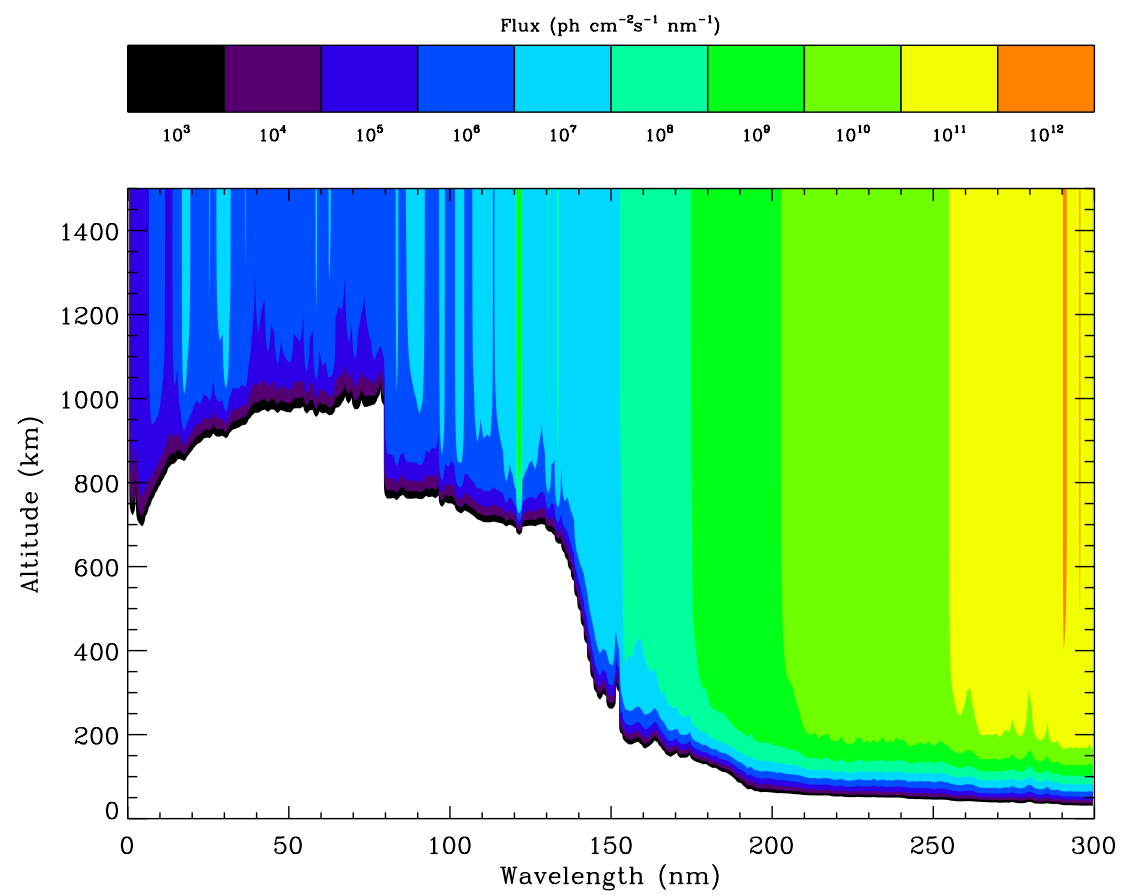

Figure 16: Photon flux versus altitude and wavelength, between 1.5 and $300 \mathrm{~nm}$. White denotes no flux (complete absorption).
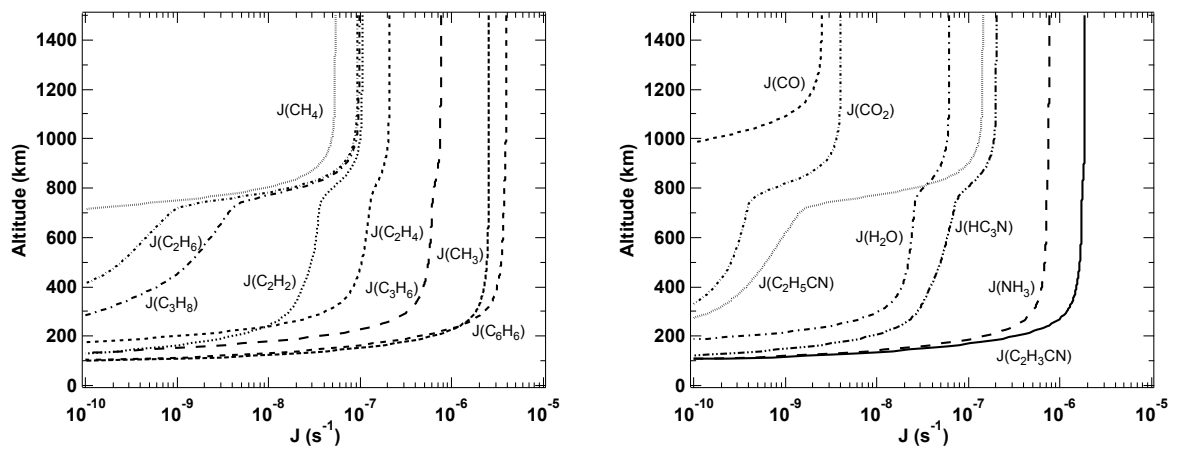

Figure 17: Photolysis rate coefficients ( $\mathrm{J}$ values) versus altitude for selected hydrocarbons (left panel), and nitrogen and oxygen bearing species (right panel). 
The model calculated photolysis rate coefficients for the most important hydrocarbons (left panel), and nitrogen and oxygen bearing species (right panel) are shown in Figure 17. A secondary photolysis region below $700 \mathrm{~km}$ for some of the species is due to their extended absorption cross section profiles above the methane dissociation limit at $145 \mathrm{~nm}$ and their presence in significant amounts.

\subsubsection{Galactic Cosmic Rays}

GCR, due to their high energy, penetrate deep in the atmosphere and deposit their energy in the lower stratosphere and troposphere (Capone et al., 1983; Molina-Cuberos et al., 2001). The alpha particles of the cosmic shower as well as the cascade of secondary electrons generated are degraded in energy along their path, leading to the ionization and dissociation of the main gaseous compounds. For the production rates of the different $\mathrm{N}_{2}$ and $\mathrm{CH}_{4}$ fragments (cf. Figure 18 and Table B.13), we use the results from the energy deposition models described in Gronoff et al. $(2009,2011)$ and Lavvas et al. (2014). Molina-Cuberos et al. (2000) discussed the formation of negative ions from hypothetical electrophilic species and concluded that one would not expect there to be a large anion population in the lower atmosphere. As a consequence, we do not consider the formation of negative ions in the GCR region.
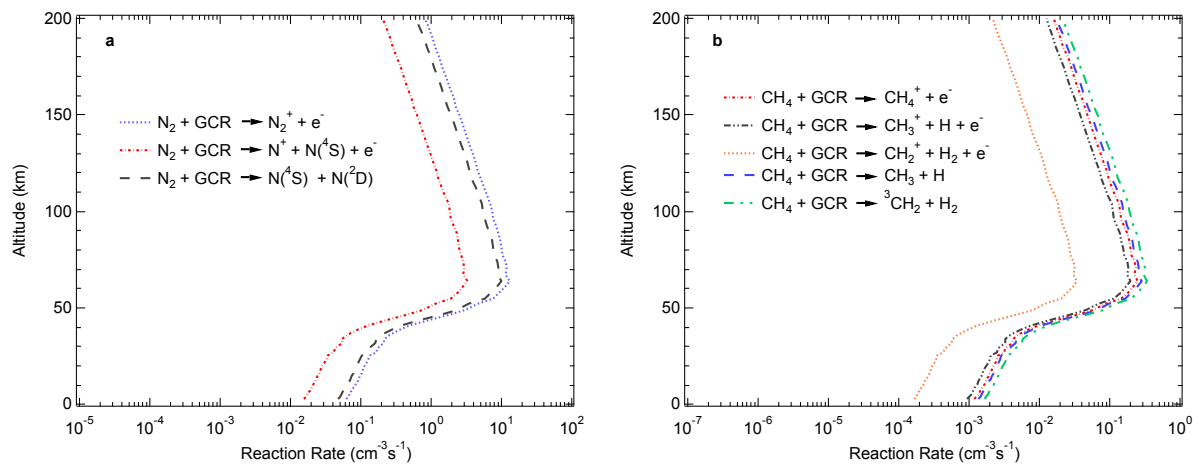

Figure 18: Production rates from Galactic Cosmic Rays for $\mathrm{N}_{2}$ (left panel) and $\mathrm{CH}_{4}$ (right panel).

\subsection{Chemical Network}

\subsubsection{Equations}

The model simulations require simple representations of the temperature and pressure dependence of the rate coefficients. Here, the temperature dependence of the various rate coefficients are represented with modified Arrhenius forms:

$$
k(T)=A T^{B} \exp (C / T),
$$

where $\mathrm{T}$ is the temperature and $\mathrm{A}, \mathrm{B}$, and $\mathrm{C}$ are fitting parameters. Properly representing the pressure dependence is more difficult. The well-known, but 
rather approximate, Lindemann mechanism yields an expression for the association rate coefficient that smoothly interpolates from a linear dependence $\left(\mathrm{k}_{0}\right.$ $[\mathrm{M}]$ ) on pressure at low pressure (or density $[\mathrm{M}]$ ) to a pressure independent value at high pressure $\left(\mathrm{k}_{\infty}\right)$ :

$$
k(T,[M])=k_{\infty} k_{\circ}[M] /\left(k_{\infty}+k_{\circ}[M]\right),
$$

Master equation simulations long ago showed that at intermediate pressures the association rate coefficient is generally lower than predicted by the Lindemann expression. This deviation from the low and high pressure limits is commonly accounted for through the introduction of an empirical broadening factor $\mathrm{F}$ :

$$
k(T,[M])=F k_{\infty} k_{\circ}[M] /\left(k_{\infty}+k_{\circ}[M]\right)
$$

In the combustion literature, the broadening factor $\mathrm{F}$ is generally represented with the empirical Troe form (Troe, 1979, 1983; Gilbert et al., 1983),

$$
\log _{10}(F)=\log _{10}\left(F_{c}\right) / 1+\left[\left(\log _{10}\left(P_{r}\right)+C\right) /\left(N-0.14\left(\log _{10}\left(P_{r}\right)+C\right)\right)\right]^{2}
$$

with the reduced pressure, $P_{r}$, given by $k_{\circ}[M] / k_{\infty}, N=0.75-1.27 \log _{10}\left(F_{c}\right)$, and $C=-0.4-0.67 \log _{10}\left(F_{c}\right)$. The fitting parameter $F_{c}$ generally takes values between 0 and 1 . For reactions where radiative stabilization is unimportant, we use E11 to represent the pressure dependence with separate modified Arrhenius representations for the rate coefficients $k_{\circ}$ and $k_{\infty}$. The $F_{c}$ parameter is taken as a constant independent of temperature.

Radiative stabilization complicates the representation of the pressure dependence, as the association rate coefficient then becomes independent of pressure in the low-pressure limit, rather than linearly dependent on pressure (Vuitton et al., 2012). To see this, consider the modified strong collider/TST derivation of the rate constant (which provides a qualitatively useful description). The pressure dependent stabilization rate constant may be written as

$$
k(T,[M])=\int k_{a s s}(E) P_{s t a b}(E) P_{B o l t z}(E) d E,
$$

where $k_{a s s}(E)$ is the microcanonical rate constant for the capture rate, $P_{\text {stab }}(E)$ is the probability that the initially formed complex is stabilized, and $P_{B o l t z}(E)$ is the Boltzmann probability, $\rho(E) \exp (-\beta E) / Q(T)$, with $\rho(E)$ as the density of states of the reactants, $\beta=1 /\left(k_{B} T\right), k_{B}$ is Boltzmann's constant, and $\mathrm{Q}(\mathrm{T})$ is the partition function of the reactants. Within transition state theory, $k_{\text {ass }}(E)=N^{\dagger}(E) /[h \rho(E)]$, where $N^{\dagger}$ is the number of available states for motion on the transition state dividing surface separating reactants from products, and h is Planck's constant. With the modified strong collider assumption the stabilization probability is given by the probability of a stabilizing collision occurring during the lifetime of the collision complex:

$$
P_{s t a b}(E)=\beta_{c} k_{c}[M] /\left(\beta_{c} k_{c}[M]+k_{d i s s}(E)\right),
$$


where $\beta_{c}$ is the probability that any one collision is stabilizing, and $k_{c}$ is the inelastic collision rate. Allowing for an energy dependent radiative emission rate, $k_{R}^{\prime}(E)$, modifies the stabilization probability:

$$
P_{s t a b}(E)=\left(\beta_{c} k_{c}[M]+k_{R}^{\prime}(E)\right) /\left(\beta_{c} k_{c}[M]+k_{R}^{\prime}(E)+k_{d i s s}(E)\right)
$$

48 The pressure dependent association rate constant is then expressed as

$k(T,[M])=[1 / h Q(T)] \int N^{\dagger}(E) \exp (-\beta E)\left(\beta_{c} k_{c}[M]+k_{R}^{\prime}(E)\right) /\left(\beta_{c} k_{c}[M]+k_{R}^{\prime}(E)+k_{d i s s}(E)\right) d E$

$$
k_{R}(T)=[1 / h Q(T)] \int N^{\dagger}(E) \exp (-\beta E) k_{R}^{\prime}(E) /\left(k_{R}^{\prime}(E)+k_{d i s s}(E)\right) d E
$$

Unfortunately, there does not appear to be a standard literature form for representing the effect of radiative emission on the pressure dependence. We employ a simple modification to the Troe form:

$$
k(T,[M])=k_{R}+F k_{\infty}^{\prime} k_{\circ}[M] /\left(k_{\infty}^{\prime}+k_{\circ}[M]\right)
$$

where $k_{\infty}^{\prime}=k_{\infty}-k_{R}$. This form approaches $k_{R}$ in the low pressure limit, retains the Troe form when $k_{R}$ is small, and approaches $k_{\infty}$ in the high pressure limit or when $k_{R}$ is large (cf. Figure 19). At intermediate pressures, the parameter $F_{c}$, which determines the broadening factor $\mathrm{F}$, allows for a reasonable reproduction of our explicit master equation calculations. A representative illustration of the quality of the fit provided by Eq. (E18) is given in Figure 20 for the $\mathrm{C}_{2} \mathrm{H}_{5}+\mathrm{CH}_{3}$ recombination reaction at $140 \mathrm{~K}$. 


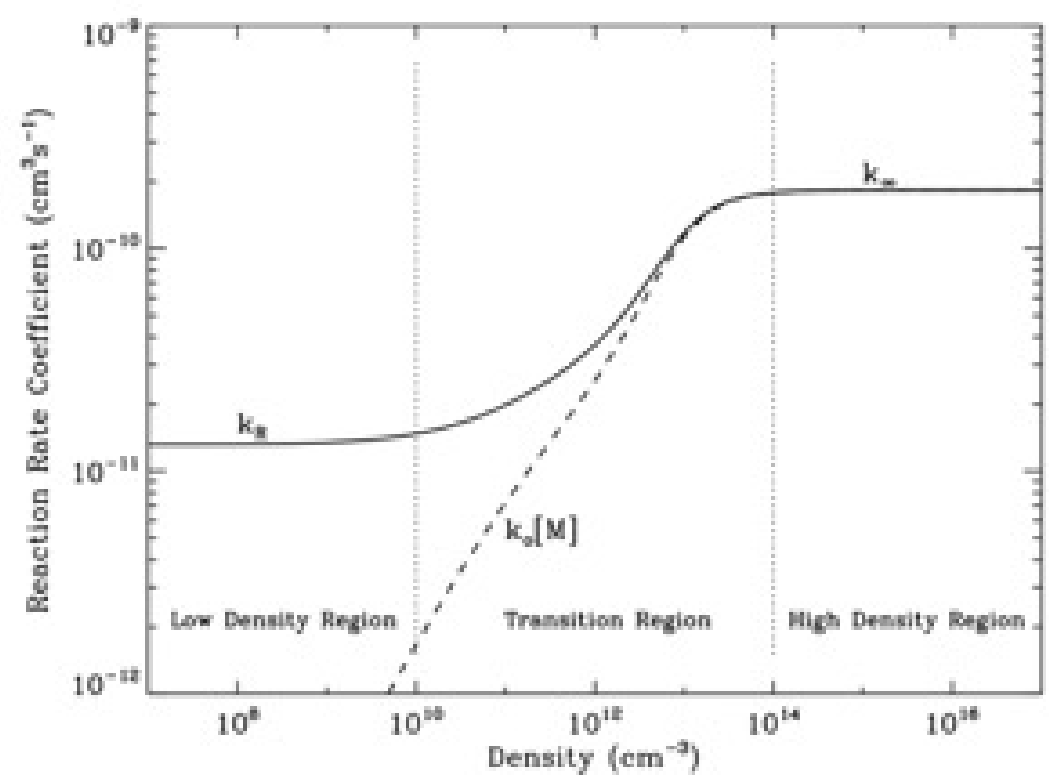

Figure 19: Representative illustration of an association reaction rate coefficient versus density. 


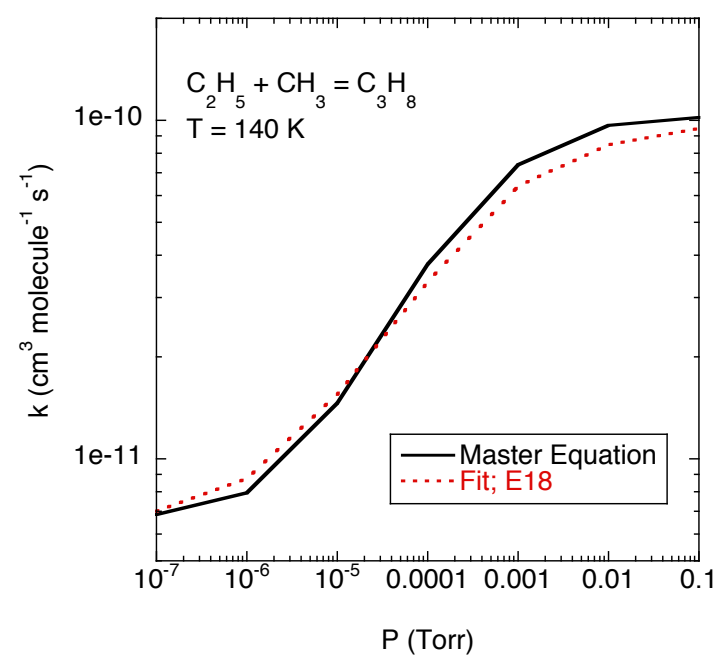

Figure 20: Plot of the pressure dependence of the master equation based theoretical predictions for the $\mathrm{C}_{2} \mathrm{H}_{5}+\mathrm{CH}_{3}$ recombination rate constant together with the fit based on Eq. (E18).

\subsubsection{Species}

2.7.2.1. Neutrals. The chemically active neutral species in our model are listed in Table B.3 and consist of 38 molecules and 45 radicals (not counting ${ }^{15} \mathrm{~N}$ isotopologues, see section 2.7.4). We consider a complete reaction set for the $\mathrm{C}$ through $\mathrm{C}_{4}$ hydrocarbons as well as the polyyne $\mathrm{C}_{6} \mathrm{H}_{2}$ and the aromatic compounds $\mathrm{C}_{6} \mathrm{H}_{6}$ and $\mathrm{C}_{7} \mathrm{H}_{8}$. We also consider several species involving $\mathrm{N}$ and $\mathrm{O}$ but deliberately focus exclusively on those species for which observational constraints are available. We include coupling between oxygen and nitrogen chemistries. We do not consider sulfur-bearing species since their source is not well constrained and expected to be small and unimportant for the abundance profiles of other species (Nixon et al., 2013; Hickson et al., 2014).

The fact that some products are not tracked in this work does represent a minor inconsistency in conservation of elements in the model, but as later discussed in section 5.2, the column-integrated rate of these reactions sum to $\sim 5 \times 10^{8} \mathrm{~cm}^{-2} \mathrm{~s}^{-1}$. Even though a fast catalytic recycling of $\mathrm{C}-\mathrm{C}_{4}$ species from $\mathrm{C}_{5}$ or higher hydrocarbons cannot be ruled out, the non-detection of the latter implies that their abundances are small, so that their effect on the system is probably of minor importance.

Molecules having more than two atoms exhibit several isomers. Since we expect the less stable forms to be less abundant, we have deliberately chosen to 
Table 4: Lifetime of some metastable states. Those included in the model are in bold.

\begin{tabular}{lll}
\hline \hline Species & Lifetime & Reference \\
\hline \hline $\mathrm{N}_{2}\left(\mathrm{~A}^{3} \Sigma_{\mathrm{u}}^{+}\right)$ & $2.4 \mathrm{~s}$ & Piper (1993) \\
$\mathbf{N}\left({ }^{2} \mathbf{D}\right)$ & $14 \mathrm{~h}$ and $37 \mathrm{~h}^{(a)}$ & Ralchenko et al. (2011) \\
$\mathrm{N}\left({ }^{2} \mathrm{P}\right)$ & $11 \mathrm{~s}$ and $10 \mathrm{~s}^{(b)}$ & Ralchenko et al. (2011) \\
$\mathbf{C H}_{2}\left(\mathbf{a}^{1} \mathbf{A}_{1}\right)$ & $18 \mathrm{~s}$ & Jacox (2003) \\
$\mathrm{C}\left({ }^{1} \mathrm{D}\right)$ & $54 \mathrm{~min}$ & Schofield (1979) \\
$\mathbf{O}\left({ }^{1} \mathbf{D}\right)$ & $2.5 \mathrm{~min}$ & Okabe (1978) \\
$\mathrm{O}\left({ }^{1} \mathrm{~S}\right)$ & $770 \mathrm{~ms}$ & Koyano et al. (1975) \\
\hline \hline
\end{tabular}

${ }^{(a)}$ Lifetimes for the $\mathrm{N}\left({ }^{2} \mathrm{D}_{3 / 2}\right)$ and $\mathrm{N}\left({ }^{2} \mathrm{D}_{5 / 2}\right)$ states, respectively.

${ }^{(b)}$ Lifetimes for the $\mathrm{N}\left({ }^{2} \mathrm{P}_{1 / 2}\right)$ and $\mathrm{N}\left({ }^{2} \mathrm{P}_{3 / 2}\right)$ states, respectively.

consider only the most stable isomer. There are two exceptions to this: $\mathrm{C}_{3} \mathrm{H}_{4}$ and $\mathrm{HCN}$ for which a less stable form has been (at least tentatively) detected on Titan: methylacetylene, $\mathrm{CH}_{3} \mathrm{CCH} /$ allene, $\mathrm{CH}_{2} \mathrm{CCH}_{2}$ (Roe et al., 2011), and hydrogen cyanide HCN / hydrogen isocyanide $\mathrm{HNC}$ (Moreno et al., 2011). We discuss $\mathrm{CH}_{3} \mathrm{CCH} / \mathrm{CH}_{2} \mathrm{CCH}_{2}$ in section 4.2.3.1 and $\mathrm{HCN} / \mathrm{HNC}$ in section 4.2.3.2.

Hébrard et al. (2013) revisited the chemistry of $\mathrm{C}_{3} \mathrm{H}_{x}$ hydrocarbons and included in their chemical network two $\mathrm{C}_{3} \mathrm{H}$ isomers: 2-cyclopropyn-1-yl $\left(\mathrm{c}-\mathrm{C}_{3} \mathrm{H}\right)$ and 2-propynylidyne $\left(\mathrm{l}_{3} \mathrm{C}\right)$, and three $\mathrm{C}_{3} \mathrm{H}_{2}$ isomers: cyclopropenylidene (c$\mathrm{C}_{3} \mathrm{H}_{2}$ ), propadienylidene $\left(\mathrm{l}-\mathrm{C}_{3} \mathrm{H}_{2}\right)$ and propynylidene $\left(\mathrm{t}-\mathrm{C}_{3} \mathrm{H}_{2}\right)$. However, because none of the reactions involving these species are identified as key reactions responsible for uncertainties on major $\mathrm{C}_{3} \mathrm{H}_{x}$ compounds (cf. their Table 3), we decided to only consider one isomer for both species. We indeed find that neither $\mathrm{C}_{3} \mathrm{H}$ or $\mathrm{C}_{3} \mathrm{H}_{2}$ has any impact on the overall chemistry because of their low production rate.

2.7.2.2. Metastable Species. It was soon recognized that electronically excited atomic nitrogen $\left(\mathrm{N}\left({ }^{2} \mathrm{D}\right)\right.$, the ground state is $\left.\mathrm{N}\left({ }^{4} \mathrm{~S}\right)\right)$, methylene $\left(\mathrm{CH}_{2}\left(\mathrm{a}^{1} \mathrm{~A}_{1}\right)\right.$, the ground state is $\left.\mathrm{X}^{3} \mathrm{~B}_{1}\right)$ and atomic oxygen $\left(\mathrm{O}\left({ }^{1} \mathrm{D}\right)\right.$, the ground state is $\left.\mathrm{O}\left({ }^{3} \mathrm{P}\right)\right)$, formed in the photodissociation of $\mathrm{N}_{2}, \mathrm{CH}_{4}, \mathrm{H}_{2} \mathrm{O}$ and $\mathrm{CO}$, respectively, have a radiative lifetime long enough to contribute to Titan's atmospheric chemistry (Yung et al., 1984; Toublanc et al., 1995). The production of excited $\mathrm{N}\left({ }^{2} \mathrm{D}\right)$ atoms is an important step in the chemical evolution of the upper atmosphere of Titan, as nitrogen atoms in this state are much more reactive than those in the ground ${ }^{4} \mathrm{~S}$ state and are not efficiently quenched by collisions with $\mathrm{N}_{2}$ (the room temperature recommended value is $\mathrm{k}=1.7 \times 10^{-14} \mathrm{~cm}^{3} \mathrm{~s}^{-1}$ Herron (1999)). $\mathrm{N}\left({ }^{2} \mathrm{D}\right), \mathrm{CH}_{2}\left(\mathrm{a}^{1} \mathrm{~A}_{1}\right)$ and $\mathrm{O}\left({ }^{1} \mathrm{D}\right)$ are to date the only excited states that have been included in Titan photochemical models. However, four other excited species are metastable and could have some impact on the chemistry as well: $\mathrm{N}_{2}\left(\mathrm{~A}^{3} \Sigma_{\mathrm{u}}^{+}\right), \mathrm{N}\left({ }^{2} \mathrm{P}\right), \mathrm{C}\left({ }^{1} \mathrm{D}\right)$ and $\mathrm{O}\left({ }^{1} \mathrm{~S}\right)$. Their lifetimes are given in Table 4.

The production of $\mathrm{N}_{2}\left(\mathrm{~A}^{3} \Sigma_{\mathrm{u}}^{+}\right), \mathrm{N}\left({ }^{2} \mathrm{D}\right)$ and $\mathrm{N}\left({ }^{2} \mathrm{P}\right)$ by interaction with photons and photoelectrons of $\mathrm{N}_{2}$ is discussed in Lavvas et al. $(2011 \mathrm{a}, 2015) . \mathrm{N}\left({ }^{2} \mathrm{D}\right)$ 
and $\mathrm{N}\left({ }^{2} \mathrm{P}\right)$ are also formed in the electron-ion recombination of $\mathrm{N}_{2}{ }^{+}$but their production rate through this pathway is small since most $\mathrm{N}_{2}{ }^{+}$react with $\mathrm{CH}_{4}$. $\mathrm{N}_{2}\left(\mathrm{~A}^{3} \Sigma_{\mathrm{u}}^{+}\right)$and $\mathrm{N}\left({ }^{2} \mathrm{P}\right)$ are quite nonreactive with $\mathrm{N}_{2}, \mathrm{H}_{2}$ and $\mathrm{CH}_{4}$, quenching into $\mathrm{N}_{2}(\mathrm{X})$ and $\mathrm{N}\left({ }^{2} \mathrm{D}\right)$, respectively being the main channels. Reaction of $\mathrm{N}_{2}\left(\mathrm{~A}^{3} \Sigma_{\mathrm{u}}^{+}\right)$with unsaturated hydrocarbons leads to the dissociation of the latter (Herron, 1999; Dutuit et al., 2013) but the mole fraction of $\mathrm{N}_{2}\left(\mathrm{~A}^{3} \Sigma_{\mathrm{u}}^{+}\right)$has been calculated to be only about $10^{-9}$ (Lavvas et al., 2015) and we therefore did not include this species in the chemical network. The production of $\mathrm{N}\left({ }^{2} \mathrm{P}\right)$ relative to $\mathrm{N}\left({ }^{2} \mathrm{D}\right)$ is not well constrained by measurements both for photons and photoelectrons. Based on this argument, and also because the reaction products of $\mathrm{N}\left({ }^{2} \mathrm{P}\right)$ are unknown (Herron, 1999; Dutuit et al., 2013), we assume in the calculations that all $\mathrm{N}\left({ }^{2} \mathrm{P}\right)$ formed results in $\mathrm{N}\left({ }^{2} \mathrm{D}\right)$ (section 2.6.3). $\mathrm{C}\left({ }^{1} \mathrm{D}\right)$ is a product in the photodissociation of $\mathrm{CO}$ as shown in Table B.5. CO being efficiently shielded by $\mathrm{N}_{2}$, the production rate of $\mathrm{C}\left({ }^{1} \mathrm{D}\right)$ is small $\left(5 \times 10^{4} \mathrm{~cm}^{-2}\right.$ $\left.\mathrm{s}^{-1}\right)$ and we assume that the atomic carbon ground state, $\mathrm{C}\left({ }^{3} \mathrm{P}\right)$, is formed instead. We consider that the excited state of neutral oxygen $\mathrm{O}\left({ }^{1} \mathrm{~S}\right)$, produced in the reaction of $\mathrm{N}\left({ }^{2} \mathrm{D}\right)$ and $\mathrm{NO}\left(\mathrm{R}_{n} 584 \mathrm{c}\right)$, is converted very rapidly to $\mathrm{O}\left({ }^{1} \mathrm{D}\right)$.

2.7.2.3. Positive Ions. The chemical network contains 196 positive ions (from $\mathrm{m} / \mathrm{z}=1$ to $\sim 100$, which corresponds to the mass range of INMS) containing carbon, nitrogen, oxygen and hydrogen that are listed in Table B.4. It is based on Vuitton et al. (2007) but has been improved in several ways:

- In order to better describe the oxygen chemistry in the upper atmosphere, 14 new oxygen bearing species $\left(\mathrm{O}^{+}, \mathrm{OH}^{+}, \mathrm{CO}^{+}, \mathrm{HCO}^{+}, \mathrm{HOC}^{+}, \mathrm{CH}_{2} \mathrm{O}^{+}\right.$, $\mathrm{CH}_{2} \mathrm{OH}^{+}, \mathrm{HC}_{2} \mathrm{O}^{+}, \mathrm{CH}_{2} \mathrm{CO}^{+}, \mathrm{CH}_{3} \mathrm{CO}^{+}, \mathrm{CH}_{3} \mathrm{COH}^{+}, \mathrm{HC}_{3} \mathrm{O}^{+}, \mathrm{C}_{2} \mathrm{H}_{2} \mathrm{CO}^{+}$ and $\left.\mathrm{C}_{2} \mathrm{H}_{3} \mathrm{CO}^{+}\right)$and 4 new oxygen and nitrogen bearing species $\left(\mathrm{NO}^{+}\right.$, $\mathrm{HNO}^{+}, \mathrm{NCO}^{+}$and $\mathrm{HNCO}^{+}$) are considered.

- In order to couple the ion and neutral chemistry, the neutral products of electron recombination reactions have been included.

- In order not to lose ions in a bottomless sink, ions with a mass-to-charge ratio above 100 all fall in a generic bin called " $\mathrm{C}_{x} \mathrm{H}_{y} \mathrm{~N}_{z}^{+}$". This virtual species is not involved in further ion-molecule reactions but does recombine with electrons to produce untracked neutral species.

- In order to describe the lower ionospheric layer induced by GCR, we include cluster ions.

Because of the lack of kinetic data, we do not distinguish different isomers, with the exception of linear/cyclic $\mathrm{C}_{3} \mathrm{H}_{3}{ }^{+}, \mathrm{HCN}^{+} / \mathrm{HNC}^{+}, \mathrm{CNC}^{+} / \mathrm{C}_{2} \mathrm{~N}^{+}$and $\mathrm{HCO}^{+} / \mathrm{HOC}^{+}$, for which some information is available.

Nicolas et al. (2003b) estimated that in the photoionization of $\mathrm{N}_{2}$ about $15 \%$ of the $\mathrm{N}^{+}$ions are produced in the first excited state, $\mathrm{N}^{+}\left({ }^{1} \mathrm{D}\right)$, which has a lifetime of $258 \mathrm{~s}$ (Wiese and Fuhr, 2007). Even though the branching ratios of the products formed by $\mathrm{N}^{+}\left({ }^{1} \mathrm{D}\right)$ with methane are quite different from the products formed by $\mathrm{N}^{+}\left({ }^{3} \mathrm{P}\right)$ (Alcaraz et al., 2004), a sensitivity study showed 
that taking into account the $\mathrm{N}^{+}\left({ }^{1} \mathrm{D}\right)$ state has no relevant effect on the model predictions (Carrasco et al., 2008) and therefore we decided to ignore it.

Lilensten et al. (2005) investigated the presence of a $\mathrm{N}_{2}{ }^{++}$layer in the upper atmosphere of Titan, considering production by double photoionization and photoelectron impact of $\mathrm{N}_{2}$ and loss by dissociative recombination with electrons and chemical reactions with $\mathrm{N}_{2}$ and $\mathrm{CH}_{4}$. They predicted a maximum density of $10^{-2} \mathrm{~cm}^{-3}$ at $1100 \mathrm{~km}$. Because this represents only about $10^{-5}$ of the total ion density and because the predicted emissions (fluorescence from $\mathrm{D}^{1} \Sigma_{u}^{+}$to $\mathrm{X}^{1} \Sigma_{g}^{+}$) have not been detected so far by UVIS (Ajello et al., 2007), we do not consider $\mathrm{N}_{2}^{++}$in the chemical network.

2.7.2.4. Negative Ions. The chemical network is similar to that described in Vuitton et al. (2009). It contains 12 negative ions listed in Table B.4. A few cross-sections and rate coefficients have been updated and a few new reactions have been added, as described in section 2.7.3.3. Also, we include specific products for ion recombination and associative detachment reactions, in order to couple the ion to the neutral chemistry. However, the negative ion density is low, at the ionospheric peak typically $1 \%$ of the positive ion density, and the presence of negative ions does not have any impact on the abundance of neutral or positive ion species.

\subsubsection{Gas Phase Reactions}

The chemical network is based on the ion chemistry presented in Vuitton et al. (2007) and Vuitton et al. (2009), and the neutral chemistry presented in Hörst et al. (2008), Yelle et al. (2010) and Vuitton et al. (2012) for oxygen bearing species, nitrogen bearing species and hydrocarbons, respectively. In the present work, many rate coefficients have been updated and numerous reactions have been added. The reaction network has been last updated in December 2016 and reflects the state-of-the-art of the laboratory measurements and theoretical calculations available at that time. The different families of reactions included in the model are listed in Table 5 and are further described in the next sections.

\subsubsection{Neutrals.}

General Description. The model includes 572 reactions among neutral species. These are listed in Table B.15 along with the parameters for calculation of the rate coefficients as a function of pressure and temperature. The reaction list has been compiled mainly for a carbon, nitrogen-rich and oxygen poor atmosphere, for temperatures at or below $300 \mathrm{~K}$. It consists of reactions we have identified, based on available kinetics or thermodynamics, as being important for the understanding of the chemistry in the atmosphere of Titan. We rule out endothermic processes and reactions known to exhibit an energy barrier, at the exception of a few cases where they have been specifically studied down to low temperature (see further down). Kinetic parameters are primarily taken from databases and we refer to the compilations of Baulch et al. (2005) and Sander et al. (2011). A smaller number come from the online 
Table 5: Classes of reactions included in the photochemical model.

\begin{tabular}{ll}
\hline \hline Reaction class & Chemical equation \\
\hline \hline & Neutral Species \\
Photodissociation & $A B+h \nu \rightarrow A+B$ \\
Electron dissociation & $A B+e_{S}^{-} \rightarrow A+B+e^{-}$ \\
Bimolecular reactions & $A+B C \rightarrow A B+C$ \\
Termolecular association & $A+B+M \rightarrow A B+M$ \\
Radiative association & $A+B \rightarrow A B+h \nu$ \\
\hline & Positive ions \\
(Dissociative) photoionization & $A B+h \nu \rightarrow A B^{+}\left(A^{+}+B\right)+e^{-}$ \\
(Dissociative) electron ionization & $A B+e_{S}^{-} \rightarrow A B^{+}\left(A^{+}+B\right)+2 e^{-}$ \\
Bimolecular reactions & $A^{+}+B C \rightarrow A B^{+}+C$ \\
Termolecular association & $A^{+}+B+M \rightarrow A B^{+}+M$ \\
Radiative association & $A^{+}+B \rightarrow A B^{+}+h \nu$ \\
Dissociative recombination & $A B^{+}+e_{T}^{-} \rightarrow A+B$ \\
Radiative recombination & $A^{+}+e_{T}^{-} \rightarrow A+h \nu$ \\
\hline & \\
Ion-pair formation & Negative ions \\
Dissociative attachment & $A B+h \nu \rightarrow A^{-}+B^{+}$ \\
Radiative attachment & $A+e_{S}^{-} \rightarrow A^{-}+B$ \\
Bimolecular reactions & $A^{-}+B C \rightarrow A B^{-}+C$ \\
Photodetachment & $A^{-}+h \nu \rightarrow A+e^{-}$ \\
Ion recombination & $A^{-}+B C^{+} \rightarrow A B+C$ \\
Associative detachment & $A^{-}+B \rightarrow A B+e^{-}$ \\
\hline \hline
\end{tabular}

$e_{S}^{-}$and $e_{T}^{-}$stand for supra-thermal and thermal electrons, respectively. 
database KIDA (Wakelam et al. (2012, 2015), http://kida.obs.u-bordeaux1.fr), where a small subset of reactions has been evaluated by a panel of physical chemists. For those reactions that were not referenced in these evaluations or when more recent experiments or calculations have been undertaken, we had to make choices among the different measurements available in the National Institute of Standards and Technology (NIST) Chemical Kinetics Database (http://kinetics.nist.gov/kinetics/index.jsp), an uncritical compilation, and in the recent literature. For some important reactions for which there was inadequate kinetic data, we have performed theoretical estimates based on transition state theory. This is described further below and in section Appendix A.

Laboratory experiments are usually performed at room temperature (300 $\mathrm{K}$ ) and do not provide any information on the variation of the rate coefficient with temperature. When a temperature dependence is investigated, it is often motivated by the importance of the given reaction in combustion studies (500-2000 K). Consequently, it is important to keep in mind that the majority of published results are obtained at higher temperatures than are found in the atmosphere of Titan (75-200 K). Fortunately, in the 90's, there was renewed interest in astronomy in the physical-chemistry community and experiments based on the CRESU technique (a French acronym for Cinétique de Réaction en Ecoulement Supersonique Uniforme) were designed to reach the low temperatures encountered in planetary atmospheres and the interstellar medium. Building on these results, Smith et al. (2006) correlated the values of the rate coefficients for the reactions between radicals and unsaturated hydrocarbons and their dependence on temperature with differences between the ionization energy (I.E.) of the molecular reagent and the electron affinity (E.A.) of the radical. They came to the conclusion that when (I.E. - E.A.) is less than $\sim 8.75$ $\mathrm{eV}$, then it is likely that the reaction will remain rapid at low temperature.

When no experimental or theoretical studies are available for a given reaction, we applied the extremely valuable approach from Smith et al. (2006) to determine if the reaction has to be included in the model or not. As a first approximation, it can be considered that the reaction of $\mathrm{H}, \mathrm{C}, \mathrm{CH},{ }^{1} \mathrm{CH}_{2}, \mathrm{C}_{2}$, $\mathrm{C}_{2} \mathrm{H}, \mathrm{C}_{4} \mathrm{H}, \mathrm{N}\left({ }^{2} \mathrm{D}\right), \mathrm{NH}, \mathrm{CN}, \mathrm{C}_{3} \mathrm{~N}, \mathrm{O}\left({ }^{1} \mathrm{D}\right)$ and $\mathrm{OH}$ with unsaturated species is fast. If room temperature data are available, we used the long-range capture theory to extend those measurements towards low temperature (Faure et al., 2009). If no kinetic data are available, we estimate the rate coefficient on the basis of chemical similarity with the one or more of the relatively small number of reactions for which rate coefficients have been measured at low temperatures. For example, the reaction of ${ }^{1} \mathrm{CH}_{2}$ with $\mathrm{C}_{2} \mathrm{H}_{2}$ and $\mathrm{C}_{2} \mathrm{H}_{4}$ has been studied, even at low temperatures, and rate coefficients were found to be close to the collision limit (Gannon et al., 2010a). We assume that this is also true for reactions of ${ }^{1} \mathrm{CH}_{2}$ with larger alkenes and alkynes.

Radical-radical association reactions are amongst those that are the least constrained experimentally at low pressure and temperature. For many of these reactions only the high pressure limit rate coefficient $\left(k_{\infty}\right)$ is available and the buffer gas is rarely $\mathrm{N}_{2}$. Moreover, we showed that radiative association is efficient for adducts having as little as four $\mathrm{C}$ atoms, a process that can only be 
evaluated with state-of-the-art ab initio calculations (Vuitton et al., 2012). We follow our previous approach for hydrocarbons and extend it to nitrogen and oxygen containing species.

We estimate $k_{\infty}$ from the following rule: for $\mathrm{H}+$ radical, $k_{\infty}=2 \times 10^{-10}$ $\mathrm{cm}^{3} \mathrm{~s}^{-1}$; for cross reactions (two different radicals), $k_{\infty}=8 \times 10^{-11} \mathrm{~cm}^{3}$ $\mathrm{s}^{-1}$ and for self-reactions (two identical radicals), $k_{\infty}=5 \times 10^{-11} \mathrm{~cm}^{3} \mathrm{~s}^{-1}$. These empirical estimates are based on prior theoretical studies of $\mathrm{H}+$ alkyl radical reactions (Harding et al., 2005) and alkyl + alkyl reactions (Klippenstein et al., 2006), and from our knowledge of the low-temperature behavior of rate coefficients (Georgievskii and Klippenstein, 2005). For $k_{\circ}$ and $k_{R}$, we consider that the rate coefficient depends primarily on the number $N$ of heavy atoms $(\mathrm{C}$, $\mathrm{N}, \mathrm{O})$ involved. The formulae, $k_{R}=6 \times 10^{-16} N^{7.5}$ and $k_{\circ}=8 \times 10^{-30} e^{4.7 N}$, are fits to the theoretically predicted rate coefficients for five reactions (cf. Table 1 and Figure 1 in Vuitton et al. (2012)).

The identities of product species and associated branching ratios are rarely determined along with the measurements of kinetic rate coefficients, even at room temperature. Only a handful have been obtained at low temperature (Gannon et al., 2007, 2010a). Collision free experiments are the principal source of information when it comes to products (cf. for example Jones et al. (2010) and Zhang et al. (2009)) but they are often performed at high collision energy (10-50 kJ mol $\left.{ }^{-1}\right)$. Unlike rate coefficients, branching ratios are difficult to guess from first principles (beyond the fact that the reaction should be exothermic) and a new generation of experiments is mandatory. Therefore, estimates of the probable products are offered based on various chemical arguments.

For many unsaturated molecules, there are multiple stable isomers that may be formed, and the barriers between them are large enough to prohibit thermal isomerization. For example, for $\mathrm{CH}_{3} \mathrm{CCH} / \mathrm{CH}_{2} \mathrm{CCH}_{2}$ the barrier separating the two species is $65.1 \mathrm{kcal} / \mathrm{mol}$ (Alnama et al., 2007), while for $\mathrm{HCN} / \mathrm{HNC}$ the barrier is $30.2 \mathrm{kcal} / \mathrm{mol}$ (Herbst et al., 2000). For simplicity, unless otherwise specified, we presume the formation of the most stable of these isomers. In many cases, the reaction that produces a given species is exothermic enough that there is a rapid equilibration of the isomers prior to stabilization. In this instance, the most stable isomer will be the dominant species. Furthermore, in many cases, the sequence of radical (e.g., H atoms) addition, followed by isomerization, and then identical radical loss can serve to catalyze the conversion to the lower energy isomer. Similarly, photoexcitation followed by collisional or radiative cooling, will also generally lead to production of the most stable isomer.

Theoretical Calculations of Rate Coefficients. For 70 neutral reactions we have used the ab initio transition state theory based master equation (AITSTME) approach (Harding et al., 2007; Truhlar et al., 1996; Miller and Klippenstein, 2006; Klippenstein, 2017) to predict the temperature and pressure dependence of the rate coefficient. We chose the most significant and/or less constrained reactions as discussed in section 3.2. A list of these reactions can be found in Tables A.1-A.3. 
The AITSTME approach involves three distinct calculations. First, the stationary points on the potential energy surface connecting reactants and products are explored with ab initio electronic structure theory. Then, transition state theory is used to predict the microcanonical rate constants for each of the chemical processes occurring on that potential energy surface. Next, these microcanonical rate constants are coupled with simple models for collisional energy transfer and radiative emission to form a linear master equation describing the time dependence of the energy resolved species populations. Finally, the eigensolution for this master equation directly yields the global phenomenological rate coefficients at arbitrary temperatures and pressures (Miller and Klippenstein, 2006). The chemical model employs simple representations of these theoretically predicted temperature (i.e., modified Arrhenius) and pressure (i.e., Troe format) dependent rate coefficients. A brief overview of the present implementation of this general methodology is provided next, with more reaction specific details provided in Appendix A.

Many of the reactions of interest have small positive barriers, and the predicted kinetics is then strongly dependent on the estimated barrier height and tunneling frequency. Here, for many species we have employed the coupled cluster method with singles doubles and perturbative triples $[\operatorname{CCSD}(\mathrm{T})]$ method (Raghavachari et al., 1989) with the cc-pVTZ basis set (Dunning, 1989) to predict the rovibrational properties of the saddle point and other stationary points of relevance to the kinetics. This method provides high accuracy partition functions, tunneling frequencies, and geometries. For some of the larger species computational limitations lead us to instead employ B3LYP density function theory (Becke, 1993) rovibrational evaluations. Higher accuracy barrier height estimates are generally obtained here from further $\operatorname{CCSD}(\mathrm{T})$ calculations with larger basis sets in an attempt to produce the CCSD(T)/CBS (complete basis set) limit. For some cases, we include a number of high level corrections, such as the $\operatorname{CCSDT}(\mathrm{Q})$ correction for quadruple excitations, core-valence, relativistic, diagonal Born-Oppenheimer, and vibrational anharmonicity corrections. A number of these higher level energy evaluations employ an early version of the ANL0 scheme (Klippenstein et al., 2017), which has been shown to yield $2 \sigma$ uncertainties on the order of 0.2 to $0.3 \mathrm{kcal} / \mathrm{mol}$, as do other high level ab initio methods, such as the HEAT (Bomble et al., 2006) and W4 (Karton et al., 2006) protocols.

For radical-radical reactions, and any other reactions where the barrier is submerged below the reactants, we implement the variable-reaction coordinate transition state theory (VRC-TST) approach (Klippenstein, 1992; Georgievskii and Klippenstein, 2003) with direct multireference wavefunction based potential energy surface evaluations (Harding et al., 2005). This approach yields highpressure capture rates that appear to generally be accurate to within about $20 \%$ (Klippenstein et al., 2006). Typically, the direct orientational sampling is performed at the CASPT2 level employing modest basis sets (e.g., cc-pVTZ) with one-dimensional corrections incorporated from higher-level calculations [larger basis sets, larger active spaces, multi-reference configuration interaction (MRCI) evaluations, and/or including geometry relaxation effects] along the minimum 
energy path. Our kinetic analyses for the small barrier reactions generally employ Eckart tunneling corrections, one-dimensional hindered rotor treatments, and variational transition state theory treatments as appropriate. For the barrierless reactions, the TST analysis is implemented at the energy E and angular momentum J resolved level. For reactions with a barrier, and in all the master equation treatments, the analyses are implemented at the microcanonical level. For many cases, these kinetic analyses are either identical to or are slightly modified from earlier analyses carried out for the higher temperatures of relevance to combustion. In some instances these prior studies included modest adjustments of barrier heights (e.g. a few tenths of a $\mathrm{kcal} / \mathrm{mol}$ ) to improve agreement with experimental data.

The pressure dependence of the kinetics was obtained from the one-dimensional master equation employing Lennard-Jones collision frequencies and the exponential down model for energy transfer. The exponential parameter $\alpha$, which correlates closely with the average downwards energy transfer $\left\langle\Delta E_{\text {down }}\right\rangle$, was generally represented with the form $A(T / 300)^{n}$, with the parameters A and $n$ empirically chosen from experience, from prior reaction specific studies, or from consideration of related trajectory analyses (Jasper and Miller, 2011; Jasper et al., 2015). The radiative emission rates were obtained from an average over the harmonic approximation to the radiative emission rate for a given vibrational state and the statistical distribution of vibrational state populations (Klippenstein et al., 1996).

The present $\operatorname{CCSD}(\mathrm{T}), \mathrm{CASPT}$, and MRCI calculations were performed with MOLPRO (Werner et al., 2012), with the CCSDT(Q) corrections obtained with Kallays add-on to MOLPRO (Kállay and Surján, 2001). The density functional calculations were obtained with Gaussian09 (Frisch et al., 2009), while the diagonal Born-Oppenheimer corrections were obtained with CFOUR (Stanton et al., 2010). The variable reaction coordinate transition state theory calculations were performed with VaReCoF (Georgievskii et al., 2016). The master equation analyses were mostly performed with the VariFlex code (Klippenstein et al., 2011), with a few of the more recent analyses employing the MESS code (Georgievskii et al., 2013; Georgievskii and Klippenstein, 2016).

It is generally difficult to ascertain the uncertainty in theoretical predictions, especially for reactions occurring at low temperatures over complex multiple well potential energy surfaces. In general, the uncertainties in our predicted bimolecular rate coefficients are larger the smaller the predicted rate coefficient. If it is near the collision limit, as for many of the radical-radical reactions with larger radicals, the uncertainty in the predictions may be as low as 20 or $30 \%$. In contrast, when the rate coefficients are as low as $10^{-17} \mathrm{~cm}^{3} \mathrm{~s}^{-1}$, it is likely that our uncertainties are closer to an order of magnitude. For rate coefficients in the $10^{-13} \mathrm{~cm}^{3} \mathrm{~s}^{-1}$ neighborhood, the uncertainty in the predictions is perhaps a factor of three. For a recent study on how the uncertainties in reaction rates and branching ratios propagate in Titan models and how they affect the model output, see Dobrijevic et al. (2014) and previous work from the same group.

In the end, the chemical network consists of 727 neutral reactions (not including ${ }^{15} \mathrm{~N}$ isotopologues), amongst which 497 rate coefficients had to be estimated 
because no experiments or calculations were available (cf. Table B.15). We will discuss later those unconstrained but nevertheless important reactions.

\subsubsection{Positive Ions.}

Ion-Neutral Reactions. The chemical network contains 1882 ion-neutral reactions that are presented in Table B.16. They mostly originate from the compilations by Anicich (1993), McEwan and Anicich (2007), Wakelam et al. (2012, 2015) and Dutuit et al. (2013), although a specific literature search has been conducted for many other reactions that were not listed in those papers. New relevant data that have been obtained since 2007 can be found in Edwards et al. (2008, 2009), Fondren et al. (2009), Gerlich and Borodi (2009), Stavish et al. (2009), Žabka et al. (2009), Adams et al. (2010), Dryahina et al. (2011), Gerlich et al. (2011), Mathews and Adams (2011), Plašil et al. (2011), Demarais et al. (2013) and Lin et al. (2013). They represent about 170 reactions.

In Vuitton et al. (2007), we pointed out that the production and loss reactions of most nitrogen bearing ions were not well characterized in the literature. There is however convincing theoretical evidence that proton transfer in ion molecule reactions can have no barrier when the reactions are exothermic (Lindinger et al., 2000). We therefore made the general hypothesis that protons flow from species with a low proton affinity (typically hydrocarbons) to species having a high proton affinity (typically nitrogen bearing species) and assumed that these reactions occurred with a rate $\mathrm{k}=3 \times 10^{-9} \mathrm{~cm}^{3} \mathrm{~s}^{-1}$, a simple and often reasonable approximation of the rate coefficient in the capture limit. However, better capture theory estimates for rate coefficients can be made rather easily, as long as the relevant parameters are known.

For non-polar neutral species $\left(\mathrm{H}_{2}, \mathrm{CH}_{4}, \mathrm{C}_{2} \mathrm{H}_{4}\right.$, etc.), we use the Langevin expression $k_{L}$ :

$$
k_{L}=2 \pi e(\alpha / \mu)^{1 / 2},
$$

where $e$ is the electronic charge of the ion, $\alpha$ is the dipole polarizability of the neutral and $\mu$ is the reduced mass of the reactants.

For neutrals having a permanent dipole moment (such as nitrogen bearing species), the Langevin expression no longer applies and we use the SuChesnavich expression instead (Woon and Herbst, 2009). The ion-dipole rate coefficient $k_{D}$ can be referenced to the Langevin value via the equations:

$$
k_{D} / k_{L}=0.4767 x+0.6200 \text { if } x \geq 2
$$

and

$$
k_{D} / k_{L}=(x+0.5090)^{2} / 10.526+0.9754 \text { if } x<2,
$$

with $x$ defined as:

$$
x=\frac{\mu_{D}}{(2 \alpha k T)^{1 / 2}},
$$


where $\mu_{D}$ is the dipole moment of the neutral, $k$ is the Boltzmann constant and $T$ is the temperature. $\alpha$ and $\mu_{D}$ are obtained from Woon and Herbst (2009).

Although the Langevin rate is temperature independent, the Su-Chesnavich expression exhibits a complex temperature dependence with $k_{D}$ increasing with decreasing temperature. We did not implement this temperature dependence in the model but used a temperature independent rate coefficient, calculated for $\mathrm{T}=150 \mathrm{~K}$. Using expression (E22), it can be shown that decreasing the temperature from 300 to $150 \mathrm{~K}$ leads to an increase of the rate coefficient by a few tens of percent at best. Therefore, for those reactions that occur at the capture rate, the temperature dependence of the rate coefficient is expected to be small. The temperature dependence can however, be much stronger for those reactions that are rather slow at room temperature and we made sure to use temperature dependent rate coefficients when they were available in the literature. Unfortunately, a large majority of the rate coefficients have only been measured at $300 \mathrm{~K}$.

Ion production by cosmic rays is now included in the model and is a source of ion-neutral chemistry below $100 \mathrm{~km}$. Three-body reactions are efficient in the lower atmosphere and 118 new chemical reactions to calculate the concentration of 15 new ions are considered. Most of these reactions were compiled in previous models by Capone et al. $(1976,1981)$ and Molina-Cuberos et al. (1999a, 2002), and originate from review papers by Anicich and McEwan (1997), Anicich et al. (2000) and McEwan and Anicich (2007). Other sources of information include the works of Smith et al. (1978a), Dheandhanoo et al. (1984), McEwan and Anicich (1995), Speller et al. (1995), Vacher et al. (1997), Milligan et al. (2001) and Hiraoka et al. (2003). We assume that protonated hydrocarbon ions (such as $\mathrm{CH}_{5}{ }^{+}$and $\mathrm{C}_{2} \mathrm{H}_{7}{ }^{+}$) form $\mathrm{C}_{n} \mathrm{H}_{m}^{+} \cdot \mathrm{CH}_{4}$ electrostatic clusters with methane rather than additional $\mathrm{C}-\mathrm{C}$ covalent bond type ions such as $\mathrm{C}_{3} \mathrm{H}_{11}{ }^{+}$(Capone et al., 1979).

Positive Ion Loss. The ultimate loss for positive ions is radiative or dissociative electron recombination. The 492 recombination reactions present in the network are listed in Table B.17. Rate constants originate from a thorough literature search. Since 2007, new data have been obtained for $\mathrm{CH}_{4}{ }^{+}$ (Thomas et al., 2013), $\mathrm{CH}_{5}{ }^{+}$(Kamińska et al., 2010), $\mathrm{C}_{4} \mathrm{H}_{4}{ }^{+}, \mathrm{C}_{5} \mathrm{H}_{6}{ }^{+}$and $\mathrm{C}_{7} \mathrm{H}_{7}{ }^{+}$ (Fournier et al., 2013), $\mathrm{C}_{6} \mathrm{H}_{6}{ }^{+}$and $\mathrm{C}_{6} \mathrm{H}_{7}{ }^{+}$(Hamberg et al., 2011), $\mathrm{C}_{7} \mathrm{H}_{9}{ }^{+}$(Osborne et al., 2011), $\mathrm{C}_{2} \mathrm{H}_{3} \mathrm{NH}^{+}$(Vigren et al., 2008), $\mathrm{HC}_{3} \mathrm{~N}^{+}$and $\mathrm{HC}_{3} \mathrm{NH}^{+}$(Vigren et al., 2012a), $\mathrm{C}_{2} \mathrm{H}_{3} \mathrm{CNH}^{+}$(Vigren et al., 2009), $\mathrm{C}_{3} \mathrm{H}_{5} \mathrm{NH}^{+}$(Vigren et al., 2010a), $\mathrm{C}_{4} \mathrm{H}_{5} \mathrm{NH}^{+}$(Osborne et al., 2011), $\mathrm{C}_{5} \mathrm{H}_{5} \mathrm{NH}^{+}$(Adams et al., 2010; Osborne et al., 2011), $\mathrm{N}_{2} \mathrm{H}^{+}$(Vigren et al., 2012b) and $\mathrm{CH}_{3} \mathrm{CHO}^{+}$(Vigren et al., 2010b). 


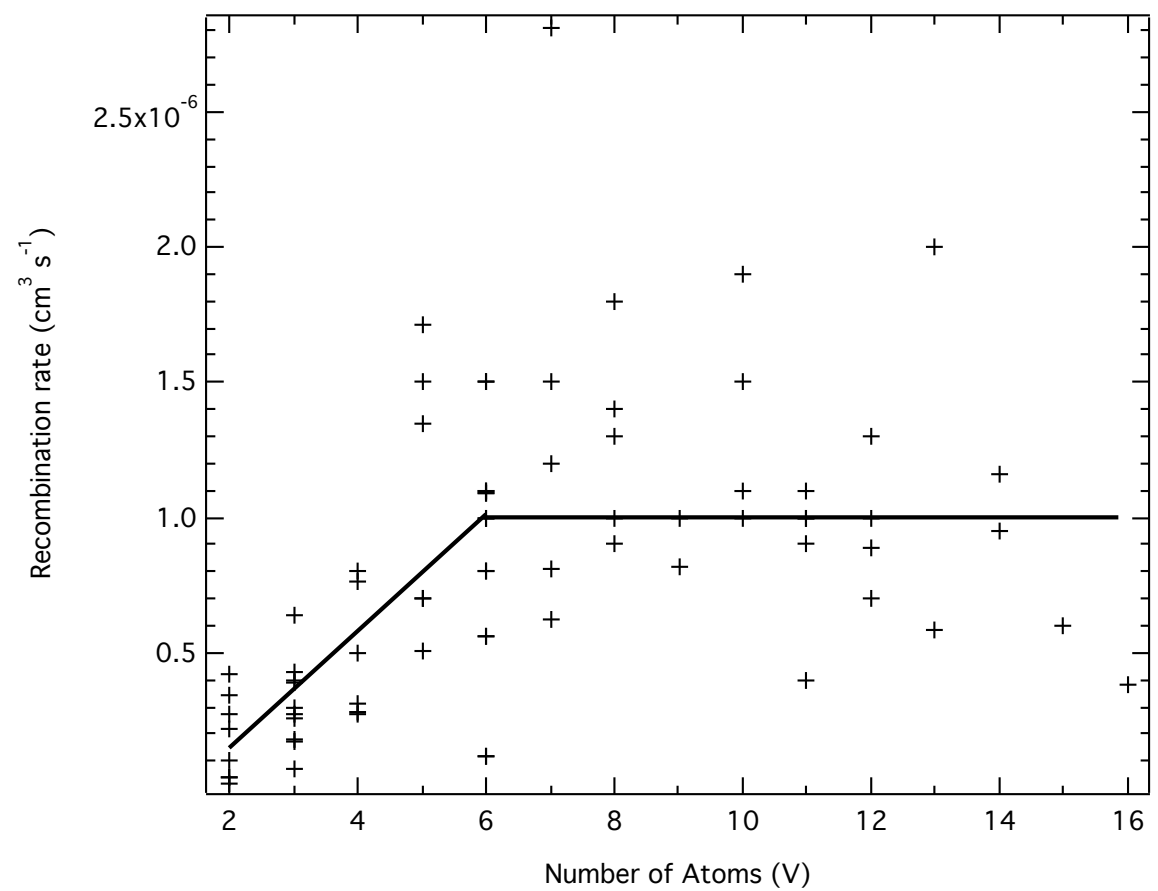

Figure 21: Dissociative recombination rate at an electron temperature of $300 \mathrm{~K}$ against the number of atoms constituting the ion.

When no literature data were available, we assumed, in Vuitton et al. (2007), a rate $\alpha=7.0 \times 10^{-7} \mathrm{~cm}^{3} \mathrm{~s}^{-1}$ at $T_{e}=300 \mathrm{~K}$, which corresponded to an average of the rates that had been measured at that time. However, Vigren et al. (2013) pointed out that for simple ions, there is a trend of increasing rate coefficient with the number of atoms in the recombining ion. Building on this approach, we present in Figure 21 the recombination rate at $300 \mathrm{~K}$ of the 67 ions present in the reaction network for which a rate is available in the literature, against the number of atoms present in the ion, $V$. This plot confirms the clear trend of increasing rate coefficient up to about 6 atoms, followed by a leveling off for more complex ions. To implement unknown recombination rates in the network, we therefore use a linear fit of the data in Figure 21 for $2 \leq V \leq 6$, given by:

$$
\alpha_{300}(V)=(2.15 \times V-2.80) \times 10^{-7},
$$

and a constant value $\alpha_{300}=10^{-6} \mathrm{~cm}^{3} \mathrm{~s}^{-1}$ for $V>6$. The dependence with electron temperature is set to $T_{e}^{-0.7}$, which corresponds to the average of the available temperature dependences in our reaction network. The number of electrons is set equal to the total modeled positive ion content minus the total modeled negative ion content.

Small clusters recombine with electrons with a high efficiency. For those formed in Earth's stratosphere, $\mathrm{H}_{3} \mathrm{O}^{+} \cdot\left(\mathrm{H}_{2} \mathrm{O}\right)_{n}$ and $\mathrm{NH}_{4}^{+} \cdot\left(\mathrm{NH}_{3}\right)_{n}$, recombination 
rates as large as $6.0 \times 10^{-6} \mathrm{~cm}^{3} \mathrm{~s}^{-1}$ have been found, with the rate increasing with the cluster complexity (Mitchell, 1990). For the GCR induced layer of Titan, a general recombination coefficient with the same order of magnitude (4-5 $\left.\times 10^{-6} \mathrm{~cm}^{3} \mathrm{~s}^{-1}\right)$, has been applied by Borucki et al. (1987) and MolinaCuberos et al. (1999a). In the absence of experimental values relevant to Titan's atmosphere, we assume a mean dissociative recombination rate of $5 \times 10^{-6} \mathrm{~cm}^{3}$ $\mathrm{s}^{-1}$ for clusters.

For product branching ratios, the situation is much more complex than for rate coefficients. Experiments are not always available and the proposed theoretical models fail to provide correct predictions of dissociative recombination branching ratios for most of the poly-atomic molecules considered, or even appropriate guidance for their determination. The frequent assumptions to consider $\mathrm{H}$ loss or the most exothermic products as the only channel has been shown to be incorrect for most studied hydrocarbon or nitrogen bearing ions. Instead, dissociative recombination tends to break bonds between heavy atoms and generate multiple fragments (up to four), giving many different product channels. Recently, Chabot et al. (2013) put forward that fragmentation-branching ratios of electronically excited molecular species (as in dissociative recombination) exhibit a statistical behavior. Therefore, they could be deduced from a combination of the large set of experimental data measured in high velocity collisions and semi empirical models. However, this approach has only been applied to a subset of ions so far, amongst which only three are relevant to Titan.

In the absence of clearly established empirical rules correlating breakup patterns to easily estimated physical properties, we made some gross assumptions to estimate product branching ratios:

- The structure of the ions is taken from the Kinetic Database for Astrochemistry (cf. Wakelam et al. (2012, 2015) or http://kida.obs.u-bordeaux1.fr/).

- H loss occurs for every reaction with a branching ratio of $50 \%$. We do not consider loss of multiple $\mathrm{H}$ atoms, or loss of $\mathrm{H}_{2}$ (cf. bullet 3).

- Breakage of bounds between heavy atoms (C-C, C-N, C-O) accounts for the rest of the channels, with the same branching ratio.

- For a given set of products only one bound is allowed to break, which implies that double and triple bonds stay intact and that there are no multiple products.

- We minimize rearrangement of the atoms, especially when it comes to choosing between different isomers (such as $\mathrm{HCN}$ versus HNC). When the situation is unclear, we assume the formation of $50 \%$ of each isomer.

We acknowledge that this is overly simplistic and probably quite different from reality. Pernot et al. (2011) proposed a novel approach, based on probabilistic trees, to fully account for partially available information. However, this method requires some a priori information and has not been implemented for totally unconstrained reactions. 


\subsubsection{Negative Ions.}

Negative Ion Production. Production mechanisms for negative ions include photoionization (ion-pair formation) as well as dissociative and radiative electron attachment. Photoionization is presented in Table B.10, dissociative electron attachment in Table B.11 and radiative electron attachment in Table B.14.

The dissociative electron attachment cross-sections for $\mathrm{CH}_{4}, \mathrm{C}_{2} \mathrm{H}_{2}, \mathrm{C}_{4} \mathrm{H}_{2}$ and $\mathrm{HCN}$ have been updated since Vuitton et al. (2009). For $\mathrm{CH}_{4}$, we prefer the measurements of Rawat et al. (2008) to that of Sharp and Dowell (1967). In both studies, a broad peak centered at $9.8 \mathrm{eV}$ is attributed to the $\mathrm{H}^{-}$channel, while a one order of magnitude smaller and relatively narrow peak centered at $10.4 \mathrm{eV}$ is attributed to the $\mathrm{CH}_{2}{ }^{-}$channel. However, it appears that the crosssections for $\mathrm{H}^{-}$have been underestimated by a factor of almost 20 by Sharp and Dowell (1967), which Rawat et al. (2008) rationalize in terms of kinetic energy discrimination. The contribution for the $\mathrm{CH}_{2}{ }^{-}$channel is also higher in Rawat et al. (2008) but differs only by a factor of about 2 .

For $\mathrm{C}_{2} \mathrm{H}_{2}$, the studies by May et al. $(2008,2009)$ supersede the data obtained by Rutkowski et al. (1980). The main difference is that the peak close to 7.9 $\mathrm{eV}$ and previously assigned to $\mathrm{C}_{2} \mathrm{H}^{-}$is now attributed to $\mathrm{H}^{-}$. Also, the crosssections are a factor of about two smaller.

Because of the absence of any study available at that time, we used in Vuitton et al. (2009) the $\mathrm{C}_{2} \mathrm{H}_{2}$ data for $\mathrm{C}_{4} \mathrm{H}_{2}$ and $\mathrm{C}_{6} \mathrm{H}_{2}$. The absolute cross-sections obtained by May et al. (2008) for the two former species are clearly different from one another (cf. Table B.11) and do not support our previous assumption. As a consequence, we use the values now available for $\mathrm{C}_{4} \mathrm{H}_{2}$ and in the absence of a reliable way to estimate the cross-sections, no longer consider dissociative electron attachment to $\mathrm{C}_{6} \mathrm{H}_{2}$ (or $\mathrm{HC}_{5} \mathrm{~N}$, previously assumed to behave as $\mathrm{HC}_{3} \mathrm{~N}$ ).

For HCN, we prefer the recent values obtained by May et al. (2010) to that of Inoue (1966). The peak of the $\mathrm{CN}^{-}$band is at somewhat lower energy (1.9 versus $2.5 \mathrm{eV}$ ), which can be explained by the absence of electron monochromator and the relatively crude method of energy scale calibration of the early work. Also, the cross-section is about one order of magnitude lower than the value previously estimated $\left(9.4 \times 10^{-18}\right.$ versus $\left.2.0 \times 10^{-16} \mathrm{~cm}^{2}\right)$. Finally, dissociative electron attachment to $\mathrm{H}_{2}$ (Krishnakumar et al., 2011), $\mathrm{CH}_{3} \mathrm{CCH}$ (Janečková et al., 2012), $\mathrm{NH}_{3}$ (Rawat et al., 2008), $\mathrm{HNC}$ (Chourou and Orel, 2009) and $\mathrm{C}_{4} \mathrm{~N}_{2}$ (Graupner et al., 2008) are now included, as some data became available since our previous publication.

The radiative electron attachment rate coefficients are essentially the same as those presented in Table 2 of Vuitton et al. (2009), with the exception of those for $\mathrm{H}$ (Stancil and Dalgarno, 1998), $\mathrm{C}_{4} \mathrm{H}, \mathrm{C}_{6} \mathrm{H}$ (Carelli et al., 2013) and $\mathrm{C}_{5} \mathrm{~N}$ (Walsh et al., 2009), which have been updated. The changes made for the radiative electron attachment rates are not significant (factor of 2 at most). 
Ion-Neutral Reactions. Ion-neutral reactions are presented in Table B.18. Some reaction rates have been updated since Vuitton et al. (2009): Biennier et al. (2014); Martinez et al. (2010), Su \& Chesnavich at $150 \mathrm{~K}$ (cf. positive ions).

Mackay et al. (1977) measured the rate coefficient for the proton transfer reaction of $\mathrm{H}^{-}$with $\mathrm{C}_{2} \mathrm{H}_{2}$ and reported $\mathrm{k}=4.4 \pm 1.1 \times 10^{-9} \mathrm{~cm}^{3} \mathrm{~s}^{-1}$, a value that we used in Vuitton et al. (2009). Recently, Martinez et al. (2010) obtained $\mathrm{k}=3.1 \pm 0.9 \times 10^{-9} \mathrm{~cm}^{3} \mathrm{~s}^{-1}$ with a similar technique. Although the measurements overlap within combined error bars, the faster rate of Mackay et al. (1977) can be rationalized by the presence of acetone (used to safely store acetylene) as a contaminant in their experiment and we prefer the recent rate of Martinez et al. (2010).

Because $\mathrm{HCN}$ is less acidic than $\mathrm{HC}_{3} \mathrm{~N}$, we assumed in Vuitton et al. (2009) that the products of the reaction between $\mathrm{CN}^{-}$and $\mathrm{HC}_{3} \mathrm{~N}$ are $\mathrm{C}_{3} \mathrm{~N}^{-}$and $\mathrm{HCN}$ and that the reaction occurs at every collision. This assumption has since been validated experimentally (Biennier et al., 2014).

We finally include the reaction of $\mathrm{C}_{4} \mathrm{H}^{-}$and $\mathrm{C}_{6} \mathrm{H}^{-}$with $\mathrm{N}$ atoms, which primarily form $\mathrm{CN}^{-}$but are rather slow, with rate coefficients close to $10^{-11}$ $\mathrm{cm}^{3} \mathrm{~s}^{-1}$ (Eichelberger et al., 2007).

Negative Ion Loss. Loss mechanisms for negative ions include photodetachment (cf. Table B.12), recombination with positive ions (cf. Table B.17) and associative detachment with neutrals (cf. Table B.19).

For the photodetachment calculations, we adopt a cross-section $\sigma\left(\mathrm{cm}^{2}\right)$ that depends on the photon energy $\epsilon(\mathrm{eV})$, according to the empirical formula:

$$
\text { for } \epsilon \geq \mathrm{EA}, \sigma=\sigma_{\infty}(1-E A / \epsilon)^{0.5},
$$

where $\sigma_{\infty}$ denotes the asymptotic cross-section $\left(\mathrm{cm}^{2}\right)$ for large photon energies and EA the electron affinity $(\mathrm{eV})$ of the corresponding neutral (Millar et al., 2007). Ion traps have recently been successfully employed to study absolute photodetachment cross-sections for $\mathrm{O}^{-}$and $\mathrm{OH}^{-}$(Hlavenka et al., 2009), $\mathrm{C}_{2} \mathrm{H}^{-}$, $\mathrm{C}_{4} \mathrm{H}^{-}$and $\mathrm{C}_{6} \mathrm{H}^{-}$(Best et al., 2011), and $\mathrm{CN}^{-}$and $\mathrm{C}_{3} \mathrm{~N}^{-}$(Kumar et al., 2013). For these ions, $\sigma_{\infty}$ is derived from fits to the measured cross-sections using Equation (E24) and literature electron affinities. For the other ions for which no experimental data are available, we assume $\sigma_{\infty}$ to be equal to $10^{-17} \mathrm{~cm}^{2}$. The electron affinities and asymptotic cross-section values are given in Table (B.12).

The parameterization of Hickman (1979) for the recombination of negative with positive ions has been revised by Miller et al. (2012), following a new set of measurements. We therefore use the updated expression,

$$
k=2.8 \times 10^{-7} E A^{-0.13} \mu^{-0.5}(T / 300)^{-0.9} \mathrm{~cm}^{3} \mathrm{~s}^{-1},
$$

where $E A$ is the electron affinity of the corresponding neutral, $\mu$ is the reduced mass of the collision partners and $T$ is the temperature of the gas. For the ions 
of interest here, we obtain rate coefficients varying from $5 \times 10^{-8}$ to $3 \times 10^{-7}$ $\mathrm{cm}^{3} \mathrm{~s}^{-1}$ at $300 \mathrm{~K}$.

We now consider reaction products and, in the absence of data, we use the general scheme: $A^{-}+B H^{+} \rightarrow A+B+H$. In the case of $\mathrm{HCNH}^{+}$, it is assumed that the product is only $\mathrm{HCN}$ (no HNC), the most stable isomer, as explained in section 2.7.3.1. We elaborate on possible $\mathrm{HCNH}^{+}$states in the discussion, section 5.1.

We consider associative detachment with $\mathrm{H}$ and $\mathrm{CH}_{3}$ as in Vuitton et al. (2009), and now also include reaction with $\mathrm{N}$ atoms that exhibit an abundance similar to $\mathrm{CH}_{3}$ in the ionosphere. Gerlich et al. (2012) studied the formation of $\mathrm{H}_{2}$ via associative detachment in $\mathrm{H}^{-}+\mathrm{H}$ collisions between 10 and $135 \mathrm{~K}$ and reports a rate coefficient of $5.5 \times 10^{-9} \mathrm{~cm}^{3} \mathrm{~s}^{-1}$ at $135 \mathrm{~K}$, which we prefer to the room temperature value of Fehsenfeld et al. (1973).

We also update the rate coefficients for $\mathrm{CN}^{-}, \mathrm{C}_{3} \mathrm{~N}^{-}$and $\mathrm{C}_{5} \mathrm{~N}^{-}$with $\mathrm{H}$ atoms (Yang et al., 2011; Snow et al., 2009). For $\mathrm{CN}^{-}$, the reported rate coefficient $\left(6.3 \times 10^{-10} \mathrm{~cm}^{3} \mathrm{~s}^{-1}\right)$ agrees well with the previous results of Fehsenfeld et al. (1973): $8 \times 10^{-10} \mathrm{~cm}^{3} \mathrm{~s}^{-1} \pm$ factor of 2. We note that in Vuitton et al. (2009), we reproduced an incorrect value $\left(1.3 \times 10^{-9} \mathrm{~cm}^{3} \mathrm{~s}^{-1}\right)$ cited in Fehsenfeld (1975). For $\mathrm{C}_{3} \mathrm{~N}^{-}$and $\mathrm{C}_{5} \mathrm{~N}^{-}$, the rate coefficient is a factor of $\sim 2$ smaller than the assumption (after Petrie and Herbst (1997)) reported in our previous paper.

The reactions with $\mathrm{N}$ atoms use the rate coefficients reported in Eichelberger et al. (2007) and Ferguson (1973) for $\mathrm{C}_{2} \mathrm{H}^{-}$and $\mathrm{OH}^{-}$, respectively. For the other ions, we assume that the rate coefficient is the same as that for the reaction with $\mathrm{H}$ atoms.

Again, we now consider reaction products and, in the absence of data, use the general scheme: $A^{-}+B \rightarrow A B+e^{-}$. In the case of $\mathrm{C}_{3} \mathrm{H}_{4}$, it is assumed that the products are equal amounts of $\mathrm{CH}_{3} \mathrm{CCH}$ and $\mathrm{CH}_{2} \mathrm{CCH}_{2}$ (cf. Table B.14).

\subsection{4. ${ }^{15} \mathrm{~N}$ Species}

In order to take into account ${ }^{15} \mathrm{~N}$ bearing species, we start from our ${ }^{14} \mathrm{~N}$ chemistry and generate analogous reactions in which ${ }^{14} \mathrm{~N}$ is replaced by ${ }^{15} \mathrm{~N}$. Because they do not impact nitrogen chemistry, we do not include reactions of ${ }^{15} \mathrm{~N}$ species with oxygen species and negative ions. Reactions in which both reactants contain nitrogen or in which a species contains more than one nitrogen atom create special problems and some general rules have to be applied. First of all, we leave out reactions where ${ }^{15} \mathrm{~N}$ species would react with each other (i.e. $\mathrm{C}^{15} \mathrm{~N}+\mathrm{HC}^{14} \mathrm{~N}$ and $\mathrm{C}^{14} \mathrm{~N}+\mathrm{HC}^{15} \mathrm{~N}$ are taken into account but not $\mathrm{C}^{15} \mathrm{~N}$ $\left.+\mathrm{HC}^{15} \mathrm{~N}\right)$. If the reaction consists in some exchange reaction $\left(\mathrm{H}^{+}, \mathrm{H}^{-}\right.$and $\mathrm{e}^{-}$ transfer), we assume that there is no rearrangement and that the ${ }^{15} \mathrm{~N}$ is not allowed to change molecule during reaction, as shown in the three following examples:

- $\mathrm{H}^{-}$transfer:

$$
\mathrm{N}^{+}+{ }^{15} \mathrm{NH}_{3} \rightarrow{ }^{15} \mathrm{NH}_{2}^{+}+\mathrm{NH} \quad\left(\mathrm{R}_{c n}^{15} 602 \mathrm{a}\right)
$$

- $\mathrm{e}^{-}$transfer:

$$
\mathrm{N}^{+}+\mathrm{HC}^{15} \mathrm{~N} \rightarrow \mathrm{HC}^{15} \mathrm{~N}^{+}+\mathrm{N} \quad\left(\mathrm{R}_{c n}^{15} 603 \mathrm{a}\right)
$$


- $\mathrm{H}^{+}$transfer:

$$
\mathrm{NH}^{+}+{ }^{15} \mathrm{NH}_{3} \rightarrow{ }^{15} \mathrm{NH}_{4}^{+}+\mathrm{N} \quad\left(\mathrm{R}_{c n}^{15} 624 \mathrm{~b}\right)
$$

If the reaction is some more intricate process, which may involve an intermediate complex with possible rearrangement, we arbitrarily assign statistical branching ratios to the individual channels. Thus, for the two product channels in the reaction below, a statistical ratio of 0.5 is applied to each.

- Intermediate complex:

$$
\begin{aligned}
\mathrm{N}^{+}+\mathrm{CH}_{3} \mathrm{C}^{15} \mathrm{~N} & \rightarrow \mathrm{C}^{15} \mathrm{~N}^{+}+\mathrm{CH}_{2} \mathrm{NH} & \left(\mathrm{R}_{c n}^{15 a} 605 \mathrm{~d}\right) \\
& \rightarrow \mathrm{CN}^{+}+\mathrm{CH}_{2}^{15} \mathrm{NH} & \left(\mathrm{R}_{c n}^{15 b} 605 \mathrm{~d}\right)
\end{aligned}
$$

Fortunately, we find that the only reactions that turn out to be important are proton exchange reactions and that therefore the assumption made above does not have any impact.

Once the list of reactions of ${ }^{15} \mathrm{~N}$ species has been established, it remains to evaluate their rate coefficients. Substitution of an atom in the reactants of a chemical reaction with one of its isotopes results in a change in rate coefficient, a mechanism referred to as a kinetic isotope effect. The difference can be rationalized by noting that the mass of an atom affects the vibrational frequency of the chemical bond that it forms, even if the electron configuration is nearly identical. Heavier atoms will lead to lower vibration frequencies, or, viewed quantum mechanically, will have lower zero-point energy. With a lower zeropoint energy, more energy must be supplied to break the bond, resulting in a higher activation energy for bond cleavage, which in turn lowers the measured rate coefficient according to the Arrhenius equation.

A direct consequence is that rate coefficient changes are most pronounced when the relative mass change of the isotopes is greatest. For instance, substituting a hydrogen atom to deuterium represents a $100 \%$ increase in mass and the rate coefficient of a reaction involving a $\mathrm{C}-\mathrm{H}$ bond is typically $6-10$ times faster than for the corresponding C-D bond. However, when replacing ${ }^{12} \mathrm{C}$ with ${ }^{13} \mathrm{C}$, the mass increases by only $8 \%$ and a ${ }^{12} \mathrm{C}$ reaction is only $\sim 1.04$ times faster than the corresponding ${ }^{13} \mathrm{C}$ reaction (even though, in both cases, the isotope is one atomic mass unit heavier). Since replacing a ${ }^{14} \mathrm{~N}$ with ${ }^{15} \mathrm{~N}$ represents only a $7 \%$ increase in mass, we neglect mass-dependent kinetic isotope effects when generating our ${ }^{15} \mathrm{~N}$ reaction network. This assumption is supported by recent laboratory experiments (Kuga et al., 2014; Sebree et al., 2016), which show little nitrogen isotopic fractionation in tholins, ${ }^{15} \mathrm{~N}$ being depleted by $\delta^{15} \mathrm{~N}=+0.8 /-$ $25 \%$ relative to the initial $\mathrm{N}_{2}$ gas. This is to be compared to the $\delta^{15} \mathrm{~N} \sim+2000 \%$ o fractionation observed between $\mathrm{HCN}$ and $\mathrm{N}_{2}$.

Another possible isotopic fractionation mechanism is equilibrium fractionation, which is the partial separation of isotopes between two or more substances in chemical equilibrium, owing to exothermicity. In an isotopic exchange reaction such as: 


$$
{ }^{n} \mathrm{AB}+{ }^{m} \mathrm{AC} \rightarrow{ }^{m} \mathrm{AB}+{ }^{n} \mathrm{AC},
$$

where $\mathrm{A}, \mathrm{B}$, and $\mathrm{C}$ are chemical elements and $m$ and $n$ refer to the atomic masses of two isotopes of $\mathrm{A}$, the equilibrium coefficient $K(T)$ can be written as:

$$
K(T)=k_{f} / k_{r}=f(B, m) \exp \left(\Delta E_{0} / k T\right),
$$

where $k_{f}$ and $k_{r}$ are the forward and reverse rate coefficients and $\Delta E_{0}$, the zero-point vibrational energy difference between the reactants and the products, is defined to be positive for exothermic reactions. $f(B, m)$ refers to ratios of partition functions and, in most cases, is close to the molecular symmetry factor, which depends on the rotational constants, masses and symmetries of the reactants and products. Equilibrium fractionation is therefore strongest at low temperatures and isotopes of light elements (hydrogen, carbon, nitrogen, oxygen, sulfur) tend to be separated to a greater degree. However, the exothermicity of isotopic exchange reactions being typically less than $40 \mathrm{~K}$ (Terzieva and Herbst, 2000), we neglect this mechanism in our reaction network.

At the end, we have 26 neutrals, 72 positive ions, 285 neutral reactions, 1453 ion-neutral reactions, and 169 ion-electron recombination reactions involving the ${ }^{15} \mathrm{~N}$ isotope.

\subsubsection{Heterogeneous Processes}

The presence of aerosols in the atmosphere can interfere with the purely gas phase chemistry described so far. Heterogeneous processes that can occur on the surfaces of aerosol particles include:

1. Simple adsorption of gaseous species.

2. Recombination of gaseous species at the surface of aerosols and release of a new species in the gas phase.

3. Reaction of gaseous species with the chemical constituents of the aerosols with subsequent release in the gas phase.

However, heterogeneous processes have been poorly described in photochemical models so far, the only species ever considered being atomic hydrogen, following Sekine et al. (2008a) who investigated heterogeneous reactions of atomic hydrogen in conditions relevant to Titan (tholins material, low temperature). They showed that hydrogenation of tholins (process 1) is about one order of magnitude more important than $\mathrm{H}_{2}$ recombination (process 2), with a reaction probability $\eta_{\text {hydro }}=2.08( \pm 0.64) \times \exp (-1000 / \mathrm{T})$.

In this model, we include a loss of atomic hydrogen to the aerosols with a reaction probability of $2.65 \times 10^{-3}$, which corresponds to $\eta_{\text {hydro }}$ at $150 \mathrm{~K}$ (Sekine et al., 2008a). For the aerosol distribution (cf. Figure 22), we use the mean particle size and density predicted by the microphysical model of Lavvas et al. (2010), which are consistent with the haze optical properties retrieved by the DISR observations (Tomasko et al., 2008). 


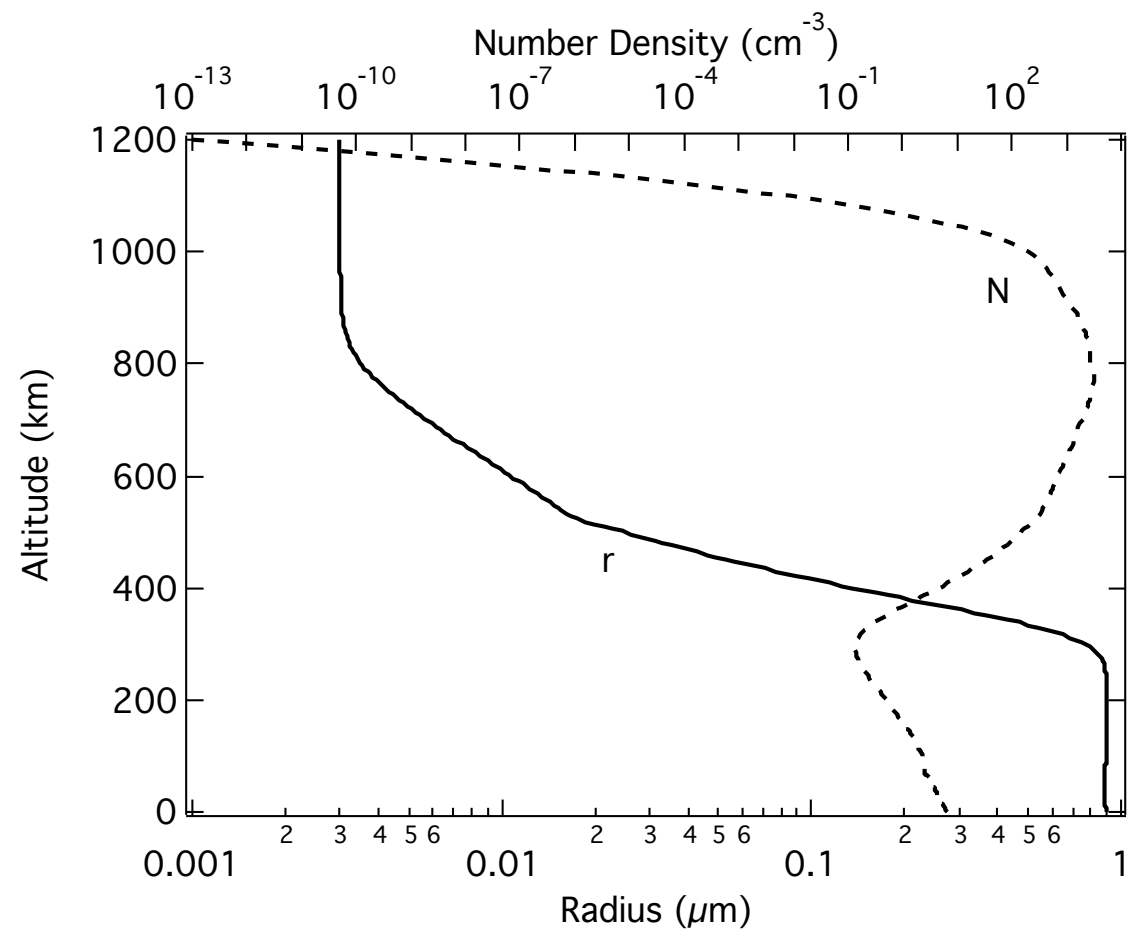

Figure 22: Altitude variation of the aerosol radius and density.

\section{Chemical Composition}

\subsection{The Upper Ionosphere}

\subsubsection{Positive Ions}

Solar radiation and associated photoelectrons are mostly deposited above 600 $\mathrm{km}$ and are at the origin of Titan's upper ionosphere. We review here our current knowledge on the major ion-molecule reactions involved in the chemistry. We focus first on the primary ions $\left(\mathrm{N}_{2}{ }^{+}, \mathrm{N}^{+}\right.$and $\left.\mathrm{CH}_{4}{ }^{+}\right)$and follow the chemical evolution to complex hydrocarbon ions and nitrogen-bearing ions, and finish with oxygen-bearing ions. All the numbers listed are from the nominal model, as described in section 4.2.3.

3.1.1.1. Primary Ions. Primary ions are produced upon interaction of $\mathrm{N}_{2}$ and $\mathrm{CH}_{4}$ with solar photons and suprathermal photo- and secondary electrons. Table 6 presents the $\mathrm{N}_{2}$ and $\mathrm{CH}_{4}$ loss rates from photons, photoelectrons and chemistry. Our calculated rates are similar to those presented in Lavvas et al. (2011a), which was to be expected since we use similar cross-sections. Lavvas et al. (2011a) compared the primary $\mathrm{N}_{2}$ loss rates obtained by different photochemical models and we do not repeat this discussion here.

From Table $6, \mathrm{~N}_{2}{ }^{+}, \mathrm{N}^{+}$and $\mathrm{CH}_{4}{ }^{+}$are the major primary ionization products from $\mathrm{N}_{2}$ and $\mathrm{CH}_{4}$, all the other ions being mostly formed by secondary chemical 
Table 6: Calculated column production rate $\left(\mathrm{cm}^{-2}\right)$ for the fragments of the ionization of $\mathrm{CH}_{4}$ and $\mathrm{N}_{2}$ from photons, photoelectrons and chemistry.

\begin{tabular}{lllll}
\hline \hline Products & Photons & Photoelectrons & Chemistry & Total \\
\hline \hline $\mathrm{N}_{2}{ }^{+}$ & $1.6 \times 10^{8}$ & $8.9 \times 10^{7}$ & $1.1 \times 10^{4}$ & $2.5 \times 10^{8}$ \\
$\mathrm{~N}^{+}$ & $3.8 \times 10^{7}$ & $1.1 \times 10^{7}$ & $2.0 \times 10^{3}$ & $4.8 \times 10^{7}$ \\
$\mathrm{CH}_{4}{ }^{+}$ & $2.4 \times 10^{7}$ & $2.5 \times 10^{6}$ & $3.4 \times 10^{6}$ & $3.0 \times 10^{7}$ \\
$\mathrm{CH}_{3}{ }^{+}$ & $7.4 \times 10^{6}$ & $1.8 \times 10^{6}$ & $2.3 \times 10^{8}$ & $2.4 \times 10^{8}$ \\
$\mathrm{CH}_{2}{ }^{+}$ & $2.3 \times 10^{5}$ & $2.4 \times 10^{5}$ & $2.1 \times 10^{7}$ & $2.1 \times 10^{7}$ \\
$\mathrm{CH}^{+}$ & $6.9 \times 10^{4}$ & $8.9 \times 10^{4}$ & $5.7 \times 10^{5}$ & $7.3 \times 10^{5}$ \\
$\mathrm{C}^{+}$ & $1.5 \times 10^{4}$ & $2.4 \times 10^{4}$ & $1.1 \times 10^{4}$ & $5.0 \times 10^{4}$ \\
$\mathrm{H}_{2}{ }^{+}$ & $1.3 \times 10^{4}$ & $3.8 \times 10^{4}$ & $1.0 \times 10^{6}$ & $1.1 \times 10^{6}$ \\
$\mathrm{H}^{+}$ & $9.9 \times 10^{4}$ & $1.5 \times 10^{5}$ & $4.4 \times 10^{4}$ & $2.9 \times 10^{5}$ \\
\hline \hline
\end{tabular}

reactions involving these primary ions. The primary production rates of $\mathrm{N}_{2}{ }^{+}$, $\mathrm{N}^{+}$and $\mathrm{CH}_{4}{ }^{+}$are shown in Figure 11 of Lavvas et al. (2011a).

$\mathbf{N}_{2}{ }^{+}, \mathbf{N}^{+} \cdot \mathrm{N}_{2}{ }^{+}$reacts efficiently with $\mathrm{CH}_{4}$ and $\mathrm{H}_{2}$, leading directly or via $\mathrm{N}_{2} \mathrm{H}^{+}$(section 3.1.1.3) to the ionization of hydrocarbons without any net loss for $\mathrm{N}_{2}$ and therefore any production of active nitrogen:

$$
\begin{aligned}
\mathrm{N}_{2}^{+}+\mathrm{CH}_{4} & \rightarrow \mathrm{CH}_{3}^{+}+\mathrm{N}_{2}+\mathrm{H} & & \left(\mathrm{R}_{c n} 836 \mathrm{a}\right) \\
& \rightarrow \mathrm{CH}_{2}^{+}+\mathrm{N}_{2}+\mathrm{H}_{2} & & \left(\mathrm{R}_{c n} 836 \mathrm{~b}\right) \\
& \rightarrow \mathrm{N}_{2} \mathrm{H}^{+}+\mathrm{CH}_{3}, & & \left(\mathrm{R}_{c n} 836 \mathrm{c}\right) \\
\mathrm{N}_{2}^{+}+\mathrm{H}_{2} & \rightarrow \mathrm{N}_{2} \mathrm{H}^{+}+\mathrm{H} . & & \left(\mathrm{R}_{c n} 835 \mathrm{a}\right)
\end{aligned}
$$

The kinetics of the reaction of $\mathrm{N}_{2}{ }^{+}$with $\mathrm{CH}_{4}$ has been extensively studied at $300 \mathrm{~K}$ and the latest determinations are all in good agreement suggesting that the rate coefficient is equal within experimental uncertainties to the capture rate, i.e. $1.2 \times 10^{-9} \mathrm{~cm}^{3} \mathrm{~s}^{-1}$ (Dutuit et al., 2013). The rate coefficient seems unchanged over the temperature range $70-300 \mathrm{~K}$ and the recommended value is the same at $150 \mathrm{~K}$ and $300 \mathrm{~K}$ (Rowe et al., 1989).

Most studies are in agreement concerning the production of the dissociative charge transfer products, $\mathrm{CH}_{2}{ }^{+}$and $\mathrm{CH}_{3}{ }^{+}$, but there is an important disagreement concerning the production of $\mathrm{N}_{2} \mathrm{H}^{+}$, its detection being difficult because of mass overlaps. The recommended branching ratios at $300 \mathrm{~K}$ are $0.86 / 0.09 / 0.05$ for the three channels $\left(\mathrm{R}_{c n} 836 \mathrm{a}\right) /\left(\mathrm{R}_{c n} 836 \mathrm{~b}\right) /\left(\mathrm{R}_{c n} 836 \mathrm{c}\right)$ (Nicolas et al., 2003b). The branching ratios seem to be constant as a function of temperature (Gichuhi and Suits, 2011). A recent study (Xu et al., 2013) shows, however, that the branching ratios determined at $E_{c m}=0.05 \mathrm{eV}(380$ $\mathrm{K})$ are $0.68 \pm 0.05 / 0.26 \pm 0.04 / 0.06 \pm 0.01$, which is quite different from previous measurements. Since the branching ratios are found to be almost constant with collision energy (Nicolas et al., 2003b; Xu et al., 2013), it is reasonable to expect 
the same values at $300 \mathrm{~K}$. The difference seems to be due to the occurrence of secondary reactions that were not corrected for in the previous studies.

The reaction between $\mathrm{N}_{2}{ }^{+}$and $\mathrm{H}_{2}$ has been extensively studied and all rate coefficient measurements at $300 \mathrm{~K}$ are in good agreement. They give a value nearly equal to the Langevin rate $\left(1.6 \times 10^{-9} \mathrm{~cm}^{3} \mathrm{~s}^{-1}\right)$, which is the value recommended by Dutuit et al. (2013). When the temperature decreases, the rate coefficient goes through a minimum and increases again at lower temperature. The recommended value at $150 \mathrm{~K}$ is $1.3 \times 10^{-9} \mathrm{~cm}^{3} \mathrm{~s}^{-1}$ (Rowe et al., 1989; Randeniya and Smith, 1991).

The products have been the subject of extensive experimental investigations. The hydrogen transfer channel $\left(\mathrm{R}_{c n} 835 \mathrm{a}\right)$ is strongly favored over the charge transfer channel $\left(\mathrm{R}_{c n} 835 \mathrm{~b}\right)$, with recommended branching ratios at $300 \mathrm{~K}$ of 0.99/0.01 (Dutuit et al. (2013) and references therein). There are no studies available at lower temperature.

$\mathrm{N}^{+}$is essentially formed by photoionization above $900 \mathrm{~km}$ and by photoelectron impact down to $700 \mathrm{~km}$. Like $\mathrm{N}_{2}{ }^{+}$, it reacts efficiently with $\mathrm{CH}_{4}$ and $\mathrm{H}_{2}$, but leading to the formation of reactive nitrogen-bearing ions $\left(\mathrm{HCNH}^{+}, \mathrm{NH}^{+}\right.$, $\left.\mathrm{HCN}^{+}\right)$and radicals $\left(\mathrm{NH}, \mathrm{N}\left({ }^{4} \mathrm{~S}\right)\right)$.

$$
\begin{aligned}
\mathrm{N}^{+}+\mathrm{CH}_{4} & \rightarrow \mathrm{CH}_{3}^{+}+\mathrm{NH} & \left(\mathrm{R}_{c n} 593 \mathrm{a}\right) \\
& \rightarrow \mathrm{HCNH}^{+}+\mathrm{H}_{2} & \left(\mathrm{R}_{c n} 593 \mathrm{~b}\right) \\
& \rightarrow \mathrm{HCN}^{+}+\mathrm{H}_{2}+\mathrm{H} & \left(\mathrm{R}_{c n} 593 \mathrm{c}\right) \\
& \rightarrow \mathrm{CH}_{4}^{+}+\mathrm{N}, & \left(\mathrm{R}_{c n} 593 \mathrm{~d}\right) \\
\mathrm{N}^{+}+\mathrm{H}_{2} & \rightarrow \mathrm{NH}^{+}+\mathrm{H} . & \left(\mathrm{R}_{c n} 592\right)
\end{aligned}
$$

The recommended total rate coefficient at $300 \mathrm{~K}$ for the reaction of $\mathrm{N}^{+}$with $\mathrm{CH}_{4}$ is the mean value of the available experimental values, i.e. $1.2 \times 10^{-9}$ $\mathrm{cm}^{3} \mathrm{~s}^{-1}$ (McEwan and Anicich, 2007; Dutuit et al., 2013). The rate coefficient seems to decrease with decreasing temperature (Rowe et al., 1985) and a rate coefficient of $1.0 \times 10^{-9} \mathrm{~cm}^{3} \mathrm{~s}^{-1}$ is recommended at $150 \mathrm{~K}$ (Dutuit et al., 2013).

The recommended branching ratios at $300 \mathrm{~K}$ are $0.50 / 0.35 / 0.10 / 0.05$ for the channels $\left(\mathrm{R}_{c n} 593 \mathrm{a}\right) /\left(\mathrm{R}_{c n} 593 \mathrm{~b}\right) /\left(\mathrm{R}_{c n} 593 \mathrm{c}\right) /\left(\mathrm{R}_{c n} 593 \mathrm{~d}\right)$, respectively (McEwan and Anicich, 2007; Dutuit et al., 2013). The branching ratios of $\left(\mathrm{R}_{c n} 593 \mathrm{~b}\right)$ and $\left(\mathrm{R}_{c n} 593 \mathrm{c}\right)$ increase when the collision energy decreases from 0.2 to 0.05 $\mathrm{eV}$, at the expense of channels $\left(\mathrm{R}_{c n} 593 \mathrm{a}\right)$ and $\left(\mathrm{R}_{c n} 593 \mathrm{~d}\right)$ (Dheandhanoo et al., 1984).

In order to obtain the branching ratios at $150 \mathrm{~K}$, Carrasco et al. (2008) extrapolated the data of Figure 5 in Dheandhanoo et al. (1984) assuming an exponential variation and for channels $\left(\mathrm{R}_{c n} 593 \mathrm{a}\right) /\left(\mathrm{R}_{c n} 593 \mathrm{~b}\right) /\left(\mathrm{R}_{c n} 593 \mathrm{c}\right) /\left(\mathrm{R}_{c n} 593 \mathrm{~d}\right)$ obtained $0.47 / 0.40 / 0.12 / 0.02$, respectively. However, they showed that using the $150 \mathrm{~K}$ extrapolated branching ratios in their chemical model instead of the values at $300 \mathrm{~K}$ has almost no impact on the density of major ions. Because of this result and because the extrapolation of kinetic parameters outside their 
range of validity is always subject to caution, we decide to use the $300 \mathrm{~K}$ branching ratios in the model.

The reaction between $\mathrm{N}^{+}$and $\mathrm{H}_{2}$ has been extensively studied both experimentally and theoretically, at room temperature and below. It is endothermic by $17 \mathrm{meV}$ and is therefore very sensitive to temperature (cf. Zymak et al. (2013) and references therein). For the rate coefficient at $300 \mathrm{~K}$, extensive literature reviews were performed by McEwan and Anicich (2007) and Dutuit et al. (2013) who recommend a value of $5.0 \times 10^{-10} \mathrm{~cm}^{3} \mathrm{~s}^{-1}$ and $3.8 \times 10^{-10} \mathrm{~cm}^{3} \mathrm{~s}^{-1}$, respectively. Here, we choose to use the temperature dependent rate coefficient retrieved from the Marquette et al. (1988) data obtained between 8 and $163 \mathrm{~K}$, that is $4.2 \times 10^{-10} \exp (-41.9 / \mathrm{T}) \mathrm{cm}^{3} \mathrm{~s}^{-1}$.

$\mathbf{C H}_{4}{ }^{+} \cdot \mathrm{CH}_{4}{ }^{+}$is mostly formed by photoionization of $\mathrm{CH}_{4}$, photoelectron impact representing only about $10 \%$ of the total production rate, as does reaction between $\mathrm{N}^{+}$and $\mathrm{CH}_{4}\left(\mathrm{R}_{c n} 593 \mathrm{~d}\right)$. It is mostly lost by reaction with methane:

$$
\mathrm{CH}_{4}^{+}+\mathrm{CH}_{4} \rightarrow \mathrm{CH}_{5}^{+}+\mathrm{CH}_{3} \text {. }
$$

The evaluated rate coefficient at $300 \mathrm{~K}$, i.e. $1.1 \times 10^{-9} \mathrm{~cm}^{3} \mathrm{~s}^{-1}$, is based on an extensive literature review (McEwan and Anicich (2007) after Anicich et al. (2003)). The rate coefficient seems to slightly decrease with decreasing temperature since a value of $9.0 \times 10^{-10} \mathrm{~cm}^{3} \mathrm{~s}^{-1}$ has been measured at $90 \mathrm{~K}$ (Bowers et al., 1977).

\subsubsection{Hydrocarbon Ions.}

$\mathbf{C H}_{3}{ }^{+}, \mathbf{C H}_{5}{ }^{+}$. The chemistry of $\mathrm{CH}_{3}{ }^{+}$is extremely simple, as it is almost exclusively produced by dissociative charge transfer from the major primary ion, $\mathrm{N}_{2}{ }^{+}$, to methane $\left(\mathrm{R}_{c n} 836 \mathrm{a}\right)$ and lost through reaction with methane:

$$
\mathrm{CH}_{3}^{+}+\mathrm{CH}_{4} \rightarrow \mathrm{C}_{2} \mathrm{H}_{5}^{+}+\mathrm{H}_{2} \text {. }
$$

The evaluated rate coefficient at $300 \mathrm{~K}$, i.e. $1.1 \times 10^{-9} \mathrm{~cm}^{3} \mathrm{~s}^{-1}$, is based on an extensive literature review (McEwan and Anicich (2007) after Anicich et al. (2003)). The rate coefficient has not been measured at low temperature but since it is fast at room temperature, the temperature dependence is expected to be small.

The $\mathrm{CH}_{5}{ }^{+}$production is similar to that described in Vuitton et al. (2007). It is closely linked to the ionization of both $\mathrm{CH}_{4}$ through reaction $\left(\mathrm{R}_{c n} 162\right)$ and $\mathrm{N}_{2}$ through the intermediate species $\mathrm{N}_{2} \mathrm{H}^{+}$(formed through reactions $\left(\mathrm{R}_{c n} 835 \mathrm{a}\right.$ ) and $\left(\mathrm{R}_{c n} 836 \mathrm{c}\right)$, section 3.1.1.3):

$$
\mathrm{N}_{2} \mathrm{H}^{+}+\mathrm{CH}_{4} \rightarrow \mathrm{CH}_{5}^{+}+\mathrm{N}_{2} \text {. }
$$

As in Vuitton et al. (2007), this ion is mostly lost through proton transfer reactions to $\mathrm{HCN}, \mathrm{C}_{2} \mathrm{H}_{4}$ and $\mathrm{C}_{2} \mathrm{H}_{2}\left(\mathrm{R}_{c n} 194, \mathrm{R}_{c n} 180, \mathrm{R}_{c n} 179\right)$, and through dissociative electron recombination $\left(\mathrm{R}_{e r} 14\right)$. 
The reaction between $\mathrm{CH}_{5}{ }^{+}$and $\mathrm{HCN}$ has never been studied. Keller et al. (1992) made the assumption that the only channel is a proton transfer from the ion to the neutral and that the rate coefficient is equal to the Langevin rate. It is now understood that for neutrals having a permanent dipole moment, such as HCN, the Langevin expression does not apply and we use the Su-Chesnavich expression instead (Woon and Herbst, 2009). Using literature values for the dipole polarizability and dipole moment for HCN (CRC Handbook of Chemistry and Physics, 87th Edition) leads to a rate coefficient at $150 \mathrm{~K}$ of $5.8 \times 10^{-9}$ $\mathrm{cm}^{3} \mathrm{~s}^{-1}$, five times higher than the rate used in Keller et al. (1992).

The evaluated rate coefficients at $300 \mathrm{~K}$ for $\mathrm{CH}_{5}{ }^{+}+\mathrm{C}_{2} \mathrm{H}_{4}$ and $\mathrm{CH}_{5}{ }^{+}+\mathrm{C}_{2} \mathrm{H}_{2}$ (McEwan and Anicich, 2007), i.e. $1.5 \times 10^{-9} \mathrm{~cm}^{3} \mathrm{~s}^{-1}$ in both cases, are based on the measurements of Fiaux et al. (1974) and Mackay et al. (1977), and close to the Langevin rate, as should be expected for such proton transfer reactions.

$\mathrm{CH}_{5}{ }^{+}$dissociative recombination experiments have been performed at both a heavy-ion storage ring (CRYRING) and in a flowing afterglow and they present some significant discrepancies in terms of both rate coefficient and branching fractions of the products. While the former set of studies reports the dominance of $\mathrm{CH}_{3}+\mathrm{H}+\mathrm{H}$ (Semaniak et al., 1998; Kamińska et al., 2010), the latter observes $\mathrm{CH}_{4}+\mathrm{H}$ as the major products (Adams et al., 2009; Molek et al., 2009). A possible explanation is that a two-step process via the production of a $\mathrm{CH}_{4}$ intermediate with sufficient energy to fragment further to $\mathrm{CH}_{3}+\mathrm{H}$ plays an important role. The disagreement could therefore be due to a different pressure in these two experimental set-ups $\left(<10^{-11}\right.$ mbar in CRYRING versus $\sim 1$ mbar in the flow tube). The storage ring conditions being closer to that of Titan's upper atmosphere, we prefer the values presented in Kamińska et al. (2010).

$\mathrm{C}_{2} \mathrm{H}_{2}{ }^{+}, \mathrm{C}_{2} \mathrm{H}_{3}{ }^{+}, \mathbf{C}_{2} \mathrm{H}_{4}{ }^{+}, \mathrm{C}_{2} \mathrm{H}_{5}{ }^{+}, \mathrm{C}_{2} \mathrm{H}_{7}{ }^{+}, \mathrm{C}_{2} \mathrm{H}_{2}{ }^{+}$is formed by (dissociative) charge transfer to ethylene and acetylene:

$$
\begin{aligned}
& \mathrm{N}_{2}^{+}+\mathrm{C}_{2} \mathrm{H}_{4} \rightarrow \mathrm{C}_{2} \mathrm{H}_{2}^{+}+\mathrm{N}_{2}+\mathrm{H}_{2} \text {, } \\
& \left(\mathrm{R}_{c n} 838 \mathrm{~b}\right) \\
& \mathrm{N}_{2}^{+}+\mathrm{C}_{2} \mathrm{H}_{2} \rightarrow \mathrm{C}_{2} \mathrm{H}_{2}^{+}+\mathrm{N}_{2} \text {, } \\
& \left(\mathrm{R}_{c n} 837 \mathrm{a}\right) \\
& \mathrm{N}^{+}+\mathrm{C}_{2} \mathrm{H}_{2} \rightarrow \mathrm{C}_{2} \mathrm{H}_{2}^{+}+\mathrm{N} \text {, } \\
& \left(\mathrm{R}_{c n} 594 \mathrm{a}\right) \\
& \mathrm{CH}_{4}^{+}+\mathrm{C}_{2} \mathrm{H}_{2} \rightarrow \mathrm{C}_{2} \mathrm{H}_{2}^{+}+\mathrm{CH}_{4} \text {. }
\end{aligned}
$$

The evaluated rate coefficient of the reaction between $\mathrm{N}_{2}{ }^{+}$and $\mathrm{C}_{2} \mathrm{H}_{4}$ is equal to $1.3 \times 10^{-9} \mathrm{~cm}^{3} \mathrm{~s}^{-1}$, which corresponds to the Langevin rate and is therefore temperature independent (Dutuit et al. (2013) after McEwan et al. (1998)). The three studies that focus on the product branching ratios of this reaction, including that of Gichuhi and Suits (2011) at $\sim 45 \mathrm{~K}$, agree that $\mathrm{C}_{2} \mathrm{H}_{3}{ }^{+}+\mathrm{H}+\mathrm{N}_{2}$ are the main products and that a secondary channel gives $\mathrm{C}_{2} \mathrm{H}_{2}{ }^{+}+\mathrm{H}_{2}+\mathrm{N}_{2}$. A third channel leading to a small amount of $\mathrm{N}_{2} \mathrm{H}^{+}+\mathrm{C}_{2} \mathrm{H}_{3}$ remains to be confirmed (Dutuit et al., 2013).

The evaluated rate coefficient of the $\mathrm{N}_{2}{ }^{+}+\mathrm{C}_{2} \mathrm{H}_{2}$ reaction $\left(4.2 \times 10^{-10} \mathrm{~cm}^{3}\right.$ $\mathrm{s}^{-1}$ at $\left.300 \mathrm{~K}\right)$ is significantly smaller than the Langevin rate and decreases 
with increasing temperature according to $E_{C M}^{-1 / 2}$, as expected for charge transfer reactions. Extrapolation of the reaction cross section obtained at $928 \mathrm{~K}$ down to $150 \mathrm{~K}$ gives a rate coefficient of $1.0 \times 10^{-9} \mathrm{~cm}^{3} \mathrm{~s}^{-1}$ (Dutuit et al., 2013). All studies concur that $\mathrm{C}_{2} \mathrm{H}_{2}{ }^{+}+\mathrm{N}_{2}$ are the major products; the high $\mathrm{N}_{2} \mathrm{H}^{+}+\mathrm{C}_{2} \mathrm{H}$ branching ratio mentioned in the compilation of McEwan and Anicich (2007), disagreeing with Anicich et al. (2004), is probably an error (Dutuit et al., 2013).

The evaluated rate coefficient of the reaction of $\mathrm{N}^{+}$with $\mathrm{C}_{2} \mathrm{H}_{2}\left(1.4 \times 10^{-9}\right.$ $\mathrm{cm}^{3} \mathrm{~s}^{-1}$ ) is very close to the Langevin rate (Dutuit et al. (2013) after McEwan et al. (1998)). The McEwan and Anicich (2007) recommendation gives $\mathrm{C}_{2} \mathrm{H}_{2}{ }^{+}+$ $\mathrm{N}$ as the major products at room temperature. At $150 \mathrm{~K}$, the yield of the other products, which originate from a chemical reaction requiring a long-lived complex intermediate, is expected to increase relatively to the $\mathrm{C}_{2} \mathrm{H}_{2}{ }^{+}$ions, coming from charge transfer. In the absence of data, we nevertheless use the branching ratios resulting from the experimental outputs at $300 \mathrm{~K}$.

$\mathrm{C}_{2} \mathrm{H}_{2}{ }^{+}$is lost through reaction with methane:

$$
\begin{aligned}
\mathrm{C}_{2} \mathrm{H}_{2}^{+}+\mathrm{CH}_{4} & \rightarrow \mathrm{C}_{3} \mathrm{H}_{4}^{+}+\mathrm{H}_{2} \\
& \rightarrow \mathrm{C}_{3} \mathrm{H}_{5}^{+}+\mathrm{H} .
\end{aligned}
$$

$\mathrm{C}_{2} \mathrm{H}_{3}{ }^{+}$is produced through a variety of reactions involving $\mathrm{H}^{+}, \mathrm{e}^{-}, \mathrm{H}^{-}$and $\mathrm{H}$ transfer:

$$
\begin{aligned}
\mathrm{CH}_{5}^{+}+\mathrm{C}_{2} \mathrm{H}_{2} & \rightarrow \mathrm{C}_{2} \mathrm{H}_{3}^{+}+\mathrm{CH}_{4}, & \left(\mathrm{R}_{c n} 179\right) \\
\mathrm{N}_{2}^{+}+\mathrm{C}_{2} \mathrm{H}_{4} & \rightarrow \mathrm{C}_{2} \mathrm{H}_{3}^{+}+\mathrm{N}_{2}, & \left(\mathrm{R}_{c n} 838 \mathrm{a}\right) \\
\mathrm{CH}_{3}^{+}+\mathrm{C}_{2} \mathrm{H}_{4} & \rightarrow \mathrm{C}_{2} \mathrm{H}_{3}^{+}+\mathrm{CH}_{4}, & \left(\mathrm{R}_{c n} 134 \mathrm{a}\right) \\
\mathrm{N}_{2} \mathrm{H}^{+}+\mathrm{C}_{2} \mathrm{H}_{2} & \rightarrow \mathrm{C}_{2} \mathrm{H}_{3}^{+}+\mathrm{N}_{2} . & \left(\mathrm{R}_{c n} 863\right)
\end{aligned}
$$

It is lost through condensation and proton transfer reactions:

$$
\begin{array}{rlrl}
\mathrm{C}_{2} \mathrm{H}_{3}^{+}+\mathrm{CH}_{4} & \rightarrow \mathrm{C}_{3} \mathrm{H}_{5}^{+}+\mathrm{H}_{2}, & \left(\mathrm{R}_{c n} 258 \mathrm{a}\right) \\
\mathrm{C}_{2} \mathrm{H}_{3}^{+}+\mathrm{C}_{2} \mathrm{H}_{4} \rightarrow \mathrm{C}_{2} \mathrm{H}_{5}^{+}+\mathrm{C}_{2} \mathrm{H}_{2}, & \left(\mathrm{R}_{c n} 260 \mathrm{a}\right) \\
\mathrm{C}_{2} \mathrm{H}_{3}^{+}+\mathrm{HCN} \rightarrow \mathrm{HCNH}^{+}+\mathrm{C}_{2} \mathrm{H}_{2} . & \left(\mathrm{R}_{c n} 270\right)
\end{array}
$$

$\mathrm{C}_{2} \mathrm{H}_{4}{ }^{+}$is formed through one condensation reaction and through one charge transfer reaction:

$$
\begin{aligned}
\mathrm{CH}_{2}^{+}+\mathrm{CH}_{4} & \rightarrow \mathrm{C}_{2} \mathrm{H}_{4}^{+}+\mathrm{H}_{2}, & \left(\mathrm{R}_{c n} 116 \mathrm{a}\right) \\
\mathrm{CH}_{4}^{+}+\mathrm{C}_{2} \mathrm{H}_{4} \rightarrow \mathrm{C}_{2} \mathrm{H}_{4}^{+}+\mathrm{CH}_{4} . & & \left(\mathrm{R}_{c n} 164 \mathrm{a}\right)
\end{aligned}
$$

It is lost through electron recombination above $1200 \mathrm{~km}\left(\mathrm{R}_{e r} 20\right)$ and some ionneutral reactions below: 


$$
\begin{aligned}
\mathrm{C}_{2} \mathrm{H}_{4}^{+}+\mathrm{C}_{2} \mathrm{H}_{4} & \rightarrow \mathrm{C}_{3} \mathrm{H}_{5}^{+}+\mathrm{CH}_{3}, \\
\mathrm{C}_{2} \mathrm{H}_{4}^{+}+\mathrm{C}_{2} \mathrm{H}_{2} & \rightarrow \mathrm{c}^{-} \mathrm{C}_{3} \mathrm{H}_{3}^{+}+\mathrm{CH}_{3}, \\
\mathrm{C}_{2} \mathrm{H}_{4}^{+}+\mathrm{H} & \rightarrow \mathrm{C}_{2} \mathrm{H}_{3}^{+}+\mathrm{H}_{2} .
\end{aligned}
$$

The production and loss terms of $\mathrm{C}_{2} \mathrm{H}_{5}{ }^{+}$have been discussed in Vuitton et al. (2007). Production is related to the ionization of both $\mathrm{N}_{2}\left(\right.$ via $\mathrm{CH}_{3}{ }^{+}$) and $\mathrm{CH}_{4}\left(\right.$ via $\left.\mathrm{CH}_{5}^{+}\right)$:

$$
\begin{array}{rlrl}
\mathrm{CH}_{3}^{+}+\mathrm{CH}_{4} & \rightarrow \mathrm{C}_{2} \mathrm{H}_{5}^{+}+\mathrm{H}_{2}, & \left(\mathrm{R}_{c n} 132\right) \\
\mathrm{CH}_{5}^{+}+\mathrm{C}_{2} \mathrm{H}_{4} \rightarrow \mathrm{C}_{2} \mathrm{H}_{5}^{+}+\mathrm{CH}_{4} . & \left(\mathrm{R}_{c n} 180\right)
\end{array}
$$

Loss occurs through proton transfer to $\mathrm{HCN}$ and $\mathrm{HNC}\left(\mathrm{R}_{c n} 316, \mathrm{R}_{c n} 317\right)$, dissociative electron attachment $\left(\mathrm{R}_{e r} 21\right)$ and reaction with $\mathrm{C}_{2} \mathrm{H}_{4}$ :

$$
\mathrm{C}_{2} \mathrm{H}_{5}^{+}+\mathrm{C}_{2} \mathrm{H}_{4} \rightarrow \mathrm{C}_{3} \mathrm{H}_{5}^{+}+\mathrm{CH}_{4} \text {. }
$$

The evaluated rate coefficient at $300 \mathrm{~K}$ for the reaction $\mathrm{C}_{2} \mathrm{H}_{5}{ }^{+}+\mathrm{HCN}\left(\mathrm{R}_{c n} 316\right)$, i.e. $2.7 \times 10^{-9} \mathrm{~cm}^{3} \mathrm{~s}^{-1}$ (McEwan and Anicich, 2007), is based on the only study available to date (Mackay et al., 1980). The capture rate at $300 \mathrm{~K}$ is $3.7 \times 10^{-9}$ $\mathrm{cm}^{3} \mathrm{~s}^{-1}$, which implies that the measured rate coefficient corresponds to $73 \%$ of the capture rate. The rate coefficient for the reaction of $\mathrm{C}_{2} \mathrm{H}_{5}{ }^{+}$with $\mathrm{HNC}$ $\left(\mathrm{R}_{c n} 317\right)$ has never been studied and we assume that it is equal to the capture rate, i.e. $5.2 \times 10^{-9} \mathrm{~cm}^{3} \mathrm{~s}^{-1}$ at $150 \mathrm{~K}$, which according to what has been observed for $\mathrm{C}_{2} \mathrm{H}_{5}{ }^{+}+\mathrm{HCN}$, might be slightly overestimated.

The rate coefficient at $300 \mathrm{~K}$ for the reaction of $\mathrm{C}_{2} \mathrm{H}_{5}{ }^{+}$with $\mathrm{C}_{2} \mathrm{H}_{4}\left(\mathrm{R}_{c n} 302 \mathrm{a}\right)$, i.e. $3.6 \times 10^{-10} \mathrm{~cm}^{3} \mathrm{~s}^{-1}$, is based on a literature review (McEwan and Anicich (2007) after Anicich (1993)). The rate coefficient is rather slow and might exhibit some temperature dependence but no studies are available at low temperature.

Three older studies suggest that the recombination rate of $\mathrm{C}_{2} \mathrm{H}_{5}{ }^{+}$with electrons is quite slow at $300 \mathrm{~K}$, i.e. $6.0-9.0 \times 10^{-7} \mathrm{~cm}^{3} \mathrm{~s}^{-1}$ (Adams and Smith, 1988b; Gougousi et al., 1997; Lehfaoui et al., 1997). McLain et al. (2004) obtain a slightly faster rate coefficient at room temperature (i.e. $1.2 \times 10^{-6} \mathrm{~cm}^{3}$ $\mathrm{s}^{-1}$ ) and studied its evolution with temperature. The rate exhibits two distinct temperature dependencies, i.e. $\mathrm{T}^{-0.8}$ for $80<\mathrm{T}_{e}<300 \mathrm{~K}$ and $\mathrm{T}^{-1.2}$ for $300<$ $\mathrm{T}_{e}<600 \mathrm{~K}$, which seem consistent with theoretical models.

Since the RPWS LP reports electron temperatures above typically $500 \mathrm{~K}$, we use the McLain et al. (2004) temperature dependence appropriate for the higher temperature range (i.e. $\mathrm{T}^{-1.2}$ ). However, if the electron temperature is closer to the neutral temperature as suggested by Richard et al. (2011), the temperature dependence for the lower temperature range (i.e. $\mathrm{T}^{-0.8}$ ) would be more appropriate for altitudes below $\sim 1150 \mathrm{~km}$.

The branching ratios for channels $\left(\mathrm{R}_{e r} 21 \mathrm{a}\right) /\left(\mathrm{R}_{e r} 21 \mathrm{~b}\right) /\left(\mathrm{R}_{e r} 21 \mathrm{c}\right) /\left(\mathrm{R}_{e r} 21 \mathrm{~d}\right) /\left(\mathrm{R}_{e r} 21 \mathrm{e}\right)$ are $0.29 / 0.27 / 0.17 / 0.13 / 0.12$ according to the only available study, which was 
made on $\mathrm{C}_{2} \mathrm{D}_{5}{ }^{+}$(Geppert et al., 2004a). The branching ratios have been divided by 0.98 for the sum to be equal to one (W. Geppert, private communication, 2013). Although lighter isotopologues react much faster than heavier ones, branching ratios in dissociative recombination reactions have not shown pronounced effects on deuteration (see e.g. Geppert et al. (2006)). Therefore, using the branching ratios for $\mathrm{C}_{2} \mathrm{D}_{5}{ }^{+}$should be mostly accurate.

The chemistry of $\mathrm{C}_{2} \mathrm{H}_{7}{ }^{+}$is poorly characterized. In our model, it is mostly an intermediate in the proton transfer chain as ethane takes a proton from $\mathrm{CH}_{5}{ }^{+}$ $\left(\mathrm{R}_{c n} 181 \mathrm{~b}\right)$ to transfer it to hydrogen cyanide $\left(\mathrm{R}_{c n} 352 \mathrm{a}\right)$. Above $1150 \mathrm{~km}$, the main loss is dissociative electron recombination $\left(\mathrm{R}_{e r} 24\right)$ but both rate coefficient and products are unknown and had to be estimated.

$\mathbf{C}_{3} \mathbf{H}_{3}{ }^{+}, \mathbf{C}_{3} \mathbf{H}_{5}{ }^{+}, \mathbf{C}_{3} \mathbf{H}_{7}{ }^{+} . \mathrm{C}_{3} \mathrm{H}_{3}{ }^{+}$has two low-energy isomers, the cyclopropenylium ion $\mathrm{c}-\mathrm{C}_{3} \mathrm{H}_{3}{ }^{+}\left(\Delta H_{f}^{\circ}=1075 \mathrm{~kJ} \mathrm{~mol}^{-1}\right)$ and the linear propargyl ion $1-\mathrm{C}_{3} \mathrm{H}_{3}{ }^{+}\left(\Delta H_{f}^{\circ}=1179 \mathrm{~kJ} \mathrm{~mol}^{-1}\right)$ (Lias et al., 1988). Because of their very different reactivity, it is important to discriminate these two species in chemical models (Carrasco et al., 2008). The major source and sink for $1-\mathrm{C}_{3} \mathrm{H}_{3}{ }^{+}$are:

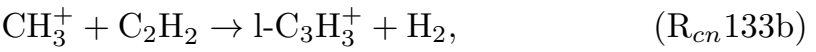

$$
\begin{aligned}
& \mathrm{l}_{-} \mathrm{C}_{3} \mathrm{H}_{3}^{+}+\mathrm{C}_{2} \mathrm{H}_{4} \rightarrow \mathrm{C}_{5} \mathrm{H}_{5}^{+}+\mathrm{H}_{2} \quad\left(\mathrm{R}_{c n} 390 \mathrm{a}\right) \\
& \rightarrow \mathrm{C}_{5} \mathrm{H}_{7}^{+}+\mathrm{h} \nu . \quad\left(\mathrm{R}_{c n} 390 \mathrm{~b}\right)
\end{aligned}
$$

Although $\mathrm{c}-\mathrm{C}_{3} \mathrm{H}_{3}{ }^{+}$is also produced in reaction $\left(\mathrm{R}_{c n} 133 \mathrm{a}\right)$, it is essentially formed in reactions that do not produce $1-\mathrm{C}_{3} \mathrm{H}_{3}{ }^{+}$(McEwan and Anicich (2007) and M.J. McEwan, private communication):

$$
\begin{aligned}
& \mathrm{C}_{2} \mathrm{H}_{5}^{+}+\mathrm{C}_{2} \mathrm{H}_{2} \rightarrow \mathrm{c}_{-} \mathrm{C}_{3} \mathrm{H}_{3}^{+}+\mathrm{CH}_{4}, \quad\left(\mathrm{R}_{c n} 301 \mathrm{a}\right)
\end{aligned}
$$

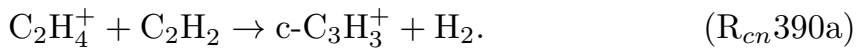

In contrast with $1-\mathrm{C}_{3} \mathrm{H}_{3}{ }^{+}, \mathrm{c}-\mathrm{C}_{3} \mathrm{H}_{3}{ }^{+}$is nonreactive with most hydrocarbons and its main loss is dissociative electron recombination $\left(\mathrm{R}_{e r} 29\right)$.

As in Vuitton et al. (2007), $\mathrm{C}_{3} \mathrm{H}_{5}{ }^{+}$is efficiently produced in reactions with methane and ethylene:

$$
\begin{aligned}
\mathrm{C}_{2} \mathrm{H}_{3}^{+}+\mathrm{CH}_{4} & \rightarrow \mathrm{C}_{3} \mathrm{H}_{5}^{+}+\mathrm{H}_{2}, & & \left(\mathrm{R}_{c n} 258 \mathrm{a}\right) \\
\mathrm{C}_{2} \mathrm{H}_{5}^{+}+\mathrm{C}_{2} \mathrm{H}_{4} & \rightarrow \mathrm{C}_{3} \mathrm{H}_{5}^{+}+\mathrm{CH}_{4} . & & \left(\mathrm{R}_{c n} 302 \mathrm{a}\right)
\end{aligned}
$$

Following the suggestion by Demarais et al. (2013), we include the proton transfer reaction between $\mathrm{HCNH}^{+}$and methylacetylene $\left(\mathrm{R}_{c n} 697\right)$, which, with our estimated rate coefficient, substantially contributes to the formation of $\mathrm{C}_{3} \mathrm{H}_{5}{ }^{+}$ as well. Above $1000 \mathrm{~km}, \mathrm{C}_{3} \mathrm{H}_{5}{ }^{+}$is mostly lost by reactions with acetylene and ethylene: 


$$
\begin{array}{ll}
\mathrm{C}_{3} \mathrm{H}_{5}^{+}+\mathrm{C}_{2} \mathrm{H}_{2} \rightarrow \mathrm{C}_{5} \mathrm{H}_{5}^{+}+\mathrm{H}_{2}, & \left(\mathrm{R}_{c n} 422 \mathrm{a}\right) \\
\mathrm{C}_{3} \mathrm{H}_{5}^{+}+\mathrm{C}_{2} \mathrm{H}_{4} \rightarrow \mathrm{C}_{5} \mathrm{H}_{7}^{+}+\mathrm{H}_{2} . & \left(\mathrm{R}_{c n} 423 \mathrm{a}\right)
\end{array}
$$

Below $1000 \mathrm{~km}$, electron dissociative recombination $\left(\mathrm{R}_{e r} 32\right)$ becomes the main loss process, as in Vuitton et al. (2007). The electron recombination of $\mathrm{C}_{3} \mathrm{H}_{5}{ }^{+}$ has not been investigated experimentally. Using general concepts based on the data available for other $\mathrm{C}_{2,3} \mathrm{H}_{y}^{+}$ions Janev and Reiter (2004) estimated both its rate coefficient and product branching ratios. The rate coefficient is expressed as a function of the number of hydrogen atoms for a given number of carbon atoms and possesses a somewhat complex temperature dependence. The values calculated with this expression are not necessarily in agreement with experimental data obtained since 2004 (for $\mathrm{C}_{2} \mathrm{H}_{5}{ }^{+}, \mathrm{C}_{3} \mathrm{H}_{3}{ }^{+}$and $\mathrm{C}_{3} \mathrm{H}_{4}{ }^{+}$, for example) and as a consequence, we stick to our own fitting expression that uses a wider dataset. For comparison, for $\mathrm{C}_{3} \mathrm{H}_{5}{ }^{+}$, we obtain a value of $10^{-6} \mathrm{~cm}^{3} \mathrm{~s}^{-1}$ while the Janev and Reiter (2004) expression would give $7.2 \times 10^{-7} \mathrm{~cm}^{3} \mathrm{~s}^{-1}$ for an electron temperature of $300 \mathrm{~K}$. In the absence of a better approach, we however use their branching ratios, which consist mostly of multiple hydrogen loss and to a lesser extent, carbon bond break-up.

$\mathrm{C}_{3} \mathrm{H}_{7}{ }^{+}$is essentially formed through reaction of protonated hydrogen cyanide with propene $\left(\mathrm{R}_{c n} 699\right)$. It is lost through dissociative electron recombination $\left(\mathrm{R}_{e r} 34\right)$ above $950 \mathrm{~km}$ and reaction with ethane below:

$$
\mathrm{C}_{3} \mathrm{H}_{7}^{+}+\mathrm{C}_{2} \mathrm{H}_{6} \rightarrow \mathrm{C}_{4} \mathrm{H}_{9}^{+}+\mathrm{CH}_{4} \text {. }
$$

$\mathrm{C}_{4} \mathrm{H}_{2}{ }^{+}, \mathrm{C}_{4} \mathrm{H}_{3}{ }^{+}, \mathrm{C}_{4} \mathrm{H}_{5}{ }^{+}, \mathrm{C}_{4} \mathrm{H}_{7}{ }^{+}, \mathrm{C}_{4} \mathrm{H}_{9}{ }^{+} . \mathrm{C}_{4} \mathrm{H}_{2}{ }^{+}$is essentially formed through a reaction involving $\mathrm{C}_{2} \mathrm{H}_{2}{ }^{+}$ions:

$$
\mathrm{C}_{2} \mathrm{H}_{2}^{+}+\mathrm{C}_{2} \mathrm{H}_{2} \rightarrow \mathrm{C}_{4} \mathrm{H}_{2}^{+}+\mathrm{H}_{2} \text {. }
$$

It is lost by electron recombination $\left(\mathrm{R}_{e r} 38\right)$ above $1100 \mathrm{~km}$ and reaction with $\mathrm{C}_{2} \mathrm{H}_{4}$ below:

$$
\begin{aligned}
\mathrm{C}_{4} \mathrm{H}_{2}^{+}+\mathrm{C}_{2} \mathrm{H}_{4} & \rightarrow \mathrm{C}_{6} \mathrm{H}_{5}^{+}+\mathrm{H} & & \left(\mathrm{R}_{c n} 465 \mathrm{c}\right) \\
& \rightarrow \mathrm{C}_{4} \mathrm{H}_{4}^{+}+\mathrm{C}_{2} \mathrm{H}_{2} . & & \left(\mathrm{R}_{c n} 465 \mathrm{a}\right)
\end{aligned}
$$

As in Vuitton et al. (2007, 2008), $\mathrm{C}_{4} \mathrm{H}_{3}{ }^{+}$is formed by proton transfer from $\mathrm{HCNH}^{+}$and $\mathrm{C}_{2} \mathrm{H}_{5}{ }^{+}$to diacetylene $\left(\mathrm{R}_{c n} 700, \mathrm{R}_{c n} 308\right)$ in the bulk of the ionosphere, while above $1300 \mathrm{~km}$, a condensation reaction kicks in:

$$
\mathrm{C}_{2} \mathrm{H}_{3}^{+}+\mathrm{C}_{2} \mathrm{H}_{2} \rightarrow \mathrm{C}_{4} \mathrm{H}_{3}^{+}+\mathrm{H}_{2} .
$$

It is lost through electron dissociative recombination $\left(\mathrm{R}_{e r} 39\right)$ above $1000 \mathrm{~km}$ and several condensation reactions below: 


$$
\begin{aligned}
\mathrm{C}_{4} \mathrm{H}_{3}^{+}+\mathrm{C}_{2} \mathrm{H}_{4} & \rightarrow \mathrm{C}_{6} \mathrm{H}_{5}^{+}+\mathrm{H}_{2}, & \left(\mathrm{R}_{c n} 479 \mathrm{a}\right) \\
\mathrm{C}_{4} \mathrm{H}_{3}^{+}+\mathrm{C}_{2} \mathrm{H}_{2} & \rightarrow \mathrm{C}_{6} \mathrm{H}_{5}^{+}+\mathrm{h} \nu, & \left(\mathrm{R}_{c n} 478 \mathrm{a}\right) \\
\mathrm{C}_{4} \mathrm{H}_{3}^{+}+\mathrm{CH}_{3} \mathrm{CCH} & \rightarrow \mathrm{C}_{5} \mathrm{H}_{5}^{+}+\mathrm{C}_{2} \mathrm{H}_{2}, & \left(\mathrm{R}_{c n} 480\right) \\
\mathrm{C}_{4} \mathrm{H}_{3}^{+}+\mathrm{C}_{4} \mathrm{H}_{2} & \rightarrow \mathrm{C}_{6} \mathrm{H}_{3}^{+}+\mathrm{C}_{2} \mathrm{H}_{2} . & \left(\mathrm{R}_{c n} 481 \mathrm{a}\right)
\end{aligned}
$$

$\mathrm{C}_{4} \mathrm{H}_{5}{ }^{+}$is formed through a branch of the reaction between $\mathrm{C}_{2} \mathrm{H}_{5}{ }^{+}$and acetylene:

$$
\mathrm{C}_{2} \mathrm{H}_{5}^{+}+\mathrm{C}_{2} \mathrm{H}_{2} \rightarrow \mathrm{C}_{4} \mathrm{H}_{5}^{+}+\mathrm{H}_{2} .
$$

It is lost through dissociative electron recombination $\left(\mathrm{R}_{e r} 42\right)$ below $1000 \mathrm{~km}$ and reaction with ethylene and acetylene above:

$$
\begin{aligned}
& \mathrm{C}_{4} \mathrm{H}_{5}^{+}+\mathrm{C}_{2} \mathrm{H}_{4} \rightarrow \mathrm{C}_{6} \mathrm{H}_{7}^{+}+\mathrm{H}_{2}, \\
& \mathrm{C}_{4} \mathrm{H}_{5}^{+}+\mathrm{C}_{2} \mathrm{H}_{2} \rightarrow \mathrm{C}_{6} \mathrm{H}_{5}^{+}+\mathrm{H}_{2} .
\end{aligned}
$$

10

$\mathrm{C}_{4} \mathrm{H}_{7}{ }^{+}$is formed through two condensation reactions:

$$
\begin{array}{ll}
\mathrm{C}_{3} \mathrm{H}_{5}^{+}+\mathrm{C}_{3} \mathrm{H}_{6} \rightarrow \mathrm{C}_{4} \mathrm{H}_{7}^{+}+\mathrm{C}_{2} \mathrm{H}_{4}, & \left(\mathrm{R}_{c n} 425 \mathrm{a}\right) \\
\mathrm{C}_{2} \mathrm{H}_{4}^{+}+\mathrm{C}_{2} \mathrm{H}_{4} \rightarrow \mathrm{C}_{4} \mathrm{H}_{7}^{+}+\mathrm{H} . & \left(\mathrm{R}_{c n} 282 \mathrm{c}\right)
\end{array}
$$

${ }_{1871} \mathrm{C}_{4} \mathrm{H}_{9}{ }^{+}$is formed by two reactions involving ethane, below and above 1000 $1872 \mathrm{~km}$, respectively:

$$
\begin{aligned}
& \mathrm{C}_{2} \mathrm{H}_{5}^{+}+\mathrm{C}_{2} \mathrm{H}_{6} \rightarrow \mathrm{C}_{4} \mathrm{H}_{9}^{+}+\mathrm{H}_{2}, \\
& \mathrm{C}_{3} \mathrm{H}_{7}^{+}+\mathrm{C}_{2} \mathrm{H}_{6} \rightarrow \mathrm{C}_{4} \mathrm{H}_{9}^{+}+\mathrm{CH}_{4} .
\end{aligned}
$$

$\mathrm{C}_{4} \mathrm{H}_{7}{ }^{+}$and $\mathrm{C}_{4} \mathrm{H}_{9}{ }^{+}$are both lost by dissociative electron recombination $\left(\mathrm{R}_{e r} 44, \mathrm{R}_{e r} 46\right)$.

$\mathbf{C}_{5} \mathbf{H}_{3}{ }^{+}, \mathbf{C}_{5} \mathbf{H}_{\mathbf{5}}{ }^{+}, \mathbf{C}_{\mathbf{5}} \mathbf{H}_{\mathbf{7}}{ }^{+} \cdot \mathrm{C}_{5} \mathrm{H}_{3}{ }^{+}$is formed by three condensation reactions involving diacetylene:

$$
\begin{aligned}
& \mathrm{C}_{2} \mathrm{H}_{4}^{+}+\mathrm{C}_{4} \mathrm{H}_{2} \rightarrow \mathrm{C}_{5} \mathrm{H}_{3}^{+}+\mathrm{CH}_{3}, \quad\left(\mathrm{R}_{c n} 287 \mathrm{a}\right) \\
& \text { l- } \mathrm{C}_{3} \mathrm{H}_{3}^{+}+\mathrm{C}_{4} \mathrm{H}_{2} \rightarrow \mathrm{C}_{5} \mathrm{H}_{3}^{+}+\mathrm{C}_{2} \mathrm{H}_{2}, \quad\left(\mathrm{R}_{c n} 393 \mathrm{~b}\right) \\
& \mathrm{CH}_{3}^{+}+\mathrm{C}_{4} \mathrm{H}_{2} \rightarrow \mathrm{C}_{5} \mathrm{H}_{3}^{+}+\mathrm{H}_{2} . \quad\left(\mathrm{R}_{c n} 138 \mathrm{~b}\right)
\end{aligned}
$$

$\mathrm{C}_{5} \mathrm{H}_{5}{ }^{+}$and $\mathrm{C}_{5} \mathrm{H}_{7}{ }^{+}$are almost exclusively formed through the reactions: 


$$
\begin{array}{ll}
\mathrm{C}_{3} \mathrm{H}_{5}^{+}+\mathrm{C}_{2} \mathrm{H}_{2} \rightarrow \mathrm{C}_{5} \mathrm{H}_{5}^{+}+\mathrm{H}_{2}, & \left(\mathrm{R}_{c n} 422 \mathrm{a}\right) \\
\mathrm{C}_{3} \mathrm{H}_{5}^{+}+\mathrm{C}_{2} \mathrm{H}_{4} \rightarrow \mathrm{C}_{5} \mathrm{H}_{7}^{+}+\mathrm{H}_{2} . & \left(\mathrm{R}_{c n} 423 \mathrm{a}\right)
\end{array}
$$

$\mathrm{C}_{5} \mathrm{H}_{3}{ }^{+}, \mathrm{C}_{5} \mathrm{H}_{5}{ }^{+}$and $\mathrm{C}_{5} \mathrm{H}_{7}{ }^{+}$are lost through dissociative electron recombination $\left(\mathrm{R}_{e r} 49, \mathrm{R}_{e r} 51, \mathrm{R}_{e r} 53\right)$.

$\mathbf{C}_{6} \mathrm{H}_{3}{ }^{+}, \mathrm{C}_{6} \mathrm{H}_{5}{ }^{+}, \mathrm{C}_{6} \mathrm{H}_{7}{ }^{+} \cdot \mathrm{C}_{6} \mathrm{H}_{3}{ }^{+}$is mostly formed through proton exchange between the most abundant ions and $\mathrm{C}_{6} \mathrm{H}_{2}\left(\mathrm{R}_{c n} 702, \mathrm{R}_{c n} 310\right)$, and the reaction:

$$
\mathrm{C}_{4} \mathrm{H}_{3}^{+}+\mathrm{C}_{4} \mathrm{H}_{2} \rightarrow \mathrm{C}_{6} \mathrm{H}_{3}^{+}+\mathrm{C}_{2} \mathrm{H}_{2} .
$$

It is lost through dissociative electron attachment $\left(\mathrm{R}_{e r} 60\right)$ above $1000 \mathrm{~km}$ and radiative association to acetylene below:

$$
\mathrm{C}_{6} \mathrm{H}_{3}^{+}+\mathrm{C}_{2} \mathrm{H}_{2} \rightarrow \mathrm{C}_{8} \mathrm{H}_{5}^{+}+\mathrm{h} \nu \text {. }
$$

$\mathrm{C}_{6} \mathrm{H}_{5}{ }^{+}$is formed through reactions of $\mathrm{C}_{4} \mathrm{H}_{3}{ }^{+}$and $\mathrm{C}_{4} \mathrm{H}_{5}{ }^{+}$with ethylene and acetylene $\left(\mathrm{R}_{c n} 479 \mathrm{a}, \mathrm{R}_{c n} 478 \mathrm{a}, \mathrm{R}_{c n} 496 \mathrm{a}\right)$. This is somewhat different from the scheme presented in Vuitton et al. (2008) where $\left(\mathrm{R}_{c n} 496 \mathrm{a}\right)$ was not a major production reaction. We nevertheless still expect that $\mathrm{C}_{6} \mathrm{H}_{5}{ }^{+}$is the phenylium ion, the ground state cyclic isomer, as discussed in Vuitton et al. (2008) and use rate coefficients corresponding to $\mathrm{c}^{-} \mathrm{C}_{6} \mathrm{H}_{5}{ }^{+}$, if available.

$\mathrm{C}_{6} \mathrm{H}_{5}{ }^{+}$is mostly lost by dissociative electron recombination $\left(\mathrm{R}_{e r} 62\right)$, as well as by reaction with methane and hydrogen:

$$
\begin{aligned}
\mathrm{C}_{6} \mathrm{H}_{5}^{+}+\mathrm{CH}_{4} & \rightarrow \mathrm{C}_{7} \mathrm{H}_{7}^{+}+\mathrm{H}_{2}, \\
\mathrm{C}_{6} \mathrm{H}_{5}^{+}+\mathrm{H}_{2} & \rightarrow \mathrm{C}_{6} \mathrm{H}_{7}^{+}+\mathrm{h} \nu .
\end{aligned}
$$$$
\left(\mathrm{R}_{c n} 553\right)
$$

$\mathrm{C}_{6} \mathrm{H}_{7}{ }^{+}$is formed through multiple reactions having about the same importance:

$$
\begin{aligned}
\mathrm{C}_{3} \mathrm{H}_{5}^{+}+\mathrm{CH}_{3} \mathrm{CCH} & \rightarrow \mathrm{C}_{6} \mathrm{H}_{7}^{+}+\mathrm{H}_{2}, & \left(\mathrm{R}_{c n} 424 \mathrm{a}\right) \\
\mathrm{C}_{6} \mathrm{H}_{5}^{+}+\mathrm{H}_{2} & \rightarrow \mathrm{C}_{6} \mathrm{H}_{7}^{+}+\mathrm{h} \nu, & \left(\mathrm{R}_{c n} 552\right) \\
\mathrm{C}_{4} \mathrm{H}_{5}^{+}+\mathrm{C}_{2} \mathrm{H}_{4} & \rightarrow \mathrm{C}_{6} \mathrm{H}_{7}^{+}+\mathrm{H}_{2} . & \left(\mathrm{R}_{c n} 497\right)
\end{aligned}
$$

It is lost through dissociative electron recombination $\left(\mathrm{R}_{e r} 64\right)$, whose rate coefficient, including its evolution with electron temperature, has been recently determined (Hamberg et al., 2011). At the exception of reaction $\left(\mathrm{R}_{c n} 424 \mathrm{a}\right)$, this is the same set of reactions as in Vuitton et al. (2008). 
$\mathbf{C}_{\mathbf{7}} \mathbf{H}_{\mathbf{7}}{ }^{+}, \mathbf{C}_{\mathbf{7}} \mathbf{H}_{\mathbf{9}}{ }^{+} \cdot \mathrm{C}_{7} \mathrm{H}_{7}{ }^{+}$is lost through electron dissociative recombination $\left(\mathrm{R}_{e r} 75\right)$ above $1000 \mathrm{~km}$ and reaction with ethylene below:

$$
\mathrm{C}_{7} \mathrm{H}_{7}^{+}+\mathrm{C}_{2} \mathrm{H}_{4} \rightarrow \mathrm{C}_{9} \mathrm{H}_{11}^{+}+\mathrm{h} \nu \text {. }
$$

$\mathrm{C}_{7} \mathrm{H}_{7}{ }^{+}$has been famously observed as both the tropylium (7 member ring) and benzylium ( 6 member ring) isomers. $\mathrm{C}_{7} \mathrm{H}_{7}{ }^{+}$dissociative recombination has been studied by Rebrion-Rowe et al. (2000) and Fournier et al. (2013) who found a very different reactivity for the two states, tropylium ions recombining with a rate coefficient of $3.2 \times 10^{-7} \mathrm{~cm}^{3} \mathrm{~s}^{-1}$ and benzylium ions with a rate coefficient of $11.6 \times 10^{-7} \mathrm{~cm}^{3} \mathrm{~s}^{-1}$. In our case, $\mathrm{C}_{7} \mathrm{H}_{7}{ }^{+}$ions are mostly formed in the reaction of phenylium ions $\left(\mathrm{c}^{-} \mathrm{C}_{6} \mathrm{H}_{5}{ }^{+}\right)$with methane $\left(\mathrm{R}_{c n} 553\right)$ and were identified as tropylium ions because of their lack of reactivity with aromatic molecules (Ausloos, 1982; Ausloos et al., 1989).

$\mathrm{C}_{7} \mathrm{H}_{9}{ }^{+}$is formed by proton transfer from the most abundant ions to toluene $\left(\mathrm{R}_{c n} 705, \mathrm{R}_{c n} 313\right)$ as well as through a condensation reaction:

$$
\mathrm{C}_{4} \mathrm{H}_{7}^{+}+\mathrm{CH}_{3} \mathrm{CCH} \rightarrow \mathrm{C}_{7} \mathrm{H}_{9}^{+}+\mathrm{H}_{2} .
$$

It is exclusively lost by dissociative electron recombination $\left(\mathrm{R}_{e r} 77\right)$. Osborne et al. (2011) have studied the dissociative electron-ion recombination of the protonated six membered ring toluene $\mathrm{C}_{6} \mathrm{H}_{5} \mathrm{CH}_{4}{ }^{+}$and have obtained a room temperature rate coefficient of $3.8 \times 10^{-7} \mathrm{~cm}^{3} \mathrm{~s}^{-1}$. Although the nature of the $\mathrm{C}_{7} \mathrm{H}_{9}{ }^{+}$isomer formed in reaction $\left(\mathrm{R}_{c n} 504\right)$ is unknown, it is likely that protonation of toluene gives $\mathrm{C}_{6} \mathrm{H}_{5} \mathrm{CH}_{4}{ }^{+}$.

\subsubsection{Nitrogen-Bearing Ions.}

$\mathbf{N}_{2} \mathbf{H}^{+}$. As mentioned in section 3.1.1.2, $\mathrm{N}_{2} \mathrm{H}^{+}$is an important intermediate in the formation of $\mathrm{CH}_{5}{ }^{+}$. It is mostly formed by reaction of $\mathrm{N}_{2}{ }^{+}$with hydrogen $\left(\mathrm{R}_{c n} 835 \mathrm{a}\right)$ and methane $\left(\mathrm{R}_{c n} 836 \mathrm{c}\right)$, and lost through reaction with methane $\left(\mathrm{R}_{c n} 862\right)$.

$\mathbf{N H}_{4}{ }^{+}$. The formation of $\mathrm{NH}_{4}{ }^{+}$is discussed in Yelle et al. (2010). A channel based on the addition of $\mathrm{H}$ onto $\mathrm{NH}_{x}^{+}(x=0-3)$ by reaction with $\mathrm{CH}_{4}$ and $\mathrm{H}_{2}$ has been proposed (Atreya, 1986) but most of the $\mathrm{NH}^{+}$formed by reaction of $\mathrm{N}^{+}$with $\mathrm{H}_{2}$ reacts with $\mathrm{N}_{2}$ to form $\mathrm{N}_{2} \mathrm{H}^{+}$, therefore short-circuiting the production of $\mathrm{NH}_{4}{ }^{+}$. Instead, $\mathrm{NH}_{4}^{+}$is created by reaction of $\mathrm{NH}_{3}$ with other protonated molecules, especially $\mathrm{HCNH}^{+}$and $\mathrm{C}_{2} \mathrm{H}_{5}{ }^{+}\left(\mathrm{R}_{c n} 707, \mathrm{R}_{c n} 315\right)$, and lost by electron recombination $\left(\mathrm{R}_{e r} 84\right)$.

$\mathbf{H C N H}^{+}, \mathbf{C H}_{2} \mathrm{NH}_{2}{ }^{+}$. Proton exchange of $\mathrm{C}_{2} \mathrm{H}_{5}{ }^{+}$and $\mathrm{CH}_{5}{ }^{+}$with hydrogen cyanide and isocyanide $\left(\mathrm{R}_{c n} 316, \mathrm{R}_{c n} 194, \mathrm{R}_{c n} 317, \mathrm{R}_{c n} 195\right)$ are responsible for the $\mathrm{HCNH}^{+}$production, various ion-neutral reactions contributing to only $\sim 10 \%$ of the total production (Vuitton et al., 2007). These reactions have never been studied. We make the assumption that the only channel is a proton transfer 
from the ion to the neutral and that the rate constant is equal to the capture rate. Using literature values for the dipole polarizability and dipole moment for HCN (CRC Handbook of Chemistry and Physics, 87th Edition) and HNC (Blackman et al., 1976; Woon and Herbst, 2009), leads to a rate constant at 150 $\mathrm{K}$ of $5.8 \times 10^{-9} \mathrm{~cm}^{3} \mathrm{~s}^{-1}$ and $6.0 \times 10^{-9} \mathrm{~cm}^{3} \mathrm{~s}^{-1}$, respectively.

Again, below $1050 \mathrm{~km}$, electron dissociative recombination is its main loss:

$$
\begin{aligned}
& \mathrm{HCNH}^{+}+\mathrm{e}^{-} \rightarrow \mathrm{HCN}+\mathrm{H} \quad\left(\mathrm{R}_{e r} 88 \mathrm{a}\right) \\
& \rightarrow \mathrm{HNC}+\mathrm{H} \quad\left(\mathrm{R}_{e r} 88 \mathrm{~b}\right) \\
& \rightarrow \mathrm{CN}+\mathrm{H}+\mathrm{H} \quad\left(\mathrm{R}_{e r} 88 \mathrm{c}\right) \\
& \rightarrow \mathrm{CN}+\mathrm{H}_{2} . \quad\left(\mathrm{R}_{e r} 88 \mathrm{~d}\right)
\end{aligned}
$$

The electron recombination of $\mathrm{HCNH}^{+}$has been investigated with two different experimental techniques: the flowing-afterglow langmuir probe (FALP) at the University of Georgia (Adams and Smith, 1988a; Adams et al., 1991; McLain et al., 2009; McLain and Adams, 2009) and the heavy ion storage ring CRYRING (Semaniak et al., 2001). In McLain et al. (2009) and McLain and Adams (2009), $\mathrm{HCNH}^{+}$is created by the reactions:

$$
\begin{array}{lr}
\mathrm{H}_{3}^{+}+\mathrm{HCN} \rightarrow\left[\mathrm{HCNH}^{+}\right]^{* *}+\mathrm{H}_{2} & \left(\mathrm{R}_{c n}^{* *} 57\right) \\
{\left[\mathrm{HCNH}^{+}\right]^{* *}+\mathrm{HCN} \rightarrow \mathrm{HCNH}^{+}+\mathrm{HCN}^{* *}} & \left(\mathrm{R}_{c n}^{* *} 711\right)
\end{array}
$$

where $^{* *}$ indicates rovibronic internal excitation. The authors argue that before recombination occurs, resonant proton transfer, reaction $\left(\mathrm{R}_{c n}^{* *} 711\right)$ rapidly quenches any rovibronically excited species created by the exothermicity of reaction $\left(\mathrm{R}_{c n}^{* *} 57\right)$. In Semaniak et al. (2001), the cross-sections are measured after $5.5 \mathrm{~s}$ of storage time, which is estimated to be long enough to allow the ions to be vibrationally relaxed.

The values measured for the rate coefficient at $300 \mathrm{~K}$ are in good agreement with each other within experimental uncertainties and give a value of $\sim 3.0$ $\times 10^{-7} \mathrm{~cm}^{3} \mathrm{~s}^{-1}$ (McLain et al., 2009; Semaniak et al., 2001). However, the dependence with electron temperature is significantly different: $\mathrm{T}_{e}^{-1.38}$ for 180 $<\mathrm{T}_{e}<600 \mathrm{~K}$ (McLain and Adams, 2009) against $\mathrm{T}_{e}^{-0.65}$ for $\mathrm{T}_{e}<1000 \mathrm{~K}$ (Semaniak et al., 2001).

Adams et al. (1991) report a fraction of $\mathrm{H}$ atoms of $0.6 \pm 0.2$, suggesting that the dissociation channel giving $\mathrm{CN}+\mathrm{H}_{2}$ must be quite efficient. The branching ratios retrieved later with the CRYRING experiment, i.e. $(0.68 \pm 0.02) /(0.32 \pm 0.03) /(0.00 \pm 0.02)$ for channels $\left(\mathrm{R}_{e r} 88 \mathrm{a}+\mathrm{R}_{e r} 88 \mathrm{~b}\right) /\left(\mathrm{R}_{e r} 88 \mathrm{c}\right) /\left(\mathrm{R}_{e r} 88 \mathrm{~d}\right)$ give a fractional $\mathrm{H}$ contribution of 1.32 (Semaniak et al., 2001) in strong disagreement with the earlier results. The experimental measurements cannot discriminate between the HCN and HNC isomers but theoretical calculations suggest that they are both formed in equivalent amount (Shiba et al., 1998; Talbi and Ellinger, 1998). We will return to this in section 3.2.2, while discussing the formation of HNC. 
In the KIDA framework, W. Geppert produced an evaluation datasheet for this reaction. He recommends using the CRYRING data because (i) the electron temperature range investigated is wider, and (ii) the FALP measurements are indirect and therefore subject to larger uncertainties. We follow this recommendation and use a rate coefficient of $2.8 \times 10^{-7} \times\left(\mathrm{T}_{e} / 300\right)^{-0.65} \mathrm{~cm}^{3} \mathrm{~s}^{-1}$ and branching ratios of $0.34 / 0.34 / 0.32 / 0.00$ for channels $\left.\left(\mathrm{R}_{e r} 88 \mathrm{a}\right) /\left(\mathrm{R}_{e r} 88 \mathrm{~b}\right) /\left(\mathrm{R}_{e r} 88 \mathrm{c}\right) /\left(\mathrm{R}_{e r} 88 \mathrm{~d}\right)\right)$.

Below $1050 \mathrm{~km}$, proton transfer to a variety of different molecules with a high proton affinity $\left(\mathrm{C}_{4} \mathrm{H}_{2}, \mathrm{C}_{2} \mathrm{H}_{3} \mathrm{CN}\right.$, etc.) becomes the dominant $\mathrm{HCNH}^{+}$loss process. The evaluated rate coefficient at $300 \mathrm{~K}$ for $\mathrm{HCNH}^{+}+\mathrm{C}_{4} \mathrm{H}_{2}$, i.e. 1.8 $\times 10^{-9} \mathrm{~cm}^{3} \mathrm{~s}^{-1}$ (McEwan and Anicich, 2007), is based on the study of Petrie et al. (1991b). The Langevin rate for this reaction is $1.5 \times 10^{-9} \mathrm{~cm}^{3} \mathrm{~s}^{-1}$ and we use this value as the rate coefficient instead.

The ion-molecule chemistry of acrylonitrile has been investigated by Petrie et al. (1991a, 1992), who find a rate coefficient at $300 \mathrm{~K}$ of $4.5 \times 10^{-9} \mathrm{~cm}^{3} \mathrm{~s}^{-1}$ for its reaction with $\mathrm{HCNH}^{+}$, which is equal to the capture rate coefficient.

$\mathrm{CH}_{2} \mathrm{NH}_{2}{ }^{+}$has a very high proton affinity $(852.9 \mathrm{~kJ} / \mathrm{mol})$ and as a consequence is produced by proton exchange reactions with most of the protonated species in Titan's atmosphere and lost by electron recombination (Yelle et al., 2010). We emphasize that although the rate coefficient for the proton transfer from $\mathrm{HCNH}^{+}$and $\mathrm{C}_{2} \mathrm{H}_{5}{ }^{+}$to $\mathrm{CH}_{2} \mathrm{NH}$ has now been determined (Edwards et al., 2008), both rate coefficient and products for the electron recombination of $\mathrm{CH}_{2} \mathrm{NH}_{2}{ }^{+}$still need to be measured.

$\mathbf{C H}_{3} \mathbf{C N H}^{+}$. As in Vuitton et al. (2007), $\mathrm{CH}_{3} \mathrm{CNH}^{+}$is efficiently produced by proton transfer from $\mathrm{HCNH}^{+}$and $\mathrm{C}_{2} \mathrm{H}_{5}{ }^{+}\left(\mathrm{R}_{c n} 713, \mathrm{R}_{c n} 320\right)$ and lost by electron recombination $\left(\mathrm{R}_{e r} 100\right)$. However, a new reaction (section 2.7.3.1) is responsible for about half of the $\mathrm{CH}_{3} \mathrm{CNH}^{+}$production:

$$
\mathrm{HCNH}^{+}+\mathrm{CH}_{3} \rightarrow \mathrm{CH}_{3} \mathrm{CNH}^{+}+\mathrm{H} \text {. }
$$

$\mathbf{H C}_{3} \mathbf{N H}^{+}, \mathbf{C}_{2} \mathbf{H}_{3} \mathbf{C N H}^{+}, \mathbf{C}_{2} \mathbf{H}_{5} \mathbf{C N H}^{+}$. As in Vuitton et al. (2007), $\mathrm{HC}_{3} \mathrm{NH}^{+}$is mostly formed by proton transfer from $\mathrm{HCNH}^{+}$and $\mathrm{C}_{2} \mathrm{H}_{5}{ }^{+}$to $\mathrm{HC}_{3} \mathrm{~N}\left(\mathrm{R}_{c n} 714, \mathrm{R}_{c n} 321\right)$. However, below $1150 \mathrm{~km}$, a new reaction in our scheme surpasses dissociative electron recombination $\left(\mathrm{R}_{e r} 104\right)$ as the main loss:

$$
\mathrm{HC}_{3} \mathrm{NH}^{+}+\mathrm{C}_{2} \mathrm{H}_{4} \rightarrow \mathrm{C}_{5} \mathrm{H}_{5} \mathrm{~N}^{+}+\mathrm{H} . \quad\left(\mathrm{R}_{c n} 790\right)
$$

$\mathrm{C}_{2} \mathrm{H}_{3} \mathrm{CNH}^{+}$is mostly formed by proton transfer from $\mathrm{HCNH}^{+}$and $\mathrm{C}_{2} \mathrm{H}_{5}{ }^{+}$ to $\mathrm{C}_{2} \mathrm{H}_{3} \mathrm{CN}\left(\mathrm{R}_{c n} 715, \mathrm{R}_{c n} 322\right)$ and lost by electron recombination $\left(\mathrm{R}_{e r} 106\right)$. We note that since Vuitton et al. (2007), both rate coefficient and products of the electron recombination of $\mathrm{C}_{2} \mathrm{H}_{3} \mathrm{CNH}^{+}$have been determined experimentally (Geppert et al., 2007; Vigren et al., 2009). Below $950 \mathrm{~km}$, proton transfer reactions from $\mathrm{C}_{2} \mathrm{H}_{3} \mathrm{CNH}^{+}$to $\mathrm{C}_{2} \mathrm{H}_{5} \mathrm{CN}$ and $\mathrm{CH}_{2} \mathrm{NH}\left(\mathrm{R}_{c n} 816, \mathrm{R}_{c n} 812\right)$ become important as well.

$\mathrm{C}_{2} \mathrm{H}_{5} \mathrm{CNH}^{+}$is mostly formed by proton transfer from $\mathrm{HCNH}^{+}$and $\mathrm{C}_{2} \mathrm{H}_{5}{ }^{+}$ to $\mathrm{C}_{2} \mathrm{H}_{5} \mathrm{CN}\left(\mathrm{R}_{c n} 716, \mathrm{R}_{c n} 323\right)$ and lost by electron recombination $\left(\mathrm{R}_{e r} 108\right)$. We 
note that since Vuitton et al. (2007), the proton transfer reaction from $\mathrm{HCNH}^{+}$ to $\mathrm{C}_{2} \mathrm{H}_{5} \mathrm{CN}$ has now been confirmed experimentally and is fast, with a rate coefficient of $4.2 \times 10^{-9} \mathrm{~cm}^{3} \mathrm{~s}^{-1}$ (Edwards et al., 2008). The temperature dependence of the rate coefficient as well as the products of the electron recombination of $\mathrm{C}_{2} \mathrm{H}_{5} \mathrm{CNH}^{+}$have also been determined (Vigren et al., 2010a). Below $800 \mathrm{~km}$, proton transfer from $\mathrm{C}_{2} \mathrm{H}_{5} \mathrm{CNH}^{+}$to $\mathrm{CH}_{2} \mathrm{NH}\left(\mathrm{R}_{c n} 819\right)$ becomes a competitive loss process for $\mathrm{C}_{2} \mathrm{H}_{5} \mathrm{CNH}^{+}$.

$\mathbf{H C}_{5} \mathbf{N H}^{+}$. $\mathrm{HC}_{5} \mathrm{NH}^{+}$is mostly formed by proton transfer from $\mathrm{HCNH}^{+}$ and $\mathrm{C}_{2} \mathrm{H}_{5}{ }^{+}$to $\mathrm{C}_{2} \mathrm{H}_{5} \mathrm{CN}\left(\mathrm{R}_{c n} 719, \mathrm{R}_{c n} 326\right)$ and lost by electron recombination $\left(\mathrm{R}_{e r} 122\right)$. We note that since Vuitton et al. (2007), the proton transfer reactions from $\mathrm{C}_{2} \mathrm{H}_{5}{ }^{+}$and $\mathrm{HCNH}^{+}$to $\mathrm{C}_{2} \mathrm{H}_{5} \mathrm{CN}$ have now been confirmed experimentally and are fast, with rate coefficients of 4.7-4.8 $\times 10^{-9} \mathrm{~cm}^{3} \mathrm{~s}^{-1}$ (Edwards et al., 2009).

Other ions. Other ions that can be identified in the INMS spectrum are $\mathrm{C}_{4} \mathrm{H}_{3} \mathrm{NH}^{+}, \mathrm{C}_{4} \mathrm{H}_{5} \mathrm{NH}^{+}, \mathrm{C}_{5} \mathrm{H}_{5} \mathrm{NH}^{+}, \mathrm{C}_{6} \mathrm{H}_{3} \mathrm{NH}^{+}$and $\mathrm{C}_{6} \mathrm{H}_{7} \mathrm{NH}^{+}$(Vuitton et al., 2007). As we do not track the associated neutrals, we adjust their density for the ions to match the INMS data.

3.1.1.4. Oxygen-Bearing Ions. We only discuss the three ions that happen to have predicted densities above $0.1 \mathrm{~cm}^{-3}$. All other oxygen ions, including those containing both oxygen and nitrogen are unimportant species.

As in Vuitton et al. (2007), $\mathrm{H}_{3} \mathrm{O}^{+}$is mostly formed by proton transfer from $\mathrm{C}_{2} \mathrm{H}_{5}{ }^{+}$and $\mathrm{CH}_{5}{ }^{+}$to $\mathrm{H}_{2} \mathrm{O}\left(\mathrm{R}_{c n} 333, \mathrm{R}_{c n} 211\right)$ and lost by proton transfer to HCN and electron recombination $\left(\mathrm{R}_{c n} 955, \mathrm{R}_{e r} 157\right)$ (Neau et al., 2000).

The chemistry of $\mathrm{HCO}^{+}$in our model is fairly simple. It is mostly an intermediate in the proton transfer chain as carbon monoxide takes a proton from $\mathrm{CH}_{5}{ }^{+}\left(\mathrm{R}_{c n} 212\right)$ to transfer it to hydrogen cyanide $\left(\mathrm{R}_{c n} 983\right)$ and acetylene $\left(\mathrm{R}_{c n} 980\right)$.

The chemistry of $\mathrm{CH}_{2} \mathrm{OH}^{+}$(protonated formaldehyde) is pretty well constrained. In our model, $\mathrm{H}_{2} \mathrm{CO}$ takes a proton from $\mathrm{C}_{2} \mathrm{H}_{5}{ }^{+}\left(\mathrm{R}_{c n} 334\right)$ and its main loss is dissociative electron recombination $\left(\mathrm{R}_{e r} 165\right)$ (Hamberg et al., 2007). $\mathrm{H}_{2} \mathrm{CO}$ has the peculiarity to have almost the same proton affinity as HCN and therefore, a proton can cycle back and forth between both species $\left(\mathrm{R}_{c n} 725 / \mathrm{R}_{c n} 1001\right)$, although the overall flux is towards $\mathrm{HCNH}^{+}$.

\subsubsection{Negative Ions}

$\mathbf{H}^{-}, \mathbf{C H}_{2}{ }^{-}, \mathbf{C H}_{3}{ }^{-} \cdot \mathrm{H}^{-}$and $\mathrm{CH}_{2}{ }^{-}$are essentially formed by dissociative electron attachment to $\mathrm{CH}_{4}\left(\mathrm{~J}_{\text {dea }} 2\right)$ with a column integrated rate of $9.4 \times 10^{4}$ and $6.9 \times 10^{3} \mathrm{~cm}^{2} \mathrm{~s}^{-1}$, respectively. $\mathrm{CH}_{3}{ }^{-}$is exclusively formed by radiative attachment to $\mathrm{CH}_{3}$, with a total column integrated rate of $3.2 \times 10^{3} \mathrm{~cm}^{2} \mathrm{~s}^{-1}$. All three are mostly lost by proton abstraction from HCN and associative detachment with $\mathrm{H}$ atoms. Above $1300 \mathrm{~km}$, photodetachment becomes a competitive loss process for $\mathrm{CH}_{2}{ }^{-}$and $\mathrm{CH}_{3}{ }^{-}$because of their small electron affinity (cf. Table B.12). 
$\mathbf{C}_{2} \mathbf{H}^{-}, \mathbf{C}_{4} \mathbf{H}^{-}, \mathbf{C}_{6} \mathbf{H}^{-} \cdot \mathrm{C}_{2} \mathrm{H}^{-}$is mostly formed by proton abstraction from $\mathrm{C}_{2} \mathrm{H}_{2}$ by $\mathrm{H}^{-}$with a column integrated rate of $1.0 \times 10^{4} \mathrm{~cm}^{2} \mathrm{~s}^{-1}$. Because of their larger number of carbon atoms, radiative electron attachment to $\mathrm{C}_{4} \mathrm{H}$ and $\mathrm{C}_{6} \mathrm{H}$ is efficient and is the main formation process of $\mathrm{C}_{4} \mathrm{H}^{-}$and $\mathrm{C}_{6} \mathrm{H}^{-}\left(3.0 \times 10^{4}\right.$ and $5.7 \times 10^{4} \mathrm{~cm}^{2} \mathrm{~s}^{-1}$, respectively). Like other hydrocarbon ions, they are mostly lost by proton abstraction from $\mathrm{HCN}$ and associative detachment with $\mathrm{H}$ atoms.

$\mathbf{C N}^{-}, \mathbf{C}_{3} \mathbf{N}^{-}, \mathbf{C}_{5} \mathbf{N}^{-}$. The major production reactions of $\mathrm{CN}^{-}$are proton transfers from $\mathrm{HCN}$ by $\mathrm{H}^{-}$(mostly above $1100 \mathrm{~km}$ ), $\mathrm{C}_{6} \mathrm{H}^{-}$and to a lesser extent $\mathrm{C}_{4} \mathrm{H}^{-}$. Dissociative electron attachment to $\mathrm{HCN}\left(\mathrm{J}_{\text {dea }} 22\right)$ also contributes over the whole altitude range. These are the same processes as in Vuitton et al. (2009), although the relative importance of the proton exchange reactions has increased because of the larger abundance of $\mathrm{H}^{-}$and $\mathrm{C}_{6} \mathrm{H}^{-}$and the lower dissociative electron attachment cross-section to $\mathrm{HCN}$ in the present model (cf. section 2.7.3.3).

The main production reactions of $\mathrm{C}_{3} \mathrm{~N}^{-}$are proton abstraction from $\mathrm{HC}_{3} \mathrm{~N}$ by several anions $\left(\mathrm{CN}^{-}, \mathrm{C}_{6} \mathrm{H}^{-}, \mathrm{H}^{-}, \mathrm{C}_{4} \mathrm{H}^{-}\right.$by decreasing order of importance). Again the higher density of $\mathrm{H}^{-}$and $\mathrm{C}_{6} \mathrm{H}^{-}$here compared to Vuitton et al. (2009) induces an increased importance of proton transfer in the production of $\mathrm{C}_{3} \mathrm{~N}^{-}$ but the main production processes remain the same.

$\mathrm{C}_{5} \mathrm{~N}^{-}$is almost exclusively formed by radiative electron attachment to $\mathrm{C}_{5} \mathrm{~N}$. The main loss for all three species is associative detachment to $\mathrm{H}$ atoms.

$\mathbf{O}^{-}, \mathbf{O H}^{-} \cdot \mathrm{O}^{-}$is formed by dissociative electron attachment to $\mathrm{CO}\left(\mathrm{J}_{\text {dea }} 3\right)$ and $\mathrm{H}_{2} \mathrm{O}\left(\mathrm{J}_{\text {dea }} 33 \mathrm{~b}\right)$. It is lost by proton abstraction from $\mathrm{C}_{2} \mathrm{H}_{2}$ and $\mathrm{CH}_{4}$, the latter being the major production reaction of $\mathrm{OH}^{-}$along with radiative attachment to $\mathrm{OH}$ and dissociative electron attachment to $\mathrm{H}_{2} \mathrm{O}\left(\mathrm{J}_{\text {dea }} 33 \mathrm{a}\right) \cdot \mathrm{OH}^{-}$is lost by proton abstraction from $\mathrm{HCN}$ and $\mathrm{C}_{2} \mathrm{H}_{2}$ and by associative detachment with $\mathrm{H}$ atoms.

\subsection{Neutral Atmosphere}

The neutral chemistry is initiated by the destruction of methane and nitrogen and the subsequent formation of radicals that are reactive species. The process by which this occurs depends on the atmospheric level. As we will show, above $600 \mathrm{~km}$, the driving mechanism is direct photolysis of methane and nitrogen, which leads to the production of numerous species, including $\mathrm{C}_{2} \mathrm{H}_{2}$ and HCN. These species are transported downward to the stratosphere where the photolysis of acetylene is indirectly responsible for the dissociation of methane, through catalytic processes. The peaks of radical production are at $\sim 800$ and $\sim 200 \mathrm{~km}$, respectively. For each $\mathrm{CH}_{x}$ fragment, the mole fractions are shown in Figure 23, while the column production rates are presented in Table 7.

\subsubsection{Hydrocarbons}

Methane $\left(\mathrm{CH}_{4}\right)$ and photofragments $\left(\mathrm{CH}_{3},{ }^{1} \mathrm{CH}_{2},{ }^{3} \mathrm{CH}_{2}, \mathrm{CH}, \mathrm{C}\right)$. 
Table 7: Primary and secondary column loss or production rates for $\mathrm{CH}_{x}$ species above and below $600 \mathrm{~km}$. Primary loss / production refers to the dissociation of $\mathrm{CH}_{4}$ by photons. Secondary production refers to reactions involving the primary products (see text for further details). Values are given in $\mathrm{cm}^{-2} \mathrm{~s}^{-1}$ scaled to the surface.

\begin{tabular}{lllllll}
\hline \hline & \multicolumn{3}{c}{ Above 600 km } & \multicolumn{3}{c}{ Below 600 km } \\
Products & Primary & Secondary & Total & Primary & Secondary & Total \\
\hline \hline $\mathrm{CH}_{4}$ & $-2.6 \times 10^{9}$ & $-2.5 \times 10^{9}$ & $-5.1 \times 10^{9}$ & $-3.7 \times 10^{7}$ & $-1.6 \times 10^{10}$ & $-1.6 \times 10^{10}$ \\
$\mathrm{CH}_{3}$ & $1.1 \times 10^{9}$ & $5.8 \times 10^{8}$ & $1.7 \times 10^{9}$ & $1.9 \times 10^{7}$ & $1.1 \times 10^{10}$ & $1.1 \times 10^{10}$ \\
${ }^{1} \mathrm{CH}_{2}$ & $1.0 \times 10^{9}$ & $7.2 \times 10^{8}$ & $1.8 \times 10^{9}$ & $1.9 \times 10^{7}$ & $1.7 \times 10^{8}$ & $1.9 \times 10^{8}$ \\
${ }^{3} \mathrm{CH}_{2}$ & $4.1 \times 10^{8}$ & $1.1 \times 10^{9}$ & $1.6 \times 10^{9}$ & 0 & $1.4 \times 10^{8}$ & $1.4 \times 10^{8}$ \\
$\mathrm{CH}$ & $1.4 \times 10^{8}$ & $7.2 \times 10^{8}$ & $1.7 \times 10^{9}$ & 0 & $9.5 \times 10^{7}$ & $9.5 \times 10^{7}$ \\
$\mathrm{C}$ & 0 & $3.4 \times 10^{6}$ & $3.4 \times 10^{6}$ & 0 & $4.1 \times 10^{3}$ & $4.1 \times 10^{3}$ \\
\hline \hline
\end{tabular}




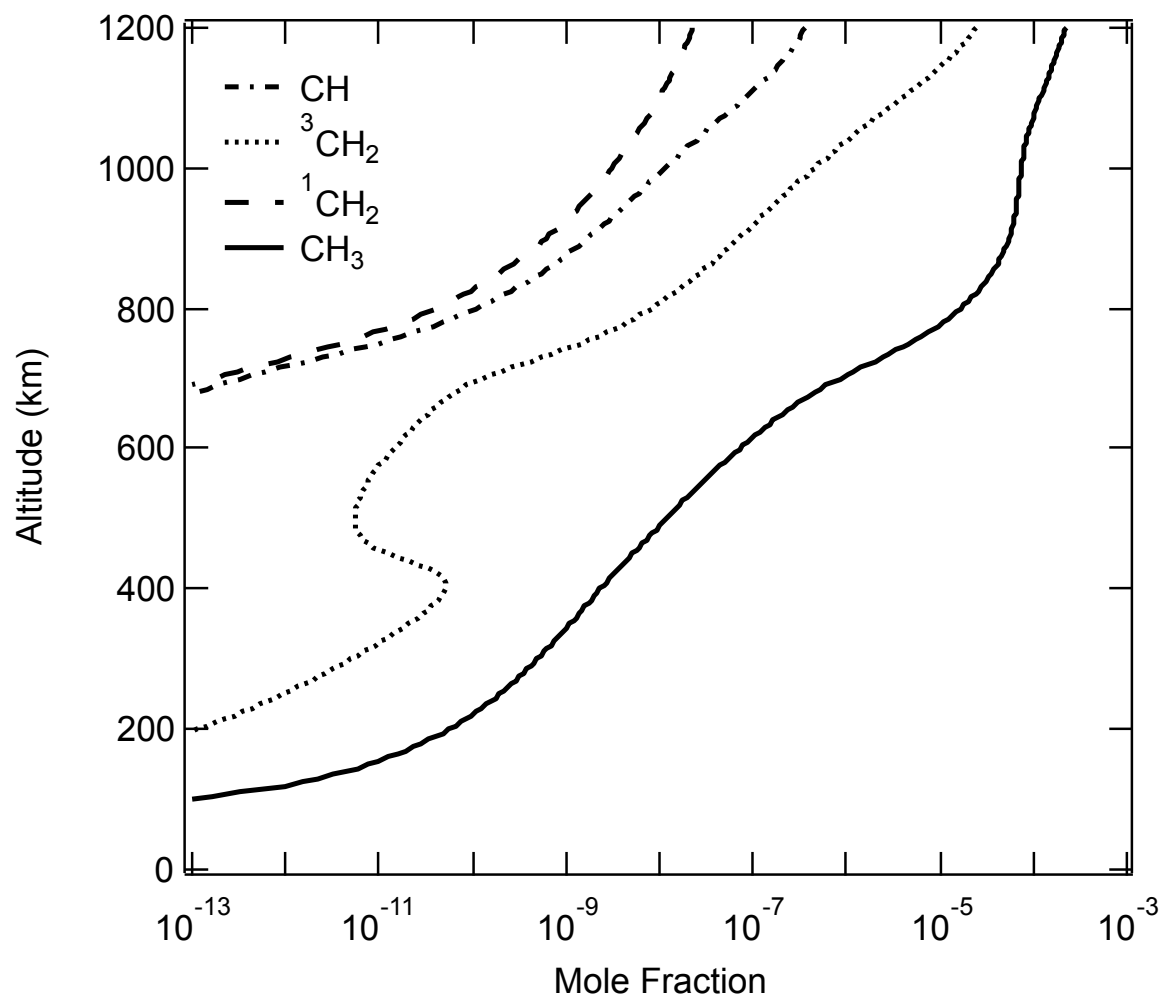

Figure 23: Model results for the mole fraction of the $\mathrm{CH},{ }^{3} \mathrm{CH}_{2},{ }^{1} \mathrm{CH}_{2}$, and $\mathrm{CH}_{3}$ radicals.

The reactive species formed from methane essentially react with each other and with methane to form more complex molecules. Methyl $\left(\mathrm{CH}_{3}\right)$, methylene $\left(\mathrm{CH}_{2}\right.$ in both ground $\mathrm{X}^{3} \mathrm{~B}_{1}$ and first excited $\alpha^{1} \mathrm{~A}_{1}$ electronic states) and methylidyne $(\mathrm{CH})$ radicals are all produced in the photolysis of $\mathrm{CH}_{4}\left(\mathrm{~J}_{d} 2\right)$, which represents $50 \%$ of its loss above $600 \mathrm{~km}$. Secondary reactions of these primary products are competitive loss pathways for $\mathrm{CH}_{4}$ and formation pathways for the radicals, as presented in Table 7 and detailed below.

In the upper atmosphere, most of the methyl radicals originate from the photolysis of methane $\left(\mathrm{J}_{d} 2 \mathrm{a}\right)$ but about $35 \%$ are formed through the reaction of excited methylene radicals with methane:

$$
{ }^{1} \mathrm{CH}_{2}+\mathrm{CH}_{4} \rightarrow \mathrm{CH}_{3}+\mathrm{CH}_{3} \text {. }
$$

The excited state of methylene $\left({ }^{1} \mathrm{CH}_{2}\right)$ is however recycled through photolysis of methyl radicals:

$$
\mathrm{CH}_{3}+\mathrm{h} \nu \rightarrow{ }^{1} \mathrm{CH}_{2}+\mathrm{H} \text {. }
$$

This reaction represents $40 \%$ of the ${ }^{1} \mathrm{CH}_{2}$ production rate in the upper atmosphere, the rest being attributed to the photolysis of methane $\left(\mathrm{J}_{d} 2 \mathrm{~b}\right)$. Ground 
state methylene radicals $\left({ }^{3} \mathrm{CH}_{2}\right)$ are efficiently produced through quenching of the excited state by $\mathrm{N}_{2}$ or through its radiative emission:

$$
\begin{aligned}
{ }^{1} \mathrm{CH}_{2}+\mathrm{N}_{2} & \rightarrow{ }^{3} \mathrm{CH}_{2}+\mathrm{N}_{2}, \\
{ }^{1} \mathrm{CH}_{2} & \rightarrow{ }^{3} \mathrm{CH}_{2}+\mathrm{h} \nu .
\end{aligned}
$$

These processes represent most of the ${ }^{3} \mathrm{CH}_{2}$ production, the photolysis of methane $\left(\mathrm{J}_{d} 2 \mathrm{~d}\right)$ only accounting for $25 \%$. Once formed, the ground state methylene radicals react with the most abundant radicals in the upper atmosphere, hydrogen atoms, to produce methylidyne radicals:

$$
\mathrm{H}+{ }^{3} \mathrm{CH}_{2} \rightarrow \mathrm{CH}+\mathrm{H}_{2} \text {. }
$$

The photolysis of methane $\left(\mathrm{J}_{d} 2 \mathrm{c}\right)$ accounts for $15 \%$ of the total methylidyne formation in the upper atmosphere. Carbon atoms are not formed by direct methane photolysis but instead through a complex set of secondary reactions involving nitrogen-bearing species:

$$
\begin{aligned}
\mathrm{N}\left({ }^{2} \mathrm{D}\right)+\mathrm{C}_{2} \mathrm{H}_{2} & \rightarrow \mathrm{HC}_{2} \mathrm{~N}+\mathrm{H}, \\
\mathrm{HC}_{2} \mathrm{~N}+\mathrm{H} & \rightarrow \mathrm{C}_{2} \mathrm{~N}+\mathrm{H}_{2}, \\
\mathrm{C}_{2} \mathrm{~N}+\mathrm{H} & \rightarrow \mathrm{HCN}+\mathrm{C} .
\end{aligned}
$$

However, the total $\mathrm{C}$ production rate is small (cf. Table 7) and although, as first pointed out in Hébrard et al. (2013), it is at the origin of $\mathrm{C}_{3}$ and $\mathrm{C}_{3} \mathrm{H}$, these radicals have a very minor impact on the overall hydrocarbon chemistry.

The photons that can dissociate methane $(\lambda<145 \mathrm{~nm})$ are all absorbed above $600 \mathrm{~km}$ (cf. section 2.6.4) but in the lower atmosphere, the photodissociation of acetylene (and to a lower extent, of the other polyynes, $\mathrm{C}_{4} \mathrm{H}_{2}$ and $\mathrm{C}_{6} \mathrm{H}_{2}$ ) leads to a secondary dissociation of $\mathrm{CH}_{4}$, as originally proposed by Allen et al. (1980) and validated experimentally by Vuitton et al. (2006a):

$$
\begin{aligned}
\mathrm{C}_{2} \mathrm{H}_{2}+\mathrm{h} \nu & \rightarrow \mathrm{C}_{2} \mathrm{H}+\mathrm{H}, \\
\mathrm{C}_{2} \mathrm{H}+\mathrm{CH}_{4} & \rightarrow \mathrm{C}_{2} \mathrm{H}_{2}+\mathrm{CH}_{3} .
\end{aligned}
$$

This scheme is the main driver of the chemistry in the lower atmosphere, through production of methyl radicals. Methylidyne and methylene radicals are formed locally through a similar set of secondary reactions as in the upper atmosphere (reactions $\left(\mathrm{J}_{d} 38\right),\left(\mathrm{R}_{n} 62\right)$ and $\left.\left(\mathrm{R}_{n} 3\right)\right)$. Less than $5 \%$ of the methane destroyed by the above mentioned mechanisms is recycled back throughout the atmosphere:

$$
\begin{aligned}
\mathrm{CH}_{3}+\mathrm{C}_{3} \mathrm{H}_{7} & \rightarrow \mathrm{C}_{3} \mathrm{H}_{6}+\mathrm{CH}_{4}, \\
\mathrm{CH}_{3}+\mathrm{C}_{2} \mathrm{H}_{5} & \rightarrow \mathrm{C}_{2} \mathrm{H}_{4}+\mathrm{CH}_{4}, \\
\mathrm{CH}_{3}+\mathrm{C}_{2} \mathrm{H}_{3} & \rightarrow \mathrm{C}_{2} \mathrm{H}_{2}+\mathrm{CH}_{4}, \\
\mathrm{CH}_{3}+\mathrm{H}_{2} \mathrm{CN} & \rightarrow \mathrm{HCN}+\mathrm{CH}_{4} .
\end{aligned}
$$


To our knowledge, reaction $\left(\mathrm{R}_{n} 396\right)$ has never been studied either experimentally or theoretically. We assume that the rate coefficient of the isoelectronic $\mathrm{NH}_{2}+\mathrm{H}_{2} \mathrm{CN}$ reaction makes a good surrogate.

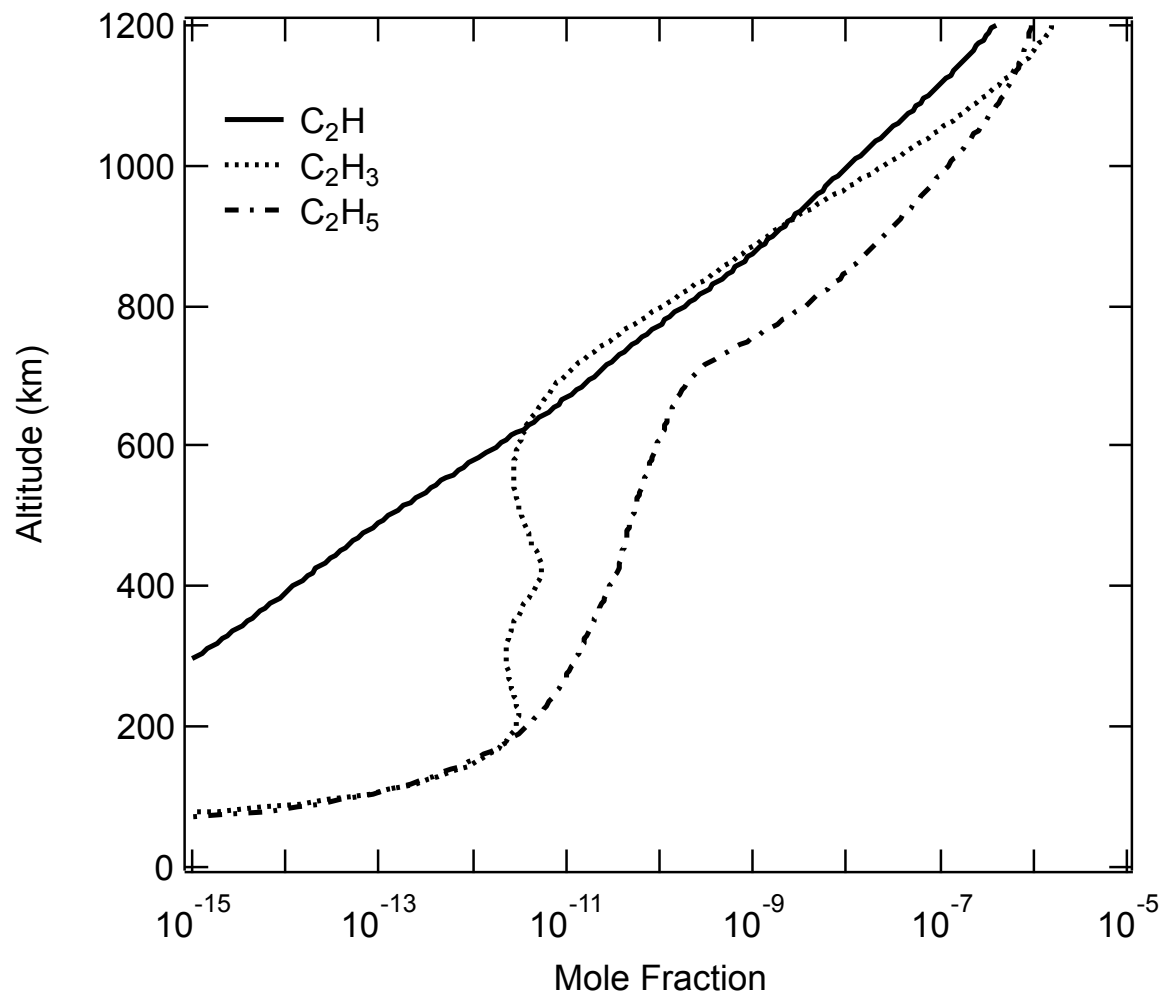

Figure 24: Model results for the mole fraction of the $\mathrm{C}_{2} \mathrm{H}, \mathrm{C}_{2} \mathrm{H}_{3}$ and $\mathrm{C}_{2} \mathrm{H}_{5}$ radicals.

Ethane $\left(\mathrm{C}_{2} \mathbf{H}_{6}\right)$, Ethylene $\left(\mathrm{C}_{2} \mathbf{H}_{4}\right)$, Acetylene $\left(\mathrm{C}_{2} \mathbf{H}_{2}\right)$. The rate coefficient of the recombination of methyl radicals has been calculated in the present work (cf. Figure A.3c) and this reaction is the origin of essentially all $\mathrm{C}_{2} \mathrm{H}_{6}$ :

$$
\mathrm{CH}_{3}+\mathrm{CH}_{3} \stackrel{\mathrm{M}}{\rightarrow} \mathrm{C}_{2} \mathrm{H}_{6} \text {. }
$$

The production maxima are centered around 800 and $200 \mathrm{~km}$, which corresponds to the peak of methane and acetylene photolysis, respectively, and subsequent $\mathrm{CH}_{3}$ production as described above.

After production, the majority of $\mathrm{C}_{2} \mathrm{H}_{6}$ flows downward until it condenses near the tropopause (cf. Table 19). A small fraction diffuses upward and is responsible for the presence of ethane above $900 \mathrm{~km}$. There, ethane is mostly 
lost by photodissociation $\left(\mathrm{J}_{d} 7\right)$ and to some minor extent, by reaction with $\mathrm{CH}$ and ${ }^{1} \mathrm{CH}_{2}$ radicals, and $\mathrm{CH}_{5}{ }^{+}$ions:

$$
\begin{aligned}
\mathrm{CH}+\mathrm{C}_{2} \mathrm{H}_{6} & \rightarrow \mathrm{C}_{2} \mathrm{H}_{4}+\mathrm{CH}_{3}, \\
{ }^{1} \mathrm{CH}_{2}+\mathrm{C}_{2} \mathrm{H}_{6} & \rightarrow \mathrm{C}_{2} \mathrm{H}_{5}+\mathrm{CH}_{3}, \\
\mathrm{CH}_{5}^{+}+\mathrm{C}_{2} \mathrm{H}_{6} & \rightarrow \mathrm{C}_{2} \mathrm{H}_{7}^{+}+\mathrm{CH}_{4} .
\end{aligned}
$$

The main bulk of ethylene is formed in the upper atmosphere, by methylidyne reacting with methane:

$$
\mathrm{CH}+\mathrm{CH}_{4} \rightarrow \mathrm{C}_{2} \mathrm{H}_{4}+\mathrm{H} \text {. }
$$

A secondary channel involves the reaction of ground state methylene radicals with methyl radicals:

$$
{ }^{3} \mathrm{CH}_{2}+\mathrm{CH}_{3} \rightarrow \mathrm{C}_{2} \mathrm{H}_{4}+\mathrm{H} .
$$

$$
\begin{array}{rlrl}
\mathrm{C}_{2} \mathrm{H}_{5}^{+}+\mathrm{HCN} & \rightarrow \mathrm{C}_{2} \mathrm{H}_{4}+\mathrm{HCNH}^{+}, & \left(\mathrm{R}_{c n} 316\right) \\
\mathrm{C}_{2} \mathrm{H}_{5}^{+}+\mathrm{C}_{2} \mathrm{H}_{4} \rightarrow \mathrm{CH}_{4}+\mathrm{C}_{3} \mathrm{H}_{5}^{+} . & \left(\mathrm{R}_{c n} 302 \mathrm{a}\right)
\end{array}
$$

The presence of ethylene below $700 \mathrm{~km}$ is a combination of downward flux from the upper atmosphere and local production through the formation of ethyl radicals $\left(\mathrm{C}_{2} \mathrm{H}_{5}\right.$, cf. Figure 24$)$ and their subsequent reaction with atomic hydrogen:

$$
\begin{aligned}
{ }^{1} \mathrm{CH}_{2}+\mathrm{CH}_{4} & \rightarrow \mathrm{C}_{2} \mathrm{H}_{5}+\mathrm{H}, \\
\mathrm{H}+\mathrm{C}_{2} \mathrm{H}_{5} & \rightarrow \mathrm{C}_{2} \mathrm{H}_{4}+\mathrm{H}_{2} .
\end{aligned}
$$

However, below $500 \mathrm{~km}$, the reaction of hydrogen atoms with ethylene becomes more important than reaction $\left(\mathrm{R}_{n} 10 \mathrm{~b}\right)$ and along with photodissociation (J6) becomes the dominant loss of ethylene to reform ethyl radicals:

$$
\mathrm{H}+\mathrm{C}_{2} \mathrm{H}_{4} \stackrel{\mathrm{M}}{\longrightarrow} \mathrm{C}_{2} \mathrm{H}_{5} .
$$

The rate coefficient of reaction $\left(\mathrm{R}_{n} 9 \mathrm{a}\right)$ is from Vuitton et al. (2012) (cf. Figure A.1b). Around $200 \mathrm{~km}$, the photolysis of butadiene (J14a) becomes a significant production of ethylene.

The photolysis of ethylene is, in turn, the main source of acetylene in the bulk of the atmosphere $\left(\mathrm{J}_{d} 6\right)$. In the upper atmosphere, hydrogen abstraction from vinyl radicals $\left(\mathrm{C}_{2} \mathrm{H}_{3}\right.$, cf. Figure 24) and as first highlighted by Plessis et al. (2012) (based on the T19 INMS ion densities and the diurnally averaged model 
of Hébrard et al. (2009)), dissociative recombination of $\mathrm{C}_{2} \mathrm{H}_{5}{ }^{+}\left(\mathrm{R}_{e r} 21 \mathrm{a}, \mathrm{d}\right)$, are the dominant production of $\mathrm{C}_{2} \mathrm{H}_{2}$ :

$$
\mathrm{H}+\mathrm{C}_{2} \mathrm{H}_{3} \rightarrow \mathrm{C}_{2} \mathrm{H}_{2}+\mathrm{H}_{2} \text {. }
$$

The rate coefficient of the addition channel $\left(\mathrm{R}_{n} 8 \mathrm{a}\right)$ has been calculated in the present work and remains negligible throughout the atmosphere (cf. Figure A.3a).

At these altitudes, vinyl radicals originate from several reactions, including electron recombination of $\mathrm{C}_{2} \mathrm{H}_{5}{ }^{+}\left(\mathrm{R}_{e r} 21 \mathrm{~b}\right)$ and $\mathrm{C}_{2} \mathrm{H}_{3} \mathrm{CNH}^{+}\left(\mathrm{R}_{e r} 106 \mathrm{c}\right)$, and neutral-neutral reaction of imidogen radicals (NH, cf. Figure 31) and ethylene $\left(\mathrm{R}_{n} 332\right)$. Interestingly, $\mathrm{NH}$ radicals are essentially being formed by ion chemistry $\left(\mathrm{R}_{c n} 593 \mathrm{a}\right)$.

Downward transport, photolysis $\left(\mathrm{J}_{d} 5\right)$ and reaction with methylidyne $\left(\mathrm{R}_{n} 48\right)$ and cyano radicals $\left(\mathrm{R}_{n} 370\right)$ are acetylene's main losses above $800 \mathrm{~km}$. Ion chemistry also contributes to the loss of acetylene through its reaction with $\mathrm{C}_{3} \mathrm{H}_{5}^{+}\left(\mathrm{R}_{c n} 422\right)$.

Although the addition of reactive hydrogen atoms with acetylene to produce $\mathrm{C}_{2} \mathrm{H}_{3}$ is not very efficient (Vuitton et al. (2012) and Figure A.1a), it does become the main loss of $\mathrm{C}_{2} \mathrm{H}_{2}$ below $500 \mathrm{~km}$ :

$$
\mathrm{H}+\mathrm{C}_{2} \mathrm{H}_{2} \stackrel{\mathrm{M}}{\longrightarrow} \mathrm{C}_{2} \mathrm{H}_{3} .
$$

However, a substantial fraction of vinyl radicals further reacts with atomic hydrogen $\left(\mathrm{R}_{n} 8 \mathrm{~b}\right)$ and methyl radicals $\left(\mathrm{R}_{n} 92 \mathrm{c}\right)$ to reform acetylene. 


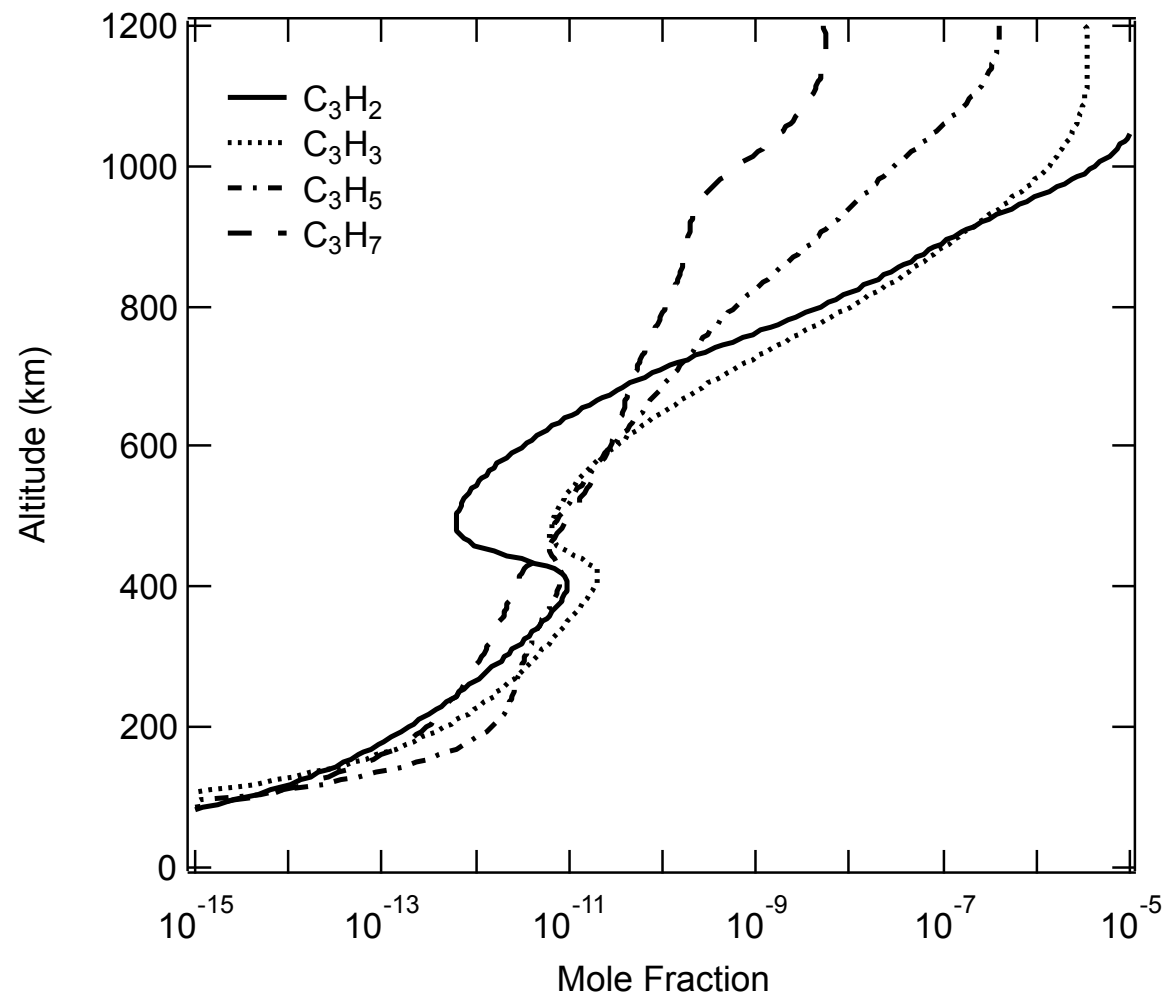

Figure 25: Model results for the mole fraction of the $\mathrm{C}_{3} \mathrm{H}_{2}, \mathrm{C}_{3} \mathrm{H}_{3}, \mathrm{C}_{3} \mathrm{H}_{5}$ and $\mathrm{C}_{3} \mathrm{H}_{7}$ radicals. 

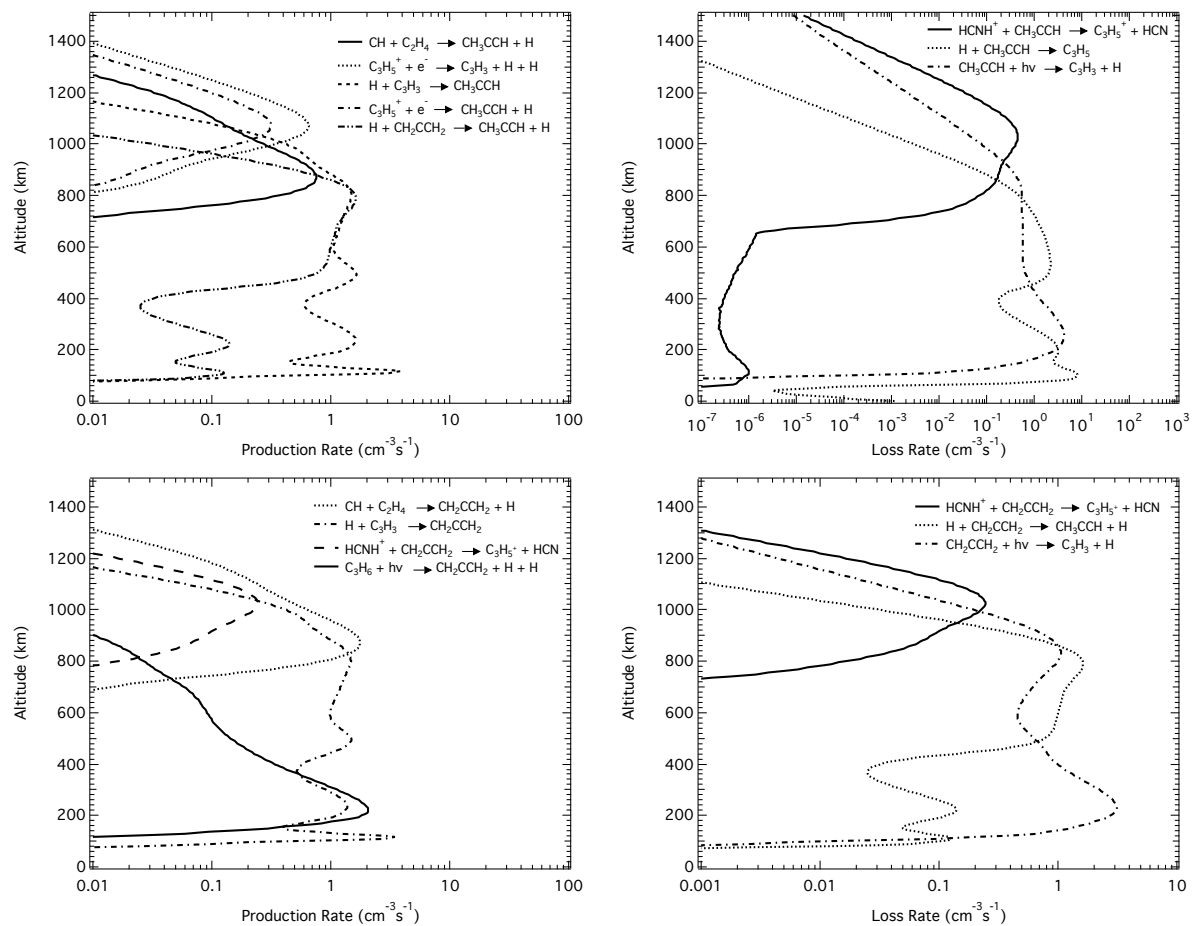

Figure 26: Production (left panel) and loss (right panel) rates of methylacetylene (top panel) and allene (bottom panel).

Methylacetylene $\left(\mathrm{CH}_{3} \mathrm{CCH}\right)$, Allene $\left(\mathrm{CH}_{2} \mathrm{CCH}_{2}\right)$, Propylene $\left(\mathrm{C}_{3} \mathrm{H}_{6}\right)$, Propane $\left(\mathbf{C}_{3} \mathbf{H}_{\mathbf{8}}\right)$. In the upper atmosphere, methylacetylene and its isomer, allene, are formed through electron recombination of $\mathrm{C}_{3} \mathrm{H}_{5}{ }^{+}\left(\mathrm{R}_{e r} 32 \mathrm{~b}\right)$ and the reaction of methylidyne radicals with ethylene (cf. left panels in Figure 26):

$$
\begin{aligned}
\mathrm{CH}+\mathrm{C}_{2} \mathrm{H}_{4} & \rightarrow \mathrm{CH}_{3} \mathrm{CCH}+\mathrm{H} \\
& \rightarrow \mathrm{CH}_{2} \mathrm{CCH}_{2}+\mathrm{H} .
\end{aligned}
$$

Propargyl radicals $\left(\mathrm{C}_{3} \mathrm{H}_{3}\right.$, cf. Figure 25$)$, formed through reactions $\left(\mathrm{R}_{e r} 32 \mathrm{a}, \mathrm{c}\right)$ and photodissociation of butadiene $\left(\mathrm{J}_{d} 14 \mathrm{c}\right)$, contribute as well to the production of methylacetylene and allene, via their addition of hydrogen atoms:

$$
\begin{aligned}
\mathrm{H}+\mathrm{C}_{3} \mathrm{H}_{3} & \rightarrow \mathrm{CH}_{3} \mathrm{CCH}+\mathrm{H} \\
& \rightarrow \mathrm{CH}_{2} \mathrm{CCH}_{2}+\mathrm{H} .
\end{aligned}
$$

Neither species is efficiently destroyed above $1100 \mathrm{~km}$ and they mostly flow downward. There is a main loss channel at the peak of the ionosphere (around $1000 \mathrm{~km}$ ) due to proton transfer from $\mathrm{HCNH}^{+}$to both isomers, which we assume 


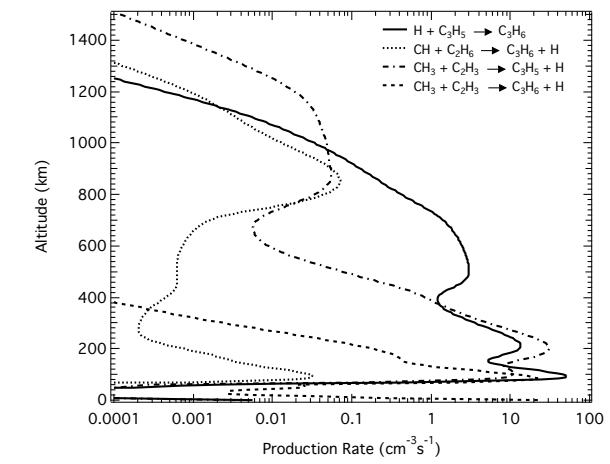
26): $\mathrm{CH}_{2} \mathrm{CCH}_{2}$ into $\mathrm{CH}_{3} \mathrm{CCH}$ (cf. Figure A.1d): ( $\left.\mathrm{R}_{n} 14 \mathrm{a}\right)$.

is efficient based on their respective proton affinity (cf. right panels in Figure

$$
\begin{aligned}
\mathrm{CH}_{3} \mathrm{CCH}+\mathrm{HCNH}^{+} & \rightarrow \mathrm{C}_{3} \mathrm{H}_{5}^{+}+\mathrm{HCN}, \\
\mathrm{CH}_{2} \mathrm{CCH}_{2}+\mathrm{HCNH}^{+} & \rightarrow \mathrm{C}_{3} \mathrm{H}_{5}^{+}+\mathrm{HCN} .
\end{aligned}
$$

Photodissociation, although extremely efficient, does not lead to a net loss of methylacetylene since the major channel is the formation of propargyl $\left(\mathrm{J}_{d} 8 \mathrm{~b}\right)$, and subsequent reformation of $\mathrm{CH}_{3} \mathrm{CCH}$ and $\mathrm{CH}_{2} \mathrm{CCH}_{2}$ through $\left(\mathrm{R}_{n} 14\right)$.

The addition of hydrogen atoms onto allene is efficient below $200 \mathrm{~km}$ but above that altitude the main outcome of this reaction is the isomerization of

$$
\mathrm{H}+\mathrm{CH}_{2} \mathrm{CCH}_{2} \rightarrow \mathrm{H}+\mathrm{CH}_{3} \mathrm{CCH} \text {. }
$$

A more complex isomerization scheme involves the photodissociation of allene to form propargyl radicals $\left(\mathrm{J}_{d} 9 \mathrm{~b}\right)$ and their subsequent reaction with hydrogen

Methylacetylene is finally lost by reaction with hydrogen atoms to produce allyl radicals $\left(\mathrm{C}_{3} \mathrm{H}_{5}\right.$, cf. Figure 25), addition being the main channel throughout the atmosphere, thanks to the radiative component (cf. Figure A.1c):

$$
\mathrm{H}+\mathrm{CH}_{3} \mathrm{CCH} \stackrel{\mathrm{M}}{\longrightarrow} \mathrm{C}_{3} \mathrm{H}_{5} \text {. }
$$

Figure 27: Production (left panel) and loss (right panel) rates of propylene.

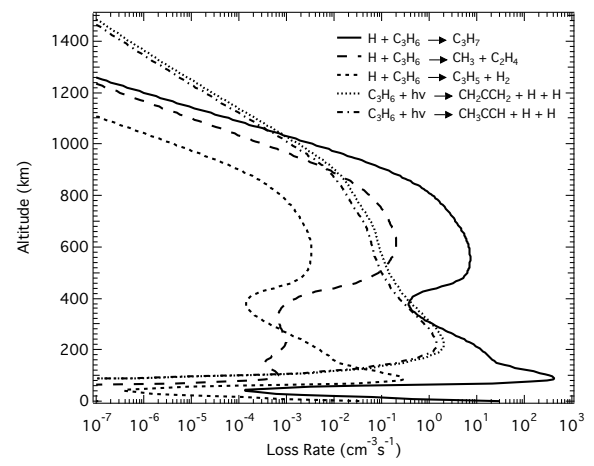

In the upper atmosphere, the main reactions that contribute to the formation of propylene are (cf. left panel in Figure 27):

$$
\begin{gathered}
\mathrm{CH}+\mathrm{C}_{2} \mathrm{H}_{6} \rightarrow \mathrm{C}_{3} \mathrm{H}_{6}+\mathrm{H}, \\
\mathrm{H}+\mathrm{C}_{3} \mathrm{H}_{5} \stackrel{\mathrm{M}}{\rightarrow} \mathrm{C}_{3} \mathrm{H}_{6} .
\end{gathered}
$$


Below $850 \mathrm{~km}$, only reaction $\left(\mathrm{R}_{n} 17 \mathrm{a}\right)$ is important for forming propylene and, as discussed above, $\mathrm{C}_{3} \mathrm{H}_{5}$ is produced by addition of $\mathrm{H}$ to $\mathrm{CH}_{3} \mathrm{CCH}\left(\mathrm{R}_{n} 15 \mathrm{a}, \mathrm{b}\right)$. Above $1000 \mathrm{~km}$ and around $200 \mathrm{~km}$, the recombination of methyl and vinyl radicals $\left(\mathrm{R}_{n} 92 \mathrm{~b}\right)$ is responsible for the production of allyl radicals; between 1000 and $800 \mathrm{~km}$, it is the reaction between excited methylene and ethylene $\left(\mathrm{R}_{n} 68\right)$ :

$$
\begin{aligned}
\mathrm{CH}_{3}+\mathrm{C}_{2} \mathrm{H}_{3} & \rightarrow \mathrm{C}_{3} \mathrm{H}_{5}+\mathrm{H}, \\
{ }^{1} \mathrm{CH}_{2}+\mathrm{C}_{2} \mathrm{H}_{4} & \rightarrow \mathrm{C}_{3} \mathrm{H}_{5}+\mathrm{H} .
\end{aligned}
$$

The rate coefficient of reaction $\left(\mathrm{R}_{n} 92\right)$ has been calculated (cf. Figure A.3d). Bimolecular product formation $\left(\mathrm{C}_{3} \mathrm{H}_{5}+\mathrm{H}\right.$, channel b) is the major channel, adduct stabilization $\left(\mathrm{C}_{3} \mathrm{H}_{6}\right.$, channel a) only kicking in below $150 \mathrm{~km}$.

Gannon et al. (2010a,b) experimentally studied reaction $\left(\mathrm{R}_{n} 68\right)$ at low pressure ( 1 mbar) and over a broad temperature range (195-498 K). Removal of ${ }^{1} \mathrm{CH}_{2}$ occurs by electronic relaxation to ground state triplet methylene (channel a) and chemical reaction (channel b). The $\mathrm{H}$ atom yield doubles from $195 \mathrm{~K}$ to $298 \mathrm{~K}$, while the total rate coefficient exhibits a negative temperature dependence. We adopt this temperature dependent rate coefficient with branching ratios obtained at $195 \mathrm{~K}$. We neglect collisional stabilization that has been calculated to compete with the other channels only at pressures above 260 mbar.

The fate of propylene is to recycle back to methylacetylene and allene via photodissociation and to form propyl radicals $\left(\mathrm{C}_{3} \mathrm{H}_{7}\right.$, cf. Figure 25$)$ by addition of hydrogen atoms (cf. right panel in Figure 27):

$$
\begin{aligned}
& \mathrm{C}_{3} \mathrm{H}_{6}+\mathrm{h} \nu \rightarrow \mathrm{CH}_{3} \mathrm{CCH}+\mathrm{H}_{2} \\
& \rightarrow \mathrm{CH}_{2} \mathrm{CCH}_{2}+\mathrm{H}_{2}, \\
& \mathrm{H}+\mathrm{C}_{3} \mathrm{H}_{6} \stackrel{\mathrm{M}}{\rightarrow} \mathrm{C}_{3} \mathrm{H}_{7} .
\end{aligned}
$$

Hébrard et al. (2013) highlighted $\left(\mathrm{R}_{n} 18\right)$ as another key reaction responsible for uncertainties on $\mathrm{C}_{3} \mathrm{H}_{6}$ at 600 and $200 \mathrm{~km}$ and the rate coefficient of this reaction has again been calculated in the present work (cf. Figure A.1e). Adduct stabilization leading to $\mathrm{i}-\mathrm{C}_{3} \mathrm{H}_{7}$ (channel b) is dominant throughout the atmosphere, bimolecular product formation $\left(\mathrm{C}_{2} \mathrm{H}_{4}+\mathrm{CH}_{3}\right.$ and $\mathrm{C}_{3} \mathrm{H}_{5}+\mathrm{H}_{2}$, channels $\mathrm{c}$ and $\mathrm{d}$ ) being essentially negligible.

The production of ethyl radicals by reaction $\left(\mathrm{R}_{n} 66 \mathrm{c}\right)$ opens a pathway for producing propane in the upper atmosphere:

$$
\mathrm{CH}_{3}+\mathrm{C}_{2} \mathrm{H}_{5} \stackrel{\mathrm{M}}{\longrightarrow} \mathrm{C}_{3} \mathrm{H}_{8} .
$$

Hébrard et al. (2013) highlighted $\left(\mathrm{R}_{n} 93 \mathrm{a}\right)$ as a key reaction responsible for uncertainties on $\mathrm{C}_{3} \mathrm{H}_{8}$ throughout the atmosphere. The rate coefficient of this reaction has therefore been calculated in the present work (cf. Figure A.3e). Adduct stabilization leading to $\mathrm{C}_{3} \mathrm{H}_{8}$ (channel a) is important throughout the 
atmosphere, bimolecular product formation $\left(\mathrm{C}_{2} \mathrm{H}_{4}+\mathrm{CH}_{4}\right.$, channel b) being nevertheless significant above $800 \mathrm{~km}$.

A secondary pathway is through the addition of hydrogen atoms onto propyl radicals (formed through $\left.\left(\mathrm{R}_{n} 18 \mathrm{a}, \mathrm{b}\right)\right)$ :

$$
\mathrm{H}+\mathrm{C}_{3} \mathrm{H}_{7} \stackrel{\mathrm{M}}{\rightarrow} \mathrm{C}_{3} \mathrm{H}_{8} \text {. }
$$

Propane efficiently reacts with ethynyl radicals (formed through $\left(\mathrm{J}_{d} 5\right)$ ) to produce propyl radicals:

$$
\mathrm{C}_{2} \mathrm{H}+\mathrm{C}_{3} \mathrm{H}_{8} \rightarrow \mathrm{C}_{3} \mathrm{H}_{7}+\mathrm{C}_{2} \mathrm{H}_{2} .
$$

However, since propyl radicals mostly react with hydrogen atoms to reform propane $\left(\mathrm{R}_{n} 19 \mathrm{a}\right)$, this reaction does not lead to a net loss of propane. Instead, propane is lost by photolysis to reform propylene $\left(\mathrm{J}_{d} 11 \mathrm{~b}\right)$ and by downward flow that ultimately leads to condensation (cf. Table 19).

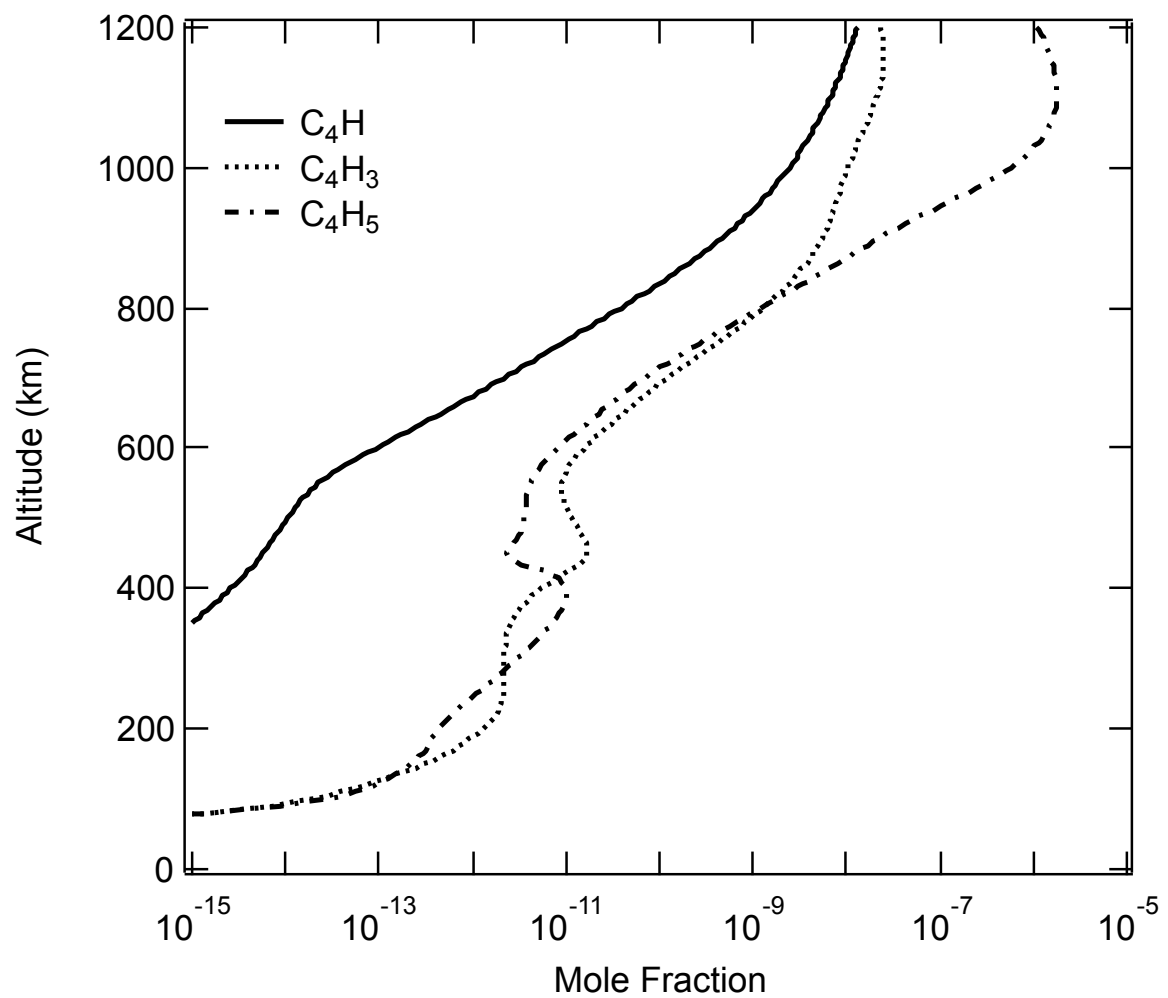

Figure 28: Model results for the mole fraction of the $\mathrm{C}_{4} \mathrm{H}, \mathrm{C}_{4} \mathrm{H}_{3}$ and $\mathrm{C}_{4} \mathrm{H}_{5}$ radicals.

Diacetylene $\left(\mathrm{C}_{4} \mathrm{H}_{2}\right)$, Vinylacetylene $\left(\mathrm{C}_{4} \mathrm{H}_{4}\right)$, Butadiene $\left(\mathrm{C}_{4} \mathrm{H}_{6}\right)$, Butene $\left(\mathbf{C}_{\mathbf{4}} \mathbf{H}_{\mathbf{8}}\right)$, Butane $\left(\mathbf{C}_{\mathbf{4}} \mathbf{H}_{\mathbf{1 0}}\right)$. Above $1200 \mathrm{~km}$, diacetylene is formed 
through a complex scheme involving $\mathrm{C}_{4} \mathrm{H}_{3}$ radicals (cf. Figure 28), which are produced via the reaction of methylene and propadienylidene radicals $\left(\mathrm{C}_{3} \mathrm{H}_{2}\right.$, cf. Figure 25). The latter are formed by photolysis of propargyl radicals and reaction of methylidyne radicals with acetylene:

$$
\begin{aligned}
\mathrm{C}_{3} \mathrm{H}_{3}+\mathrm{h} \nu & \rightarrow \mathrm{C}_{3} \mathrm{H}_{2}+\mathrm{H}, \\
\text { and } \mathrm{CH}+\mathrm{C}_{2} \mathrm{H}_{2} & \rightarrow \mathrm{C}_{3} \mathrm{H}_{2}+\mathrm{H} \\
\text { followed by }{ }^{3} \mathrm{CH}_{2}+\mathrm{C}_{3} \mathrm{H}_{2} & \rightarrow \mathrm{C}_{4} \mathrm{H}_{3}+\mathrm{H} \\
\text { and } \mathrm{CH}_{3}+\mathrm{C}_{4} \mathrm{H}_{3} & \rightarrow \mathrm{C}_{4} \mathrm{H}_{2}+\mathrm{CH}_{4} .
\end{aligned}
$$

In the bulk of the atmosphere, the chemistry of diacetylene is closely related to that of vinylacetylene and a direct consequence of the presence of acetylene. Diacetylene and vinylacetylene are mostly produced through the reaction of ethynyl radicals (the main product of acetylene photolysis $\left(\mathrm{J}_{d} 5\right)$ ) with acetylene and ethylene, respectively:

$$
\begin{aligned}
\mathrm{C}_{2} \mathrm{H}+\mathrm{C}_{2} \mathrm{H}_{2} & \rightarrow \mathrm{C}_{4} \mathrm{H}_{2}+\mathrm{H} \\
\text { and } \mathrm{C}_{2} \mathrm{H}+\mathrm{C}_{2} \mathrm{H}_{4} & \rightarrow \mathrm{C}_{4} \mathrm{H}_{4}+\mathrm{H} .
\end{aligned}
$$

At the turn of the century, the rate coefficients for the reaction of $\mathrm{C}_{2} \mathrm{H}$ (cf. Figure 24) with $\mathrm{C}_{2} \mathrm{H}_{2}$ and $\mathrm{C}_{2} \mathrm{H}_{4}\left(\mathrm{R}_{n} 120, \mathrm{R}_{n} 122\right)$ have been determined at Titanrelevant temperatures and were found to be near the collision limit (Opansky and Leone, 1996; Chastaing et al., 1998; Vakhtin et al., 2001a,b). However, it has only been recently confirmed experimentally that the only products were diacetylene and vinylacetylene, even at low temperature (Kovács et al., 2010; Bouwman et al., 2012).

Vinylacetylene and diacetylene are efficiently recycled into one another through a scheme involving $\mathrm{C}_{4} \mathrm{H}_{3}$ radicals:

$$
\begin{aligned}
& \qquad \begin{array}{c}
\mathrm{C}_{4} \mathrm{H}_{4}+\mathrm{h} \nu \rightarrow \mathrm{C}_{4} \mathrm{H}_{2}+\mathrm{H}_{2}, \\
\text { followed by } \mathrm{H}+\mathrm{C}_{4} \mathrm{H}_{2} \stackrel{\mathrm{M}}{\rightarrow} \mathrm{C}_{4} \mathrm{H}_{3} \\
\text { and } \mathrm{H}+\mathrm{C}_{4} \mathrm{H}_{3} \stackrel{\mathrm{M}}{\rightarrow} \mathrm{C}_{4} \mathrm{H}_{4} .
\end{array}
\end{aligned}
$$

The rate coefficients for both reaction $\left(\mathrm{R}_{n} 21\right)$ and $\left(\mathrm{R}_{n} 22 \mathrm{a}\right)$ were presented in Vuitton et al. (2012). The addition of hydrogen atoms onto diacetylene is quite efficient $\left(\sim 10^{-13} \mathrm{~cm}^{3} \mathrm{~s}^{-1}\right)$ throughout the atmosphere thanks to radiative stabilization. $\mathrm{H}+\mathrm{C}_{4} \mathrm{H}_{3}$ leads to two product channels with similar importance, addition and abstraction, the former dominating below $600 \mathrm{~km}$ and the latter above (cf. Figure A.3b).

Above $1100 \mathrm{~km}$, vinylacetylene and diacetylene are mostly lost through downward transport but in the bulk of the atmosphere, they are consumed by reaction with $\mathrm{H}$ atoms and photodissociation, respectively: 


$$
\begin{gathered}
\mathrm{H}+\mathrm{C}_{4} \mathrm{H}_{4} \stackrel{\mathrm{M}}{\rightarrow} \mathrm{C}_{4} \mathrm{H}_{5} \\
\text { and } \mathrm{C}_{4} \mathrm{H}_{2}+\mathrm{h} \nu \\
\rightarrow \mathrm{C}_{4} \mathrm{H}+\mathrm{H} .
\end{gathered}
$$

The rate coefficient of reaction $\left(\mathrm{R}_{n} 23\right)$ has been calculated in the present work (cf. Appendix A) and is close to $10^{-13} \mathrm{~cm}^{3} \mathrm{~s}^{-1}$ throughout the atmosphere, thanks to radiative association.

Similar to $\mathrm{C}_{2} \mathrm{H} / \mathrm{C}_{2} \mathrm{H}_{2}$, a large fraction $\left(75 \%\right.$ ) of $\mathrm{C}_{4} \mathrm{H}$ (cf. Figure 28) is recycled back to $\mathrm{C}_{4} \mathrm{H}_{2}$ through reaction with methane, despite the fact that the rate coefficient is quite small at $150 \mathrm{~K}\left(\sim 5 \times 10^{-13} \mathrm{~cm}^{3} \mathrm{~s}^{-1}\right)$, according to Berteloite et al. (2008, 2010b). However, because of the low abundance of $\mathrm{C}_{4} \mathrm{H}$ this cycle is only responsible for $\sim 10 \%$ of the methane loss:

$$
\mathrm{C}_{4} \mathrm{H}+\mathrm{CH}_{4} \rightarrow \mathrm{C}_{4} \mathrm{H}_{2}+\mathrm{CH}_{3} .
$$

Once $\mathrm{C}_{4} \mathrm{H}_{5}$ (cf. Figure 28) is produced by reaction $\left(\mathrm{R}_{n} 23\right.$ ), butadiene can be efficiently formed through the reaction of $\mathrm{C}_{4} \mathrm{H}_{5}$ with $\mathrm{H}$ atoms:

$$
\mathrm{C}_{4} \mathrm{H}_{5}+\mathrm{H} \stackrel{\mathrm{M}}{\longrightarrow} \mathrm{C}_{4} \mathrm{H}_{6} .
$$

Photodissociation of butadiene $\left(\mathrm{J}_{d} 14\right)$ is its almost exclusive loss process but about one third of the butadiene destroyed is formed back through the addition of methyl and propargyl radicals:

$$
\mathrm{CH}_{3}+\mathrm{C}_{3} \mathrm{H}_{3} \stackrel{\mathrm{M}}{\longrightarrow} \mathrm{C}_{4} \mathrm{H}_{6} .
$$

Butene is formed through the addition of methyl and allyl radicals and lost by photodissociation:

$$
\begin{aligned}
\mathrm{CH}_{3}+\mathrm{C}_{3} \mathrm{H}_{5} & \stackrel{\mathrm{M}}{\rightarrow} \mathrm{C}_{4} \mathrm{H}_{8}, \\
\mathrm{C}_{4} \mathrm{H}_{8}+\mathrm{h} \nu & \rightarrow \mathrm{C}_{4} \mathrm{H}_{7}+\mathrm{H} \\
& \rightarrow \mathrm{C}_{3} \mathrm{H}_{5}+\mathrm{CH}_{3} .
\end{aligned}
$$

About $20 \%$ of the $\mathrm{C}_{4} \mathrm{H}_{7}$ radicals formed by photodissociation recycle back to butene through addition with $\mathrm{H}$ atoms:

$$
\mathrm{H}+\mathrm{C}_{4} \mathrm{H}_{7} \stackrel{\mathrm{M}}{\rightarrow} \mathrm{C}_{4} \mathrm{H}_{8} .
$$

The formation of butane occurs essentially through the addition of methyl and propyl radicals, the latter being formed via reaction $\left(\mathrm{R}_{n} 128\right)$ and addition of hydrogen atoms on propylene:

$$
\begin{array}{rr}
\mathrm{H}+\mathrm{C}_{3} \mathrm{H}_{6} \stackrel{\mathrm{M}}{\longrightarrow} \mathrm{C}_{3} \mathrm{H}_{7} & \left(\mathrm{R}_{n} 18 \mathrm{a}, \mathrm{b}\right) \\
\text { followed by } \mathrm{C}_{3} \mathrm{H}_{7}+\mathrm{CH}_{3} \stackrel{\mathrm{M}}{\longrightarrow} \mathrm{C}_{4} \mathrm{H}_{10} . & \left(\mathrm{R}_{n} 99 \mathrm{a}\right)
\end{array}
$$




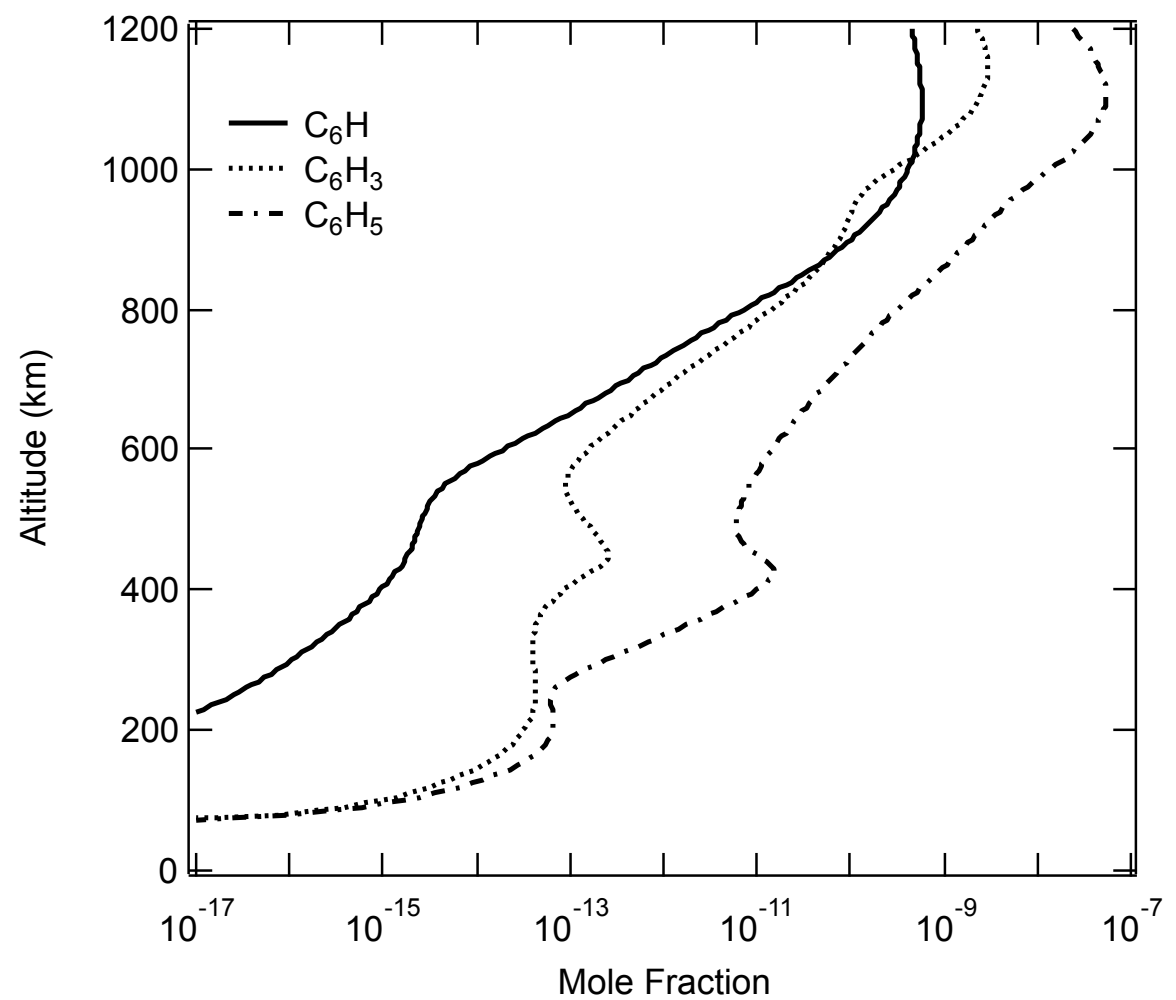

Figure 29: Model results for the mole fraction of the $\mathrm{C}_{6} \mathrm{H}, \mathrm{C}_{6} \mathrm{H}_{3}$ and $\mathrm{C}_{6} \mathrm{H}_{5}$ radicals.

Triacetylene $\left(\mathrm{C}_{6} \mathbf{H}_{2}\right)$, Benzene $\left(\mathbf{C}_{6} \mathbf{H}_{6}\right)$. The production of triacetylene occurs almost exclusively through:

$$
\mathrm{C}_{4} \mathrm{H}+\mathrm{C}_{2} \mathrm{H}_{2} \rightarrow \mathrm{C}_{6} \mathrm{H}_{2}+\mathrm{H} .
$$

In early models, the rate coefficient of this reaction was taken as equal to that of $\mathrm{C}_{2} \mathrm{H}+\mathrm{C}_{2} \mathrm{H}_{2}$. Measurements by Berteloite et al. (2008, 2010b) show that the rate coefficient for $\mathrm{C}_{4} \mathrm{H}+\mathrm{C}_{2} \mathrm{H}_{2}$ is actually about two times faster than the rate coefficient measured by Chastaing et al. (1998) and Vakhtin et al. (2001b) for $\mathrm{C}_{2} \mathrm{H}+\mathrm{C}_{2} \mathrm{H}_{2}$, which essentially validates the assumption that those rate coefficients should be similar. A theoretical study of the $\mathrm{C}_{6} \mathrm{H}_{3}$ (cf. Figure 29) potential energy surface predicts that the $\mathrm{C}_{4} \mathrm{H}+\mathrm{C}_{2} \mathrm{H}_{2}$ reaction readily produces triacetylene $+\mathrm{H}$ (Landera et al., 2008).

The main loss of $\mathrm{C}_{6} \mathrm{H}_{2}$ is essentially through photodissociation $\left(\mathrm{J}_{d} 17\right)$, but below $500 \mathrm{~km}$, most of the $\mathrm{C}_{6} \mathrm{H}$ radicals (cf. Figure 29) formed in the process recycle back to triacetylene by reaction with methane: 


$$
\begin{aligned}
\mathrm{C}_{6} \mathrm{H}_{2}+\mathrm{h} \nu & \rightarrow \mathrm{C}_{6} \mathrm{H}+\mathrm{H}, \\
\mathrm{C}_{6} \mathrm{H}+\mathrm{CH}_{4} & \rightarrow \mathrm{C}_{6} \mathrm{H}_{2}+\mathrm{CH}_{3} .
\end{aligned}
$$

As highlighted in past studies (Wilson et al., 2003; Vuitton et al., 2008), electron recombination of $\mathrm{C}_{6} \mathrm{H}_{7}{ }^{+}\left(\mathrm{R}_{e r} 64\right)$ controls benzene production in the upper atmosphere. We note that the assumption that benzene is the sole product of the electron recombination of $\mathrm{C}_{6} \mathrm{H}_{7}{ }^{+}$has now been validated experimentally (Hamberg et al., 2011).

The main loss of benzene is downward transport above $1000 \mathrm{~km}$ and photodissociation $\left(\mathrm{J}_{d} 18\right)$ to phenyl radicals $\left(\mathrm{C}_{6} \mathrm{H}_{5}\right.$, cf. Figure 29) throughout the bulk of the atmosphere. However, radiative association of hydrogen atoms with phenyl radicals makes this reaction pressure independent and extremely fast $\left(1.6 \times 10^{-10} \mathrm{~cm}^{3} \mathrm{~s}^{-1}\right)$ throughout the atmosphere (Vuitton et al., 2012), leading to the recycling of $\sim 80 \%$ of the benzene destroyed:

$$
\mathrm{H}+\mathrm{C}_{6} \mathrm{H}_{5} \stackrel{\mathrm{M}}{\longrightarrow} \mathrm{C}_{6} \mathrm{H}_{6} .
$$

Below $400 \mathrm{~km}$, two radical-radical reactions dominate the production of benzene:

$$
\begin{aligned}
& \mathrm{C}_{2} \mathrm{H}_{3}+\mathrm{C}_{4} \mathrm{H}_{3} \stackrel{\mathrm{M}}{\longrightarrow} \mathrm{C}_{6} \mathrm{H}_{6}, \\
& \mathrm{C}_{3} \mathrm{H}_{3}+\mathrm{C}_{3} \mathrm{H}_{3} \stackrel{\mathrm{M}}{\longrightarrow} \mathrm{C}_{6} \mathrm{H}_{6} .
\end{aligned}
$$

The addition reaction $\mathrm{C}_{2} \mathrm{H}_{3}+\mathrm{C}_{4} \mathrm{H}_{3}\left(\mathrm{R}_{n} 144\right)$ has never been studied and had to be estimated (Appendix A). This general scheme is similar to what has been extensively described in Vuitton et al. (2008).

\subsubsection{Nitrogen-Bearing Species}

Table 8: Column production rates for nitrogen atoms from different energy sources.

\begin{tabular}{lllll}
\hline \hline Products & Photons & Photoelectrons & Chemistry & Total \\
\hline \hline $\mathrm{N}\left({ }^{2} \mathrm{D}\right)$ & $5.2 \times 10^{7}$ & $3.4 \times 10^{7}$ & $2.2 \times 10^{6}$ & $8.8 \times 10^{7}$ \\
$\mathrm{~N}\left({ }^{4} \mathrm{~S}\right)$ & $6.7 \times 10^{7}$ & $4.5 \times 10^{7}$ & $4.4 \times 10^{7}$ & $1.6 \times 10^{8}$ \\
\hline \hline
\end{tabular}

$\mathbf{N}_{2}$ photofragments: $\mathbf{N}\left({ }^{2} \mathbf{D}\right), \mathbf{N}\left({ }^{4} \mathbf{S}\right)$. Table 8 and Figure 30 summarize the major sources and production rates for nitrogen fragments. Their formation is initiated by the dissociation and ionization of molecular nitrogen above 600 $\mathrm{km}$. The photodissociation of $\mathrm{N}_{2}$ is effective between 80 and $100 \mathrm{~nm}$ (cf. Figure 15) and it is a dominant formation mechanism of both excited $\left(\mathrm{N}\left({ }^{2} \mathrm{D}\right)\right)$ and ground-state $\left(\mathrm{N}\left({ }^{4} \mathrm{~S}\right)\right)$ nitrogen atoms. Nevertheless, dissociative ionization of $\mathrm{N}_{2}$, which only occurs below $80 \mathrm{~nm}$, is the major formation channel of $\mathrm{N}\left({ }^{4} \mathrm{~S}\right)$. The production of $\mathrm{N}\left({ }^{2} \mathrm{D}\right)$ and $\mathrm{N}\left({ }^{4} \mathrm{~S}\right)$ due to dissociation by photoelectrons is only $\sim 1.5$ times less than the production due to direct EUV photolysis. $\mathrm{N}\left({ }^{4} \mathrm{~S}\right)$ 


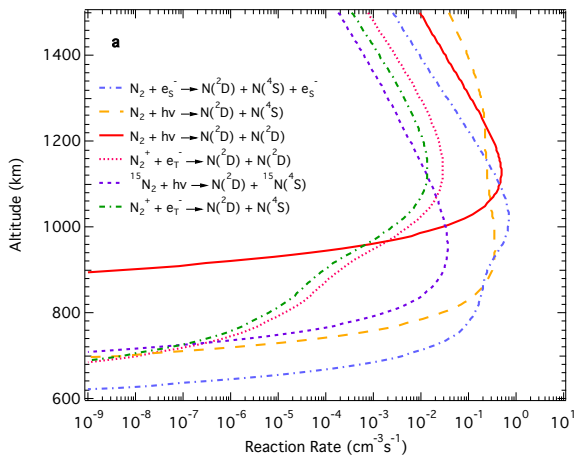

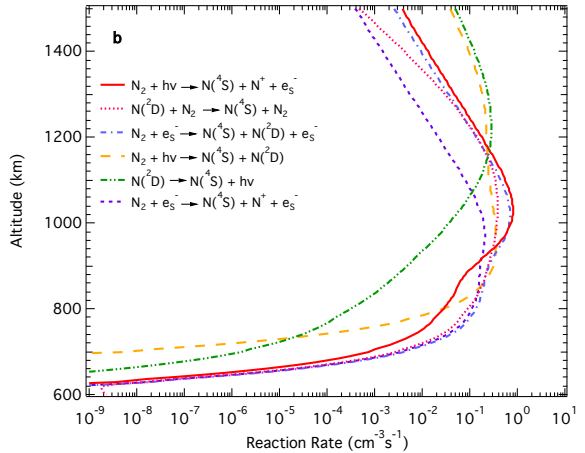

Figure 30: Major production rates of nitrogen fragments $\mathrm{N}\left({ }^{2} \mathrm{D}\right)$ (left panel) and $\mathrm{N}\left({ }^{4} \mathrm{~S}\right)$ (right panel). 


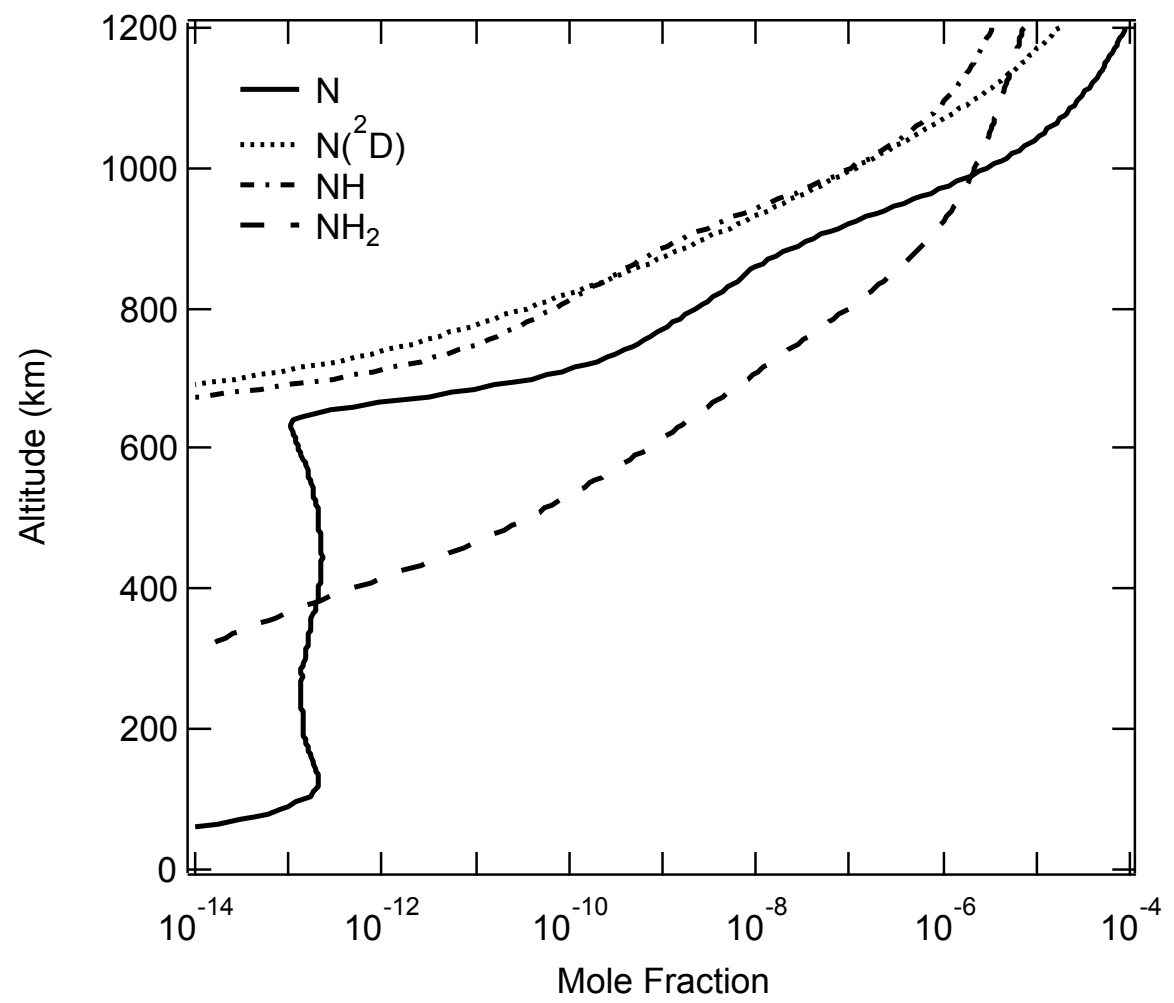

Figure 31: Model results for the mole fraction of the $\mathrm{N}\left({ }^{4} \mathrm{~S}\right), \mathrm{N}\left({ }^{2} \mathrm{D}\right), \mathrm{NH}$ and $\mathrm{NH}_{2}$ radicals.

Ammonia $\left(\mathbf{N H}_{\mathbf{3}}\right)$, Hydrazine $\left(\mathbf{N}_{\mathbf{2}} \mathbf{H}_{\mathbf{4}}\right)$. Figure 3 in Yelle et al. (2010) illustrates the chemical pathways leading to the formation of ammonia. It is produced primarily through reaction of amino $\left(\mathrm{NH}_{2}\right.$, cf. Figure 31$)$ with methyleneamidogen $\left(\mathrm{H}_{2} \mathrm{CN}\right.$, cf. Figure 33) radicals:

$$
\begin{aligned}
\mathrm{NH}+\mathrm{C}_{2} \mathrm{H}_{4} \rightarrow \mathrm{NH}_{2}+\mathrm{C}_{2} \mathrm{H}_{3}, \\
\mathrm{CH}_{2} \mathrm{NH}_{2}^{+}+\mathrm{e}^{-} \rightarrow \mathrm{NH}_{2}+{ }^{3} \mathrm{CH}_{2} \\
\text { and } \\
\mathrm{N}\left({ }^{4} \mathrm{~S}\right)+\mathrm{CH}_{3} \rightarrow \mathrm{H}_{2} \mathrm{CN}+\mathrm{H} \\
\text { lead to } \\
\mathrm{NH}_{2}+\mathrm{H}_{2} \mathrm{CN} \rightarrow \mathrm{NH}_{3}+\mathrm{HCN} .
\end{aligned}
$$

There is only one available experimental study of the $\mathrm{N}\left({ }^{4} \mathrm{~S}\right)+\mathrm{CH}_{3}$ reaction (Marston et al., 1989a,b). A rate coefficient with a strong and complex temperature dependence has been measured over the temperature range 200-423 K, with a value of $(6.4 \pm 2.1) \times 10^{-11} \mathrm{~cm}^{3} \mathrm{~s}^{-1}$ at $200 \mathrm{~K}$. The results suggest that at colder temperatures, the rate coefficient will not be substantially lower than 
that value. We therefore follow Hébrard et al. (2012) and Dutuit et al. (2013) who recommend the use of $6.2 \times 10^{-11} \mathrm{~cm}^{3} \mathrm{~s}^{-1}$ in the $150-200 \mathrm{~K}$ range.

Product measurements lead to a major channel $\left(\mathrm{H}_{2} \mathrm{CN}+\mathrm{H}\right)$ and a minor production of $\mathrm{HCN}$, which can either originate from some $\mathrm{H}_{2} \mathrm{CN}$ having enough internal energy to further dissociate, or from secondary reactions of $\mathrm{H}_{2} \mathrm{CN}$ with nitrogen atoms. Taking into account the various uncertainties, we use the branching ratios recommended by Hébrard et al. (2012) and Dutuit et al. (2013), that is $90 \%$ for $\mathrm{H}_{2} \mathrm{CN}+\mathrm{H}$ and $10 \%$ for $\mathrm{HCN}+\mathrm{H}+\mathrm{H}$.

As already explained in section 3.1.1.3, $\mathrm{NH}_{3}$ quickly reacts with protonated molecules to create $\mathrm{NH}_{4}{ }^{+}\left(\mathrm{R}_{c n} 707, \mathrm{R}_{c n} 315\right)$ but electron recombination of the latter reforms $\mathrm{NH}_{2}$ and $\mathrm{NH}_{3}\left(\mathrm{R}_{e r} 84\right)$, and therefore these reactions do not lead to a net loss of ammonia. Instead, it is mostly lost by photodissociation $\left(\mathrm{J}_{d} 20\right)$.

The amino radical is also responsible for the production of hydrazine:

$$
\mathrm{NH}_{2}+\mathrm{NH}_{2} \stackrel{\mathrm{M}}{\longrightarrow} \mathrm{N}_{2} \mathrm{H}_{4} \text {. }
$$

Photodissociation $\left(\mathrm{J}_{d} 21\right)$ is hydrazine's main loss. Its ion chemistry is poorly known but its proton affinity $(853.2 \mathrm{~kJ} / \mathrm{mol})$ is such that it should efficiently react with most of the ionospheric ions to produce $\mathrm{N}_{2} \mathrm{H}_{5}{ }^{+}$, which will recombine with electrons. This latter process is unlikely to lead solely to $\mathrm{N}_{2} \mathrm{H}_{4}+\mathrm{H}$ and will therefore induce an extra loss for $\mathrm{N}_{2} \mathrm{H}_{4}$ in the upper atmosphere.

Methanimine $\left(\mathrm{CH}_{2} \mathrm{NH}\right)$, Methylamine $\left(\mathrm{CH}_{3} \mathbf{N H}_{2}\right)$. Methanimine is produced by two main reactions, which dominate above and below $800 \mathrm{~km}$, respectively:

$$
\begin{aligned}
\mathrm{N}\left({ }^{2} \mathrm{D}\right)+\mathrm{CH}_{4} & \rightarrow \mathrm{CH}_{2} \mathrm{NH}+\mathrm{H}, \\
\mathrm{CH}_{3} \mathrm{NH}_{2}+\mathrm{h} \nu & \rightarrow \mathrm{CH}_{2} \mathrm{NH}+\mathrm{H}+\mathrm{H} .
\end{aligned}
$$

The latter reaction $\left(\mathrm{J}_{d} 25\right)$ is the main loss for methylamine, while it is formed by the addition of the methyl and amino radicals:

$$
\mathrm{NH}_{2}+\mathrm{CH}_{3} \stackrel{\mathrm{M}}{\longrightarrow} \mathrm{CH}_{3} \mathrm{NH}_{2} \text {. }
$$

As mentioned in section 3.1.1.3, $\mathrm{CH}_{2} \mathrm{NH}$ can abstract a proton from most of the protonated species in Titan's atmosphere. Electron recombination of $\mathrm{CH}_{2} \mathrm{NH}_{2}{ }^{+}$ is the major loss for $\mathrm{CH}_{2} \mathrm{NH}$ above $1000 \mathrm{~km}$ because, although the products are unknown, it is unlikely that they are $100 \% \mathrm{CH}_{2} \mathrm{NH}$. Down to $400 \mathrm{~km}$, reaction with hydrogen atoms is methanimine's main loss, while further below it is mostly destroyed by photodissociation:

$$
\begin{aligned}
\mathrm{H}+\mathrm{CH}_{2} \mathrm{NH} & \rightarrow \mathrm{H}_{2} \mathrm{CN}+\mathrm{H}_{2} \\
& \stackrel{\mathrm{M}}{\rightarrow} \mathrm{CH}_{2} \mathrm{NH}_{2}, \\
\mathrm{CH}_{2} \mathrm{NH}+\mathrm{h} \nu & \rightarrow \mathrm{HCN}+\mathrm{H}+\mathrm{H} .
\end{aligned}
$$


We calculated the rate coefficient of reaction $\left(\mathrm{R}_{n} 256\right)$ (cf. A.2c). Bimolecular product formation $\left(\mathrm{H}_{2} \mathrm{CN}+\mathrm{H}_{2}\right.$, channel a), is dominant throughout the atmosphere, adduct stabilization leading to $\mathrm{CH}_{2} \mathrm{NH}_{2}$ (channel b) and to a lesser extent $\mathrm{CH}_{3} \mathrm{NH}$ (channel c) being nevertheless significant. Because of the lack of information on the differential reactivity of $\mathrm{CH}_{2} \mathrm{NH}_{2}$ versus $\mathrm{CH}_{3} \mathrm{NH}$, we do not differentiate the two isomers. As emphasized in Lavvas et al. (2008b), the photodissociation cross-section of $\mathrm{CH}_{2} \mathrm{NH}$ has only been measured in the wavelength range 235-260 $\mathrm{nm}$.
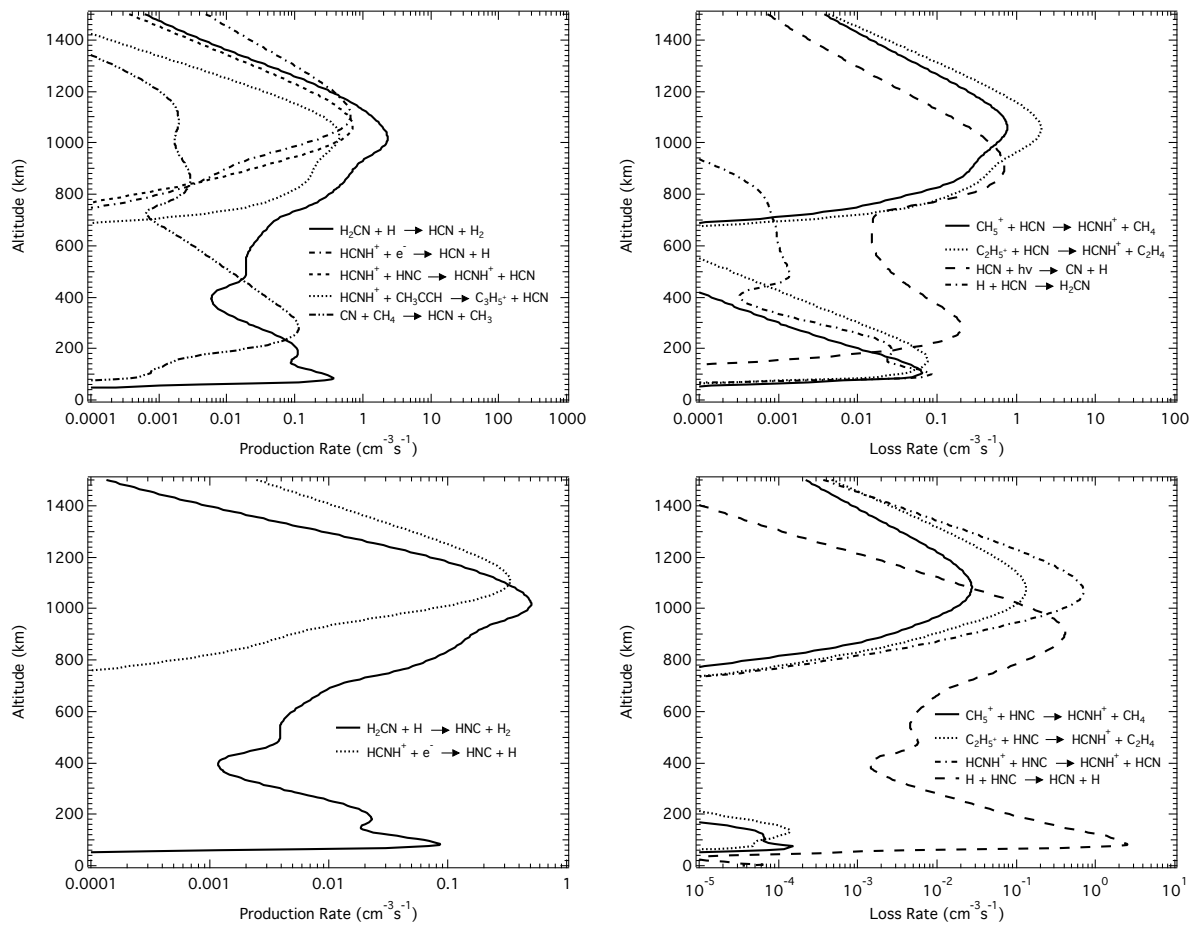

Figure 32: Production and loss rates of hydrogen cyanide and hydrogen isocyanide.

Hydrogen Cyanide (HCN), Hydrogen Isocyanide (HNC), Acetonitrile $\left(\mathbf{C H}_{\mathbf{3}} \mathbf{C N}\right)$. The main production channel of both $\mathrm{HCN}$ and its isomer $\mathrm{HNC}$ is the reaction of hydrogen atoms with methylene-amidogen radicals, whose production mechanism has been described in the previous subsection (cf. left panels in Figure 32):

$$
\begin{aligned}
\mathrm{H}+\mathrm{H}_{2} \mathrm{CN} & \rightarrow \mathrm{HCN}+\mathrm{H}_{2}, \\
& \rightarrow \mathrm{HNC}+\mathrm{H}_{2} .
\end{aligned}
$$

Once formed, the triple $\mathrm{C} \equiv \mathrm{N}$ bond in $\mathrm{HCN}$ is extremely stable and difficult to 
break up. As a consequence, its main fate is to be incorporated in more complex nitrile species.

Hébrard et al. (2012) argue that the discrepancy between their calculated and observed HNC mole fraction can be partly explained by the poor knowledge of this reaction. We therefore performed theoretical calculations (cf. A.3f) that indicated that channel (a) was dominant over channel (b) with rate coefficients of $\sim 2.3 \times 10^{-10}$ and $\sim 5.0 \times 10^{-11} \mathrm{~cm}^{3} \mathrm{~s}^{-1}$, respectively and little temperature variation. These values are a factor $4-5$ higher than those presented in Hébrard et al. (2012). The adduct channel giving $\mathrm{CH}_{2} \mathrm{NH}$ is negligible.

Around the ionospheric peak, both isomers efficiently react with protonated ions, whose associated neutrals have a lower proton affinity $(\mathrm{PA}(\mathrm{HCN})=712.9$ $\mathrm{kJ} / \mathrm{mol}$ and $\mathrm{PA}(\mathrm{HNC})=772.0 \mathrm{~kJ} / \mathrm{mol})$, such as $\mathrm{CH}_{5}{ }^{+}$and $\mathrm{C}_{2} \mathrm{H}_{5}{ }^{+}$:

$$
\begin{array}{rlrl}
\mathrm{CH}_{5}^{+}+\mathrm{HCN} / \mathrm{HNC} & \rightarrow \mathrm{HCNH}^{+}+\mathrm{CH}_{4}, & \left(\mathrm{R}_{c n} 194, \mathrm{R}_{c n} 195\right) \\
\mathrm{C}_{2} \mathrm{H}_{5}^{+}+\mathrm{HCN} / \mathrm{HNC} \rightarrow \mathrm{HCNH}^{+}+\mathrm{C}_{2} \mathrm{H}_{4} . & \left(\mathrm{R}_{c n} 316, \mathrm{R}_{c n} 317\right)
\end{array}
$$

$\mathrm{HCNH}^{+}$can in turn react with neutrals having a higher proton affinity $\left(\mathrm{R}_{c n} 697, \mathrm{R}_{c n} 715\right)$ and recombine with electrons $\left(\mathrm{R}_{c n} 88\right)$, which induces the recycling of $\mathrm{HCN}$ and HNC:

$$
\begin{aligned}
\mathrm{HCNH}^{+}+\mathrm{CH}_{3} \mathrm{CCH} & \rightarrow \mathrm{HCN}+\mathrm{C}_{3} \mathrm{H}_{5}^{+}, & \left(\mathrm{R}_{c n} 697\right) \\
\mathrm{HCNH}^{+}+\mathrm{C}_{2} \mathrm{H}_{3} \mathrm{CN} & \rightarrow \mathrm{HCN}+\mathrm{C}_{2} \mathrm{H}_{3} \mathrm{CNH}^{+}, & \left(\mathrm{R}_{c n} 715\right) \\
\mathrm{HCNH}^{+}+\mathrm{e}^{-} & \rightarrow \mathrm{HCN}+\mathrm{H} & \left(\mathrm{R}_{e r} 88 \mathrm{a}\right) \\
& \rightarrow \mathrm{HNC}+\mathrm{H} & \left(\mathrm{R}_{e r} 88 \mathrm{~b}\right)
\end{aligned}
$$

The overall rate coefficient for the electron recombination of $\mathrm{HCNH}^{+}$measured by different techniques can be regarded as similar but the branching fraction between the different product channels is still under debate (cf. KIDA data sheet 2815 V1 for a brief review). For our purpose, an important question is the distribution between the HCN and HNC production. Theoretical calculations conclude that they are produced in equal amounts (Talbi and Ellinger, 1998; Hickman et al., 2005). This is supported by an experiment showing that the internal energy of the fragments is extremely high, far exceeding the isomerization barrier, which implies an isomeric production ratio in a narrow range near unity (Mendes et al., 2012).

On Titan, the lifetime of HNC is short (cf. section 5.4) as it quickly isomerizes to HCN through the following reactions (cf. lower right panel in Figure $32)$ :

$$
\begin{aligned}
& \mathrm{HCNH}^{+}+\mathrm{HNC} \rightarrow \mathrm{HCN}+\mathrm{HCNH}^{+}, \\
& \mathrm{H}+\mathrm{HNC} \rightarrow \mathrm{HCN}+\mathrm{H} .
\end{aligned}
$$


Reaction $\left(\mathrm{R}_{c n} 712\right)$, although tentative, is based on the suggestion by Cotton et al. (2013) that the interaction of $\mathrm{HCNH}^{+}$with $\mathrm{HNC}$ would immediately proceed through a barrier-less proton transfer reaction to isomerize HNC to $\mathrm{HCN}$.

Hébrard et al. (2012) performed a global sensitivity analysis to study how the poor knowledge of this second reaction rate coefficient propagates in their model and reached the conclusion that it limits strongly the accuracy of the model. This is highlighted for HNC on their Figure 2. We therefore performed theoretical calculations (cf. Figure A.2b) to reduce the uncertainty associated to this reaction and found a rate coefficient of $\sim 10^{-13} \mathrm{~cm}^{3} \mathrm{~s}^{-1}$, that is one order of magnitude faster than the recommended value of Hébrard et al. (2012).

A third channel in the electron recombination of $\mathrm{HCNH}^{+}$as well as photolysis do lead to a net loss of HCN (cf. upper right panel in Figure 32):

$$
\begin{gathered}
\mathrm{HCNH}^{+}+\mathrm{e}^{-} \rightarrow \mathrm{CN}+\mathrm{H}+\mathrm{H}, \\
\mathrm{HCN}+\mathrm{h} \nu \rightarrow \mathrm{CN}+\mathrm{H},
\end{gathered}
$$

but its main fate is to diffuse down. Below $650 \mathrm{~km}$, the reaction between hydrogen atoms and HCN becomes important:

$$
\mathrm{H}+\mathrm{HCN} \stackrel{\mathrm{M}}{\longrightarrow} \mathrm{H}_{2} \mathrm{CN} .
$$

Theoretical calculations for this reaction are described in Appendix A. The rate coefficient is temperature and pressure dependent and reaches a maximum of $\sim 10^{-17} \mathrm{~cm}^{3} \mathrm{~s}^{-1}$ around $200 \mathrm{~km}$ (cf. Figure A.2a). Although this is extremely slow, it is significantly faster than the rate coefficient presented in Hébrard et al. (2013) $\left(<10^{-23} \mathrm{~cm}^{3} \mathrm{~s}^{-1}\right)$. The low value of the rate coefficient is counterbalanced by the large density of hydrogen atoms, which makes this reaction non-negligible.

This reaction cancels out the production through reaction $\mathrm{H}_{2} \mathrm{CN}+\mathrm{H}\left(\mathrm{R}_{n} 395 \mathrm{a}\right)$ and therefore, $\mathrm{H}+\mathrm{HNC}\left(\mathrm{R}_{n} 255\right)$ becomes the major $\mathrm{HCN}$ source. Similarly, the cyano radicals (CN, cf. Figure 33 ) produced by photodissociation of HCN $\left(\mathrm{J}_{d} 22\right)$ react with methane to recycle back some $\mathrm{HCN}$ :

$$
\mathrm{CN}+\mathrm{CH}_{4} \rightarrow \mathrm{HCN}+\mathrm{CH}_{3} .
$$

Finally, condensation near the tropopause amounts to about $40 \%$ of the HCN total loss (cf. Table 19). 


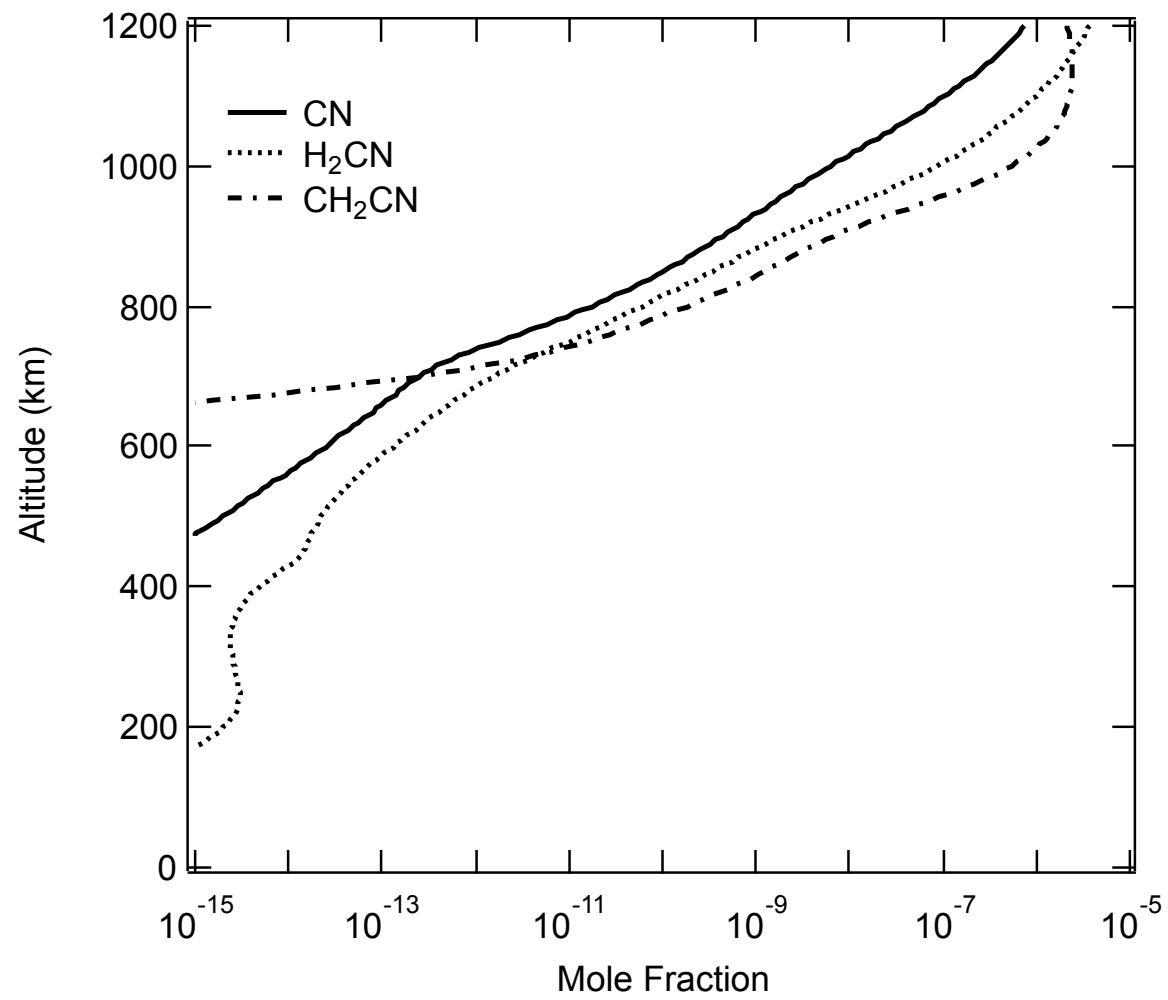

Figure 33: Model results for the mole fraction of the $\mathrm{CN}, \mathrm{H}_{2} \mathrm{CN}$, and $\mathrm{CH}_{2} \mathrm{CN}$ radicals.

Acetonitrile is formed by two major channels:

$$
\begin{gathered}
\mathrm{H}+\mathrm{CH}_{2} \mathrm{CN} \stackrel{\mathrm{M}}{\longrightarrow} \mathrm{CH}_{3} \mathrm{CN}, \\
\mathrm{N}\left({ }^{2} \mathrm{D}\right)+\mathrm{C}_{2} \mathrm{H}_{4} \rightarrow \mathrm{CH}_{3} \mathrm{CN}+\mathrm{H} .
\end{gathered}
$$

It has been established that the $\mathrm{C}_{2} \mathrm{H}_{3} \mathrm{~N}$ products formed in reaction $\left(\mathrm{R}_{n} 309 \mathrm{a}\right)$ are not $\mathrm{CH}_{3} \mathrm{CN}$ but two other isomers, cyclic- $\mathrm{CH}_{2} \mathrm{NCH}$ and $\mathrm{CH}_{2}(\mathrm{~N}) \mathrm{CH}$ (Balucani et al., 2012). Nevertheless, because the reactivity of these species is unknown, we assume that they eventually rearrange to acetonitrile by collisions with other species. We emphasize that this reaction represents $\sim 30 \%$ of the total acetonitrile production above $600 \mathrm{~km}$ and that setting this production to zero would not drastically affect the $\mathrm{CH}_{3} \mathrm{CN}$ profile.

Cyanomethyl radicals $\left(\mathrm{CH}_{2} \mathrm{CN}\right.$, cf. Figure 33$)$ are formed through reaction of imidogen radicals with acetylene, of nitrogen atoms with vinyl radicals and through electron recombination of $\mathrm{CH}_{3} \mathrm{CNH}^{+}$and $\mathrm{C}_{2} \mathrm{H}_{5} \mathrm{CNH}^{+}$: 


$$
\begin{aligned}
\mathrm{NH}+\mathrm{C}_{2} \mathrm{H}_{2} & \rightarrow \mathrm{CH}_{2} \mathrm{CN}+\mathrm{H}, \\
\mathrm{N}\left({ }^{4} \mathrm{~S}\right)+\mathrm{C}_{2} \mathrm{H}_{3} & \rightarrow \mathrm{CH}_{2} \mathrm{CN}+\mathrm{H}, \\
\mathrm{CH}_{3} \mathrm{CNH}^{+}+\mathrm{e}^{-} & \rightarrow \mathrm{CH}_{2} \mathrm{CN}+\mathrm{H}+\mathrm{H}, \\
\mathrm{C}_{2} \mathrm{H}_{5} \mathrm{CNH}^{+}+\mathrm{e}^{-} & \rightarrow \mathrm{CH}_{2} \mathrm{CN}+\mathrm{CH}_{3}+\mathrm{H} .
\end{aligned}
$$

The dissociative recombination of protonated propionitrile has been recently investigated at CRYRING (Vigren et al., 2010a, 2012a). Due to insufficient resolution of the detector, it was not possible to do a detailed investigation into the relative importance of the product channels; only the significance of different breakups or retention of the CCCN chain could be determined. In $\sim 43 \%$ of the reactions the four heavy atoms (a $\mathrm{C}$ atom or an $\mathrm{N}$ atom, denoted $X$ ) remain in the same product fragment. An equal portion of the reactions leads to products where one of the heavy atoms is split off from the other three and $\sim 14 \%$ result in a breakup into two heavy fragments containing two heavy atoms each. For the first channel leading to $X_{4}$, the only energetically allowed channel is $\mathrm{CH}_{3} \mathrm{CH}_{2} \mathrm{CN}+\mathrm{H}$, while for the third channel giving $X_{2}+X_{2}$, we prefer the fragmentation of one single $\mathrm{C}-\mathrm{C}$ bond giving $\mathrm{C}_{2} \mathrm{H}_{5}+\mathrm{HNC}$. Finally, the authors believe that it is a fission of a $\mathrm{C}-\mathrm{C}$ bond that dominates the $X_{3}+X$ type of fragmentations, possibly leading to the stable $\mathrm{CH}_{3}$ and $\mathrm{CH}_{2} \mathrm{CN}$ radicals alongside a hydrogen atom and we stick to this supposition.

Acetonitrile is mostly lost by downward transport until it reaches the ionospheric peak where its affinity for protons $\left(\mathrm{R}_{c n} 713, \mathrm{R}_{c n} 320\right)$ becomes its prominent fate. Although most $\mathrm{CH}_{3} \mathrm{CNH}^{+}$recycles back to $\mathrm{CH}_{3} \mathrm{CN}$ through proton transfer reactions $\left(\mathrm{R}_{c n} 775, \mathrm{R}_{c n} 776\right)$ and the two major electron recombination channels $\left(\mathrm{R}_{e r} 100 \mathrm{a}, \mathrm{b}\right)$, about $20 \%$ is lost through the two minor electron recombination channels:

$$
\begin{aligned}
\mathrm{CH}_{3} \mathrm{CNH}^{+}+\mathrm{e}^{-} & \rightarrow \mathrm{HNC}+\mathrm{CH}_{3} & & \left(\mathrm{R}_{e r} 100 \mathrm{c}\right) \\
& \rightarrow \mathrm{HCN}+{ }^{3} \mathrm{CH}_{2}+\mathrm{H} . & & \left(\mathrm{R}_{e r} 100 \mathrm{~d}\right)
\end{aligned}
$$

Below $700 \mathrm{~km}$, the only source of acetonitrile is diffusion from the upper atmosphere and its major loss is photodissociation $\left(\mathrm{J}_{d} 26\right)$ and condensation near the tropopause (cf. Table 19). 


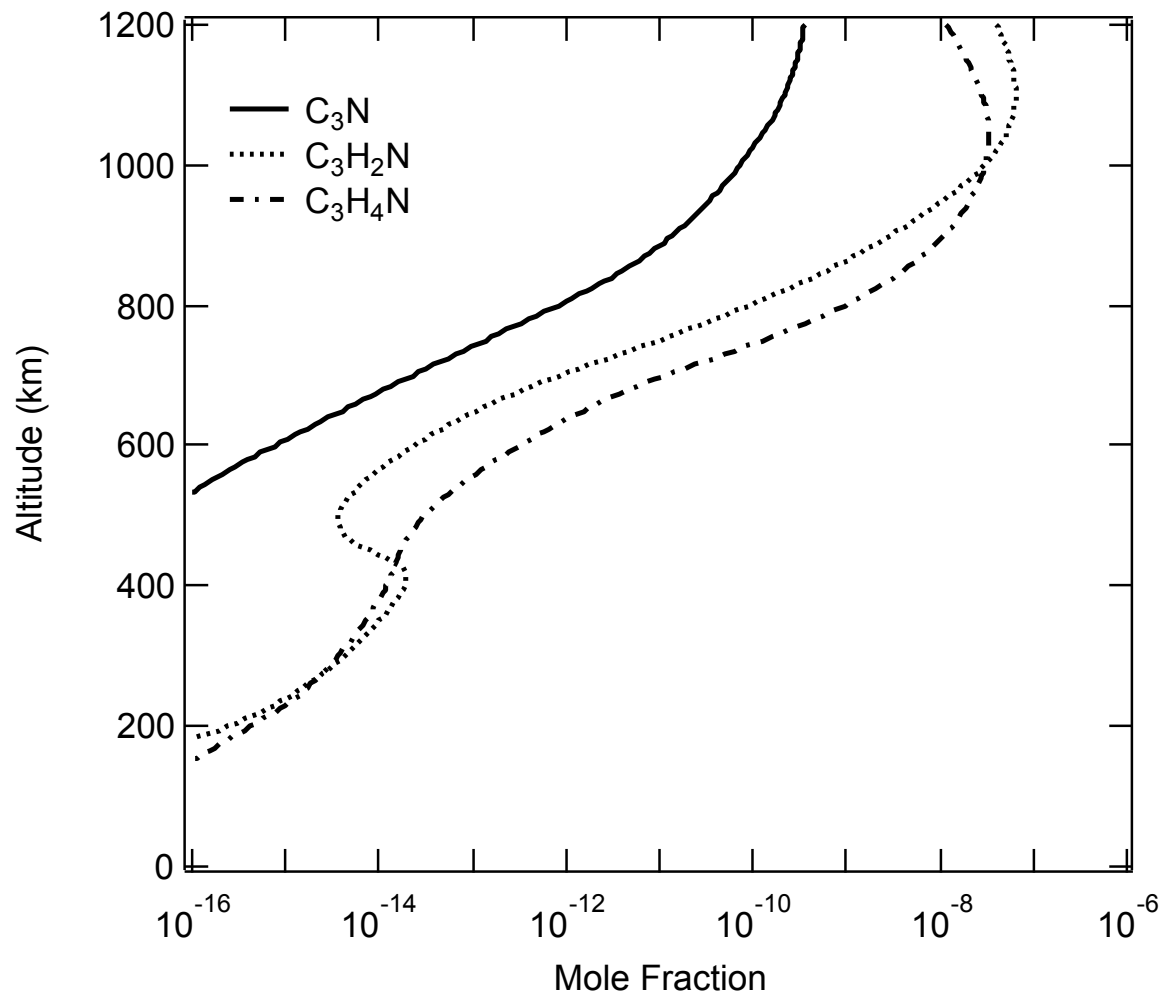

Figure 34: Model results for the mole fraction of the $\mathrm{C}_{3} \mathrm{~N}, \mathrm{H}_{2} \mathrm{C}_{3} \mathrm{~N}$, and $\mathrm{C}_{2} \mathrm{H}_{4} \mathrm{CN}$ radicals.

Cyanoacetylene $\left(\mathrm{HC}_{3} \mathrm{~N}\right)$, Acrylonitrile $\left(\mathrm{C}_{2} \mathrm{H}_{3} \mathrm{CN}\right)$, Propionitrile $\left(\mathbf{C}_{2} \mathbf{H}_{5} \mathbf{C N}\right)$. Above $1000 \mathrm{~km}$, cyanoacetylene is formed by the reaction between cyano radicals and acetylene:

$$
\mathrm{CN}+\mathrm{C}_{2} \mathrm{H}_{2} \rightarrow \mathrm{HC}_{3} \mathrm{~N}+\mathrm{H} \text {. }
$$

Like $\mathrm{CH}_{3} \mathrm{CN}$, it diffuses down to a level $(\sim 1000 \mathrm{~km})$ where proton transfer from $\mathrm{HCNH}^{+}$and $\mathrm{C}_{2} \mathrm{H}_{5}{ }^{+}\left(\mathrm{R}_{c n} 714, \mathrm{R}_{c n} 321\right)$ followed by electron recombination of $\mathrm{HC}_{3} \mathrm{NH}^{+}$become its most important loss:

$$
\begin{aligned}
\mathrm{HC}_{3} \mathrm{NH}^{+}+\mathrm{e}^{-} & \rightarrow \mathrm{CN}+\mathrm{C}_{2} \mathrm{H}_{2} & \left(\mathrm{R}_{e r} 104 \mathrm{~b}\right) \\
& \rightarrow \mathrm{C}_{3} \mathrm{~N}+\mathrm{H}_{2} . & \left(\mathrm{R}_{e r} 104 \mathrm{c}\right)
\end{aligned}
$$

Geppert et al. (2004b) and Vigren et al. (2012a) presented results on the electron recombination of protonated cyanoacetylene: the rate coefficient at electron temperatures relevant for Titan's upper atmosphere as well as the product branching ratios. Instead of $\mathrm{HC}_{3} \mathrm{NH}^{+}, \mathrm{DC}_{3} \mathrm{ND}^{+}$was used for reasons concerning the detection of the fragments, but previously measured branching ratios 

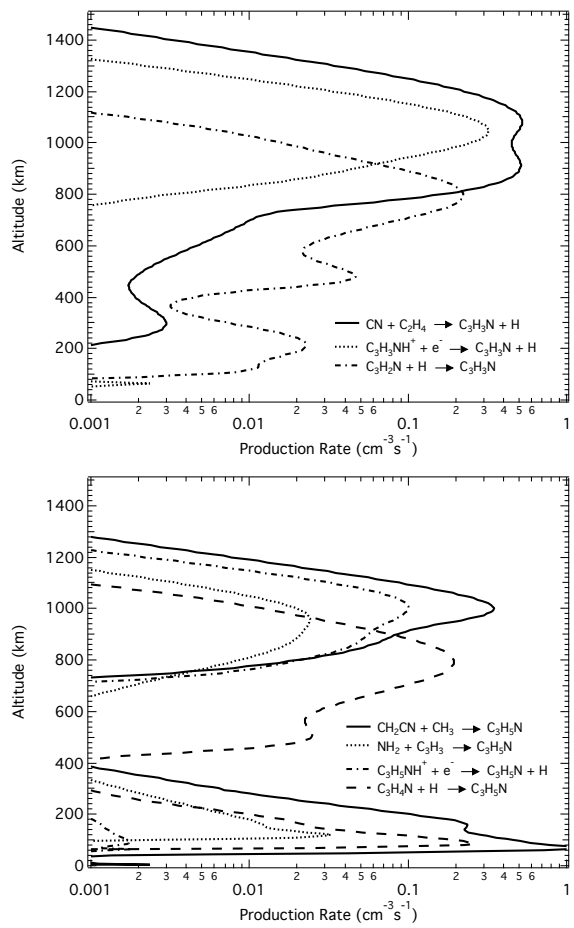
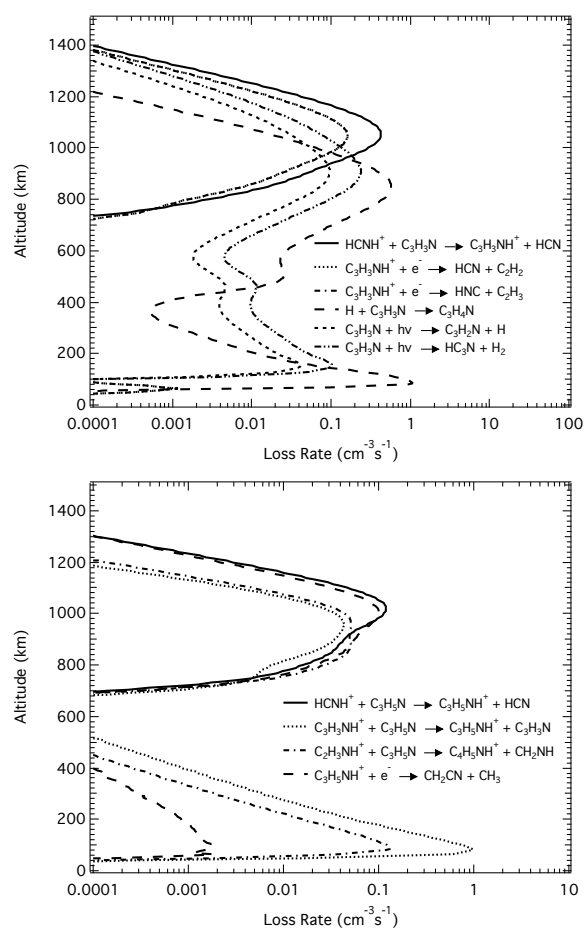

Figure 35: Production (left panels) and loss (right panels) rates of acrylonitrile (upper panels) and propionitrile (lower panels).

on other small polyatomic ions (Neau et al., 2000; Jensen et al., 2000; Geppert et al., 2006; Kamińska et al., 2010) were similar for the deuterated and hydrogenated species. The channels preserving the carbon chain $\left(\mathrm{D}_{x} \mathrm{C}_{3} \mathrm{~N}+(\mathrm{D})\right)$ or producing two fragments with a pair of heavy atoms each $\left(\mathrm{C}_{2} \mathrm{D}_{x}+\mathrm{CND}_{y}+\right.$ (D)) are detected with about equal fractions. Although the latter channel does lead to a net loss of $\mathrm{HC}_{3} \mathrm{~N}$, the first one essentially recycles back $\mathrm{HC}_{3} \mathrm{~N}$ through reaction of $\mathrm{C}_{3} \mathrm{~N}$ (cf. Figure 34) with $\mathrm{CH}_{4}\left(\mathrm{R}_{n} 438\right)$.

Between 1000 and $700 \mathrm{~km}$, photodissociation of acrylonitrile $\left(\mathrm{J}_{d} 28 \mathrm{a}\right)$ becomes the major formation pathway of cyanoacetylene. It diffuses further down where it is lost by photodissociation $\left(\mathrm{J}_{d} 27\right)$ and addition of hydrogen atoms, which subsequently leads to the production of acrylonitrile (see further below). Less than $10 \%$ of $\mathrm{HC}_{3} \mathrm{~N}$ is lost by condensation (cf. Table 19).

Above $800 \mathrm{~km}$, acrylonitrile is almost exclusively produced by the reaction between cyano radicals and ethylene (cf. upper left panel of Figure 35):

$$
\mathrm{CN}+\mathrm{C}_{2} \mathrm{H}_{4} \rightarrow \mathrm{C}_{2} \mathrm{H}_{3} \mathrm{CN}+\mathrm{H} \text {. }
$$

It is lost essentially through downward transport with the exception of around the ionospheric peak where, like the other nitriles, it is mostly destroyed by 
proton transfer reactions from $\mathrm{HCNH}^{+}$and $\mathrm{C}_{2} \mathrm{H}_{5}{ }^{+}\left(\mathrm{R}_{c n} 715, \mathrm{R}_{c n} 322\right)$, and subsequent fragmentation of $\mathrm{C}_{2} \mathrm{H}_{3} \mathrm{CNH}^{+}$via electron recombination (cf. upper right panel of Figure 35):

$$
\begin{array}{rlrl}
\mathrm{C}_{2} \mathrm{H}_{3} \mathrm{CNH}^{+}+\mathrm{e}^{-} & \rightarrow \mathrm{HNC}+\mathrm{C}_{2} \mathrm{H}_{3} & \left(\mathrm{R}_{e r} 106 \mathrm{~b}\right) \\
& \rightarrow \mathrm{HCN}+\mathrm{C}_{2} \mathrm{H}_{2}+\mathrm{H} . & & \left(\mathrm{R}_{e r} 106 \mathrm{c}\right)
\end{array}
$$

Below $800 \mathrm{~km}, \mathrm{C}_{2} \mathrm{H}_{3} \mathrm{CN}$ is a secondary product of $\mathrm{HC}_{3} \mathrm{~N}$ through additions of hydrogen atoms:

$$
\begin{aligned}
& \mathrm{H}+\mathrm{HC}_{3} \mathrm{~N} \stackrel{\mathrm{M}}{\longrightarrow} \mathrm{H}_{2} \mathrm{C}_{3} \mathrm{~N}, \\
& \text { followed by } \\
& \mathrm{H}+\mathrm{H}_{2} \mathrm{C}_{3} \mathrm{~N} \stackrel{\mathrm{M}}{\longrightarrow} \mathrm{C}_{2} \mathrm{H}_{3} \mathrm{CN} .
\end{aligned}
$$

Acrylonitrile is efficiently photodissociated but, with the main products being $\mathrm{HC}_{3} \mathrm{~N}$ and $\mathrm{C}_{3} \mathrm{H}_{2} \mathrm{~N}$ (cf. Figure 34), this process does not result in a major sink for $\mathrm{C}_{2} \mathrm{H}_{3} \mathrm{CN}$ :

$$
\begin{aligned}
\mathrm{C}_{2} \mathrm{H}_{3} \mathrm{CN}+\mathrm{h} \nu & \rightarrow \mathrm{HC}_{3} \mathrm{~N}+\mathrm{H}_{2} \\
& \rightarrow \mathrm{C}_{3} \mathrm{H}_{2} \mathrm{~N}+\mathrm{H} .
\end{aligned}
$$

Instead, it is mostly lost by addition of $\mathrm{H}$ atoms $\left(\mathrm{R}_{n} 259\right)$.

There are no reactions known to produce propionitrile in conditions relevant to Titan. We therefore searched for radical associations that could reasonably produce $\mathrm{C}_{2} \mathrm{H}_{5} \mathrm{CN}$ and estimated their rate coefficient based on the number of heavy atoms involved (section 2.7.3.1 and Vuitton et al. (2012)). The reaction of excited nitrogen atoms with propylene $\left(\mathrm{R}_{n} 313\right)$ is quite fast at room temperature (Herron, 1999) and can also be reasonably expected to produce propionitrile.

Within these assumptions, our model results indicate that above $900 \mathrm{~km}$, propionitrile is formed by addition of methyl and cyanomethyl radicals and to a lower extent of propargyl with amino radicals (cf. lower left panel of Figure $35)$ :

$$
\begin{array}{r}
\mathrm{CH}_{3}+\mathrm{CH}_{2} \mathrm{CN} \stackrel{\mathrm{M}}{\longrightarrow} \mathrm{C}_{2} \mathrm{H}_{5} \mathrm{CN}, \\
\mathrm{C}_{3} \mathrm{H}_{3}+\mathrm{NH}_{2} \stackrel{\mathrm{M}}{\longrightarrow} \mathrm{C}_{2} \mathrm{H}_{5} \mathrm{CN} .
\end{array}
$$

In this region, it is almost exclusively lost via proton attachment $\left(\mathrm{R}_{c n} 716, \mathrm{R}_{c n} 323\right)$ followed by electron recombination of $\mathrm{C}_{2} \mathrm{H}_{5} \mathrm{CNH}^{+}$, and ion-molecule reactions (cf. lower right panel of Figure 35):

$$
\begin{aligned}
& \left.\mathrm{C}_{2} \mathrm{H}_{5} \mathrm{CNH}^{+}+\mathrm{e}^{-} \rightarrow \mathrm{CH}_{2} \mathrm{CN}+\mathrm{CH}_{3}+\mathrm{H}, \quad \text { (Rer } 108 \mathrm{~b}\right) \\
& \mathrm{CH}_{3} \mathrm{CNH}^{+}+\mathrm{C}_{2} \mathrm{H}_{5} \mathrm{CN} \rightarrow \mathrm{C}_{3} \mathrm{H}_{5} \mathrm{CNH}^{+}+\mathrm{CH}_{2} \mathrm{NH} . \quad\left(\mathrm{R}_{c n} 776\right)
\end{aligned}
$$


Unlike for neutral chemistry, these reactions have been fairly well constrained experimentally (Edwards et al., 2008; Vigren et al., 2010a). From 900 km down to $400 \mathrm{~km}$, propionitrile is found to be formed by a sequence of addition of hydrogen atoms to acrylonitrile and lost by transport downward ultimately reaching the condensation level (cf. Table 19):

$$
\begin{aligned}
& \mathrm{H}+\mathrm{C}_{2} \mathrm{H}_{3} \mathrm{CN} \stackrel{\mathrm{M}}{\longrightarrow} \mathrm{C}_{2} \mathrm{H}_{4} \mathrm{CN}, \\
& \mathrm{H}+\mathrm{C}_{2} \mathrm{H}_{4} \mathrm{CN} \stackrel{\mathrm{M}}{\longrightarrow} \mathrm{C}_{2} \mathrm{H}_{5} \mathrm{CN} .
\end{aligned}
$$

Cyanodiacetylene $\left(\mathbf{H C}_{5} \mathbf{N}\right)$. Similarly to cyanoacetylene, above $800 \mathrm{~km}$, cyanodiacetylene is formed by the reaction between cyanoethynyl radicals $\left(\mathrm{C}_{3} \mathrm{~N}\right)$ and acetylene, and between cyano radicals and diacetylene:

$$
\begin{aligned}
\mathrm{C}_{3} \mathrm{~N}+\mathrm{C}_{2} \mathrm{H}_{2} & \rightarrow \mathrm{HC}_{5} \mathrm{~N}+\mathrm{H}, \\
\mathrm{CN}+\mathrm{C}_{4} \mathrm{H}_{2} & \rightarrow \mathrm{HC}_{5} \mathrm{~N}+\mathrm{H} .
\end{aligned}
$$

Cyanoethynyl radicals are formed in the electron recombination of protonated cyanoacetylene above $1000 \mathrm{~km}$ and the photodissociation of cyanoacetylene $\left(\mathrm{J}_{d} 27 \mathrm{a}\right)$ below.

The rate coefficients of reactions $\left(\mathrm{R}_{n} 439\right)$ and $\left(\mathrm{R}_{n} 377\right)$ have recently been measured at low temperature by Sims et al. (Cheikh Sid Ely, 2012; Fournier, 2014). A common assumption in the literature is to assume that the rate coefficient for reaction $\left(\mathrm{R}_{n} 439\right)$ is the same as that for $\mathrm{C}_{4} \mathrm{H}+\mathrm{C}_{2} \mathrm{H}_{2}\left(\mathrm{R}_{n} 195\right)$, which proves to be a fairly valid assumption $\left(\mathrm{k}\left(\mathrm{R}_{n} 439\right)=3.4 \times 10^{-10}\right.$ versus $\mathrm{k}\left(\mathrm{R}_{n} 195\right)=2.4 \times 10^{-10} \mathrm{~cm}^{-3} \mathrm{~s}^{-1}$ at $\left.150 \mathrm{~K}\right)$. Since the products have never been investigated, we assume that the formation of $\mathrm{HC}_{5} \mathrm{~N}$ and a $\mathrm{H}$ atom, which is more exothermic than the $\mathrm{H}$ abstraction and reformation of $\mathrm{HC}_{3} \mathrm{~N}$, is the only channel.

The reactivity of the $\mathrm{CN}$ radical (cf. Figure 24$)$ with $\mathrm{C}_{4} \mathrm{H}_{2}\left(\mathrm{R}_{n} 377\right)$ is found to be very close to its reactivity with $\mathrm{C}_{2} \mathrm{H}_{2}\left(\mathrm{R}_{n} 370\right)$, with a mild negative temperature dependence. It is worthwhile mentioning that the semi-empirical capture model of Faure et al. (2009) reproduces extremely well the experimental measurements. The products have never been investigated experimentally but ab initio calculations demonstrate that the reactions of the $\mathrm{CN}$ radical with polyynes lead to the formation of cyanopolyyne chains $\mathrm{HC}_{2 n} \mathrm{CN}$, here $\mathrm{HC}_{5} \mathrm{~N}$ (Fukuzawa et al., 1998).

Production of cyanodiacetylene is balanced by photolysis $\left(\mathrm{J}_{d} 30\right)$ and ion chemistry (proton exchange reactions and subsequent electron recombination) in the upper atmosphere, as for $\mathrm{HC}_{3} \mathrm{~N}$. The magnitude of these loss processes is quite uncertain since data are neither available for the photodissociation branching ratios or electron recombination (both rate coefficient and products).

Cyanogen $\left(\mathrm{C}_{2} \mathrm{~N}_{2}\right)$ and dicyanoacetylene $\left(\mathrm{C}_{4} \mathrm{~N}_{2}\right)$. From quantum chemical calculations by Petrie and Osamura (2004), the main source of cyanogen 
and dicyanogen is, respectively:

$$
\begin{array}{r}
\mathrm{CN}+\mathrm{HNC} \rightarrow \mathrm{C}_{2} \mathrm{~N}_{2}+\mathrm{H}, \\
\mathrm{C}_{3} \mathrm{~N}+\mathrm{HCN} \rightarrow \mathrm{C}_{4} \mathrm{~N}_{2}+\mathrm{H} .
\end{array}
$$

2568

Dicyanoacetylene is also formed through $\mathrm{CN}$ addition and $\mathrm{H}$ atom elimination:

$$
\mathrm{CN}+\mathrm{HC}_{3} \mathrm{~N} \rightarrow \mathrm{C}_{4} \mathrm{~N}_{2}+\mathrm{H} .
$$

The first experimental measurements of this reaction below room temperature show only a slight increase in the rate coefficient from room temperature to 150 $\mathrm{K}$ (from $1.8 \times 10^{-11}$ to $2.8 \times 10^{-11} \mathrm{~cm}^{3} \mathrm{~s}^{-1}$ ) (Cheikh Sid Ely et al., 2013), falling short of capture theory calculations, which predicted a value of $5.5 \times 10^{-11} \mathrm{~cm}^{3}$ $\mathrm{s}^{-1}$ at $150 \mathrm{~K}$ (Faure et al., 2009).

Both $\mathrm{C}_{2} \mathrm{~N}_{2}$ and $\mathrm{C}_{4} \mathrm{~N}_{2}$ are lost by $\mathrm{H}$ atom addition and to a lower extent photodissociation:

$$
\begin{gathered}
\mathrm{H}+\mathrm{C}_{2} \mathrm{~N}_{2} \stackrel{\mathrm{M}}{\longrightarrow} \mathrm{HC}_{2} \mathrm{~N}_{2} \\
\text { followed by } \\
\mathrm{H}+\mathrm{HC}_{2} \mathrm{~N}_{2} \rightarrow \mathrm{HCN}+\mathrm{HCN}, \\
\text { and } \\
\mathrm{H}+\mathrm{C}_{4} \mathrm{~N}_{2} \stackrel{\mathrm{M}}{\longrightarrow} \mathrm{HC}_{4} \mathrm{~N}_{2} \\
\text { followed by } \\
\mathrm{H}+\mathrm{HC}_{4} \mathrm{~N}_{2} \rightarrow \mathrm{HC}_{3} \mathrm{~N}+\mathrm{HCN} .
\end{gathered}
$$

\subsubsection{O-bearing Species}

A crucial reaction for the distribution of O-bearing species is that between $\mathrm{OH}$ and $\mathrm{CH}_{3}\left(\mathrm{R}_{n} 526\right)$ but the nature of the products has been a long standing debate. For many years, $\mathrm{CO}+2 \mathrm{H}_{2}$ were thought to be the main products but laboratory experiments performed in the 90's suggested that instead they were $\mathrm{H}_{2} \mathrm{O}+{ }^{1} \mathrm{CH}_{2}$ in the bimolecular regime and $\mathrm{CH}_{3} \mathrm{OH}$ in the termolecular regime (Baulch et al., 1994; De Avillez Pereira et al., 1997). In Appendix A we present calculations based on transition state theory that confirm that $\mathrm{H}_{2} \mathrm{O}+{ }^{1} \mathrm{CH}_{2}$ is indeed an important channel but that the major products are actually $\mathrm{CHOH}$ $+\mathrm{H}_{2}$, where $\mathrm{CHOH}$ is an isomer of formaldehyde $\left(\mathrm{H}_{2} \mathrm{CO}\right)$ (cf. Figure A.4).

With this scheme, the fate of an $\mathrm{OH}$ (hydroxy radicals) influx into Titan's upper atmosphere can be divided into three basic pathways that are further described below:

- To restore $\mathrm{H}_{2} \mathrm{O}$ in reactions with $\mathrm{CH}_{3}, \mathrm{CH}_{4}$, and $\mathrm{C}_{2} \mathrm{H}_{6}$.

- To form $\mathrm{CO}$ in reactions with $\mathrm{CH}_{3}, \mathrm{C}_{2} \mathrm{H}_{4}$, and ${ }^{3} \mathrm{CH}_{2}$ because the products in these reactions decompose further to $\mathrm{CO}$. 
- To form $\mathrm{CO}_{2}$ by removing $\mathrm{CO}$.

As a consequence, $\mathrm{OH}$ can lead to the formation of the three major O-bearing species $\left(\mathrm{CO}, \mathrm{H}_{2} \mathrm{O}\right.$ and $\left.\mathrm{CO}_{2}\right)$. This is fundamentally different than the Hörst et al. (2008) paper where both $\mathrm{O}$ and $\mathrm{OH}$ were required.

The $\mathrm{OH}$ radicals deposited in the upper atmosphere are also quickly transformed to $\mathrm{H}_{2} \mathrm{O}$ molecules by reaction with abundant species, such as $\mathrm{CH}_{3}$ and $\mathrm{CH}_{4}$ :

$$
\begin{aligned}
& \mathrm{OH}+\mathrm{CH}_{3} \rightarrow \mathrm{H}_{2} \mathrm{O}+{ }^{1} \mathrm{CH}_{2} \\
& \mathrm{OH}+\mathrm{CH}_{4} \rightarrow \mathrm{H}_{2} \mathrm{O}+\mathrm{CH}_{3},
\end{aligned}
$$

but $\mathrm{OH}$ is rapidly recycled by photolysis:

$$
\mathrm{H}_{2} \mathrm{O}+h \nu \rightarrow \mathrm{OH}+\mathrm{H} .
$$

Water is ultimately lost by condensation near the tropopause.

The OH flux $\left(10^{7} \mathrm{~cm}^{-2} \mathrm{~s}^{-1}\right)$ is mostly spent to form $\mathrm{CO}$ in reactions with $\mathrm{CH}_{3}$ :

$$
\mathrm{OH}+\mathrm{CH}_{3} \rightarrow \mathrm{CHOH}+\mathrm{H}_{2} .
$$

$\mathrm{CHOH}$ mainly reacts with $\mathrm{H}$ to form formaldehyde $\left(\mathrm{H}_{2} \mathrm{CO}\right)$ :

$$
\mathrm{CHOH}+\mathrm{H} \rightarrow \mathrm{H}_{2} \mathrm{CO}+\mathrm{H} .
$$

Formaldehyde easily photodissociates forming $\mathrm{CO}$ either directly:

$$
\mathrm{H}_{2} \mathrm{CO}+h \nu \rightarrow \mathrm{CO}+\mathrm{H}_{2} / 2 \mathrm{H},
$$

or through a 2-step process involving HCO:

$$
\begin{aligned}
\mathrm{H}_{2} \mathrm{CO}+h \nu & \rightarrow \mathrm{HCO}+\mathrm{H} \\
\mathrm{HCO}+\mathrm{H} / \mathrm{CH}_{3} & \rightarrow \mathrm{CO}+\mathrm{H}_{2} / \mathrm{CH}_{4} .
\end{aligned}
$$

$\mathrm{CO}$ is an extremely stable species in the conditions of the atmosphere of Titan, with the only loss mechanism being the reaction with $\mathrm{OH}$ radicals to produce $\mathrm{CO}_{2}$ :

$$
\mathrm{OH}+\mathrm{CO} \rightarrow \mathrm{CO}_{2}+\mathrm{H} .
$$

$\mathrm{CO}_{2}$ major loss is condensation near the tropopause.

Above $\sim 800 \mathrm{~km}$, proton transfer reactions are not a main loss for $\mathrm{H}_{2} \mathrm{O}$ and $\mathrm{CO}$ as electron recombinations recycle them efficiently. The relevant ion-neutral chemistry is given in section 3.1.1.4. The coupled nitrogen-oxygen chemistry only impacts the density of the major O-bearing species to the $1 \%$ level at most. 


\subsection{The GCR Layer}

GCR can penetrate to the deeper atmosphere to altitude levels where solar radiation and saturnian electrons cannot reach and are the main ionization source below $400 \mathrm{~km}$. The main product following GCR impact is $\mathrm{N}_{2}{ }^{+}$(cf. Figure 18). Unlike in the upper ionosphere, it can efficiently add to $\mathrm{N}_{2}$ by three-body collisions to form $\mathrm{N}_{4}{ }^{+}$(Anicich et al., 2000; McEwan and Anicich, 2007), which immediately reacts with methane:

$$
\begin{aligned}
\mathrm{N}_{2}^{+}+\mathrm{N}_{2} \stackrel{\mathrm{M}}{\rightarrow} \mathrm{N}_{4}^{+} & \left(\mathrm{R}_{c n} 854\right) \\
\mathrm{N}_{4}^{+}+\mathrm{CH}_{4} \rightarrow \mathrm{CH}_{4}^{+}+\mathrm{N}_{2}+\mathrm{N}_{2} . & \left(\mathrm{R}_{c n} 901\right)
\end{aligned}
$$

This process rather than direct ionization is actually the main source of $\mathrm{CH}_{4}{ }^{+}$. $\mathrm{CH}_{3}{ }^{+}$is a direct product of $\mathrm{N}_{2}{ }^{+}$and $\mathrm{CH}_{4}\left(\mathrm{R}_{c n} 836 \mathrm{a}\right)$ and the chemistry then goes on to $\mathrm{CH}_{5}{ }^{+}\left(\mathrm{R}_{c n} 162\right), \mathrm{C}_{2} \mathrm{H}_{5}{ }^{+}\left(\mathrm{R}_{c n} 132\right)$ and $\mathrm{HCNH}^{+}\left(\mathrm{R}_{c n} 316, \mathrm{R}_{c n} 194\right)$, as in the upper ionosphere. Nevertheless, a third channel contributes as well to the formation of $\mathrm{HCNH}^{+}$(Dutuit et al., 2013):

$$
\mathrm{N}^{+}+\mathrm{CH}_{4} \rightarrow \mathrm{HCNH}^{+}+\mathrm{H}_{2} .
$$

Because of the high-pressure at low altitude, the main loss of $\mathrm{HCNH}^{+}$occurs through a 3-body reaction with nitrogen (Capone et al., 1981) to form an adduct that can subsequently react with acetylene (Capone et al., 1981) and ethane (Vacher et al., 2000):

$$
\begin{array}{cc}
\mathrm{HCNH}^{+}+\mathrm{N}_{2} \stackrel{\mathrm{M}}{\rightarrow} \mathrm{HCNH}^{+} \cdot \mathrm{N}_{2} & \left(\mathrm{R}_{c n} 723\right) \\
\mathrm{HCNH}^{+} \cdot \mathrm{N}_{2}+\mathrm{C}_{2} \mathrm{H}_{2} \stackrel{\mathrm{M}}{\rightarrow} \mathrm{C}_{3} \mathrm{H}_{3} \mathrm{NH}^{+}+\mathrm{N}_{2} & \left(\mathrm{R}_{c n} 728\right) \\
\mathrm{HCNH}^{+} \cdot \mathrm{N}_{2}+\mathrm{C}_{2} \mathrm{H}_{6} \stackrel{\mathrm{M}}{\rightarrow} \mathrm{C}_{3} \mathrm{H}_{7} \mathrm{NH}^{+}+\mathrm{N}_{2} & \left(\mathrm{R}_{c n} 729\right)
\end{array}
$$

$\mathrm{CH}_{5}{ }^{+}$ions are at the origin of a suite of hydrocarbon ions, via $\mathrm{HCO}^{+}$:

$$
\begin{aligned}
\mathrm{CH}_{5}^{+}+\mathrm{CO} & \rightarrow \mathrm{HCO}^{+}+\mathrm{CH}_{4} \\
\mathrm{HCO}^{+}+\mathrm{C}_{2} \mathrm{H}_{2} & \rightarrow \mathrm{C}_{2} \mathrm{H}_{3}^{+}+\mathrm{CO}
\end{aligned}
$$

After reacting with $\mathrm{CH}_{4}, \mathrm{C}_{2} \mathrm{H}_{3}{ }^{+}$can either lead to $\mathrm{C}_{7} \mathrm{H}_{9}{ }^{+}$:

$$
\begin{array}{rr}
\mathrm{C}_{2} \mathrm{H}_{3}^{+}+\mathrm{CH}_{4} \rightarrow \mathrm{C}_{3} \mathrm{H}_{5}^{+}+\mathrm{H}_{2} & \left(\mathrm{R}_{c n} 258 \mathrm{a}\right) \\
\mathrm{C}_{3} \mathrm{H}_{5}^{+}+\mathrm{C}_{2} \mathrm{H}_{2} \rightarrow \mathrm{C}_{5} \mathrm{H}_{5}^{+}+\mathrm{H}_{2} & \left(\mathrm{R}_{c n} 422 \mathrm{a}\right) \\
\mathrm{C}_{5} \mathrm{H}_{5}^{+}+\mathrm{C}_{2} \mathrm{H}_{4} \stackrel{\mathrm{M}}{\rightarrow} \mathrm{C}_{7} \mathrm{H}_{9}^{+} & \left(\mathrm{R}_{c n} 528\right)
\end{array}
$$

or to $\mathrm{C}_{4} \mathrm{H}_{9}{ }^{+}$: 


$$
\begin{aligned}
& \mathrm{C}_{2} \mathrm{H}_{3}^{+}+\mathrm{CH}_{4} \stackrel{\mathrm{M}}{\rightarrow} \mathrm{C}_{3} \mathrm{H}_{7}^{+} \\
& \mathrm{C}_{3} \mathrm{H}_{7}^{+}+\mathrm{C}_{2} \mathrm{H}_{6} \rightarrow \mathrm{C}_{4} \mathrm{H}_{9}^{+}+\mathrm{CH}_{4}
\end{aligned}
$$

Then $\mathrm{C}_{4} \mathrm{H}_{9}{ }^{+}$can either lead to $\mathrm{C}_{6} \mathrm{H}_{9}{ }^{+}$or $\mathrm{C}_{6} \mathrm{H}_{11}{ }^{+}$(Burt et al., 1970):

$$
\begin{aligned}
\mathrm{C}_{4} \mathrm{H}_{9}^{+}+\mathrm{C}_{2} \mathrm{H}_{4} & \rightarrow \mathrm{C}_{6} \mathrm{H}_{9}^{+}+\mathrm{H}_{2}+\mathrm{H}_{2} & & \left(\mathrm{R}_{c n} 506 \mathrm{a}\right) \\
& \rightarrow \mathrm{C}_{6} \mathrm{H}_{11}^{+}+\mathrm{H}_{2} & & \left(\mathrm{R}_{c n} 506 \mathrm{~b}\right)
\end{aligned}
$$

Nevertheless, the main loss of $\mathrm{HCO}^{+}$is not proton transfer to $\mathrm{C}_{2} \mathrm{H}_{2}$ but association to $\mathrm{H}_{2}$ (McEwan and Anicich, 2007; Molina-Cuberos et al., 1999a):

$$
\mathrm{HCO}^{+}+\mathrm{H}_{2} \stackrel{\mathrm{M}}{\longrightarrow} \mathrm{HCO}^{+} \cdot \mathrm{H}_{2}
$$

As in the upper ionosphere, the presence of $\mathrm{NH}_{3}$ leads to the formation of $\mathrm{NH}_{4}{ }^{+}$, which is thus an intermediate in the chain of proton transfer reactions, eventually leading to heavier nitrogen-bearing ions with a large proton affinity.

The chemistry induced by cosmic rays is also an important source of neutral species. It is well established that electron recombination of $\mathrm{C}_{3} \mathrm{H}_{3} \mathrm{NH}^{+}$ $\left(\mathrm{R}_{e r} 106 \mathrm{a}\right)$ leads to acrylonitrile. Similarly, some fraction of the electron recombination of $\mathrm{C}_{3} \mathrm{H}_{7} \mathrm{NH}^{+}\left(\mathrm{R}_{e r} 109 \mathrm{c}\right)$ may lead to ammonia.

The photodissociation of ammonia $\left(\mathrm{J}_{d} 20\right)$ as well as the reaction of $\mathrm{NH}$ radicals with ethane (Mullen and Smith, 2005) are the main sources of $\mathrm{NH}_{2}$ :

$$
\mathrm{NH}+\mathrm{C}_{2} \mathrm{H}_{6} \rightarrow \mathrm{C}_{2} \mathrm{H}_{5}+\mathrm{NH}_{2}
$$

$\mathrm{NH}_{2}$ radicals are at the origin of methylamine $\left(\mathrm{R}_{n} 349\right)$ and hydrazine $\left(\mathrm{R}_{n} 361 \mathrm{~b}\right)$.

The photodissociation of $\mathrm{CH}_{3} \mathrm{NH}_{2}\left(\mathrm{~J}_{d} 25 \mathrm{a}\right)$ and the reaction of excited nitrogen atoms with methane $\left(\mathrm{R}_{n} 307 \mathrm{a}\right)$ are the principal channels forming methanimine.

The $\mathrm{N}\left({ }^{2} \mathrm{D}\right)$ peak in the GCR layer is at the origin of the production of HCCN $\left(\mathrm{R}_{n} 308\right)$, which by reaction with $\mathrm{N}$ atoms forms $\mathrm{C}_{2} \mathrm{~N}_{2}\left(\mathrm{R}_{n} 300\right)$.

The production of $\mathrm{N}^{+}$by GCR impact and the presence of heavy nitrogen bearing species (cf. Figure 55) lead to the formation of $\mathrm{H}_{2} \mathrm{CN}$ (Stavish et al., 2009):

$$
\begin{aligned}
\mathrm{N}^{+}+\mathrm{C}_{4} \mathrm{H}_{5} \mathrm{~N} & \rightarrow \mathrm{C}_{3} \mathrm{H}_{3} \mathrm{~N}^{+}+\mathrm{H}_{2} \mathrm{CN} \\
& \rightarrow \mathrm{c}_{-} \mathrm{C}_{3} \mathrm{H}_{3}{ }^{+}+\mathrm{H}_{2} \mathrm{CN}+\mathrm{N}
\end{aligned}
$$$$
\left(\mathrm{R}_{c n} 609 \mathrm{a}\right)
$$

As in the upper ionosphere, the formation of $\mathrm{H}_{2} \mathrm{CN}$ and $\mathrm{N}$ atoms subsequently leads to the production of $\mathrm{HNC}\left(\mathrm{R}_{n} 298\right)$. 


\section{Comparison to Observations and Other Models}

In this section, we compare the density profiles obtained with this latest update of our photochemical model to the available observations and to the models developed by other groups. We first focus on the positively charged hydrocarbon ions and negatively charged species present in the upper ionosphere. In a second part, we discuss neutral species, including hydrocarbons, nitrogen (including ${ }^{15} \mathrm{~N}$ ) and oxygen bearing species. The protonated nitrogen species are also discussed here because their abundance is closely related to the presence of the neutral form of those species. In addition to the nominal model discussed up to this point, we have run a number of additional models to test the sensitivity of certain molecules to various parameters. These models are shown in the relevant figures. Finally, we finish with a tentative composition for the GCR produced layer.

\subsection{The Upper Ionosphere}

4.1.1. Positive Ions

4.1.1.1. Previous Models. Beside our own work, the groups of Cravens and that based at SwRI have been focusing extensively on the composition and density of ions in Titan's atmosphere (cf. Table 8 in Mandt et al. (2012a), and the reviews by Galand et al. (2014) and Vuitton et al. (2014)). Cravens et al. (2009) and Richard et al. (2015a) focus on the nightside ionosphere (T5, T21, T55-T59) while Robertson et al. (2009), Westlake et al. (2012), Mandt et al. (2012a) and Richard et al. (2015b) are interested in the dayside (T17, T18, T40, T48). These models use neutral background atmospheres usually based on the INMS observations available at that time or previous photochemical models. Cravens et al. (2009) and Richard et al. (2015a) used magnetospheric electron fluxes measured by CAPS ELS as boundary conditions at the top of the atmosphere for the downward electron flux in a two-stream model (Gan et al., 1992), the model of Robertson et al. (2009) includes ionization by solar radiation and photoelectrons, while in Westlake et al. (2012), the primary ionization is initiated by solar photons and magnetospheric electrons. Mandt et al. (2012a) improves on these models by including high resolution photoabsorption crosssections for $\mathrm{N}_{2}$ and ${ }^{15} \mathrm{~N}^{14} \mathrm{~N}$, while Richard et al. (2015b) makes the simplified assumption that only $50 \%$ of the photons between 80 and $100 \mathrm{~nm}$ interact with $\mathrm{N}_{2}$. In all models, the chemical network relies heavily on the reaction list published in Vuitton et al. (2007). The models achieve somewhat different success in fitting various species in altitude regions of interest, but despite all differences in input parameters, one common conclusion to all these models is that $\mathrm{CH}_{5}{ }^{+}, \mathrm{C}_{2} \mathrm{H}_{5}{ }^{+}$and $\mathrm{HCNH}^{+}$are overestimated by a factor of typically $2-4$. We will return to this in section 5.1.

4.1.1.2. Observations. INMS and CAPS performed the first and only ion density measurements of Titan's upper atmosphere, most of which cannot be obtained with any other technique. INMS detected roughly 50 ions at or above the detection threshold in the mass range 1-100 u. Qualitatively the dayside and 
nightside ion composition are similar, but quantitative differences exist, particularly for the species with a relatively short chemical lifetime $\left(\mathrm{CH}_{5}{ }^{+}, \mathrm{HCNH}^{+}\right.$, $\mathrm{C}_{2} \mathrm{H}_{5}{ }^{+}$) that are more abundant for the dayside than for the nightside. On the other hand, the day-to-night variations for terminal ion species with long lifetimes $\left(\mathrm{NH}_{4}^{+}, \mathrm{C}_{6} \mathrm{H}_{7}{ }^{+}, \mathrm{C}_{2} \mathrm{H}_{3} \mathrm{CNH}^{+}\right)$are rather small (Cui et al., 2009a).

The first ion observations by INMS were made deep into the nightside (solar zenith angle of $127^{\circ}$ or more) during the outbound portion of the T5 flyby. It later appeared that they occurred during a strong precipitation event of magnetospheric electrons and that the ionization rate exceeded the "typical" one by a factor of 10 (Ågren et al., 2007; Cravens et al., 2008). Therefore, although largely studied, T5 appears as a peculiar encounter with total ion densities somewhere in between that for a mean nightside $\left(400-1000 \mathrm{~cm}^{-3}\right)$ and for a dayside pass (2500-3500 $\mathrm{cm}^{-3}$ ) (Ågren et al., 2009).

We choose to compare our ion density profiles to the T40 flyby because it is a dayside flyby (solar zenith angle of $65^{\circ}$ at $1500 \mathrm{~km}$ inbound, $38^{\circ}$ at closest approach and $14^{\circ}$ at $1500 \mathrm{~km}$ outbound) that occurred at mid-latitudes $\left(-21^{\circ}\right.$ at $1500 \mathrm{~km}$ inbound, $-12^{\circ}$ at closest approach and $0^{\circ}$ at $1500 \mathrm{~km}$ outbound) and in which high quality INMS and CAPS data were retrieved. The ion densities are obtained using the method described in Mandt et al. (2012a). Teolis et al. (2015) corrected the original INMS calibration model and accordingly, the previously published ion densities are revised upward by a correction factor of $1.55 \pm 0.33$.

4.1.1.3. Density Profiles. The calculated ion densities are given in Table 9 and the model spectrum is compared to the INMS spectrum obtained during the T40 flyby in Figure 36. For the ions that are closely related to a neutral (section 3), we discuss them in conjunction with the associated neutral in section 4.2.3. Because our model corresponds to globally averaged conditions while T40 is a dayside pass, we expect the predicted densities for the primary ions to underestimate the measured densities. We will see that this is indeed the case for the ionization products of $\mathrm{N}_{2}$ and $\mathrm{CH}_{4}$, whose density is in agreement with the observations if multiplied by a factor of two. This does not support the idea that there is a problem with our understanding of the production of primary ions on the dayside. Because the model conveniently matches the primary ion densities, we expect that the subsequent chemistry should be well described. 
Table 9: Calculated ion densities $\left(\mathrm{cm}^{-3}\right)$ at the peak of the ionosphere $(1125 \mathrm{~km})$. Only ions with a density above $10^{-1} \mathrm{~cm}^{-3}$ and major isotopologues are shown.

\begin{tabular}{|c|c|c|c|c|c|c|c|c|}
\hline $\mathrm{m} / \mathrm{z}$ & Species & Density & Species & Density & Species & Density & Species & Density \\
\hline 13 & $\mathrm{CH}^{+}$ & $1.2 \times 10^{-1}$ & & & & & & \\
\hline 14 & $\mathrm{CH}_{2}^{+}$ & $7.8 \times 10^{0}$ & $\mathrm{~N}^{+}$ & $7.1 \times 10^{0}$ & & & & \\
\hline 15 & $\mathrm{CH}_{3}^{+}$ & $3.1 \times 10^{1}$ & & & & & & \\
\hline 16 & $\mathrm{CH}_{4}^{+}$ & $1.9 \times 10^{0}$ & & & & & & \\
\hline 17 & $\mathrm{CH}_{5}^{+}$ & $3.6 \times 10^{1}$ & & & & & & \\
\hline 18 & $\mathrm{NH}_{4}^{+}$ & $6.0 \times 10^{0}$ & & & & & & \\
\hline 19 & $\mathrm{H}_{3} \mathrm{O}^{+}$ & $6.4 \times 10^{-1}$ & & & & & & \\
\hline 26 & $\mathrm{C}_{2} \mathrm{H}_{2}^{+}$ & $1.2 \times 10^{0}$ & & & & & & \\
\hline 27 & $\mathrm{C}_{2} \mathrm{H}_{3}^{+}$ & $1.4 \times 10^{1}$ & $\mathrm{HCN}^{+}$ & $8.8 \times 10^{-1}$ & & & & \\
\hline 28 & $\mathrm{HCNH}^{+}$ & $1.6 \times 10^{3}$ & $\mathrm{C}_{2} \mathrm{H}_{4}^{+}$ & $1.2 \times 10^{2}$ & $\mathrm{~N}_{2}^{+}$ & $3.7 \times 10^{1}$ & & \\
\hline 29 & $\mathrm{C}_{2} \mathrm{H}_{5}{ }^{+}$ & $4.2 \times 10^{2}$ & $\mathrm{HC}^{15} \mathrm{NH}^{+}$ & $1.8 \times 10^{1}$ & $\mathrm{~N}_{2} \mathrm{H}^{+}$ & $6.3 \times 10^{0}$ & $\mathrm{HCO}^{+}$ & $9.8 \times 10^{-1}$ \\
\hline 30 & $\mathrm{CH}_{2} \mathrm{NH}_{2}^{+}$ & $7.6 \times 10^{1}$ & $\mathrm{C}_{2} \mathrm{H}_{6}^{+}$ & $7.6 \times 10^{-1}$ & & & & \\
\hline 31 & $\mathrm{CH}_{2}^{15} \mathrm{NH}_{2}^{+}$ & $2.0 \times 10^{0}$ & $\mathrm{C}_{2} \mathrm{H}_{7}^{+}$ & $1.6 \times 10^{0}$ & $\mathrm{CH}_{2} \mathrm{OH}^{+}$ & $2.9 \times 10^{-1}$ & & \\
\hline 32 & $\mathrm{CH}_{3} \mathrm{NH}_{3}{ }^{+}$ & $7.1 \times 10^{-1}$ & & & & & & \\
\hline 33 & $\mathrm{CH}_{3} \mathrm{OH}_{2}^{+}$ & $6.0 \times 10^{-1}$ & & & & & & \\
\hline 38 & $\mathrm{CNC}^{+}$ & $2.8 \times 10^{-1}$ & & & & & & \\
\hline 39 & $\mathrm{c}-\mathrm{C}_{3} \mathrm{H}_{3}^{+}$ & $1.4 \times 10^{2}$ & $1-\mathrm{C}_{3} \mathrm{H}_{3}^{+}$ & $8.5 \times 10^{0}$ & $\mathrm{HC}_{2} \mathrm{~N}^{+}$ & $2.1 \times 10^{0}$ & & \\
\hline 40 & $\mathrm{HC}_{2} \mathrm{NH}^{+}$ & $1.3 \times 10^{1}$ & $\mathrm{C}_{3} \mathrm{H}_{4}^{+}$ & $4.3 \times 10^{0}$ & & & & \\
\hline 41 & $\mathrm{C}_{3} \mathrm{H}_{5}^{+}$ & $4.8 \times 10^{2}$ & & & & & & \\
\hline 42 & $\mathrm{CH}_{3} \mathrm{CNH}^{+}$ & $1.8 \times 10^{2}$ & & & & & & \\
\hline 43 & $\mathrm{CH}_{3} \mathrm{C}^{15} \mathrm{NH}^{+}$ & $2.8 \times 10^{0}$ & $\mathrm{C}_{3} \mathrm{H}_{7}^{+}$ & $2.5 \times 10^{0}$ & & & & \\
\hline 50 & $\mathrm{C}_{4} \mathrm{H}_{2}^{+}$ & $1.1 \times 10^{-1}$ & & & & & & \\
\hline 51 & $\mathrm{C}_{4} \mathrm{H}_{3}^{+}$ & $7.1 \times 10^{1}$ & & & & & & \\
\hline
\end{tabular}


Table 9 - Continued from previous page

\begin{tabular}{|c|c|c|c|c|c|c|c|c|}
\hline $\mathrm{m} / \mathrm{z}$ & Species & Density $\left(\mathrm{cm}^{-3}\right)$ & Species & Density $\left(\mathrm{cm}^{-3}\right)$ & Species & Density $\left(\mathrm{cm}^{-3}\right)$ & Species & Density $\left(\mathrm{cm}^{-3}\right)$ \\
\hline 52 & $\mathrm{PHC}_{3} \mathrm{NH}^{+}$ & $3.8 \times 10^{1}$ & $\mathrm{C}_{4} \mathrm{H}_{4}{ }^{+}$ & $2.1 \times 10^{-1}$ & & & & \\
\hline 53 & $\mathrm{C}_{4} \dot{\mathrm{H}}_{5}^{+}$ & $4.7 \times 10^{1}$ & $\mathrm{HC}_{2} \mathrm{~N}_{2}^{+}$ & $1.1 \times 10^{-2}$ & & & & \\
\hline 54 & $\mathrm{C}_{2} \mathrm{H}_{3} \mathrm{CNH}^{+}$ & $8.4 \times 10^{1}$ & & & & & & \\
\hline 55 & $\mathrm{C}_{4} \mathrm{H}_{7}{ }^{+}$ & $9.3 \times 10^{0}$ & $\mathrm{C}_{2} \mathrm{H}_{3} \mathrm{C}^{15} \mathrm{NH}^{+}$ & $1.0 \times 10^{0}$ & $\mathrm{C}_{2} \mathrm{H}_{3} \mathrm{CO}^{+}$ & $5.1 \times 10^{-1}$ & & \\
\hline 56 & $\mathrm{C}_{2} \mathrm{H}_{5} \mathrm{CNH}^{+}$ & $1.7 \times 10^{1}$ & & & & & & \\
\hline 57 & $\mathrm{C}_{4} \mathrm{H}_{9}^{+}$ & $1.2 \times 10^{0}$ & & & & & & \\
\hline 58 & $\mathrm{C}_{3} \mathrm{H}_{5} \mathrm{NH}_{3}^{+}$ & $1.2 \times 10^{0}$ & & & & & & \\
\hline 63 & $\mathrm{C}_{5} \mathrm{H}_{3}^{+}$ & $1.6 \times 10^{0}$ & & & & & & \\
\hline 65 & $\mathrm{C}_{5} \mathrm{H}_{5}{ }^{+}$ & $1.4 \times 10^{2}$ & & & & & & \\
\hline 66 & $\mathrm{C}_{4} \mathrm{H}_{3} \mathrm{NH}^{+}$ & $4.2 \times 10^{1}$ & & & & & & \\
\hline 67 & $\mathrm{C}_{5} \mathrm{H}_{7}^{+}$ & $7.6 \times 10^{1}$ & & & & & & \\
\hline 68 & $\mathrm{C}_{4} \mathrm{H}_{5} \mathrm{NH}^{+}$ & $6.5 \times 10^{0}$ & & & & & & \\
\hline 70 & $\mathrm{C}_{4} \mathrm{H}_{7} \mathrm{NH}^{+}$ & $7.7 \times 10^{0}$ & & & & & & \\
\hline 75 & $\mathrm{C}_{6} \mathrm{H}_{3}^{+}$ & $1.7 \times 10^{0}$ & & & & & & \\
\hline 76 & $\mathrm{HC}_{5} \mathrm{NH}^{+}$ & $1.5 \times 10^{0}$ & $\mathrm{C}_{6} \mathrm{H}_{4}^{+}$ & $1.0 \times 10^{0}$ & & & & \\
\hline 77 & $\mathrm{C}_{6} \mathrm{H}_{5}{ }^{+}$ & $5.9 \times 10^{0}$ & $\mathrm{C}_{5} \mathrm{H}_{3} \mathrm{~N}^{+}$ & $5.4 \times 10^{-1}$ & & & & \\
\hline 78 & $\mathrm{C}_{5} \mathrm{H}_{3} \mathrm{NH}^{+}$ & $4.4 \times 10^{-1}$ & & & & & & \\
\hline 79 & $\mathrm{C}_{6} \mathrm{H}_{7}^{+}$ & $9.9 \times 10^{0}$ & & & & & & \\
\hline 80 & $\mathrm{C}_{5} \mathrm{H}_{5} \mathrm{NH}^{+}$ & $4.4 \times 10^{0}$ & & & & & & \\
\hline 81 & $\mathrm{C}_{6} \mathrm{H}_{9}{ }^{+}$ & $3.6 \times 10^{-1}$ & & & & & & \\
\hline 83 & $\mathrm{C}_{6} \mathrm{H}_{11}+$ & $3.6 \times 10^{-1}$ & & & & & & \\
\hline 89 & $\mathrm{C}_{7} \mathrm{H}_{5}^{+}$ & $8.4 \times 10^{-1}$ & & & & & & \\
\hline 90 & $\mathrm{C}_{6} \mathrm{H}_{3} \mathrm{NH}^{+}$ & $3.3 \times 10^{0}$ & & & & & & \\
\hline 91 & $\mathrm{C}_{7} \mathrm{H}_{7}^{+}$ & $3.0 \times 10^{1}$ & & & & & & \\
\hline 92 & $\mathrm{C}_{6} \mathrm{H}_{5} \mathrm{NH}^{+}$ & $1.2 \times 10^{0}$ & & & & & & \\
\hline
\end{tabular}


Table 9 - Continued from previous page

\begin{tabular}{|c|c|c|c|c|c|c|}
\hline $\mathrm{m} / \mathrm{z}$ & Species & Density $\left(\mathrm{cm}^{-3}\right) \quad$ Species & Density $\left(\mathrm{cm}^{-3}\right) \quad$ Species & Density $\left(\mathrm{cm}^{-3}\right)$ & Species & Density $\left(\mathrm{cm}^{-3}\right)$ \\
\hline 93 & $\mathrm{C}_{7} \mathrm{H}_{9}{ }^{+}$ & $2.0 \times 10^{0}$ & & & & \\
\hline 94 & $\mathrm{C}_{6} \mathrm{H}_{7} \mathrm{NH}^{+}$ & $6.4 \times 10^{-1}$ & & & & \\
\hline 96 & $\mathrm{C}_{6} \mathrm{H}_{9} \mathrm{NH}^{+}$ & $1.4 \times 10^{0}$ & & & & \\
\hline
\end{tabular}



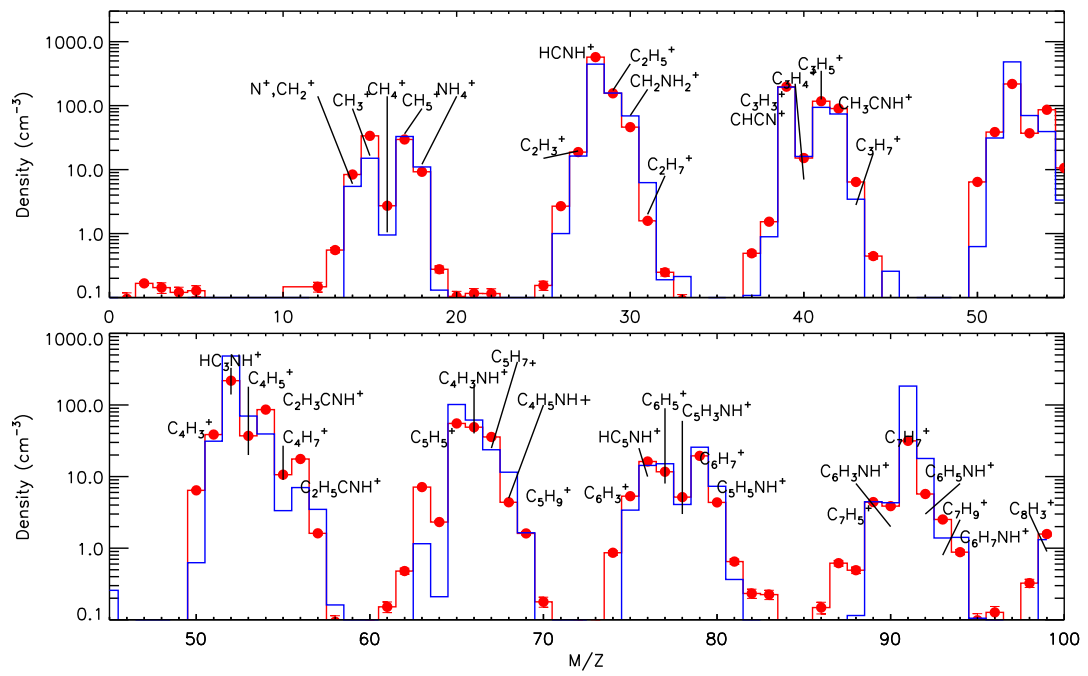

Figure 36: INMS observed during T40 (symbols) and modeled (lines) mass spectrum at 1100 $\mathrm{km}$.

${ }_{2733} \mathrm{~N}^{+} / \mathrm{CH}_{2}{ }^{+}, \mathbf{C H}_{3}{ }^{+}, \mathbf{C H}_{4}{ }^{+}, \mathbf{C H}_{5}{ }^{+}$. The ion species in the model con2734 taining one carbon atom are $\mathrm{C}^{+}, \mathrm{CH}^{+}, \mathrm{CH}_{2}{ }^{+}, \mathrm{CH}_{3}{ }^{+}, \mathrm{CH}_{4}{ }^{+}$and $\mathrm{CH}_{5}{ }^{+}$. The 2735 production rate of $\mathrm{C}^{+}$and $\mathrm{CH}^{+}$is too small for these ions to be detectable by 2736 INMS. $\mathrm{CH}_{2}{ }^{+}$at $\mathrm{m} / \mathrm{z}=14$ has a similar abundance as $\mathrm{N}^{+}$, while $\mathrm{CH}_{3}{ }^{+}, \mathrm{CH}_{4}{ }^{+}$ ${ }_{2737}$ and $\mathrm{CH}_{5}{ }^{+}$can be constrained by the observations. 


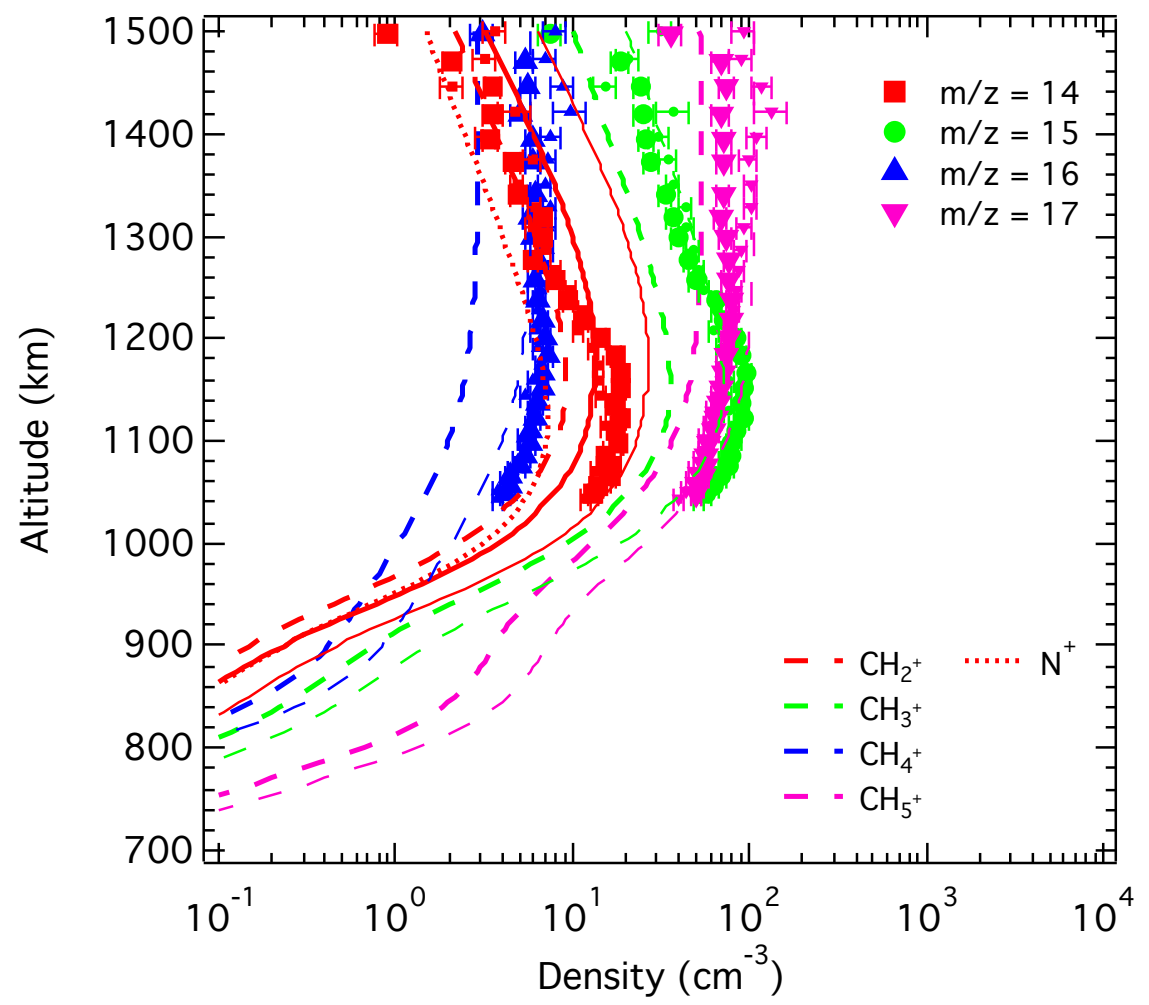

Figure 37: Model results for the density of $\mathrm{N}^{+} / \mathrm{CH}_{2}{ }^{+}, \mathrm{CH}_{3}{ }^{+}, \mathrm{CH}_{4}{ }^{+}$and $\mathrm{CH}_{5}{ }^{+}$and comparison to the T40 INMS ion density measurements at $\mathrm{m} / \mathrm{z} 14,15,16$ and 17 . The thin lines correspond to the model results multiplied by a factor of two, to approximate a daytime calculation.

The simulated profiles for $\mathrm{CH}_{2}{ }^{+} / \mathrm{N}^{+}, \mathrm{CH}_{3}{ }^{+}, \mathrm{CH}_{4}{ }^{+}$and $\mathrm{CH}_{5}{ }^{+}$are illustrated in Figure 37. In order to compare the model with data we scale the model by a factor of 2 to convert from global average to dayside values. The sum of the $\mathrm{N}^{+}$and $\mathrm{CH}_{2}{ }^{+}$profiles agrees roughly in magnitude and shape with the $\mathrm{m} / \mathrm{z}$ $=14$ densities in the vicinity of the peak with slightly worse agreement at high altitudes. This could be due to the lack of diffusion or magnetic forces in the model. "Dayside" $\mathrm{CH}_{3}{ }^{+}, \mathrm{CH}_{4}{ }^{+}$and $\mathrm{CH}_{5}{ }^{+}$are within the INMS error bars for $\mathrm{m} / \mathrm{z}=15,16$ and 17 , respectively.

$\mathrm{N}^{+}, \mathrm{N}_{2}{ }^{+}$and $\mathrm{CH}_{4}{ }^{+}$are the direct ionization products of $\mathrm{N}_{2}$ and $\mathrm{CH}_{4}$, respectively. Since $\mathrm{HCNH}^{+}$is the dominant species at $\mathrm{m} / \mathrm{z}=28$ (Cravens et al. (2009) and section 4.2.3.2), $\mathrm{CH}_{3}{ }^{+}$, the main product of the reaction of $\mathrm{N}_{2}{ }^{+}$ with $\mathrm{CH}_{4}$ (section 3.1.1.2), is the best tracer of the $\mathrm{N}_{2}{ }^{+}$density. The production rates depend on both the $\mathrm{CH}_{4}$ and $\mathrm{N}_{2}$ ionizing schemes, through the $\mathrm{N}_{2}$ high-resolution cross-sections, as first suggested by Lavvas et al. (2011a). Therefore, the perfect agreement between the observations and the simulated 
$\mathrm{CH}_{4}{ }^{+}$and $\mathrm{CH}_{3}{ }^{+}$densities when multiplied by a factor of 2 suggests that the primary ionization products of $\mathrm{N}_{2}$ are calculated accurately.

The $\mathrm{N}^{+}$modeled densities of Mandt et al. (2012a) are a factor of $\sim 5$ too high when compared to the data, while Westlake et al. (2012) and Dobrijevic et al. (2016) do not overpredict this ion. The results for the hydrocarbon ions in the simulations by Robertson et al. (2009), Mandt et al. (2012a), Westlake et al. (2012), Richard et al. (2015b) and Dobrijevic et al. (2016) exhibit densities close to the INMS measurements for $\mathrm{CH}_{3}{ }^{+}$and $\mathrm{CH}_{4}{ }^{+}$but densities too high by a factor of 2-3 for $\mathrm{CH}_{5}{ }^{+}$. Mandt et al. (2012a) emphasize that the use of the Lavvas et al. (2011a) high-resolution cross-sections for the photodissociation of $\mathrm{N}_{2}$ (80-100 nm) produce better results for both $\mathrm{CH}_{4}{ }^{+}$and $\mathrm{CH}_{3}{ }^{+}$. Although the strong effect that the high-resolution cross-sections have on the $\mathrm{CH}_{4}{ }^{+}$production rates has already been put forward and explained by Lavvas et al. (2011a), it is unclear why they should have some impact on $\mathrm{CH}_{3}{ }^{+}$as this ion is mostly formed through the reaction of $\mathrm{N}_{2}{ }^{+}$with $\mathrm{CH}_{4}\left(\mathrm{R}_{c n} 836 \mathrm{a}\right)$ and not through direct photoionization of $\mathrm{CH}_{4}$, which accounts only for $5 \%$ of the total $\mathrm{CH}_{3}{ }^{+}$production rate.

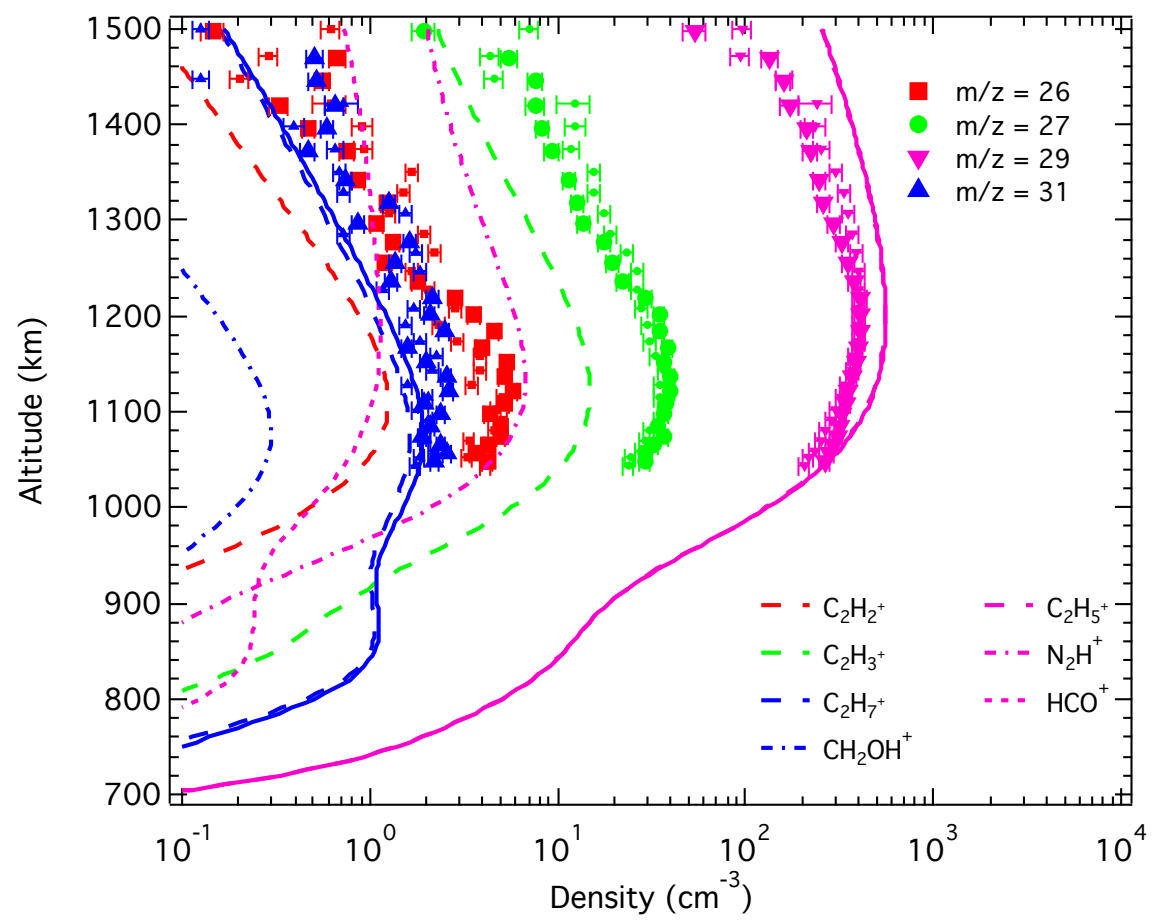

Figure 38: Model results for the density of $\mathrm{C}_{2} \mathrm{H}_{2}{ }^{+}, \mathrm{C}_{2} \mathrm{H}_{3}{ }^{+}, \mathrm{C}_{2} \mathrm{H}_{5}{ }^{+} / \mathrm{N}_{2} \mathrm{H}^{+} / \mathrm{HCO}^{+}$and $\mathrm{C}_{2} \mathrm{H}_{7}{ }^{+} / \mathrm{CH}_{2} \mathrm{OH}^{+}$and comparison with the T40 INMS ion density measurements at $\mathrm{m} / \mathrm{z}$ $=26,27,29,31$. 


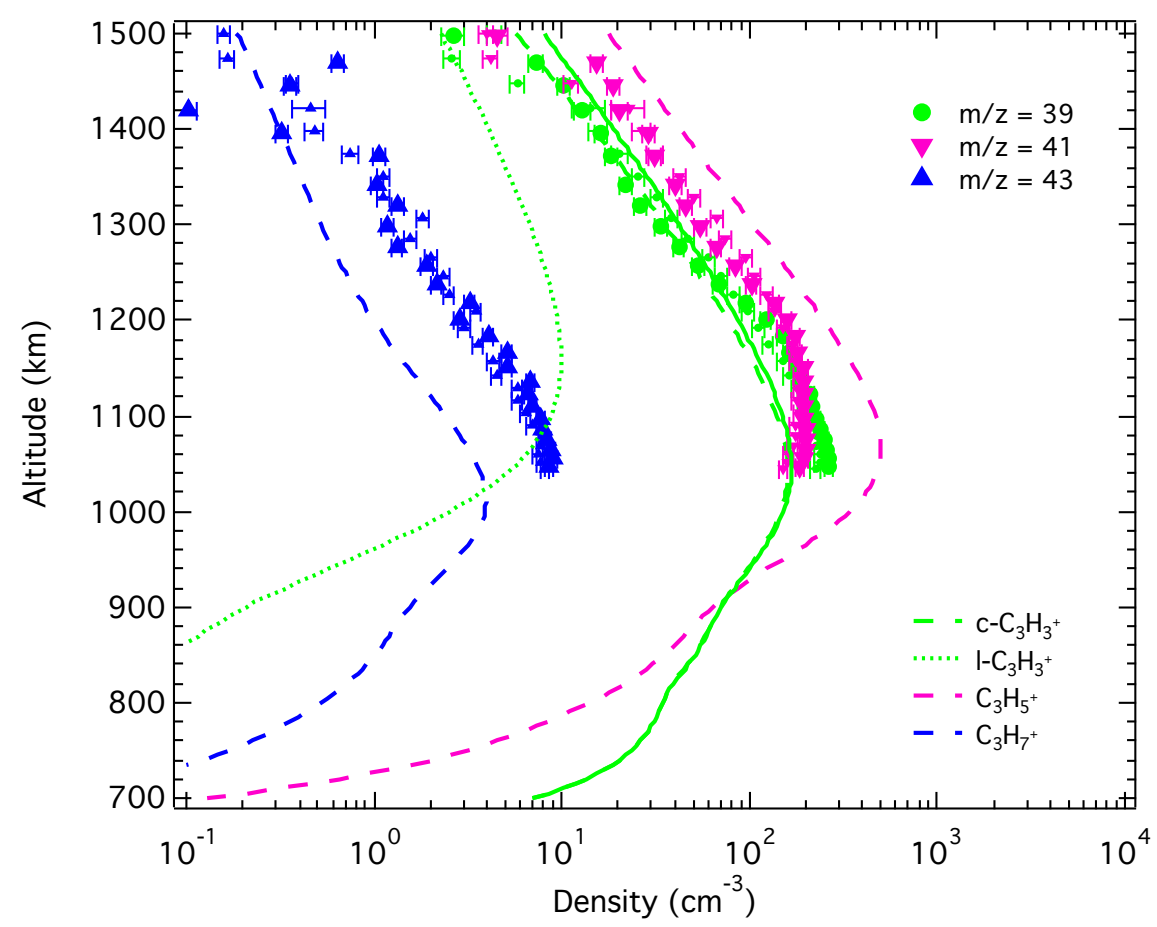

Figure 39: Model results for the density of c,l- $\mathrm{C}_{3} \mathrm{H}_{3}{ }^{+}, \mathrm{C}_{3} \mathrm{H}_{5}{ }^{+}$and $\mathrm{C}_{3} \mathrm{H}_{7}{ }^{+}$and comparison with the T40 INMS ion density measurements at $\mathrm{m} / \mathrm{z}=39,41,43$.

$\mathbf{C}_{\mathbf{3}} \mathbf{H}_{\mathbf{3}}{ }^{+}, \mathbf{C}_{\mathbf{3}} \mathbf{H}_{\mathbf{5}}{ }^{+}, \mathbf{C}_{\mathbf{3}} \mathbf{H}_{\mathbf{7}}{ }^{+}$. The signal at $\mathrm{m} / \mathrm{z}=39$ is attributed to $\mathrm{C}_{3} \mathrm{H}_{3}{ }^{+}$ and is well reproduced by the model (cf. Figure 39). At the top of the ionosphere, the $\mathrm{l}_{-} \mathrm{C}_{3} \mathrm{H}_{3}{ }^{+} / \mathrm{c}_{-} \mathrm{C}_{3} \mathrm{H}_{3}{ }^{+}$ratio is about one but because of the high reactivity of $1-\mathrm{C}_{3} \mathrm{H}_{3}{ }^{+}$with hydrocarbons, the ratio drops significantly below 1400 $\mathrm{km}$ to reach a value of $\sim 10 \%$ at the $\mathrm{c}_{-} \mathrm{C}_{3} \mathrm{H}_{3}{ }^{+}$peak at $1050 \mathrm{~km}$. Dobrijevic et al. (2016) slightly underestimates the $\mathrm{C}_{3} \mathrm{H}_{3}{ }^{+}$measured densities, which is consistent with the fact that they do not differentiate $\mathrm{l}_{-} \mathrm{C}_{3} \mathrm{H}_{3}{ }^{+}$and $\mathrm{c}-\mathrm{C}_{3} \mathrm{H}_{3}{ }^{+}$. Indeed, Carrasco et al. (2008) showed that the assumption that both isomers are identically reactive leads to an under prediction of $\mathrm{C}_{3} \mathrm{H}_{3}{ }^{+}$of the same magnitude (cf. their Figure 4).

The signal at $\mathrm{m} / \mathrm{z}=41$ is attributed to $\mathrm{C}_{3} \mathrm{H}_{5}{ }^{+}$(cf. Figure 39). The INMS ion density is overestimated by the model by a factor of $\sim 2$ above $1200 \mathrm{~km}$ and a factor of $\sim 4$ at the peak at $1060 \mathrm{~km}$. This can be partly explained by the overestimation of $\mathrm{C}_{2} \mathrm{H}_{5}{ }^{+}$, which contributes to $30 \%$ of the $\mathrm{C}_{3} \mathrm{H}_{5}{ }^{+}$production through its reaction with $\mathrm{C}_{2} \mathrm{H}_{4}\left(\mathrm{R}_{c n} 302 \mathrm{a}\right)$.

Finally, using the rate coefficient from Herbst et al. (2010), initial calculations showed that the radiative association reaction involving $\mathrm{C}_{3} \mathrm{H}_{5}{ }^{+}$and $\mathrm{H}_{2}$ was an efficient source of protonated propene: 


$$
\mathrm{C}_{3} \mathrm{H}_{5}^{+}+\mathrm{H}_{2} \rightarrow \mathrm{C}_{3} \mathrm{H}_{7}^{+}+\mathrm{h} \nu .
$$

This reaction lead to a large overestimation of the signal at $\mathrm{m} / \mathrm{z}=43$. However, a combination of experiments and more refined quantum chemical ab initio calculations reported later on (Lin et al., 2013) does not corroborate the Herbst et al. (2010) rate coefficient. Indeed, the latter approach shows that this reaction does not occur efficiently at cold temperatures due to activation energy barriers. Without this reaction, the signal at $\mathrm{m} / \mathrm{z}=43$ is now well reproduced by the model (cf. Figure 39).

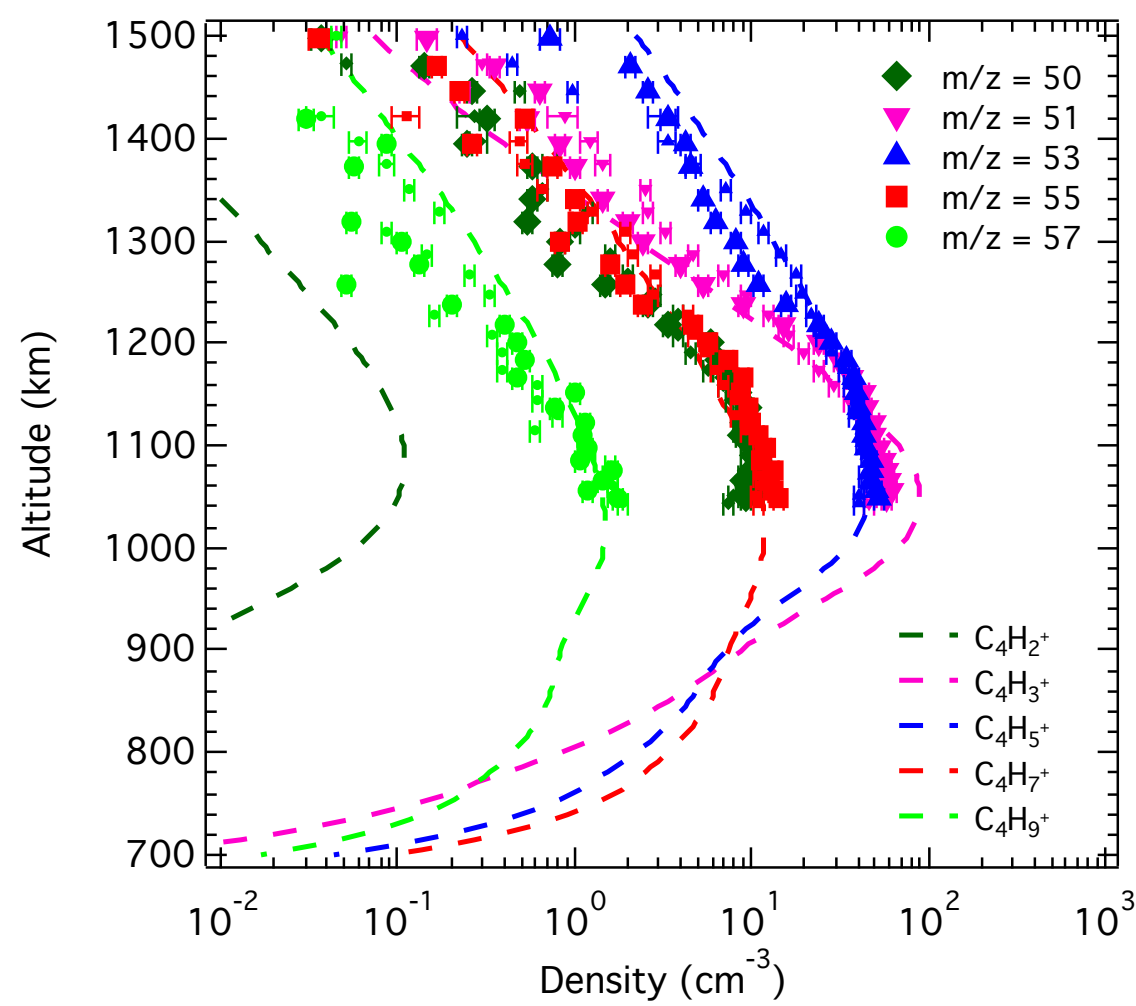

Figure 40: Model results for the density of $\mathrm{C}_{4} \mathrm{H}_{2}{ }^{+}, \mathrm{C}_{4} \mathrm{H}_{3}{ }^{+}, \mathrm{C}_{4} \mathrm{H}_{5}{ }^{+}, \mathrm{C}_{4} \mathrm{H}_{7}{ }^{+}$and $\mathrm{C}_{4} \mathrm{H}_{9}{ }^{+}$and comparison with the T40 INMS ion density measurements at $\mathrm{m} / \mathrm{z}=50,51,53,55,57$.

$\mathbf{C}_{4} \mathrm{H}_{2}{ }^{+}, \mathrm{C}_{4} \mathrm{H}_{3}{ }^{+}, \mathrm{C}_{4} \mathrm{H}_{5}{ }^{+}, \mathrm{C}_{4} \mathrm{H}_{7}{ }^{+}, \mathrm{C}_{4} \mathbf{H}_{9}{ }^{+}$. As already discussed in Vuitton et al. (2007), our model cannot reproduce the observed ion density at $\mathrm{m} / \mathrm{z}=50$ (cf. Figure 40). The major ion at this channel is $\mathrm{C}_{4} \mathrm{H}_{2}{ }^{+}$but its density at the peak is a factor of 45 lower than measured. $\mathrm{C}_{4} \mathrm{H}_{2}{ }^{+}$is formed by charge transfer from $\mathrm{C}_{2} \mathrm{H}_{2}$ to $\mathrm{C}_{4} \mathrm{H}_{2}$ (McEwan and Anicich, 2007): 


$$
\mathrm{C}_{2} \mathrm{H}_{2}^{+}+\mathrm{C}_{4} \mathrm{H}_{2} \rightarrow \mathrm{C}_{4} \mathrm{H}_{2}^{+}+\mathrm{C}_{2} \mathrm{H}_{2} . \quad\left(\mathrm{R}_{c n} 240 \mathrm{a}\right)
$$

As discussed above, the model underestimates $\mathrm{C}_{4} \mathrm{H}_{2}$ in the thermosphere, which implies that the production rate of $\mathrm{C}_{4} \mathrm{H}_{2}^{+}$through this reaction is underestimated, as shown in Figure 40. However, this difference cannot account for the factor of 40 between observed and modeled densities. In low-pressure plasma conditions, reaction of $\mathrm{C}_{2} \mathrm{H}^{+}$with $\mathrm{C}_{2} \mathrm{H}_{2}$ serves as one of the main routes to formation of $\mathrm{C}_{4} \mathrm{H}_{2}{ }^{+}$, after a loss of $\mathrm{H}$ atom (Benedikt, 2010). An association reaction of $\mathrm{C}_{2}{ }^{+}$with $\mathrm{C}_{2} \mathrm{H}_{2}$ can also form some $\mathrm{C}_{4} \mathrm{H}_{2}{ }^{+}$as discussed in Bera et al. (2015). But neither $\mathrm{C}_{2} \mathrm{H}^{+}$or $\mathrm{C}_{2}{ }^{+}$are detected by INMS (Vuitton et al., 2007) and it is still unclear what production reaction(s) are missing for $\mathrm{C}_{4} \mathrm{H}_{2}{ }^{+}$.

The signal at $\mathrm{m} / \mathrm{z}=51$ is attributed to $\mathrm{C}_{4} \mathrm{H}_{3}{ }^{+}$and is well reproduced by the model (cf. Figure 40). This might seem surprising since $\mathrm{C}_{4} \mathrm{H}_{3}{ }^{+}$is closely related to $\mathrm{C}_{4} \mathrm{H}_{2}$ through proton transfer reactions $\left(\mathrm{R}_{c n} 700, \mathrm{R}_{c n} 308\right)$ and since the model profile of $\mathrm{C}_{4} \mathrm{H}_{2}$ is not in very good agreement with the observations in the thermosphere. However, both $\mathrm{HCNH}^{+}$and $\mathrm{C}_{2} \mathrm{H}_{5}{ }^{+}$, the precursor ions of $\mathrm{C}_{4} \mathrm{H}_{3}{ }^{+}$, are overestimated, which essentially compensates the underestimation of $\mathrm{C}_{4} \mathrm{H}_{2}$.

The signals at $\mathrm{m} / \mathrm{z}=53,55$ and 57 are attributed to $\mathrm{C}_{4} \mathrm{H}_{5}{ }^{+}, \mathrm{C}_{4} \mathrm{H}_{7}{ }^{+}$and $\mathrm{C}_{4} \mathrm{H}_{9}{ }^{+}$and are all very well reproduced by the model (cf. Figure 40). 


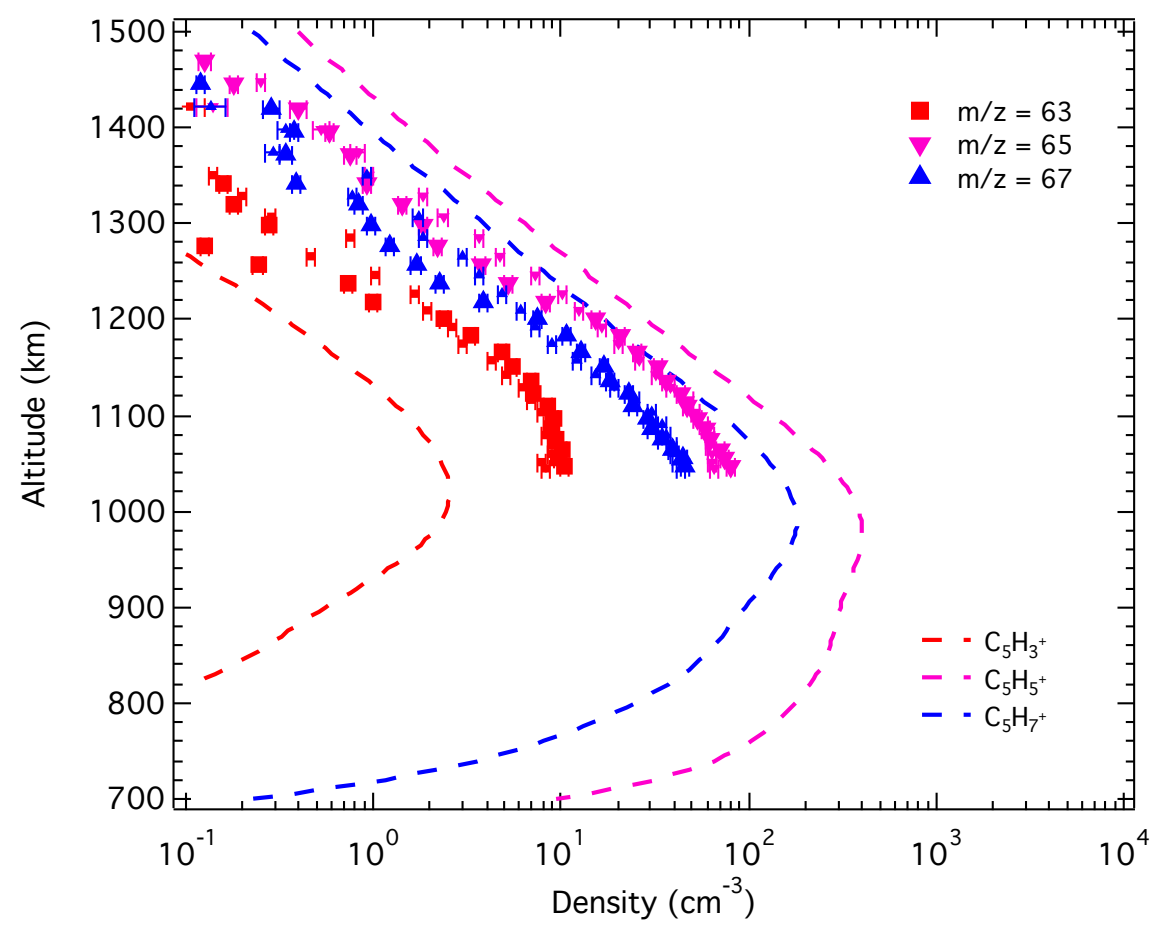

Figure 41: Model results for the density of $\mathrm{C}_{5} \mathrm{H}_{3}{ }^{+}, \mathrm{C}_{5} \mathrm{H}_{5}{ }^{+}$, and $\mathrm{C}_{5} \mathrm{H}_{7}{ }^{+}$and comparison with the T40 INMS ion density measurements at $\mathrm{m} / \mathrm{z}=63,65,67$.

$\mathbf{C}_{5} \mathbf{H}_{3}{ }^{+}, \mathbf{C}_{\mathbf{5}} \mathbf{H}_{\mathbf{5}}{ }^{+}, \mathbf{C}_{5} \mathbf{H}_{\mathbf{7}}{ }^{+}$. The signal at $\mathrm{m} / \mathrm{z}=63$ is attributed to $\mathrm{C}_{5} \mathrm{H}_{3}{ }^{+}$ but is underestimated in the model by a factor of about 5 (cf. Figure 41). $\mathrm{C}_{5} \mathrm{H}_{3}{ }^{+}$ formation reactions all involve $\mathrm{C}_{4} \mathrm{H}_{2}\left(\mathrm{R}_{c n} 287 \mathrm{a}, \mathrm{R}_{c n} 393 \mathrm{~b}, \mathrm{R}_{c n} 138 \mathrm{~b}\right)$ and again its underestimation can mostly explain the incapacity of the model to properly predict the $\mathrm{C}_{5} \mathrm{H}_{3}{ }^{+}$density.

The signals at $\mathrm{m} / \mathrm{z}=65$ and 67 are attributed to $\mathrm{C}_{5} \mathrm{H}_{5}{ }^{+}$and $\mathrm{C}_{5} \mathrm{H}_{7}{ }^{+}$, respectively. They both are overestimated by the model by a factor of about 2 (cf. Figure 41), which is still a fair agreement since the rate coefficient for the main loss processes, electron recombination $\left(\mathrm{R}_{e r} 51, \mathrm{R}_{e r} 53\right)$, is an estimate. 


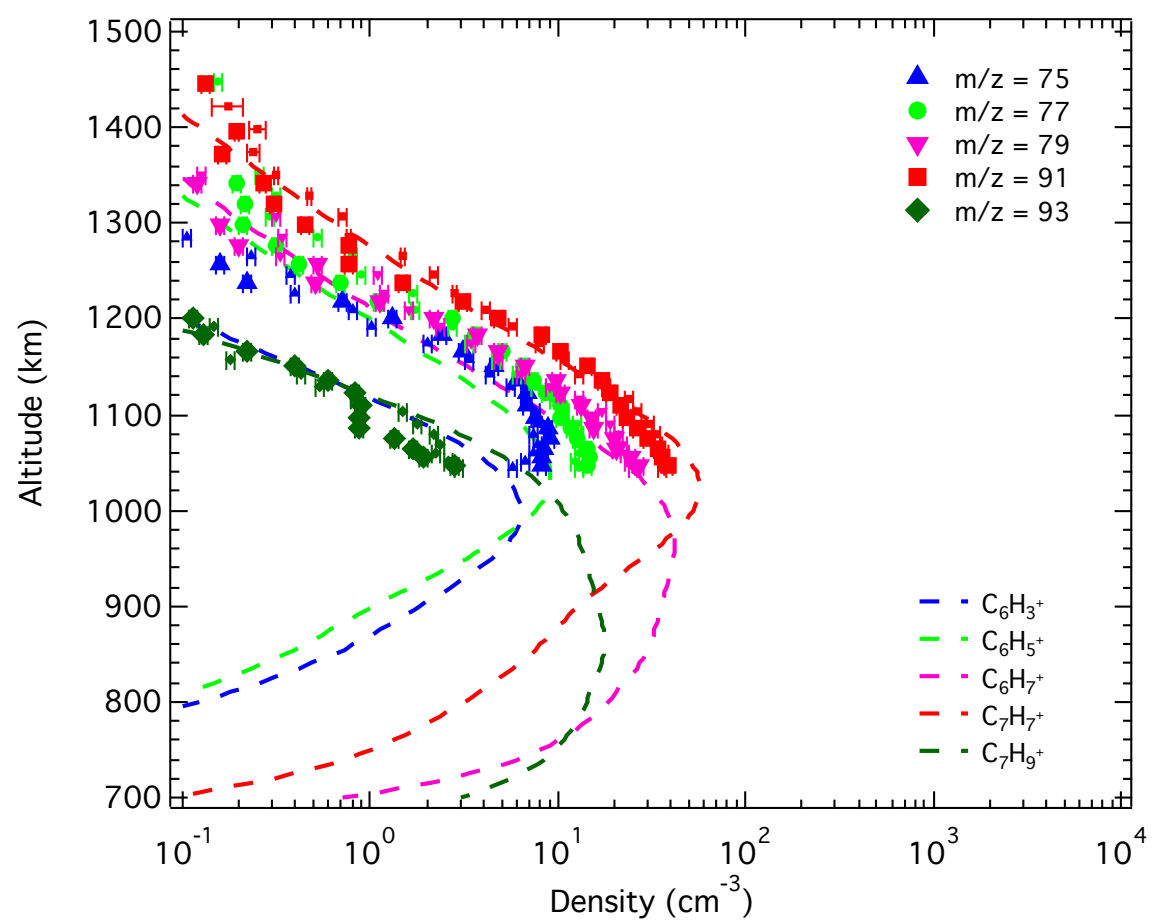

Figure 42: Model results for the density of $\mathrm{C}_{6} \mathrm{H}_{3}{ }^{+}, \mathrm{C}_{6} \mathrm{H}_{5}{ }^{+}, \mathrm{C}_{6} \mathrm{H}_{7}{ }^{+}, \mathrm{C}_{7} \mathrm{H}_{7}{ }^{+}$and $\mathrm{C}_{7} \mathrm{H}_{9}{ }^{+}$and comparison with the T40 INMS ion density measurements at $\mathrm{m} / \mathrm{z}=75,77,79,91,93$.

$\mathbf{C}_{6} \mathbf{H}_{3}{ }^{+}, \mathbf{C}_{6} \mathbf{H}_{5}{ }^{+}, \mathbf{C}_{6} \mathbf{H}_{\mathbf{7}}{ }^{+}, \mathbf{C}_{\mathbf{7}} \mathbf{H}_{\mathbf{7}}{ }^{+}, \mathbf{C}_{\mathbf{7}} \mathbf{H}_{\mathbf{9}}{ }^{+}$. The signal at $\mathrm{m} / \mathrm{z}=75$ is attributed to $\mathrm{C}_{6} \mathrm{H}_{3}{ }^{+}$and is underestimated by the model by a factor of 4 (cf. Figure 42). The production of $\mathrm{C}_{6} \mathrm{H}_{3}{ }^{+}$is directly related to the $\mathrm{C}_{4} \mathrm{H}_{2}$ density through reaction $\left(\mathrm{R}_{c n} 481 \mathrm{a}\right)$ and the underestimation of $\mathrm{C}_{6} \mathrm{H}_{3}{ }^{+}$may be interpreted as a consequence of the underestimation of $\mathrm{C}_{4} \mathrm{H}_{2}$. Another possible explanation relies on the observation that the loss processes for $\mathrm{C}_{6} \mathrm{H}_{3}{ }^{+}$, reaction with acetylene and electron recombination, both suffer from large errors. Radiative association of $\mathrm{C}_{6} \mathrm{H}_{3}^{+}$to $\mathrm{C}_{2} \mathrm{H}_{2}$ is reported in McEwan and Anicich (2007) to be extremely efficient with a rate coefficient of $2.3 \times 10^{-10} \mathrm{~cm}^{3} \mathrm{~s}^{-1}$. However, two different structures of $\mathrm{C}_{6} \mathrm{H}_{3}{ }^{+}$are known to exist, one reactive with $\mathrm{C}_{2} \mathrm{H}_{2}$ and one unreactive (Anicich et al., 2003, 2006). It is possible that the isomer produced in reaction $\left(\mathrm{R}_{c n} 481 \mathrm{a}\right)$ is mostly the unreactive one and that the loss of $\mathrm{C}_{6} \mathrm{H}_{3}{ }^{+}$with acetylene is largely overestimated in the model. Also, both rate coefficient and products of the $\mathrm{C}_{6} \mathrm{H}_{3}{ }^{+}$recombination with electrons are unknown and have been estimated, and the actual reactivity may be lower.

The signals at $\mathrm{m} / \mathrm{z}=77$ and 79 are attributed to $\mathrm{C}_{6} \mathrm{H}_{5}{ }^{+}$and $\mathrm{C}_{6} \mathrm{H}_{7}{ }^{+}$, respectively, and both channels are very well reproduced by the model and this despite the fact that the model does not match the $\mathrm{C}_{4} \mathrm{H}_{2}{ }^{+}$densities (cf. Figure 42). In Vuitton et al. (2008), $\mathrm{C}_{4} \mathrm{H}_{2}{ }^{+}$was identified as the major precursor of 
$\mathrm{C}_{6} \mathrm{H}_{5}{ }^{+}$(through reaction $\left(\mathrm{R}_{c n} 465 \mathrm{c}\right)$ ) and had to be set to the observation values for the model to reproduce the abundance of $\mathrm{C}_{6} \mathrm{H}_{5}{ }^{+}$. Here, the model matches the $\mathrm{C}_{6} \mathrm{H}_{5}{ }^{+}$observations, even though the $\mathrm{C}_{4} \mathrm{H}_{2}{ }^{+}$predictions are about one order of magnitude off. This is particularly interesting because both species are important intermediates in the production of $\mathrm{C}_{6} \mathrm{H}_{6}$ (section 3.1.1.2 and Vuitton et al. (2008)).

The signals at $\mathrm{m} / \mathrm{z}=91$ and 93 are attributed to $\mathrm{C}_{7} \mathrm{H}_{7}{ }^{+}$and $\mathrm{C}_{7} \mathrm{H}_{9}{ }^{+}$and are well reproduced by the model (cf. Figure 42).

\subsubsection{Negative Ions}

4.1.2.1. Observations. Although it was not designed to detect ions, CAPS-ELS showed evidence for negatively charged ions up to $\sim 10,000 \mathrm{u}$ including three distinct low mass peaks (Coates et al., 2007). Because of the poor energy resolution of the analyzer, the exact mass-to-charge ratio of the negative ions cannot be retrieved and only broad mass ranges giving the approximate location of the peaks can be determined. The mass range of the three first mass groups are provided in Table 10. Wellbrock et al. (2013) presented, for 34 Titan encounters, the negative ion density trend versus altitude for each mass group. They found that the average peak altitude for the three low mass groups is about $1060 \mathrm{~km}$ and that the peak density varies by about one order of magnitude from flyby to flyby, with densities reaching up to 112,92 and $36 \mathrm{~cm}^{-3}$ for mass group 1 , 2 and 3, respectively. The total negative ion densities inferred from the Radio and Plasma Wave Science (RPWS) Langmuir Probe for 47 flybys (Shebanits et al., 2013) are in general agreement with the values retrieved by CAPS-ELS, although a specific comparison of both datasets is still warranted.

Table 10: Negative ions mass groups.

\begin{tabular}{lll}
\hline \hline Mass group & Mass range $(\mathrm{u})$ & Negative ions \\
\hline \hline 1 & $12-30$ & $\mathrm{H}^{-}, \mathrm{CH}_{2}^{-}, \mathrm{CH}_{3}^{-}, \mathrm{C}_{2} \mathrm{H}^{-}, \mathrm{CN}^{-}, \mathrm{O}^{-}, \mathrm{OH}^{-}$ \\
2 & $30-55$ & $\mathrm{C}_{4} \mathrm{H}^{-}, \mathrm{C}_{3} \mathrm{~N}^{-}$ \\
3 & $55-95$ & $\mathrm{C}_{6} \mathrm{H}^{-}, \mathrm{C}_{5} \mathrm{~N}^{-}$ \\
\hline \hline
\end{tabular}

4.1.2.2. Density Profiles. The calculated densities of the 11 negative ion species included in our model are given in Figure 43. The 7 ions falling into mass group 1 are shown in panel a, while the ions falling into mass groups 2 and 3 are shown in panel b (cf. Table 10). As in Vuitton et al. (2009), $\mathrm{CN}^{-}$is the most prominent ion with a peak density of $\sim 1 \mathrm{~cm}^{-3}$ at $1050 \mathrm{~km}$ and it contributes almost entirely to the total density in mass group $1 . \mathrm{H}^{-}, \mathrm{CH}_{2}{ }^{-}$and $\mathrm{CH}_{3}{ }^{-}$reach peak densities of $10^{-1}$ (at $1345 \mathrm{~km}$ ), $2 \times 10^{-2}$ (at $1260 \mathrm{~km}$ ) and $10^{-3} \mathrm{~cm}^{-3}$ (at $1165 \mathrm{~km}$ ), respectively. This $\mathrm{H}^{-}$density is a factor of about 200 higher than that calculated in Vuitton et al. (2009), which is mostly explained by the much higher $\mathrm{CH}_{4}$ dissociative electron attachment cross-section used in the current study (cf. section 2.7.3.3). The $\mathrm{C}_{2} \mathrm{H}^{-}$density reaches $3.6 \times 10^{-2} \mathrm{~cm}^{-3}$ at 1270 $\mathrm{km}$, which is higher by about one order of magnitude compared to Vuitton et al. 
(2009). Because $\mathrm{C}_{2} \mathrm{H}^{-}$originates mostly from $\mathrm{H}^{-}$, the higher $\mathrm{H}^{-}$density in this model compared to Vuitton et al. (2009) is directly responsible for the higher $\mathrm{C}_{2} \mathrm{H}^{-}$density. This is in line with the results of Dobrijevic et al. (2016). $\mathrm{O}^{-}$ and $\mathrm{OH}^{-}$both reach a negligible peak density of about $7 \times 10^{-6} \mathrm{~cm}^{-3}$ close to $1200 \mathrm{~km}$.

Both $\mathrm{C}_{4} \mathrm{H}^{-}$and $\mathrm{C}_{3} \mathrm{~N}^{-}$contribute to the density in mass group 2, with $\mathrm{C}_{4} \mathrm{H}^{-}$ dominating above $1140 \mathrm{~km}$ and $\mathrm{C}_{3} \mathrm{~N}^{-}$below, with peak densities of $5.7 \times 10^{-2}$ $\mathrm{cm}^{-3}$ (at $\left.1165 \mathrm{~km}\right)$ and $1.1 \times 10^{-1} \mathrm{~cm}^{-3}$ (at $1050 \mathrm{~km}$ ), respectively. For mass group $3, \mathrm{C}_{6} \mathrm{H}^{-}$and $\mathrm{C}_{5} \mathrm{~N}^{-}$are almost equally abundant below $1040 \mathrm{~km}$ but $\mathrm{C}_{5} \mathrm{~N}^{-}$dominates in the upper ionosphere. Their peak density is $7.3 \times 10^{-2}$ $\mathrm{cm}^{-3}$ (at $1065 \mathrm{~km}$ ) and $2.7 \times 10^{-1} \mathrm{~cm}^{-3}$ (at $1145 \mathrm{~km}$ ), respectively. This is somewhat different from Vuitton et al. (2009), where the nitrile ions $\left(\mathrm{C}_{3} \mathrm{~N}^{-}\right.$, $\left.\mathrm{C}_{5} \mathrm{~N}^{-}\right)$dominated over the hydrocarbon ions $\left(\mathrm{C}_{4} \mathrm{H}^{-}, \mathrm{C}_{6} \mathrm{H}^{-}\right)$. Indeed, the $\mathrm{C}_{6} \mathrm{H}^{-}$ densities are higher by about one order of magnitude in this model compared to Vuitton et al. (2009). It is the higher $\mathrm{C}_{6} \mathrm{H}$ density in this model compared to Vuitton et al. (2009) that translates into a higher density for this ion. In Vuitton et al. (2009), the abundances of the neutral species originated from the Lavvas et al. (2008b) model scaled to match the INMS observations, while they are now calculated self-consistently. Desai et al. (2017) recently reported that the third peak appears compatible with $\mathrm{C}_{5} \mathrm{~N}^{-} / \mathrm{C}_{6} \mathrm{H}^{-}$at higher altitudes but that at lower altitudes this peak widens, extending as high as $\sim 94 \mathrm{u}$ in some instances. This indicates the presence of anionic structures other than linear chains and Desai et al. (2017) suggest a number of possible anions in this range.

The negative ion production is initiated by dissociative and radiative electron attachment, which depend on the energetic and thermal electron distributions, respectively. These distributions heavily depend on solar illumination conditions (Ågren et al., 2009), which probably explains at least some of the variability in density and altitude of the peaks observed in the CAPS-ELS data (cf. Figure 2 of Wellbrock et al. (2013)). Our model is meant to reproduce globally averaged conditions and the calculated negative ion profiles should not be too far from the average altitudes and densities that can be retrieved from the observations. Indeed, the altitude of the calculated density peak for the three mass groups (cf. right panel in Figure 43) is in fair agreement with the CAPS-ELS data. However, as already observed at $1015 \mathrm{~km}$ for the T40 encounter, our calculated ion densities fall short by one to two orders of magnitude compared to the observations, a conclusion also shared by Dobrijevic et al. (2016).

A first possibility is that we are missing a source of light negative ions in our models. It seems quite difficult to believe that our bottom-up approach to generate negative ions could be enhanced by such a large factor. However, it may be possible that once formed, heavy ions fragment back to light ions in a top-down process. Pirim et al. (2015) reported $\mathrm{H}^{-}$desorption during lowenergy $(3-15 \mathrm{eV})$ electron irradiation of tholin materials maintained at $130 \mathrm{~K}$. Another explanation is related to the fact that the efficiency of the CAPS-ELS microchannel plates was never tested using negative ions. A value of $5 \%$ was used based upon the extensive work by Fraser (2002) for species detected at $>1$ $\mathrm{keV}$. Studies have however shown that at energies of $<1 \mathrm{keV}$ this can be up to 
an order or magnitude larger for negative ions (Peko and Stephen, 2000), and calibration studies should be performed to confirm the densities published so far. Although the total ion densities inferred from RPWS-LP (Shebanits et al., 2013) support those provided by CAPS-ELS, heavy ions dominate over light ions at the ionospheric peak, which implies that the RPWS-LP dataset does not provide any constrain for the light ions.

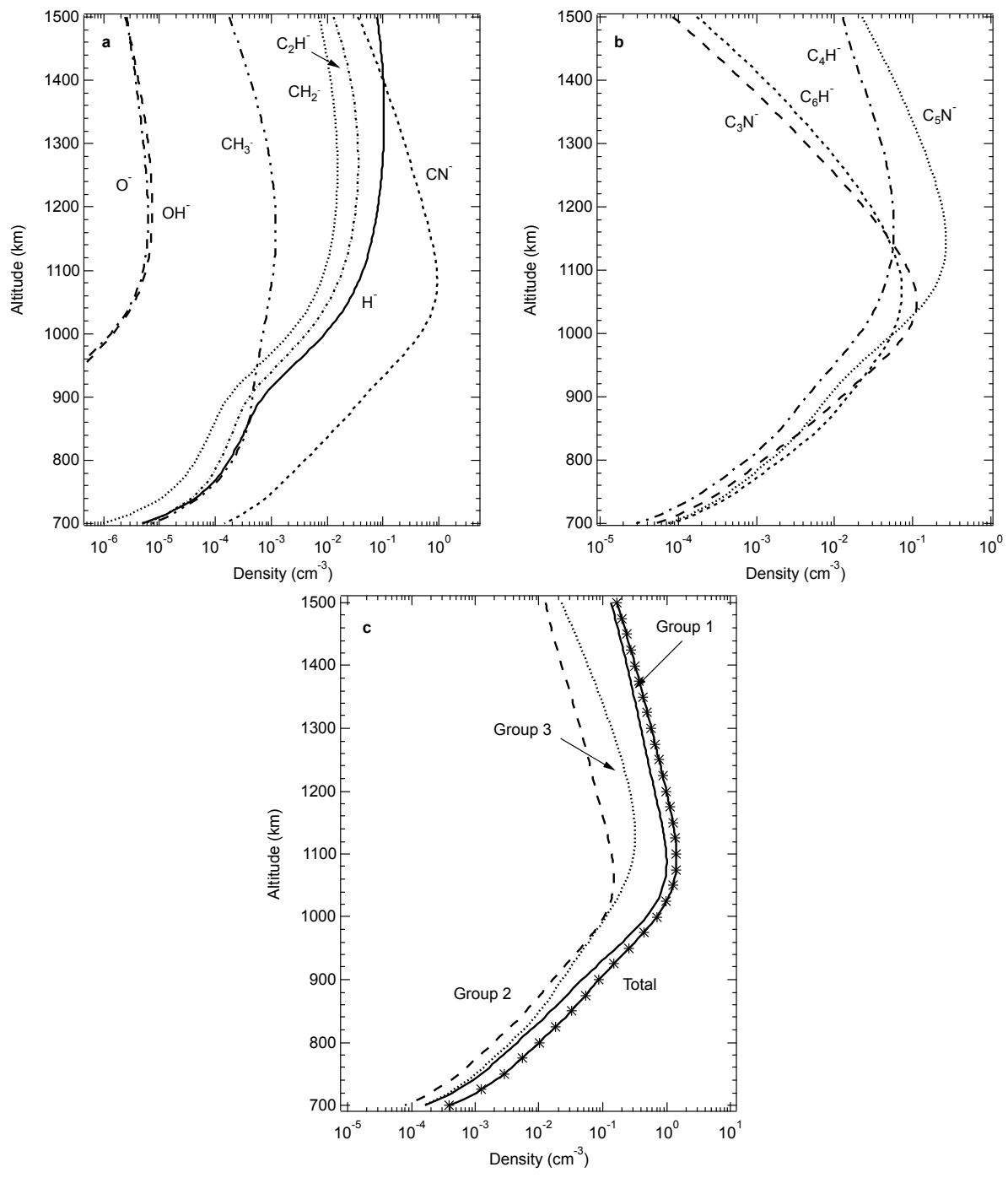

Figure 43: Calculated negative ion densities (upper left panel) $\mathrm{H}^{-}, \mathrm{CH}_{2}{ }^{-}, \mathrm{CH}_{3}{ }^{-}, \mathrm{O}^{-}, \mathrm{OH}^{-}$, (upper right panel) $\mathrm{C}_{2} \mathrm{H}^{-}, \mathrm{C}_{4} \mathrm{H}^{-}, \mathrm{C}_{6} \mathrm{H}^{-}, \mathrm{CN}^{-}, \mathrm{C}_{3} \mathrm{~N}^{-}, \mathrm{C}_{5} \mathrm{~N}^{-}$and (lower panel) sum of the ions constituting the three mass groups as defined in Table 10. 


\subsection{Neutral Atmosphere}

\subsubsection{Previous Models}

Four other teams have been working on the understanding of Titan's neutral atmospheric chemistry through photochemical models: Krasnopolsky (2009, 2010, 2012, 2014) constructed a model where the neutral and ion chemistry are fully coupled; the Bordeaux team (Hébrard et al., 2012, 2013; Dobrijevic et al., 2014; Loison et al., 2015; Dobrijevic et al., 2016) exhibited a model whose main specificity is its capacity to study the propagation of uncertainties; Lara (Moreno et al., 2012; Lara et al., 2014) updated an older neutral model first described in Lara et al. (1994) in support of the Herschel observations; the Caltech group revived the pioneering work of Yung et al. (1984) to study hydrocarbon and nitrogen (including isotopologues) chemistry (Liang et al., 2007; Li et al., 2014, 2015; Willacy et al., 2016).

Although Krasnopolsky $(2009,2010,2014)$ and Dobrijevic et al. (2016) discuss the chemistry as a whole, some of these models focus on a specific topic or class of molecules. For example, Hébrard et al. (2012) discuss the neutral production of hydrogen isocyanide (HNC) and hydrogen cyanide (HCN), while Loison et al. (2015) and Willacy et al. (2016) are mostly interested in N-bearing species. Hébrard et al. (2013) put the emphasis on $\mathrm{C}_{3} \mathrm{H}_{p}$ hydrocarbons and Krasnopolsky (2012), Moreno et al. (2012), Dobrijevic et al. (2014) and Lara et al. (2014) on O-bearing species, especially $\mathrm{H}_{2} \mathrm{O}$.

In general, these models take into account the same physical and chemical processes (dissociation of $\mathrm{N}_{2}$ and $\mathrm{CH}_{4}$ by photons and charged particles followed by a complex chemistry, transport by eddy mixing and molecular diffusion, condensation close to the tropopause, etc.) and only differ on the quantitative treatment of these processes, such as different rate coefficients, eddy mixing coefficients, saturation vapor pressure expressions, etc. Because of the large number of degrees of freedom, it is often extremely difficult to track down why the model outputs differ sometimes significantly from each other. Nevertheless, we will in the next section compare the results obtained by the different models and discuss these differences, when possible.

\subsubsection{Observations}

The abundant measurements from Cassini-Huygens and Earth-based efforts over the past 13 years have substantially increased the amount of information that we have about the composition of Titan's atmosphere and its spatial and temporal variations. The completeness in observational coverage (both spatially and temporally) varies substantially by molecule. Rather than compare our model calculations to every observation, we have compiled a set of representative observations with a preference for measurements that provide altitude profiles from Cassini-Huygens when possible. There appears to be little, if any, inter-annual variability detected when comparing Voyager and Cassini observations (Coustenis et al., 2013, 2016). For molecules where observations appear to disagree, as is the case for $\mathrm{H}_{2} \mathrm{O}$, we include them all. We also note that some measurements were published as a function of altitude, while others were 
published as a function of pressure. Although pressure (or number density) is the more relevant parameter, it was easier to provide a consistent comparison between observations using altitude. Representative values and references are listed in Table 11, which focuses on the equatorial and mid-latitudes as the observations allow. We include upper limit measurements, shown in Table 12, only when detections at a similar altitude have not been published and avoid disk-integrated measurements unless they are the only measurements available.

At the surface and for most of the troposphere, composition measurements are limited exclusively to the Gas Chromatograph/Mass Spectrometer (GCMS) (Niemann et al., 2005, 2010). In the stratosphere and the mesosphere, observations come from a combination of spacecraft measurements (Voyager, Cassini) and Earth-based observations (e.g., Infrared Space Observatory (ISO), Atacama Large Millimeter Array (ALMA), Institut de radioastronomie millimtrique (IRAM), Herschel). For Voyager, the measurements were obtained by the Infrared Interferometer Spectrometer (IRIS), which covered the upper troposphere/stratosphere. Cassini remote sensing composition measurements cover the upper troposphere (Cassini Composite Infrared Spectrometer CIRS), stratosphere (CIRS and Visible Infrared Mapping Spectrometer (VIMS)), and mesosphere (VIMS and Ultraviolet Imaging Spectrograph UVIS) (cf. Figure 1 in Hörst (2017)). It is important to note that the altitude ranges covered by these instruments are not well-defined as the ability to observe molecules depends on their abundances, so observations of trace species are sometimes possible only for smaller altitude ranges (or limited to specific latitudes). The neutral ionospheric composition is provided by measurements from the closed source neutral mode (CSN) of the Ion and Neutral Mass Spectrometer (INMS) carried by Cassini. The CSN INMS measurements we use for comparison come from averaging a number of flybys to increase signal to noise; these measurements provide altitude profiles but average out any latitude variations.

Due to the length of the mission, Cassini CIRS has provided substantial information about variations with latitude/season for a number of molecules. The model presented here is 1-D and therefore does not capture these types of variations. The strength of seasonal variations in Titan's atmospheric composition depends on the chemical lifetime of the species (c.f. Lavvas et al. (2008a) and section 5.4). Long-lived species (e.g., $\mathrm{C}_{2} \mathrm{H}_{2}, \mathrm{C}_{2} \mathrm{H}_{6}, \mathrm{C}_{3} \mathrm{H}_{8}, \mathrm{CO}_{2}$ ) exhibit very little seasonal (latitudinal) variation compared to short-lived species (e.g., $\left.\mathrm{C}_{2} \mathrm{H}_{4}, \mathrm{C}_{3} \mathrm{H}_{4}, \mathrm{C}_{4} \mathrm{H}_{2}, \mathrm{C}_{6} \mathrm{H}_{6}, \mathrm{HC}_{3} \mathrm{~N}\right)$. In assessing the performance of the model, we compare the model to the equatorial and mid-latitude observations where little to no seasonal variation is observed. However, as possible, we include observations for a variety of latitudes to provide a sense of the variations that are observed for a given molecule when plotting the model output profiles compared to observations. For a small number of molecules (e.g., $\mathrm{C}_{6} \mathrm{H}_{6}$ ), the abundance is only sufficiently high to be detected by Cassini CIRS limb observations at the winter high latitudes where the stratosphere is enriched by downwelling from the descending branch of the global circulation. We do not expect to reproduce these observations, as we do not capture this type of dynamical behavior, but include the observations for completeness. 
Table 11: Composition of Titan's neutral atmosphere

\begin{tabular}{|c|c|c|c|c|c|}
\hline & & Stratosphere & & Mesosphere & Thermosphere \\
\hline Formula & Ground Based $^{(a)}$ & ISO $^{(g)} /$ Herschel & $\operatorname{CIRS}^{(m)}$ & $\operatorname{UVIS}^{(s)}$ & INMS $(\mathrm{CSN})^{(u)}$ \\
\hline $\begin{array}{l}\mathrm{H}_{2} \\
40 \mathrm{Ar}\end{array}$ & & & $9.6 \pm 2.4 \times 10^{-4(n)}$ & & $\begin{array}{l}3.9 \pm 0.01 \times 10^{-3} \\
1.1 \pm 0.03 \times 10^{-5}\end{array}$ \\
\hline $\mathrm{C}_{2} \mathrm{H}_{2}$ & & $5.5 \pm 0.5 \times 10^{-6}$ & $2.97 \times 10^{-6}$ & $5.9 \pm 0.6 \times 10^{-5}$ & $3.1 \pm 1.1 \times 10^{-4}$ \\
\hline $\mathrm{C}_{2} \mathrm{H}_{4}$ & & $1.2 \pm 0.3 \times 10^{-7}$ & $1.2 \times 10^{-7}$ & $1.6 \pm 0.7 \times 10^{-6}$ & $3.1 \pm 1.1 \times 10^{-4}$ \\
\hline $\mathrm{C}_{2} \mathrm{H}_{6}$ & & $2.0 \pm 0.8 \times 10^{-5}$ & $7.3 \times 10^{-6}$ & & $7.3 \pm 2.6 \times 10^{-5}$ \\
\hline $\mathrm{CH}_{3} \mathrm{C}_{2} \mathrm{H}$ & & $1.2 \pm 0.4 \times 10^{-8}$ & $4.8 \times 10^{-9}$ & & $1.4 \pm 0.9 \times 10^{-4}$ \\
\hline $\mathrm{C}_{3} \mathrm{H}_{6}$ & & & $2.6 \pm 1.6 \times 10^{-9(o)}$ & & $2.3 \pm 0.2 \times 10^{-6(v)}$ \\
\hline $\mathrm{C}_{3} \mathrm{H}_{8}$ & $6.2 \pm 1.2 \times 10^{-7(b)}$ & $2.0 \pm 1.0 \times 10^{-7}$ & $4.5 \times 10^{-7}$ & & $<4.8 \times 10^{-5}$ \\
\hline $\mathrm{C}_{4} \mathrm{H}_{2}$ & & $2.0 \pm 0.5 \times 10^{-9}$ & $1.12 \times 10^{-9}$ & $7.6 \pm 0.9 \times 10^{-7}$ & $6.4 \pm 2.7 \times 10^{-5}$ \\
\hline $\mathrm{C}_{6} \mathrm{H}_{6}$ & & $4.0 \pm 3.0 \times 10^{-10}$ & $2.2 \times 10^{-10}$ & $2.3 \pm 0.3 \times 10^{-7}$ & $8.95 \pm 0.44 \times 10^{-7}$ \\
\hline $\mathrm{HCN}$ & $5 \times 10^{-7}$ & $3.0 \pm 0.5 \times 10^{-7}$ & $6.7 \times 10^{-8}$ & $1.6 \pm 0.7 \times 10^{-5}$ & \\
\hline $\mathrm{HNC}$ & $4.9 \pm 0.3 \times 10^{-9(c)}$ & $4.5 \pm 1.2 \times 10^{-9(h)}$ & & & \\
\hline $\mathrm{HC}_{3} \mathrm{~N}$ & $3 \times 10^{-11}$ & $5.0 \pm 3.5 \times 10^{-10}$ & $2.8 \times 10^{-10}$ & $2.4 \pm 0.3 \times 10^{-6}$ & $3.2 \pm 0.7 \times 10^{-5}$ \\
\hline $\mathrm{CH}_{3} \mathrm{CN}$ & $8 \times 10^{-9}$ & & $<1.1 \times 10^{-7(p)}$ & & $3.1 \pm 0.7 \times 10^{-5}$ \\
\hline $\mathrm{C}_{2} \mathrm{H}_{3} \mathrm{CN}$ & $3.6 \times 10^{-10(d)}$ & & & & $<1.8 \times 10^{-5}$ \\
\hline $\mathrm{C}_{2} \mathrm{H}_{5} \mathrm{CN}$ & $2.8 \times 10^{-10(e)}$ & & & & \\
\hline $\mathrm{C}_{2} \mathrm{~N}_{2}$ & & & $9 \times 10^{-10(q)}$ & & $4.8 \pm 0.8 \times 10^{-5}$ \\
\hline $\mathrm{NH}_{3}$ & & $<1.9 \times 10^{-10(i)}$ & $<1.3 \times 10^{-9(p)}$ & & $2.99 \pm 0.22 \times 10^{-5}$ \\
\hline $\mathrm{CO}$ & $5.1 \pm 0.4 \times 10^{-5(f)}$ & $4.0 \pm 5 \times 10^{-5(j)}$ & $4.7 \pm 0.8 \times 10^{-5(r)}$ & $5.4 \pm 3.2 \times 10^{-5(t)}$ & \\
\hline $\mathrm{H}_{2} \mathrm{O}$ & & $8 \times 10^{-9(k)} / 7 \times 10^{-10(l)}$ & $4.5 \pm 1.5 \times 10^{-10(r)}$ & & $<3.42 \times 10^{-6}$ \\
\hline $\mathrm{CO}_{2}$ & & $2.0 \pm 0.2 \times 10^{-8}$ & $1.1 \times 10^{-8}$ & & $<8.49 \times 10^{-7}$ \\
\hline
\end{tabular}


(at $200 \mathrm{~km})^{(b)}$ Roe et al. (2003) ${ }^{(c)}$ Cordiner et al. (2014) (constant profile at $\left.400 \mathrm{~km}\right)^{(d)}$ Palmer et al. (2017) (at 297 km, using fractional scale height model) ${ }^{(e)}$ Cordiner et al. (2015b) (gradient profile at $\left.200 \mathrm{~km}\right){ }^{(f)}$ Gurwell (2004) ${ }^{(g)}$ Coustenis et al. (2003) ${ }^{(h)}$ Moreno et al. (2011) (constant profile at $\left.400 \mathrm{~km}\right)^{(i)}$ Teanby et al. (2013) (3- $\sigma$ upper limit, peak sensitivity at $\left.75 \mathrm{~km}\right){ }^{(j)}$ Courtin et al. (2011) ${ }^{(k)}$ Coustenis et al. (1998) (at $\left.400 \mathrm{~km}\right){ }^{(l)}$ Moreno et al. (2012) (from the $\mathrm{S}_{a}$ profile at $\left.400 \mathrm{~km}\right){ }^{(m)}$ Coustenis et al. (2010) (at $5^{\circ} \mathrm{S} ; \mathrm{HCN}, \mathrm{C}_{6} \mathrm{H}_{6}, \mathrm{C}_{3} \mathrm{H}_{8}, \mathrm{C}_{4} \mathrm{H}_{2}$, and $\mathrm{HC}_{3} \mathrm{~N}$ exhibit latitudinal variations (Coustenis et al., 2007)), values averaged over TB-T44 assuming constant vertical profiles, for vertical variations see Vinatier et al. (2010)) ${ }^{(n)}$ Courtin et al. (2007) ${ }^{(o)}$ Nixon et al. (2010) $\left(3-\sigma\right.$ upper limits at $\left.25^{\circ} \mathrm{S}\right){ }^{(p)}$ Teanby et al. (2006) $\left(3-\sigma \text { upper limit at } 50^{\circ} \mathrm{N}\right)^{(q)}$ Nixon et al. (2013) (at $\left.225 \mathrm{~km}\right){ }^{(r)} \mathrm{Cottini}$ et al. $(2012)$ (using constant VMR at $230 \mathrm{~km}){ }^{(s)}$ Koskinen et al. $(2011)($ at $\sim 600 \mathrm{~km}){ }^{(t)}$ Fabiano et al. (2017) VIMS (at $\left.450 \mathrm{~km}\right){ }^{(u)} \mathrm{Cui}$ et al. (2009b) (at $1077 \mathrm{~km}$ ) from T5, 16, 18, 19, 21, 23, 25-30, 32, 36, 37 using INMS CSN mode, italics indicates corrected values, signals from $\mathrm{C}_{2} \mathrm{H}_{2}$ and $\mathrm{C}_{2} \mathrm{H}_{4}$ are difficult to separate so the reported value is for both species combined) ${ }^{(v)}$ Magee et al. (2009) (global average at $1050 \mathrm{~km}$ ). 
Table 12: Upper limits on the composition of Titan's neutral atmosphere

\begin{tabular}{|c|c|c|c|c|}
\hline \multirow[b]{2}{*}{ Formula } & \multicolumn{3}{|c|}{ Stratosphere } & \multirow{2}{*}{$\begin{array}{l}\text { Thermosphere } \\
\text { INMS (CSN) })^{(g)}\end{array}$} \\
\hline & Voyager & $\begin{array}{l}\text { ISO/Ground } \\
\text { Based }\end{array}$ & CIRS & \\
\hline$\overline{\mathrm{CH}_{2} \mathrm{CCH}_{2}}$ & & $<2 \times 10^{-9(d)}$ & $<9 \times 10^{-10(f)}$ & \\
\hline $\mathrm{C}_{4} \mathrm{H}_{6}$ & & & & $<9.5 \times 10^{-6}$ \\
\hline $\mathrm{C}_{6} \mathrm{H}_{2}$ & $<6.0 \times 10^{-10(a)}$ & & & \\
\hline $\mathrm{C}_{7} \mathrm{H}_{8}$ & & & & $<1.32 \times 10^{-7}$ \\
\hline $\mathrm{C}_{4} \mathrm{H}_{3} \mathrm{~N}$ & $<2.5 \times 10^{-7(b)}$ & & & \\
\hline $\mathrm{C}_{4} \mathrm{H}_{5} \mathrm{~N}$ & $<4.0 \times 10^{-8(b)}$ & & & $<3.0 \times 10^{-7}$ \\
\hline $\mathrm{C}_{4} \mathrm{~N}_{2}$ & $<5.3 \times 10^{-10(c)}$ & & & \\
\hline $\mathrm{HC}_{5} \mathrm{~N}$ & & $<4 \times 10^{-10(e)}$ & & \\
\hline $\mathrm{H}_{2} \mathrm{CO}$ & & & $<1.6 \times 10^{-8(f)}$ & \\
\hline
\end{tabular}

All values come from the work referenced in the table header unless otherwise noted in the table. ${ }^{(a)}$ Delpech et al. (1994) ${ }^{(b)}$ Cerceau et al. (1985) ${ }^{(c)}$ Jolly et al. $(2015){ }^{(d)}$ Coustenis et al. (2003) ${ }^{(e)}$ Marten et al. (2002) (at $\left.200 \mathrm{~km}\right){ }^{(f)}$ Nixon et al. (2010) (3- $\sigma$ upper limits) ${ }^{(g)}$ Cui et al. (2009b) (at $1077 \mathrm{~km}$ from T5, 16,18, 19, 21, 23, 25-30, 32, 36, 37 using INMS Closed Source Neutral mode). 

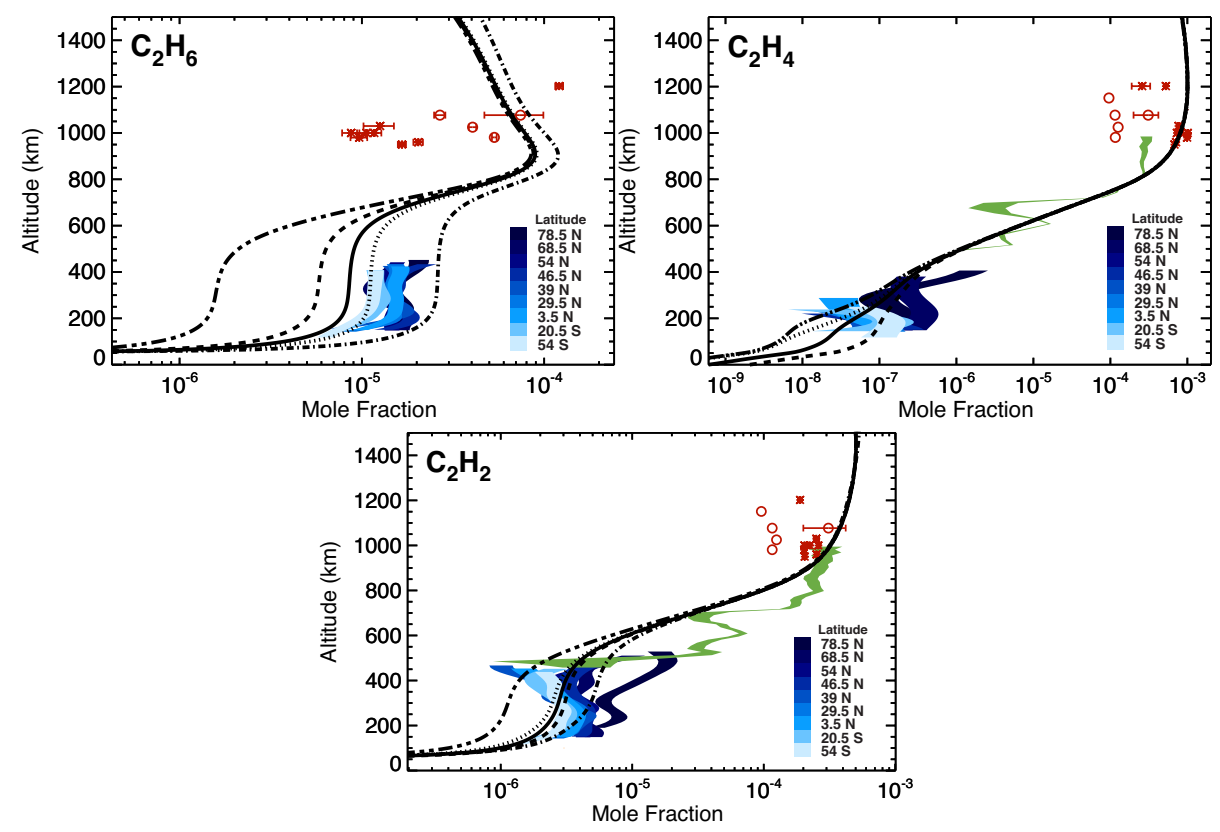

Figure 44: Calculated and observed mole fractions of $\mathrm{C}_{2} \mathrm{H}_{6}$ (upper left panel), $\mathrm{C}_{2} \mathrm{H}_{4}$ (upper right panel) and $\mathrm{C}_{2} \mathrm{H}_{2}$ (lower panel). Solid line: nominal; dash-dot and long dash - double short dash line: eddy mixing coefficient $K_{0}=10^{2}$ and $10^{3} \mathrm{~cm}^{-2}$, respectively, section 2.4; dotted and dashed lines: aerosol optical depth multiplied by 0.5 and 2, respectively, section 5.3.1; long dash-dot-dot line: no $\mathrm{H}$ heterogeneous loss, section 5.3.2. The data shown is from CIRS (Vinatier et al., 2010); UVIS (Koskinen et al., 2011); INMS (Cui et al., 2009b) (circles no error bars are uncorrected, circle with error bar is corrected) $\mathrm{C}_{2} \mathrm{H}_{2}$ joint with $\mathrm{C}_{2} \mathrm{H}_{4}$, (Waite et al., 2007) asterisks.

Acetylene $\left(\mathbf{C}_{2} \mathbf{H}_{2}\right)$, Ethylene $\left(\mathbf{C}_{2} \mathbf{H}_{4}\right)$, Ethane $\left(\mathbf{C}_{2} \mathbf{H}_{6}\right)$. The model profile of ethane resulting from the chemistry described in section 3.2.1, is presented in the upper left panel of Figure 44. The $\mathrm{C}_{2} \mathrm{H}_{6}$ abundance at 1000-1100 $\mathrm{km}$ in the model falls in between the uncorrected and corrected mole fractions retrieved from the INMS observations $\left(2-7 \times 10^{-5}\right)$. CIRS limb observations favor a constant ethane mole fraction of $\sim 15 \mathrm{ppm}$ from 150 to $400 \mathrm{~km}$ (cf. Vinatier et al. (2010)) and the difference between these data and the model is a factor of about 2.

All the models (Lavvas et al., 2008b; Krasnopolsky, 2014; Li et al., 2015; Dobrijevic et al., 2016) are in fairly good agreement with each other and with the observations, despite using quite different rate coefficients for the recombination of $\mathrm{CH}_{3}$ radicals above $800 \mathrm{~km}$ (cf. Figure A.3c). We note that Dobrijevic et al. (2016) mention that for INMS data they applied a correction factor of $2.2 \pm 0.5$ to the mixing ratios (cf. their Figure 7 for $\mathrm{C}_{2} \mathrm{H}_{6}$ but this applies to all their figures). However, this correction applies to the absolute density of all molecules and therefore cancels out for mixing ratios (Teolis et al., 2015).

Ethylene and acetylene are amongst the best constrained species with the combination of INMS, UVIS and CIRS observations providing an almost unin- 
terrupted profile from the thermosphere down to the troposphere (Cui et al., 2009b; Magee et al., 2009; Koskinen et al., 2011; Vinatier et al., 2010). The Krasnopolsky (2014) (their Figure 2) and Lavvas et al. (2008b) (their Figure 4a) profiles agree with the INMS data but underestimate the $\mathrm{C}_{2} \mathrm{H}_{4}$ abundance by about one order of magnitude at $200 \mathrm{~km}$, as do all previous photochemical models. In order to match the observations, $\mathrm{Li}$ et al. (2014) suggest a new expression for the rate coefficient of the key reaction, $\mathrm{H}+\mathrm{C}_{2} \mathrm{H}_{4}+\mathrm{M} \rightarrow \mathrm{C}_{2} \mathrm{H}_{5}+\mathrm{M}$. The new reaction rate coefficient is estimated to be $\sim 10$ times lower than that used by Moses et al. (2005) for giant planets (and by other Titan models), see Figure A.1b. Instead, our calculations yield a rate coefficient about one order of magnitude larger in the stratosphere (Vuitton et al., 2012). Nevertheless, the model simulation for $\mathrm{C}_{2} \mathrm{H}_{4}$ is in general agreement with the INMS and UVIS observations and provides values in good agreement with the CIRS retrieved mole fractions (cf. upper right panel of Figure 44). A possible explanation for the difference with our profile is the important production of ethylene below 400 $\mathrm{km}$ via the photodissociation of butadiene in our model.

Although our model as well as Dobrijevic et al. (2016) (their Figure 8) reproduce the observed $\mathrm{C}_{2} \mathrm{H}_{4}$ abundance, they do not capture the negative vertical gradient observed by CIRS at mid-latitudes. This peculiar behavior has been explained by the fact that ethylene does not condense, allowing the return branch of the global circulation cell to bring air enriched in $\mathrm{C}_{2} \mathrm{H}_{4}$ from the winter pole towards the equator, a circulation pattern that cannot be modeled with 1-dimensional models (Crespin et al., 2008).

Acetylene's vertical profile is presented in the lower panel of Figure 44. The observed acetylene mole fraction is near $3 \times 10^{-4}$ at $1000 \mathrm{~km}$ and gradually decreases until reaching a constant value of $5 \times 10^{-6}$ below $500 \mathrm{~km}$. Hébrard et al. (2013) and Li et al. (2015) predict the right stratospheric abundance of $\mathrm{C}_{2} \mathrm{H}_{2}$ within a factor of about 2, while the profile of Krasnopolsky (2014) is too high by one order of magnitude at $600 \mathrm{~km}$, probably because of his choice of eddy mixing coefficient. Other calculated $\mathrm{C}_{2} \mathrm{H}_{2}$ profiles (cf. Figure $4 \mathrm{~b}$ in Lavvas et al. (2008b) and Figures 7 in Loison et al. (2015) and Dobrijevic et al. (2016)), including ours, agree very well with the INMS, UVIS and CIRS mid-latitude results.

Methylacetylene $\left(\mathrm{CH}_{3} \mathrm{CCH}\right)$, Allene $\left(\mathrm{CH}_{2} \mathrm{CCH}_{2}\right)$, Propylene $\left(\mathrm{C}_{3} \mathrm{H}_{6}\right)$, Propane $\left(\mathbf{C}_{\mathbf{3}} \mathbf{H}_{\mathbf{8}}\right)$. The mole fractions of $\mathrm{CH}_{3} \mathrm{CCH}, \mathrm{CH}_{2} \mathrm{CCH}_{2}, \mathrm{C}_{3} \mathrm{H}_{6}$ and $\mathrm{C}_{3} \mathrm{H}_{8}$ obtained with the current model are shown in Figure 45. 

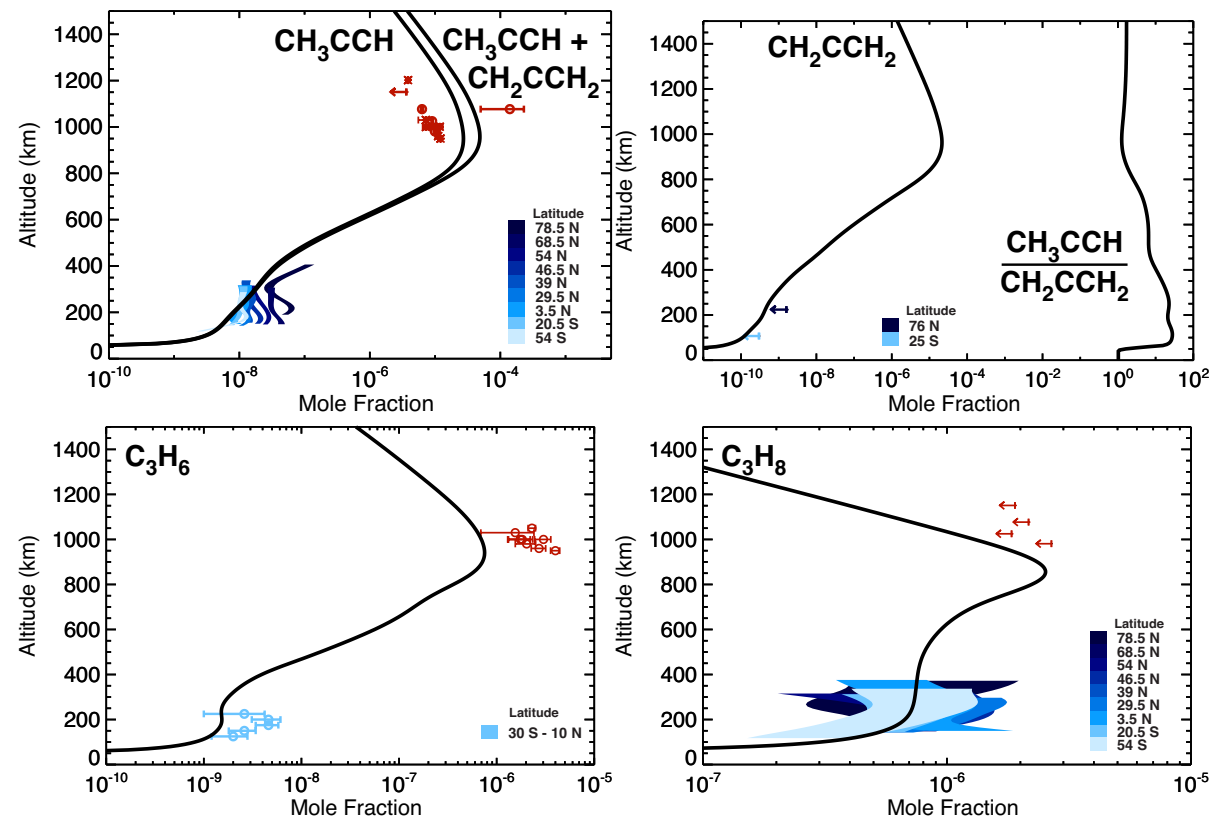

Figure 45: Calculated and observed mole fractions of $\mathrm{CH}_{3} \mathrm{CCH}$ and $\mathrm{CH}_{2} \mathrm{CCH}_{2}$ (top panels), $\mathrm{C}_{3} \mathrm{H}_{6}$ (lower left panel), and $\mathrm{C}_{3} \mathrm{H}_{8}$ (lower right panel). The sum and ratio of $\mathrm{CH}_{3} \mathrm{CCH}$ and $\mathrm{CH}_{2} \mathrm{CCH}_{2}$ are shown on panels (a) and (b), respectively. The data shown for $\mathrm{CH}_{3} \mathrm{CCH}$ is from Vinatier et al. (2010), Cui et al. (2009b) (circles) and Waite et al. (2007) (asterisks), for $\mathrm{CH}_{2} \mathrm{CCH}_{2}$ from Nixon et al. (2010), for $\mathrm{C}_{3} \mathrm{H}_{6}$ from Nixon et al. (2013) (CIRS) and Waite et al. (2007) (circles) and for $\mathrm{C}_{3} \mathrm{H}_{8}$ from Vinatier et al. (2010) and Cui et al. (2009b).

Methylacetylene $\left(\mathrm{CH}_{3} \mathrm{CCH}\right)$ and its isomer allene $\left(\mathrm{CH}_{2} \mathrm{CCH}_{2}\right)$ are presented in the upper left panel of Figure 45. Because CIRS observations show evidence for $\mathrm{CH}_{3} \mathrm{CCH}$ (mole fraction of $\sim 10^{-8}$, Vinatier et al. (2010)) and not for $\mathrm{CH}_{2} \mathrm{CCH}_{2}$ (upper limit of $3 \times 10^{-10}$ at $107 \mathrm{~km}$, Nixon et al. (2010)) and because $\mathrm{CH}_{3} \mathrm{CCH}$ is more stable than $\mathrm{CH}_{2} \mathrm{CCH}_{2}$, Cui et al. (2009b) only consider $\mathrm{CH}_{3} \mathrm{CCH}$ in their analysis of the INMS spectra. They provide uncorrected and corrected mole fractions of $(6.31 \pm 0.24) \times 10^{-6}$ and $(1.4 \pm 0.9) \times 10^{-4}$ at $1077 \mathrm{~km}$, respectively (Cui et al., 2009b). However, they mention that strictly speaking, the derived abundances of $\mathrm{CH}_{3} \mathrm{CCH}$ should refer to the sum of $\mathrm{CH}_{3} \mathrm{CCH}$ and $\mathrm{CH}_{2} \mathrm{CCH}_{2}$ due to the similarity of their cracking patterns for mass channels 39 and 40 from which the $\mathrm{CH}_{3} \mathrm{CCH}$ abundance is constrained.

The current model indicates that in the upper atmosphere $\mathrm{CH}_{3} \mathrm{CCH}$ and $\mathrm{CH}_{2} \mathrm{CCH}_{2}$ have similar abundances, with mole fractions at $1075 \mathrm{~km}$ of $1.5 \times 10^{-5}$ and $1.8 \times 10^{-5}$ for $\mathrm{CH}_{3} \mathrm{CCH}$ and $\mathrm{CH}_{2} \mathrm{CCH}_{2}$, respectively. Contrary to the assumption made by Cui et al. (2009b), it therefore seems that both species contribute to the INMS signal, a conclusion already reached by Lavvas et al. (2008b). We plot the sum of the mole fractions of $\mathrm{CH}_{3} \mathrm{CCH}$ and $\mathrm{CH}_{2} \mathrm{CCH}_{2}$ in the upper right panel of Figure 45. The combined contribution at $1075 \mathrm{~km}$ $\left(3.3 \times 10^{-5}\right)$ falls in between the uncorrected and corrected mole fractions from 
INMS. In the lower atmosphere, the $\mathrm{CH}_{3} \mathrm{CCH}$ model profile reproduces well the CIRS mid-latitude abundance (Vinatier et al., 2010), while the modeled $\mathrm{CH}_{2} \mathrm{CCH}_{2}$ mole fraction at 225 and $105 \mathrm{~km}$ is just in agreement with the CIRS $3 \sigma$ upper limits (Nixon et al., 2010). These results are in line with previous models, which with the exception of Lavvas et al. (2008b) in the stratosphere (their Figure 5a), provide a good fit to both INMS and CIRS data (cf. Figure 3 in Krasnopolsky (2014), Figure 2 in Li et al. (2015) and Figure 8 in Dobrijevic et al. (2016)).

$\mathrm{C}_{3} \mathrm{H}_{6}$ has been tentatively detected in Titan's thermosphere by INMS with a mole fraction of about $3 \times 10^{-6}$ (Magee et al., 2009) and for the first time in the stratosphere by CIRS with a mole fraction peaking at $5 \times 10^{-9}$ at 200 $\mathrm{km}$ (Nixon et al., 2013). The profile of propylene from our model along with the INMS and CIRS detections are shown in the lower left panel of Figure 45, and the agreement of the prediction with the observations is within a factor of two. Other models also reasonably match the $\mathrm{C}_{3} \mathrm{H}_{6}$ abundance in both the thermosphere and stratosphere (cf. Figure 4 in Krasnopolsky (2014), Figures 2,3 and discussion in Li et al. (2015) and Figure 7 in Dobrijevic et al. (2016)).

The modeled mole fraction of $\mathrm{C}_{3} \mathrm{H}_{8}$ around $1000 \mathrm{~km}$ is consistent with upper limits from INMS $\left(<5 \times 10^{-5}\right)$. The CIRS stratospheric mole fraction is more or less constant from 100 to $300 \mathrm{~km}$ with a value of $8 \times 10^{-7}$. Our model reproduces a similar constant profile with a value of $\sim 5 \times 10^{-7}$ (cf. lower right panel of Figure 45). While Lavvas et al. (cf. Figure 5b in Lavvas et al. (2008b)) overestimates the propane mole fraction in the stratosphere, Krasnopolsky (cf. Figure 3 in Krasnopolsky (2014)), Yung et al. (cf. Figure 2 in Li et al. (2015)) and Dobrijevic et al. (cf. Figure 7 in Loison et al. (2015) and Figure 8 in Dobrijevic et al. (2016)) obtain an almost perfect match with the observations.
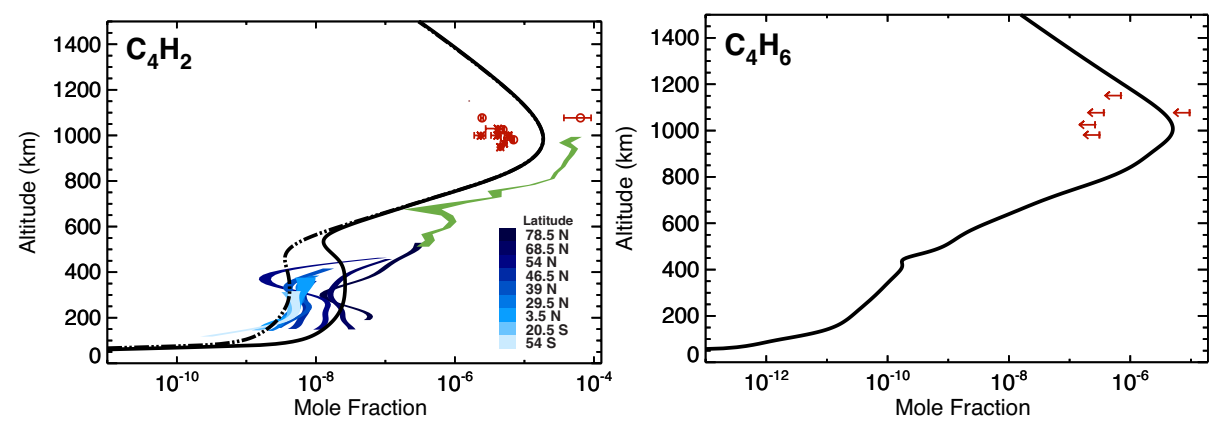

Figure 46: Calculated and observed mole fractions of $\mathrm{C}_{4} \mathrm{H}_{2}$ (left panel) and $\mathrm{C}_{4} \mathrm{H}_{6}$ (right panel). Solid line: nominal; long dash-dot-dot line: no $\mathrm{H}$ heterogeneous loss, section 5.3.2. The data shown for $\mathrm{C}_{4} \mathrm{H}_{2}$ is from CIRS (Vinatier et al., 2010); UVIS (Koskinen et al., 2011); INMS (Cui et al., 2009b) (circles no error bars are uncorrected, circle with error bar is corrected), (Waite et al., 2007) asterisks. The upper limits for $\mathrm{C}_{4} \mathrm{H}_{6}$ are from INMS (Cui et al., 2009b). 


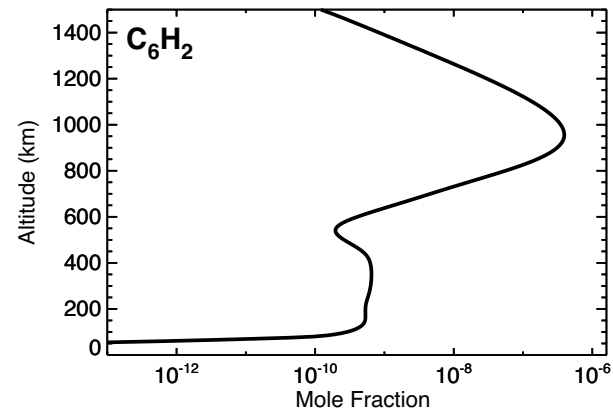
2010) by a factor of about 5 .

Diacetylene $\left(\mathrm{C}_{4} \mathbf{H}_{2}\right)$, Butadiene $\left(\mathrm{C}_{4} \mathbf{H}_{6}\right)$. The calculated vertical profile of diacetylene is compared to the INMS, UVIS and CIRS observations in the left panel of Figure 46. The observed mole fractions are close to $6 \times 10^{-5}$ at $1050 \mathrm{~km}$ (corrected INMS value from Cui et al. (2009b) that merges nicely with the UVIS data at lower altitude) and decrease gradually to $3 \times 10^{-9}$ at $200 \mathrm{~km}$, where they exhibit some strong latitudinal variations. Photochemical models have never been fully successful at reproducing the diacetylene observations. The Lavvas et al. (2008b) vertical profile (their Figure 5c) underestimates the INMS observations by more than one order of magnitude, the Krasnopolsky (2014) profile (his Figure 2) has a significantly different altitude variation than the UVIS retrieved profile, while the Hébrard et al. (2013) profile (their Figure 1) underestimates the CIRS observations by a factor 10 or more. Only the Dobrijevic et al. (2016) profile is in good agreement with the stratospheric data. It is unclear to us what improvement lead to such a different profile from Hébrard et al. (2013) to Dobrijevic et al. (2016). The reactions related to the production and loss of $\mathrm{C}_{4} \mathrm{H}_{2}$ (according to our model) are mostly the same in both versions of the Dobrijevic et al. model and a change in the chemistry cannot explain the difference (unless some unexpected reaction controls the $\mathrm{C}_{4} \mathrm{H}_{2}$ abundance in these models). Our calculated vertical profile of diacetylene falls short from the INMS corrected values and the UVIS profile (Koskinen et al., 2011) by a factor of a few and overestimates the mid-latitude CIRS data (Vinatier et al.,

$\mathrm{C}_{4} \mathrm{H}_{6}$ has neither been detected on Titan or described in previous photochemical models, with the exception of the mole fraction profiles given in Figure 19 of Dobrijevic et al. (2016). Our model profile (cf. right panel of Figure 46) agrees with the INMS upper limit of $10^{-5}$ (Cui et al., 2009b).

Figure 47: Calculated and observed mole fractions of $\mathrm{C}_{6} \mathrm{H}_{2}$ (left panel), and $\mathrm{C}_{6} \mathrm{H}_{6}$ and $\mathrm{C}_{6} \mathrm{H}_{5}$ (right panel). Solid line: nominal; long dash-dot-dot line: no $\mathrm{H}$ heterogeneous loss, section 5.3.2. The data shown for $\mathrm{C}_{6} \mathrm{H}_{6}$ is from CIRS (Vinatier et al., 2010); UVIS (Koskinen et al., 2011); INMS (Cui et al., 2009b) (circles no error bars are uncorrected, circle with error bar is corrected), (Waite et al., 2007) asterisks.

Triacetylene $\left(\mathrm{C}_{6} \mathbf{H}_{2}\right)$, Benzene $\left(\mathrm{C}_{6} \mathbf{H}_{6}\right)$. Presented in the left panel of Figure 47 , is the vertical profile of triacetylene, which exhibits a sharp peak at 
$950 \mathrm{~km}$ (mole fraction of $4 \times 10^{-7}$ ) and a broader one around $250 \mathrm{~km}$ (mole fraction of $\left.6 \times 10^{-10}\right)$. There are no observations available to constrain its altitude variation. In previous photochemical models, Lavvas et al. (2008b) has provided a vertical profile similar to ours, while Krasnopolsky (2012)'s high altitude peak is quite different with a mole fraction of $\sim 10^{-5}$.

In Vuitton et al. (2008), we reported that the $\mathrm{C}_{6} \mathrm{H}_{6}$ signal measured by INMS (at $\mathrm{m} / \mathrm{z}=78$ and 77 ) exhibits a peculiar time dependence, peaking $\sim 20 \mathrm{~s}$ after closest approach. We explained this behavior by recombination of $\mathrm{C}_{6} \mathrm{H}_{5}$ radicals with $\mathrm{H}$ atoms on the walls of the instrument and argued that the measured signal was a combination of (i) $\mathrm{C}_{6} \mathrm{H}_{6}$ from the atmosphere and (ii) $\mathrm{C}_{6} \mathrm{H}_{6}$ formed within the instrument. This interpretation was backed-up by our photochemical model, which predicted that $\mathrm{C}_{6} \mathrm{H}_{5}$ radicals were $\sim 3$ times as abundant as benzene.

Unlike in our 2008 paper, our newest modeling results indicate that the abundance of phenyl radicals $\left(\mathrm{C}_{6} \mathrm{H}_{5}\right)$ is small compared to that of benzene (cf. right panel of Figure 47). This is explained by the inclusion of radiative association in our chemical scheme (Vuitton et al., 2012). $\mathrm{C}_{6} \mathrm{H}_{5}$ does not react with closed-shell molecules and its main loss is addition to the most abundant radicals, such as $\mathrm{H}\left(\mathrm{R}_{n} 30\right)$ and $\mathrm{CH}_{3}\left(\mathrm{R}_{n} 105\right)$. In 2008 , we only considered threebody association reactions, $\left(\mathrm{R}_{n} 30\right)$ and $\left(\mathrm{R}_{n} 105\right)$ were inefficient in the upper atmosphere, leading to rather long chemical lifetime and therefore high density for $\mathrm{C}_{6} \mathrm{H}_{5}$. Consideration of radiative association enhances the rate coefficient of reaction $\left(\mathrm{R}_{n} 30\right)$ by seven orders of magnitude at $1000 \mathrm{~km}$, resulting in the fast recycling of $\mathrm{C}_{6} \mathrm{H}_{5}$ into $\mathrm{C}_{6} \mathrm{H}_{6}$ even at high altitude, and as a consequence small $\mathrm{C}_{6} \mathrm{H}_{5}$ densities.

Further analysis of the INMS data revealed that the $\mathrm{C}_{6} \mathrm{H}_{6}$ time behavior is actually observed for most species and that it is difficult to disentangle heterogeneous surface chemistry of radicals from adsorption/desorption of the parent molecule (Cui et al., 2009b). The low radical abundance obtained with our improved chemical network tends to point towards the latter explanation. This implies that the densities inferred by our previous analyses of the INMS data should be attributed to $\mathrm{C}_{6} \mathrm{H}_{6}$ only and that the associated error bars are large. The UVIS mole fraction at $900 \mathrm{~km}$ is close to $10^{-5}$, which is a factor of about two higher than the values retrieved from the INMS data at 950-1000 km (Koskinen et al., 2011). In the stratosphere, both CIRS limb and nadir data present some evidence for strong latitudinal variations, with mole fractions at mid-latitudes of about $2 \times 10^{-10}$ at $150 \mathrm{~km}$ (Coustenis et al., 2010; Vinatier et al., 2010).

Within this paradigm, the predicted $\mathrm{C}_{6} \mathrm{H}_{6}$ profile is in good agreement with the INMS data in the $950-1050 \mathrm{~km}$ altitude range and also with the UVIS data in the 550-900 km range. The benzene abundance in the Lavvas et al. (2008b) and Hébrard et al. (2013) models is significantly smaller than the INMS derived one, which is expected since $\mathrm{C}_{6} \mathrm{H}_{6}$ formation in the upper atmosphere is related to ion chemistry, which is not taken into account in these models. 


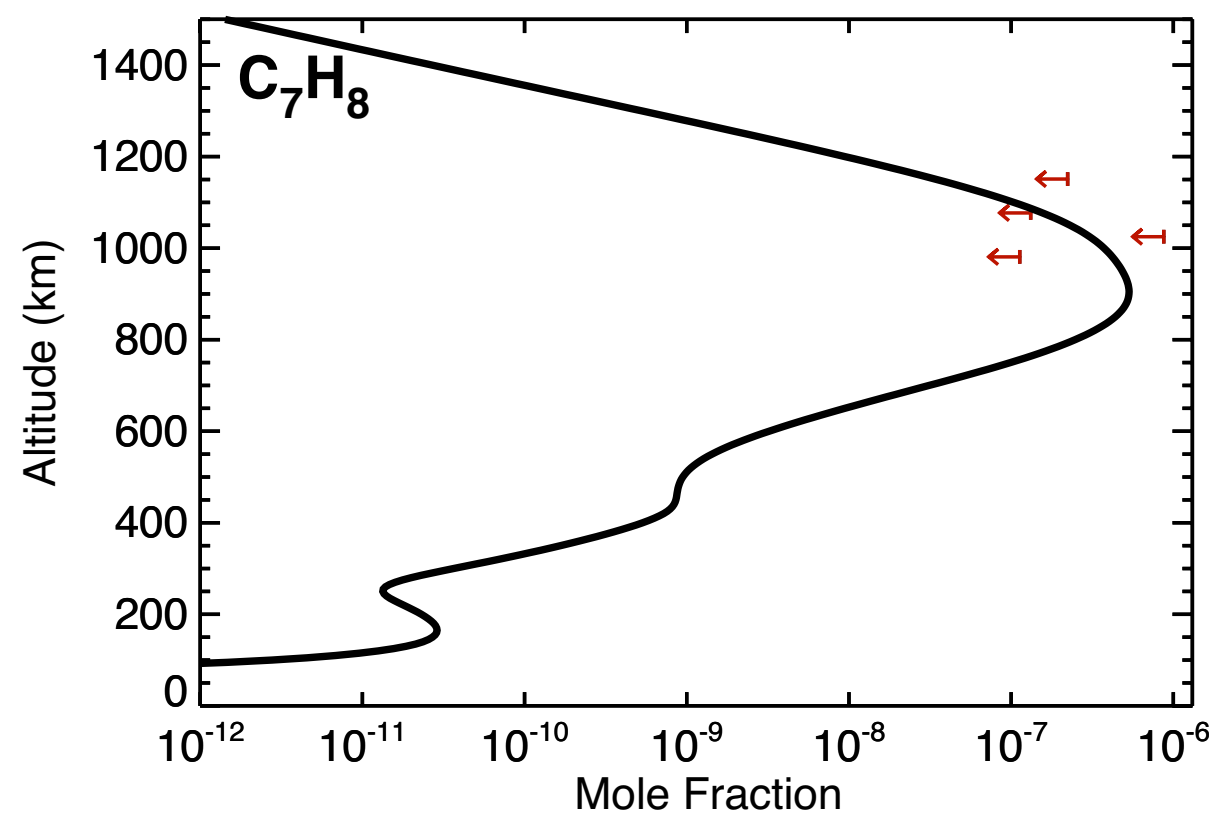

Figure 48: Calculated and observed mole fractions of $\mathrm{C}_{7} \mathrm{H}_{8}$. The upper limits shown are from INMS (Cui et al., 2009b).

Toluene $\left(\mathbf{C}_{\mathbf{7}} \mathbf{H}_{\mathbf{8}}\right)$. We described explicitly chemical reactions and photolysis of $\mathrm{C}_{7} \mathrm{H}_{8}$ in Vuitton et al. (2008) but no other photochemical model has presented results for this molecule to date. $\mathrm{C}_{7} \mathrm{H}_{8}$ is not detected on Titan. Although peaks at channels 91-92 in some of the INMS outbound spectra have been attributed to $\mathrm{C}_{7} \mathrm{H}_{8}$ molecules, such a feature can be reasonably explained by the wall effects in the INMS antechamber (Vuitton et al., 2008). $\mathrm{C}_{7} \mathrm{H}_{8}$ is not detected at $3 \sigma$ significance level in any individual inbound spectrum (Cui et al., 2009b). Our current profile in the upper atmosphere is similar to that shown in our previous paper with a peak mole fraction of $5 \times 10^{-7}$ at $900 \mathrm{~km}$ (cf. Figure 48), which is in the ball park of the INMS upper limits. 


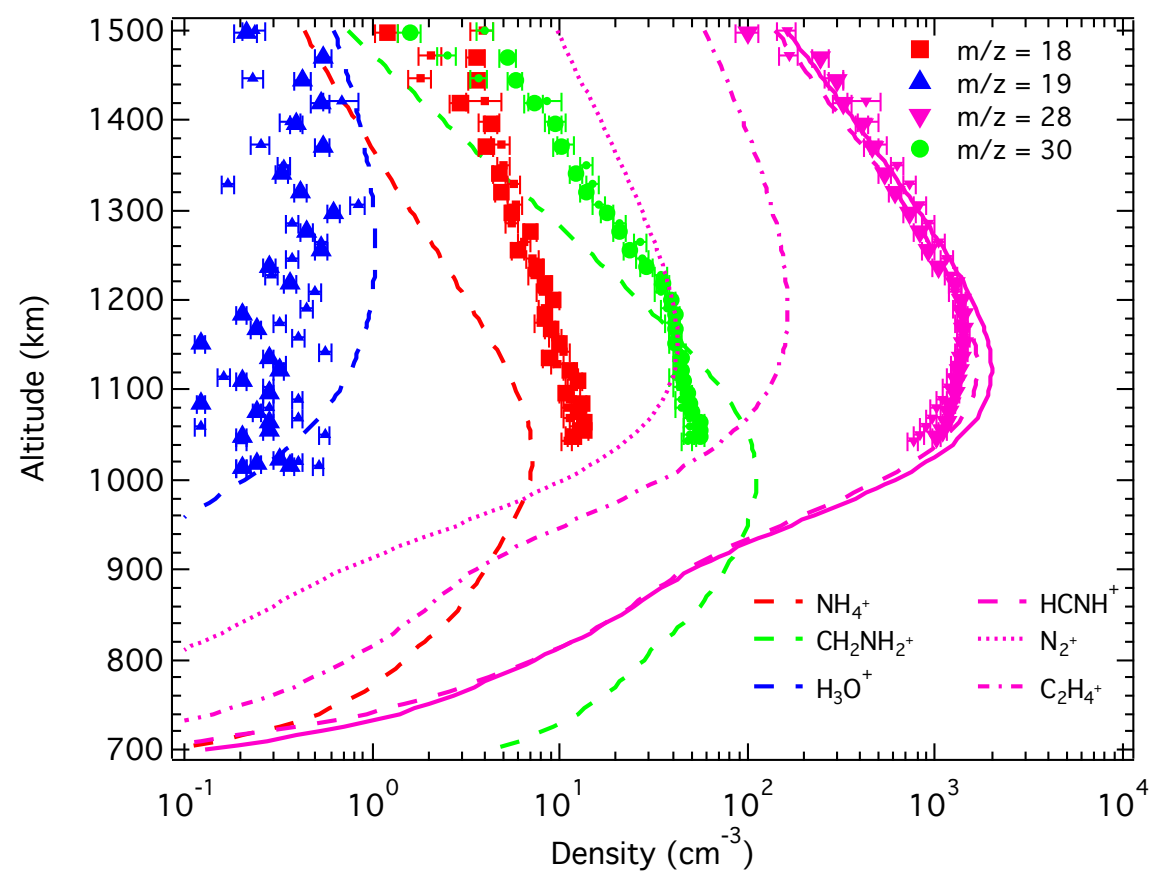

Figure 49: Model results for the density of $\mathrm{NH}_{4}{ }^{+}, \mathrm{H}_{3} \mathrm{O}^{+}, \mathrm{HCNH}^{+} / \mathrm{N}_{2}{ }^{+} / \mathrm{C}_{2} \mathrm{H}_{4}{ }^{+}$and $\mathrm{CH}_{2} \mathrm{NH}_{2}{ }^{+}$, and comparison with the T40 INMS ion density measurements at $\mathrm{m} / \mathrm{z}=18$, $19,28,30$.

\subsubsection{Nitrogen-Bearing Species.}
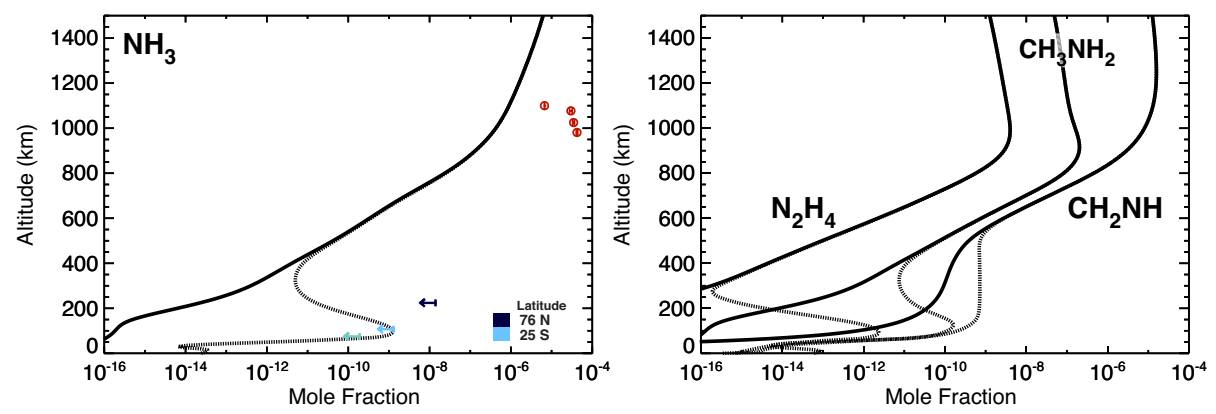

Figure 50: Calculated and observed mole fractions of $\mathrm{NH}_{3}$ (left panel), and $\mathrm{N}_{2} \mathrm{H}_{4}, \mathrm{CH}_{2} \mathrm{NH}$ and $\mathrm{CH}_{3} \mathrm{NH}_{2}$ (right panel). Solid line: nominal; hair line: with GCR. The data shown for $\mathrm{NH}_{3}$ is from CIRS $25 \mathrm{~S}$ and $76 \mathrm{~N}$ (Nixon et al., 2010) (blue), Herschel (Teanby et al., 2012) (green) and INMS (Cui et al., 2009b).

Ammonia $\left(\mathrm{NH}_{\mathbf{3}} / \mathbf{N H}_{\mathbf{4}}{ }^{+}\right)$. The presence of about $10 \mathrm{ppm}$ of $\mathrm{NH}_{3}$ in the upper atmosphere (Vuitton et al., 2007; Cui et al., 2009b), is not reproduced by 
photochemical models (Lavvas et al., 2008b; Krasnopolsky, 2010; Loison et al., 2015; Dobrijevic et al., 2016; Willacy et al., 2016). Lavvas et al. (2008b) suggest that the photolysis of ethylenimine $\left(\mathrm{C}_{2} \mathrm{H}_{5} \mathrm{~N}\right.$, produced from the reaction between $\mathrm{N}\left({ }^{2} \mathrm{D}\right)$ and $\left.\mathrm{C}_{2} \mathrm{H}_{6}\right)$ is the main source of $\mathrm{NH}_{3}$. However, this hypothesis implies densities of protonated ethylenimine $\left(\mathrm{C}_{2} \mathrm{H}_{5} \mathrm{NH}^{+}\right)$that are incompatible with the small signal detected at $\mathrm{m} / \mathrm{z}=44$ in the INMS ion spectra. The abundance of $\mathrm{NH}_{3}$ in Figure 6 of Krasnopolsky (2010) is about one order of magnitude lower than the values retrieved from the INMS ion and neutral spectra. Although this model includes the new $\mathrm{NH}_{3}$ production reaction suggested by Yelle et al. (2010), $\mathrm{NH}_{2}+\mathrm{H}_{2} \mathrm{CN} \rightarrow \mathrm{NH}_{3}+\mathrm{HCN}\left(\mathrm{R}_{n} 362\right)$, the rate coefficient is taken equal to that of $\mathrm{N}+\mathrm{H}_{2} \mathrm{CN} \rightarrow \mathrm{NH}+\mathrm{HCN}$, that is at $150 \mathrm{~K}$, a factor of three lower than the rate calculated in Yelle et al. (2010), which probably partly explains the inability of the Krasnopolsky (2010) model to reproduce the observations. The Loison et al. (2015) $\mathrm{NH}_{3}$ profile falls short of the INMS retrieved values by about two orders of magnitude (cf. their Figure 13), which they attribute to the lack of ion chemistry in their model to produce $\mathrm{NH}_{2}$, one of the major precursors of $\mathrm{NH}_{3}$, according to the chemical pathways described in Yelle et al. (2010) and section 3.2.2. This interpretation is supported by Willacy et al. (2016), whose model abundances in the upper atmosphere are a factor of 10 lower than the observations (their Figure 3).

Our calculated $\mathrm{NH}_{4}^{+}$densities slightly underestimate the INMS ion spectrum, as shown in Figure 49. This may be due to the fact that we do not consider the proton transfer reactions from $\mathrm{C}_{2} \mathrm{H}_{5}{ }^{+}$and $\mathrm{HCNH}^{+}$to $\mathrm{NH}_{2}$ leading to $\mathrm{NH}_{3}{ }^{+}$and then to $\mathrm{NH}_{4}{ }^{+}$, through the $\mathrm{NH}_{3}{ }^{+}+\mathrm{CH}_{4}$ reaction (Dobrijevic et al., 2016). However, our model underestimates the abundance of $\mathrm{NH}_{3}$ by one to two orders of magnitude compared to the value inferred by Cui et al. (2009b), as illustrated in the left panel of Figure 50. The $\mathrm{NH}_{4}{ }^{+}$and $\mathrm{NH}_{3}$ densities found in the model of Dobrijevic et al. (2016) are similar to our simulations, which puts into question the validity of the INMS NH 3 detection. Actually, Dobrijevic et al. (2016) suggest that most of the $\mathrm{NH}_{3}$ does not come from Titan's atmospheric chemistry but either from reaction of ambient $\mathrm{N}$ and $\mathrm{H}$ on the chamber walls or from spent hydrazine fuel (Cui et al., 2009b; Magee et al., 2009). In the stratosphere, the calculated $\mathrm{NH}_{3}$ mole fraction is consistent with the upper limits from CIRS and Herschel (Nixon et al., 2010; Teanby et al., 2012).

Methanimine $\left(\mathrm{CH}_{2} \mathbf{N H} / \mathbf{C H}_{2} \mathbf{N H}_{2}{ }^{+}\right)$. Methanimine is a particularly interesting molecule because, unlike all the other nitrogen-bearing species detected on Titan with the exception of ammonia, its nitrogen is not locked up in a triple bond and can therefore be more easily incorporated in prebiotic molecules, such as amino acids and nucleobases. Protonated methanimine $\left(\mathrm{CH}_{2} \mathrm{NH}_{2}{ }^{+}\right)$has been identified in INMS ion spectra, resulting in a $\mathrm{CH}_{2} \mathrm{NH}$ mole fraction of about $10^{-5}$ at $1100 \mathrm{~km}$ (Vuitton et al., 2006b, 2007; Yelle et al., 2010).

The chemical network of Lavvas et al. (2008a) was the first one to follow the destruction of $\mathrm{CH}_{2} \mathrm{NH}$. Yet their calculated $\mathrm{CH}_{2} \mathrm{NH}$ abundance is larger than the one retrieved from the INMS measurements, which the authors attribute to 
the poor knowledge of photodissociation cross-sections and more generally of loss processes for this species. However, it seems likely that the absence of ion chemistry is responsible for the discrepancy between model and observations in the upper atmosphere, as suggested by Dobrijevic et al. who face the same issue with their own photochemical model (Loison et al., 2015).

Calculated densities for $\mathrm{CH}_{2} \mathrm{NH}_{2}{ }^{+}$are shown in Figure 49, along with the measured densities. $\mathrm{CH}_{2} \mathrm{NH}$ mole fractions are presented in the right panel of Figure 50. Their order of magnitude is adequate over most of the altitude range but the shape of the profile is insufficient, especially below $1200 \mathrm{~km}$. Although the primary production and loss mechanisms for $\mathrm{CH}_{2} \mathrm{NH}_{2}{ }^{+}$and $\mathrm{CH}_{2} \mathrm{NH}$ have been identified in Yelle et al. (2010), we should keep in mind the large uncertainties on the dissociative recombination reaction of $\mathrm{CH}_{2} \mathrm{NH}_{2}{ }^{+}$.
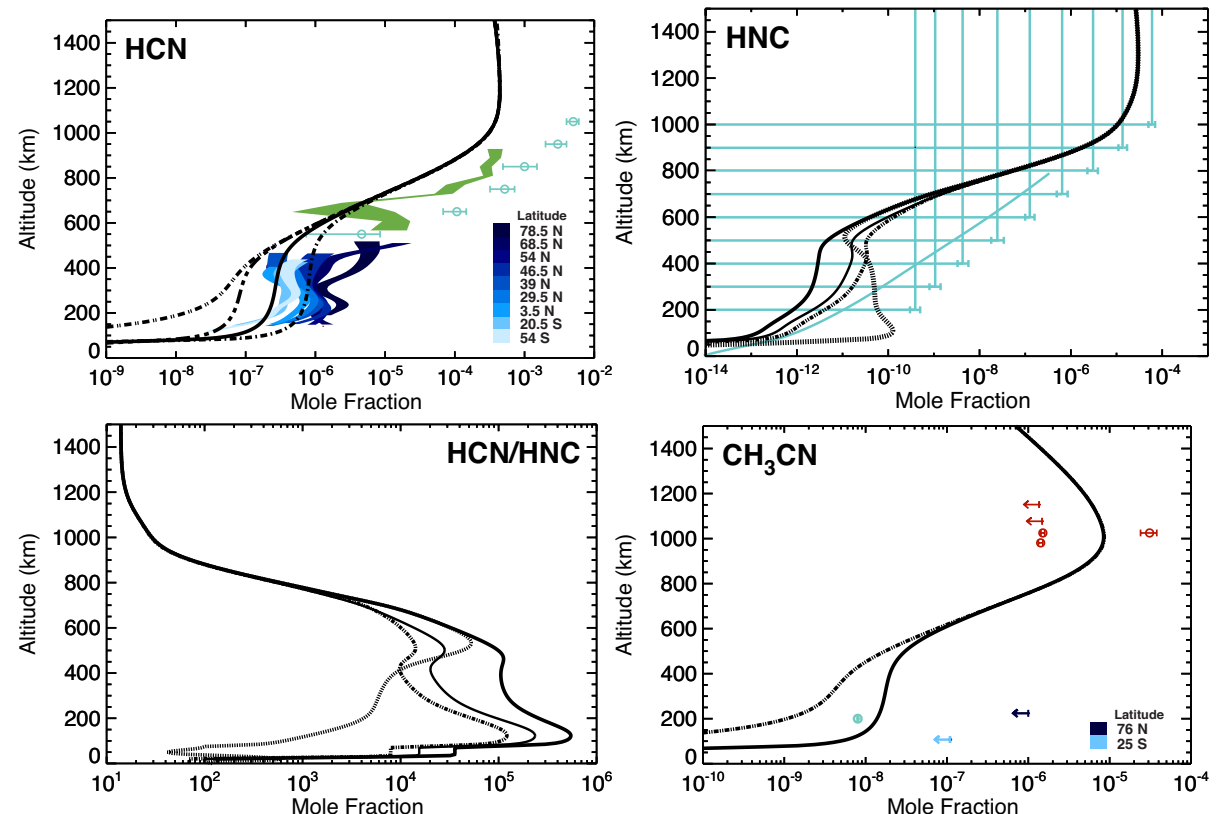

Figure 51: Calculated and observed mole fractions of HCN (upper left panel), HNC (upper right panel), $\mathrm{HCN} / \mathrm{HNC}$ ratio (lower left panel), and $\mathrm{CH}_{3} \mathrm{CN}$ (lower right panel). Solid line: nominal; short dash-dot-dot and thin lines: $\mathrm{HC}_{3} \mathrm{~N}$ sensitivity test, Table 13 ; dotted line: with GCR; dash-dot and long dash - double short dash line: eddy mixing coefficient $K_{0}=10^{2}$ and $10^{3} \mathrm{~cm}^{-2}$, respectively, section 2.4; dash-dot-dot line: with nitriles heterogeneous chemistry, section 5.3.3. Some of the lines are displayed in some plots and not in others. The data shown for HCN is from CIRS (Vinatier et al., 2010), UVIS (Koskinen et al., 2011), VIMS (Adriani et al., 2011); for HNC is from Herschel HIFI (Moreno et al., 2011) (lines that assume constant profiles), ALMA (Cordiner et al., 2014) (line is their gradient model); for $\mathrm{CH}_{3} \mathrm{CN}$ is from IRAM (Marten et al., 2002) (green), CIRS (Nixon et al., 2010) (blue upper limits), INMS (Cui et al., 2009b). 
Hydrogen (iso)cyanide ( $\left.\mathbf{H C N}, \mathbf{H N C} / \mathbf{H C N H}^{+}\right)$. Titan ionospheric models have long predicted that $\mathrm{HCNH}^{+}$should be one of the most abundant ions with a peak density on the order of $1000 \mathrm{~cm}^{-3}$ around 1000-1200 km (Ip, 1990; Keller et al., 1998). Nevertheless, it is a recurrent problem amongst Titan ionospheric models (from either the Cravens, Waite or Yelle groups) that they predict an $\mathrm{HCNH}^{+}$density that is $2-3$ times higher than observed by INMS. The Dobrijevic et al. (2016) model result agrees better with INMS data than those previous results. This would be explained by the introduction of the reaction $\mathrm{N}\left({ }^{2} \mathrm{D}\right)+\mathrm{HCN} \rightarrow \mathrm{CH}+\mathrm{N}_{2}$ that would efficiently consume HCN, according to the authors. Despite including this reaction in our model with a similar rate coefficient, it turns out to only be a minor $(<5 \%)$ loss process for HCN in our model. Therefore, our current findings are in line with Westlake et al. (2012), Mandt et al. (2012a) and Richard et al. (2015a), as our calculated globally averaged $\mathrm{HCNH}^{+}$profile overestimates the T40 observations by 1.4 at 1100 $\mathrm{km}$. This issue is probably related to the overproduction of electrons in dayside models, as discussed in Vigren et al. (2013) and Sagnières et al. (2015). We will return to this complicated topic in section 5.1.

The HCN molecule is the main nitrile in Titan's atmosphere. There is a relatively wide consensus for its main formation and loss pathways despite the fact that most post-Cassini models tend to underestimate its abundance (Lavvas et al., 2008b; Krasnopolsky, 2014; Loison et al., 2015). One important difference with the recent model of Dobrijevic et al. (Loison et al., 2015; Dobrijevic et al., 2016) is that we find that a large fraction of the $\mathrm{CN}$ radicals reacts with $\mathrm{C}_{2} \mathrm{H}_{4}$ and $\mathrm{C}_{2} \mathrm{H}_{2}$ leading to $\mathrm{C}_{2} \mathrm{H}_{3} \mathrm{CN}$ and $\mathrm{HC}_{3} \mathrm{~N}$ production, while only a small fraction $(\sim 10 \%)$ reacts with $\mathrm{CH}_{4}$ and $\mathrm{C}_{2} \mathrm{H}_{6}$ leading back to $\mathrm{HCN}$. Willacy et al. (2016) report that below $200 \mathrm{~km}, \mathrm{HCN}$ destruction is by reaction with $\mathrm{C}_{2} \mathrm{H}_{3}$ but calculations performed in the present work (cf. Appendix A) indicate that the rate coefficient of this reaction is extremely small $\left(\sim 10^{-22} \mathrm{~cm}^{3} \mathrm{~s}^{-1}\right.$ at 150 $\mathrm{K})$.

Our model vertical profile of $\mathrm{HCN}$ is presented in the upper left panel of Figure 51. HCN being the major product of the photodissociation of $\mathrm{N}_{2}$, it is a particularly good test for the $\mathrm{N}_{2}$ dissociation scheme derived from Lavvas et al. (2011a). The model gives a simulated HCN abundance in good agreement with INMS in the thermosphere and CIRS in the stratosphere, but it is smaller than the abundance derived from UV absorption in the mesosphere. Despite the differences in formation and destruction processes mentioned above, the profiles obtained in the present work, Dobrijevic et al. (2016) and Willacy et al. (2016) are similar.

After high-level ab initio quantum chemical investigations concurred that $\mathrm{HNC}$ is a major product of the dissociative recombination of $\mathrm{HCNH}^{+}$(Talbi and Ellinger, 1998), it was soon recognized that HNC could have a significant density in Titan's upper thermosphere (Petrie, 2001). Simple calculations assuming formation through electron recombination of $\mathrm{HCNH}^{+}$, loss through proton exchange reactions, and using the atmospheric properties known at that time, lead to an HNC column density of $7.0 \times 10^{11}-5.2 \times 10^{12} \mathrm{~cm}^{-2}$, which is somewhat marginally consistent with a column density in the range $(0.6-1.5) \times 10^{13} \mathrm{~cm}^{-2}$ 
from observations of the HIFI instrument on the Herschel Space Observatory (Moreno et al., 2011) and $(1.2-1.9) \times 10^{13} \mathrm{~cm}^{-2}$ using ALMA (Cordiner et al., 2014).

Our model vertical profile of $\mathrm{HNC}$ is presented in the upper right panel of Figure 51. With improved production and loss rates (Appendix A), we find that the bulk of the $\mathrm{HNC}$ is produced above $800 \mathrm{~km}$, with a density profile reaching a peak of $\sim 10^{5} \mathrm{~cm}^{-3}$ at an altitude of $950-1000 \mathrm{~km}$. This translates into a constant mole fraction of $\sim 2 \times 10^{-5}$ above 1050-1100 km (cf. lower left panel of Figure 51) and a total column density of $8.9 \times 10^{12} \mathrm{~cm}^{-2}$, which is the column density retrieved from the observations assuming a constant mole fraction profile above $700 \mathrm{~km}$. While the simple comparison of the column density can give us a feeling that our distribution is adequate overall, a definitive test of the modeling would require the computation of a synthetic spectrum based on our calculated mixing profile and comparison against the HIFI and ALMA data.

Following its detection, Hébrard et al. (2012) investigated the production of $\mathrm{HNC}$ via neutral reactions. They find that $\mathrm{HNC}$ is mostly produced from $\mathrm{H}_{2} \mathrm{CN}$ $+\mathrm{H} \rightarrow \mathrm{HNC}+\mathrm{H}$ and to a lesser extent from $\mathrm{N}\left({ }^{4} \mathrm{~S}\right)+{ }^{3} \mathrm{CH}_{2} \rightarrow \mathrm{HNC}+\mathrm{H}$, which are also the two major processes involving neutrals in our model. It is mainly destroyed by its reaction with $\mathrm{N}\left({ }^{2} \mathrm{D}\right)$ above $1000 \mathrm{~km}$ and $\mathrm{H}$ at lower altitude. We agree with the latter but find that the former process is a marginal loss. Since Hébrard et al. (2012) estimated the rate coefficient of this reaction to be about one order of magnitude faster than the rate coefficient that we calculated, their $\mathrm{N}\left({ }^{2} \mathrm{D}\right)$ density must be about three orders of magnitude higher than ours, probably because of a different dissociation scheme for $\mathrm{N}_{2}$. The column density obtained is $3.4 \times 10^{13} \mathrm{~cm}^{-2}$, which is about 3 times higher than the value derived from the observations.

In Dobrijevic et al. (2016) where neutral and ion chemistries are coupled, the dissociative recombination of $\mathrm{HCNH}^{+}$now produces a significant fraction of $\mathrm{HNC}$, in good agreement with our findings. Its relative abundance reaches a value of $7 \times 10^{-5}$ above $1200 \mathrm{~km}$. Although the authors claim that their $\mathrm{HNC}$ profile is in good agreement with the Herschel/HIFI observations, they do not provide the column density obtained and the mole fraction profile presented in their Figure 26 does not seem to match with Cordiner et al. (2014). Although the same main production and loss reactions are at play in both the present and Dobrijevic et al. (2016) models, different rate coefficients explain the distinct HNC profiles. Willacy et al. (2016) are only able to reasonably match the ALMA best-fit profile with a model where an extra loss leading to haze is applied to $\mathrm{HNC}$ at lower altitudes. 


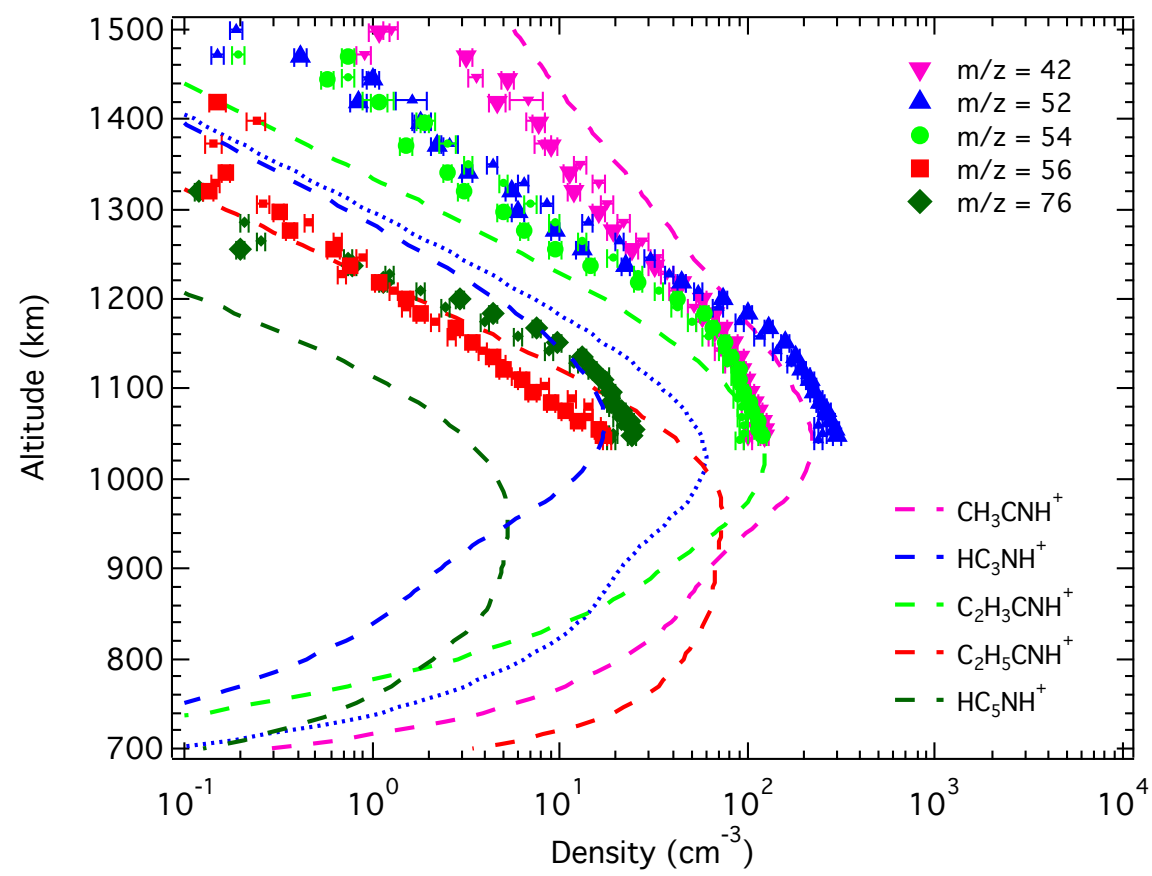

Figure 52: Model results for the density of $\mathrm{CH}_{3} \mathrm{CNH}^{+}, \mathrm{HC}_{3} \mathrm{NH}^{+}, \mathrm{C}_{2} \mathrm{H}_{3} \mathrm{CNH}^{+}, \mathrm{C}_{2} \mathrm{H}_{5} \mathrm{CNH}^{+}$ and $\mathrm{HC}_{5} \mathrm{NH}^{+}$, and comparison with the T40 INMS ion density measurements at $\mathrm{m} / \mathrm{z}=42$, $52,54,56,76$. The $\mathrm{HC}_{3} \mathrm{NH}^{+}$dotted line corresponds to the sensitivity test described in the text.

Acetonitrile $\left(\mathbf{C H}_{3} \mathbf{C N} / \mathbf{C H}_{3} \mathbf{C N H}^{+}\right)$. Our model vertical profile of acetonitrile is presented in the lower right panel of Figure 51. Like for all model profiles (Krasnopolsky, 2014; Loison et al., 2015; Dobrijevic et al., 2016; Willacy et al., 2016), the resulting $\mathrm{CH}_{3} \mathrm{CN}$ abundance is slightly below the observed values in the thermosphere (Vuitton et al., 2007; Cui et al., 2009b), while the situation reverses in the stratosphere where our calculated abundance is slightly above the the ground-based data (Marten et al., 2002). Our calculated profile for $\mathrm{CH}_{3} \mathrm{CNH}^{+}$(Figure 52) overestimates the INMS abundance by a factor of 3 at $1100 \mathrm{~km}$. Since $\mathrm{CH}_{3} \mathrm{CNH}^{+}$is mostly formed by reactions involving $\mathrm{HCNH}^{+}$ $\left(\mathrm{R}_{c n} 692, \mathrm{R}_{c n} 713\right)$, the overestimation of the latter directly translates into the overestimation of the former. The situation is reversed in Dobrijevic et al. (2016), where the $\mathrm{CH}_{3} \mathrm{CNH}^{+}$ion is underestimated.

Our main $\mathrm{CH}_{3} \mathrm{CN}$ neutral production and loss pathways are similar to those discussed in the models of Lavvas et al. (2008b), Krasnopolsky (2014), and Loison et al. (2015). However, we find that ion chemistry also plays an important role in the thermosphere through the production of $\mathrm{CH}_{2} \mathrm{CN}$, which further adds to $\mathrm{H}$ atoms to form $\mathrm{CH}_{3} \mathrm{CN}\left(\mathrm{R}_{n} 423\right)$ and through loss by proton transfer reaction (cf. section 3.1.1.3). 

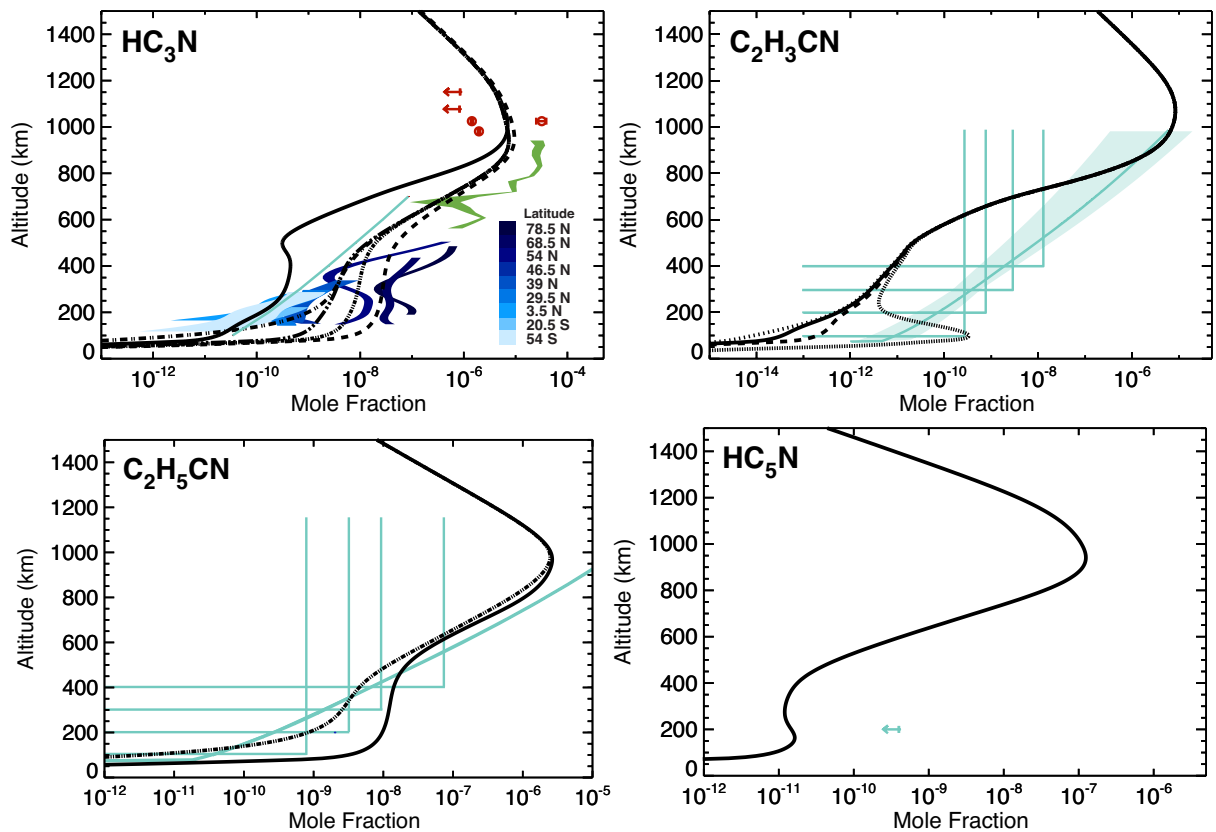

Figure 53: Calculated and observed mole fractions of $\mathrm{HC}_{3} \mathrm{~N}$ (upper left panel), $\mathrm{C}_{2} \mathrm{H}_{3} \mathrm{CN}$ (upper right panel), $\mathrm{C}_{2} \mathrm{H}_{5} \mathrm{CN}$ (lower left panel) and $\mathrm{HC}_{5} \mathrm{~N}$ (lower right panel). Solid line: nominal; thin line: $\mathrm{HC}_{3} \mathrm{~N}$ sensitivity test, Table 13; hair line: with GCR; dotted and dashed lines: aerosol optical depth multiplied by 0.5 and 2 , respectively, section 5.3 .1 ; long dash-dotdot line: no $\mathrm{H}$ heterogeneous loss, section 5.3.2; dash-dot-dot line: with nitriles heterogeneous chemistry, section 5.3.3. The data shown for $\mathrm{HC}_{3} \mathrm{~N}$ is from ALMA (Cordiner et al., 2014) (green line, gradient model), CIRS (Vinatier et al., 2010); UVIS (Koskinen et al., 2011); INMS (Cui et al., 2009b) (circles no error bars are uncorrected, circle with error bar is corrected); for $\mathrm{C}_{2} \mathrm{H}_{3} \mathrm{CN}$ is from ALMA (Palmer et al., 2017) (the line with error bars is their fractional scale height model. The other lines are just assuming a constant value above a certain height); for $\mathrm{C}_{2} \mathrm{H}_{5} \mathrm{CN}$ is from ALMA (Cordiner et al., 2014) (line is gradient model, others are assuming constant with height); for $\mathrm{HC}_{5} \mathrm{~N}$ is from IRAM (Marten et al., 2002).

Cyanoacetylene $\left(\mathbf{H C}_{3} \mathbf{N} / \mathbf{H C}_{3} \mathbf{N H}^{+}\right)$. Like that of diacetylene, the cyanoacetylene vertical profile is well constrained with available observations from INMS, UVIS and CIRS. In the thermosphere, the UVIS data support the corrected value from Cui et al. (2009b). In the stratosphere, the abundance presents some drastic latitudinal variations, with polar abundances at $200 \mathrm{~km}$ being three orders of magnitude higher than the equatorial ones. 
Table 13: Parameters used in the nominal and $\mathrm{HC}_{3} \mathrm{~N}$ sensitivity test runs.

\begin{tabular}{lll}
\hline \hline Reaction & Nominal Run & Test Run \\
\hline \hline $\mathrm{HC}_{3} \mathrm{NH}^{+}+\mathrm{C}_{2} \mathrm{H}_{4} \rightarrow \mathrm{C}_{5} \mathrm{H}_{5} \mathrm{~N}^{+}+\mathrm{H}\left(\mathrm{R}_{c n} 790\right)$ & Upper Limit & 0 \\
$\mathrm{HC}_{3} \mathrm{NH}^{+}+\mathrm{e}^{-} \rightarrow \mathrm{HC}_{3} \mathrm{~N}+\mathrm{H} / \mathrm{C}_{3} \mathrm{~N}+\mathrm{H}_{2}\left(\mathrm{R}_{e r} 104 \mathrm{a}, \mathrm{c}\right)$ & $0.5 / 0.5$ & $1 / 0$ \\
$\mathrm{H}+\mathrm{HC}_{3} \mathrm{~N} \rightarrow \mathrm{H}_{2} \mathrm{C}_{3} \mathrm{~N}\left(\mathrm{R}_{n} 258\right)$ & This Work & est. $\left(\mathrm{H}+\mathrm{C}_{4} \mathrm{H}_{2}\right)$ \\
\hline \hline
\end{tabular}


Our model vertical profile of $\mathrm{HC}_{3} \mathrm{~N}$ and $\mathrm{HC}_{3} \mathrm{NH}^{+}$are presented in Figures 53 (upper left panel) and 52, respectively. In the upper atmosphere, both $\mathrm{HC}_{3} \mathrm{~N}$ and $\mathrm{HC}_{3} \mathrm{NH}^{+}$are too low by a factor of about five and twenty, respectively. In the stratosphere, $\mathrm{HC}_{3} \mathrm{~N}$ is one to two orders of magnitude higher than CIRS mid-latitude observations. Therefore, we obtain a similar underestimation in the thermosphere / overestimation in the stratosphere for $\mathrm{C}_{4} \mathrm{H}_{2}$ and $\mathrm{HC}_{3} \mathrm{~N}$.

Our main $\mathrm{HC}_{3} \mathrm{~N}$ production and loss pathways are similar to those discussed in previous models (cf. section 3.2.2) but the chemistry of $\mathrm{HC}_{3} \mathrm{NH}^{+}$is somewhat uncertain. The reaction with $\mathrm{C}_{2} \mathrm{H}_{4}\left(\mathrm{R}_{c n} 790\right)$, which is its main loss, has a rate coefficient that is given as an upper limit in McEwan and Anicich (2007). The rate coefficient of its second main loss, recombination with electrons, is well constrained but the exact products are unknown with the channel preserving the carbon chain being either $\mathrm{HC}_{3} \mathrm{~N}+\mathrm{H}$ or $\mathrm{C}_{3} \mathrm{~N}+\mathrm{H}_{2}\left(\mathrm{R}_{e r} 104 \mathrm{a}, \mathrm{c}\right)$. We performed a sensitivity test (cf. Table 13), in which we attempted to increase the $\mathrm{HC}_{3} \mathrm{NH}^{+} / \mathrm{HC}_{3} \mathrm{~N}$ density by (i) setting $\left(\mathrm{R}_{c n} 790\right)$ to 0 and (ii) assuming that the electron recombination of $\mathrm{HC}_{3} \mathrm{NH}^{+}$does not produce any $\mathrm{C}_{3} \mathrm{~N}+\mathrm{H}_{2}$ and that instead $100 \%$ of the products of the channel preserving the carbon chain are $\mathrm{HC}_{3} \mathrm{~N}+\mathrm{H}\left(\mathrm{R}_{e r} 104 \mathrm{a}\right)$. With this new scheme, the $\mathrm{HC}_{3} \mathrm{NH}^{+}$density increases by a factor of about three (thin line in Figure 52), which implies that it still falls short from the observations by a factor of $\sim 5$. The impact on $\mathrm{HC}_{3} \mathrm{~N}$ is minor, as its density increases by only about $30 \%$. This is due to the fact that ion chemistry is a minor player for $\mathrm{HC}_{3} \mathrm{~N}$, as discussed in section 3.1.1.3.

We actually realized that an older version of the model, where we had the rate coefficients of the main loss reaction along with photodissociation, $\mathrm{H}+\mathrm{HC}_{3} \mathrm{~N}$ $\left(\mathrm{R}_{n} 258\right)$, set equal to that of the $\mathrm{H}+\mathrm{C}_{4} \mathrm{H}_{2}$ reaction $\left(\mathrm{R}_{n} 21\right)$, provided quite a good fit below $300 \mathrm{~km}$. This is reproduced here as the thin line in the upper left panel of Figure 53. However, our present calculations predict that the $\mathrm{H}+\mathrm{HC}_{3} \mathrm{~N}$ reaction is quite a bit slower than the $\mathrm{H}+\mathrm{C}_{4} \mathrm{H}_{2}$ reaction, with the high pressure limits differing by a factor of 30 at $140 \mathrm{~K}$ (cf. Figure A.2d). Note, however, since the reaction is tunneling dominated, there is considerable uncertainty in the present rate estimates. For example, decreasing the imaginary frequency by $100 \mathrm{~cm}^{-1}$ lowers the rate constant by a factor of 2 at $140 \mathrm{~K}$, as does increasing the barrier height by $0.2 \mathrm{kcal} / \mathrm{mol}$. This strong sensitivity may be the cause of either the overprediction of the $\mathrm{HC}_{3} \mathrm{~N}$ concentration at low altitudes or the underprediction at high altitudes. However, it is difficult to see how the predicted rate can be both too low at low altitudes and too high at high altitudes, since we predict only a modest pressure dependence. It has been postulated that because of their polar nature, the nitriles present in the gas phase could efficiently stick to the aerosols present in particular in the stratosphere, adding an extra heterogeneous loss for these species. We will return to this in section 5.3.3.

Earlier models have not been very successful at matching the cyanoacetylene mole fraction at all levels of the atmosphere. Both the Lavvas et al. and Loison et al. calculated profiles (cf. Figure 9c in Lavvas et al. (2008b) and top of Figure 10 in Loison et al. (2015)) are below the retrieved abundance in the upper atmosphere, yet they are closer to the polar profiles retrieved from the CIRS ob- 
servations than from the mid-latitudes ones. The cyanoacetylene abundance in the model from Krasnopolsky (cf. Figure 3 in Krasnopolsky (2014)) agrees with that from INMS neutral and ion spectra but significantly exceeds both UVIS and CIRS data. The Dobrijevic et al. (2016) model results in an overestimate of the $\mathrm{HC}_{3} \mathrm{~N}$ abundance compared to the observations in all regions (their Figure 10), while their $\mathrm{HC}_{3} \mathrm{NH}^{+}$profile is in fair agreement with INMS (their Figure 12). Finally, Willacy et al. (2016) tend to under predict the $\mathrm{HC}_{3} \mathrm{~N}$ abundance above $700 \mathrm{~km}$, while below $500 \mathrm{~km}$, it is in agreement with the observations (their Figure 3 bottom left) only if it is assumed that $\mathrm{HC}_{3} \mathrm{~N}$ forms aerosols and thus is permanently removed from the gas.

Acrylonitrile $\left(\mathbf{C}_{\mathbf{2}} \mathbf{H}_{\mathbf{3}} \mathbf{C N} / \mathbf{C}_{\mathbf{2}} \mathbf{H}_{\mathbf{3}} \mathbf{C N H}{ }^{+}\right)$. Our calculated $\mathrm{C}_{2} \mathrm{H}_{3} \mathrm{CNH}^{+}$ densities between 1000 and $1400 \mathrm{~km}$ altitude are in good agreement with the INMS observations (cf. Figure 52). The abundance profile of the neutral from our chemical model is shown in the upper right panel of Figure 53. Its overall shape is consistent with ALMA (the fractional scale height model, with a smoothly varying altitude dependence), but its magnitude is slightly too low. Indeed, the vertical column densities implied by the observations lie in the range of $(0.37-1.4) \times 10^{14} \mathrm{~cm}^{-2}$ (Palmer et al., 2017), while our nominal model gives a value of $3 \times 10^{13} \mathrm{~cm}^{-2}$. This is nevertheless better than the modeled abundance profile of Dobrijevic et al. that is consistent with the observed line shapes, provided that it is scaled up by a constant factor of 4.7 (Palmer et al., 2017). Our main neutral production and loss processes are mostly consistent with those discussed in (Loison et al., 2015). The need for an enhanced $\mathrm{C}_{2} \mathrm{H}_{3} \mathrm{CN}$ production may imply that the rate coefficient of the reaction $\mathrm{CN}+\mathrm{C}_{2} \mathrm{H}_{4} \rightarrow \mathrm{C}_{2} \mathrm{H}_{3} \mathrm{CN}+$ $\mathrm{H}\left(\mathrm{R}_{n} 371\right)$ is underestimated in both models. It might also imply that there is a polar enhancement for $\mathrm{C}_{2} \mathrm{H}_{3} \mathrm{CN}$, which would result in the disc-averaged measurement being higher than the equatorial models (Palmer et al., 2017).

The models calculated profiles are generally in good agreement with the observed abundance in the upper atmosphere (cf. Figure 9b in Lavvas et al. (2008b), Figure 1d in Krasnopolsky (2012), Figure 11 in Loison et al. (2015) and Figure 4 in Willacy et al. (2016)). There is more variability in the predicted profiles in the lower atmosphere, some of them (Krasnopolsky, 2012; Willacy et al., 2016) being well above the detection limit placed by ground-based observations $\left(\sim 2 \times 10^{-9}\right.$, Marten et al. (2002)). Also, the Krasnopolsky profile produces strong pressure-broadened line wings that do not match with the very recent ALMA observations (Palmer et al., 2017). This is essentially because of the reaction of hydrogen cyanide with vinyl radicals $\left(\mathrm{C}_{2} \mathrm{H}_{3}\right)$, which was suggested to lead to the formation of acrylonitrile, but whose suggested rate below room temperature was too high $\left(2.7 \times 10^{-15} \mathrm{~cm}^{3} \mathrm{~s}^{-1}\right.$ instead of $1.4 \times 10^{-22} \mathrm{~cm}^{3} \mathrm{~s}^{-1}$ at $150 \mathrm{~K}$, see Appendix A). Actually, this reaction turns out to be negligible with our newly calculated rate coefficient. In a few photochemical models (Lavvas et al., 2008b; Willacy et al., 2016), some chemical loss to the haze counterbalanced the excessive production of acrylonitrile in the lower atmosphere through this reaction. 
Propionitrile $\left(\mathrm{C}_{2} \mathrm{H}_{5} \mathbf{C N} / \mathbf{C}_{2} \mathbf{H}_{5} \mathbf{C N H}^{+}\right)$. Our calculated densities for $\mathrm{C}_{2} \mathrm{H}_{5} \mathrm{CNH}^{+}$between 1000 and $1400 \mathrm{~km}$ altitude are in relatively good agreement with the INMS observations but the $\mathrm{C}_{2} \mathrm{H}_{5} \mathrm{CN}$ vertical profile (cf. lower left panel of Figure 53) leads to a column density of $1.6 \times 10^{16} \mathrm{~cm}^{-2}$, which is about a factor of 50 higher than that deduced from the ALMA observations (Cordiner et al., 2015b). None of the photochemical models (Krasnopolsky, 2012; Loison et al., 2015; Dobrijevic et al., 2016; Willacy et al., 2016) that present predictions for $\mathrm{C}_{2} \mathrm{H}_{5} \mathrm{CN}$ are in agreement with the recent ALMA observations that imply a $\mathrm{C}_{2} \mathrm{H}_{5} \mathrm{CN}$ column density in the range $(2.1-5.1) \times 10^{14} \mathrm{~cm}^{-2}$ (Cordiner et al., 2015b). The model from Krasnopolsky (2009), that produces $\mathrm{C}_{2} \mathrm{H}_{5} \mathrm{CN}$ through the reaction of excited nitrogen atoms with $\mathrm{C}_{3} \mathrm{H}_{6}$, generates a stratospheric abundance about two orders of magnitude too low, which indicates that (an)other production channel(s) is required. Conversely, the $\mathrm{C}_{2} \mathrm{H}_{5} \mathrm{CN}$ abundance calculated with the model from Dobrijevic et al. (Loison et al., 2015; Dobrijevic et al., 2016) are, between 100 and $300 \mathrm{~km}$ altitude, from one to three orders of magnitude higher than the observations. In this case, $\mathrm{C}_{2} \mathrm{H}_{5} \mathrm{CN}$ is produced by the ternary association of $\mathrm{CH}_{2} \mathrm{CN}$ and $\mathrm{CH}_{3}\left(\mathrm{R}_{n} 424\right)$, whose rate coefficient has never been measured. Only the model of Willacy et al. (2016) reaches an agreement with the ALMA data by including an additional sticking of $\mathrm{C}_{2} \mathrm{H}_{5} \mathrm{CN}$ to the aerosols. The trend is similar in the upper atmosphere, with the Krasnopolsky (2012) model underestimating the INMS data, while the Loison et al. (2015) and Willacy et al. (2016) models overestimate the abundance of $\mathrm{C}_{2} \mathrm{H}_{5} \mathrm{CN}$, probably because they do not include ion chemistry, a feature that was later added in Dobrijevic et al. (2016), providing a good agreement with the measurements.

The main limitation of these models is a lack of knowledge of the production and loss mechanisms for $\mathrm{C}_{2} \mathrm{H}_{5} \mathrm{CN}$. The only reactions that have been studied are $\mathrm{N}\left({ }^{2} \mathrm{D}\right)+\mathrm{C}_{3} \mathrm{H}_{6} \rightarrow \mathrm{C}_{2} \mathrm{H}_{5} \mathrm{CN}+\mathrm{H}$ and the radiative association of $\mathrm{CH}_{3} \mathrm{CN}$ with $\mathrm{CH}_{3}{ }^{+}$. The latter reaction produces $\mathrm{C}_{2} \mathrm{H}_{5} \mathrm{CNH}^{+}$and this species being in equilibrium with $\mathrm{C}_{2} \mathrm{H}_{5} \mathrm{CN}$ through proton transfer reactions and the electronic recombination of $\mathrm{C}_{2} \mathrm{H}_{5} \mathrm{CNH}^{+}$, the production of $\mathrm{C}_{2} \mathrm{H}_{5} \mathrm{CNH}^{+}$ultimately leads to some $\mathrm{C}_{2} \mathrm{H}_{5} \mathrm{CN}$ as well. However, our calculations show that, with our estimated rate coefficients, radical-radical reactions $\left(\mathrm{CH}_{2} \mathrm{CN}+\mathrm{CH}_{3}\left(\mathrm{R}_{n} 424\right), \mathrm{NH}_{2}\right.$ $+\mathrm{C}_{3} \mathrm{H}_{3}\left(\mathrm{R}_{n} 352\right)$, etc.) are the main source of $\mathrm{C}_{2} \mathrm{H}_{5} \mathrm{CN}$. Loss occurs mostly through proton transfer reactions above $700 \mathrm{~km}$, by radical reactions below 400 $\mathrm{km}$, and by photodissociation in between (cf. section 3.2.2).

Cyanodiacetylene $\left(\mathbf{H C}_{5} \mathbf{N} / \mathbf{H C}_{5} \mathbf{N H}^{+}\right)$. We obtain a peak at $\sim 3 \times$ $10^{-7}$ in the thermosphere, associated to a $\mathrm{HC}_{5} \mathrm{NH}^{+}$that underestimates the INMS signal at $\mathrm{m} / \mathrm{z}=76$ by a factor of about 5 . In the stratosphere, the modeled mole fraction decreases to reach a constant value of $\sim 10^{-11}$, in good agreement with the Marten et al. (2002) upper limit of $4 \times 10^{-10}$ (cf. lower right panel of Figure 53). The Dobrijevic et al. model is the only one to present mole fraction profiles of $\mathrm{HC}_{5} \mathrm{~N}$ (top panel in Figure 14 from Loison et al. (2015)). It peaks with a similar magnitude as our model at $1000 \mathrm{~km}$ and in the stratosphere, 

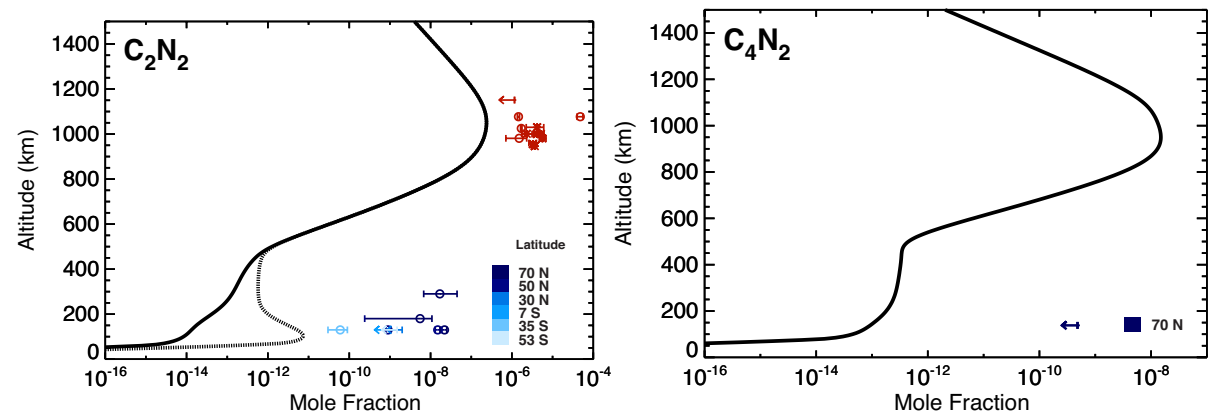

Figure 54: Calculated and observed mole fractions of $\mathrm{C}_{2} \mathrm{~N}_{2}$ (left panel) and $\mathrm{C}_{4} \mathrm{~N}_{2}$ (right panel). Solid line: nominal; hair line: with GCR. The data shown for $\mathrm{C}_{2} \mathrm{~N}_{2}$ is from IRIS (Coustenis et al., 1991; Coustenis and Bézard, 1995), CIRS (Teanby et al., 2006; Teanby et al., 2009), INMS (Cui et al., 2009b) (circles no error bars are uncorrected, circle with error bar is corrected) (Waite et al., 2007) (asterisks); for $\mathrm{C}_{4} \mathrm{~N}_{2}$ is from CIRS (Jolly et al., 2015).

Cyanogen $\left(\mathbf{C}_{2} \mathbf{N}_{2}\right)$. Our model under predicts the observations by one order of magnitude in the thermosphere and almost four orders of magnitude in the stratosphere (cf. left panel of Figure 54). Actually, the only CIRS detections are at pretty high latitudes, implying that $\mathrm{C}_{2} \mathrm{~N}_{2}$ is probably enriched at the poles and that the values on the Figure are higher than what we expect at the equator. Also, the chemistry of $\mathrm{C}_{2} \mathrm{~N}_{2}$ is poorly constrained and its principal production and loss reactions had to be estimated (cf. section 3.2.2).

Most of the previous models underestimate the cyanogen abundance in the upper atmosphere as well. In the stratosphere, the Lavvas et al. profile is well below the CIRS observations for the low latitude regions (cf. Figure 9d in Lavvas et al. (2008b)) while both the Krasnopolsky and Dobrijevic et al. models overestimate them (cf. Figure 5 in Krasnopolsky (2014) and top panel of Figure 12 in Loison et al. (2015)). Unlike them, the most recent model of the Yung et al. group is in very good agreement with both the INMS and CIRS datasets (cf. top left panel of Figure 4 in Willacy et al. (2016)). It is unfortunate that the values and origin of the rate coefficients used in this model are not clearly stated.

Dicyanogen $\left(\mathrm{C}_{4} \mathbf{N}_{2}\right)$. Our calculated profile for $\mathrm{C}_{4} \mathrm{~N}_{2}$ and that from previous models are all well below the upper limit retrieved close to $100 \mathrm{~km}$. This is shown on the right panel of Figure 54 for this work but see also Figure 9d in Lavvas et al. (2008b), Figure 1d in Krasnopolsky (2012) and bottom panel of Figure 12 in Loison et al. (2015). 

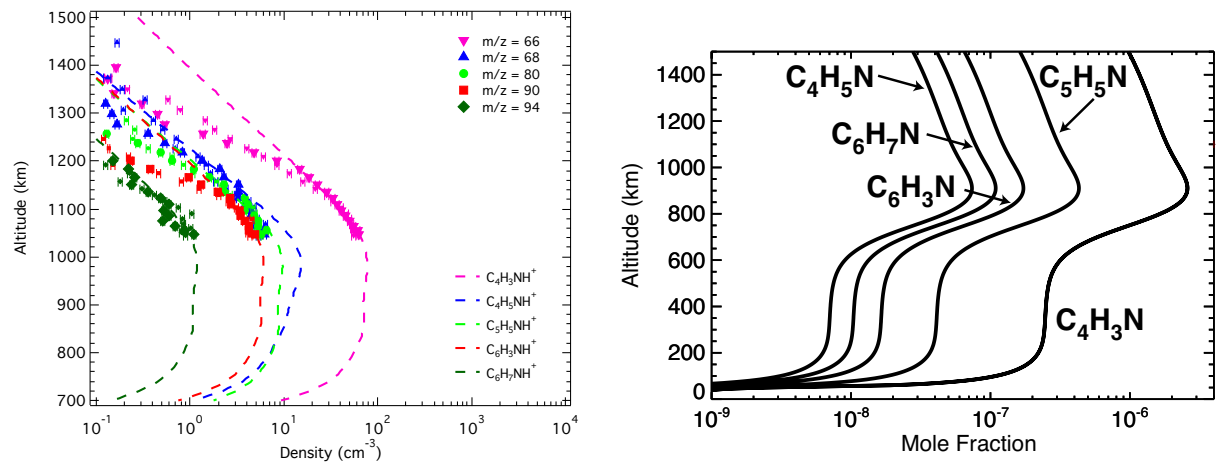

Figure 55: Model results for the density of $\mathrm{C}_{4} \mathrm{H}_{3} \mathrm{NH}^{+}, \mathrm{C}_{4} \mathrm{H}_{5} \mathrm{NH}^{+}, \mathrm{C}_{5} \mathrm{H}_{5} \mathrm{NH}^{+}, \mathrm{C}_{6} \mathrm{H}_{3} \mathrm{NH}^{+}$ and $\mathrm{C}_{6} \mathrm{H}_{7} \mathrm{NH}^{+}$, and comparison with the T40 INMS ion density measurements at $\mathrm{m} / \mathrm{z}=66$, $68,80,90,94$ (left panel). Inferred vertical profile for associated neutrals (right panel).

Table 14: Inferred neutrals mole fraction at $1100 \mathrm{~km}$.

\begin{tabular}{lll}
\hline \hline & \multicolumn{3}{c}{ Mixing Ratio } \\
Species & Vuitton et al. (2007) & This Work \\
\hline \hline $\mathrm{C}_{4} \mathrm{H}_{3} \mathrm{~N}$ & $4.0 \times 10^{-6}$ & $2.0 \times 10^{-6}$ \\
$\mathrm{C}_{4} \mathrm{H}_{5} \mathrm{~N}$ & $<3.0 \times 10^{-7}$ & $5.0 \times 10^{-8}$ \\
$\mathrm{C}_{5} \mathrm{H}_{5} \mathrm{~N}$ & $4.0 \times 10^{-7}$ & $3.0 \times 10^{-7}$ \\
$\mathrm{C}_{6} \mathrm{H}_{3} \mathrm{~N}$ & $3.0 \times 10^{-7}$ & $1.0 \times 10^{-7}$ \\
$\mathrm{C}_{6} \mathrm{H}_{7} \mathrm{~N}$ & $1.0 \times 10^{-7}$ & $8.0 \times 10^{-8}$ \\
\hline \hline
\end{tabular}

Heavies. The nitrogen rule states that protonated organic compounds with an even nominal mass have an odd number of nitrogen atoms. This general principal allowed us to attribute the ions at $\mathrm{m} / \mathrm{z}=66,68,80,90$ and 94 to $\mathrm{C}_{4} \mathrm{H}_{3} \mathrm{NH}^{+}, \mathrm{C}_{4} \mathrm{H}_{5} \mathrm{NH}^{+}, \mathrm{C}_{5} \mathrm{H}_{5} \mathrm{NH}^{+}, \mathrm{C}_{6} \mathrm{H}_{3} \mathrm{NH}^{+}$and $\mathrm{C}_{6} \mathrm{H}_{7} \mathrm{NH}^{+}$. Moreover, we showed that they depend closely on the composition of the neutral atmosphere and that measurement of their density coupled with simple chemical schemes provides a sensitive determination of the abundance of their associated neutral species (Vuitton et al., 2007). Because the production and loss pathways for $\mathrm{C}_{4} \mathrm{H}_{3} \mathrm{~N}, \mathrm{C}_{4} \mathrm{H}_{5} \mathrm{~N}, \mathrm{C}_{5} \mathrm{H}_{5} \mathrm{~N}, \mathrm{C}_{6} \mathrm{H}_{3} \mathrm{~N}$ and $\mathrm{C}_{6} \mathrm{H}_{7} \mathrm{~N}$ are largely undetermined, we do not track them specifically but instead stick to our previous approach to estimate their abundance in the upper atmosphere. Mixing ratios at $1100 \mathrm{~km}$ inferred from the T40 ion densities (this model) are given in Table 14, along with the values inferred from the T5 ion densities (Vuitton et al., 2007). Both set of values are in good agreement although the T40 ones tend to be smaller by a factor of about 2 .

${ }^{15} \mathrm{~N}$ Isotopologues The ${ }^{14} \mathrm{~N} /{ }^{15} \mathrm{~N}$ ratio in $\mathrm{N}_{2}$ has been retrieved from INMS and GCMS measurements (cf. Table 15). INMS gives a ratio of 172 to 215 from the analysis of an average spectrum for the altitude range of 1230 
to $1174 \mathrm{~km}$ during TA, which translates into an estimated value at the surface of 168-211 (Waite et al., 2005). A later fit of the INMS ${ }^{14} \mathrm{~N}^{14} \mathrm{~N}$ and ${ }^{14} \mathrm{~N}^{15} \mathrm{~N}$ vertical profiles between 1100 and $1500 \mathrm{~km}$ averaged over 16 passes gives a ${ }^{14} \mathrm{~N} /{ }^{15} \mathrm{~N}$ ratio of $147.5 \pm 7.5$ below the homopause level (Mandt et al., 2009). GCMS measurements in the troposphere indicate a ratio of $167.7 \pm 0.6$ averaged through the entire altitude range 144-16 km (Niemann et al., 2010). (Niemann et al., 2010) is meant to supersede the earlier ratio of (Niemann et al., 2005) $(183 \pm 5)$ anywhere they disagree.

The ${ }^{14} \mathrm{~N} /{ }^{15} \mathrm{~N}$ ratio in $\mathrm{HCN}$ has been determined from ground-based observations (Marten et al., 2002; Gurwell, 2004), as well as from CIRS (Vinatier et al., 2007b) and Herschel (Courtin et al., 2011) data, which all probe the stratosphere. The values are somewhat scattered, ranging from $56 \pm 8$ for CIRS to $94 \pm 13$ for the Sub-Millimeter Array using the Lellouch (1990) temperature profile. The ${ }^{14} \mathrm{~N} /{ }^{15} \mathrm{~N}$ ratio in $\mathrm{CH}_{3} \mathrm{CN}$ has been retrieved from ALMA data (Cordiner et al., 2015a) but it may be subject to revision based on more accurate observations and modeling. This preliminary ratio is in excellent agreement with the CIRS value for $\mathrm{HCN}$. The $\mathrm{HCN}$ - and $\mathrm{CH}_{3} \mathrm{CN}$-derived values are therefore a factor of 2-3 lower than the $\mathrm{N}_{2}$-derived values, showing enrichment of the heavier isotope of nitrogen in $\mathrm{HCN}$ and $\mathrm{CH}_{3} \mathrm{CN}$. 
Table 15: Observed ${ }^{14} \mathrm{~N} /{ }^{15} \mathrm{~N}$ isotopic ratio in $\mathrm{N}_{2}, \mathrm{HCN}$ and $\mathrm{CH}_{3} \mathrm{CN}$.

\begin{tabular}{|c|c|c|c|c|}
\hline$\overline{\overline{\mathrm{N}_{2}} \text { (thermosphere) }}$ & $19193.5 \pm 21.5^{(a)}$ & & & \\
\hline $\mathrm{N}_{2}$ (troposphere $)$ & $188 \pm 16^{(b)}$ & $147.5 \pm 7.5^{(c)}$ & $183 \pm 5^{(d)}$ & $167.7 \pm 0.6^{(e)}$ \\
\hline $\mathrm{HCN}$ (stratosphere) & $65 \pm 5^{(f)}$ & $72 \pm 9^{*} ; 94 \pm 13^{* *(g)}$ & $56 \pm 8^{(h)}$ & $76 \pm 6^{(i)}$ \\
\hline $\mathrm{CH}_{3} \mathrm{CN}$ (stratosphere) & $58 \pm 8^{(j)}$ & & & \\
\hline
\end{tabular}

${ }^{(a)}$ INMS (Waite et al., 2005); ${ }^{(b)}$ INMS extrapolated to surface (Waite et al., 2005); ${ }^{(c)}$ INMS extrapolated to surface (Mandt et al., 2009); ${ }^{(d)}$ GCMS (Niemann et al., 2005); ${ }^{(e)}$ GCMS (Niemann et al., 2010); ${ }^{(f)}$ IRAM (Marten et al., 2002); ${ }^{(g)}$ SMA (Gurwell, 2004) *assuming the temperature profile from (Lellouch, 1990), ${ }^{* *}$ assuming the temperature profile from (Coustenis and Bézard, 1995); ${ }^{(h)}$ CIRS (Vinatier et al., 2007b); ${ }^{(i)}$ Herschel (Courtin et al., 2011); ${ }^{(j)}$ ALMA (Cordiner et al., 2015a). 


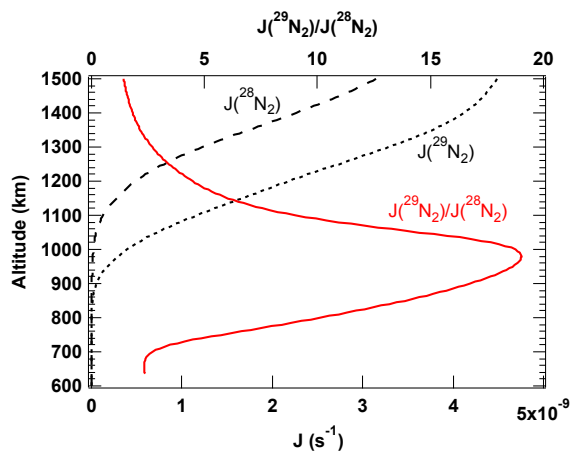
to be reevaluated. to $970 \mathrm{~km}$.

Photo-induced isotopic fractionation has been put forward to explain the ${ }^{15} \mathrm{~N}$ enrichment in $\mathrm{HC}^{15} \mathrm{~N}$ (Liang et al., 2007; Mandt et al., 2012a). However, the original calculations by Liang et al. (2007) required an additional source of atomic nitrogen in the upper atmosphere to obtain the observed $\mathrm{HC}^{14} \mathrm{~N} / \mathrm{HC}^{15} \mathrm{~N}$ ratio. With a ${ }^{14} \mathrm{~N} /{ }^{15} \mathrm{~N}=183-260$, the $\mathrm{N}$ input was constrained to be in the range $(1-2) \times 10^{9} \mathrm{~cm}^{-2} \mathrm{~s}^{-1}$. This value, in agreement with the understanding of $\mathrm{N}_{2}$ dissociation by Saturnian magnetospheric ion/electron impact at that time, is now believed to be overestimated by about two orders of magnitude (Lavvas et al., 2011a), which implies that the origin of the ${ }^{14} \mathrm{~N} /{ }^{15} \mathrm{~N}$ fractionation needs

As presented in section 2.6.3, photoabsorption cross sections for ${ }^{28} \mathrm{~N}_{2}$ and ${ }^{29} \mathrm{~N}_{2}$ are highly structured in the $80-100 \mathrm{~nm}$ region and can induce some isotopeselective shielding, resulting in a higher photolytic efficiency for ${ }^{29} \mathrm{~N}_{2}$ than for ${ }^{28} \mathrm{~N}_{2}$, therefore leading to a higher $\mathrm{HC}^{15} \mathrm{~N}$ production rate. This is illustrated in the left panel of Figure 56, which shows the profiles of the photodissociation rate coefficients (J-value, defined in Eq. E8) for ${ }^{28} \mathrm{~N}_{2}$ and ${ }^{29} \mathrm{~N}_{2}$. Shifts in the rovibrational transition energies associated with differences in line intensities due to isotopic substitution and selective overlap with solar lines cause $J\left({ }^{29} \mathrm{~N}_{2}\right)$ to be larger than $\mathrm{J}\left({ }^{28} \mathrm{~N}_{2}\right)$, the ratio reaching a maximum of 19 at $980 \mathrm{~km}$.

The calculated photodissociation rates for ${ }^{28} \mathrm{~N}_{2}$ and ${ }^{29} \mathrm{~N}_{2}$ are shown in the right panel of Figure 56. Unlike ${ }^{28} \mathrm{~N}_{2}$, where the rate peaks at $1130 \mathrm{~km}$, the isotope-selective photodissociation allows more dissociative photons for ${ }^{29} \mathrm{~N}_{2}$ to penetrate deeper into Titan's atmosphere and moves the ${ }^{29} \mathrm{~N}_{2}$ maximum down

Figure 56: ${ }^{28} \mathrm{~N}_{2}$ and ${ }^{29} \mathrm{~N}_{2}$ photodissociation rate coefficient (left panel) and photodissociation rate (right panel).

Higher photodissociation rate coefficients for ${ }^{29} \mathrm{~N}_{2}$ than for ${ }^{28} \mathrm{~N}_{2}$ translate into the production of more ${ }^{15} \mathrm{~N}$ than ${ }^{14} \mathrm{~N}$ and therefore higher ${ }^{15} \mathrm{~N}$ densities (both $\mathrm{N}\left({ }^{2} \mathrm{D}\right)$ and $\mathrm{N}\left({ }^{4} \mathrm{~S}\right)$ ), as illustrated in Figure 57. 


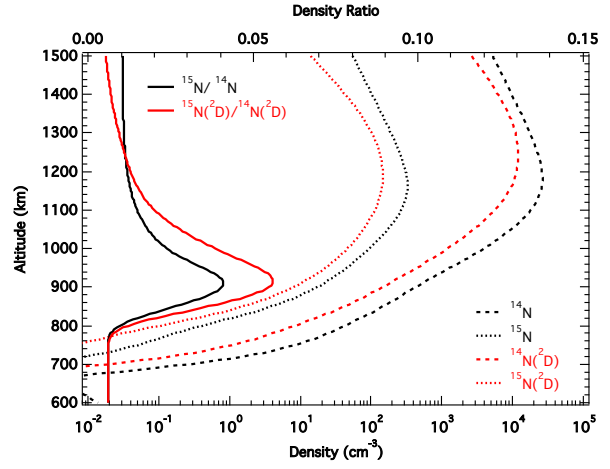

Figure 57: Model results for the density of nitrogen atoms.

The subsequent nitrogen chemistry is described in section 3.2.2. The resulting abundances of the ${ }^{15} \mathrm{~N}$ isotopologues of $\mathrm{HCN}, \mathrm{CH}_{3} \mathrm{CN}$ and $\mathrm{HC}_{3} \mathrm{~N}$ are presented in Figure 58. The ${ }^{14} \mathrm{~N} /{ }^{15} \mathrm{~N}$ ratio for those three species is shown in those same plots and corresponding values at 1200 and $200 \mathrm{~km}$ are listed in Table 16. For all three species, the ${ }^{14} \mathrm{~N} /{ }^{15} \mathrm{~N}$ ratio is constant above $1100 \mathrm{~km}$ and then decreases to reach again a pretty constant value close to 55 below $800 \mathrm{~km}$. Because the ${ }^{29} \mathrm{~N}_{2}$ photolysis peaks at lower altitude (cf. Figure 56), the ${ }^{15} \mathrm{~N}$ fraction increases with decreasing altitude eventually reaching a plateau near $800 \mathrm{~km}$ (cf. Figure 57). The ${ }^{14} \mathrm{~N} /{ }^{15} \mathrm{~N}$ ratio varies from 76 for $\mathrm{CH}_{3} \mathrm{CN}$ to 103 for $\mathrm{HC}_{3} \mathrm{~N}$ and is a direct consequence of the primary source of nitrogen leading to the formation of the molecule. Since $N\left({ }^{2} D\right)$ is more enriched in ${ }^{15} \mathrm{~N}$ than $N\left({ }^{4} \mathrm{~S}\right)$ (cf. Figure 57), the species originating from $\mathrm{N}\left({ }^{2} \mathrm{D}\right)$, such as $\mathrm{CH}_{3} \mathrm{CN}$ should be more enriched in ${ }^{15} \mathrm{~N}$ than the species originating from $\mathrm{N}\left({ }^{4} \mathrm{~S}\right)$, such as $\mathrm{HCN}$. This translates into a higher ${ }^{14} \mathrm{~N} /{ }^{15} \mathrm{~N}$ in $\mathrm{HCN}$ than in $\mathrm{CH}_{3} \mathrm{CN}$. As the molecules diffuse downward, several recycling pathways and secondary production of (not fractioned) reactive nitrogen by galactic cosmic rays tend to homogenize the ${ }^{14} \mathrm{~N} /{ }^{15} \mathrm{~N}$ ratio. Therefore, the model predicts at $200 \mathrm{~km}$ a ratio of $\sim 55$ for all three nitrile species, which is in good agreement with the CIRS and ALMA value for $\mathrm{HCN}$ and $\mathrm{CH}_{3} \mathrm{CN}$, respectively. This provides an overall check on the reaction network and its multiple inputs. We emphasize that our calculations are self-consistent and do not require any additional source of atomic nitrogen in the upper atmosphere, unlike Liang et al. (2007). 
Table 16: Modeled ${ }^{14} \mathrm{~N} /{ }^{15} \mathrm{~N}$ isotopic ratio in $\mathrm{HCN}, \mathrm{CH}_{3} \mathrm{CN}$ and $\mathrm{HC}_{3} \mathrm{~N}$ at 1200 and $200 \mathrm{~km}$.

\begin{tabular}{llll}
\hline \hline & $\mathrm{HCN}$ & $\mathrm{CH}_{3} \mathrm{CN}$ & $\mathrm{HC}_{3} \mathrm{~N}$ \\
\hline \hline at $1200 \mathrm{~km}$ & 88 & 76 & 103 \\
at $200 \mathrm{~km}$ & 55 & 54 & 52 \\
\hline \hline
\end{tabular}
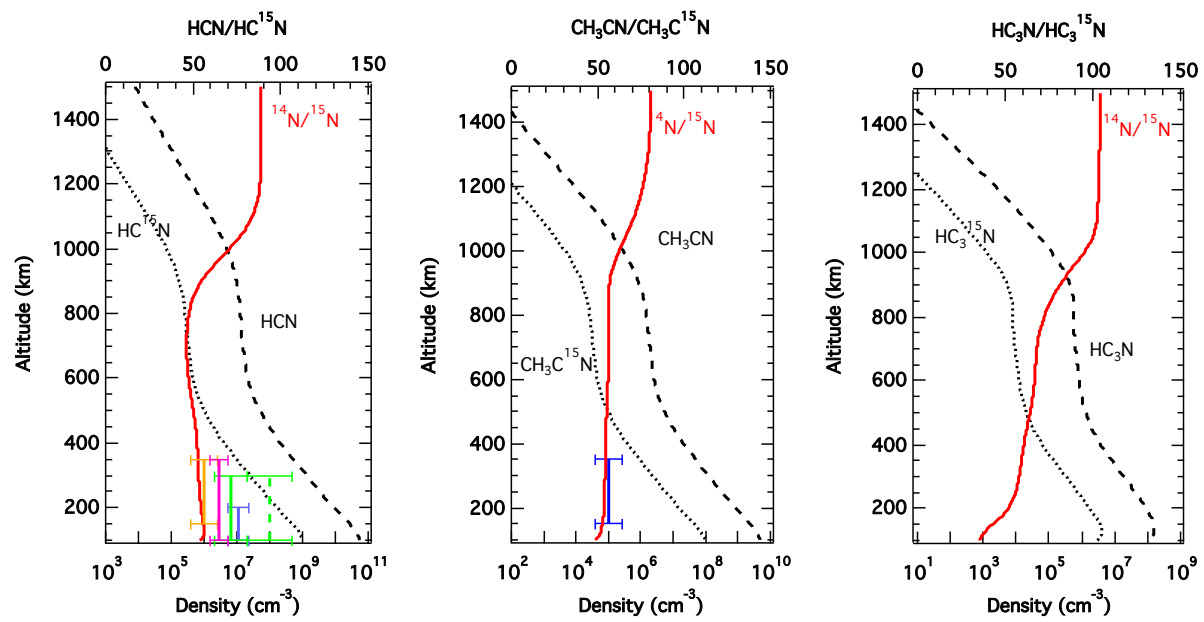

Figure 58: Modeled density of the three major nitrile species and their ${ }^{15} \mathrm{~N}$ isotopologue versus altitude and associated observations as listed in Table 15. HCN, pink: Marten et al. (2002), green: Gurwell (2004), red: Vinatier et al. (2007b), blue: Courtin et al. (2011) (left panel); $\mathrm{CH}_{3} \mathrm{CN}$ (middle panel); $\mathrm{HC}_{3} \mathrm{~N}$ (right panel). 

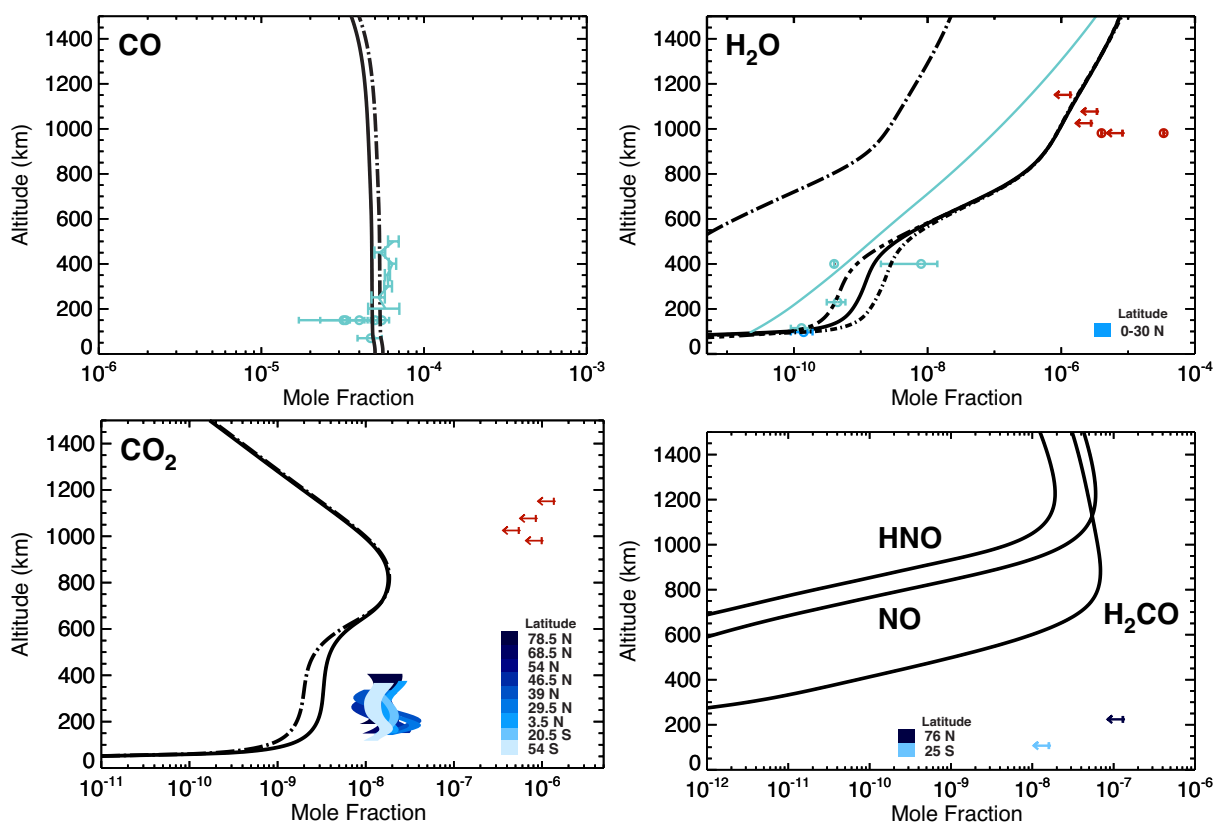

Figure 59: Calculated and observed mole fractions of $\mathrm{CO}$ (upper left panel), $\mathrm{H}_{2} \mathrm{O}$ (upper right panel), $\mathrm{CO}_{2}$ (lower left panel) and other minor oxygen-bearing species (lower right panel). Solid line: nominal (OH flux of $10^{7} \mathrm{~cm}^{-2} \mathrm{~s}^{-1}$ ); dash-dot and long dash - double short dash line: eddy mixing coefficient $K_{0}=10^{2}$ and $10^{3} \mathrm{~cm}^{-2}$, respectively, section 2.4 ; long dashed dotted line: CO fixed, no external flux. The data shown for CO is from CIRS (De Kok et al., 2007; Teanby et al., 2010), VIMS (Baines et al., 2006; Bellucci et al., 2009; Fabiano et al., 2017), Herschel SPIRE (Courtin et al., 2011), Herschel PACS (Rengel et al., 2014); for $\mathrm{H}_{2} \mathrm{O}$ is from ISO (Coustenis et al., 1998) (two circles at $400 \mathrm{~km}$, one with small error bar is assuming constant profile, the one with large error bars is the scaled Lara profile), CIRS (Cottini et al., 2012) (lower 3 circles), INMS (Cui et al., 2009b) (upper limits are average of all the flybys, circles are individual detections from single flybys), Herschel (Moreno et al., 2012) (green line, SA profile); for $\mathrm{CO}_{2}$ is from CIRS (Vinatier et al., 2010), INMS (Cui et al., 2009b); for $\mathrm{H}_{2} \mathrm{CO}$ is from CIRS (Nixon et al., 2010).

4.2.3.3. Oxygen-Bearing Species. Unlike $\mathrm{N}_{2}$ and $\mathrm{CH}_{4}$, which are at the origin of all the minor nitriles and hydrocarbons detected on Titan, it became apparent from early on that CO, the main reservoir of oxygen on Titan, cannot be the single source of oxygen in this atmosphere. Specifically, an external flux of oxygen is necessary to produce the $\mathrm{OH}$ atoms required for the $\mathrm{CO}_{2}$ formation (cf. section 3.2.3). This is illustrated in Figure 59, where a test run with $\mathrm{CO}$ fixed and no external oxygen flux produces $\mathrm{H}_{2} \mathrm{O}$ densities that underestimate the observations by 4 orders of magnitude.

Early investigations about external sources of oxygen suggested that $\mathrm{CO}_{2}$ could be produced through a chemical reaction scheme that begins with an influx of $\mathrm{H}_{2} \mathrm{O}$ into the upper atmosphere from micrometeorite ablation (Lara et al., 1996; Toublanc et al., 1995; Yung et al., 1984). However, these models had some difficulty in reproducing the abundance of all three observed species and a different approach for the origin of $\mathrm{O}$ on Titan was needed. 
The detection of $\mathrm{O}^{+}$precipitating into Titan's atmosphere by CAPS (Hartle et al., 2006) has offered a valid alternative to those suggestions. A photochemical model was presented by Hörst et al. (2008) in which the oxygen ions are incorporated into $\mathrm{CO}$ and $\mathrm{CO}_{2}$ and the observed abundances of $\mathrm{CO}, \mathrm{CO}_{2}$ and $\mathrm{H}_{2} \mathrm{O}$ are simultaneously reproduced using an oxygen flux of $\sim 10^{6} \mathrm{~cm}^{-2} \mathrm{~s}^{-1}$ consistent with the CAPS observations during TA and an $\mathrm{OH}$ flux in agreement with predicted production from micrometeorite ablation (cf. Figure 3 in Hörst et al. (2008)).

The $\mathrm{O}^{+}$ions are stopped as neutral $\mathrm{O}$ atoms, in the ground state $\left(\mathrm{O}\left({ }^{3} \mathrm{P}\right)\right)$ or in some excited state $\left(\mathrm{O}\left({ }^{1} \mathrm{D}\right), \mathrm{O}\left({ }^{1} \mathrm{~S}\right)\right) \cdot \mathrm{H}_{2} \mathrm{O}$ can easily be converted (through photolysis or reactions with radicals) into $\mathrm{OH}$ radicals. Therefore, a steady state between $\mathrm{OH}$ and $\mathrm{H}_{2} \mathrm{O}$ is established down to the stratosphere. In this model, the main production peak for $\mathrm{CO}$ occurs at $\sim 1100 \mathrm{~km}$, where $\mathrm{O}\left({ }^{3} \mathrm{P}\right)$ is primarily deposited. A second peak at $\sim 200 \mathrm{~km}$ is, instead, due to the $\mathrm{CO}_{2}$ photolysis. It is essential to have sources of both atomic oxygen and $\mathrm{OH}$ or $\mathrm{H}_{2} \mathrm{O}$ : $\mathrm{O}$ alone would produce only $\mathrm{CO}$, while input of $\mathrm{OH}$ or $\mathrm{H}_{2} \mathrm{O}$ alone does not produce $\mathrm{CO}$ and leads only to the production of $\mathrm{CO}_{2}$ if $\mathrm{CO}$ is already present.

At that time, the only observation available for $\mathrm{H}_{2} \mathrm{O}$ was that from ISO, which provided a fairly loose constraint with a mole fraction of $8_{-4}^{+6} \times 10^{-9}$ in the stratosphere, assuming a profile constant with height above the condensation level (Coustenis et al., 1998). More recent $\mathrm{H}_{2} \mathrm{O}$ observations from CIRS and Herschel show that the $\mathrm{H}_{2} \mathrm{O}$ relative abundance strongly increases with altitude with mole fractions of $4.5 \times 10^{-10}$ at $230 \mathrm{~km}$ and $7 \times 10^{-10}$ at $400 \mathrm{~km}$ (Cottini et al., 2012; Moreno et al., 2012). Both observations indicate that the Hörst et al. (2008) model is too "water-rich" with a too shallow slope over 100-300 $\mathrm{km}$.

As a follow-up, Moreno et al. (2012) reconsidered the oxygen chemistry by updating the Lara et al. photochemical model. They fixed $\mathrm{CO}$ at the lower boundary but they were unable to match both $\mathrm{H}_{2} \mathrm{O}$ and $\mathrm{CO}_{2}$ with a given $\mathrm{OH} / \mathrm{H}_{2} \mathrm{O}$ flux. Their $\mathrm{OH}$ flux required to match the Herschel $\mathrm{H}_{2} \mathrm{O}$ observations is about $3 \times 10^{5} \mathrm{~cm}^{-2} \mathrm{~s}^{-1}$ but this leads to a $\mathrm{CO}_{2}$ mole fraction one order of magnitude short below the measured value. They observed that neither the $\mathrm{OH}$ versus $\mathrm{H}_{2} \mathrm{O}$ form of the water input or the deposition profile is important. They envisaged a direct injection of $\mathrm{CO}_{2}$ but the required $\mathrm{CO}_{2} / \mathrm{H}_{2} \mathrm{O}$ ratio is inconsistent with cometary composition. They also considered some additional chemical loss for $\mathrm{H}_{2} \mathrm{O}$ but given that the formation of $\mathrm{CO}_{2}$ occurs predominantly from $\mathrm{OH}$, the associated $\mathrm{CO}_{2}$ profile remained inadequate. Subsequently, Krasnopolsky (2012) did manage to match $\mathrm{CO}, \mathrm{CO}_{2}$ and $\mathrm{H}_{2} \mathrm{O}$ pretty well (cf. Figure 3 in Krasnopolsky (2012)) with a $\mathrm{H}_{2} \mathrm{O}$ and $\mathrm{O}^{+}$flux of $4.78 \times 10^{6}$ and $3.46 \times 10^{6} \mathrm{~cm}^{-2}$ $\mathrm{s}^{-1}$, respectively, albeit with the wrong chemistry as discussed in the following. In order to tackle this problem, we have calculated new rates for the $\mathrm{OH}+$ $\mathrm{CH}_{3}$ reaction (cf. section 3.2.3). The main difference between our new scheme and the one in Hörst et al. (2008) is that it gives mostly $\mathrm{CO}$ in our case via $\mathrm{CHOH}$, while it gives mostly $\mathrm{H}_{2} \mathrm{O}$ in their case. As a matter of fact, we are essentially back to last century's models, where this reaction was considered to produce $\mathrm{CO}+2 \mathrm{H}_{2}$ (Wong et al., 2002), and therefore to form a C-O bond 
instead of recycling back $\mathrm{OH}$ to $\mathrm{H}_{2} \mathrm{O}$.

With the new reaction rates, it became virtually impossible to reproduce the observations with the previous $\mathrm{O}\left({ }^{3} \mathrm{P}\right) / \mathrm{OH}$ fluxes. As a matter of fact, an $\mathrm{O}\left({ }^{3} \mathrm{P}\right)$ input is no longer required as $\mathrm{OH}$ alone can now lead to $\mathrm{H}_{2} \mathrm{O}, \mathrm{CO}$ and $\mathrm{CO}_{2}$. The mole fractions of the most abundant oxygen-bearing species calculated with an $\mathrm{OH} / \mathrm{H}_{2} \mathrm{O}$ flux of $10^{7} \mathrm{~cm}^{-2} \mathrm{~s}^{-1}$ (and no atomic oxygen) are shown in Figure 59. Although $\mathrm{H}_{2} \mathrm{O}$ and $\mathrm{CO}_{2}$ are overestimated and underestimated, respectively by a factor of 10, this is the best fit that we could achieve with any combination of $\mathrm{O}\left({ }^{3} \mathrm{P}\right) / \mathrm{OH} / \mathrm{H}_{2} \mathrm{O} / \mathrm{CO}_{2}$, irrespectively of observational constrains regarding the flux. This $\mathrm{OH}$ flux is consistent with the INMS observations of $\mathrm{H}_{2} \mathrm{O}$ as well as $\mathrm{H}_{3} \mathrm{O}^{+}$(cf. Figure 49) in the upper atmosphere.

Interestingly enough, recent studies (Sillanpää and Johnson, 2015; Snowden et al., 2018) seem to indicate that the $\mathrm{O}^{+}$flux in Titan's plasma wake during TA was unusually high and that the average $\mathrm{O}^{+}$flux is around $5 \times 10^{4} \mathrm{~cm}^{-2}$ $\mathrm{s}^{-1}$, instead of $10^{6} \mathrm{~cm}^{-2} \mathrm{~s}^{-1}$ as reported in Hartle et al. (2006). Some test runs indicate that as long as the $\mathrm{O}^{+}$flux stays below $10^{5} \mathrm{~cm}^{-2} \mathrm{~s}^{-1}$, it does not have any impact on the abundance of the major species. However, Hartogh et al. (2011) inferred that neither Enceladus' activity or micrometeorites are likely to provide a flux large enough for them to be the ultimate source of water in the upper atmosphere of Titan.

The difficulty of finding the origin of Titan's water with the current known external sources supports the idea first put forward by Moreno et al. (2012) of a variable oxygen input. This hypothesis is based on the fact that $\mathrm{CO}, \mathrm{CO}_{2}$ and $\mathrm{H}_{2} \mathrm{O}$ have very different lifetimes (in our model $\sim 2$ Myrs., $\sim 2$ kyrs., $\sim 3$ yrs., Titan years). To follow on this idea, Lara et al. (2014) used a time-dependent model where they fixed $\mathrm{CO}$ at the lower boundary and initialized the oxygen compounds to the steady state solution profiles. Then, the oxygen sources were cut to zero and the oxygen species evolved over time according to $\phi(\mathrm{OH})=$ $\phi_{0}(\mathrm{OH}) \times \exp (-(\mathrm{t} / \tau))$. They obtained a satisfactory set of solutions $(\phi(\mathrm{OH})=$ $1-2 \times 10^{7} \mathrm{~cm}^{-2} \mathrm{~s}^{-1}, \mathrm{t}_{0}=290-360$ yrs., $\tau=100 \mathrm{yrs}$.) if the targeted water profile was the one retrieved from CIRS, whereas the approach was unsuccessful if the targeted water profile was the one retrieved from Herschel, unless some additional loss to the haze was added.

Subsequently, Dobrijevic et al. (2014) investigated two different scenarios, one where $\mathrm{CO}$ is from an internal source and one where it originates from external $\mathrm{O}$ and $\mathrm{OH} / \mathrm{H}_{2} \mathrm{O}$. In the first case, they could match both $\mathrm{H}_{2} \mathrm{O}$ and $\mathrm{CO}_{2}$ with an $\mathrm{OH}$ flux of $2.6 \times 10^{6} \mathrm{~cm}^{-2} \mathrm{~s}^{-1}$ and $\mathrm{CO}$ fixed at the lower boundary. In the second case, they could match everything with $\mathrm{OH} / \mathrm{O}$ fluxes of $2.6 \times 10^{6}$ and $1.6 \times 10^{6} \mathrm{~cm}^{-2} \mathrm{~s}^{-1}$, respectively and an integration time adjusted to $10^{16}$ $\mathrm{s}$ to get to the agreement for CO. They concluded that the oxygen input (both $\mathrm{OH}$ from micrometeorites and $\mathrm{O}$ from Enceladus) must have started 300 Myrs. ago, a point already raised in Hörst et al. (2008).

In order to definitively clarify the source of oxygen on Titan, future work includes:

- Analysis of the CAPS and CDA data to better constrain the external 
sources of oxygen $\left(\mathrm{OH}_{x}^{+}\right.$from Enceladus, $\mathrm{H}_{2} \mathrm{O}$ from micrometeorites) in order to evaluate their average flux and variability with time.

- Analysis of the INMS data to better constrain the density and variability of $\mathrm{H}_{2} \mathrm{O}$ and $\mathrm{H}_{3} \mathrm{O}^{+}$in the upper atmosphere, and new observations of $\mathrm{H}_{2} \mathrm{O}$ in the lower atmosphere to clarify the discrepancy between CIRS and Herschel. The detection of a $4^{\text {th }}$ oxygen species would also be a valuable asset. According to our model results, $\mathrm{H}_{2} \mathrm{CO}$ appears as the best candidate.

- Investigation of a range of possible chemical evolutionary tracks through the development of time dependent photochemical models with state-ofthe-art chemistry.

\subsection{The GCR Layer}

The permittivity, waves and altimetry (PWA) experiment onboard the Huygens probe measured a peak electron density of $\sim 650 \mathrm{~cm}^{-3}$ at $65 \mathrm{~km}$ (Hamelin et al., 2007; López-Moreno et al., 2008). In our study, the ion density peaks at $90 \mathrm{~km}$, with a magnitude of $\sim 2000 \mathrm{~cm}^{-3}$, which implies that it is not so consistent with the electron densities measured by Huygens. Our model does not take into account electron attachment onto aerosols or electrophilic species, which could reduce the electron densities by significant factors (Borucki et al., 1987; Borucki and Whitten, 2008). Molina-Cuberos et al. (1999a,b) calculated the vertical distributions of ions resulting from the impact of cosmic radiation and subsequent ion-neutral chemistry. The GCR ionization peak is similar in both altitude and magnitude with that obtained in our work.

The chemistry resulting from GCR is essentially initiated by nitrogen atoms $\left(\mathrm{N}\left({ }^{4} \mathrm{~S}\right)\right.$ and $\left.\mathrm{N}\left({ }^{2} \mathrm{D}\right)\right)$ and to a lower extent ions $\left(\mathrm{N}_{2}{ }^{+}\right.$and $\left.\mathrm{N}^{+}\right)$, the production of $\mathrm{CH}_{4}$ fragments being lower by a factor of $\sim 30$ (cf. section 2.6 ). $\mathrm{N}\left({ }^{4} \mathrm{~S}\right.$ ) leads to $\mathrm{HNC}\left(\mathrm{R}_{n} 289 \mathrm{~b}\right)$ and to the secondary formation of many nitrogen radicals, including $\mathrm{NH}\left(\mathrm{R}_{n} 293 \mathrm{a}\right)$ followed by $\mathrm{NH}_{2}\left(\mathrm{R}_{n} 333\right)$, and $\mathrm{H}_{2} \mathrm{CN}\left(\mathrm{R}_{n} 290 \mathrm{a}\right) . \mathrm{NH}_{2}$ radicals are responsible for the subsequent production of $\mathrm{NH}_{3}\left(\mathrm{R}_{n} 350 \mathrm{~b}\right), \mathrm{N}_{2} \mathrm{H}_{4}$ $\left(\mathrm{R}_{n} 361 \mathrm{~b}\right)$ and $\mathrm{CH}_{3} \mathrm{NH}_{2}\left(\mathrm{R}_{n} 349\right)$, and $\mathrm{H}_{2} \mathrm{CN}$ for that of $\mathrm{HNC}\left(\mathrm{R}_{n} 395 \mathrm{~b}\right)$. The production of $\mathrm{N}\left({ }^{2} \mathrm{D}\right)$ leads directly to $\mathrm{CH}_{2} \mathrm{NH}\left(\mathrm{R}_{n} 307 \mathrm{a}\right)$ and also to the formation of the $\mathrm{HC}_{2} \mathrm{~N}$ radicals $\left(\mathrm{R}_{n} 308\right)$. The latter, in combination with $\mathrm{N}\left({ }^{4} \mathrm{~S}\right)$, produce $\mathrm{C}_{2} \mathrm{~N}_{2}\left(\mathrm{R}_{n} 300\right)$.

Our column-integrated abundances of some selected species with and without GCR impact are presented in Table 17. We do observe an impact around $100 \mathrm{~km}$ on some minor nitrogen-bearing species such as $\mathrm{NH}_{3}, \mathrm{HNC}, \mathrm{CH}_{2} \mathrm{NH}$, $\mathrm{CH}_{3} \mathrm{NH}_{2}, \mathrm{~N}_{2} \mathrm{H}_{4}, \mathrm{C}_{2} \mathrm{H}_{3} \mathrm{CN}$ and $\mathrm{C}_{2} \mathrm{~N}_{2} . \mathrm{C}_{6} \mathrm{H}_{6}$ and $\mathrm{C}_{4} \mathrm{~N}_{2}$ do not exhibit any significant change in their vertical profile, with a column-integrated abundance increase of about $30 \%$ only. The addition of GCR brings $\mathrm{C}_{2} \mathrm{~N}_{2}$ in better agreement with the observations (see left panel of Figure 54) although as we mentioned earlier, we do not have an appropriate observation to compare to. $\mathrm{NH}_{3}$ is very close to the available upper limits at low latitude (cf. left panel of Figure 50), while the HNC column-integrated abundance exhibits a 50-fold increase 
and is therefore no longer in agreement with the observations (cf. Table 11). This is linked to the presence of $\mathrm{C}_{4} \mathrm{H}_{3} \mathrm{NH}^{+}$that forms $\mathrm{HNC}$ through electron recombination $\left(\mathrm{R}_{e r} 115 \mathrm{c}\right)$ but we highlight that both rate and products have been estimated. The photodissociation cross sections of HNC have very recently been calculated and it was found that HNC is photodissociated faster than HCN by one order of magnitude for the solar radiation field, at 1 au (Aguado et al., 2017). Future models should test these cross sections to check their impact on the HNC abundance in the atmosphere of Titan. It is also possible that HNC efficiently forms aggregates, a process that could lead to a significant decrease of its density.

Table 17: Calculated column-integrated abundances (molecules $\mathrm{cm}^{-2}$ ) of the species most affected by GCR.

\begin{tabular}{lll}
\hline \hline Species & Model without GCR & Model with GCR \\
\hline \hline $\mathrm{NH}_{3}$ & $8.9 \times 10^{11}$ & $5.1 \times 10^{15}$ \\
$\mathrm{HNC}$ & $8.9 \times 10^{12}$ & $4.5 \times 10^{14}$ \\
$\mathrm{CH}_{2} \mathrm{NH}$ & $4.1 \times 10^{13}$ & $1.3 \times 10^{15}$ \\
$\mathrm{CH}_{3} \mathrm{NH}_{2}$ & $1.7 \times 10^{12}$ & $4.9 \times 10^{14}$ \\
$\mathrm{~N}_{2} \mathrm{H}_{4}$ & $1.2 \times 10^{10}$ & $2.4 \times 10^{13}$ \\
$\mathrm{C}_{2} \mathrm{H}_{3} \mathrm{CN}$ & $3.2 \times 10^{13}$ & $9.6 \times 10^{14}$ \\
$\mathrm{C}_{2} \mathrm{~N}_{2}$ & $4.7 \times 10^{11}$ & $2.9 \times 10^{13}$ \\
\hline \hline
\end{tabular}

In both the Lavvas et al. and Dobrijevic et al. models, the inclusion of nitrogen atoms from GCR impact induces some drastic increase of the $\mathrm{CH}_{2} \mathrm{NH}$ mole fraction and to a lesser extent of $\mathrm{C}_{4} \mathrm{~N}_{2}$ (cf. Figures 9d and 12a in Lavvas et al. (2008b), Figure 16 in Loison et al. (2015)). Loison et al. (2015) also reports the increase of several other nitrogen compounds, such as $\mathrm{NH}_{3}, \mathrm{CH}_{3} \mathrm{NH}_{2}, \mathrm{HNC}$, $\mathrm{C}_{2} \mathrm{H}_{3} \mathrm{CN}$, and $\mathrm{C}_{2} \mathrm{~N}_{2}$. Krasnopolsky (2014) observes a sharp peak in the $\mathrm{C}_{6} \mathrm{H}_{6}$ mole fraction at $50 \mathrm{~km}$ resulting from GCR.

While the neutral pathways are fairly well constrained, the chemistry resulting from ion formation is very uncertain. As in the upper atmosphere, the ion chemistry heavily depends on the presence of heavy nitrogen-bearing species, whose profiles in the lower stratosphere cannot be constrained from observations and are therefore largely unknown (cf. section 4.2.3.2). With our current chemical network and assumed vertical profiles, the density of $\mathrm{NH}_{3}$ and $\mathrm{HNC}$ relies on the abundance of $\mathrm{C}_{3} \mathrm{H}_{7} \mathrm{~N} / \mathrm{C}_{5} \mathrm{H}_{5} \mathrm{~N}$, and $\mathrm{C}_{4} \mathrm{H}_{3} \mathrm{~N}$, respectively.

Figure 60 shows the predicted density of cations at the peak, with values higher than $10^{-1} \mathrm{~cm}^{-3}$. The major species at the peak are the nitrogen-bearing ions $\mathrm{C}_{3} \mathrm{H}_{7} \mathrm{NH}^{+}(\mathrm{m} / \mathrm{z}=58)$ and $\mathrm{C}_{5} \mathrm{H}_{5} \mathrm{NH}^{+}(\mathrm{m} / \mathrm{z}=80)$ and the hydrocarbon ions $\mathrm{C}_{6} \mathrm{H}_{9}{ }^{+}(\mathrm{m} / \mathrm{z}=81), \mathrm{C}_{6} \mathrm{H}_{11}{ }^{+}(\mathrm{m} / \mathrm{z}=83)$ and $\mathrm{C}_{7} \mathrm{H}_{9}{ }^{+}(\mathrm{m} / \mathrm{z}=93)$. Below the peak, the cluster ion $\mathrm{HCO}^{+} \cdot \mathrm{H}_{2}$ increases. In Molina-Cuberos et al. (1999a,b) model, the most abundant ions above the peak are purely covalent hydrocarbon ions with four or more carbon atoms and a few nitrogen-bearing ions. Below the peak, the ionosphere is mainly composed of cluster ions such as $\mathrm{CH}_{5}{ }^{+} \cdot \mathrm{CH}_{4}$, $\mathrm{HCO}^{+} \cdot \mathrm{H}_{2}, \mathrm{HCNH}^{+} \cdot \mathrm{C}_{2} \mathrm{H}_{4}$ and $\mathrm{CH}_{4}^{+} \cdot \mathrm{N}_{2}$, formed by three-body processes. 
Unfortunately, 3-body reaction rate coefficients are poorly known as well as loss processes for adduct species. There are no observations that constrain the ion composition in the lower atmosphere.

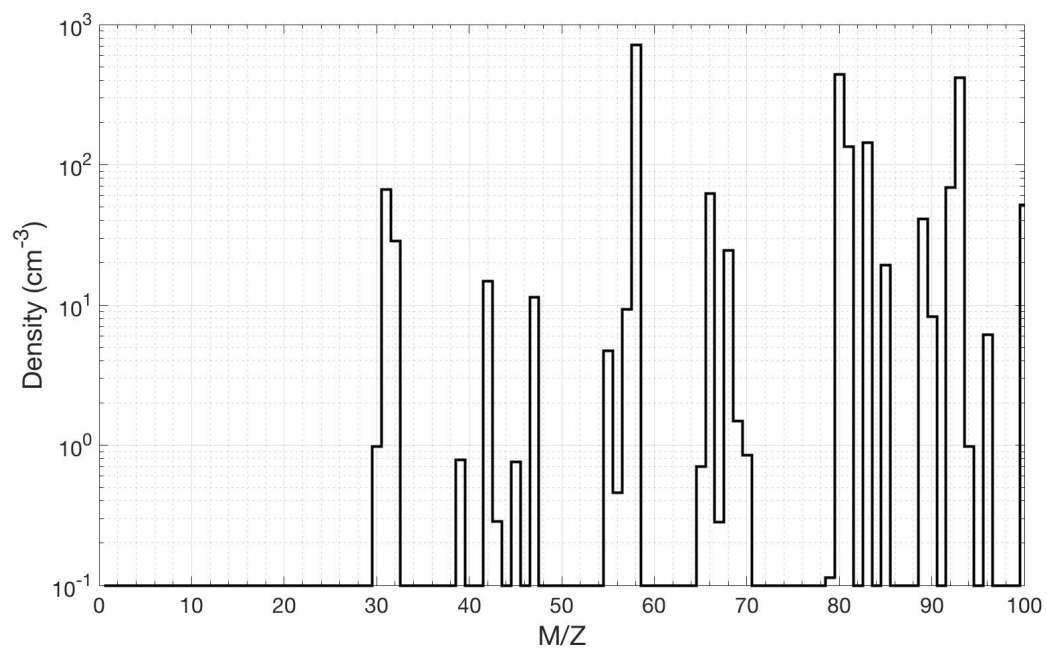

Figure 60: Calculated ion mass spectrum at $90 \mathrm{~km}$.

\section{Discussion}

\subsection{The Puzzle of the Overestimation of $\mathrm{C}_{2} \mathrm{H}_{5}{ }^{+}$and $\mathrm{HCNH}^{+}$}

We return here to the results described in section 3.1.1.3, especially to those ions whose density in Titan's upper atmosphere is smaller than predictions from theoretical models, i.e. the dominant $\mathrm{HCNH}^{+}$ion but also $\mathrm{C}_{2} \mathrm{H}_{5}{ }^{+}$. All other studies do find the same discrepancy, thereby suggesting a lack in our understanding of the reactions that control the first steps of the chemistry (Robertson et al., 2009; Mandt et al., 2012a; Westlake et al., 2012; Sagnières et al., 2015).

A mechanism that would lead to a reduction of the electron density in Titan's ionosphere appears necessary as well. An analysis of Cassini observations demonstrates that the observed electron densities are lower by a factor of 2 than densities calculated with photochemical models under the conditions of the observations (Vigren et al., 2013). This problem seems to appear only during the daytime where energy deposition is dominated by solar photons. During night-time, the observed electron densities are found to be in good agreement with predictions from models of magnetospheric electron ionization. Similarly, on the night-side the densities of the primary ions are in agreement with the INMS measurements (Vigren et al., 2015). LP data shows electrons soaked up by aerosols and the negative charge on these exceed that of the ions. However, electrons are preferentially soaked up in the nightside (Shebanits et al., 2016) while electron densities are overestimated by models only on the dayside (Vigren 
3855 et al., 2013, 2015). This therefore doesn't seem to be a possible explanation. 3856 Moreover, having electrons soaked up by aerosols on the dayside would actually 3857 worsen the model overestimation of the $\mathrm{C}_{2} \mathrm{H}_{5}{ }^{+}$and $\mathrm{HCNH}^{+}$densities because 3858 the recombination of positive ions with charged aerosols is not as efficient as 3859 with electrons (see e.g. Vigren et al. (2014)). 


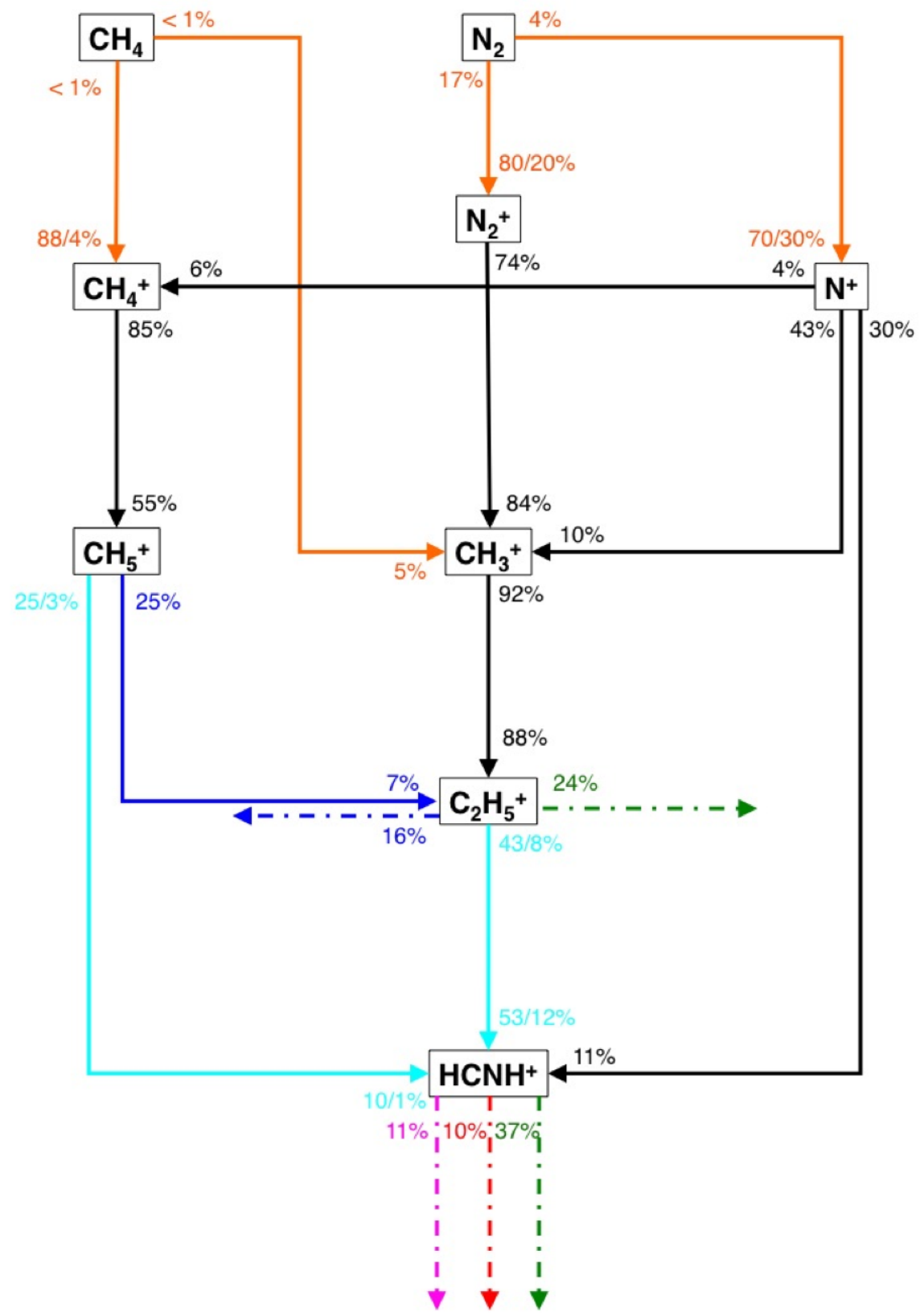

Figure 61: Flowchart highlighting the very first steps of the ionospheric chemistry. Orange: $\mathrm{h} \nu / \mathrm{e}_{s}^{-}$, green: $\mathrm{e}_{T}^{-}$, black: $\mathrm{CH}_{4}$, blue: $\mathrm{C}_{2} \mathrm{H}_{4}$, pink: $\mathrm{C}_{4} \mathrm{H}_{2}$, cyan: $\mathrm{HCN} / \mathrm{HNC}$, red: $\mathrm{C}_{2} \mathrm{H}_{3} \mathrm{CN}$. The percentages represent the calculated contribution of the given channel to the total production/loss of the given species for a globally average model. 


\subsubsection{Reactivity and Nature of $\mathrm{HCNH}^{+}$}

Westlake et al. (2012) analyzed the processes producing and consuming $\mathrm{HCNH}^{+}$in order to determine the cause of the discrepancy between calculations and observations. They identified four potential culprits for the model overestimation that are detailed below. We repeated numerical experiments (1)-(3) and essentially reached the same conclusions.

1. Errors in the HCN atmospheric densities: The HCN mole fraction is poorly constrained by INMS and there is a large range of acceptable values $(3 \times$ $\left.10^{-4}-3 \times 10^{-3}\right)$. However, while decreasing the HCN density has the desired effect of decreasing the $\mathrm{HCNH}^{+}$density, it does so at the expense of the $\mathrm{CH}_{5}{ }^{+}$and $\mathrm{C}_{2} \mathrm{H}_{5}{ }^{+}$densities, which are as a consequence overestimated (cf. Figure 61 and section 3.1.1.2). Therefore, the HCN profile cannot be held responsible for the overestimation of the ionospheric densities.

2. Errors in the electron density, electron temperature, or electron recombination coefficients of $\mathrm{HCNH}^{+}$: Electron recombination is the major loss of $\mathrm{HCNH}^{+}$(cf. Figure 61). The electron recombination rate depends on both the electron density and temperature (cf. section 3.1.1.2). Runs using different realistic sets of values for the electron density (taken as either the LP total electron density or the INMS total ion density) and temperature (taken as either the measured LP electron temperature or the calculated electron temperature of Richard et al. (2011)) show that these parameters have some effect on the $\mathrm{HCNH}^{+}$density but cannot completely solve the problem. Both electron recombination rate constants available in the literature (cf. section 3.1.1.2) were also tested but none is fast enough to lead to a sufficient loss of $\mathrm{HCNH}^{+}$.

3. Missing reactions with heavy neutrals or negative ions: Large amounts of heavy neutrals could lead to a significant loss of $\mathrm{HCNH}^{+}$through proton exchange reactions. However, the expected scale height of such species is incompatible with the large excess of $\mathrm{HCNH}^{+}$still observed at higher altitude. The positive - negative ion recombination rates and observed negative ion densities are too small to be competitive with ion - neutral reactions.

4. Missing reactions with light species: The reaction of $\mathrm{HCNH}^{+}$with $\mathrm{H}_{2}$, $\mathrm{CH}_{4}, \mathrm{C}_{2} \mathrm{H}_{2}$ or $\mathrm{C}_{2} \mathrm{H}_{4}$ would lead to enough destruction of $\mathrm{HCNH}^{+}$were the rate constants fast enough. Although these reactions have all been investigated experimentally or theoretically and have been found to be slow (typically $\mathrm{k}<5 \times 10^{-13} \mathrm{~cm}^{3} \mathrm{~s}^{-1}$ ), Westlake et al. (2012) argue that the pressures and temperatures used were not relevant to the Titan's ionosphere and that the rate constants could actually be much faster. They finally compute that rate constants equal to $6 \times 10^{-11}, 1 \times 10^{-11}, 2 \times$ $10^{-10}$ and $2 \times 10^{-10} \mathrm{~cm}^{3} \mathrm{~s}^{-1}$ for $\mathrm{H}_{2}, \mathrm{CH}_{4}, \mathrm{C}_{2} \mathrm{H}_{2}$ or $\mathrm{C}_{2} \mathrm{H}_{4}$, respectively would solve the $\mathrm{HCNH}^{+}$issue.

In response to item (4), Demarais et al. (2013) investigated these reactions experimentally and theoretically to probe for the specific products suggested 
by Westlake et al. (2012). In all cases, the products were not observed experimentally, which is rationalized computationally by the presence of large energy barriers. However, association products for the reactions with $\mathrm{C}_{2} \mathrm{H}_{2}$ and $\mathrm{C}_{2} \mathrm{H}_{4}$ have been found. In the experimental conditions, the adducts are stabilized by 3-body collisions but in the Titan ionosphere, only radiative association could be efficient.

Following this, we investigated theoretically the association rate for $\mathrm{HCNH}^{+}+$ $\mathrm{C}_{2} \mathrm{H}_{4}$ (section 2.7.3.2) and found that $k_{R}$ is only $4 \times 10^{-15} \mathrm{~cm}^{3} \mathrm{~s}^{-1}$ at $150 \mathrm{~K}$ and this rate is too low for this reaction to be an efficient pathway for the loss of $\mathrm{HCNH}^{+}$. The $\mathrm{HCNH}^{+}$addition to $\mathrm{C}_{2} \mathrm{H}_{2}$ (and $\mathrm{HCN}$, another major neutral in the thermosphere) will certainly not be faster because the binding energy is much less and there are fewer vibrational modes. Therefore, we use a rate constant of $1.5 \times 10^{-15} \mathrm{~cm}^{3} \mathrm{~s}^{-1}$ for both these reactions, based on Herbst et al. (1989). Again, this is too slow to significantly impact the $\mathrm{HCNH}^{+}$density.

It is interesting to mention that the most stable form of this ion is the singlet linear $\mathrm{HCNH}^{+}\left(\mathrm{H}-\mathrm{C}^{+}=\mathrm{N}-\mathrm{H}\right)$. The singlet methylenimine cation $\mathrm{H}_{2} \mathrm{CN}^{+}\left(\mathrm{H}_{2^{-}}\right.$ $\mathrm{C}=\mathrm{N}^{+}$), which energetically lies $3.6 \mathrm{eV}$ above $\mathrm{HCNH}^{+}$, is inherently unstable. Four triplet isomers with stable geometries have been computed with energies at least $5.4 \mathrm{eV}$ higher than the linear ion $\mathrm{HCNH}^{+}: \mathrm{H}_{2} \mathrm{NC}^{+}$, cis- $\mathrm{HCNH}^{+}, \mathrm{H}_{2} \mathrm{CN}^{+}$ and trans-HCNH${ }^{+}$(Holzmeier et al., 2013). One could entertain the idea that the isomer formed in the ionosphere is not the one studied in the laboratory and that their different reactivity could explain the observed discrepancy.

Semaniak et al. (2001) give an extensive discussion supported by theoretical calculations on the nature of the $\mathrm{HCNH}^{+}$ions present in the storage ring for dissociative recombination measurements. The conclusion is that the formaldehyde-like $\mathrm{H}_{2} \mathrm{CN}^{+}$ions cannot be present in the ion source for stability or thermodynamic reasons. However, a combined crossed-beam (at 2 collision energies close to $4 \mathrm{eV})$ and theoretical study suggests that reaction $\left(\mathrm{R}_{c n} 593 \mathrm{c}\right)$ leads preferentially $(\sim 70 \%)$ to the triplet $\mathrm{H}_{2} \mathrm{CN}^{+}$isomer $\left(+\mathrm{H}_{2}\right)$ over both singlet $(+2 \mathrm{H})$ and triplet $\left(+\mathrm{H}_{2}\right) \mathrm{HCNH}^{+}$isomers (Žabka et al., 2010). Clearly, new investigations are required to clarify the nature of the $\mathrm{HCNH}^{+}$isomers and their respective reactivity before any further conclusion can be made.

Since it seems difficult to stretch the chemistry currently present in models by enough to achieve our purpose, i.e. decrease the predicted $\mathrm{HCNH}^{+}$densities by a significant factor, we turn to other chemical processes that are missing from the models but nonetheless could significantly alter the chemistry.

\subsubsection{Vibrationally Excited Nitrogen}

Lavvas et al. (2015) calculated the vibrational distribution of the ground state levels $\mathrm{N}_{2}^{+}\left(X^{2} \Sigma_{g}^{+}\right)$and $\mathrm{N}_{2}\left(X^{1} \Sigma_{g}^{+}\right)$and we use this investigation as a starting point to discuss the ramifications of vibrational excited nitrogen for the ionospheric densities.

The $\mathrm{N}_{2}{ }^{+}\left(\mathrm{X}, \nu^{*}\right)$ density demonstrates a rapid drop with increasing vibrational level. For example, at $1000 \mathrm{~km}$ only the $\nu=1$ level has a fraction higher than $1 \%$ of the ground level. For any chemical reaction involving hot molecules 
to be competitive, the magnitude of the rate of the hot molecules should therefore be 100 times higher than that of the ground state.

The main fate of $\mathrm{N}_{2}^{+}\left(\mathrm{X}, \nu^{*}\right)$ is to collide with $\mathrm{N}_{2}$ and four possible outcomes can be considered:

$$
\begin{aligned}
\mathrm{N}_{2}^{+}\left(X, \nu^{*}\right)+\mathrm{N}_{2} & \rightarrow \mathrm{N}_{2}^{+}\left(X, \nu<\nu^{*}\right)+\mathrm{N}_{2} & & \left(\mathrm{R}_{c n}^{*} 854 \mathrm{a}\right) \\
& \rightarrow \mathrm{N}_{2}\left(X, \nu^{*}\right)+\mathrm{N}_{2}^{+} & & \left(\mathrm{R}_{c n}^{*} 854 \mathrm{~b}\right) \\
& \rightarrow \mathrm{N}_{3}^{+}+\mathrm{N} & & \left(\mathrm{R}_{c n}^{*} 854 \mathrm{c}\right) \\
& \rightarrow \mathrm{N}_{4}^{+}+\mathrm{h} \nu & & \left(\mathrm{R}_{c n}^{*} 854 \mathrm{~d}\right)
\end{aligned}
$$

Collisions of vibrationally excited $\mathrm{N}_{2}{ }^{+}$ions $(\nu=0-4)$ can lead to vibrational deactivation $\left(\mathrm{R}_{c n}^{*} 854 \mathrm{a}\right)$ as well as charge transfer $\left(\mathrm{R}_{c n}^{*} 854 \mathrm{~b}\right)$, which essentially only replenishes the $\mathrm{N}_{2}{ }^{+}$ground state. The formation of $\mathrm{N}_{3}{ }^{+}\left(\mathrm{R}_{c n}^{*} 854 \mathrm{c}\right)$ is endothermic by $4.97 \mathrm{eV}$ (Dutuit et al., 2013) and is inaccessible to levels with moderate excitation. The formation of $\mathrm{N}_{4}{ }^{+}\left(\mathrm{R}_{c n}^{*} 854 \mathrm{~d}\right.$ ), even if fast enough (a reaction rate of $10^{-11} \mathrm{~cm}^{3} \mathrm{~s}^{-1}$ is required), is unlikely to affect the ionospheric balance because its main fate will be reaction with methane to form $\mathrm{CH}_{4}^{+}$and ultimately $\mathrm{HCNH}^{+}$(cf. Figure 61 and Lavvas et al. (2015)).

The second major process defining the abundance of $\mathrm{N}_{2}^{+}\left(\mathrm{X}, \nu^{*}\right)$ is reaction with methane:

$$
\begin{aligned}
& \mathrm{N}_{2}^{+}\left(X, \nu^{*}\right)+\mathrm{CH}_{4} \rightarrow \mathrm{CH}_{3}^{+}+\mathrm{N}_{2}+\mathrm{H} \\
& \left(\mathrm{R}_{c n}^{*} 836 \mathrm{a}\right) \\
& \rightarrow \mathrm{CH}_{2}^{+}+\mathrm{N}_{2}+\mathrm{H}_{2} \\
& \left(\mathrm{R}_{c n}^{*} 836 \mathrm{~b}\right) \\
& \rightarrow \mathrm{N}_{2} \mathrm{H}^{+}+\mathrm{CH}_{3} \\
& \left(\mathrm{R}_{c n}^{*} 836 \mathrm{c}\right)
\end{aligned}
$$

After taking into account the experimental uncertainties, the branching ratios for the three channels are found to be independent of the $\nu$ state of reactant $\mathrm{N}_{2}{ }^{+}$for $\nu=0-4$ (Dutuit et al., 2013; Xu et al., 2013) and this reaction cannot differentially affect the ion densities.

The variation of the recombination rate of $\mathrm{N}_{2}{ }^{+}$with vibrational level has also been considered in Lavvas et al. (2015) but this process is not efficient either because the recombination rate of the hot ions is smaller than the rate for the ground ion level (Bates and Mitchell, 1991). Therefore, a reduction of the ionospheric densities due to loss of the primary $\mathrm{N}_{2}{ }^{+}$ions seems unlikely from the processes we have considered.

A significant fraction of $\mathrm{N}_{2}$ is in some vibrationally excited state of the ground electronic state, about $10 \%$ for $\nu=1,2$ and $1 \%$ for $\nu>2$ (Lavvas et al., 2015). These states have a lifetime of $10^{7}-10^{11} \mathrm{~s}$ and are up to a few $\mathrm{eV}$ above the ground state implying that some reactions considered endothermic could actually occur with some fraction of the $\mathrm{N}_{2}$ population. Lavvas et al. (2015) considered the chemical reactivity of $\mathrm{C}_{2} \mathrm{H}_{5}{ }^{+}$and $\mathrm{HCNH}^{+}$with hot nitrogen molecules but the population of those having enough internal energy is 
too small to affect the overall abundance of these major ions. Vibrational energy transfer is another possible outcome of those collisions but for polyatomic ions the recombination rate is not expected to be significantly affected by their vibrational excitation and will not affect the ionospheric balance either.

\subsubsection{Electronically Excited Nitrogen}

The ionization cross sections of $\mathrm{N}_{2}$ are well constrained but a key issue is the description of the electronic state of the dissociation fragments ( $\mathrm{R}$. Thissen, private communication, 2013). For example, the dissociative ionization of $\mathrm{N}_{2}$ is known to lead to $\mathrm{N}^{+}\left({ }^{3} \mathrm{P}\right)+\mathrm{N}\left({ }^{4} \mathrm{~S}\right), \mathrm{N}^{+}\left({ }^{1} \mathrm{D}\right)+\mathrm{N}\left({ }^{4} \mathrm{~S}\right)$ and $\mathrm{N}^{+}\left({ }^{3} \mathrm{P}\right)+\mathrm{N}\left({ }^{2} \mathrm{D}\right)$, depending on the photon energy. However, our calculations only consider the production in the $\mathrm{N}^{+}\left({ }^{3} \mathrm{P}\right)+\mathrm{N}\left({ }^{2} \mathrm{D}\right)$ states.

The $\mathrm{N}^{+}\left({ }^{1} \mathrm{D}\right)$ state lies $1.9 \mathrm{eV}$ above the $\mathrm{N}^{+}\left({ }^{3} \mathrm{P}\right)$ ground state and has a lifetime of $258 \mathrm{~s}$ (Dutuit et al., 2013). If $\mathrm{N}^{+}\left({ }^{1} \mathrm{D}\right)$ is formed in sufficient amount and its reactivity is significantly different from that of $\mathrm{N}^{+}\left({ }^{3} \mathrm{P}\right)$, its consideration in the calculations could alter the $\mathrm{N}^{+}$density and have some impact on the subsequent chemistry.

$$
\begin{aligned}
\mathrm{N}^{+}\left({ }^{1} \mathrm{D}\right)+\mathrm{N}_{2} & \rightarrow \mathrm{N}^{+}\left({ }^{3} \mathrm{P}\right)+\mathrm{N}_{2} & & \left(\mathrm{R}_{c n}^{*} 612 \mathrm{a}\right) \\
& \rightarrow \mathrm{N}_{2}^{+}+\mathrm{N}\left({ }^{2} \mathrm{D}\right) & & \left(\mathrm{R}_{c n}^{*} 612 \mathrm{~b}\right)
\end{aligned}
$$

The quenching of $\mathrm{N}^{+}\left({ }^{1} \mathrm{D}\right)$ by collisions with $\mathrm{N}_{2}$, channel $\left(\mathrm{R}_{c n}^{*} 612 \mathrm{a}\right)$, is negligible due to its spin forbidden nature (Dutuit et al. (2013) after Freysinger et al. (1994)). Channel $\left(\mathrm{R}_{c n}^{*} 612 \mathrm{~b}\right)$ is exothermic by $0.9 \mathrm{eV}$ but the first spin-allowed channel is endothermic by $1.53 \mathrm{eV}$ (Dutuit et al., 2013). One obvious possibility is that it does react with $\mathrm{N}_{2}\left(\mathrm{X}, \nu^{*}\right)$ but the relevant hot states are not abundant enough for this reaction to have an impact on $\mathrm{N}^{+}$(Lavvas et al., 2015).

$$
\begin{array}{rlrl}
\mathrm{N}^{+}\left({ }^{1} \mathrm{D}\right)+\mathrm{CH}_{4} & \rightarrow \mathrm{CH}_{3}^{+}+\mathrm{NH} & \left(\mathrm{R}_{c n}^{*} 593 \mathrm{a}\right) \\
& \rightarrow \mathrm{HCNH}^{+}+\mathrm{H}_{2} & & \left(\mathrm{R}_{c n}^{*} 612 \mathrm{~b}\right) \\
& \rightarrow \mathrm{HNC}^{+}+\mathrm{H}_{2}+\mathrm{H} & & \left(\mathrm{R}_{c n}^{*} 612 \mathrm{c}\right) \\
& \rightarrow \mathrm{CH}_{4}^{+}+\mathrm{N}\left({ }^{4} \mathrm{~S}\right) & & \left(\mathrm{R}_{c n}^{*} 612 \mathrm{~d}\right)
\end{array}
$$

The total rate constant seems to be the same as for the $\mathrm{N}^{+}\left({ }^{3} \mathrm{P}\right)$ state, i.e. $1.2 \times$ $10^{-9} \mathrm{~cm}^{3} \mathrm{~s}^{-1}$ at $300 \mathrm{~K}$. The product branching ratios $\left(\mathrm{R}_{c n}^{*} 593 \mathrm{a}\right) /\left(\mathrm{R}_{c n}^{*} 593 \mathrm{~b}\right) /\left(\mathrm{R}_{c n}^{*} 593 \mathrm{c}\right) /\left(\mathrm{R}_{c n}^{*} 593\right) \mathrm{d}$ are equal to $0.09 / 0.18 / 0.33 / 0.40$. In comparison with the reactivity of $\mathrm{N}^{+}\left({ }^{3} \mathrm{P}\right)$, the ratio $\left(\mathrm{CH}_{3}{ }^{+}+\mathrm{CH}_{4}^{+}\right) /\left(\mathrm{HCN}^{+}+\mathrm{HCNH}^{+}\right)$decreases from 1.6 to 0.96 , corresponding to an increase in the yield of the nitrogen-containing compounds. The strongest effect is a complete inversion of the ratio $\mathrm{CH}_{3}{ }^{+} / \mathrm{CH}_{4}{ }^{+}$, from 4.4 to 0.22 (Dutuit et al. (2013) after Tichý et al. (1979) and Alcaraz et al. (2004)). $\mathrm{N}^{+}\left({ }^{4} \mathrm{~S}\right)$ also reacts with $\mathrm{H}_{2}$ and all the major hydrocarbons but these are minor loss processes for $\mathrm{N}^{+}$and these reactions cannot substantially affect its density. 
Carrasco et al. (2008) evaluated the effect on model predictions of the different reactivity of $\mathrm{N}^{+}\left({ }^{1} \mathrm{D}\right)$ than $\mathrm{N}^{+}\left({ }^{3} \mathrm{P}\right)$ with methane. They made a test by considering the extreme scenario in which $100 \%$ of the $\mathrm{N}^{+}$ions would be in the excited state. The effect is globally weak, the biggest difference consisting in the increase of the $\mathrm{CH}_{5}{ }^{+}$and $\mathrm{C}_{2} \mathrm{H}_{3}{ }^{+}$densities by tens of percent. $\mathrm{N}^{+}$is not affected (the total rate constant is identical for both states) while the effect on $\mathrm{HCNH}^{+}$is almost negligible (cf. their Figure 3).

This result is consistent with our observation that the production of $\mathrm{HCNH}^{+}$ directly from channel $\left(\mathrm{R}_{c n}^{*} 593 \mathrm{c}\right)$ and indirectly from channel $\left(\mathrm{R}_{c n}^{*} 593 \mathrm{~d}\right)$ followed by the reaction of $\mathrm{HCN}^{+}$with $\mathrm{CH}_{4}$ is only $15 \%$. Moreover, following the study of Nicolas et al. (2003a), Carrasco et al. (2008) estimate that the actual fraction of $\mathrm{N}^{+}\left({ }^{1} \mathrm{D}\right)$ produced in the photoionization of $\mathrm{N}_{2}$ represents only about $15 \%$ of the $\mathrm{N}^{+}$population. Therefore, it appears that the $\mathrm{N}^{+}\left({ }^{1} \mathrm{D}\right)$ state has no relevant effect on $\mathrm{HCNH}^{+}$(and the model as a whole) and it seems reasonable to ignore it.

Finally, the first two excited states of $\mathrm{N}_{2}{ }^{+},\left(A^{2} \Pi_{u}\right)$ and $\left(B^{2} \Sigma_{u}^{+}\right)$, have lifetimes of $\sim 10 \mathrm{~ms}$ and $\sim 70 \mathrm{~ns}$, respectively (Dutuit et al., 2013) and cannot play any active role in the chemistry.

\subsection{Molecular Weight Growth}

There is much uncertainty in the growth mechanisms of Titan's aerosols, particularly how simple precursors form larger molecules that ultimately seed haze particles. Generalized mechanisms focusing on radical chemistry have been put forward for four chemical families: polyynes, nitriles, copolymers (mixed hydrocarbons and nitriles), and aromatics (Lebonnois et al., 2002; Wilson and Atreya, 2003; Lavvas et al., 2008a).

Wilson and Atreya (2003) and Lebonnois (2005) found that the aerosol production is concentrated around $200 \mathrm{~km}$ and that the main contribution comes from $\mathrm{C}_{6} \mathrm{H}_{5}+\mathrm{C}_{2} \mathrm{H}_{2}$ and $\mathrm{H}_{2} \mathrm{CN}+\mathrm{HCN}$. Note that the rate coefficient of both reactions was not available at the Titan temperatures and was extrapolated from combustion studies for the first reaction (Wang and Frenklach, 1994) and estimated for the second one. Our calculations show that the entrance barrier of these reactions is high and consequently the predicted rates are small (cf. Appendix A).

Lavvas et al. (2008b) obtain a secondary aerosol production in the upper atmosphere dominated by copolymers $\left(\mathrm{C}_{3} \mathrm{~N}+\mathrm{C}_{4} \mathrm{H}_{2}, \mathrm{R}_{n} 446\right)$ around $800 \mathrm{~km}$ and nitriles $\left(\mathrm{CN}+\mathrm{C}_{2} \mathrm{H}_{3} \mathrm{CN}, \mathrm{R}_{n} 392\right)$ above $1000 \mathrm{~km}$. The existence of this secondary peak is supported by the Descent Imager/Spectral Radiometer (DISR) haze extinction profile (Tomasko et al., 2005) and the Huygens Atmosphere Structure Instrument (HASI) temperature profile (Fulchignoni et al., 2005). The total column production rate from both low and high altitude production is $1.3 \times 10^{-14}$ $\mathrm{g} \mathrm{cm}^{-2} \mathrm{~s}^{-1}$ for monomers of $7.25 \AA$ radius.

The model of Krasnopolsky reproduces this high altitude peak, although in this case, reactions of $\mathrm{C}_{6} \mathrm{H}$ and $\mathrm{C}_{3} \mathrm{~N}$ with $\mathrm{C}_{4} \mathrm{H}_{2}\left(\mathrm{R}_{n} 236, \mathrm{R}_{n} 446\right)$ are significant (cf. Figure 7 in Krasnopolsky (2014)), with a total production of $10^{-13}$ 
$\mathrm{g} \mathrm{cm}^{-2} \mathrm{~s}^{-1}$. It also considers for the first time recombination of heavy ions $\left(\mathrm{C}_{9} \mathrm{H}_{11}{ }^{+}, \mathrm{C}_{10} \mathrm{H}_{9}{ }^{+}\right.$and $\left.\mathrm{C}_{10} \mathrm{H}_{11}{ }^{+}\right)$as a source of haze, although this turns out to be negligible (less than $1 \%$ of the neutral polymerization channels).

Lavvas et al. (2011c) consider formation of polycyclic aromatic compounds through neutral reactions starting from benzene and involving the $\mathrm{CN}, \mathrm{C}_{2} \mathrm{H}$ and HCCN radicals. However, the main focus of this investigation is to identify the main processes that can affect the aerosol evolution and these pathways are mostly proxies for the possible contribution of radicals.

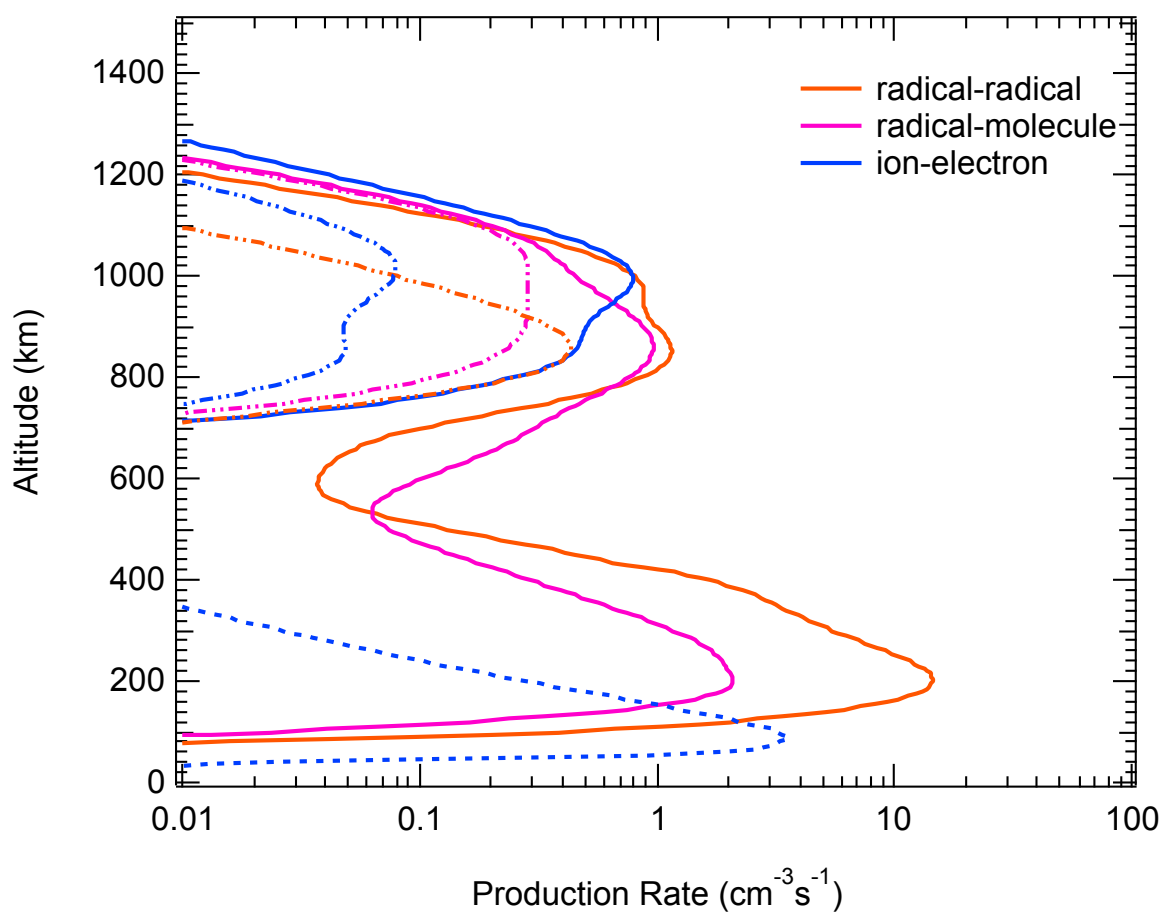

Figure 62: Calculated production rate of species that are considered as aerosol seeds. Dotdashed lines represent the production rate of nitrogen-bearing species. The dotted line shows the production rate from the electron recombination of cluster ions resulting from GCR impact; they all contain nitrogen.

Table 18: Calculated column-integrated production rates (molecules $\mathrm{cm}^{-2} \mathrm{~s}^{-1}$ ) of the three generic channels.

\begin{tabular}{llll}
\hline \hline Channels & Thermosphere & Stratosphere & Total \\
\hline \hline Radical-Radical & $6.6 \times 10^{7}$ & $2.9 \times 10^{8}$ & $3.6 \times 10^{8}$ \\
Radical-Molecule & $4.6 \times 10^{7}$ & $4.4 \times 10^{7}$ & $9.0 \times 10^{7}$ \\
Ion-Electron & $3.6 \times 10^{7}$ & $4.1 \times 10^{6}$ & $4.0 \times 10^{7}$ \\
\hline Total & $1.5 \times 10^{8}$ & $3.4 \times 10^{8}$ & $4.9 \times 10^{8}$ \\
\hline \hline
\end{tabular}


Photochemical models as they currently exist are not well suited to predicting haze production rates. Our understanding of the pathways leading to the observed aerosols is still at a preliminary stage and it is likely that those proposed so far are only a small fraction of a multitude of chemical processes acting simultaneously. As a result, models can at best provide an upper limit from the sum of the production rate of all the species that do not have any loss process. Our total aerosol flux of $\sim 5 \times 10^{8} \mathrm{~cm}^{-2} \mathrm{~s}^{-1}$ (cf. Table 18) translates to a mass production of $\sim 6 \times 10^{-14} \mathrm{~g} \mathrm{~cm}^{-2} \mathrm{~s}^{-1}$, if considering that the transition occurs at a mean molecular mass of $70-80 \mathrm{~g} \mathrm{~mol}^{-1}$, which corresponds to a molecule having 5 or 6 carbon atoms. A total aerosol mass production of $3 \times 10^{-14} \mathrm{~g}$ $\mathrm{cm}^{-2} \mathrm{~s}^{-1}$ is required to match the haze optical properties retrieved by the DISR observations (Lavvas et al., 2010). This implies that about $50 \%$ of the species that we do not track should further decompose back to smaller molecules while $50 \%$ should evolve to aerosol particles.

We find that the copolymers and nitriles contribution to the radical-molecule channel is negligible, with a total rate of $\sim 3 \times 10^{4} \mathrm{~cm}^{-2} \mathrm{~s}^{-1}$. The polyyne type pathways are somewhat significant with $\mathrm{C}_{6} \mathrm{H}+\mathrm{C}_{2} \mathrm{H}_{2},\left(\mathrm{R}_{n} 229\right)$ and $\mathrm{C}_{4} \mathrm{H}+\mathrm{C}_{2} \mathrm{H}_{4}$, $\left(\mathrm{R}_{n} 196\right)$ representing $\sim 15 \%$ of the total production. Electron recombination of nitrogen-bearing cluster ions formed in the GCR layer leads to the production of many nitrile species with a total integrated production rate of $3 \times 10^{-7} \mathrm{~cm}^{-2}$ $\mathrm{s}^{-1}$, which is similar to the value calculated by Molina-Cuberos et al. (2002). To summarize, although our total aerosol mass production is of the same order of magnitude as previous models (Lavvas et al., 2008b; Krasnopolsky, 2014), the specific molecular growth reactions are very different.

Instead of discussing further specific reaction pathways, we adopt an alternative approach consisting in grouping reactions in three generic channels: radicalradical, radical-molecule and ion-electron (cf. Figure 62). Not surprisingly, production rates peak where the energy deposition is maximum, i.e. around 900 and $200 \mathrm{~km}$. Our major source of aerosols in the stratosphere originates from the recombination of hydrocarbon radicals (amongst which the most efficient are $\mathrm{CH}_{3}+\mathrm{C}_{4} \mathrm{H}_{n}$ ), a process that had never been put forward. The unprecedented efficiency of this process in our model $\left(2.9 \times 10^{8} \mathrm{~cm}^{-2} \mathrm{~s}^{-1}\right)$ is attributed to the consideration of radiative association reactions that become extremely efficient $\left(\mathrm{k}=8 \times 10^{-11} \mathrm{~cm}^{3} \mathrm{~s}^{-1}\right)$ when the number of carbon atoms involved reaches 4 or 5 (Vuitton et al., 2012). In the thermosphere, all three channels have a similar contribution to the total production, values are given in Table 18.

\subsection{Effects of Stratospheric Aerosols}

The aerosols in the stratosphere of Titan have four important roles on the (photo)chemistry: (1) shielding of the UV flux, (2) recombination of $\mathrm{H}$ atoms on their surface, (3) heterogeneous removal of polar species, and (4) condensation nuclei.

\subsubsection{UV Shielding}

Aerosols are the dominant source of opacity for wavelengths $\geq 180 \mathrm{~nm}$ in the stratosphere (cf. Figure 14) and aerosol shielding has a profound impact on 
the photochemistry. In our nominal model, aerosol opacities are derived from the single scattering albedo and extinction retrieved by DISR (Lavvas et al., 2010). However, a newer analysis of DISR data (Doose et al., 2016) implies a different aerosol scale height above $80 \mathrm{~km}$. The impact of this recent study on the aerosol opacity has not been investigated yet. Can lack of fits for $\mathrm{HC}_{3} \mathrm{~N}$ and $\mathrm{C}_{2} \mathrm{H}_{5} \mathrm{CN}$, i.e. species that are mostly lost by photodissociation, be resolved with small adjustments in aerosol opacity?

To answer this question, we performed two model runs where the nominal aerosol opacity was either multiplied or divided by a factor of 2. Figures 44 and 53 show the sensitivity for $\mathrm{C} 2$ hydrocarbons and some nitriles $\left(\mathrm{C}_{2} \mathrm{H}_{3} \mathrm{CN}\right.$ and $\mathrm{C}_{2} \mathrm{H}_{5} \mathrm{CN}$ ), respectively. The different choices of opacity do not significantly change the results. The mole fraction of $\mathrm{C}_{2} \mathrm{H}_{2}, \mathrm{C}_{2} \mathrm{H}_{4}$ and the nitriles (not shown for $\mathrm{HC}_{3} \mathrm{~N}$ to avoid overcrowding the plot) increases by a few tens of percent from the low to high opacity run because of the increase in aerosol shielding and subsequent decrease in photodissociation rate for these species. Not surprisingly, the most sensitive species is $\mathrm{C}_{2} \mathrm{H}_{6}$, which can be explained by the less effective photodissociation of $\mathrm{C}_{2} \mathrm{H}_{2}$ leading to a lower abundance of $\mathrm{C}_{2} \mathrm{H}$ radicals and subsequently of $\mathrm{CH}_{3}$ radicals, the precursors of $\mathrm{C}_{2} \mathrm{H}_{6}$, as detailed in section 3.2.1.

\subsubsection{Atomic and Molecular Hydrogen Budget}

Since $\mathrm{CH}_{4}$ is the ultimate source of all carbon atoms, the production of the more complex carbon species must be accompanied by comparable quantities of $\mathrm{H}$ or $\mathrm{H}_{2}$. Indeed, molecular hydrogen is the third most abundant species in Titan's atmosphere, with a mole fraction of $10^{-3}$ at the tropopause (cf. Table 11).

Sekine et al. (2008b) introduced heterogeneous chemistry of hydrogen atoms in the model of Lebonnois (2005) and observed dramatic impacts on atomic hydrogen and heavy hydrocarbon profiles, as expected from the high reaction probabilities inferred in the laboratory (Sekine et al. (2008a), see section 2.7.5). According to their calculations, heterogeneous reactions increase the mole fractions of $\mathrm{C}_{4} \mathrm{H}_{2}$ and $\mathrm{C}_{6} \mathrm{H}_{5}$ by more than 2 orders of magnitude around $400 \mathrm{~km}$. Although they did not perform a thorough comparison of their computed profiles with available observations, they mention that the $\mathrm{C}_{4} \mathrm{H}_{2}$ profile obtained with heterogeneous processes is in better agreement with the CIRS observations (Vinatier et al., 2007a), a conclusion also shared by Lavvas et al. (2008b). Krasnopolsky (2009) considers the same processes as Sekine et al. (2008b) and finds that formation of $\mathrm{H}_{2}$ in the heterogeneous recombination of $\mathrm{H}$ with the haze is insignificant.

In our model, the $\mathrm{H}$ production in the upper atmosphere peaks around 800 $\mathrm{km}$ and occurs through direct photodissociation of $\mathrm{CH}_{4}\left(\mathrm{~J}_{d} 2 \mathrm{a}, \mathrm{c}, \mathrm{d}\right)$ and $\mathrm{CH}_{3}$ $\left(\mathrm{J}_{d} 38\right)$, and reaction of $\mathrm{CH}$ with $\mathrm{CH}_{4}\left(\mathrm{R}_{n} 47\right) . \mathrm{H}_{2}$ is mostly produced by reaction of $\mathrm{H}$ with ${ }^{3} \mathrm{CH}_{2}\left(\mathrm{R}_{n} 3\right)$ and photodissociation of $\mathrm{CH}_{4}\left(\mathrm{~J}_{d} 2 \mathrm{~b}\right)$. In the stratosphere, a secondary production of hydrogen atoms occurs through photodissociation of abundant unsaturated species such as $\mathrm{C}_{2} \mathrm{H}_{2}\left(\mathrm{~J}_{d} 5\right), \mathrm{C}_{2} \mathrm{H}_{4}\left(\mathrm{~J}_{d} 6 \mathrm{~b}\right), \mathrm{C}_{4} \mathrm{H}_{2}\left(\mathrm{~J}_{d} 12 \mathrm{a}\right)$ 
and $\mathrm{C}_{6} \mathrm{H}_{2}\left(\mathrm{~J}_{d} 17 \mathrm{a}\right)$. Molecular hydrogen is essentially formed by photodissociation of $\mathrm{C}_{2} \mathrm{H}_{4}\left(\mathrm{~J}_{d} 6 \mathrm{a}\right)$ and $\mathrm{C}_{4} \mathrm{H}_{4}\left(\mathrm{~J}_{d} 13 \mathrm{c}\right)$, and reaction of $\mathrm{H}$ with $\mathrm{C}_{2} \mathrm{H}_{3}\left(\mathrm{R}_{n} 8 \mathrm{~b}\right)$ and $\mathrm{C}_{2} \mathrm{H}_{5}\left(\mathrm{R}_{n} 10 \mathrm{~b}\right)$. All the $\mathrm{H}_{2}$ produced eventually escapes hydrodynamically from the exosphere (Strobel, 2010). Similarly, the hydrogen atoms formed in the thermosphere subsequently flow upward and escape the atmosphere at a rate of $3.0 \times 10^{9} \mathrm{~cm}^{-2} \mathrm{~s}^{-1}$.

Below $500 \mathrm{~km}$, the heterogeneous reactions of atomic hydrogen atoms on the surface of aerosol particles become their dominant removal process and a sharp decrease in the atomic hydrogen density in the region centered around $400 \mathrm{~km}$ is evident as shown in the left panel of Figure 63 . About $60 \%$ of the total $\mathrm{H}$ produced ends up adsorbed onto aerosols, a conclusion also shared by Sekine et al. (2008b). Here, hydrogen atoms are not recycled back to the gas phase and therefore, this process represents a net loss for hydrogen in the model and as a consequence, a decrease in the $\mathrm{H}_{2}$ mole fraction is observed (cf. right panel of Figure 63). This is the opposite effect as reported in Lavvas et al. (2008b) because the latter include recombination of $\mathrm{H}$ atoms on the aerosols but not their hydrogenation. However, because $\mathrm{H}_{2}$ is practically inert in the atmosphere, the actual fate of hydrogen atoms (sequestration at the surface of the aerosols or release in the atmosphere as $\mathrm{H}_{2}$ ) has little impact on the chemistry, including haze formation, the essential point being their removal from the gas phase.
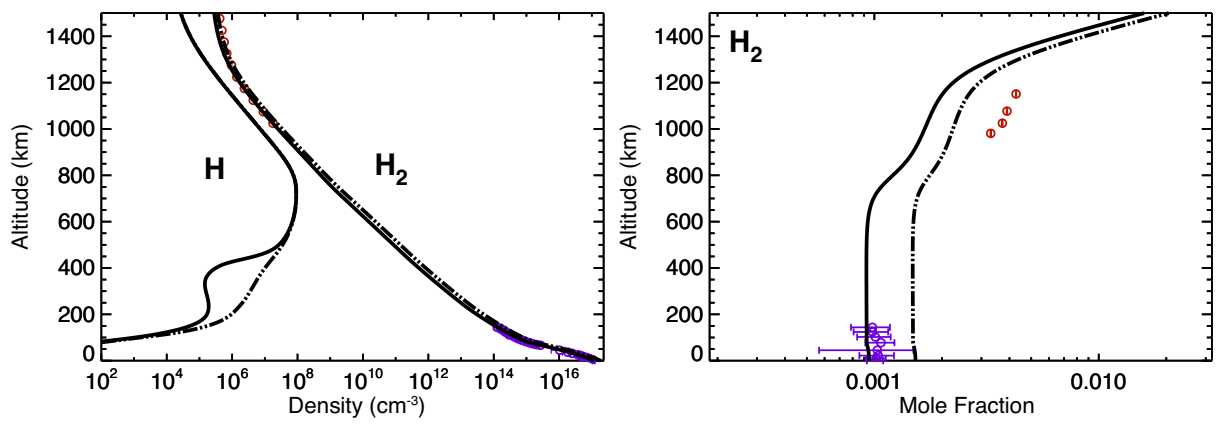

Figure 63: Calculated densities of $\mathrm{H}$ and $\mathrm{H}_{2}$ (left panel). Calculated and observed mole fractions of $\mathrm{H}_{2}$ with (solid line) and without (long dash-dot-dot line) atomic hydrogen heterogeneous loss (right panel).

Combination of $\mathrm{H}$ with $\mathrm{C}_{2} \mathrm{H}_{2}, \mathrm{C}_{2} \mathrm{H}_{4}, \mathrm{C}_{4} \mathrm{H}_{2}$ and $\mathrm{HC}_{3} \mathrm{~N}\left(\mathrm{R}_{n} 7 \mathrm{a}, \mathrm{R}_{n} 9 \mathrm{a}, \mathrm{R}_{n} 21\right.$, $\mathrm{R}_{n} 258$ ) are critical loss reactions for these species (section 2.7.3.1) and therefore removal of $\mathrm{H}$ atoms strongly raises their stratospheric abundance. The vertical profiles of $\mathrm{C}_{2} \mathrm{H}_{2}$ and $\mathrm{C}_{2} \mathrm{H}_{4}$ with and without the atomic hydrogen heterogeneous loss process are shown in Figure 44. Their mole fractions increase by a factor of $\sim 2$ and $\sim 5$, respectively and are found to be closer to that of the CIRS midlatitude profiles when this heterogeneous chemistry is included. However, the decrease in the atomic hydrogen population, allows for more diacetylene and cyanoacetylene, with the simulated profiles (solid lines in Figure 47 (left panel) and 53 (upper left panel)) being in worse agreement with the CIRS retrieved 
vertical profiles. Benzene is decreased by a factor of $\sim 20$ at $300 \mathrm{~km}$ (cf. right panel of Figure 47) because combination of $\mathrm{H}$ with $\mathrm{C}_{6} \mathrm{H}_{5}\left(\mathrm{R}_{n} 30\right)$, its main recycling reaction, has been reduced.

It is interesting that the $\mathrm{H}+\mathrm{C}_{2} \mathrm{H}_{2}\left(\mathrm{R}_{n} 7 \mathrm{~b}\right)$ and $\mathrm{H}+\mathrm{C}_{2} \mathrm{H}_{4}\left(\mathrm{R}_{n} 9 \mathrm{~b}\right)$ hydrogen abstractions do not efficiently convert hydrogen atoms into molecular hydrogen, unlike stated in previous models. We note that the $\mathrm{H}+\mathrm{C}_{4} \mathrm{H}_{2}$ and $\mathrm{H}+\mathrm{HC}_{3} \mathrm{~N}$ abstraction reactions are endothermic.

\subsubsection{Nitriles Heterogeneous Chemistry}

Lara et al. (1999) reported difficulties in matching with their photochemical model the observed nitrile and hydrocarbon profiles and suggested that an additional chemical loss to the aerosols is required for HCN. Our nominal model does not include the heterogeneous reactions of molecules onto aerosols, with the exception of hydrogen atoms. Sticking should apply to polar molecules, such as nitriles and water. We performed a sensitivity test on putative adsorption reactions for $\mathrm{HCN}, \mathrm{HNC}, \mathrm{CH}_{3} \mathrm{CN}, \mathrm{HC}_{3} \mathrm{~N}, \mathrm{C}_{2} \mathrm{H}_{3} \mathrm{CN}, \mathrm{C}_{2} \mathrm{H}_{5} \mathrm{CN}, \mathrm{HC}_{5} \mathrm{~N}$ and $\mathrm{H}_{2} \mathrm{O}$. The adsorption rates are assumed to be proportional to the physical collision rate between molecules and aerosols; the aerosol radius and density are from Figure 22. The efficiency for this absolute loss is taken as $10^{-7}$.

The densities of $\mathrm{HCN}, \mathrm{CH}_{3} \mathrm{CN}, \mathrm{HC}_{3} \mathrm{~N}$ and $\mathrm{C}_{2} \mathrm{H}_{5} \mathrm{CN}$ with the heterogeneous loss process described above are shown in Figures 51 and 53. This brings the model abundances of $\mathrm{HC}_{3} \mathrm{~N}$ and $\mathrm{C}_{2} \mathrm{H}_{5} \mathrm{CN}$ (column-integrated density of $5.7 \times 10^{14} \mathrm{~cm}^{-2} \mathrm{~s}^{-1}$ ) into better conformance with the observations but $\mathrm{HCN}$ and $\mathrm{CH}_{3} \mathrm{CN}$ are now largely underestimated. $\mathrm{CH}_{3} \mathrm{CN}, \mathrm{HC}_{3} \mathrm{~N}, \mathrm{C}_{2} \mathrm{H}_{3} \mathrm{CN}$ and $\mathrm{C}_{2} \mathrm{H}_{5} \mathrm{CN}$ all have a similar dipole moment $(3.7-4.1 \mathrm{D})$ and there is no obvious reason suggesting that their sticking efficiency should differ significantly. There is no impact on $\mathrm{HNC}$ because of its very large reactivity with $\mathrm{H}$ atoms. The contribution of this process to aerosol mass loading comes essentially from $\mathrm{HCN}$ with an adsorption rate of $6 \times 10^{-15} \mathrm{~g} \mathrm{~cm}^{-2} \mathrm{~s}^{-1}$. This is to be compared to the aerosol mass flux from the upper atmosphere of $3 \times 10^{-14} \mathrm{~g} \mathrm{~cm}^{-2} \mathrm{~s}^{-1}$ (Lavvas et al., 2010).

Even with a conservatively small adsorption probability to the aerosols, the implied loss for the species considered is enough to drastically affect their abundance. We emphasize that there are significant uncertainties on adsorption rates and that stronger constraints are needed to know if heterogeneous chemistry can resolve the issues we are encountering for some nitriles. Laboratory measurements and/or theoretical computations for the sticking efficiency of molecules onto tholins are required to verify this process and to provide a better constraint on its impact in Titan's stratosphere.

\subsubsection{Condensation}

As mentioned previously, all the minor species (with the exception of $\mathrm{C}_{2} \mathrm{H}_{4}$ ) are subject to condensation near the tropopause followed by precipitation to the surface. Willacy et al. (2016) investigated the condensation and sublimation with treatments of the sedimentation of the aerosols and their condensates, and the formation of haze particles. They found that these processes do affect 
the abundances of nitrogen-bearing species, particularly $\mathrm{HCN}, \mathrm{HNC}, \mathrm{HC}_{3} \mathrm{~N}$, $\mathrm{C}_{2} \mathrm{H}_{3} \mathrm{CN}$ and $\mathrm{C}_{2} \mathrm{H}_{5} \mathrm{CN}$. Lavvas et al. (2011b) had followed a similar approach but only considered the effects of the condensation of methane, ethane and hydrogen cyanide and found that the latter one followed its saturation mole fraction.

The column integrated mass fluxes for the six species that are the major sources of condensates are presented in Table 19. The major liquid condensate is $\mathrm{C}_{2} \mathrm{H}_{6}$ with a precipitation flux of $3.5 \times 10^{9} \mathrm{~cm}^{-2} \mathrm{~s}^{-1}$ (or $1.7 \times 10^{-13} \mathrm{~g} \mathrm{~cm}^{-2}$ $\mathrm{s}^{-1}$ ), while the major solid condensate is $\mathrm{C}_{2} \mathrm{H}_{2}$ with a precipitation flux of $1.1 \times 10^{9} \mathrm{~cm}^{-2} \mathrm{~s}^{-1}$ (or $4.6 \times 10^{-14} \mathrm{~g} \mathrm{~cm}^{-2} \mathrm{~s}^{-1}$ ). In general, the values from our model are similar to that in previous models (cf. Table 7.6 in Vuitton et al. (2014) and Table 4 in Willacy et al. (2016)).

Table 19: Calculated condensation flux of major species. For each species, the fraction of its condensation rate to its total loss rate as well as the associated deposition rate of material on the surface are given.

\begin{tabular}{|c|c|c|c|}
\hline$\overline{\text { Species }}$ & Flux ${ }^{(a)}\left(\mathrm{g} \mathrm{cm}^{-2} \mathrm{~s}^{-1}\right)$ & Fraction of the total loss & 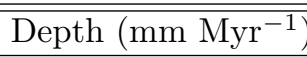 \\
\hline \multicolumn{4}{|c|}{ Liquids } \\
\hline $\mathrm{C}_{2} \mathrm{H}_{6}$ & $1.7 \times 10^{-13}$ & $88 \%$ & 55 \\
\hline $\mathrm{C}_{3} \mathrm{H}_{8}$ & $2.6 \times 10^{-14}$ & $81 \%$ & 8.1 \\
\hline $\mathrm{C}_{4} \mathrm{H}_{10}$ & $2.7 \times 10^{-15}$ & $71 \%$ & 0.85 \\
\hline Total & $2.0 \times 10^{-13}$ & - & 64 \\
\hline \multicolumn{4}{|c|}{ Solids } \\
\hline $\mathrm{C}_{2} \mathrm{H}_{2}$ & $4.6 \times 10^{-14}$ & $11 \%$ & 14 \\
\hline $\mathrm{HCN}$ & $5.9 \times 10^{-15}$ & $41 \%$ & 1.9 \\
\hline $\mathrm{HC}_{3} \mathrm{~N}$ & $9.8 \times 10^{-16}$ & $8 \%$ & 0.31 \\
\hline Total & $5.3 \times 10^{-14}$ & - & 17 \\
\hline \multicolumn{4}{|c|}{ 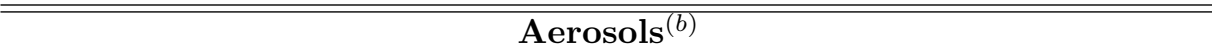 } \\
\hline & $3.0 \times 10^{-14}$ & - & 10 \\
\hline
\end{tabular}

${ }^{(a)}$ The density of all compounds is taken as $1 \mathrm{~g} \mathrm{~cm}^{-3}$.

${ }^{(b)}$ From Lavvas et al. (2010).

The above fluxes can be used to calculate the depth of material accumulated on the surface of Titan over a given period of time. This is presented in Table 19 in $\mathrm{mm} \mathrm{Myr}^{-1}$. The mass flux of the solid condensates is equivalent to $5.3 \times 10^{-14}$ $\mathrm{g} \mathrm{cm}^{-2} \mathrm{~s}^{-1}$, which is about twice as much as the aerosol mass flux. Liquids $\left(\mathrm{C}_{2} \mathrm{H}_{6}, \mathrm{C}_{3} \mathrm{H}_{8}, \mathrm{C}_{4} \mathrm{H}_{10}\right)$ dominate over solid deposits (condensates + aerosols) by a factor of $\sim 2$, with a deposition rate of $6.4 \mathrm{~cm} \mathrm{Myr}^{-1}$. If this deposition rate has been constant over the age of the solar system, the equivalent of $290 \mathrm{~m}$ of liquid material and $120 \mathrm{~m}$ of solid material should have accumulated on the surface. This is to be compared to the $0.2-2.0 \mathrm{~m}$ equivalent depth of liquid currently present in the lakes, while dunes are consistent with an organic component that would be 2 to $8 \mathrm{~m}$ thick if evenly spread on the surface, as inferred from Cassini RADAR observations (Lorenz et al., 2008). These values support the ideas 
that: (i) the concentration of atmospheric methane has varied over the age of the solar system and may be a recent addition to the atmosphere (Tobie et al., 2006), 75-300 Myrs being enough to produce some 2-8 m thick dune material, (ii) $\mathrm{C}_{2} \mathrm{H}_{6}$ is lost to the crust or deeper interior in the form of clathrates (Lunine, 2010).

\subsection{Lifetimes}

Minor species have a large range of lifetimes, from days to several thousand years, which means that they are sensitive to atmospheric motions over a range of timescales and different gases can be used to study different aspects of the dynamics. If the only processes occurring are photochemical reactions and vertical atmospheric motion, the polar enrichment observed in the stratosphere during winter should be proportional to the species vertical gradient, i.e. inversely proportional to its lifetime. This is explained by the presence of a circulation cell with downwelling in the polar regions, which causes the lower stratosphere to be greatly enriched in short lifetime species compared to the equator.

Figure 64 shows the lifetime of several compounds as a function of altitude. The species lifetime is the shortest of their chemical and transport time constant, as defined in section 2.4. The diurnal/ annual time constants are equal to the length of a day/ year (in seconds) divided by $2 \pi$. The solar time constant corresponds to the solar cycle of 11 Earth years (cf. next section). 

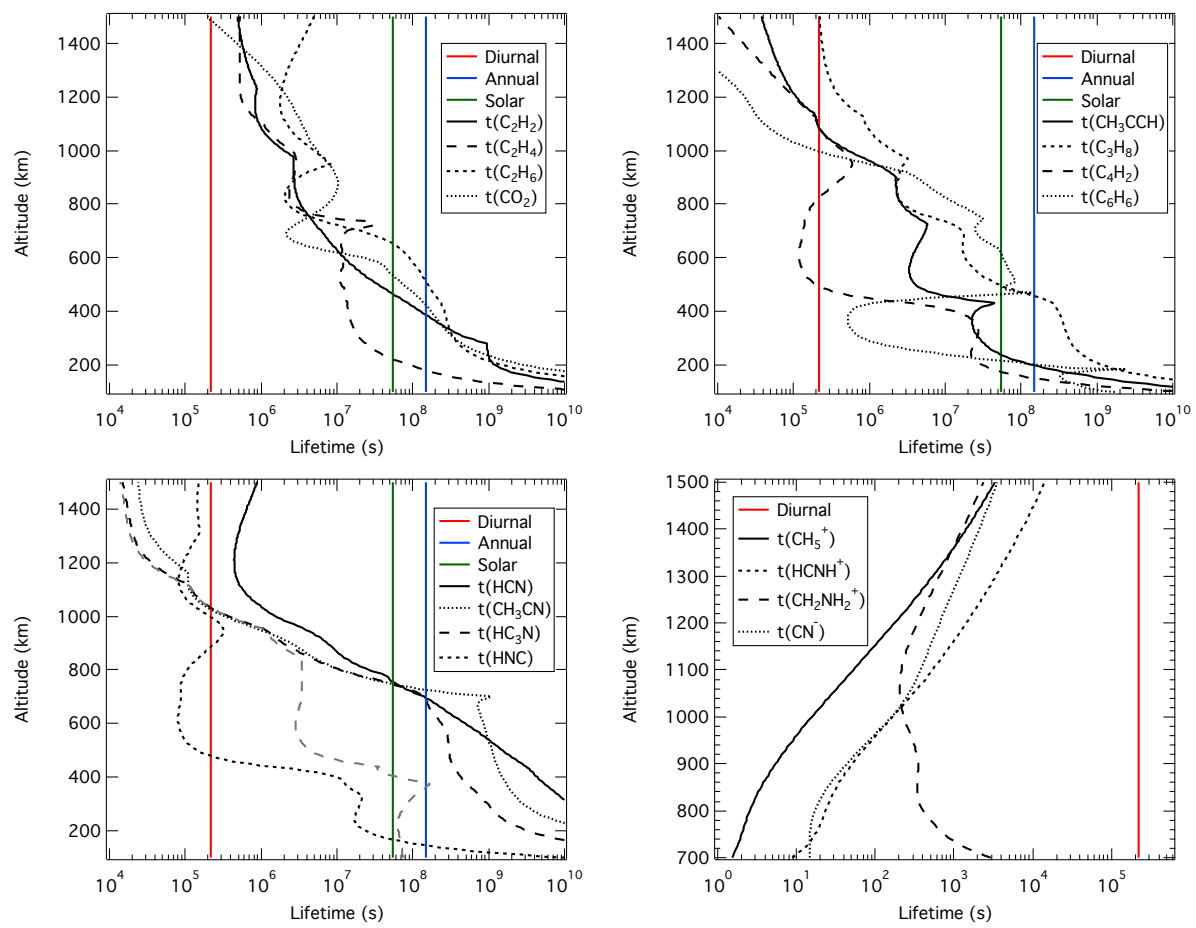

Figure 64: Calculated lifetime of $\mathrm{C}_{2} \mathrm{H}_{2}, \mathrm{C}_{2} \mathrm{H}_{4}, \mathrm{C}_{2} \mathrm{H}_{6}$, and $\mathrm{CO}_{2}$ (upper left panel), $\mathrm{CH}_{3} \mathrm{CCH}$, $\mathrm{C}_{3} \mathrm{H}_{8}, \mathrm{C}_{4} \mathrm{H}_{2}$ and $\mathrm{C}_{6} \mathrm{H}_{6}$ (upper right panel), $\mathrm{HCN}, \mathrm{HNC}, \mathrm{CH}_{3} \mathrm{CN}$ and $\mathrm{HC}_{3} \mathrm{~N}$ (lower left panel), $\mathrm{CH}_{5}{ }^{+}, \mathrm{HCNH}^{+}, \mathrm{CH}_{2} \mathrm{NH}_{2}{ }^{+}$and $\mathrm{CN}^{-}$(lower right panel). Characteristic diurnal/annual/solar times are also presented. 
Table 20: Calculated chemical $\left(\mathrm{t}_{C}\right)$ and transport $\left(\mathrm{t}_{K}\right)$ lifetime of various species at $300 \mathrm{~km}$.

\begin{tabular}{lll}
\hline \hline Species & $\mathrm{t}_{C}(\mathrm{~s})$ & $\mathrm{t}_{K}(\mathrm{~s})$ \\
\hline \hline $\mathrm{C}_{2} \mathrm{H}_{2}$ & $9.1 \times 10^{8}$ & $5.6 \times 10^{8}$ \\
$\mathrm{C}_{2} \mathrm{H}_{4}$ & $2.0 \times 10^{8}$ & $2.0 \times 10^{7}$ \\
$\mathrm{C}_{2} \mathrm{H}_{6}$ & $4.0 \times 10^{9}$ & $3.5 \times 10^{8}$ \\
$\mathrm{CH}_{3} \mathrm{CCH}$ & $1.9 \times 10^{8}$ & $2.8 \times 10^{7}$ \\
$\mathrm{C}_{3} \mathrm{H}_{8}$ & $1.9 \times 10^{9}$ & $4.2 \times 10^{8}$ \\
$\mathrm{C}_{4} \mathrm{H}_{2}$ & $2.6 \times 10^{7}$ & $6.2 \times 10^{7}$ \\
$\mathrm{C}_{6} \mathrm{H}_{6}$ & $6.2 \times 10^{8}$ & $8.2 \times 10^{5}$ \\
$\mathrm{HCN}$ & $3.8 \times 10^{11}$ & $1.1 \times 10^{10}$ \\
$\mathrm{HNC}$ & $2.0 \times 10^{7}$ & $4.9 \times 10^{7}$ \\
$\mathrm{CH}_{3} \mathrm{CN}$ & $8.9 \times 10^{11}$ & $2.9 \times 10^{9}$ \\
$\mathrm{HC}_{3} \mathrm{~N}^{(a)}$ & $8.2 \times 10^{7}$ & $9.2 \times 10^{7}$ \\
$\mathrm{C}_{2} \mathrm{~N}_{2}$ & $3.6 \times 10^{7}$ & $4.6 \times 10^{7}$ \\
$\mathrm{H}_{2} \mathrm{O}$ & $3.7 \times 10^{10}$ & $1.1 \times 10^{8}$ \\
$\mathrm{CO}_{2}$ & $6.9 \times 10^{15}$ & $3.5 \times 10^{8}$ \\
\hline \hline
\end{tabular}

${ }^{(a)}$ Values obtained with the nitriles heterogeneous chemistry turned on, see section 5.3.3.

Generally, as species settle towards the surface, their lifetime increases from typically one Titan day in the thermosphere to one Titan year in the stratosphere. Nevertheless, there is some large variability between species, with HCN having a lifetime of $\sim 200$ Titan yrs. and $\mathrm{C}_{6} \mathrm{H}_{6}$ of $\sim 4$ days at $300 \mathrm{~km}$ (cf. Table 20 ). In the ionosphere, the nitriles are efficiently lost by ion chemistry. Because the reaction rates are all fast and quite similar, the chemical lifetime of all species is about the same. It is a different story in the stratosphere. For example, $\mathrm{HNC}$ isomerizes very quickly to $\mathrm{HCN}$ by collision with $\mathrm{H}$ atoms and its lifetime is quite short. However, the nitriles do not react efficiently with any of the radicals present there and their main chemical loss is photodissociation. Because $\mathrm{HC}_{3} \mathrm{~N}$ absorbs to much longer wavelengths (where there are more photons available) than $\mathrm{CH}_{3} \mathrm{CN}$, its loss rate is higher and therefore its chemical lifetime shorter. Actually, the chemical loss for $\mathrm{CH}_{3} \mathrm{CN}$ is so inefficient that its main actual loss is vertical transport (downwelling) to the tropopause where it condenses (cf. Table 20). In fact, for most species, depending on the altitude, regions dominated by photochemical equilibrium alternate with regions dominated by dynamical equilibrium. At $300 \mathrm{~km}$, the lifetime of most species is dominated by transport, with the exception of the few having a short chemical time constant, such as $\mathrm{C}_{4} \mathrm{H}_{2}, \mathrm{HNC}$ and $\mathrm{C}_{2} \mathrm{~N}_{2}$.

CIRS shows that north-polar $\mathrm{C}_{4} \mathrm{H}_{2}$ and $\mathrm{HC}_{3} \mathrm{~N}$ enrichment is much greater than that of HCN (Teanby et al., 2008b). This is consistent with the much shorter photochemical lifetime of $\mathrm{C}_{4} \mathrm{H}_{2}$ and $\mathrm{HC}_{3} \mathrm{~N}$ ( $\sim 1 \mathrm{yr}$.) compared to $\mathrm{HCN}$ ( 1000 yrs.), which prevents them from mixing with air at lower latitude (cf. Figure 4 in (Teanby et al., 2008b)). However, nitriles appear to be anomalously enriched compared to hydrocarbons (cf. Figure 8 in Teanby et al. (2010)). Teanby et al. (2010) suggests that an additional loss mechanism is required for 
nitrile species. However, our stratospheric $\mathrm{HCN}$ profile is already on the lower edge of the observations and any additional loss would lead to an inconsistent HCN abundance (unless the production rate is somehow increased as well). For $\mathrm{HC}_{3} \mathrm{~N}$, adding some heterogeneous loss to the aerosols in order to match the observations leads to a decrease of its chemical lifetime from $1.6 \times 10^{9}$ to $8.2 \times 10^{7}$ s.

At the ionospheric peak, the lifetime of ions is typically $10^{2}-10^{3} \mathrm{~s}$. It is largely dominated by their chemical loss as their reactivity is high and generally increases with decreasing altitude, therefore leading to a decreasing lifetime, as shown in the bottom right panel of Figure 64 .

\subsection{Solar Cycle}

11-year variations in the Sun's magnetic field significantly affect the shorter EUV/UV range of the solar output, making these changes directly relevant to Titan's photochemistry. The Cassini prime mission encountered an extended solar minimum, with a rising phase of solar activity starting in 2011. As shown in Figure 64, lifetimes of most constituents are considerably shorter than one solar cycle above the stratosphere and are thus sensitive to the changes in solar flux over the course of that cycle in that region.

In Titan's atmosphere, constituents above $500 \mathrm{~km}$ are typically destroyed chemically within one solar cycle, while for certain stable constituents, like $\mathrm{C}_{4} \mathrm{H}_{2}$, their relatively short lifetimes may allow changes in solar output to affect their lower stratospheric abundances, as well. With this in mind, it is constructive to analyze the effect of variations in solar flux on the distribution of various key constituents in Titan's atmosphere.

Westlake et al. (2014) and Madanian et al. (2016) studied the effects of the solar cycle based on a total of twelve years (2004-2015) of measurements by INMS. They reported a declining trend in the $\mathrm{CH}_{4}, \mathrm{~N}_{2}$ and $\mathrm{C} 2$ hydrocarbon abundance during solar maximum. For ions, the solar maximum densities are significantly greater than their solar minimum counterparts (at similar solar zenith angles), with a decrease in altitude of the ionospheric peak.

Combining these data with modeling, Westlake et al. (2014) relate the diminution of $\mathrm{CH}_{4}$ during high solar activity periods to an increased $\mathrm{CH}_{4}$ photodestruction because of the increased photon flux. They also suggested that ion-molecule reactions producing large hydrocarbons are responsible for an enhanced loss of C2 hydrocarbons during solar maximum and therefore declining mole fractions in the thermosphere. Luspay-Kuti et al. (2016) investigated as well the influence on minor hydrocarbons of variations in the solar photons in response to the solar cycle. Their calculations indicate a decrease of $\mathrm{CH}_{3}, \mathrm{C}_{2} \mathrm{H}_{6}$ and $\mathrm{C}_{3} \mathrm{H}_{8}$ above $\sim 900-950 \mathrm{~km}$ during solar maximum, which they explain by the role of $\mathrm{N}\left({ }^{4} \mathrm{~S}\right)$. Higher abundance of $\mathrm{N}\left({ }^{4} \mathrm{~S}\right)$ would increase the importance of the $\mathrm{CH}_{3}+\mathrm{N}\left({ }^{4} \mathrm{~S}\right)$ reaction, decreasing the $\mathrm{CH}_{3}$ abundance and affecting the overall hydrocarbon chemistry. 

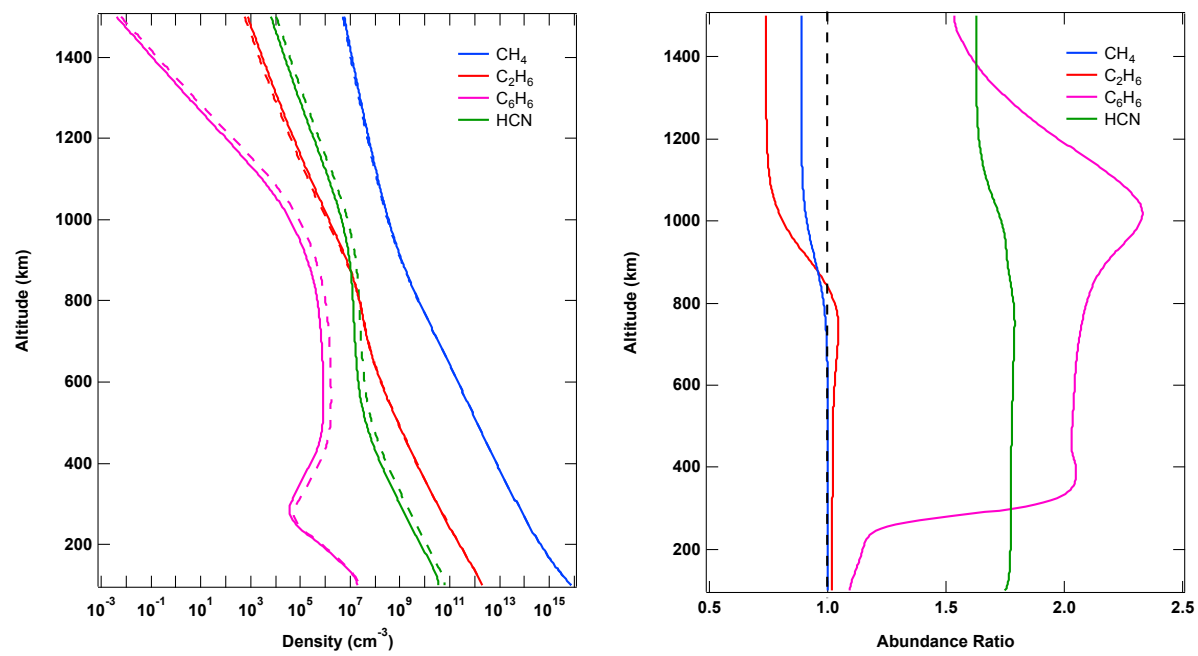

Figure 65: Calculated $\mathrm{CH}_{4}, \mathrm{C}_{2} \mathrm{H}_{6}, \mathrm{C}_{6} \mathrm{H}_{6}, \mathrm{HCN}$ density (left panel) at solar maximum (dashed) / solar minimum (solid) and abundance ratio (right panel).

A comparison between our solar maximum and solar minimum profiles (absolute and relative abundances) of selected neutrals is shown in Figure 65. Methane and ethane show a reduction in density above $\sim 900 \mathrm{~km}$ during solar maximum compared to solar minimum conditions. $\mathrm{C}_{6} \mathrm{H}_{6}$ and $\mathrm{HCN}$ show increased densities during solar maximum over the entire altitude range. The magnitude of the variation varies among species from about $10 \%\left(\mathrm{CH}_{4}\right)$ to as much as a factor of more than $2\left(\mathrm{C}_{6} \mathrm{H}_{6}\right) \cdot \mathrm{C}_{2} \mathrm{H}_{2}$ and $\mathrm{C}_{2} \mathrm{H}_{4}$ exhibit a very moderate increase of $20 \%$ and $5 \%$, respectively (not shown). 

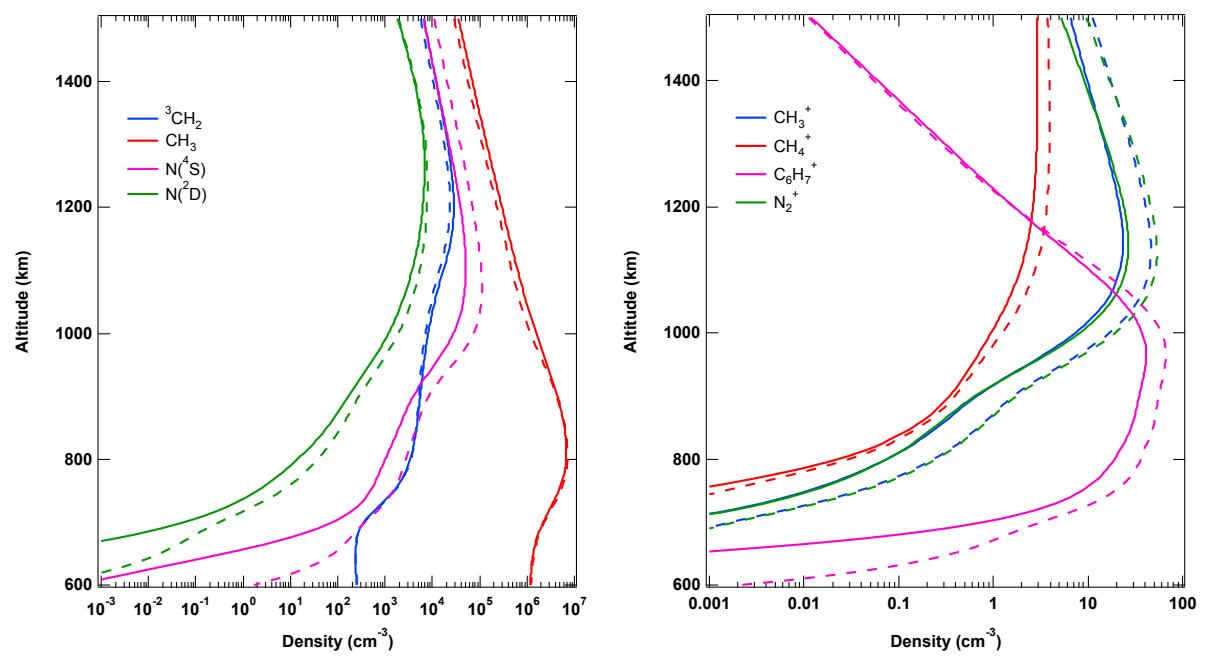

Figure 66: Calculated solar maximum (dashed) / solar minimum (solid) abundance of ${ }^{3} \mathrm{CH}_{2}$, $\mathrm{CH}_{3}, \mathrm{~N}\left({ }^{4} \mathrm{~S}\right), \mathrm{N}\left({ }^{2} \mathrm{D}\right)$ (left panel) and $\mathrm{CH}_{3}{ }^{+}, \mathrm{CH}_{5}{ }^{+}, \mathrm{N}_{2}{ }^{+}, \mathrm{HCNH}^{+}$(right panel).

The observed solar maximum depletion in $\mathrm{CH}_{4}$ is in agreement with its increased photodestruction (11\%) during solar maximum conditions in response to the increased photon flux. Since $\mathrm{CH}_{3}$ is a primary product of $\mathrm{CH}_{4}$ photodissociation $\left(\mathrm{J}_{d} 38 \mathrm{a}\right)$, a higher peak abundance $(6 \%)$ is observed during solar maximum as a result of increased production (cf. left panel of Figure 66). Similarly, the peak abundance of $\mathrm{N}\left({ }^{4} \mathrm{~S}\right)$ increases at solar maximum (factor of $\sim 2$ ). A drop in peak altitude $(30 \mathrm{~km})$ is due to the relative enhancement of the photon flux at shorter wavelengths (cf. Figure 16), where the $\mathrm{N}_{2}$ cross-sections decrease sharply. The higher $\mathrm{N}\left({ }^{4} \mathrm{~S}\right)$ abundance during solar maximum boosts reactions between $\mathrm{N}\left({ }^{4} \mathrm{~S}\right)$ and $\mathrm{CH}_{3}$, resulting in higher $\mathrm{CH}_{3}$ loss rates and a lower $\mathrm{CH}_{3}$ abundance during solar maximum than during solar minimum above $880 \mathrm{~km}$. This general trend is the same as observed by Luspay-Kuti et al. (2016), although their very small $\mathrm{CH}_{3}$ abundance ratio above $1000 \mathrm{~km}$ in their Figure 1 does not seem realistic to us, especially looking at their $\mathrm{C}_{2} \mathrm{H}_{6}$ profile. $\mathrm{CH}_{3}$ plays a lead role in the photochemistry of Titan's upper atmosphere, and is especially important for the production of $\mathrm{C}_{2} \mathrm{H}_{6}$ and $\mathrm{HCN}\left(\mathrm{R}_{n} 90, \mathrm{R}_{n} 290 \mathrm{~b}\right)$. Hence, the enriched solar maximum $\mathrm{C}_{2} \mathrm{H}_{6}$ abundance below $850 \mathrm{~km}$ and depleted above. The effect of $\mathrm{N}\left({ }^{4} \mathrm{~S}\right)$ is observed on the density of $\mathrm{HCN}$, which is higher by up to about $80 \%$ during solar maximum because of increasing production.

Ion-molecule chemistry becomes enhanced during solar maximum compared with solar minimum due to the increased EUV flux that initiates ion production (cf. right panel of Figure 66). The density enhancement is best seen in the primary ion species $\mathrm{N}_{2}{ }^{+}$and $\mathrm{CH}_{3}{ }^{+}$that increase by a factor of about 2 . The density of $\mathrm{CH}_{4}{ }^{+}$exhibits a smaller increase of $\sim 40 \%$ between high and low solar activity, while that of $\mathrm{HCNH}^{+}$increases by $\sim 60 \%$. The ion peak altitudes are slightly lower $(10-15 \mathrm{~km})$ for higher activity, again because of the relative 
increase in solar flux at shorter wavelengths. Since ion-molecule chemistry dominates the production of $\mathrm{C}_{6} \mathrm{H}_{6}$ at high altitudes, its higher density is primarily due to the increased reaction rates. Although we do observe an enhanced loss of $\mathrm{C}_{2} \mathrm{H}_{2}$ and $\mathrm{C}_{2} \mathrm{H}_{4}$ from ion-molecule reactions, this process is not competitive and does not support the conclusion of Westlake et al. (2014).

\section{Conclusions and Perspectives}

We developed and improved a photochemical model extending from Titan's surface to the exobase. Solar EUV/UV, photoelectrons and cosmic rays are considered as energy sources. The chemical network includes hydrocarbons, nitrogen and oxygen-bearing species, in the form of neutrals as well as positive and negative ions. It has become apparent that:

- New constraints from Cassini on the background atmosphere, eddy diffusion coefficient and haze opacity significantly reduce uncertainties in the photochemical model.

- The neutral composition is fairly well understood, with the notable exception of oxygen-bearing species. Neutral and ion chemistry are intimately coupled and the latter is a substantial source of neutral species through electron recombination reactions.

- The model provides assignments for the complex positive and negative ions present in the thermosphere, up to $\mathrm{m} / \mathrm{z} \sim 100$. We notably infer that ions are nitrogen rich. Reproducing the measured density of major ions (both positive and negative) with photochemical models remains an issue.

- Reaction pathways leading to the macromolecules constituting the aerosols as well as the impact of heterogeneous reactions are largely unknown but likely involve ion as well as neutral species.

Investigations into the nature of Titan's chemistry can be extended in several ways:

- Analysis of more Cassini data, to further characterize temporal (solar zenith angle, season) and spatial (altitude, latitude, magnetospheric interaction geometry) variations. Neutral mole fractions are well constrained by CIRS in the stratosphere but extensive analysis of the UVIS occultation data and INMS data are necessary to constrain neutral abundances in the upper atmosphere. INMS and CAPS positive and negative ion mass spectra should be interpreted for a large number of flybys.

- Diurnal variations as well as transport based on realistic general circulation models should be included in photochemical models. A time-variable model may be required to solve the oxygen photochemistry discrepancy. 
- Collection of more chemical data, from both experimental and theoretical investigations, at the temperature and pressure conditions relevant for Titan, with emphasis on nitrogen-bearing species. Special attention should be paid to product branching ratios at low temperature and isomers identification. Of highest interest are electron recombination and association (both collisional and radiative) reactions as well as negative ion chemistry in general. Radical-radical and ion-radical reactions should be given the highest priority. Laboratory investigations of heterogeneous processes on tholins should also be developed.

- Construction of state-of-the-art laboratory experiments in order to get some insight into the intermediate steps involved in molecular growth (from $\mathrm{m} / \mathrm{z}=100$ to 1000 ). Detection should be performed in situ and allow probing intermediate species such as radicals and ions as a function of time. EUV/UV light should be preferred and ultra-high vacuum reactors should be used to limit atmospheric contamination. Temperature and pressure should be representative of Titan as far as possible.

- Remote sensing (ALMA, Herschel, JWST, etc.) observational campaigns should target more complex molecules (nitriles, aromatics, oxygen species, including ${ }^{15} \mathrm{~N}$ and ${ }^{18} \mathrm{O}$ isotopes) not detected by Cassini. The mystery of the aerosol composition will not be conclusively resolved, however, until next generation space instruments are sent to the Saturn system. Very high-resolution mass spectrometers optimized for the detection of complex organics, both in the volatile and solid phases, should be developed for that critical endeavor.

\section{Acknowledgements}

VV acknowledges support from the French space agency (CNES) through a Cassini Participating Scientist grant. The contribution from SJK is based upon work supported through NASA Planetary Atmospheres Program grant number NNH09AK24I and by the U.S. Department of Energy, Office of Science, Office of Basic Energy Sciences, Division of Chemical Sciences, Geosciences, and Biosciences under Contract No. DE-AC02-06CH11357 (BES Gas Phase Chemical Physics Core Program). PL acknowledges support from the PNP under program AMG and from the CNRS PICS program TAC. The authors thank Alexandre Faure, Wolf Geppert, Murray McEwan, Darci Snowden, Roland Thissen and Erik Vigren for useful discussions.

\section{References}

Abouelaziz, H., Gomet, J., Pasquerault, D., Rowe, B., Mitchell, J., 1993. Measurements of $\mathrm{C}_{3} \mathrm{H}_{3}{ }^{+}, \mathrm{C}_{5} \mathrm{H}_{3}{ }^{+}, \mathrm{C}_{6} \mathrm{H}_{6}{ }^{+}, \mathrm{C}_{7} \mathrm{H}_{5}{ }^{+}$, and $\mathrm{C}_{10} \mathrm{H}_{8}{ }^{+}$dissociative recombination rate coefficients. J. Chem. Phys. 99, 237-243. 
Adachi, H., Basco, N., James, D., 1981. The acetyl radicals $\mathrm{CH}_{3} \mathrm{CO}$ and $\mathrm{CD}_{3} \mathrm{CO}$ studied by flash photolysis and kinetic spectroscopy. Int. J. Chem. Kin. 13, $1251-1276$.

Adam, L., Hack, W., Zhu, H., Qu, Z., Schinke, R., 2005. Experimental and theoretical investigation of the reaction $\mathrm{NH}\left(\mathrm{X}^{3} \Sigma^{-}\right)+\mathrm{H}\left({ }^{2} \mathrm{~S}\right) \rightarrow \mathrm{N}\left({ }^{4} \mathrm{~S}\right)+$ $\mathrm{H}_{2}\left(\mathrm{X}^{1} \Sigma_{g}^{+}\right)$. J. Chem. Phys. 122, 114301-114307.

Adams, N., 1996. Gas phase ionic reactions, in: Drake, G. (Ed.), Atomic, molecular \& optical physics handbook. American Institute of Physics, Woodbury, New York, pp. 441-451.

Adams, N., Herd, C., Geoghegan, M., Smith, D., Canosa, A., Gomet, J., Rowe, B., Queffelec, J., Morlais, M., 1991. Laser induced fluorescence and vacuum ultraviolet spectroscopic studies of $\mathrm{H}$-atom production in the dissociative recombination of some protonated ions. J. Chem. Phys. 94, 4852-4857.

Adams, N., Mathews, L., Osborne Jr., D., 2010. Laboratory chemistry relevant to understanding and modeling the ionosphere of Titan. Faraday Discuss. $147,323-335$.

Adams, N., Molek, C., McLain, J., 2009. New flowing afterglow technique for determining products of dissociative recombination: $\mathrm{CH}_{5}{ }^{+}$and $\mathrm{N}_{2} \mathrm{H}^{+} . \mathrm{J}$. Phys.: Conf. Ser. 192, \#012004.

Adams, N., Smith, D., 1978. Reactions of CHn+ ions with molecules at $300 \mathrm{~K}$. Chem. Phys. Lett. 54, 530-534.

Adams, N., Smith, D., 1988a. Laboratory studies of dissociative recombination and mutual neutralization and their relevance to interstellar chemistry, in: Millar, T., Williams, D. (Eds.), Rate coefficients in astrochemistry. Kluwer Academic Publishers, pp. 173-192.

Adams, N., Smith, D., 1988b. Measurements of the dissociative recombination coefficients for several polyatomic ion species at $300 \mathrm{~K}$. Chem. Phys. Lett. $144,11-14$.

Adams, N., Smith, D., Paulson, J., 1980. An experimental survey of the reactions of $\mathrm{NH}_{n}^{+}$ions $(\mathrm{n}=0$ to 4$)$ with several diatomic and polyatomic molecules at 300 K. J. Chem. Phys. 72, 288-297.

Adriani, A., Dinelli, B., López-Puertas, M., García-Comas, M., Moriconi, M., D'Aversa, E., Funke, B., Coradini, A., 2011. Distribution of HCN in Titan's upper atmosphere from Cassini/VIMS observations at $3 \mu \mathrm{m}$. Icarus 214, 584595 .

Ågren, K., Wahlund, J.E., Garnier, P., Modolo, R., Cui, J., Galand, M., MüllerWodarg, I., 2009. On the ionospheric structure of Titan. Planet. Space Sci. 57, 1821-1827. 
Ågren, K., Wahlund, J.E., Modolo, R., Lummerzheim, D., Galand, M., MüllerWodarg, I., Canu, P., Kurth, W., Cravens, T., Yelle, R., Waite Jr., J., Coates, A., Lewis, G., Young, D., Bertucci, C., Dougherty, M., 2007. On magnetospheric electron impact ionisation and dynamics in Titan's ram-side and polar ionosphere - a Cassini case study. Ann. Geophys. 25, 2359-2369.

Aguado, A., Roncero, O., Zanchet, A., Agúndez, M., Cernicharo, J., 2017. The photodissociation of HCN and HNC: Effects on the HNC/HCN abundance ratio in the interstellar medium. Astrophys. J. 838, \#33.

Ahmed, M., Peterka, D., Suits, A., 1999. The photodissociation of the vinyl radical $\left(\mathrm{C}_{2} \mathrm{H}_{3}\right)$ at $243 \mathrm{~nm}$ studied by velocity map imaging. J. Chem. Phys. 110, 4248-4253.

Ajello, J., Stevens, M., Stewart, I., Larsen, K., Esposito, L., Coldwell, J., McClintock, W., Holsclaw, G., Gustin, J., Pryor, W., 2007. Titan airglow spectra from Cassini Ultraviolet Imaging spectrograph (UVIS): EUV analysis. Geophys. Res. Lett. 34, \#L24202.

Akimoto, H., Obi, K., Tanaka, I., 1965. Primary process in the photolysis of ethane at 1236 A. J. Chem. Phys. 42, 3864-3868.

Albritton, D., 1978. Ion-neutral reaction rate constants measured in flow reactors through 1977. At. Data Nucl. Data Tables 22, 1-101.

Alcaraz, C., Nicolas, C., Thissen, R., Žabka, J., Dutuit, O., 2004. ${ }^{15} \mathrm{~N}^{+}+$ $\mathrm{CD}_{4}$ and $\mathrm{O}^{+}+{ }^{13} \mathrm{CO}_{2}$ state-selected ion-molecule reactions relevant to the chemistry of planetary ionospheres. J. Phys. Chem. A 108, 9998-10009.

Alge, E., Adams, N., Smith, D., 1983. Measurements of the dissociative recombination coefficients of $\mathrm{O}_{2}{ }^{+}, \mathrm{NO}^{+}$and $\mathrm{NH}_{4}{ }^{+}$in the temperature range 200-600 K. J. Phys. B: At. Mol. Phys. 16, 1433-1444.

Allen, M., Pinto, J., Yung, Y., 1980. Titan: Aerosol photochemistry and variations related to the sunspot cycle. Astrophys. J. 242, L125-L128.

Alnama, K., Boyé-Péronne, S., Douin, S., Innocenti, F., O'Reilly, J., Roche, A.L., Shafizadeh, N., Zuin, L., Gauyacq, D., 2007. Photolysis of allene and propyne in the $7-30 \mathrm{eV}$ region probed by the visible fluorescence of their fragments. J. Chem. Phys. 126, \#044304.

Amitay, Z., Zajfman, D., Forck, P., Hechtfischer, U., Seidel, B., Grieser, M., Habs, D., Repnow, R., Schwalm, D., Wolf, A., 1996. Dissociative recombination of $\mathrm{CH}^{+}$: Cross section and final states. Phys. Rev. A 54, 4032-4050.

Angelova, G., Novotny, O., Mitchell, J., Rebrion-Rowe, C., Le Garrec, J., Bluhme, H., Seiersen, K., Andersen, L., 2004a. Branching ratios for the dissociative recombinaion of hydrocarbon ions II. The cases of $\mathrm{C}_{4} \mathrm{H}_{\mathrm{n}}^{+}(\mathrm{n}=$ 1-9). Int. J. Mass Spectrom. 232, 195-203. 
Angelova, G., Novotny, O., Mitchell, J., Rebrion-Rowe, C., Le Garrec, J., Bluhme, H., Svendsen, A., Andersen, L., 2004b. Branching ratios for the dissociative recombinaion of hydrocarbon ions III: the cases of $\mathrm{C}_{3} \mathrm{H}_{n}^{+}(\mathrm{n}=$ 1-8). Int. J. Mass Spectrom. 235, 7-13.

Anicich, V., 1993. Evaluated bimolecular ion-molecule gas phase kinetics of positive ions for use in modeling planetary atmospheres, cometary comae, and interstellar clouds. J. Phys. Chem. Ref. Data 22, 1469-1569.

Anicich, V., McEwan, M., 1997. Ion-molecule chemistry in Titan's ionosphere. Planet. Space Sci. 45, 897-921.

Anicich, V., Milligan, D., Fairley, D., McEwan, M., 2000. Termolecular ionmolecule reactions in Titan's atmosphere. I: Principal ions with principal neutrals. Icarus 146, 118-124.

Anicich, V., Wilson, P., McEwan, M., 2003. Termolecular ion-molecule reactions in Titan's atmosphere. IV: A search made at up to 1 micron in pure hydrocarbons. J. Am. Soc. Mass Spectrom. 14, 900-915.

Anicich, V., Wilson, P., McEwan, M., 2004. A SIFT ion-molecule study of some reactions in Titan's atmosphere. Reactions of $\mathrm{N}^{+}, \mathrm{N}_{2}{ }^{+}$, and $\mathrm{HCN}^{+}$with $\mathrm{CH}_{4}$, $\mathrm{C}_{2} \mathrm{H}_{2}$, and $\mathrm{C}_{2} \mathrm{H}_{4}$. J. Am. Soc. Mass Spectrom. 15, 1148-1155.

Anicich, V., Wilson, P., McEwan, M., 2006. An ICR study of ion-molecules reactions in Titan's atmosphere: An investigation of binary hydrocarbon mixtures up to 1 micron. J. Am. Soc. Mass Spectrom. 17, 544-561.

Aoto, T., Ito, K., Hikosaka, Y., Shibasaki, A., Hirayama, R., Yamamono, N., Miyoshi, E., 2006. Inner-valence states of $\mathrm{N}_{2}{ }^{+}$and the dissociation dynamics studied by threshold photoelectron spectroscopy and configuration interaction calculation. J. Chem. Phys. 124, 6.

Arnold, S., Dotan, I., Willliams, S., Viggiano, A., Morris, R., 2000. Selected ion flow tube studies of air plasma cations reacting with alkylbenzenes. J. Phys. Chem. A 104, 928-934.

Arnold, S., Williams, S., Dotan, I., Midey, A., Morris, R., Viggiano, A., 1999. Flow tube studies of benzene charge transfer reactions from 250 to $1400 \mathrm{~K}$. J. Phys. Chem. A 103, 8421-8432.

Ashfold, M., Dixon, R., Kono, M., Mordaunt, D., Reed, C., 1997. Near ultraviolet photolysis of ammonia and methylamine studied by H Rydberg atom photofragment translational spectroscopy. Phil. Trans. Roy. Soc. London A $355,1659-1676$.

Asvany, O., Savić, I., Schlemmer, S., Gerlich, D., 2004. Variable temperature ion trap studies of $\mathrm{CH}_{4}{ }^{+}+\mathrm{H}_{2}$, $\mathrm{HD}$ and $\mathrm{D}_{2}$ : negative temperature dependence and significant isotope effect. Chem. Phys. 298, 97-105. 
Atkinson, D., Hudgens, J., 1999. Rate coefficients for the propargyl radical self-reaction and oxygen addition reaction measured using ultraviolet cavity ring-down spectroscopy. J. Phys. Chem. A 103, 4242-4252.

Atreya, S., 1986. Atmospheres and ionospheres of the outer planets and their satellites. volume 15 of Physics and Chemistry in Space. Springer-Verlag, New York.

Au, J., Cooper, G., Burton, G., Olney, T., Brion, C., 1993. The valence shell photoabsorption of the linear alkanes $\mathrm{C}_{n} \mathrm{H}_{2 n+2}(\mathrm{n}=1-8)$ : Absolute oscillator strenghts (7-220 eV). Chem. Phys. 173, 209-239.

Ausloos, P., 1982. Structure and isomerization of $\mathrm{C}_{7} \mathrm{H}_{7}{ }^{+}$ions formed in the charge-transfer-induced fragmentation of ethylbenzene, toluene, and norbornadiene. J. Am. Chem. Soc. 104, 5259-5265.

Ausloos, P., Jackson, J.A., Lias, S., 1980. Reactions of benzyl ions with alkanes, alkenes, and aromatic compounds. Int. J. Mass Spectrom. Ion Processes 33, 269-283.

Ausloos, P., Lias, S., Buckley, T., Rogers, E., 1989. Concerning the formation and the kinetics of phenylium ions. Int. J. Mass Spectrom. 92, 65-77.

Baines, K., Drossart, P., Lopez-Valverde, M., Atreya, S., Sotin, C., Momary, T., Brown, R., Buratti, B., Clark, R., Nicholson, P., 2006. On the discovery of CO nighttime emissions on Titan by Cassini/VIMS: Derived stratospheric abundances and geological implications. Planet. Space Sci. 54, 1552-1562.

Balko, B.A., Zhang, J., Lee, Y.T., 1992. Photodissociation of ethylene at 193 nm. J. Chem. Phys. 97, 935-942.

Balucani, N., Asvany, O., Kaiser, R., Osamura, Y., 2002. Formation of three $\mathrm{C}_{4} \mathrm{H}_{3} \mathrm{~N}$ isomers from the reaction of $\mathrm{CN}\left(\mathrm{X}^{2} \Sigma^{+}\right)$with allene, $\mathrm{H}_{2} \mathrm{CCCH}_{2}$ $\left(\mathrm{X}^{1} \mathrm{~A}_{1}\right)$, and methylacetylene, $\mathrm{CH}_{3} \mathrm{CCH}\left(\mathrm{X}^{1} \mathrm{~A}_{1}\right)$ : A combined crossed beam and ab initio study. J. Phys. Chem. A 106, 4301-4311.

Balucani, N., Leonori, F., Petrucci, R., Stazi, M., Skouteris, D., Rosi, M., Casavecchia, P., 2010. Formation of nitriles and imines in the atmosphere of Titan: Combined crossed-beam and theoretical studies on the reaction dynamics of excited nitrogen atoms $\mathrm{N}\left({ }^{2} \mathrm{D}\right)$ with ethane. Faraday Discuss. 147, $1-28$.

Balucani, N., Skouteris, D., Leonori, F., Petrucci, R., Hamberg, M., Geppert, W., Casavecchia, P., 2012. Combined crossed beam and theoretical studies of the $\mathrm{N}\left({ }^{2} \mathrm{D}\right)+\mathrm{C}_{2} \mathrm{H}_{4}$ reaction and implications for atmospheric models of Titan. J. Phys. Chem. A 116, 10467-10479.

Bampasidis, G., Coustenis, A., Achterberg, R., Vinatier, S., Lavvas, P., Nixon, C., Jennings, D., Teanby, N., Flasar, F., Carlson, R., Moussas, X., PrekaPapadema, P., Romani, P., Guandique, E., Stamogiorgos, S., 2012. Thermal 
and chemical structure variations in Titan's stratosphere during the Cassini mission. Astrophys. J. 760, \#144.

Banaszkiewicz, M., Lara, L., Rodrigo, R., López-Moreno, J., Molina-Cuberos, G., 2000. A coupled model of Titan's atmosphere and ionosphere. Icarus 147, 386-404.

Barckholtz, C., Snow, T., Bierbaum, V., 2001. Reactions of $\mathrm{C}_{n}^{-}$and $\mathrm{C}_{n} \mathrm{H}^{-}$with atomic and molecular hydrogen. Astrophys. J. 547, L171-L174.

Bartels, M., Edelbuttel-Einhaus, J., Hoyermann, K., 1991. The detection of $\mathrm{CH}_{3} \mathrm{CO}, \mathrm{C}_{2} \mathrm{H}_{5}$, and $\mathrm{CH}_{3} \mathrm{CHO}$ by REMPI/mass spectrometry and the application to the study of the reactions $\mathrm{H}+\mathrm{CH}_{3} \mathrm{CO}$ and $\mathrm{O}+\mathrm{CH}_{3} \mathrm{CO}$. Symp. Int. Combust. Proc. 23, 131-138.

Bartels, M., Hoyermann, K., Sievert, R., 1982. Elementary reactions in the oxidation of ethylene: the reaction of $\mathrm{OH}$ radicals with ethylene and the reaction of $\mathrm{C} 2 \mathrm{H} 4 \mathrm{OH}$ radicals with $\mathrm{H}$ atoms. Symp. Int. Combust. Proc. 19, 61.

Bartmess, J., 1982. Gas-phase ion chemistry of 5-methylene-1,3-cyclohexadiene (o-isotoluene) and 3-methylene-1,4-cyclohexadiene (p-isotoluene). J. Am. Chem. Soc. 104, 335-337.

Bastian, M., Dressler, R., Murad, E., Arnold, S., Viggiano, A., 1996. Detailed study of the dynamics of the $\mathrm{O}^{+}\left({ }^{4} \mathrm{~S}\right)+\mathrm{HCN}$ reaction: a case study of ionmolecule reactions in the spacecraft environment. J. Chem. Soc., Faraday Trans. 92, 2659-2670.

Bates, D., Mitchell, J., 1991. Rate coefficients for $\mathrm{N}_{2}^{+}(\nu)$ dissociative recombination. Planet. Space Sci. 39, 1297-1300.

Baulch, D., Bowman, C., Cobos, C., Cox, R., Just, T., Kerr, J., Pilling, M., Stocker, D., Troe, J., Tsang, W., Walker, R., Warnatz, J., 2005. Evaluated kinetic data for combustion modeling: Supplement II. J. Phys. Chem. Ref. Data $34,757-1397$.

Baulch, D., Cobos, C., Cox, R., Esser, C., Frank, P., Just, T., Kerr, J., Pilling, M., Troe, J., Walker, R., Warnatz, J., 1992. Evaluated kinetic data for combustion modeling. J. Phys. Chem. Ref. Data 21, 411-734.

Baulch, D., Cobos, C., Cox, R., Frank, P., Hayman, G., Just, T., Kerr, J., Murrells, T., Pilling, M., Troe, J., Walker, R., Warnatz, J., 1994. Evaluated kinetic data for combustion modeling: Supplement I. J. Phys. Chem. Ref. Data 23, 847-1033.

Becke, A., 1993. Density-functional thermochemistry. 3. The role of exact exchange. J. Chem. Phys. 98, 5648-5652. 
Bell, J., Bougher, S., Waite Jr., J., Ridley, A., Magee, B., Mandt, K., Westlake, J., DeJong, A., Bar-Nun, A., Jacovi, R., Toth, G., De La Haye, V., Gell, D., Fletcher, G., 2011. Simulating the one-dimensional structure of Titan's upper atmosphere: 3. Mechanisms determining methane escape. J. Geophys. Res. 116, \#E11002.

Bellucci, A., Sicardy, B., Drossart, P., Rannou, P., Nicholson, P., Hedman, M., Baines, K., Burrati, B., 2009. Titan solar occultation observed by Cassini/VIMS: Gas absorption and constraints on aerosol composition. Icarus 201, 198-216.

Benedikt, J., 2010. Plasma-chemical reactions: low pressure acetylene plasmas. J. Phys. D: Appl. Phys. 43, \#043001.

Bénilan, Y., Bruston, P., Raulin, F., Cossart-Magos, C., Guillemin, J.C., 1994. Mid-UV spectroscopy of propynenitrile at low temperature: Consequences on expected results from observations of Titan's atmosphere. J. Geophys. Res. 99, 17069-17074.

Bénilan, Y., Jolly, A., Raulin, F., 1999. Comparative IR and UV spectroscopic studies of allene and methylacetylene: Implication for observation and modeling of Titan atmosphere. Bull. Am. Astron. Soc. 31, \#1169.

Bénilan, Y., Smith, N., Jolly, A., Raulin, F., 2000. The long wavelength range temperature variations of the mid-UV acetylene absorption coefficient. Planet. Space Sci. 48, 463-471.

Bera, P., Head-Gordon, M., Lee, T., 2011. Initiating molecular growth in the interstellar medium via dimeric complexes of observed ions and molecules. A\&A 535, \#A74.

Bera, P., Peverati, R., Head-Gordon, M., Lee, T., 2015. Hydrocarbon growth via ion-molecule reactions: computational studies of the isomers of $\mathrm{C}_{4} \mathrm{H}_{2}{ }^{+}$, $\mathrm{C}_{6} \mathrm{H}_{2}{ }^{+}$and $\mathrm{C}_{6} \mathrm{H}_{4}{ }^{+}$and their formation paths from acetylene and its fragments. Phys. Chem. Chem. Phys. 17, 1859-1869.

Bergeat, A., Hickson, K., Daugey, N., Caubet, P., Costes, M., 2009. A low temperature investigation of the $\mathrm{N}\left(4 \mathrm{~S}^{\circ}\right)+\mathrm{NO}$ reaction. Phys. Chem. Chem. Phys. 11, 8149-8155.

Bergeat, A., Loison, J., 2001. Reaction of carbon atoms, $\mathrm{C}\left(2 \mathrm{p}^{2},{ }^{3} \mathrm{P}\right)$ with $\mathrm{C}_{2} \mathrm{H}_{2}, \mathrm{C}_{2} \mathrm{H}_{4}$ and $\mathrm{C}_{6} \mathrm{H}_{6}$ : Overall rate constant and relative atomic hydrogen production. Phys. Chem. Chem. Phys. 3, 2038-2042.

Berkowitz, J., 1996. Photoion-pair formation, in: Becker, U., Shirley, D. (Eds.), VUV and soft X-ray photoionization. Plenum Press, New York. Physics of atoms and molecules, pp. 263-289.

Berkowitz, J., Chupka, W., Walter, T., 1969. Photoionization of HCN: The electron affinity and heat of formation of CN. J. Chem. Phys. 50, 1497-1500. 
Bernard, E., Strazisar, B., Davis, H., 1999. Excited state dynamics of $\mathrm{H}_{2} \mathrm{CN}$ radicals. Chem. Phys. Lett. 313, 461-466.

Berteloite, C., Le Picard, S., Balucani, N., Canosa, A., Sims, I., 2010a. Low temperature rate coefficients for reactions of the butadiynyl radical, $\mathrm{C}_{4} \mathrm{H}$, with various hydrocarbons. Part I: reactions with alkanes $(\mathrm{CH} 4, \mathrm{C} 2 \mathrm{H} 6, \mathrm{C} 3 \mathrm{H} 8$, C4H10). Phys. Chem. Chem. Phys. 12, 3666-3676.

Berteloite, C., Le Picard, S., Balucani, N., Canosa, A., Sims, I., 2010b. Low temperature rate coefficients for reactions of the butadiynyl radical, $\mathrm{C}_{4} \mathrm{H}$, with various hydrocarbons. Part II: reactions with alkenes (ethylene, propene, 1butene), dienes (allene, 1,3-butadiene) and alkynes (acetylene, propyne and 1-butyne). Phys. Chem. Chem. Phys. 12, 3677-3689.

Berteloite, C., Le Picard, S., Birza, P., Gazeau, M.C., Canosa, A., Bénilan, Y., Sims, I., 2008. Low temperature (39-298 K) kinetics study of the reactions of the $\mathrm{C}_{4} \mathrm{H}$ radical with various hydrocarbons observed in Titan's atmosphere. Icarus 194, 746-757.

Best, T., Otto, R., Trippel, S., Hlavenka, P., von Zastrow, A., Eisenbach, S., Jézouin, S., Wester, R., Vigren, E., Hamberg, M., Geppert, W., 2011. Absolute photodetachment cross-section measurements for hydrocarbon chain anions. Astrophys. J. 742, \#63.

Bézard, B., 2014. The methane mole fraction in Titan's stratosphere from DISR measurements during the Huygens probe's descent. Icarus 242, 64-73.

Biennier, L., Carles, S., Cordier, D., Guillemin, J.C., Le Picard, S., Faure, A., 2014. Low temperature reaction kinetics of $\mathrm{CN}^{-}+\mathrm{HC}_{3} \mathrm{~N}$ and implications for the growth of anions in Titan's atmosphere. Icarus 227, 123-131.

Blackman, G., Brown, R., Godfrey, P., Gunn, H., 1976. The microwave spectrum of HNC: Identification of U90.7. Nature 261, 395-396.

Blair, A., Harrison, A., 1973. Bimolecular reactions of trapped ions. VI. Ionmolecule reactions involving $\mathrm{CH} 5+$ and $\mathrm{C} 2 \mathrm{H} 5+$. Can. J. Chem. 51, 16451654 .

Blank, D., Suits, A., Lee, Y., North, S., Hall, G., 1998. Photodissociation of acrylonitrile at $193 \mathrm{~nm}$ : A photofragment translational spectroscopy study using synchrotron radiation for product photoionization. J. Chem. Phys. 108, $5784-5794$.

Bocherel, P., Herbert, L., Rowe, B., Sims, I., Smith, I., Travers, D., 1996. Ultralow-temperature kinetics of $\mathrm{CH}\left(\mathrm{X}^{2} \Pi\right)$ reactions: Rate coefficients for reactions with $\mathrm{O}_{2}$ and $\mathrm{NO}(\mathrm{T}=13-708 \mathrm{~K})$, and with $\mathrm{NH}_{3}(\mathrm{~T}=23-295 \mathrm{~K})$. J. Phys. Chem. 100, 3063-3069. 
Bohme, D., 1975. The kinetics and energetics of proton transfer, in: Ausloos, P. (Ed.), Interaction between ions and molecules. Plenum Press, New York, N.Y. USA, pp. 489-504.

Bohme, D., Fehsenfeld, F., 1969. Thermal reactions of O- ions with saturated hydrocarbon molecules. Can. J. Chem. 47, 2717-2719.

Bohme, D., Raksit, A., Schiff, H., 1982. Reactions of ${ }^{12} \mathrm{C}^{+}$with hydrocarbons at $296 \mathrm{~K}$ : carbon-carbon bond formation. Chem. Phys. Lett. 93, 592-597.

Bomble, Y., Vázquez, J., Kállay, M., Michauk, C., Szalay, P., Császár, A., Gauss, J., Stanton, J., 2006. High-accuracy extrapolated ab initio thermochemistry. II. Minor improvements to the protocol and a vital simplification. J. Chem. Phys. 125, \#064108.

Borrell, P., Cervenka, A., Turner, J.W., 1971. Pressure effects and quantum yields in the photolysis of ethylene and propene at $185 \mathrm{~nm}$. J. Chem. Soc. B $2,2293-2298$.

Borucki, W., Levin, Z., Whitten, R., Keesee, R., Capone, L., Summers, A., Toon, O., Dubach, J., 1987. Predictions of the electrical conductivity and charging of the aerosols in Titan's atmosphere. Icarus 72, 604-622.

Borucki, W., Whitten, R., 2008. Influence of high abundances of aerosols on the electrical conductivity of the Titan atmosphere. Planet. Space Sci. 56, 19-26.

Bouwman, J., Fournier, M., Sims, I., Leone, S., Wilson, K., 2013. Reaction rate and isomer-specific product branching ratios of $\mathrm{C}_{2} \mathrm{H}+\mathrm{C}_{4} \mathrm{H}_{8}$ : 1-butene, cis-2-butene, trans-2-butene, and isobutene at 79 K. J. Phys. Chem. A 117, 5093-5105.

Bouwman, J., Goulay, F., Leone, S., Wilson, K., 2012. Bimolecular rate constant and product banching ratio measurements for the reaction of $\mathrm{C}_{2} \mathrm{H}$ with ethene and propene at 79 K. J. Phys. Chem. A 116, 3907-3917.

Bowers, M., Neilson, P., Kemper, P., Wren, A., 1977. Temperature dependence of ion-molecule collision phenomena: An application of ion cyclotron resonance spectroscopy to the determination of reactive and momentum transfer rate constants. Int. J. Mass Spectrom. 25, 103-116.

Boyé, S., Campos, A., Douin, S., Fellows, C., Gauyacq, D., Shafizadeh, N., Halvick, P., Boggio-Pasqua, M., 2002. Visible emission from the vibrationally hot $\mathrm{C}_{2} \mathrm{H}$ radical following vacuum-ultraviolet photolysis of acetylene: Experiment and theory. J. Chem. Phys. 116, 8843-8855.

Bradforth, S., Kim, E., Arnold, D., Neumark, D., 1993. Photoelectron spectroscopy of $\mathrm{CN}^{-}, \mathrm{NCO}^{-}$, and $\mathrm{NCS}^{-}$. J. Chem. Phys. 98, 800-810.

Brown, R., 1973. A measurement of the rate of the reaction $\mathrm{N}+\mathrm{H}+\mathrm{M} \rightarrow \mathrm{NH}$ + M. Int. J. Chem. Kinet. 5, 663-667. 
Brownsword, R., Canosa, A., Rowe, B., Sims, I., Smith, I., Stewart, D., Symonds, A., Travers, D., 1997a. Kinetics over a wide range of temperature (13-744 K): Rate constants for the reactions of $\mathrm{CH}(\mathrm{E}=0)$ with $\mathrm{H} 2$ and $\mathrm{D} 2$ and for the removal of $\mathrm{CH}(\mathrm{CE}=1)$ by $\mathrm{H} 2$ and $\mathrm{D} 2$. J. Chem. Phys. 106, $7662-7677$.

Brownsword, R., Sims, I., Smith, I., Stewart, D., Canosa, A., Rowe, B., 1997b. The radiative association of $\mathrm{CH}$ with $\mathrm{H} 2$ : A mechanism for formation of $\mathrm{CH} 3$ in interstellar clouds. Astrophys. J. 485, 195.

Burt, J., Dunn, J., McEwan, M., Sutton, M., Roche, A., Schiff, H., 1970. Some ion-molecule reactions of $\mathrm{H}_{3}{ }^{+}$and the proton affinity of $\mathrm{H}_{2}$. J. Chem. Phys. $52,6062-6075$.

Burton, C., Noyes, W., 1968. Electronic energy relaxation in toluene vapor. J. Chem. Phys. 49, 1705-1714.

Butterfield, M., Yu, T., Lin, M., 1993. Kinetics of CN reactions with allene, butadiene, propylene and acrylonitrile. Chem. Phys. 169, 129-134.

Cameron, M., Sivakumaran, V., Dillon, T., Crowley, J., 2002. Reaction between $\mathrm{OH}$ and $\mathrm{CH}_{3} \mathrm{CHO}$ Part 1. Primary product yields of $\mathrm{CH}_{3}(296 \mathrm{~K}), \mathrm{CH}_{3} \mathrm{CO}$ (296 K), and H (237-296 K). Phys. Chem. Chem. Phys. 4, 3628-3638.

Campbell, I., Gray, C., 1973. Rate constants for $\mathrm{O}\left({ }^{3} \mathrm{P}\right)$ recombination and association with $\mathrm{N}\left({ }^{4} \mathrm{~S}\right)$. Chem. Phys. Lett. 18, 607-609.

Canosa, A., Sims, I., Travers, D., Smith, I., Rowe, B., 1997. Reactions of the methylidine radical with $\mathrm{CH}_{4}, \mathrm{C}_{2} \mathrm{H}_{2}, \mathrm{C}_{2} \mathrm{H}_{4}, \mathrm{C}_{2} \mathrm{H}_{6}$, and but-1-ene studied between 23 and $295 \mathrm{~K}$ with a CRESU apparatus. Astron. Astrophys. 323, 644-651.

Capalbo, F., Bénilan, Y., Fray, N., Schwell, M., Champion, N., Es-sebbar, E., Koskinen, T., Lehocki, I., Yelle, R., 2016. New benzene absorption cross sections in the VUV, relevance for Titan's upper atmosphere. Icarus 265, 95-109.

Capone, L., Dubach, J., Prasad, S., Whitten, R., 1983. Galactic cosmic rays and $\mathrm{N}_{2}$ dissociation on Titan. Icarus 55, 73-82.

Capone, L., Dubach, J., Whitten, R., Prasad, S., 1979. Cosmic ray ionization of the Jovian atmosphere. Icarus 39, 433-449.

Capone, L., Dubach, J., Whitten, R., Prasad, S., Santhanam, K., 1980. Cosmic ray synthesis of organic molecules in Titan's atmosphere. Icarus 44, 72-84.

Capone, L., Prasad, S., Huntress, W., Whitten, R., Dubach, J., Santhanam, K., 1981. Formation of organic molecules on Titan. Nature 293, 45-46.

Capone, L., Whitten, R., Dubach, J., Prasad, S., Huntress Jr., W., 1976. The lower ionosphere of Titan. Icarus 28, 367-378. 
Carelli, F., Satta, M., Grassi, T., Gianturco, F., 2013. Carbon-rich molecular chains in protoplanetary and planetary atmospheres: Quantum mechanisms and electron attachment rates for anion formation. Astrophys J. 774, \#97.

Carrasco, N., Alcaraz, C., Dutuit, O., Plessis, S., Thissen, R., Vuitton, V., Yelle, R., Pernot, P., 2008. Sensitivity of a Titan ionospheric model to the ion-molecule reaction parameters. Planet. Space Sci. 56, 1644-1657.

Carty, D., Le Page, V., Sims, I., Smith, I., 2001. Low temperature rate coefficients for the reactions of $\mathrm{CN}$ and $\mathrm{C}_{2} \mathrm{H}$ radicals with allene and methyl acetylene. Chem. Phys. Lett. 344, 310-316.

Cerceau, F., Raulin, F., Courtin, R., Gautier, D., 1985. Infrared spectra of gaseous mononitriles - Application to the atmosphere of Titan. Icarus 62, 207-220.

Chabot, M., Béroff, K., Gratier, P., Jallat, A., Wakelam, V., 2013. Reactions forming $\mathrm{C}_{n=2,10}^{(0,+)}, \mathrm{C}_{n=2,4} \mathrm{H}^{(0,+)}$, and $\mathrm{C}_{3} \mathrm{H}_{2}^{(0,+)}$ in the gas phase: Semiempirical branching ratios. Astrophys. J. 771, \#90.

Chamot-Rooke, J., Mourgues, P., van der Rest, G., Audier, H., 2003. Ambident reactivity and characterization of small ionized carbenes. Int. J. Mass Spectrom. 226, 249-269.

Chan, W., Cooper, G., Brion, C., 1993a. Absolute optical oscillator strengths for discrete and continuum photoabsorption of carbon monoxide (7-200 eV) and transition moments for the X 1E+ Üí A 1E system. Chem. Phys. 170, $123-138$.

Chan, W., Cooper, G., Brion, C., 1993b. The electronic spectrum of carbon dioxide. Discrete and continuum photoabsorption oscillator strengths (6-203 eV). Chem. Phys. 178, 401-413.

Chan, W., Cooper, G., Brion, C., 1993c. The electronic spectrum of water in the discrete and continuum regions. Absolute optical oscillator strengths for photoabsorption (6-200 eV). Chem. Phys. 178, 387-401.

Chastaing, D., James, P., Sims, I., Smith, I., 1998. Neutral-neutral reactions at the temperatures of interstellar clouds. Rate coefficients for reactions of $\mathrm{C}_{2} \mathrm{H}$ radicals with $\mathrm{O}_{2}, \mathrm{C}_{2} \mathrm{H}_{2}, \mathrm{C}_{2} \mathrm{H}_{4}$ and $\mathrm{C}_{3} \mathrm{H}_{6}$ down to $15 \mathrm{~K}$. Faraday Discuss. 109, $165-181$.

Chastaing, D., James, P., Sims, I., Smith, I., 1999. Neutral-neutral reactions at the temperatures of interstellar clouds: Rate coefficients for reactions of atomic carbon $\mathrm{C}\left({ }^{3} \mathrm{P}\right)$, with $\mathrm{O}_{2}, \mathrm{C}_{2} \mathrm{H}_{2}, \mathrm{C}_{2} \mathrm{H}_{4}$ and $\mathrm{C}_{3} \mathrm{H}_{6}$ down to $15 \mathrm{~K}$. Phys. Chem. Chem. Phys. 1, 2247-2256.

Chastaing, D., Le Picard, S., Sims, I., Smith, I., 2001. Rate coefficients for the reactions of $\mathrm{C}\left({ }^{3} \mathrm{P}_{J}\right)$ atoms with $\mathrm{C}_{2} \mathrm{H}_{2}, \mathrm{C}_{2} \mathrm{H}_{4}, \mathrm{CH}_{3} \mathrm{C} \equiv \mathrm{CH}$ and $\mathrm{H}_{2} \mathrm{C}=\mathrm{C}=\mathrm{CH}_{2}$ at temperatures down to $15 \mathrm{~K}$. Astron. Astrophys. 365, 241-247. 
Cheikh Sid Ely, S., 2012. Réactivité des molécules organiques à très basses températures $(22-300 \mathrm{~K})$ pour la modélisation des milieux extra-terrestres froids. Ph.D. thesis. Université de Rennes 1.

Cheikh Sid Ely, S., Morales, S., Guillemin, J.C., Klippenstein, S., Sims, I., 2013. Low temperature rate coefficients for the reaction $\mathrm{CN}+\mathrm{HC}_{3} \mathrm{~N}$. J. Phys. Chem. A $117,12155-12164$.

Chen, F., Judge, D., Wu, C., 2000. Temperature dependent photoabsorption cross sections of allene and methylacetylene in the VUV-UV region. Chem. Phys. 260, 215-223.

Chen, F., Judge, D., Wu, C., Caldwell, J., 1999. Low and room temperature photoabsorption cross sections of $\mathrm{NH}_{3}$ in the UV region. Planet. Space Sci. $47,261-266$.

Chen, F., Wu, C., 2004. Temperature-dependent photoabsorption cross sections in the VUV-UV region. I. Methane and ethane. J. Quant. Spectrosc. Radiat. Transfer 85, 195-209.

Cheng, B.M., Lu, H.C., Chen, H.K., Bahou, M., Lee, Y.P., Mebel, A., Lee, L., Liang, M.C., Yung, Y., 2006. Absorption cross sections of $\mathrm{NH}_{3}, \mathrm{NH}_{2} \mathrm{D}, \mathrm{NHD}_{2}$, and $\mathrm{ND}_{3}$ in the spectral range $140-220 \mathrm{~nm}$ and implications for planetary isotopic fractionation. Astrophys. J. 647, 1535-1542.

Choi, Y., Lin, M., 2005. Kinetics and mechanisms for reactions of HNO with $\mathrm{CH}_{3}$ and $\mathrm{C}_{6} \mathrm{H}_{5}$ studied by quantum-chemical and statistical-theory calculations. Inter. J. Chem. Kin. 37, 261-274.

Chourou, S., Orel, A., 2009. Dissociative electron attachment to HCN and HNC. Phys. Rev. A 80, \#032709.

Chupka, W., Dehmer, P., Jivery, W., 1975. High resolution photoionization study of ion-pair formation in $\mathrm{H}_{2}, \mathrm{HD}$, and $\mathrm{D}_{2}$. J. Chem. Phys. 63, 39293944 .

Clarke, D., Ferris, J., 1995. Photodissociation of cyanoacetylene: application to the atmospheric chemistry of Titan. Icarus 115, 119-125.

Clyne, M., Stedman, D., 1967. Rate of recombination of nitrogen atoms. J. Phys. Chem. 71, 3071-3073.

Coates, A., Crary, F., Lewis, G., Young, D., Waite Jr., J., Sittler Jr., E., 2007. Discovery of heavy negative ions in Titan's ionosphere. Geophys. Res. Lett. 34, \#L22103.

Coates, A., Wellbrock, A., Lewis, G., Jones, G., Young, D., Crary, F., Waite Jr., J., 2009. Heavy negative ions in Titan's ionosphere: altitude and latitude dependence. Planet. Space Sci. 57, 1866-1871. 
Coates, A., Wellbrock, A., Lewis, G., Jones, G., Young, D., Crary, F., Waite Jr., J., Johnson, R., Hill, T., Sittler Jr., E., 2010. Negative ions at Titan and Enceladus: recent results. Faraday Discuss. 147, 293-305.

Collin, G., 1988. Photochemistry of simple olefins: Chemistry of electronic excited states or hot ground state? Adv. Photochem. 14, 135-176.

Collin, G., Deslauriers, H., Deschenes, J., 1979. Photolyse du propène et du méthyl-2-butène vers 174 et à $163 \mathrm{~nm}$. Can. J. Chem. 57, 870-875.

Collin, G., Perrin, P., 1972. Photolyse dans l'ultraviolet à vide du Cis- et du trans-butène-2. Can. J. Chem. 50, 2823-2832.

Colombatti, G., Withers, P., Ferri, F., Aboudan, A., Ball, A.J., Bettanini, C., Gaborit, V., Harri, A.M., Hathi, B., Leese, M.R., Makinen, T., Stoppato, P.L., Towner, M.C., Zarnecki, J.C., Angrilli, F., Fulchignoni, M., 2008. Reconstruction of the trajectory of the Huygens probe using the Huygens Atmospheric Structure Instrument (HASI). Planet. Space Sci. 56, 586-600.

Connors, R., Roebber, J., Weiss, K., 1974. Vacuum ultraviolet spectroscopy of cyanogen and cyanoacetylenes. J. Chem. Phys. 60, 5011-5024.

Cook, G., Metzger, P., Ogawa, M., 1965. Photoionization and absorption coefficients of CO in the 600 to $1000 \AA$ region. Can. J. Chem. 43, 1706-1722.

Cooper, G., Anderson, J., Brion, C., 1996. Absolute photoabsorption and photoionization of formaldehyde in the VUV and soft X-ray regions $(3-200 \mathrm{eV})$. Chem. Phys. 209, 61-77.

Cooper, G., Burton, G., Brion, C., 1995. Absolute UV and soft X-ray photoabsorption of acetylene by high resolution dipole (e,e) spectroscopy. J. Electron Spectrosc. Rel. Phenomena 73, 139-148.

Cordiner, M., Nixon, C., Charnley, S., Mumma, M., Serigano, J., Palmer, M., Irwin, P., Teanby, N., Kisiel, Z., 2015a. ALMA spectroscopy of Titan's atmosphere: First detections of vinyl cyanide and acetonitrile isotopologues. Bull. Am. Astron. Soc. 46, \#205.03.

Cordiner, M., Palmer, M., Nixon, C., Irwin, P., Teanby, N., Charnley, S., Mumma, M., Kisiel, Z., Serigano, J., Kuan, Y.J., Chuang, Y.L., Wang, K.S., 2015b. Ethyl cyanide on Titan: Spectroscopic detection and mapping using ALMA. Astrophys. J. 800, \#L14.

Cordiner, M.A., Nixon, C.A., Teanby, N.A., Irwin, P.G.J., Serigano, J., Charnley, S.B., Milam, S.N., Mumma, M.J., Lis, D.C., Villanueva, G., Paganini, L., Kuan, Y.J., Remijan, A.J., 2014. ALMA Measurements of the HNC and $\mathrm{HC}_{3} \mathrm{~N}$ Distributions in Titan's Atmosphere. Astrophys. J. 795, \#L30.

Costes, M., Halvick, P., Hickson, K., Daugey, N., Naulin, C., 2009. Nonthreshold, threshold, and nonadiabatic behavior of the key interstellar $\mathrm{C}+$ $\mathrm{C}_{2} \mathrm{H}_{2}$ reaction. Astrophys. J. 703, 1179-1187. 
Cottini, V., Nixon, C., Jennings, D., Anderson, C., Gorius, N., Bjoraker, G., Coustenis, A., Teanby, N., Achterberg, R., Bézard, B., de Kok, R., Lellouch, E., Irwin, P., Flasar, F., Bampasidis, G., 2012. Water vapor in Titan's stratosphere from Cassini CIRS far-infrared spectra. Icarus 220, 855-862.

Cotton, C., Francisco, J., Klemperer, W., 2013. Computational study of the linear proton bound ion-molecule complexes of $\mathrm{HCNH}^{+}$with $\mathrm{HCN}$ and $\mathrm{HNC}$. J. Chem. Phys. 139, \#014304.

Courtin, R., Swinyard, B., Moreno, R., Fulton, T., Lellouch, E., Rengel, M., Hartogh, P., 2011. First results of Herschel-SPIRE observations of Titan. Astron. Astrophys. 536, \#L2.

Courtin, R.D., Sim, C.K., Kim, S.J., Gautier, D., 2007. The tropospheric abundance of $\mathrm{H}_{2}$ on Titan from the Cassini CIRS investigation. Bull. Am. Astron. Soc. 39, \#56.05.

Coustenis, A., Achterberg, R., Conrath, B., Jennings, D., Marten, A., Gautier, D., Nixon, C., Flasar, F., Teanby, N., Bezard, B., Samuelson, R., Carlson, R., Lellouch, E., Bjoraker, G., Romani, P., Taylor, F., Irwin, P., Fouchet, T., Hubert, A., Orton, G., Kunde, V., Vinatier, S., Mondellini, J., Abbas, M., Courtin, R., 2007. The composition of Titan's stratosphere from Cassini/CIRS mid-infrared spectra. Icarus 189, 35-62.

Coustenis, A., Bampasidis, G., Achterberg, R., Lavvas, P., Jennings, D., Nixon, C., Teanby, N., Vinatier, S., Flasar, F., Carlson, R., Orton, G., Romani, P., Guandique, E., Stamogiorgos, S., 2013. Evolution of the stratospheric temperature and chemical composition over one Titanian year. Astrophys. J. $779, \# 177$.

Coustenis, A., Bézard, B., 1995. Titan's atmosphere from Voyager infrared observations IV. Latitudinal variations of temperature and composition. Icarus $115,126-140$.

Coustenis, A., Bézard, B., Gautier, D., Marten, A., Samuelson, R., 1991. Titan's atmosphere from Voyager infrared observations III. Vertical distributions of hydrocarbons and nitriles near Titan's north pole. Icarus 89, 152-167.

Coustenis, A., Jennings, D., Nixon, C., Achterberg, R., Lavvas, P., Vinatier, S., Teanby, N., Bjoraker, G., Carlson, R., Piani, L., Bampasidis, G., Flasar, F., Romani, P., 2010. Titan trace gaseous composition from CIRS at the end of the Cassini-Huygens prime mission. Icarus 207, 461-476.

Coustenis, A., Jennings, D.E., Achterberg, R.K., Bampasidis, G., Lavvas, P., Nixon, C.A., Teanby, N.A., Anderson, C., Cottini, V., Flasar, F.M., 2016. Titan's temporal evolution in stratospheric trace gases near the poles. Icarus 270, 409-420. 
Coustenis, A., Salama, A., Lellouch, E., Encrenaz, T., Bjoraker, G., Samuelson, R., de Graauw, T., Feuchtgruber, H., Kessler, M., 1998. Evidence for water vapor in Titan's atmosphere from ISO/SWS data. Astron. Astrophys. 336, L85-L89.

Coustenis, A., Salama, A., Schulz, B., Ott, S., Lellouch, E., Encrenaz, T., Gautier, D., Feuchtgruber, H., 2003. Titan's atmosphere from ISO midinfrared spectroscopy. Icarus 161, 383-403.

Crary, F., Magee, B., Mandt, K., Waite Jr., J., Westlake, J., Young, D., 2009. Heavy ions, temperatures and winds in Titan's ionosphere: Combined Cassini CAPS and INMS observations. Planet. Space Sci. 57, 1847-1856.

Cravens, T., Robertson, I., Ledvina, S., Mitchell, D., Krimigis, S., Waite Jr., J., 2008. Energetic ion precipitation at Titan. Geophys. Res. Lett. 35, \#L03103.

Cravens, T., Robertson, I., Waite Jr., J., Yelle, R., Vuitton, V., Coates, A., Wahlund, J.E., Agren, K., Richard, M., De La Haye, V., Wellbrock, A., Neubauer, F., 2009. Model-data comparisons for Titan's nightside ionosphere. Icarus 199, 174-188.

Crespin, A., Lebonnois, S., Vinatier, S., Bézard, B., Coustenis, A., Teanby, N., Achterberg, R., Rannou, P., Hourdin, F., 2008. Diagnostics of Titan's stratospheric dynamics using the Cassini/CIRS data and the 2-dimensional IPSL circulation model. Icarus 197, 556-571.

Cui, J., Galand, M., Yelle, R., Vuitton, V., Wahlund, J.E., Lavvas, P., MüllerWodarg, I., Cravens, T., Kasprzak, W., Waite Jr., J., 2009a. Diurnal variations of Titan's ionosphere. J. Geophys. Res. 114, \#A06310.

Cui, J., Yelle, R., Strobel, D., Müller-Wodarg, I., Snowden, D., Koskinen, T., Galand, M., 2012. The $\mathrm{CH}_{4}$ structure in Titan's upper atmosphere revisited. J. Geophys. Res. 117, \#E11006.

Cui, J., Yelle, R., Vuitton, V., Waite Jr., J., Kasprzak, W., Gell, D., Niemann, H., Müller-Wodarg, I., Borggren, N., Fletcher, G., Patrick, E., Raaen, E., Magee, B., 2009b. Analysis of Titan's neutral upper atmosphere from Cassini Ion Neutral Mass Spectrometer measurements. Icarus 200, 581-615.

Curdt, W., Germerott, D., Wilhelm, K., Schühle, U., Teriaca, L., Innes, D., Bocchialini, K., Lemaire, P., 2013. The SUMER data in the SOHO archive. Solar Phys. 289, 2345-2376.

Dalgarno, A., Degges, T., Williams, D.A., 1967. Dipole properties of molecular nitrogen. Proc. Phys. Soc. 92, 291-295.

Dangi, B., Maity, S., Kaiser, R., Mebel, A., 2013. A combined crossed beam and ab initio investigation of the gas phase reaction of dicarbon molecules $\left(\mathrm{C}_{2}\right.$; $\left.\mathrm{X}^{1} \Sigma_{g}^{+} / a^{3} \Pi_{u}\right)$ with propene $\left(\mathrm{C}_{3} \mathrm{H}_{6} ; \mathrm{X}^{1} \mathrm{~A}^{\prime}\right)$ : Identification of the resonantly stabilized free radicals 1- and 3-vinylpropargyl. J. Phys. Chem. A 117, 1178311793. 
Danielsson, M., Hamberg, M., Zhaunerchyk, V., Ehlerding, A., Kamińska, M., Hellberg, F., Thomas, R., Österdahl, F., af Ugglas, M., Källberg, A., Simonsson, A., Paál, A., Larsson, M., Geppert, W., 2008. The cross-section and branching fractions for dissociative recombination of the diacetylene cation $\mathrm{C}_{4} \mathrm{D}_{2}{ }^{+}$. Int. J. Mass Spectrom. 273, 111-116.

Daranlot, J., Hincelin, U., Bergeat, A., Costes, M., Loison, J.C., Wakelam, V., Hickson, K., 2012. Elemental nitrogen partitioning in dense interstellar clouds. PNAS 109, 10233-10238.

Daugey, N., Caubet, P., Bergeat, A., Costes, M., Hickson, K., 2008. Reaction kinetics to low temperatures. Dicarbon + acetylene, methylacetylene, allene and propene from $77 \leq \mathrm{T} \leq 296 \mathrm{~K}$. Phys. Chem. Chem. Phys. 10, 729-737.

Daugey, N., Caubet, P., Retail, B., Costes, M., Bergeat, A., Dorthe, G., 2005. Kinetic measurements on methylidyne radical reactions with several hydrocarbons at low temperatures. Phys. Chem. Chem. Phys. 7, 2921-2927.

Davidson, J., Schiff, H., Brown, T., Howard, C., 1978. Temperature dependence of the deactivation of $\mathrm{O}(1 \mathrm{D})$ by $\mathrm{CO}$ from 113-333 K. J. Chem. Phys. 69, 12161217 .

De Avillez Pereira, R., Baulch, D., Pilling, M., Robertson, S., Zeng, G., 1997. Temperature and pressure dependence of the multichannel rate coefficients for the $\mathrm{CH}_{3}+\mathrm{OH}$ system. J. Phys. Chem. A 101, 9681-9693.

De Bleecker, K., Bogaerts, A., Goedheer, W., 2006. Detailed modeling of hydrocarbon nanoparticle nucleation in acetylene discharges. Phys. Rev. E 73, 026405 .

De Kok, R., Irwin, P., Teanby, N., Lellouch, E., Bézard, B., Vinatier, S., Nixon, C., Fletcher, L., Howett, C., Calcutt, S., Bowles, N., Flasar, F., Taylor, F., 2007. Oxygen compounds in Titan's stratosphere as observed by Cassini CIRS. Icarus 186, 354-363.

De La Haye, V., Waite Jr., J., Cravens, T., Robertson, I., Lebonnois, S., 2008. Coupled ion and neutral rotating model of Titan's upper atmosphere. Icarus 197, 110-136.

Deakyne, C., Meot-Ner, M., Buckley, T., Metz, R., 1987. Proton affinities of diacetylene, cyanoacetylene, and cyanogen. J. Chem. Phys. 86, 2334-2342.

Delpech, C., Guillemin, J., Paillous, P., Khlifi, M., Bruston, P., Raulin, F., 1994. Infrared spectra of triacetylene in the $4000-220 \mathrm{~cm}^{-1}$ region: Absolute band intensity and implications for the atmosphere of Titan. Spectrochim. Acta 50 A, 1095-1100.

Demarais, N., Yang, Z., Snow, T., Bierbaum, V., 2013. Chemistry of $\mathrm{HCNH}^{+}$: mechanisms, structures, and relevance to Titan's atmosphere. Struct. Chem. 24, 1957-1963. 
Derecskei-Kovacs, A., North, S., 1999. The unimolecular dissociation of vinylcyanide: A theoretical investigation of a complex multichannel reaction. J. Chem. Phys. 110, 2862-2871.

Derkatch, A., Al-Khalili, A., Vikor, L., Neau, A., Shi, W., Danared, H., af Ugglas, M., Larsson, M., 1999. Branching ratios in dissociative recombination of the $\mathrm{C}_{2} \mathrm{H}_{2}{ }^{+}$molecular ion. J. Phys. B: At. Mol. Opt. Phys. 32, 3391-3398.

Desai, R.T., Coates, A.J., Wellbrock, A., Vuitton, V., Crary, F., GonzálezCaniulef, D., Shebanits, O., Jones, G.H., Lewis, G.R., Waite, J.H., Taylor, S.A., Kataria, D.O., Wahlund, J.E., Edberg, N.J.T., Sittler, E.C., 2017. Carbon chain anions and the growth of complex organic molecules in Titan's ionosphere. Astrophys. J. 844, \#L18.

DeSain, J., Taatjes, C., 2003. Infrared laser absorption measurements of the kinetics of propargyl radical self-reaction and the $193 \mathrm{~nm}$ photolysis of propyne. J. Phys. Chem. A 107, 4843-4850.

Deslauriers, H., Makulski, W., Collin, G., 1987. The $\alpha(\mathrm{C}-\mathrm{C}) / \beta(\mathrm{C}-\mathrm{H})$ ratio of the primary processes in the $184.9 \mathrm{~nm}$ photolysis of gaseous cis- and trans-2-butene. Can. J. Chem. 65, 1631-1638.

Deyerl, H.J., Fischer, I., Chen, P., 1999. Photodissociation dynamics of the allyl radical. J. Chem. Phys. 110, 1450-1462.

Deyerl, H.J., Gilbert, T., Fischer, I., Chen, P., 1997. Kinetics and dynamics in the photodissociation of the allyl radical. J. Chem. Phys. 107, 3329-3332.

Dheandhanoo, S., Forte, L., Fox, A., Bohme, D., 1986. Ion-molecule reactions with carbon chain molecules: reactions with diacetylene and the diacetylene cation. Can. J. Chem. 64, 641-648.

Dheandhanoo, S., Johnsen, R., Biondi, M., 1984. Measured ion-molecule reaction rates for modelling Titan's atmosphere. Planet. Space Sci. 32, 1301-1305.

Dibeler, V., Reese, R., Franklin, J., 1961. Mass spectrometric study of cyanogen and cyanoacetylenes. J. Am. Chem. Soc. 83, 1813-1818.

Dobrijevic, M., Hébrard, E., Loison, J., Hickson, K., 2014. Coupling of oxygen, nitrogen, and hydrocarbon species in the photochemistry of Titan's atmosphere. Icarus 228, 324-346.

Dobrijevic, M., Loison, J., Hickson, K., Gronoff, G., 2016. 1D-coupled photochemical model of neutrals, cations and anions in the atmosphere of Titan. Icarus 268, 313-339.

Dong, H., Ding, Y.H., Sun, C.C., 2005. $\mathrm{C}_{2} \mathrm{H}+\mathrm{H}_{2} \mathrm{CO}$ : A new route for formaldehyde removal. J. Chem. Phys. 122, \#204321. 
Doose, L., Karkoschka, E., Tomasko, M., Anderson, C., 2016. Vertical structure and optical properties of Titan's aerosols from radiance measurements made inside and outside the atmosphere. Icarus 270, 355-375.

Dryahina, K., Cunha de Miranda, B., Španěl, P., Žabka, J., Alcaraz, C., Herman, Z., 2011. Selected ion flow tube study of ion-molecule reactions of $\mathrm{N}^{+}\left({ }^{3} \mathrm{P}\right)$ and $\mathrm{Kr}^{+}$with $\mathrm{C}_{3}$ hydrocarbons propane, propene, and propyne. J. Phys. Chem. A 115, 7310-7315.

Dulaney, J., Biondi, M., Johnsen, R., 1987. Electron temperature dependence of the recombination of electrons with $\mathrm{NO}^{+}$ions. Phys. Rev. A 36, 1342-1350.

Dunning, T., 1989. Gaussian-basis sets for use in correlated molecular calculations. 1. The atoms boron through neon and hydrogen. J. Chem. Phys. 90, $1007-1023$.

Dutuit, O., Carrasco, N., Thissen, R., Vuitton, V., Alcaraz, C., Pernot, P., Balucani, N., Casavecchia, P., Canosa, A., Le Picard, S., Loison, J.C., Herman, Z., Žabka, J., Ascenzi, D., Tosi, P., Franceschi, P., Price, S., Lavvas, P., 2013. Critical review of $\mathrm{N}, \mathrm{N}^{+}, \mathrm{N}_{2}{ }^{+}, \mathrm{N}^{++}$and $\mathrm{N}_{2}^{++}$main production processes and reactions of relevance to Titan's atmosphere. Astrophys. J. Suppl. Ser. 204, \#20.

Eden, S., Limão-Vieira, P., Kendall, P., Mason, N., Hoffmann, S., Spyrou, S., 2003. High resolution photo-absorption studies of acrylonitrile, $\mathrm{C}_{2} \mathrm{H}_{3} \mathrm{CN}$, and acetonitrile, $\mathrm{CH}_{3}$ CN. Eur. Phys. J. D 26, 201-210.

Edwards, S., Freeman, C., McEwan, M., 2008. The ion chemistry of methylenimine and propionitrile and their relevance to Titan. Int. J. Mass Spectrom. $272,86-90$.

Edwards, S., Freeman, C., McEwan, M., 2009. Some ion chemistry of $\mathrm{HC}_{5} \mathrm{~N}$. Int. J. Mass Spectrom. 279, 82-86.

Ehlerding, A., Arnold, S., Viggiano, A., Kalhori, S., Semaniak, J., Derkatch, A., Rosén, S., af Ugglas, M., Larsson, M., 2003. Rates and products of the dissociative recombination of $\mathrm{C}_{3} \mathrm{H}_{7}{ }^{+}$in low-energy electron collisions. J. Phys. Chem. A 107, 2179-2184.

Ehlerding, A., Hellberg, F., Thomas, R., Kalhori, S., Viggiano, A., Arnold, S., Larsson, M., af Ugglas, M., 2004. Dissociative recombination of $\mathrm{C}_{2} \mathrm{H}^{+}$and $\mathrm{C}_{2} \mathrm{H}_{4}{ }^{+}$: Absolute cross sections and product branching ratios. Phys. Chem. Chem. Phys. 6, 949-954.

Eichelberger, B., Snow, T., Barckholtz, C., Bierbaum, V., 2007. Reactions of $\mathrm{H}, \mathrm{N}$, and $\mathrm{O}$ atoms with carbon chain anions of interstellar interest: An experimental study. Astrophys. J. 667, 1283-1289. 
Ellison, G., Engelking, P., Lineberger, W., 1978. An experimental determination of the geometry and electron affinity of methyl radical. J. Am. Chem. Soc. $100,2556-2558$.

English, M., Lara, L., Lorenz, R., Ratcliff, P., Rodrigo, R., 1996. Ablation and chemistry of meteoric materials in the atmosphere of Titan. Adv. Space. Res. $17,157-160$.

Erwin, D., Kunc, J., 2005. Electron-impact dissociation of the methane molecule into neutral fragments. Phys. Rev. A 72, 052719-052724.

Erwin, D., Kunc, J., 2008. Dissociation and ionization of the methane molecule by nonrelativistic electrons including the near threshold region. J. Appl. Phys. 103, \#064906.

Es-sebbar, E., Benilan, Y., Farooq, A., 2013. Temperature-dependent absorption cross-section measurements of 1-butene $\left(1-\mathrm{C}_{4} \mathrm{H}_{8}\right)$ in VUV and IR. J. Quant. Spectrosc. Radiat. Transfer 115, 1-12.

Espinosa-Garcia, J., Corchado, J., 1994. Variational transition-state theory calculation using the direct dynamics method: $\mathrm{NH}_{3}+\mathrm{H} \rightarrow \mathrm{NH}_{2}+\mathrm{H}_{2}$ reaction. J. Chem. Phys. 101, 1333-1342.

Fabiano, F., López Puertas, M., Adriani, A., Moriconi, M.L., D’Aversa, E., Funke, B., López-Valverde, M.A., Ridolfi, M., Dinelli, B.M., 2017. CO concentration in the upper stratosphere and mesosphere of Titan from VIMS dayside limb observations at $4.7 \mu \mathrm{m}$. Icarus 293, 119-131.

Fagerström, K., Lund, A., Mahmoud, G., Jodkowski, J., Ratajczak, E., 1993. Kinetics of the gas-phase reaction between ethyl and hydroxyl radicals. Chem. Phys. Lett. 208, 321-327.

Fahr, A., Hassanzadeh, P., Atkinson, D., 1998. Ultraviolet absorption spectrum and cross-sections of vinyl $\left(\mathrm{C}_{2} \mathrm{H}_{3}\right)$ radical in the $225-238 \mathrm{~nm}$ region. Chem. Phys. 236, 43-51.

Fahr, A., Hassanzadeh, P., Laszlo, B., Huie, R., 1997. Ultraviolet absorption and cross sections of propargyl $\left(\mathrm{C}_{3} \mathrm{H}_{3}\right)$ radicals in the $230-300 \mathrm{~nm}$ region. Chem. Phys. 215, 59-66.

Fahr, A., Laufer, A., 1988. Ultraviolet absorption of the vinyl radical and reaction with oxygen. J. Phys. Chem. 92, 7229-7232.

Fahr, A., Laufer, A., 1992. The 1,1-elimination of hydrogen cyanide and formation of triplet vinylidene from the photolysis of acrylonitrile. J. Phys. Chem. 96, 4217-4219.

Fahr, A., Laufer, A., Klein, R., Braun, W., 1991. Reaction rate determinations of vinyl radical reactions with vinyl, methyl, and hydrogen atoms. J. Phys. Chem. 95, 3218-3224. 
Fahr, A., Nayak, A., 1994. Temperature dependant ultraviolet absorption cross sections of 1.3-butadiene and butadiyne. Chem. Phys. 189, 725-731.

Fahr, A., Nayak, A., 1996. Temperature dependant ultraviolet absorption cross sections of propylene, methylacetylene and vinylacetylene. Chem. Phys. 203, $351-358$.

Faure, A., Vuitton, V., Thissen, R., Wiesenfeld, L., 2009. A semiempirical capture model for fast neutral reactions at low temperature. J. Phys. Chem. A $113,13694-13699$.

Fedor, J., Cicman, P., Coupier, B., Feil, S., Winkler, M., Gluch, K., Husarik, J., Jaksch, D., Farizon, B., Mason, N., Scheier, P., Märk, T., 2006. Fragmentation of transient water anions following low-energy electron capture by $\mathrm{H}_{2} \mathrm{O} / \mathrm{D}_{2} \mathrm{O}$. J. Phys. B: At. Mol. Opt. Phys. 39, 3935-3944.

Fehsenfeld, F., 1975. Interaction between ions and molecules, in: Ausloos, P. (Ed.), NATO advanced study institutes series. Series B, Physics. Plenum Press, New York. volume 6, p. 387.

Fehsenfeld, F., Howard, C., Ferguson, E., 1973. Thermal energy reactions of negative ions with $\mathrm{H}$ atoms in the gas phase. J. Chem. Phys. 58, 5841-5842.

Feng, W., Hershberger, J., 2013. Experimental and theoretical study of the product channels of the $\mathrm{C}_{2} \mathrm{H}+\mathrm{NO}$ reaction. J. Phys. Chem. A 117, 35853592 .

Fennelly, J., Torr, D., 1992. Photoionization and photoabsorption cross sections of $\mathrm{O}, \mathrm{N}_{2}, \mathrm{O}_{2}$, and $\mathrm{N}$ for aeronomic calculations. At. Data Nucl. Data Tables $51,321-363$.

Ferguson, E., 1973. Rate constants of thermal energy binary ion-molecule reactions of aeronomic interest. At. Data Nucl. Data Tables 12, 159-178.

Fiaux, A., Smith, D., Futrell, J., 1974. Reaction of $\mathrm{CH}_{5}{ }^{+}$with $\mathrm{C}_{2} \mathrm{H}_{2}, \mathrm{C}_{2} \mathrm{H}_{4}$, $\mathrm{C}_{3} \mathrm{H}_{6}$ and $\mathrm{c}-\mathrm{C}_{3} \mathrm{H}_{6}$. Int. J. Mass Spectrom. 15, 9-21.

Fisher, J., McMahon, T., 1990. Determination of rate constants for low pressure association reactions by Fourier transform-ion cyclotron resonance spectrometry. Int. J. Mass Spectrom. Ion Processes 100, 701-717.

Flad, J., Brown, S., Burkholder, J., Stark, H., Ravishankara, A., 2006. Absorption cross sections for the A $2 \mathrm{~A}^{\prime \prime}(0,90,0) \leftarrow \mathrm{X} 2 \mathrm{~A}^{\prime}(0,01,0)$ band of the HCO radical. Phys. Chem. Chem. Phys. 8, 3636-3642.

Fondren, L., Adams, N., Stavish, L., 2009. Gas phase reactions of $\mathrm{CH}_{3}{ }^{+}$with a series of homo- and heterocyclic molecules. J. Phys. Chem. A 113, 592-598.

Fondren, L., McLain, J., Jackson, D., Adams, N., Babcock, L., 2007. Studies of reactions of a series of ions with nitrogen containing heterocyclic molecules using a selected ion flow tube. Int. J. Mass Spectrom. 265, 60-67. 
Fournier, J., Shuman, N., Melko, J., Ard, S., Viggiano, A., 2013. A novel technique for measurement of thermal rate constants and temperature dependences of dissociative recombination: $\mathrm{CO}_{2}^{+}, \mathrm{CF}_{3}{ }^{+}, \mathrm{N}_{2} \mathrm{O}^{+}, \mathrm{C}_{7} \mathrm{H}_{8}{ }^{+}, \mathrm{C}_{7} \mathrm{H}_{7}{ }^{+}$, $\mathrm{C}_{6} \mathrm{H}_{6}{ }^{+}, \mathrm{C}_{6} \mathrm{H}_{5}{ }^{+}, \mathrm{C}_{5} \mathrm{H}_{6}{ }^{+}, \mathrm{C}_{4} \mathrm{H}_{4}{ }^{+}$, and $\mathrm{C}_{3} \mathrm{H}_{3}{ }^{+}$. J. Chem. Phys. 138, \#154201.

Fournier, M., 2014. Reactivity of $\mathrm{C}_{3} \mathrm{~N}$ and $\mathrm{C}_{2} \mathrm{H}$ at low temperature: applications for the Interstellar Medium and Titan. Ph.D. thesis. Université de Rennes 1.

Fox, J., Yelle, R., 1997. Hydrocarbon ions in the ionosphere of Titan. Geophys. Res. Lett. 24, 2179-2182.

Franklin, J., Wada, Y., Natalis, P., Hierl, P., 1966. Ion-molecule reactions in acetonitrile and propionitrile. J. Phys. Chem. 70, 2353-2361.

Fraser, G., 2002. The ion detection efficiency of microchannel plates (MCPs). Int. J. Mass Spectrom. 215, 13-30.

Fray, N., Bénilan, Y., Gazeau, M.C., Jolly, A., Schwell, M., Arzoumanian, E., Ferradaz, T., Es-Sebbar, E., Guillemin, J.C., 2010. Temperature-dependent photoabsorption cross section of cyanodiacetylene in the vacuum UV. J. Geophys. Res. 115, \#E06010.

Fray, N., Schmitt, B., 2009. Sublimation of ices of astrophysical interest: A bibliographic review. Planet. Space Sci. 57, 2053-2080.

Freysinger, W., Khan, F., Armentrout, P., Tosi, P., Dimitrev, O., Bassi, D., 1994. Charge-transfer reaction of ${ }^{14,15} \mathrm{~N}^{+}\left({ }^{3} \mathrm{P}_{J}\right)+\mathrm{N}_{2}\left({ }^{1} \Sigma_{g}^{+}\right)$from thermal to $100 \mathrm{eV}$. Crossed-beam and scattering-cell guided-ion beam experiments. J. Chem. Phys. 101, 3688-3695.

Frisch, M., Trucks, G., Schlegel, H., Scuseria, G., Robb, M., Cheeseman, J., Scalmani, G., Barone, V., Mennucci, B., Petersson, G., et al., 2009. Gaussian 09, Revision D.01. Gaussian, Inc., Wallingford CT.

Fröchtenicht, R., 1995. The photodissociation of toluene studied by forward photofragment translational spectroscopy. J. Chem. Phys. 102, 4850-4859.

Fukuzawa, K., Matsushita, T., Morokuma, K., Levandier, D., Chiu, Y., Dressler, R., Murad, E., Midey, A., Williams, S., Viggiano, A., 2001. An ab initio and experimental study of vibrational effects in low energy $\mathrm{O}^{+}+\mathrm{C}_{2} \mathrm{H}_{2}$ chargetransfer collisions. J. Chem. Phys. 115, 3184-3194.

Fukuzawa, K., Osamura, Y., Schaefer, H.I., 1998. Are neutral-neutral reactions effective for the carbon-chain growth of cyanopolyynes and polyacetylenes in interstellar space? Astrophys. J. 505, 278-285.

Fulchignoni, M., Ferri, F., Angrilli, F., Ball, A., Bar-Nun, A., Barucci, M., Bettanini, C., Bianchini, G., Borucki, W., Colombatti, G., Coradini, M., Coustenis, A., Debei, S., Falkner, P., Fanti, G., Flamini, E., Gaborit, V., Grard, R., Hamelin, M., Harri, A., Hathi, B., Jernej, I., Leese, M., Lehto, A., 
Lion Stoppato, P., López-Moreno, J., Mäkinen, T., McDonnell, J., McKay, C., Molina-Cuberos, G., Neubauer, F., Pirronello, V., Rodrigo, R., Saggin, B., Schwingenschuh, K., Seiff, A., Simões, F., Svedhem, H., Tokano, T., Towner, M., Trautner, R., Withers, P., Zarnecki, J., 2005. In situ measurements of the physical characteristics of Titan's environment. Nature 438, 785-791.

Fulle, D., Hippler, H., 1997. The temperature and pressure dependence of the reaction $\mathrm{CH}+\mathrm{H}_{2} \Leftrightarrow \mathrm{CH}_{3} \Leftrightarrow \mathrm{CH}_{2}+\mathrm{H}$. J. Chem. Phys. 106, 8691-8698.

Galand, M., Coates, A., Cravens, T., Wahlund, J.E., 2014. Titan's ionosphere, in: Müller-Wodarg, I., Griffith, C., Lellouch, E., Cravens, T. (Eds.), Titan: Interior, Surface, Atmosphere and Space Environment. Cambridge University Press, New York. chapter 11, pp. 376-418.

Galand, M., Lilensten, J., Toublanc, D., Maurice, S., 1999. The ionosphere of Titan: Ideal diurnal and nocturnal cases. Icarus 140, 92-105.

Galand, M., Yelle, R., Cui, J., Wahlund, J.E., Vuitton, V., Wellbrock, A., Coates, A., 2010. Ionization sources in Titan's deep ionosphere. J. Geophys. Res. 115, \#A07312.

Gan, L., Keller, C., Cravens, T., 1992. Electrons in the ionosphere of Titan. J. Geophys. Res. 97, 12137-12151.

Gannon, K., Blitz, M., Liang, C., Pilling, M., Seakins, P., Glowacki, D., 2010a. Temperature dependent kinetics (195-798 K) and $\mathrm{H}$ atom yields (298-498 K) from reactions of ${ }^{1} \mathrm{CH}_{2}$ with acetylene, ethene, and propene. J. Phys. Chem. A $114,9413-9424$.

Gannon, K., Blitz, M., Liang, C.H., Pilling, M., Seakins, P., Glowacki, D., Harvey, J., 2010b. An experimental and theoretical investigation of the competition between chemical reaction and relaxation for the reactions of ${ }^{1} \mathrm{CH}_{2}$ with acetylene and ethene: implications for the chemistry of the giant planets. Faraday Discuss. 147, 173-188.

Gannon, K., Blitz, M., Pilling, M., Seakins, P., Klippenstein, S., Harding, L., 2008. Kinetics and product branching ratios of the reaction of ${ }^{1} \mathrm{CH}_{2}$ with $\mathrm{H}_{2}$ and $\mathrm{D}_{2}$. J. Phys. Chem. A 112, 9575-9583.

Gannon, K., Glowacki, D., Blitz, M., Hughes, K., Pilling, M., Seakins, P., 2007. $\mathrm{H}$ atom yields from the reactions of $\mathrm{CN}$ radicals with $\mathrm{C}_{2} \mathrm{H}_{2}, \mathrm{C}_{2} \mathrm{H}_{4}, \mathrm{C}_{3} \mathrm{H}_{6}$, trans-2- $\mathrm{C}_{4} \mathrm{H}_{8}$, and iso- $\mathrm{C}_{4} \mathrm{H}_{8}$. J. Phys. Chem. A 111, 6679-6692.

Gans, B., Boyé-Péronne, S., Broquier, M., Delsaut, M., Douin, S., Fellows, C., Halvick, P., Loison, J.C., Lucchese, R., Gauyacq, D., 2011. Photolysis of methane revisited at $121.6 \mathrm{~nm}$ and at $118.2 \mathrm{~nm}$ : quantum yields of the primary products, measured by mass spectrometry. Phys. Chem. Chem. Phys. $13,8140-8152$. 
Gans, B., Peng, Z., Carrasco, N., Gauyacq, D., Lebonnois, S., Pernot, P., 2013. Impact of a new wavelength-dependent representation of methane photolysis branching ratios on the modeling of Titan's atmospheric photochemistry. Icarus 223, 330-343.

Gardner, E., McNesby, J., 1982. Vacuum-ultraviolet photolysis of methylamine. J. Phys. Chem. 86, 2646-2651.

Gehring, M., Hoyermann, K., Wagner, H., Wolfrum, J., 1971. Reaction of atomic hydrogen with hydrazine. Ber. Bunsenges. Phys. Chem. 75.

Georgievskii, Y., Harding, L., Klippenstein, S., 2016. VaReCoF: Variable Reaction Coordinate Flux. http://tcg.cse.anl.gov/papr/codes/varecof.html .

Georgievskii, Y., Klippenstein, S., 2003. Transition state theory for multichannel addition reactions: Multifaceted dividing surfaces. J. Phys. Chem. A 107, 9776-9781.

Georgievskii, Y., Klippenstein, S., 2005. Long-range transition state theory. J. Chem. Phys. 122, \#194103.

Georgievskii, Y., Klippenstein, S., 2016. MESS: Master Equation System Solver. http://tcg.cse.anl.gov/papr/codes/mess.html .

Georgievskii, Y., Miller, J., Burke, M., Klippenstein, S., 2013. Reformulation and solution of the master equation for multiple-well chemical reactions. J. Phys. Chem. A 117, 12146-12154.

Geppert, W., Ehlerding, A., Hellberg, F., Kalhori, S., Thomas, R., Novotny, O., Arnold, S., Miller, T., Viggiano, A., Larsson, M., 2004a. First observation of four-body breakup in electron recombination: $\mathrm{C}_{2} \mathrm{D}_{5}{ }^{+}$. Phys. Rev. Lett. 93, \#153201.

Geppert, W., Ehlerding, A., Hellberg, F., Semaniak, J., Österdahl, F., Kamińska, M., Al-Khalili, A., Zhaunerchyk, V., Thomas, R., af Ugglas, M., Källberg, A., Simonsson, A., Larsson, M., 2004b. Dissociative recombination of nitrile ions: $\mathrm{DCCCN}^{+}$and $\mathrm{DCCCND}^{+}$. Astrophys. J. 613, 1302-1309.

Geppert, W., Hamberg, M., Thomas, R., Österdahl, F., Hellberg, F., Zhaunerchyk, V., Ehlerding, A., Millar, T., Roberts, H., Semaniak, J., af Ugglas, M., Källberg, A., Simonsson, A., Kamińska, M., Larsson, M., 2006. Dissociative recombination of protonated methanol. Faraday Discuss. 133, 177-190.

Geppert, W., Thomas, R., Ehlerding, A., Hellberg, F., Österdahl, F., Hamberg, M., Semaniak, J., Zhaunerchyk, V., Kamińska, M., Källberg, A., Paál, A., Larsson, M., 2005. Dissociative recombination branching ratios and their influence on interstellar clouds. J. Phys.: Conf. Ser. 4, 26-31.

Geppert, W., Thomas, R., Ehlerding, A., Hellberg, F., Österdahl, F., af Ugglas, M., Larsson, M., 2004c. Dissociative recombination of $\mathrm{C}_{3} \mathrm{H}_{4}{ }^{+}$: preferential formation of the $\mathrm{C}_{3} \mathrm{H}_{3}$ radical. Int. J. Mass Spectrom. 237, 25-32. 
Geppert, W., Thomas, R., Ehlerding, A., Semaniak, J., Österdahl, F., af Ugglas, M., Djurić, N., Paál, A., Larsson, M., 2004d. Extraordinary branching ratios in astrophysically important dissociative recombination reactions. Faraday Discuss. 127, 425-437.

Geppert, W., Vigren, E., Hamberg, M., Zhaunerchyk, V., Thomas, R., Kamińska, M., Millar, T., Semaniak, J., Roberts, H., Hellberg, F., Österdahl, F., Ehlerding, A., Larsson, M., 2007. Formation of biomolecule precursors in space. J. Phys.: Conf. Ser. 88, \#012068.

Gerlich, D., Borodi, G., 2009. Buffer gas cooling of polyatomic ions in rf multielectrode traps. Faraday Discuss. 142, 57-72.

Gerlich, D., Borodi, G., Luca, A., Mogo, C., Smith, M., 2011. Reactions between cold $\mathrm{CH}_{x}^{+}$and slow $\mathrm{H}$ and $\mathrm{H}_{2}$. Z. Phys. Chem. 225, 475-492.

Gerlich, D., Jusku, P., Roučka, Š., Zymak, I., Plašil, R., Glosík, J., 2012. Ion trap studies of $\mathrm{H}^{-}+\mathrm{H} \rightarrow \mathrm{H}_{2}+\mathrm{e}^{-}$between 10 and $135 \mathrm{~K}$. Astrophys. J. 749, \#22.

Gerlich, D., Smith, M., 2006. Laboratory astrochemistry: Studying molecules under inter- and circumstellar conditions. Phys. Scr. 73, C25-C31.

Gichuhi, W., Suits, A., 2011. Primary branching ratios for the low-temperature reaction of state-prepared $\mathrm{N}_{2}^{+}$with $\mathrm{CH}_{4}, \mathrm{C}_{2} \mathrm{H}_{2}$, and $\mathrm{C}_{2} \mathrm{H}_{4}$. J. Phys. Chem. A $115,7105-7111$.

Gierczak, T., Gawlowski, J., Niedzielski, J., 1988. Reactions of excited $\mathrm{C}_{3} \mathrm{H}_{5}$ radicals: Implications for the photolysis of propylene at $8.4 \mathrm{eV}$. J. Photochem. Photobiol. A 43, 1-9.

Gilbert, R., Luther, K., Troe, J., 1983. Theory of thermal unimolecular reactions in the fall-off range. II. Weak collision rate constants. Ber. Bunsenges. Phys. Chem. 87, 169-177.

Gladstone, G., Allen, M., Yung, Y., 1996. Hydrocarbon photochemistry in the upper atmosphere of Jupiter. Icarus 119, 1-52.

Glicker, S., Okabe, H., 1987. Photochemistry of diacetylene. J. Phys. Chem. 91, 437-440.

Glicker, S., Stief, L., 1971. Photolysis of formaldehyde at 1470 and $1236 \AA$ A. J. Chem. Phys. 54, 2852-2857.

Glosík, J., Smith, D., Spanel, P., Freysinger, W., Lindinger, W., 1993. SIFDT studies of the reactions of $\mathrm{C}^{+}, \mathrm{CH}^{+}$and $\mathrm{CH}_{2}{ }^{+}$with $\mathrm{HCl}$ and $\mathrm{CO}_{2}$, and $\mathrm{CH}_{3}{ }^{+}$ with HCl. Int. J. Mass Spectrom. Ion Processes 129, 131-143.

Goldfarb, F., Drag, C., Chaibi, W., Kröger, S., Blondel, C., Delsart, C., 2005. Photodetachment microscopy of the $P, Q$, and $R$ branches of the $\mathrm{OH}^{-}(v=0)$ to $\mathrm{OH}(v=0)$ detachment threshold. J. Chem. Phys. 122, \#014308. 
Goncher, S., Moore, D., Sveum, N., Neumark, D., 2008. Photofragment translational spectroscopy of propargyl radicals at $248 \mathrm{~nm}$. J. Chem. Phys. 128, \#114303.

Gougousi, T., Golde, M., Johnsen, R., 1997. Electron-ion recombination rate coefficient measurements in a flowing afterglow plasma. Chem. Phys. Lett. 265, 399-403.

Goulay, F., Leone, S., 2006. Low-temperature rate coefficients for the reaction of ethynyl radical $\left(\mathrm{C}_{2} \mathrm{H}\right)$ with benzene. J. Phys. Chem. A 110, 1875-1880.

Goulay, F., Osborn, D., Taatjes, C., Zou, P., Meloni, G., Leone, S., 2007. Direct detection of polyynes formation from the reaction of ethynyl radical $\left(\mathrm{C}_{2} \mathrm{H}\right)$ with propyne $\left(\mathrm{CH}_{3}-\mathrm{C} \equiv \mathrm{CH}\right)$ and allene $\left(\mathrm{CH}_{2}=\mathrm{C}=\mathrm{CH}_{2}\right)$. Phys. Chem. Chem. Phys. 9, 4291-4300.

Goulay, F., Soorkia, S., Meloni, G., Osborn, D., Taatjes, C., Leone, S., 2011. Detection of pentatetraene by reaction of the ethynyl radical $\left(\mathrm{C}_{2} \mathrm{H}\right)$ with allene $\left(\mathrm{CH}_{2}=\mathrm{C}=\mathrm{CH}_{2}\right)$ at room temperature. Phys. Chem. Chem. Phys. 13, 20820-20827.

Goulay, F., Trevitt, A., Meloni, G., Selby, T., Osborn, D., Taatjes, C., Vereecken, L., Leone, S., 2009. Cyclic versus linear isomers produced by reaction of the methylidyne radical $(\mathrm{CH})$ with small unsaturated hydrocarbons. J. Am. Chem. Soc. 131, 993-1005.

Graupner, K., Field, T., Saunders, G., 2008. Experimental evidence for radiative attachment in astrochemistry from electron attachment to NCCCCN. Astrophys. J. 685, L95-L98.

Greenwald, E., North, S., Klippenstein, S., 2005. A two transition state model for radical-molecule reactions: A case study of the addition of $\mathrm{OH}$ to $\mathrm{C}_{2} \mathrm{H}_{4}$. J. Phys. Chem. A 109, 6031-6044.

Gronoff, G., Lilensten, J., Desorgher, L., Flückiger, E., 2009. Ionization processes in the atmosphere of Titan I. Ionization in the whole atmosphere. Astron. Astrophys. 506, 955-964.

Gronoff, G., Mertens, C., Lilensten, J., Desorgher, L., Flückiger, E., Velinov, P., 2011. Ionization processes in the atmosphere of Titan III. Ionization by high-Z nuclei cosmic rays. Astron. Astrophys. 529, \#A143.

Gu, X., Guo, Y., Mebel, A., Kaiser, R., 2006. Chemical dynamics of the formation of the 1,3-butadiynyl radical $\left(\mathrm{C}_{4} \mathrm{H}\left(\mathrm{X}^{2} \Sigma^{+}\right)\right)$and its isotopomers. J. Phys. Chem. A 110, 11265-11278.

Gu, X., Guo, Y., Zhang, F., Mebel, A., Kaiser, R., 2007. A crossed molecular beams study on the formation and energetics of the resonantly stabilized free i- $\mathrm{C}_{4} \mathrm{H}_{3}\left(\mathrm{X}^{2} \mathrm{~A}^{\prime}\right)$ radical and its isotopomers. Chem. Phys. 335, 95-108. 
Gu, X., Kaiser, R., Mebel, A., Kislov, V., Klippenstein, S., Harding, L., Liang, M.C., Yung, Y., 2009a. A crossed molecular beams study on the formation of the exotic cyanoethynyl radical in Titan's atmosphere. Astrophys. J. 701, $1797-1803$.

Gu, X., Kim, Y., Kaiser, R., Mebel, A., Liang, M.C., Yung, Y., 2009b. Chemical dynamics of triacetylene formation and implications to the synthesis of polyynes in Titan's atmosphere. PNAS 106, 16078-16083.

Guo, J., Carrington, T., Filseth, S., 2001. Energy disposal in $\mathrm{CN}\left(X^{2} \Sigma^{+}\right)$produced in the $157 \mathrm{~nm}$ photodissociation of acrylonitrile. J. Chem. Phys. 115, $8411-8417$.

Guo, Y., Gu, X., Balucani, N., Kaiser, R., 2006a. Formation of the 2,4pentadiynyl-1 radical $\left(\mathrm{H}_{2} \mathrm{CCCCCH}, \mathrm{X}^{2} \mathrm{~B}_{1}\right)$ in the crossed beams reaction of dicarbon molecules with methylacetylene. J. Phys. Chem. A 110, 6245-6249.

Guo, Y., Gu, X., Zhang, F., Mebel, A., Kaiser, R., 2006b. Unimolecular decomposition of chemically activated pentatetraene $\left(\mathrm{H}_{2} \mathrm{CCCCCH}_{2}\right)$ intermediates: A crossed beams study of dicarbon molecule reactions with allene. J. Phys. Chem. A 110, 10699-10707.

Gupta, S., Jones, E., Harrison, A., Myher, J.J., 1967. Reactions of thermal energy ions. VI. Hydrogen-transfer ion-molecule reactions involving polar molecules. Can. J. Chem. 45, 3107-3117.

Gurwell, M., 2004. Submillimeter observations of Titan: Global measures of stratospheric temperature, $\mathrm{CO}, \mathrm{HCN}, \mathrm{HC}_{3} \mathrm{~N}$, and the isotopic ratios ${ }^{12} \mathrm{C} /{ }^{13} \mathrm{C}$ and ${ }^{14} \mathrm{~N} /{ }^{15} \mathrm{~N}$. Astrophys. J. 616, L7-L10.

Hahndorf, I., Lee, H., Mebel, A., Lin, S., Lee, Y., Kaiser, R., 2000. A combined crossed beam and ab initio investigation on the reaction of carbon species with $\mathrm{C}_{4} \mathrm{H}_{6}$ isomers. I. The 1,3-butadiene molecule, $\mathrm{H}_{2} \mathrm{CCHCHCH}_{2}\left(X^{1} A^{\prime}\right)$. J. Chem. Phys. 113, 9622-9636.

Haider, N., Husain, D., 1993. Absolute rate data for the reactions of groundstate atomic carbon, $\mathrm{C}\left[{ }^{2} \mathrm{p}_{2}\left({ }^{3} \mathrm{P}_{J}\right)\right]$, with alkenes investigated by time-resolved atomic resonance absorption spectroscopy in the vacuum ultraviolet. J. Chem. Soc., Faraday Trans. 89, 7-14.

Halpern, J., Huang, Y., 1997. Radiative lifetimes, fluorescence quantum yields and photodissociation of the $\mathrm{C}_{2} \mathrm{~N}_{2}\left(\mathrm{~A}^{1} \Sigma_{u}^{-}\right)$and $\left(\mathrm{B}^{1} \Delta_{u}\right)$ states: Evidence for sterically hindered, triplet mediated crossings to the $\left(\mathrm{X}^{1} \Sigma_{g}^{+}\right)$ground state. Chem. Phys. 222, 71-86.

Halpern, J., Miller, G., Okabe, H., Nottingham, W., 1988. The UV photochemistry of cyanoacetylene. J. Photochem. Photobiol. A 42, 63-72. 
Halpern, J., Petway, L., Lu, R., Jackson, W., McCrary, V., Nottingham, W., 1990. Photochemistry of cyano- and dicyanoacetylene at $193 \mathrm{~nm}$. J. Phys. Chem. 94, 1869-1873.

Halpern, J., Tang, X., 1985. Production of cyanogen $\left(A^{2} \Pi\right)$ in the photolysis of acetonitrile at $158 \mathrm{~nm}$. Chem. Phys. Lett. 122, 294-299.

Hamberg, M., Geppert, W., Thomas, R., Zhaunerchyk, V., Österdahl, F., Ehlerding, A., Kamińska, M., Semaniak, J., af Ugglas, M., Källberg, A., Paál, A., Simonsson, A., Larsson, M., 2007. Experimental determination of dissociative recombination reaction pathways and absolute reaction cross-sections of $\mathrm{CH}_{2} \mathrm{OH}^{+}, \mathrm{CD}_{2} \mathrm{OD}^{+}$and $\mathrm{CD}_{2} \mathrm{OD}_{2}{ }^{+}$. Mol. Phys. 105, 899-906.

Hamberg, M., Vigren, E., Thomas, R., Zhaunerchyk, V., Zhang, M., Trippel, S., Kamińska, M., Kashperka, I., af Ugglas, M., Källberg, A., Simonsson, A., Paál, A., Semaniak, J., Larsson, M., Geppert, W., 2011. Experimental studies of the dissociative recombination processes for the $\mathrm{C}_{6} \mathrm{D}_{6}{ }^{+}$and $\mathrm{C}_{6} \mathrm{D}_{7}{ }^{+}$ions, in: Joblin, C., Tielens, A. (Eds.), PAHs and the Universe. EAS Publications Series. volume 46, pp. 241-249.

Hamelin, M., Béghin, C., Grard, R., López-Moreno, J., Schwingenschuh, K., Simões, F., Trautner, R., Berthelier, J., Brown, V., Chabassière, M., Falkner, P., Ferri, F., Fulchignoni, M., Jernej, I., Jeronimo, J., Molina-Cuberos, G., Rodrigo, R., Tokano, T., 2007. Electron conductivity and density profiles derived from the mutual impedance probe measurements performed during the descent of Huygens through the atmosphere of Titan. Planet. Space Sci. $55,1964-1977$.

Hampson, J.R., McNesby, J., 1965. Vacuum-ultraviolet photolysis of ethane at high temperature. J. Chem. Phys. 42, 2200-2208.

Harding, L., Georgievskii, Y., Klippenstein, S., 2005. Predictive theory for hydrogen atoms - Hydrocarbon radical association kinetics. J. Phys. Chem. A $109,4646-4656$.

Harding, L., Guadagnini, R., Schatz, G., 1993. Theoretical studies of the reactions $\mathrm{H}+\mathrm{CH} \rightarrow \mathrm{C}+\mathrm{H}_{2}$ and $\mathrm{C}+\mathrm{H}_{2} \rightarrow \mathrm{CH}_{2}$ using an ab initio global ground-state potential surface for $\mathrm{CH}_{2}$. J. Phys. Chem. 97, 5472-5481.

Harding, L., Klippenstein, S., Georgievskii, Y., 2007. On the combination reactions of hydrogen atoms with resonance-stabilized hydrocarbon radicals. J. Phys. Chem. A 111, 3789-3801.

Harding, L., Klippenstein, S., Miller, J., 2008. Kinetics of $\mathrm{CH}+\mathrm{N}_{2}$ revisited with multireference methods. J. Phys. Chem. A 112, 522-532.

Harich, S., Lee, Y., Yang, X., 2000. Photodissociation dynamics of allene at 157 nm. Phys. Chem. Chem. Phys. 2, 1187-1191. 
van Harrevelt, R., van Hemert, M., Schatz, G., 2002. The $\mathrm{CH}+\mathrm{H}$ reaction studied with quantum-mechanical and classical trajectory calculations. J. Chem. Phys. 116, 6002-6011.

Hartle, R., Sittler Jr., E., Neubauer, F., Johnson, R., Smith, H., Crary, F., McComas, D., Young, D., Coates, A., Simpson, D., Bolton, S., Reisenfeld, D., Szego, K., Berthelier, J.J., Rymer, A., Vilppola, J., Steinberg, J., Andre, N., 2006. Initial interpretation of Titan plasma interactions as observed by the Cassini Plasma Spectrometer: Comparisons with Voyager 1. Planet. Space Sci. 54, 1211-1224.

Hartogh, P., Lellouch, E., Moreno, R., Bockelée-Morvan, D., Biver, N., Cassidy, T., Rengel, M., Jarchow, C., Cavalié, T., Crovisier, J., Helmich, F., Kidger, M., 2011. Direct detection of the Enceladus water torus with Herschel. Astron. Astrophys. 532.

Hassinen, E., Kalliorinne, K., Koskikallio, J., 1990. Kinetics of reactions between methyl and acetyl radicals in gas phase produced by flash photolysis of acetic anhydride. Int. J. Chem. Kin. 22, 741-745.

Haxton, D., Rescigno, T., McCurdy, C., 2007. Dissociative electron attachment to the $\mathrm{H}_{2} \mathrm{O}$ molecule. II. Nuclear dynamics on coupled electronic surfaces within the local complex potential model. Phys. Rev. A 75, \#012711.

Hayden, C., Neumark, D., Shobatake, K., Sparks, R., Lee, Y., 1982. Methylene singlet-triplet energy splitting by molecular beam photodissociation of ketene. J. Chem. Phys. 76, 3607-3613.

Hayes, F., Gutsche, G., Lawrance, W., Staker, W., King, K., 1995. Singlet methylene removal by saturated and unsaturated hydrocarbons. Combust. Flame 100, 653-660.

Heays, A., Dickenson, G., Salumbides, E., de Oliveira, N., Joyeux, D., Nahon, L., Lewis, B., Ubachs, W., 2011. High-resolution Fourier-transform extreme ultraviolet photoabsorption spectroscopy of ${ }^{14} \mathrm{~N}^{15} \mathrm{~N}$. J. Chem. Phys. 135, \#244301.

Heber, O., Seiersen, K., Bluhme, H., Svendsen, A., Andersen, L., Maunoury, L., 2006. Dissociative recombination of small carbon cluster cations. Phys. Rev. A 73, 022712.

Hébrard, E., Dobrijevic, M., Bénilan, Y., Raulin, F., 2006. Photochemical kinetics uncertainties in modeling Titan's atmosphere: A review. J. Photochem. Photobiol. C: Photochem. Rev. 7, 211-230.

Hébrard, E., Dobrijevic, M., Loison, J., Bergeat, A., Hickson, K., 2012. Neutral production of hydrogen isocyanide (HNC) and hydrogen cyanide (HCN) in Titan's upper atmosphere. Astron. Astrophys. 541, \#A21. 
Hébrard, E., Dobrijevic, M., Loison, J., Bergeat, A., Hickson, K., Caralp, F., 2013. Photochemistry of $\mathrm{C}_{3} \mathrm{H}_{\mathrm{p}}$ hydrocarbons in Titan's stratosphere revisited. Astron. Astrophys. 552, \#A132.

Hébrard, E., Dobrijevic, M., Pernot, P., Carrasco, N., Bergeat, A., Hickson, K., Canosa, A., Le Picard, S., Sims, I., 2009. How measurements of rate coefficients at low temperature increase the predictivity of photochemical models of Titan's atmosphere. J. Phys. Chem. A 113, 11227-11237.

Hemsworth, R., Payzant, J., Schiff, H., Bohme, D., 1974. Rate constants at $297^{\circ} \mathrm{K}$ for proton transfer reactions with $\mathrm{NH}_{3}$. Comparisons with classical theories and exothermicity. Chem. Phys. Lett. 26, 417-421.

Herbst, E., Leung, C., 1989. Gas phase production of complex hydrocarbons, cyanopolyynes, and related compounds in dense interstellar clouds. Astrophys. J. Suppl. Ser. 69, 271-300.

Herbst, E., Osamura, Y., 2008. Calculations of the formation rates and mechanisms for $\mathrm{C}_{n} \mathrm{H}$ anions in interstellar and circumstellar media. Astrophys. J. 679, 1670-1679.

Herbst, E., Roueff, E., Talbi, D., 2010. Radiative association and the formation of interstellar propylene. Mol. Phys. 108, 2171-2177.

Herbst, E., Smith, D., Adams, N., McIntosh, B., 1989. Association reactions. Theoretical shortcomings. J. Chem. Soc., Faraday Trans. 2 85, 1655-1664.

Herbst, E., Terzieva, R., Talbi, D., 2000. Calculations on the rates, mechanisms, and interstellar importance of the reactions between $\mathrm{C}$ and $\mathrm{NH}_{2}$ and between $\mathrm{N}$ and $\mathrm{CH}_{2}$. Mon. Not. R. Astron. Soc. 311, 869-876.

Herron, J.T., 1999. Evaluated chemical kinetics data for reactions of $\mathrm{N}\left({ }^{2} \mathrm{D}\right)$, $\mathrm{N}\left({ }^{2} \mathrm{P}\right)$ and $\mathrm{N}_{2}\left(\mathrm{~A}^{3} \Sigma_{\mathrm{u}}^{+}\right)$in the gas phase. J. Phys. Chem. Ref. Data 28, 14531483.

Hickman, A., 1979. Approximate scaling formula for ion-ion mutual neutralization rates. J. Chem. Phys. 70, 4872-4878.

Hickman, A., Miles, R., Hayden, C., Talbi, D., 2005. Dissociative recombination of $\mathrm{e}+\mathrm{HCNH}^{+}$: Diabatic potential curves and dynamics calculations. Astron. Astrophys. 438, 31-37.

Hickson, K., Loison, J., Cavalié, T., Hébrard, E., Dobrijevic, M., 2014. The evolution of infalling sulfur species in Titan's atmosphere. Astron. Astrophys. 572, \#A58.

Hickson, K., Loison, J.C., Caubet, P., 2013. Unusual Low Temperature Reactivity of Water. The CH H2O Reaction as a Source of Interstellar Formaldehyde? J. Phys. Chem. Lett. 4, 2843. 
Hippler, H., Troe, J., Wendelken, H., 1983. UV absorption spectra of vibrationally highly excited toluene molecules. J. Chem. Phys. 78, 5351-5357.

Hiraoka, K., Shoda, T., Kudaka, I., Fujimaki, S., Mizuse, S., Yamabe, S., Wasada, H., Wasada-Tsutsui, Y., 2003. Gas phase study of the clustering reactions of $\mathrm{C}_{2} \mathrm{H}_{5}{ }^{+}, \mathrm{s}_{-} \mathrm{C}_{3} \mathrm{H}_{7}{ }^{+}$, and $\mathrm{t}-\mathrm{C}_{4} \mathrm{H}_{9}{ }^{+}$with $\mathrm{CO}_{2}$ and $\mathrm{N}_{2} \mathrm{O}$ : Isomeric structure of $\mathrm{C}_{2} \mathrm{H}_{5}{ }^{+}, \mathrm{C}_{2} \mathrm{H}_{5}{ }^{+}\left(\mathrm{CO}_{2}\right)_{n}$, and $\mathrm{C}_{2} \mathrm{H}_{5}{ }^{+}\left(\mathrm{N}_{2} \mathrm{O}\right)_{n}$. J. Phys. Chem. A $107,775-781$.

Hlavenka, P., Otto, R., Trippel, S., Mikosch, J., Weidemüller, M., Wester, R., 2009. Absolute photodetachment cross section measurements of the $\mathrm{O}^{-}$and $\mathrm{OH}^{-}$anion. J. Chem. Phys. 130, \#061105.

Ho, G., Lin, M., Wang, Y., Chang, T., 1998. Photoabsorption and photoionization of propyne. J. Chem. Phys. 109, 5868-5879.

Holland, D., Shaw, D., Hayes, M., Shpinkova, L., Rennie, E., Karlsson, L., Baltzer, P., Wannberg, B., 1997. A photoabsorption, photodissociation and photoelectron spectroscopy study of $\mathrm{C}_{2} \mathrm{H}_{4}$ and $\mathrm{C}_{2} \mathrm{D}_{4}$. Chem. Phys. 219, 91116 .

Holzmeier, F., Lang, M., Hader, K., Hemberger, P., Fischer, I., 2013. $\mathrm{H}_{2} \mathrm{CN}^{+}$ and $\mathrm{H}_{2} \mathrm{CNH}^{+}$: New insight into the structure and dynamics from massselected threshold photoelectron spectra. J. Chem. Phys. 138, \#214310.

Homayoon, Z., Vázquez, S., Rodríguez-Fernández, R., Martínez-Núñez, E., 2011. Ab initio and RRKM study of the HCN/HNC elimination channels from vinyl cyanide. J. Phys. Chem. A 115, 979-985.

Hoobler, R., Leone, S., 1997. Rate coefficients for reactions of ethynyl radical $\left(\mathrm{C}_{2} \mathrm{H}\right)$ with $\mathrm{HCN}$ and $\mathrm{CH}_{3} \mathrm{CN}$ : Implications for the formation of complex nitriles on Titan. J. Geophys. Res. 102, 28717-28723.

Hörst, S., Vuitton, V., Yelle, R., 2008. The origin of oxygen species in Titan's atmosphere. J. Geophys. Res. 113, \#E10006.

Hörst, S., Yelle, R., Buch, A., Carrasco, N., Cernogora, G., Dutuit, O., Quirico, E., Sciamma-O'Brien, E., Smith, M., Somogyi, Á., Szopa, C., Thissen, R., Vuitton, V., 2012. Formation of amino acids and nucleotide bases in a Titan atmosphere simulation experiment. Astrobiology 12, 809-817.

Hörst, S.M., 2017. Titan's atmosphere and climate. J. Geophys. Res. Planets 122, doi:10.1002/2016JE005240.

Hotop, H., Lineberger, W., 1985. Binding energies in atomic negative ions: II. J. Phys. Chem. Ref. Data 14, 731-750.

Houriet, R., Elwood, T., Futrell, J., 1978. A tandem ion cyclotron resonance study of the reactions of allyl ions with benzene and substituted benzene. J. Am. Chem. Soc. 100, 2320-2324. 
Howard, C., Fehsenfeld, F., McFarland, M., 1974. Negative ion-molecule reactions with atomic hydrogen in the gas phase at 296 K. J. Chem. Phys. 60, $5086-5089$.

Howling, A., Sansonnens, L., Dorier, J.L., Hollenstein, C., 1994. Time-resolved measurements of highly polymerized negative ions in radio frequency silane plasma deposition experiments. J. Appl. Phys. 75, 1340-1353.

Hubin-Franskin, M.J., Delwiche, J., Giuliani, A., Ska, M.P., Motte-Tollet, F., Walker, I., Mason, N., Gingell, J., Jones, N., 2002. Electronic excitation and optical cross sections of methylamine and ethylamine in the UV-VUV spectral region. J. Chem. Phys. 116, 9261-9268.

Huntress, W.T., J., Pinizzotto, R.F., J., 1973. Product distributions and rate constants for ion-molecule reactions in water, hydrogen sulfide, ammonia, and methane. J. Chem. Phys. 59, 4742-4756.

Huntress, W., 1977. Laboratory studies of bimolecular reactions of positive ions in interstellar clouds, in comets, and in planetary atmospheres of reducing composition. Astrophys. J. Suppl. Ser. 33, 495-514.

Hunziker, H., Kneppe, H., McLean, A., Siegbahn, P., Wendt, H., 1983. Visible electronic absorption-spectrum of vinyl radical. Can. J. Chem. 61, 993-995.

Husain, D., Ioannou, A., 1997. Reactions of ground-state atomic carbon, C[2 $\mathrm{p}^{2}$ $\left.\left({ }^{3} \mathrm{P}_{J}\right)\right]$, with dienes and diynes by time-resolved atomic resonance absorption spectroscopy in the vacuum ultraviolet. J. Chem. Soc. Faraday Trans. 93, $3625-3629$.

Ikeda, N., Nakashima, N., Yoshihara, K., 1984. Formation and relaxation of hot benzyl radicals in the gas phase. J. Phys. Chem. A 88, 5803-5806.

Inoue, M., 1966. Ions négatifs formés dans le cyanogène et l'acide cyanhydrique. J. Chim. Phys. PCB 63, 1061-1071.

Ip, W., 1990. Titan's upper ionosphere. Astrophys. J. 362, 354-363.

Iraqi, M., Lifshitz, C., Reuben, B., 1991. Reaction of ketene ions with ammonia. J. Phys. Chem. 95, 7742-7746.

Ismail, H., Abel, P., Green, W., Fahr, A., Jusinski, L., Knepp, A., Zádor, J., Meloni, G., Selby, T., Osborn, D., Taatjes, C., 2009. Temperature-dependent kinetics of the vinyl radical $\left(\mathrm{C}_{2} \mathrm{H}_{3}\right)$ self-reaction. J. Phys. Chem. A 113, $1278-1286$.

Itikawa, Y., 2006. Cross Sections for electron collisions with nitrogen molecules. J. Phys. Chem. Ref. Data 35, \#31.

Jackson, D., Stibrich, N., Adams, N., Babcock, L., 2005. A selected ion flow tube study of the reactions of a sequence of ions with amines. Int. J. Mass Spectrom. 243, 115-120. 
Jackson, J.A.A., Lias, S., 1974. Primary processes in the photolysis of n-butane with 8.4 and $10.0 \mathrm{eV}$ photons. J. Photochem. 3, 151-162.

Jackson, W., Anex, D., Continetti, R., Balko, B., Lee, Y., 1991. Molecular beam studies of the photolysis of allene and the secondary photodissociation of the $\mathrm{C}_{3} \mathrm{H}_{x}$ fragments. J. Chem. Phys. 95, 7327-7336.

Jacox, M., 2003. Vibrational and electronic energy levels of polyatomic transient molecules. Supplement B. J. Phys. Chem. Ref. Data 32, \#1.

Janečková, R., May, O., Fedor, J., 2012. Dissociative electron attachment to methylacetylene and dimethylacetylene: Symmetry versus proximity. Phys. Rev. A 86, \#052702.

Janev, R., Reiter, D., 2004. Collision processes of $\mathrm{C}_{2,3} \mathrm{H}_{\mathrm{y}}$ and $\mathrm{C}_{2,3} \mathrm{H}_{\mathrm{y}}^{+}$hydrocarbons with electrons and protons. Phys. Plasmas 11, 780-829.

Jasper, A., Klippenstein, S., Harding, L., 2007. Secondary kinetics of methanol decomposition: Theoretical rate coefficients for ${ }^{3} \mathrm{CH}_{2}+\mathrm{OH},{ }^{3} \mathrm{CH}_{2}+{ }^{3} \mathrm{CH}_{2}$, and ${ }^{3} \mathrm{CH}_{2}+\mathrm{CH}_{3}$. J. Phys. Chem. A 111, 8699-8707.

Jasper, A., Miller, J., 2011. Theoretical unimolecular kinetics for $\mathrm{CH}_{4}+\mathrm{M}=$ $\mathrm{CH}_{3}+\mathrm{H}+\mathrm{M}$ in eight baths, $\mathrm{M}=\mathrm{He}, \mathrm{Ne}, \mathrm{Ar}, \mathrm{Kr}, \mathrm{H}_{2}, \mathrm{~N}_{2}, \mathrm{CO}$, and $\mathrm{CH}_{4}$. J. Phys. Chem. A 115, 6438-6455.

Jasper, A., Oana, C., Miller, J., 2015. "Third-body" collision efficiencies for combustion modeling: Hydrocarbons in atomic and diatomic baths. Proc. Comb. Inst. 35, 197-204.

Jenkin, M., Murrells, T., Shalliker, S., Hayman, G., 1993. Kinetics and product study of the self-reactions of allyl and allyl peroxy radicals at 296 K. J. Chem. Soc. Faraday Trans. 89, 433-446.

Jensen, M., Bilodeau, R., Safvan, C., Seiersen, K., Andersen, L., Pedersen, H., Heber, O., 2000. Dissociative recombination and excitation of $\mathrm{H}_{3} \mathrm{O}^{+}, \mathrm{HD}_{2} \mathrm{O}^{+}$, and $\mathrm{D}_{3} \mathrm{O}^{+}$. Astrophys. J. 543, 764-774.

Jiao, C., DeJoseph, C.J., Lee, R., Garscadden, A., 2006. Kinetics of electron impact ionization and ion-molecule reactions of pyridine. Int. J. Mass Spectrom. $257,34-40$.

Jodkowski, J., Ratajczak, E., Fagerström, K., Lund, A., Stothard, N., Humpfer, R., Grotheer, H.H., 1995. Kinetics of the cross reaction between amidogen and methyl radicals. Chem. Phys. Lett. 240, 63-71.

Johnsen, R., Biondi, M., 1974. Measurements of positive ion conversion and removal reactions relating to the Jovian ionosphere. Icarus 23, 139-143.

Jolly, A., Bénilan, Y., 2008. Review of quantitative spectroscopy of polyynes. J. Quant. Spectrosc. Radiat. Transfer 109, 963-973. 
Jolly, A., Cottini, V., Fayt, A., Manceron, L., Kwabia-Tchana, F., Benilan, Y., Guillemin, J.C., Nixon, C., Irwin, P., 2015. Gas phase dicyanoacetylene $\left(\mathrm{C}_{4} \mathrm{~N}_{2}\right)$ on Titan: New experimental and theoretical spectroscopy results applied to Cassini CIRS data. Icarus 248, 340-346.

Jones, B., Zhang, F., Kaiser, R., Jamal, A., Mebel, A., Cordiner, M., Charnley, S., 2011. Formation of benzene in the interstellar medium. P. Natl. Acad. Sci. USA 108, 452-457.

Jones, B., Zhang, F., Maksyutenko, P., Mebel, A., Kaiser, R., 2010. Crossed molecular beam study on the formation of phenylacetylene and its relevance to Titan's atmosphere. J. Phys. Chem. A 114, 5256-5262.

Kajimoto, O., Fueno, T., 1979. Relative rate constants of O(1D2)-olefin reactions. Chem. Phys. Lett. 64, 445-447.

Kalhori, S., Viggiano, A., Arnold, S., Rosén, S., Semaniak, J., Derkatch, A., af Ugglas, M., Larsson, M., 2002. Dissociative recombination of $\mathrm{C}_{2} \mathrm{H}_{3}{ }^{+}$. Astron. Astrophys. 391, 1159-1165.

Kállay, M., Surján, P., 2001. Higher excitations in coupled-cluster theory. J. Chem. Phys. 115, 2945-2954.

Kameta, K., Machida, S., Kitajima, M., Ukai, M., Kouchi, N., Hatano, Y., Ito, K., 1996. Photoabsorption, photoionization, and neutral-dissociation cross sections of $\mathrm{C}_{2} \mathrm{H}_{6}$ and $\mathrm{C}_{3} \mathrm{H}_{8}$ in the extreme-uv region. J. Electron Spectrosc. Relat. Phenom. 79, 391-393.

Kamińska, M., Zhaunerchyk, V., Vigren, E., Danielsson, M., Hamberg, M., Geppert, W., Larsson, M., Rosén, S., Thomas, R., Semaniak, J., 2010. Dissociative recombination of $\mathrm{CH}_{5}{ }^{+}$and $\mathrm{CD}_{5}{ }^{+}$: Measurement of the product branching fractions and the absolute cross sections, and the breakup dynamics in the $\mathrm{CH}_{3}+\mathrm{H}+\mathrm{H}$ product channel. Phys. Rev. A 81, \#062701.

Kanda, K., Nagata, T., Ibuki, T., 1999. Photodissociation of some simple nitriles in the extreme vacuum ultraviolet region. Chem. Phys. 243, 89-96.

Karpas, Z., Huntress, W.T., J., 1978. Reactions of OH+ and H2O+ ions with some diatomic and simple polyatomic molecules. Chem. Phys. Lett. 59, 87-89.

Karton, A., Rabinovich, E., Martin, J., Ruscic, B., 2006. W4 theory for computational thermochemistry: In pursuit of confident sub-kJ/mol predictions. J. Chem. Phys. 125, \#144108.

Keller, C., Anicich, V., Cravens, T., 1998. Model of Titan's ionosphere with detailed hydrocarbon ion chemistry. Planet. Space Sci. 46, 1157-1174.

Keller, C., Cravens, T., Gan, L., 1992. A model of the ionosphere of Titan. J. Geophys. Res. 97, 12117-12135. 
Kirby, K., Constantinides, E., Babeu, S., Oppenheimer, M., Victor, G., 1979 Photoionization and photoabsorption cross sections of $\mathrm{He}, \mathrm{O}, \mathrm{N}_{2}$ and $\mathrm{O}_{2}$ for aeronomic calculations. At. Data Nucl. Data Tables 23, 63-81.

Kislov, V., Nguyen, T., Mebel, A., Lin, S., Smith, S., 2004. Photodissociation of benzene under collision-free conditions: An ab initio/Rice-RamspergerKassel-Marcus study. J. Chem. Phys. 120, 7008-7017.

Kley, D., Lawrence, G., Stone, E., 1977. The yield of $\mathrm{N}\left({ }^{2} \mathrm{D}\right)$ atoms in the dissociative recombination of $\mathrm{NO}^{+}$. J. Chem. Phys. 66, 4157-4165.

Klippenstein, S., 1992. Variational optimizations in the Rice-RamspergerKassel-Marcus theory calculations for unimolecular dissociations with no reverse barrier. J. Chem. Phys. 96, 367-371.

Klippenstein, S., 2017. From theoretical reaction dynamics to chemical modeling of combustion. Proc. Comb. Inst. 36, 77-111.

Klippenstein, S., Georgievskii, Y., Harding, L., 2002. A theoretical analysis of the $\mathrm{CH}_{3}+\mathrm{H}$ reaction: Isotope effects, the high-pressure limit, and transition state recrossing. Proc. Comb. Inst. 29, 1229-1236.

Klippenstein, S., Georgievskii, Y., Harding, L., 2006. Predictive theory for the combination kinetics of two alkyl radicals. Phys. Chem. Chem. Phys. 8, $1133-1147$.

Klippenstein, S., Harding, L., 1999. A direct transition state theory based study of methyl radical recombination kinetics. J. Phys. Chem. A 103, 9388-9398.

Klippenstein, S., Harding, L., Georgievskii, Y., 2007. On the formation and decomposition of $\mathrm{C}_{7} \mathrm{H}_{8}$. Proc. Comb. Inst. 31, 221-229.

Klippenstein, S., Harding, L., Ruscic, B., Sivaramakrishnan, R., Srinivasan, N., Su, M.C., Michael, J., 2009. Thermal decomposition of $\mathrm{NH}_{2} \mathrm{OH}$ and subsequent reactions: Ab initio transition state theory and reflected shock tube experiments. J. Phys. Chem. A 113, 10241-10259.

Klippenstein, S., Miller, J., 2005. The addition of hydrogen atoms to diacetylene and the heats of formation of $\mathrm{i}-\mathrm{C}_{4} \mathrm{H}_{3}$ and $\mathrm{n}-\mathrm{C}_{4} \mathrm{H}_{3}$. J. Phys. Chem. A 109, $4285-4295$.

Klippenstein, S., Ruscic, B., Harding, L., 2017. Ab initio computations and active thermochemical tables hand in hand: Heats of formation of core combustion species. J. Phys. Chem. A 121, 6580-6602.

Klippenstein, S., Wagner, A., Dunbar, R., Wardlaw, D., Roberston, S., Miller, J., 2011. VariFlex: Version 2.04m. Argonne National Laboratory, Argonne, IL . 
Klippenstein, S., Yang, Y.C., Ryzhov, V., Dunbar, R., 1996. Theory and modeling of ion-molecule radiative association kinetics. J. Chem. Phys. 104, 45024516.

Kloster-Jensen, E., Haink, H., Christen, H., 1974. The electronic spectra of unsubstituted mono- to penta- acetylene in the gas phase and in solution in the range 1100 to $4000 \AA$. Helv. Chim. Acta 57, 1731-1744.

Knyazev, V., Slagle, I., 2001. Kinetics of the reactions of allyl and propargyl radicals with $\mathrm{CH}_{3}$. J. Phys. Chem. A 105, 3196-3204.

Koch, E., Otto, A., 1972. Optical absorption of benzene vapour for photon energies from $6 \mathrm{eV}$ to $35 \mathrm{eV}$. Chem. Phys. Lett. 12, 476-480.

Koizumi, H., Yoshimi, T., Shinsaka, K., Ukai, M., Morita, M., Hatano, Y., 1985. VUV-optical oscillator strength distributions of $\mathrm{C}_{3} \mathrm{H}_{6}$ and $\mathrm{C}_{4} \mathrm{H}_{8}$ isomers. J. Chem. Phys. 82, 4856-4861.

Koskinen, T., Yelle, R., Snowden, D., Lavvas, P., Sandel, B., Capalbo, F., Bénilan, Y., West, R., 2011. The mesosphere and thermosphere of Titan revealed by Cassini/UVIS stellar occultations. Icarus 216, 507-534.

Kovács, T., Blitz, M., Seakins, P., 2010. H-atom yields from the photolysis of acetylene and from the reaction of $\mathrm{C}_{2} \mathrm{H}$ with $\mathrm{H}_{2}, \mathrm{C}_{2} \mathrm{H}_{2}$, and $\mathrm{C}_{2} \mathrm{H}_{4}$. J. Phys. Chem. A 114, 4735-4741.

Koyano, I., Wauchop, T., Welge, K., 1975. Relative efficiencies of $\mathrm{O}\left({ }^{1} \mathrm{~S}\right)$ production from photodissociation of $\mathrm{CO}_{2}$ between 1080 and $1160 \AA$. J. Chem. Phys. 63, 110-112.

Krasnopolsky, V., 2009. A photochemical model of Titan's atmosphere and ionosphere. Icarus 201, 226-256.

Krasnopolsky, V., 2010. The photochemical model of Titan's atmosphere and ionosphere: A version without hydrodynamic escape. Planet. Space Sci. 58, $1507-1515$.

Krasnopolsky, V., 2012. Titan's photochemical model: Further update, oxygen species, and comparison with Triton and Pluto. Planet. Space Sci. 73, 318326 .

Krasnopolsky, V., 2014. Chemical composition of Titan's atmosphere: Observations and the photochemical model. Icarus 236, 83-91.

Krishnakumar, E., Denifl, S., Čadež, I., Markelj, S., Mason, N., 2011. Dissociative electron attachment cross sections for $\mathrm{H}_{2}$ and $\mathrm{D}_{2}$. Phys. Rev. Lett. 106, \#243201.

Krummacher, S., Schmidt, V., Wuilleumier, F., 1980. Inner-shell photoionisation in molecules: the nitrogen case. J. Phys. B: Atom. Molec. Phys. 13, 3993-4005. 
Kuga, M., Carrasco, N., Marty, B., Marrocchi, Y., Bernard, S., Rigaudier, T., Fleury, B., Tissandier, L., 2014. Nitrogen isotopic fractionation during abiotic synthesis of organic solid particles. Earth Planet. Sc. Lett. 393, 2-13.

Kumar, S., Hauser, D., Jindra, R., Best, T., Roučka, Š., Geppert, W., Millar, T., Wester, R., 2013. Photodetachment as a destruction mechanism for $\mathrm{CN}^{-}$ and $\mathrm{C}_{3} \mathrm{~N}^{-}$anions in circumstellar envelopes. Astrophys. J. 776, \#25.

Landera, A., Krishtal, S., Kislov, V., Mebel, A., Kaiser, R., 2008. Theoretical study of the $\mathrm{C}_{6} \mathrm{H}_{3}$ potential energy surface and rate constants and product branching ratios of the $\mathrm{C}_{2} \mathrm{H}\left({ }^{2} \Sigma^{+}\right)+\mathrm{C}_{4} \mathrm{H}_{2}\left({ }^{1} \Sigma_{g}^{+}\right)$and $\mathrm{C}_{4} \mathrm{H}\left({ }^{2} \Sigma^{+}\right)+\mathrm{C}_{2} \mathrm{H}_{2}\left({ }^{1} \Sigma_{g}^{+}\right)$ reactions. J. Chem. Phys. 128, \#214301.

Langford, A., Petek, H., Moore, C., 1983. Collisionnal removal of $\mathrm{CH}_{2}\left({ }^{1} \mathrm{~A}_{1}\right)$ : Absolute rate constants for atomic and molecular collisional partners at 295 K. J. Chem. Phys. 78, 6650-6659.

Lara, L., Lellouch, E., González, M., Moreno, R., Rengel, M., 2014. A timedependent photochemical model for Titan's atmosphere and the origin of $\mathrm{H}_{2} \mathrm{O}$. Astron. Astrophys. 566, \#A143.

Lara, L., Lellouch, E., Shematovich, V., 1999. Titan's atmospheric haze: The case for HCN incorporation. Astron. Astrophys. 341, 312-317.

Lara, L.M., Lellouch, E., López-Moreno, J.J., Rodrigo, R., 1996. Vertical distribution of Titan's atmospheric neutral constituents. J. Geophys. Res. 101, 23261-23283.

Lara, L.M., Lorenz, R.D., Rodrigo, R., 1994. Liquids and solids on the surface of Titan: Results of a new photochemical model. Planet. Space Sci. 42, 5-14.

Larson, A., Le Padellec, A., Semaniak, J., Strömholm, C., Larsson, M., Rosén, S., Peverall, R., Danared, H., Djuric, N., Dunn, G., Datz, S., 1998. Branching fractions in dissociative recombination of $\mathrm{CH}_{2}{ }^{+}$. Astrophys. J. 505, 459-465.

Larsson, M., Ehlerding, A., Geppert, W., Hellberg, F., Kalhori, S., Thomas, R., Djuric, N., Österdahl, F., Angelova, G., Semaniak, J., Novotny, O., Arnold, S., Viggiano, A., 2005. Rate constants and branching ratios for the dissociative recombination of $\mathrm{C}_{3} \mathrm{D}_{7}{ }^{+}$and $\mathrm{C}_{4} \mathrm{D}_{9}{ }^{+}$. J. Chem. Phys. 122, 156101-156103.

Laufer, A., Fahr, A., 2004. Reactions and kinetics of unsaturated $\mathrm{C}_{2}$ hydrocarbon radicals. Chem. Rev. 104, 2813-2832.

Laufer, A., Keller, R., 1971. Lowest excited states of ketene. J. Am. Chem. Soc. 93, 61-63.

Laufer, A., McNesby, J., 1966. The chain decomposition of propane initiated by vacuum ultraviolet photolysis. J. Phys. Chem. 70, 4094-4096. 
Läuter, A., Lee, K., Jung, K., Vatsa, R., Mittal, J., Volpp, H.R., 2002. Absolute primary $\mathrm{H}$ atom quantum yield measurements in the 193.3 and $121.6 \mathrm{~nm}$ photodissociation of acetylene. Chem. Phys. Lett. 358, 314-319.

Lavvas, P., Coustenis, A., Vardavas, I., 2008a. Coupling photochemistry with haze formation in Titan's atmosphere. Part I: Model description. Planet. Space Sci. 56, 27-66.

Lavvas, P., Coustenis, A., Vardavas, I., 2008b. Coupling photochemistry with haze formation in Titan's atmosphere. Part II: Results and validation with Cassini/Huygens data. Planet. Space Sci. 56, 67-99.

Lavvas, P., Galand, M., Yelle, R., Heays, A., Lewis, B., Lewis, G., Coates, A., 2011a. Energy deposition and primary chemical products in Titan's upper atmosphere. Icarus 213, 233-251.

Lavvas, P., Griffith, C., Yelle, R., 2011b. Condensation in Titan's atmosphere at the Huygens landing site. Icarus 215, 732-750.

Lavvas, P., Sander, M., Kraft, M., Imanaka, H., 2011c. Surface chemistry and particle shape. Processes for the evolution of aerosols in Titan's atmosphere. Astrophys. J. 728, 80-90.

Lavvas, P., West, R., Gronoff, G., Rannou, P., 2014. Titan's emission processes during eclipse. Icarus 241, 397-408.

Lavvas, P., Yelle, R., Griffith, C., 2010. Titan's vertical aerosol structure at the Huygens landing site: Constraints on particle size, density, charge, and refractive index. Icarus 210, 832-842.

Lavvas, P., Yelle, R., Heays, A., Campbell, L., Brunger, M., Galand, M., Vuitton, V., 2015. $\mathrm{N}_{2}$ state population in Titan's atmosphere. Icarus 260, 29-59.

Lawrence, G., 1972. Production of $\mathrm{O}\left({ }^{1} \mathrm{~S}\right)$ from photodissociation of $\mathrm{CO}_{2}$. J. Chem. Phys. 57, 5616-5617.

Le Garrec, J.L., Carles, S., Speck, T., Mitchell, J., Rowe, B., Ferguson, E., 2003. The ion-molecule reaction of $\mathrm{O}^{+}$with $\mathrm{N}_{2}$ measured down to $23 \mathrm{~K}$. Chem. Phys. Lett. 372, 485-488.

Le Padellec, A., Mitchell, J., Al-Khalili, A., Danared, H., Källberg, A., Larson, Å., Rosén, S., af Ugglas, M., Vikor, L., Larsson, M., 1999. Storage ring measurements of the dissociative recombination and excitation of the cyanogen ion $\mathrm{CN}^{+}\left(\mathrm{X}^{1} \Sigma^{+}\right.$and a $\left.{ }^{3} \Pi, \nu=0\right)$. J. Chem. Phys. 110, 890-901.

Le Padellec, A., Sheehan, C., Talbi, D., Mitchell, J., 1997. A merged-beam study of the dissociative recombination of $\mathrm{HCO}^{+}$. J. Phys. B: At. Mol. Opt. Phys. 30, 319-327.

Lebonnois, S., 2005. Benzene and aerosol production in Titan and Jupiter's atmospheres: A sensitivity study. Planet. Space Sci. 53, 486-497. 
Lebonnois, S., Bakes, E., McKay, C., 2002. Transition from gaseous compounds to aerosols in Titan's atmosphere. Icarus 159, 505-517.

Lebonnois, S., Toublanc, D., Hourdin, F., Rannou, P., 2001. Seasonal variations of Titan's atmospheric composition. Icarus 152, 384-406.

Lee, A., Yung, Y., Cheng, B.M., Bahou, M., Chung, C.Y., Lee, Y.P., 2001. Enhancement of deuterated ethane on Jupiter. Astrophys. J. 551, L93-L96.

Lee, L., 1980. $\mathrm{CN}\left(\mathrm{A}^{2} \Pi \rightarrow \mathrm{X}^{2} \Sigma^{+}\right)$and $\mathrm{CN}\left(\mathrm{B}^{2} \Sigma^{+} \rightarrow \mathrm{X}^{2} \Sigma^{+}\right)$yields from $\mathrm{HCN}$ photodissociation. J. Chem. Phys. 72, 6414-6421.

Lee, L., Chiang, C., 1983. Fluorescence yield from photodissociation of $\mathrm{CH}_{4}$ at 1060-1420 A. J. Chem. Phys. 78, 688-691.

Lee, S.H., Lee, Y., Yang, X., 2004. Dynamics of photodissociation of ethylene and its isotopomers at $157 \mathrm{~nm}$ : Branching ratios and kinetic-energy distributions. J. Chem. Phys. 120, 10983-10991.

LeGarrec, J., Lepage, V., Rowe, B., Ferguson, E., 1997. The temperature dependence of the rate constant for $\mathrm{O}^{+}+\mathrm{NO} \rightarrow \mathrm{NO}^{+}+\mathrm{O}$ from 23 to $3000 \mathrm{~K}$. Chem. Phys. Lett. 270, 66-70.

Lehfaoui, L., Rebrion-Rowe, C., Laubé, S., Mitchell, J., Rowe, B., 1997. The dissociative recombination of hydrocarbon ions. I. Light alkanes. J. Chem. Phys. 106, 5406-5412.

Lellouch, E., 1990. Atmospheric models of Titan and Triton. Ann. Geophys. 8, 653-660.

Lellouch, E., Bézard, B., Flasar, F., Vinatier, S., Achterberg, R., Nixon, C., Bjoraker, G., Gorius, N., 2014. The distribution of methane in Titan's stratosphere from Cassini/CIRS observations. Icarus 231, 323-337.

Leonori, F., Petrucci, R., Hickson, K., Segoloni, E., Balucani, N., Le Picard, S., Foggi, P., Casavecchia, P., 2008. Crossed molecular beam study of gas phase reactions relevant to the chemistry of planetary atmospheres: the case of $\mathrm{C}_{2}+\mathrm{C}_{2} \mathrm{H}_{2}$. Planet. Space Sci. 56, 1658-1673.

Leopold, D., Murray, K., Stevens Miller, A., Lineberger, W., 1985. Methylene: A study of the $\mathrm{X}^{3} \mathrm{~B}_{1}$ and $\tilde{a}^{1} \mathrm{~A}_{1}$ states by photoelectron spectroscopy of $\mathrm{CH}_{2}{ }^{-}$ and $\mathrm{CD}_{2}{ }^{-}$. J. Chem. Phys. 83, 4849-4865.

Lewis, B., Gibson, S., Zhang, W., Lefebvre-Brion, H., Robbe, J.M., 2005. Predissociation mechanism for the lowest ${ }^{1} \Pi_{u}$ states of $\mathrm{N}_{2}$. J. Chem. Phys. 122, \#144302.

Li, C., Zhang, X., Gao, P., Yung, Y., 2015. Vertical distribution of $\mathrm{C}_{3^{-}}$ hydrocarbons in the stratosphere of Titan. Astrophys. J. 803, \#L19. 
Li, C., Zhang, X., Kammer, J., Liang, M.C., Shia, R.L., Yung, Y., 2014. A nonmonotonic eddy diffusivity profile of Titan's atmosphere revealed by Cassini observations. Planet. Space Sci. 104, 48-58.

Li, Q., Zhang, X., 2006. Direct dynamics study on the hydrogen abstraction reactions $\mathrm{N}_{2} \mathrm{H}_{4}+R \rightarrow \mathrm{N}_{2} \mathrm{H}_{3}+R \mathrm{H}\left(R=\mathrm{NH}_{2}, \mathrm{CH}_{3}\right)$. J. Chem. Phys. 125, \#064304.

Liang, M.C., Heays, A., Lewis, B., Gibson, S., Yung, Y., 2007. Source of nitrogen isotope anomaly in $\mathrm{HCN}$ in the atmosphere of Titan. Astrophys. J. 664, L115-L118.

Lias, S., Ausloos, P., 1985. Structures of $\mathrm{C}_{6} \mathrm{H}_{7}{ }^{+}$ions formed in unimolecular and bimolecular reactions. J. Chem. Phys. 82, 3613-3624.

Lias, S., Bartmess, J., Liebman, J., Holmes, J., Levin, R., Mallard, W., 1988. Gas-phase ion and neutral thermochemistry. J. Phys. Chem. Ref. Data 17, Suppl 1.

Lias, S., Buckley, T., 1984. Structures and reactions of C3H6+ ions generated in cyclopropane. Int. J. Mass Spectrom. Ion Processes 56, 123-137.

Lias, S., Collin, G., Rebbert, R., Ausloos, P., 1970. Photolysis of ethane at 11.6-11.8 eV. J. Chem. Phys. 52, 1841-1851.

Lifshitz, C., Gibson, D., Levsen, K., 1980. Structure of the gas-phase ion C6H5+. International Journal of Mass Spectrometry and Ion Processes 35, $365-370$.

Lifshitz, C., Gibson, D., Levsen, K., Dotan, I., 1981. The gas-phase chemistry of $\mathrm{C} 4 \mathrm{H} 4+$. International Journal of Mass Spectrometry and Ion Processes 40, $157-165$.

Lifshitz, C., Reuben, B., 1969. Ion-molecule reactions in aromatic systems. I. Secondary ions and reaction rates in benzene. J. Chem. Phys. 50, 951-960.

Lifshitz, C., Weiss, M., 1980a. Ion-molecule reactions in alkynes. Trapped-ion mass spectrometry of 1,5-hexadiyne. International Journal of Mass Spectrometry and Ion Processes 35, 73-81.

Lifshitz, C., Weiss, M., 1980b. Ion-molecule reactions in aromatic systems. II. Trapped ion mass spectrometry of benzene. Int. J. Mass Spectrom. Ion Processes 34, 311-315.

Lilensten, J., Witasse, O., Simon, C., Soldi-Lose, H., Dutuit, O., Thissen, R., Alcaraz, C., 2005. Prediction of a $\mathrm{N}_{2}{ }^{++}$layer in the upper atmosphere of Titan. Geophys. Res. Lett. 32, \#L03202.

Limão-Vieira, P., Eden, S., Mason, N., Hoffmann, S., 2003. Electronic state spectroscopy of acetaldehyde, $\mathrm{CH}_{3} \mathrm{CHO}$, by high-resolution VUV photoabsorption. Chem. Phys. Lett. 376, 737-747. 
Lin, Z., Talbi, D., Roueff, E., Herbst, E., Wehres, N., Cole, C., Yang, Z., Snow, T., Bierbaum, V., 2013. Can interstellar propene $\left(\mathrm{CH}_{3} \mathrm{CHCH}_{2}\right)$ be formed via gas-phase reactions? Astrophys. J. 765, \#80.

Lindinger, W., Hansel, A., Herman, Z., 2000. Ion-molecule reactions. Adv. At. Mol. Opt. Phys. 43, 243-294.

Liu, X., Shemansky, D., 2006. Analysis of electron impact ionization properties of methane. J. Geophys. Res. 111, A04303-A04319.

Loison, J., Hébrard, E., Dobrijevic, M., Hickson, K., Caralp, F., Hue, V., Gronoff, G., Venot, O., Benilan, Y., 2015. The neutral photochemistry of nitriles, amines and imines in the atmosphere of Titan. Icarus 247, 218-247.

Loison, J.C., Bergeat, A., 2004. Reaction of carbon atoms, $\mathrm{C}\left(2 \mathrm{p}^{2},{ }^{3} \mathrm{P}\right)$ with $\mathrm{C}_{3} \mathrm{H}_{4}$ (allene and methylacetylene), $\mathrm{C}_{3} \mathrm{H}_{6}$ (propylene) and $\mathrm{C}_{4} \mathrm{H}_{8}$ (transbutene): Overall rate constants and atomic hydrogen branching ratios. Phys. Chem. Chem. Phys. 6, 5396-5401.

Loison, J.C., Bergeat, A., 2009. Rate constants and the $\mathrm{H}$ atom branching ratio of the reactions of the methylidyne $\mathrm{CH}\left(\mathrm{X}^{2} \Pi\right)$ radical with $\mathrm{C}_{2} \mathrm{H}_{2}$, $\mathrm{C}_{2} \mathrm{H}_{4}, \mathrm{C}_{3} \mathrm{H}_{4}$ (methylacetylene and allene), $\mathrm{C}_{3} \mathrm{H}_{6}$ (propene) and $\mathrm{C}_{4} \mathrm{H}_{8}$ (transbutene). Phys. Chem. Chem. Phys. 11, 655-664.

Loison, J.C., Bergeat, A., Caralp, F., Hannachi, Y., 2006. Rate constants and $\mathrm{H}$ atom branching ratios of the gas-phase reactions of methylidyne $\mathrm{CH}\left(\mathrm{X}^{2} \Pi\right)$ radical with a series of alkanes. J. Phys. Chem. A 110, 13500-13506.

López-Moreno, J., Molina-Cuberos, G., Hamelin, M., Grard, R., Simões, F., Godard, R., Schwingenschuh, K., Béghin, C., Berthelier, J., Brown, V., Falkner, P., Ferri, F., Fulchignoni, M., Jernej, I., Jerónimo, J., Rodrigo, R., Trautner, R., 2008. Structure of Titan's low altitude ionized layer from the Relaxation Probe onboard HUYGENS. Geophys. Res. Lett. 35, \#L22104.

Lorenz, R., Mitchell, K., Kirk, R., Hayes, A., Aharonson, O., Zebker, H., Paillou, P., Radebaugh, J., Lunine, J., Janssen, M., Wall, S., Lopes, R., Stiles, B., Ostro, S., Mitri, G., Stofan, E., 2008. Titan's inventory of organic surface materials. Geophys. Res. Lett. 35, \#L02206.

Lunine, J., 2010. Titan and habitable planets around M-dwarfs. Faraday Discuss. 147, 405-418.

Luspay-Kuti, A., Mandt, K., Plessis, S., Greathouse, T., 2015. Effects of nitrogen photoabsorption cross section resolution on minor species vertical profiles in Titan's upper atmosphere. Astrophys. J. 801, \#L14.

Luspay-Kuti, A., Mandt, K., Westlake, J., Plessis, S., Greathouse, T., 2016. The role of nitrogen in Titan's upper atmospheric hydrocarbon chemistry over the solar cycle. Astrophys. J. 823, \#163. 
Luther, K., Oum, K., Sekiguchi, K., Troe, J., 2004. Recombination of benzyl radicals: dependence on the bath gas, temperature, and pressure. Phys. Chem. Chem. Phys. 6, 4133-4141.

Luther, K., Troe, J., Weitzel, K.M., 1990. C-C and C-H bond splits of laserexcited aromatic molecules. 2. In situ measurements of branching ratios. J. Phys. Chem. 94, 6316-6320.

Mackay, G., Betowski, D., Payzant, J., Schiff, H., Bohme, D., 1976. Rate constants at $297 \mathrm{~K}$ for proton-transfer reactions with $\mathrm{HCN}$ and $\mathrm{CH}_{3} \mathrm{CN}$. Comparisons with classical theories and exothermicity. J. Phys. Chem. 80, 2919-2922.

Mackay, G., Schiff, H., Bohme, D., 1981. A room-temperature study of the kinetics and energetics for the protonation of ethane. Can. J. Chem. 59, $1771-1778$.

Mackay, G., Tanaka, K., Bohme, D., 1977. Rate constants at 297 K for protontransfer reactions with $\mathrm{C}_{2} \mathrm{H}_{2}$ : An assessment of the average quadrupole orientation theory. Int. J. Mass Spectrom. 24, 125-136.

Mackay, G., Vlachos, G., Bohme, D., Schiff, H., 1980. Studies of reactions involving $\mathrm{C}_{2} \mathrm{H}_{x}^{+}$ions with $\mathrm{HCN}$ using a modified selected ion flow tube. Int. J. Mass Spectrom. 36, 259-270.

Madanian, H., Cravens, T., Richard, M., Waite Jr., J., Edberg, N., Westlake, J., Wahlund, J.E., 2016. Solar cycle variations in ion composition in the dayside ionosphere of Titan. J. Geophys. Res. Space Physics 121, doi:10.1002/2015JA022274.

Magee, B., Waite Jr., J., Mandt, K., Westlake, J., Bell, J., Gell, D., 2009. INMS-derived composition of Titan's upper atmosphere: Analysis methods and model comparison. Planet. Space Sci. 57, 1895-1916.

Maltagliati, L., Bézard, B., Vinatier, S.and Hedman, M., Lellouch, E., Nicholson, P., Sotin, C., de Kok, R., Sicardy, B., 2015. Titan's atmosphere as observed by Cassini/VIMS solar occultations: $\mathrm{CH}_{4}, \mathrm{CO}$ and evidence for $\mathrm{C}_{2} \mathrm{H}_{6}$ absorption. Icarus 248, 1-24.

Mandt, K., Gell, D., Perry, M., Waite Jr., J., Crary, F., Young, D., Magee, B., Westlake, J., Cravens, T., Kasprzak, W., Miller, G., Wahlund, J.E., Ågren, K., Edberg, N., Heays, A., Lewis, B., Gibson, S., de la Haye, V., Liang, M.C., 2012a. Ion densities and composition of Titan's upper atmosphere derived from the Cassini Ion Neutral Mass Spectrometer: Analysis methods and comparison of measured ion densities to photochemical model simulations. J. Geophys. Res. 117, \#E10006.

Mandt, K., Waite Jr., J., Lewis, W., Magee, B., Bell, J., Lunine, J., Mousis, O., Cordier, D., 2009. Isotopic evolution of the major constituents of Titan's atmosphere based on Cassini data. Planet. Space Sci. 57, 1917-1930. 
Mandt, K., Waite Jr., J., Teolis, B., Magee, B., Bell, J., Westlake, J., Nixon, C., Mousis, O., Lunine, J., 2012b. The ${ }^{12} \mathrm{C} /{ }^{13} \mathrm{C}$ ratio on Titan from Cassini INMS measurements and implications for the evolution of methane. Astrophys. J. 749 , \#160.

Marquette, J.B., Rebrion, C., Rowe, B.R., 1988. Reactions of $\mathrm{N}^{+}\left({ }^{3} \mathrm{P}\right)$ ions with normal, para, and deuterated hydrogens at low temperatures. J. Chem. Phys. 89, 2041-2047.

Marston, G., Nesbitt, F., Nava, D., Payne, W., Stief, L., 1989a. Temperature dependence of the reaction of nitrogen atoms with methyl radicals. J. Phys. Chem. 93, 5769-5774.

Marston, G., Nesbitt, F., Stief, L., 1989b. Branching ratios in the atomic nitrogen + methyl reaction: formation of the methylene amidogen $\left(\mathrm{H}_{2} \mathrm{CN}\right)$ radical. J. Chem. Phys. 91, 3483-3491.

Marston, G., Stief, L., 1989. Structure, spectroscopy and kinetics of the methylene amidogen $\left(\mathrm{H}_{2} \mathrm{CN}\right)$ radical. Research on Chemical Intermediates 12, 161186.

Marten, A., Hidayat, T., Biraud, Y., Moreno, R., 2002. New millimeter heterodyne observations of Titan: Vertical distributions of $\mathrm{HCN} \mathrm{HC}_{3} \mathrm{~N}, \mathrm{CH}_{3} \mathrm{CN}$, and the isotopic ratio ${ }^{15} \mathrm{~N} /{ }^{14} \mathrm{~N}$ in its atmosphere. Icarus $158,532-544$.

Martin, T., Melton, C., 1960. Hydrogen atom abstraction reactions by cyanide ion-radicals. J. Chem. Phys. 32, 700-704.

Martinez, O., Yang, Z., Demarais, N., Snow, T., Bierbaum, V., 2010. Gas-phase reactions of hydride anion, $\mathrm{H}^{-}$. Astrophys. J. 720, 173-177.

Mason, E., Marrero, T., 1970. The diffusion of atoms and molecules, in: Bates, D., Esterman, I. (Eds.), Advances in Atomic and Molecular Physics. Academic Press, New York. volume 6, pp. 155-232.

Mathews, L., Adams, N., 2011. Experimental study of the gas phase chemistry of $\mathrm{C}_{3} \mathrm{H}_{3}{ }^{+}$with several cyclic molecules. Int. J. Mass Spectrom. 299, 139-144.

May, O., Fedor, J., Allan, M., 2009. Isotope effect in dissociative electron attachment to acetylene. Phys. Rev. A 80, \#012706.

May, O., Fedor, J., Ibănescu, C., Allan, M., 2008. Absolute cross sections for dissociative electron attachment to acetylene and diacetylene. Phys. Rev. A 77, \#040701.

May, O., Kubala, D., Allan, M., 2010. Absolute cross sections for dissociative electron attachment to HCN and DCN. Phys. Rev. A 82, \#010701(R).

McEwan, M., Anicich, V., 1995. A low-pressure study of $\mathrm{C}_{2} \mathrm{~N}_{2}$ ion chemistry. J. Phys. Chem. 99, 12204-12208. 
McEwan, M., Anicich, V., 2007. Titan's ion chemistry: A laboratory perspective. Mass Spectrom. Rev. 26, 281-319.

McEwan, M., Anicich, V., Huntress, W.T., J., 1981. An ICR investigation of ion-molecule reactions of HCN. Int. J. Mass Spectrom. Ion Processes 37, $273-281$.

McEwan, M., Scott, G., Anicich, V., 1998. Ion-molecule reactions relevant to Titan's ionosphere. Int. J. Mass Spectrom. 172, 209-219.

McKee, K., Blitz, M., Hughes, K., Pilling, M., Qian, H.B., Taylor, A., Seakins, P.W., 2003. $\mathrm{H}$ atom branching ratios from the reactions of $\mathrm{CH}$ with $\mathrm{C}_{2} \mathrm{H}_{2}$, $\mathrm{C}_{2} \mathrm{H}_{4}, \mathrm{C}_{2} \mathrm{H}_{6}$, and neo- $\mathrm{C}_{5} \mathrm{H}_{12}$ at room temperature and 25 Torr. J. Phys. Chem. A $107,5710-5716$.

McLain, J., Adams, N., 2009. Flowing afterglow studies of temperature dependencies for electron dissociative recombination of $\mathrm{HCNH}^{+}, \mathrm{CH}_{3} \mathrm{CNH}^{+}$and $\mathrm{CH}_{3} \mathrm{CH}_{2} \mathrm{CNH}^{+}$and their symmetrical proton-bound dimers. Planet. Space Sci. $57,1642-1647$.

McLain, J., Molek, C., Osborne Jr., D., Adams, N., 2009. Flowing afterglow studies of the electron recombination of protonated cyanides $(\mathrm{RCN}) \mathrm{H}^{+}$and their proton-bound dimer ions $(\mathrm{RCN})_{2} \mathrm{H}^{+}$where $\mathrm{R}$ is $\mathrm{H}, \mathrm{CH}_{3}$, and $\mathrm{CH}_{3} \mathrm{CH}_{2}$. Int. J. Mass Spectrom. 282, 85-90.

McLain, J., Poterya, V., Molek, C., Babcock, L., Adams, N., 2004. Flowing afterglow studies of the temperature dependencies for dissociative recombination of $\mathrm{O}_{2}{ }^{+}, \mathrm{CH}_{5}{ }^{+}, \mathrm{C}_{2} \mathrm{H}_{5}{ }^{+}$, and $\mathrm{C}_{6} \mathrm{H}_{7}{ }^{+}$with electrons. J. Phys. Chem. A 108, 6704-6708.

McLain, J., Poterya, V., Molek, C., Jackson, D., Babcock, L., Adams, N., 2005. $\mathrm{C}_{3} \mathrm{H}_{3}{ }^{+}$isomers: Temperature dependencies of production in the $\mathrm{H}_{3}{ }^{+}$reaction with allene and loss by dissociative recombination with electrons. J. Phys. Chem. A 109, 5119-5123.

Mebel, A., Diau, E., Lin, M., Morokuma, K., 1996. Theoretical rate constants for the $\mathrm{NH}_{3}+\mathrm{NO}_{x} \rightarrow \mathrm{NH}_{2}+\mathrm{HNO}_{x}(\mathrm{x}=1,2)$ reactions by ab initio MO/VTST calculations. J. Phys. Chem. 100, 7517-7525.

Mebel, A., Georgievskii, Y., Jasper, A., Klippenstein, S., 2017. Temperature and pressure-dependent rate coefficients for the HACA pathways from benzene to naphthalene. Proc. Comb. Inst. http://dx.doi.org/10.1016/j.proci.2016.07.013.

Mebel, A., Kislov, V., Kaiser, R., 2006a. Ab initio/Rice-Ramsperger-KasselMarcus study of the singlet $\mathrm{C}_{4} \mathrm{H}_{4}$ potential energy surface and of the reactions of $\mathrm{C}_{2}\left(\mathrm{X}^{1} \Sigma_{g}^{+}\right)$with $\mathrm{C}_{2} \mathrm{H}_{4}\left(\mathrm{X}^{1} \mathrm{~A}_{g}^{1}\right)$ and $\mathrm{C}\left({ }^{1} \mathrm{D}\right)$ with $\mathrm{C}_{3} \mathrm{H}_{4}$ (allene and methylacetylene). J. Phys. Chem. A 125, 133113. 
Mebel, A., Kislov, V., Kaiser, R., 2006b. Potential energy surface and product branching ratios for the reaction of dicarbon $\mathrm{C}_{2}\left(\mathrm{X}^{1} \Sigma_{g}^{+}\right)$, with methylacetylene, $\mathrm{CH}_{3} \mathrm{CCH}\left(\mathrm{X}^{1} \mathrm{~A}_{1}\right)$ : an ab initio/RRKM study. J. Phys. Chem. A 110, 2421-2433.

Melko, J., Ard, S., Johnson, R., Shuman, N., Guo, H., Viggiano, A., 2014. Temperature-dependent kinetics of charge transfer, hydrogen-atom transfer, and hydrogen-atom expulsion in the reaction of $\mathrm{CO}^{+}$with $\mathrm{CH}_{4}$ and $\mathrm{CD}_{4}$. J. Phys. Chem. A 118, 8141-8146.

Meller, R., Moortgat, G.K., 2000. Temperature dependence of the absorption cross sections of formaldehyde between 223 and $323 \mathrm{~K}$ in the wavelength range 225-375 nm. J. Geophys. Res. 105, 7089-7102.

Mendes, M., Buhr, H., Berg, M., Froese, M., Grieser, M., Heber, O., JordonThaden, B., Krantz, C., Novotný, O., Novotny, S., Orlov, D., Petrignani, A., Rappaport, M., Repnow, R., Schwalm, D., Shornikov, A., Stützel, J., Zajfman, D., Wolf, A., 2012. Cold electron reactions producing the energetic isomer of hydrogen cyanide in interstellar clouds. Astrophys. J. 746, \#L8.

Millar, T., Walsh, C., Cordiner, M., Chuimín, R., Herbst, E., 2007. Hydrocarbon anions in interstellar clouds and circumstellar envelopes. Astrophys. J. 662, L87-L90.

Miller, J., Klippenstein, S., 2002. Solution of some one- and two-dimensional master equation models for thermal dissociation: The dissociation of methane in the low-pressure limit. J. Phys. Chem. A 106, 4904-4913.

Miller, J., Klippenstein, S., 2003. The recombination of propargyl radicals and other reactions on a $\mathrm{C}_{6} \mathrm{H}_{6}$ potential. J. Phys. Chem. A 107, 7783-7799.

Miller, J., Klippenstein, S., 2004. The $\mathrm{H}+\mathrm{C}_{2} \mathrm{H}_{2}(+\mathrm{M}) \rightleftharpoons \mathrm{C}_{2} \mathrm{H}_{3}(+\mathrm{M})$ and $\mathrm{H}+\mathrm{C}_{2} \mathrm{H}_{4}(+\mathrm{M}) \rightleftharpoons \mathrm{C}_{2} \mathrm{H}_{5}(+\mathrm{M})$ reactions: Electronic structure, variational transition-state theory, and solutions to a two-dimensional master equation. Phys. Chem. Chem. Phys. 6, 1192-1202.

Miller, J., Klippenstein, S., 2006. Master equation methods in gas phase chemical kinetics. J. Phys. Chem. A 110, 10528-10544.

Miller, J., Klippenstein, S., 2013. Dissociation of propyl radicals and other reactions on a $\mathrm{C}_{3} \mathrm{H}_{7}$ potential. J. Phys. Chem. A 117, 2718-2727.

Miller, J., Klippenstein, S., Robertson, S., 2000. A theoretical analysis of the reaction between vinyl and acetylene: Quantum chemistry and solution of the master equation. J. Phys. Chem. A 104, 7525-7536.

Miller, J., Senosiain, J., Klippenstein, S., Georgievskii, Y., 2008. Reactions over multiple, interconnected potential wells: Unimolecular and bimolecular reactions on a $\mathrm{C}_{3} \mathrm{H}_{5}$ potential. J. Phys. Chem. A 112, 9429-9438. 
Miller, T., Shuman, N., Viggiano, A., 2012. Behavior of rate coefficients for ion-ion mutual neutralization, 300-550 K. J. Chem. Phys. 136, \#204306.

Milligan, D., Fairley, D., Meot-Ner, M., McEwan, M., 1998. Proton affinity of cyanogen and association reactions of $\mathrm{C}_{2} \mathrm{~N}_{2} \mathrm{H}^{+}$and $\mathrm{C}_{2} \mathrm{~N}_{2} \mathrm{CH}_{3}{ }^{+}$. Int. J. Mass Spectrom. Ion Processes 179/180, 285-291.

Milligan, D., Freeman, C., Maclagan, R., McEwan, M., Wilson, P., Anicich, V., 2001. Termolecular ion-molecule reactions in Titan's atmosphere. II: The structure of the associato adducts of $\mathrm{HCNH}^{+}$with $\mathrm{C}_{2} \mathrm{H}_{2}$ and $\mathrm{C}_{2} \mathrm{H}_{4}$. J. Am. Soc. Mass Spectrom. 12, 557-564.

Milligan, D., McEwan, M., 2000. H3+ + O: an experimental study. Chemical Physics Letters 319, 482-485.

Milligan, D., Wilson, P., Freeman, C., Meot-Ner, M., McEwan, M., 2002. Dissociative proton transfer reactions of $\mathrm{H}_{3}{ }^{+}, \mathrm{N}_{2} \mathrm{H}^{+}$, and $\mathrm{H}_{3} \mathrm{O}^{+}$with acyclic, cyclic, and aromatic hydrocarbons and nitrogen compounds, and astrochemical implications. J. Phys. Chem. A 106, 9745-9755.

Mitchell, J., 1990. The dissociative recombination of molecular ions. Phys. Rep. $186,215-248$

Mitsuke, K., Suzuki, S., Imamura, T., Koyano, I., 1991. Negative-ion mass spectrometric study of ion-pair formation in the vacuum ultraviolet. IV. $\mathrm{CH}_{4}$ $\rightarrow \mathrm{H}^{-}+\mathrm{CH}_{3}^{+}$and $\mathrm{CD}_{4} \rightarrow \mathrm{D}^{-}+\mathrm{CD}_{3}{ }^{+}$. J. Chem. Phys. 94, 6003-6006.

Molek, C., Poterya, V., Adams, N., McLain, J., 2009. Development of a novel technique for quantitatively determining the products of electron-ion dissociative recombination. Int. J. Mass Spectrom. 285, 1-11.

Molina-Cuberos, G., Lammer, H., Stumptner, W., Schwingenschuh, K., Rucker, H., López-Moreno, J., Rodrigo, R., Tokano, T., 2001. Ionospheric layer induced by meteoric ionization in Titan's atmosphere. Planet. Space Sci. 49, $143-153$.

Molina-Cuberos, G., López-Moreno, J., Rodrigo, R., 2000. Influence of electrophilic species on the lower ionosphere of Titan. Geophys. Res. Lett. 27, $1351-1354$.

Molina-Cuberos, G., López-Moreno, J., Rodrigo, R., Lara, L., 1999a. Chemistry of the galactic cosmic ray induced ionosphere of Titan. J. Geophys. Res. 104, 21997-22024.

Molina-Cuberos, G., Schwingenschuh, K., López-Moreno, J., Rodrigo, R., Lara, L., Anicich, V., 2002. Nitriles produced by ion chemistry in the lower ionosphere of Titan. J. Geophys. Res. 107, \#5099.

Molina-Cuberos, G.J., López-Moreno, J.J., Rodrigo, R., Lara, L.M., O'Brien, K., 1999b. Ionization by cosmic rays of the atmosphere of Titan. Planet. Space Sci. 47, 1347-1354. 
Morales, S., Bennett, C., Le Picard, S., Canosa, A., Sims, I., Sun, B., Chen, P., Chang, A., Kislov, V., Mebel, A., Gu, X., Zhang, F., Maksyutenko, P., Kaiser, R., 2011. A crossed molecular beam, low-temperature kinetics, and theoretical investigation of the reaction of the cyano radical $(\mathrm{CN})$ with 1,3-butadiene $\left(\mathrm{C}_{4} \mathrm{H}_{6}\right)$. A route to complex nitrogen-bearing molecules in low-temperature extraterrestrial environments. Astrophys. J. 742, 26.

Morales, S., Le Picard, S., Canosa, A., Sims, I., 2010. Experimental measurements of low temperature rate coefficients for neutral-neutral reactions of interest for atmospheric chemistry of Titan, Pluto and Triton: Reactions of the CN radical. Faraday Discuss. 147, 155-171.

Mordaunt, D.H., Ashfold, M.N.R., Dixon, R.N., 1994. Dissociation dynamics of $\mathrm{H}_{2} \mathrm{O}\left(\mathrm{D}_{2} \mathrm{O}\right)$ following photoexcitation at the Lyman- $\alpha$ wavelength $(121.6 \mathrm{~nm})$. J. Chem. Phys. 100, 7360-7375.

Moreno, R., Lellouch, E., Lara, L., Courtin, R., Bockelée-Morvan, D., Hartogh, P., Rengel, M., Biver, N., Banaszkiewicz, M., González, A., 2011. First detection of hydrogen isocyanide (HNC) in Titan's atmosphere. Astron. Astrophys. 536, \#L12.

Moreno, R., Lellouch, E., Lara, L., Feuchtgruber, H., Rengel, M., Hartogh, P., Courtin, R., 2012. The abundance, vertical distribution and origin of $\mathrm{H}_{2} \mathrm{O}$ in Titan's atmosphere: Herschel observations and photochemical modelling. Icarus 221, 753-767.

Morgan, C., Drabbels, M., Wodtke, A., 1996. The correlated product state distribution of ketene photodissociation at $308 \mathrm{~nm}$. J. Chem. Phys. 104, $7460-7474$.

Moses, J., Allen, M., Yung, Y., 1992. Hydrocarbon nucleation and aerosol formation in Neptune's atmosphere. Icarus 99, 318-346.

Moses, J.I., Fouchet, T., Bézard, B., Gladstone, G., Lellouch, E., Feuchtgruber, H., 2005. Photochemistry and diffusion in Jupiter's stratosphere: Constraints from ISO observations and comparisons with other giant planets. J. Geophys. Res. 110, \#E08001.

Mota, R., Parafita, R., Giuliani, A., Hubin-Franskin, M.J., Lourenço, J., Garcia, G., Hoffmann, S., Mason, M., Ribeiro, P., Raposo, M., Limão-Vieira, P., 2005. Water VUV electronic state spectroscopy by synchrotron radiation. Chem. Phys. Lett. 416, 152-159.

Mount, G.H., Warden, E.S., Moos, H.W., 1977. Photoabsorption cross sections of methane from 1400 to $1850 \AA$. Astrophys. J. 214, L47-L49.

Mul, P., McGowan, J., Defrance, P., Mitchell, J., 1983. Merged electron-ion beam experiments: V. Dissociative recombination of $\mathrm{OH}^{+}, \mathrm{H}_{2} \mathrm{O}^{+}, \mathrm{H}_{3} \mathrm{O}^{+}$and $\mathrm{D}_{3} \mathrm{O}^{+}$. J. Phys. B: At. Mol. Phys. 16, 3099-3107. 
Mullen, C., Smith, M., 2005. Low temperature $\mathrm{NH}\left(\mathrm{X}^{3} \Sigma^{-}\right)$radical reactions with NO, saturated, and unsaturated hydrocarbons studied in a pulsed supersonic Laval nozzle flow reactor between 53 and 188 K. J. Phys. Chem. A 109, 1391-1399.

Murphy, J., Vakhtin, A., Leone, S., 2003. Laboratory kinetics of $\mathrm{C}_{2} \mathrm{H}$ radical reactions with ethane, propane, and n-butane at $\mathrm{T}=96-296 \mathrm{~K}$ : Implications for Titan. Icarus 163, 175-181.

Nahar, S., 1999. Electron-ion recombination rate coefficients, photoionization cross sections, and ionization fractions for astrophysically abundant elements. II. Oxygen ions. Astrophys. J. Suppl. Ser. 120, 131-145.

Nahar, S., Pradhan, A., 1997. Electron-ion recombination rate coefficients, photoionization cross sections, and ionization fractions for astrophysically abundant elements. I. Carbon and nitrogen. Astrophys. J. Suppl. Ser. 111, 339-355.

Nakajima, M., Matsugi, A., Miyoshi, A., 2009. Mechanism and kinetic isotope effect of the reaction of $\mathrm{C}_{2}\left(\mathrm{X}^{1} \Sigma_{g}^{+}\right)$radicals with $\mathrm{H}_{2}$ and $\mathrm{D}_{2}$. J. Phys. Chem. A $113,8963-8970$.

Nakayama, T., Watanabe, K., 1964. Absorption and photoionization coefficients of acetylene, propyne, and 1-butyne. J. Chem. Phys. 40, 558-561.

Naroznik, M., Niedzielski, J., 1986. Propylene photolysis at $6.7 \mathrm{eV}$ : Calculation of the quantum yields for the secondary processes. J. Photochem. 32, 281-292.

Naulin, C., Daugey, N., Hickson, K., Costes, M., 2009. Dynamics of the reactions of $\mathrm{C}\left({ }^{3} \mathrm{P}_{J}\right)$ atoms with ethylene, allene, and methylacetylene at low energy revealed by Doppler-Fizeau spectroscopy. J. Phys. Chem. A 113, 1444714457 .

Neau, A., Al-Khalili, A., Rosén, S., Le Padellec, A., Derkatch, A., Shi, W., Vikor, L., Larsson, M., Semaniak, J., Thomas, R., Någård, M., Andersson, K., Danared, H., af Ugglas, M., 2000. Dissociative recombination of $\mathrm{D}_{3} \mathrm{O}^{+}$ and $\mathrm{H}_{3} \mathrm{O}^{+}$: Absolute cross sections and branching ratios. J. Chem. Phys. 113, $1762-1770$.

Nesbitt, F., Marston, G., Stief, L., 1990. Kinetic studies of the reactions of methyleneaminylium and dideuteromethyleneaminylium radicals with nitrogen and hydrogen atoms. J. Phys. Chem. 94, 4946-4951.

Nguyen, H., Nguyen, H., Nguyen, T.N., Hoang, H., Vereecken, L., 2014. Theoretical study on the reaction of the methylidyne radical, $\mathrm{CH}\left(\mathrm{X}^{2} \Pi\right)$, with formaldehyde, $\mathrm{CH}_{2} \mathrm{O}$. J. Phys. Chem. A 118, 8861-8871.

Nguyen, M., Sengupta, D., Ha, T.K., 1996. Another look at the decomposition of methyl azide and methanimine: How is HCN formed? J. Phys. Chem. 100, 6499-6503. 
Nguyen, T., Mebel, A., Lin, S., Kaiser, R., 2001. Product branching ratios of the $\mathrm{C}\left({ }^{3} \mathrm{P}\right)+\mathrm{C}_{2} \mathrm{H}_{3}\left({ }^{2} \mathrm{~A}^{\prime}\right)$ and $\mathrm{CH}\left({ }^{2} \Pi\right)+\mathrm{C}_{2} \mathrm{H}_{2}\left({ }^{1} \Sigma_{g}^{+}\right)$reactions and photodissociation of $\mathrm{H}_{2} \mathrm{CCa}{ }^{\circ} \mathrm{CH}\left({ }^{2} \mathrm{~B}_{1}\right)$ at $193 \mathrm{~nm}$ and $242 \mathrm{~nm}$ : An ab initio/RRKM study. J. Phys. Chem. A 105, 11549-11559.

Ni, C.K., Huang, J., Chen, Y., Kung, A., Jackson, W., 1999. Photodissociation of propyne and allene at $193 \mathrm{~nm}$ with vacuum ultraviolet detection of the products. J. Chem. Phys. 110, 3320-3325.

Nicolas, C., Alcaraz, C., Thissen, R., Vervloet, M., Dutuit, O., 2003a. Dissociative photoionization of $\mathrm{N}_{2}$ in the 24-32 eV photon energy range. J. Phys. B At. Mol. Opt. Phys. 36, 2239-2251.

Nicolas, C., Torrents, R., Gerlich, D., 2003b. Integral and differential cross section measurements at low collision energies for the $\mathrm{N}_{2}{ }^{+}+\mathrm{CH}_{4} / \mathrm{CD}_{4}$ reactions. J. Chem. Phys. 118, 2723-2730.

Niedzielski, J., Wlodzimierz, M., Gawlowski, J., 1982. Gas phase photolysis of propylene at 8.4 and $10.0 \mathrm{eV}$. J. Photochem. 19, 123-131.

Niemann, H., Atreya, S., Demick, J., Gautier, D., Haberman, J., Harpold, D., Kasprzak, W., Lunine, J., Owen, T., Raulin, F., 2010. Composition of Titan's lower atmosphere and simple surface volatiles as measured by the Cassini-Huygens probe gas chromatograph mass spectrometer experiment. J. Geophys. Res. 115, \#E12006.

Niemann, H.B., Atreya, S.K., Bauer, S.J., Carignan, G.R., Demick, J.E., Frost, R.L., Gautier, D., Haberman, J.A., Harpold, D.N., Hunten, D.M., Israel, G., Lunine, J.I., Kasprzak, W., Owen, T.C., Paulkovich, M., Raulin, F., Raaen, E., Way, S.H., 2005. The abundances of constituents of Titan's atmosphere from the GCMS instrument on the Huygens probe. Nature 438, 779-784.

Nixon, C., Achterberg, R., Teanby, N., Irwin, P., Flaud, J.M., Kleiner, I., Dehayem-Kamadjeu, A., Brown, L., Sams, R., Bézard, B., Coustenis, A., Ansty, T., Mamoutkine, A., Vinatier, S., Bjoraker, G., Jennings, D., Romani, P., Flasar, F., 2010. Upper limits for undetected trace species in the stratosphere of Titan. Faraday Discuss. 147, 65-81.

Nixon, C., Jennings, D., Bézard, B., Vinatier, S., Teanby, N., Sung, K., Ansty, T., Irwin, P., Gorius, N., Cottini, V., Coustenis, A., Flasar, F., 2013. Detection of propene in Titan's stratosphere. Astrophys. J. 776, \#L14.

Nixon, C.A., Teanby, N.A., Irwin, P.G.J., Hörst, S.M., 2013. Upper limits for $\mathrm{PH}_{3}$ and $\mathrm{H}_{2} \mathrm{~S}$ in Titan's atmosphere from Cassini CIRS. Icarus 224, 253-256.

Nizamov, B., Dagdigian, P., 2003. Spectroscopic and kinetic investigation of methylene amidogen by cavity ring-down spectroscopy. J. Phys. Chem. A $107,2256-2263$. 
Nizamov, B., Leone, S., 2004a. Kinetics of $\mathrm{C}_{2} \mathrm{H}$ reactions with hydrocarbons and nitriles in the 104-296 K temperature range. J. Phys. Chem. A 108, $1746-1752$.

Nizamov, B., Leone, S., 2004b. Rate coefficients and kinetic isotope effect for the $\mathrm{C}_{2} \mathrm{H}$ reactions with $\mathrm{NH}_{3}$ and $\mathrm{ND}_{3}$ in the 104-294 $\mathrm{K}$ temperature range. J. Phys. Chem. A 108, 3766-3771.

Noller, B., Fischer, I., 2007. Photodissociation dynamics of the 2-propyl radical, $\mathrm{C}_{3} \mathrm{H}_{7}$. J. Chem. Phys. 126, \#144302.

North, S., Hall, G., 1996. The radical photodissociation channel of acrylonitrile. Chem. Phys. Lett. 263, 148-153.

Nuth, J., Glicker, S., 1982. The vacuum ultraviolet spectra of $\mathrm{HCN}, \mathrm{C}_{2} \mathrm{~N}_{2}$, and $\mathrm{CH}_{3}$ CN. J. Quant. Spectr. Rad. Trans. 28, 223-231.

Obi, K., Akimoto, H., Ogata, Y., Tanaka, I., 1971. Photolyses of propane, nbutane, and cyclobutane at Xe and $\mathrm{Kr}$ resonance lines. J. Chem. Phys. 55, $3822-3828$.

Oertel, H., Schenk, H., Baumgärtel, H., 1980. Ion pair formation from photon irradiation of $\mathrm{O}_{2}, \mathrm{NO}$ and $\mathrm{CO}$ in $17-30 \mathrm{eV}$. Chem. Phys. 46, 251-262.

Ohmori, K., Miyoshi, A., Matsui, H., Washida, N., 1990. Studies on the reaction of acetaldehyde and acetyl radicals with atomic hydrogen. J. Phys. Chem. 94, 3253-3255.

Öjekull, J., Andersson, P., Någård, M., Pettersson, J., Derkatch, A., Neau, A., Rosén, S., Thomas, R., Larsson, M., Österdahl, F., Semaniak, J., Danared, H., Källberg, A., af Ugglas, M., Marković, N., 2004. Dissociative recombination of $\mathrm{NH}_{4}^{+}$and $\mathrm{ND}_{4}{ }^{+}$ions: Storage ring experiments and ab initio molecular dynamics. J. Chem. Phys. 120, 7391-7399.

Okabe, H., 1978. Photochemistry of Small Molecules. volume 1. John Wiley \& Sons, New York. 1st edition.

Okabe, H., 1981. Photochemistry of acetylene at 1470 A. J. Chem. Phys. 75, $2772-2778$.

Okabe, H., Becker, D., 1963. Vacuum ultraviolet photochemistry. VII. Photolysis of n-butane. J. Chem. Phys. 39, 2549-2555.

Okabe, H., McNesby, J., 1962. Vacuum ultraviolet photochemistry. IV. Photolysis of propane. J. Chem. Phys. 37, 1340-1346.

Opansky, B., Leone, S., 1996. Rate coefficients of $\mathrm{C}_{2} \mathrm{H}$ with $\mathrm{C}_{2} \mathrm{H}_{4}, \mathrm{C}_{2} \mathrm{H}_{6}$, and $\mathrm{H}_{2}$ from 150 to 359 K. J. Phys. Chem. 100, 19904-19910. 
Operti, L., Rabezzana, R., Turco, F., Vaglio, G., 2004. Gas-phase ion chemistry of the propyne/ammonia and silane/propyne/ammonia systems. Int. J. Mass Spectrom. 232, 139-146.

Orkin, V., Huie, R., Kurylo, M., 1997. Rate constants for the reactions of $\mathrm{OH}$ with HFC-245cb $\left(\mathrm{CH}_{3} \mathrm{CF}_{2} \mathrm{CF}_{3}\right)$ and some fluoroalkenes $\left(\mathrm{CH}_{2} \mathrm{CHCF}_{3}\right.$, $\mathrm{CH}_{2} \mathrm{CFCF}_{3}, \mathrm{CF}_{2} \mathrm{CFCF}_{3}$, and $\mathrm{CF}_{2} \mathrm{CF}_{2}$ ). J. Phys. Chem. A 101, 9118-9124.

Osamura, Y., Petrie, S., 2004. NCCN and NCCCCN formation in Titan's atmosphere: 1. Competing reactions of precursor $\operatorname{HCCN}\left({ }^{3} \mathrm{~A}^{\prime \prime}\right)$ with $\mathrm{H}\left({ }^{2} \mathrm{~S}\right)$ and $\mathrm{CH}_{3}\left({ }^{2} \mathrm{~A}^{\prime}\right)$. J. Phys. Chem. A 108, 3615-3622.

Osborne, D.J., Lawson, P., Adams, N., 2011. Flowing afterglow studies of dissociative electron-ion recombination for a series of single ring compounds at room temperature. Int. J. Mass Spectrom. 305, 35-39.

Ozturk, F., Baykut, G., Moini, M., Eyler, J., 1987. Reactions of $\mathrm{C}_{3} \mathrm{H}_{3}{ }^{+}$with acetylene and diacetylene in the gas phase. J. Phys. Chem. 91, 4360-4364.

Ozturk, F., Moini, M., Brill, F., Eyler, J., Buckley, T., Lias, S., Ausloos, P., 1989. Reactions of $\mathrm{C}_{5} \mathrm{H}_{3}{ }^{+}$and $\mathrm{C}_{5} \mathrm{H}_{5}{ }^{+}$ions with acetylene and diacetylene. J. Phys. Chem. 93, 4038-4044.

Palmer, M.Y., Cordiner, M.A., Nixon, C.A., Charnley, S.B., Teanby, N.A., Kisiel, Z., Irwin, P.G., Mumma, M.J., 2017. ALMA detection and astrobiological potential of vinyl cyanide on Titan. Science Advances 3, \#e1700022.

Pantos, E., Philis, J., Bolovinos, A., 1978. The extinction coefficient of benzene vapor in the region 4.6 to $36 \mathrm{eV}$. J. Mol. Spectrosc. 72, 36-43.

Páramo, A., Canosa, A., Le Picard, S., Sims, I., 2008. Rate coefficients for the reactions of $\mathrm{C}_{2}\left(\mathrm{a}^{3} \Pi_{u}\right)$ and $\mathrm{C}_{2}\left(\mathrm{X}^{1} \Sigma_{g}^{+}\right)$with various hydrocarbons $\left(\mathrm{CH}_{4}\right.$, $\mathrm{C}_{2} \mathrm{H}_{2}, \mathrm{C}_{2} \mathrm{H}_{4}, \mathrm{C}_{2} \mathrm{H}_{6}$, and $\mathrm{C}_{3} \mathrm{H}_{8}$ ): A gas-phase experimental study over the temperature range 24-300 K. J. Phys. Chem. A 112, 9591-9600.

Parent, D., 1989. Reactions of $\mathrm{CnN}+$ with methane and the implications for interstellar chemistry. Astrophys. J. 347, 1183-1186.

Parent, D., 1990. Reactions of the carbene ions $\mathrm{C}_{n} \mathrm{~N}^{+}$with labeled methane: mechanistic interpretation. J. Am. Chem. Soc. 112, 5966-5973.

Parent, D., McElvany, S., 1989. Investigations of small carbon cluster ion structures by reactions with HCN. J. Am. Chem. Soc. 111, 2393-2401.

Park, J., Gheyas, S., Lin, M., 1999. Kinetics of $\mathrm{C}_{6} \mathrm{H}_{5}$ radical reactions with 2-methylpropane, 2,3-dimethylbutane and 2,3,4-trimethylpentane. Int. J. Chem. Kin. 31, 645-653. 
Parker, D., Zhang, F., Kim, Y., Kaiser, R., 2011. On the formation of resonantly stabilized $\mathrm{C}_{5} \mathrm{H}_{3}$ radicals - A crossed beam and ab initio study of the reaction of ground state carbon atoms with vinylacetylene. J. Phys. Chem. A 115, 593-601.

Parkes, D., 1972. Oxygen negative ion reactions with carbon dioxide and carbon monoxide. Part 1. J. Chem. Soc., Faraday Trans. 1 68, 627-640.

Parkinson, W., Rufus, J., Yoshino, K., 2003. Absolute absorption cross section measurements of $\mathrm{CO}_{2}$ in the wavelength region 163-2000 $\mathrm{nm}$ and the temperature dependence. Chem. Phys. 290, 251-256.

Payne, W., Monks, P., Nesbitt, F., Stief, L., 1996. The reaction between $\left.\mathrm{N}^{4} \mathrm{~S}\right)$ and $\mathrm{C}_{2} \mathrm{H}_{3}$ : Rate constant and primary reaction channels. J. Chem. Phys. 104, 9808-9815.

Peeters, J., Boullart, W., Langhans, I., 1994. Branching ratio of the $\mathrm{C} 2 \mathrm{H} 2+\mathrm{O}$ reaction at $290 \mathrm{~K}$ from kinetic modelling of relative methylene concentration versus time profiles in $\mathrm{C} 2 \mathrm{H} 2 / \mathrm{O} / \mathrm{H}$ systems. Int. J. Chem. Kin. 26, 869-886.

Peko, B., Stephen, T., 2000. Absolute detection efficiencies of low energy H, $\mathrm{H}^{-}$, $\mathrm{H}^{+}, \mathrm{H}_{2}{ }^{+}$and $\mathrm{H}_{3}{ }^{+}$incident on a multichannel plate detector. Nucl. Instrum. Meth. B 171, 597-604.

Pernot, P., Plessis, S., Carrasco, N., 2011. Probalistic representations of partial branching ratios: bridging the gap between experiments and chemical models. J. Phys.: Conf. Ser. 300, \#012027.

Peterson, J., Le Padellec, A., Danared, H., Dunn, G., Larsson, M., Larson, Å., Peverall, R., Strömholm, C., Rosén, S., af Ugglas, M., van der Zande, W., 1998. Dissociative recombination and excitation of $\mathrm{N}_{2}^{+}$: Cross sections and product branching ratios. J. Chem. Phys. 108, 1978-1988.

Petrank, A., Iraqi, M., Dotan, I., Lifshitz, C., 1992. Proton transfer reactions of $\mathrm{C}_{2} \mathrm{H}_{4}{ }^{+}$: the bond energy $\mathrm{D}\left(\mathrm{C}_{2} \mathrm{H}_{3}-\mathrm{H}\right)$. Int. J. Mass Spectrom. Ion Processes $117,223-236$.

Petrie, S., 1996. Novel pathways to $\mathrm{CN}^{-}$within interstellar clouds and circumstellar envelopes: implications for IS and CS chemistry. Mon. Not. R. Astron. Soc. $281,137-144$.

Petrie, S., 2001. Hydrogen isocyanide, HNC: A key species in the chemistry of Titan's ionosphere? Icarus 151, 196-203.

Petrie, S., 2002. Reactivity of HNC with small hydrocarbon radicals. J. Phys. Chem. A 106, 11181-11189.

Petrie, S., Chirnside, T., Freeman, C., McEwan, M., 1991a. The ion/molecule chemistry of $\mathrm{CH}_{2} \mathrm{CHCN}$. Int. J. Mass Spectrom. 107, 319-331. 
Petrie, S., Freeman, C., McEwan, M., 1992. The ion-molecule chemistry of acrylonitrile: Astrochemical implications. Mon. Not. R. Astron. Soc. 257, 438-444.

Petrie, S., Herbst, E., 1997. Some interstellar reactions involving electrons and neutral species: Attachment and isomerization. Astrophys. J. 491, 210-215.

Petrie, S., Knight, J., Freeman, C., MacLagan, R., McEwan, M., Sudkeaw, P., 1991b. The proton affinity and selected ion/molecule reactions of diacetylene. Int. J. Mass Spectrom. 105, 43-54.

Petrie, S., Osamura, Y., 2004. NCCN and NCCCCN formation in Titan's atmosphere: 2. HNC as a viable precursor. J. Phys. Chem. A 108, 36233631 .

Pino, T., Tulej, M., Güthe, F., Pachkov, M., Maier, J., 2002. Photodetachment spectroscopy of the $\mathrm{C}_{2 n} \mathrm{H}^{-}(n=2-4)$ anions in the vicinity of their electron detachment threshold. J. Chem. Phys. 116, 6126-6131.

Piper, L.G., 1993. Reevaluation of the transition-moment function and Einstein coefficients for the $\mathrm{N}_{2}\left(\mathrm{~A}^{3} \Sigma_{u}^{+}-\mathrm{X}^{1} \Sigma_{g}^{+}\right)$transition. J. Chem. Phys. 99, 31743181.

Pirim, C., Gann, R., McLain, J., Orlando, T., 2015. Electron-molecule chemistry and charging processes on organic ices and Titan's icy aerosol surrogates. Icarus 258, 109-119.

Pithawalla, Y., Meot-Ner, M., Gao, J., El Shall, M., Baranov, V., Bohme, D., 2001. Gas-phase oligomerization of propene initiated by benzene radical cation. J. Phys. Chem. A 105, 3908-3916.

Plašil, R., Mehner, T., Dohnal, P., Kotrik, T., Glosík, J., Gerlich, D., 2011. Reactions of cold trapped $\mathrm{CH}^{+}$ions with slow $\mathrm{H}$ atoms. Astrophys. J. 737, \#60.

Plessis, S., Carrasco, N., Dobrijevic, M., Pernot, P., 2012. Production of neutral species in Titan's ionosphere through dissociative recombination of ions. Icarus 219, 254-266.

Polino, D., Klippenstein, S., Harding, L., Georgievskii, Y., 2013. Predictive theory for the addition and insertion kinetics of ${ }^{1} \mathrm{CH}_{2}$ reacting with unsaturated hydrocarbons. J. Phys. Chem. A 117, 12677-12692.

Prasad, S., Huntress Jr., W., 1980. A model for gas phase chemistry in interstellar clouds: I. The basic model, library of chemical reactions, and chemistry among C, N, and O compounds. Astrophys. J. Suppl. Ser. 43, 1-35.

Prodnuk, S., Gronert, S., Bierbaum, V., DePuy, C., 1992. Gas-phase reactions of $\mathrm{C}_{3} \mathrm{H}_{n}^{+}$ions. Org. Mass Spectrom. 27, 416-422. 
Raghavachari, K., Trucks, G., Pople, J., Head-Gordon, M., 1989. A $5^{\text {th }}$-order perturbation comparison of electron correlation theories. Chem. Phys. Lett. $157,479-483$.

Raksit, A., Bohme, D., 1983. An experimental study of the influence of hydration on the reactivity of the hydroxide anion in the gas phase at room temperature. Can. J. Chem. 61, 1683-1689.

Ralchenko, Y., Kramida, A., Reader, J., Team, N.A., 2011. NIST Atomic Spectra Database (version 4.1.0) National Institute of Standards and Technology, Gaithersburg, MD Available: http://physics.nist.gov/asd.

Randeniya, L., Smith, M., 1991. Gas phase reaction rates of $\mathrm{N}_{2}{ }^{+}$with $\mathrm{CH}_{4}$, $\mathrm{O}_{2}$, and $\mathrm{n}-\mathrm{H}_{2}$ at very low temperatures. J. Chem. Phys. 94, 351-356.

Rapp, D., Briglia, D., 1965. Total cross sections for ionization and attachment in gases by electron impact. II. Negative-ion formation. J. Chem. Phys. 43, 1480-1489.

Rawat, P., Prabhudesai, V., Rahman, M., Bhargava Ram, N., Krishnakumar, E., 2008. Absolute cross sections for dissociative electron attachment to $\mathrm{NH}_{3}$ and $\mathrm{CH}_{4}$. Int. J. Mass Spectrom. 277, 96-102.

Rebrion-Rowe, C., Lehfaoui, L., Rowe, B., Mitchell, J., 1998. The dissociative recombination of hydrocarbon ions. II. Alkene and alkyne derived species. J. Chem. Phys. 108, 7185-7189.

Rebrion-Rowe, C., Mostefaoui, T., Laubé, S., Mitchell, J., 2000. The dissociative recombination of hydrocarbon ions. III. Methyl-substituted benzene ring compounds. J. Chem. Phys. 113, 3039-3045.

Rengel, M., Sagawa, H., Hartogh, P., Lellouch, E., Feuchtgruber, H., Moreno, R., Jarchow, C., Courtin, R., Cernicharo, J., Lara, L., 2014. Herschel/PACS spectroscopy of trace gases of the stratosphere of Titan. Astron. Astrophys. 561, \#A4.

Ribeiro, J., Mebel, A., 2014. Reaction mechanism and product branching ratios of the $\mathrm{CH}+\mathrm{C}_{3} \mathrm{H}_{8}$ reaction: A theoretical study. J. Phys. Chem. A 118, 9080-9086.

Richard, M., Cravens, T., Robertson, I., Waite Jr., J., Wahlund, J.E., Crary, F., Coates, A., 2011. Energetics of Titan's ionosphere: Model comparisons with Cassini data. J. Geophys. Res. 116, \#A09310.

Richard, M., Cravens, T., Wylie, C., Webb, D., Chediak, Q., Mandt, K., Waite Jr., J., Rymer, A., Bertucci, C., Wellbrock, A., Windsor, A., Coates, A., 2015a. An empirical approach to modeling ion production rates in Titan's ionosphere II: Ion production rates on the nightside. J. Geophys. Res. Space Physics 120, doi:10.1002/2014JA020343. 
Richard, M., Cravens, T., Wylie, C., Webb, D., Chediak, Q., Perryman, R., Mandt, K., Westlake, J., Waite Jr., J., Robertson, I., Magee, B., Edberg, N., 2015b. An empirical approach to modeling ion production rates in Titan's ionosphere I: Ion production rates on the dayside and globally. J. Geophys. Res. 120, doi:10.1002/2013JA019706.

Robertson, I., Cravens, T., Waite Jr., J., Yelle, R., Vuitton, V., Coates, A., Wahlund, J.E., Ågren, K., Mandt, K., Magee, B., Richard, M., Fattig, E., 2009. Structure of Titan's ionosphere: Model comparisons with Cassini data. Planet. Space Sci. 57, 1834-1846.

Robinson, J., Harris, S., Sun, W., Sveum, N., Neumark, D., 2002. Photofragment translational spectroscopy of 1,3-butadiene and 1,3-butadiene-1,1,4,4-d4 at $193 \mathrm{~nm}$. J. Am. Chem. Soc. 124, 10211-10224.

Robinson, J., Sveum, N., Goncher, S., Neumark, D., 2005. Photofragment translational spectroscopy of allene, propyne, and propyne-d3 at $193 \mathrm{~nm}$. Mol. Phys. 103, 1765-1783.

Roche, A., Sutton, M., Bohme, D., Schiff, H., 1971. Determination of proton affinity from the kinetics of proton transfer reactions. I. Relative proton affinities. J. Chem. Phys. 55, 5480-5484.

Roe, H., Greathouse, T., Richter, M., Lacy, J., 2003. Propane $\left(\mathrm{C}_{3} \mathrm{H}_{8}\right)$ on Titan. Astrophys. J. 597, L65-L68.

Roe, H.G., Greathouse, T., Tokunaga, A., 2011. Update on the TEXES Titan Mid-Infrared Spectral Survey, in: EPSC-DPS Joint Meeting 2011, p. 1398.

Romanzin, C., Louarn, E., Lemaire, J., Žabka, J., Polášek, M., Guillemin, J.C., Alcaraz, C., 2016. An experimental study of the reactivity of $\mathrm{CN}^{-}$and $\mathrm{C}_{3} \mathrm{~N}^{-}$ anions with cyanoacetylene $\left(\mathrm{HC}_{3} \mathrm{~N}\right)$. Icarus $268,242-252$.

Rosén, S., Derkatch, A., Semaniak, J., Neau, A., Al-Khalili, A., Le Padellec, A., Vikor, L., Thomas, R., Danared, H., af Ugglas, M., Larsson, M., 2000. Recombination of simple molecular ions studied in storage ring: dissociative recombination of $\mathrm{H}_{2} \mathrm{O}^{+}$. Faraday Discuss. 115, 295-302.

Rosén, S., Peverall, R., Larsson, M., Le Padellec, A., Semaniak, J., Larson, Å., Strömholm, C., van der Zande, W., Danared, H., Dunn, G., 1998. Absolute cross sections and final-state distributions for dissociative recombination and excitation of $\mathrm{CO}^{+}(\nu=0)$ using an ion storage ring. Phys. Rev. A 57, 44624451.

Rowe, B., Marquette, J., Dupeyrat, G., Ferguson, E., 1985. Reactions of $\mathrm{He}^{+}$ and $\mathrm{N}^{+}$ions with several molecules at $8 \mathrm{~K}$. Chem. Phys. Lett. 113, 403-406.

Rowe, B., Marquette, J., Rebrion, C., 1989. Mass-selected ion-molecule reactions at very low temperatures: the CRESUS apparatus. J. Chem. Soc. Faraday Trans. 2 85, 1631-1641. 
Rudolph, P., Melton, C., 1960. Ion-molecule charge transfer reactions in the alpha radiolysis of various hydrocarbons in a mass spectrometer. J. Chem. Phys. 32, 586-588.

Ruscic, B., Berkowitz, J., 1990. Photoion-pair formation and photoelectroninduced dissociative attachment in $\mathrm{C}_{2} \mathrm{H}_{2}: D_{0}(\mathrm{HCC}-\mathrm{H})$. J. Chem. Phys. 93, $5586-5593$.

Rutherford, J., Neynaber, R., Vroom, D., 1978. Measurements of selected charge transfer processes at low energies: Final report for period November 1977November 1978 (contract No. DNA 001-77-C-0202). Defense Nuclear Agency Report DNA 4695F. IRT Corporation.

Rutkowski, J., Drost, H., Spangenberg, H.J., 1980. Investigation of the inelastic interaction of slow monoenergetic electrons with simple hydrocarbon molecules. Ann. Phys. 7, 259-270.

Sagnières, L., Galand, M., Cui, J., Lavvas, P., Vigren, E., Vuitton, V., Yelle, R., Wellbrock, A., Coates, A., 2015. Influence of local ionization on ionospheric densities in Titan's upper atmosphere. J. Geophys. Res. Space Physics 120, $5899-5921$.

Sailer, W., Pelc, A., Limão-Vieira, P., Mason, N., Limtrakul, J., Scheier, P., Probst, M., Märk, T., 2003. Low energy electron attachment to $\mathrm{CH}_{3} \mathrm{CN}$. Chem. Phys. Lett. 381, 216-222.

Samson, J., Haddad, G., Masuoka, T., Pareek, P., Kilcoyne, D., 1989. Ionization yields, total absorption, and dissociative photoionization cross sections of $\mathrm{CH}_{4}$ from 110-950 A. J. Chem. Phys. 90, 6925-6932.

Samson, J., Marmo, F., Watanabe, K., 1962. Absorption and photoionization coefficients of propylene and butene- 1 in the vacuum ultraviolet. J. Chem. Phys. 36, 783-786.

Samson, J.A.R., Masuoka, T., Pareek, P.N., Angel, G.C., 1987. Total and dissociative photoionization cross sections of $\mathrm{N}_{2}$ from threshold to $107 \mathrm{eV}$. J. Chem. Phys. 86, 6128-6132.

Sander, S., Friedl, R., Barker, J., Golden, D., Kurylo, M., Wine, P., Abbatt, J., Burkholder, J., Kolb, C., Moortgat, G., Huie, R., Orkin, V., 2011. Chemical kinetics and photochemical data for use in atmospheric studies. Evaluation number 17 .

Sander, S., Friedl, R., Golden, D., Kurylo, M., Moortgat, G., Keller-Rudek, H., Wine, P., Ravishankara, A., Kolb, C., Molina, M., Finlayson-Pitts, B., Huie, R., Orkin, V., 2006. Chemical kinetics and photochemical data for use in atmospheric studies. Evaluation number 15. 
Sato, K., Misawa, K., Kobayashi, Y., Matsui, M., Tsunashima, S., Kurosaki, K., Takayanagi, T., 1999. Measurements of thermal rate constants for the reactions of $\mathrm{N}\left({ }^{2} \mathrm{D},{ }^{2} \mathrm{P}\right)$ with $\mathrm{C}_{2} \mathrm{H}_{4}$ and $\mathrm{C}_{2} \mathrm{D}_{4}$ between 225 and $292 \mathrm{~K}$. J. Phys. Chem. A 103, 8650-8656.

Sauer, M., Ward, B., 1967. The reactions of hydrogen atoms with benzene and toluene studied by pulsed radiolysis: Reaction rate constants and transient spectra in the gas phase and aqueous solution. J. Phys. Chem. 74, 3971-3983.

Savić, I., Gerlich, D., 2005. Temperature variable ion trap studies of $\mathrm{C}_{3} \mathrm{H}_{n}^{+}$with $\mathrm{H}_{2}$ and HD. Phys. Chem. Chem. Phys. 7, 1026-1035.

Schofield, K., 1979. Critically evaluated rate constants for gaseous reactions of several electronically excited species. J. Phys. Chem. Ref. Data 8, 723-798.

Schwanebeck, W., Warnatz, J., 1975. Reaktionen des butadiins. I. Die reaktion mit wasserstoffatomen. Ber. Bunsenges. Phys. Chem. 79, 530-535.

Scott, G., Fairley, D., Freeman, C., McEwan, M., 1999. $\mathrm{C}_{m} \mathrm{H}_{n}^{+}$reactions with atomic and molecular nitrogen: an experimental study. J. Phys. Chem. A 103, 1073-1077.

Scott, G., Fairley, D., Freeman, C., McEwan, M., Anicich, V., 1998. Gas-phase reactions of some positive ions with atomic and molecular nitrogen. J. Chem. Phys. 109, 9010-9014.

Scott, G., Milligan, D., Fairley, D., Freeman, C., McEwan, M., 2000. A selected ion flow tube study of the reactions of smalll $\mathrm{C}_{m} \mathrm{H}_{n}^{+}$ions with $\mathrm{O}$ atoms. J. Chem. Phys. 112, 4959-4965.

Sebree, J., Stern, J., Mandt, K., Domagal-Goldman, S., Trainer, M., 2016. ${ }^{13} \mathrm{C}$ and ${ }^{15} \mathrm{~N}$ fractionation of $\mathrm{CH}_{4} / \mathrm{N}_{2}$ mixtures during photochemical aerosol formation: Relevance to Titan. Icarus 270, 421-428.

Seki, K., He, M., Liu, R., Okabe, H., 1996. Photochemistry of cyanoacetylene at $193.3 \mathrm{~nm}$. J. Phys. Chem. 100, 5349-5353.

Seki, K., Okabe, H., 1992. Photodissociation of methylacetylene at $193 \mathrm{~nm}$. J. Phys. Chem. 96, 3345-3349.

Seki, K., Okabe, H., 1993. Photochemistry of acetylene at $193.3 \mathrm{~nm}$. J. Phys. Chem. 97, 5284-5290.

Sekine, Y., Imanaka, H., Matsui, T., Khare, B., Bakes, E., McKay, C., Sugita, S., 2008a. The role of organic haze in Titan's atmospheric chemistry I. Laboratory investigation on heterogeneous reaction of atomic hydrogen with Titan tholin. Icarus 194, 186-200. 
Sekine, Y., Lebonnois, S., Imanaka, H., Matsui, T., Bakes, E., McKay, C., Khare, B., Sugita, S., 2008b. The role of organic haze in Titan's atmospheric chemistry II. Effect of heterogeneous reaction to the hydrogen budget and chemical composition of the atmosphere. Icarus 194, 201-211.

Semaniak, J., Larson, Å., Le Padellec, A., Semaniak, J., Strömholm, C., Larsson, M., Rosén, S., Peverall, R., Danared, H., Djuric, N., Dunn, G., Datz, S., 1998. Dissociative recombination and excitation of $\mathrm{CH}_{5}{ }^{+}$: Absolute cross sections and branching fractions. Astrophys. J. 498, 886-895.

Semaniak, J., Minaev, B., Derkatch, A., Hellberg, F., Neau, A., Rosén, S., Thomas, R., Larsson, M., Danared, H., Paál, A., af Ugglas, M., 2001. Dissociative recombination of $\mathrm{HCNH}^{+}$: Absolute cross-sections and branching ratios. Astrophys. J. Suppl. Ser. 135, 275-283.

Senosiain, J., Klippenstein, S., Miller, J., 2005. The reaction of acetylene with hydroxyl radicals. J. Phys. Chem. A 109, 6045-6055.

Sharp, T., Dowell, J., 1967. Isotope effects in dissociative attachment of electrons in methane. J. Chem. Phys. 46, 1530-1531.

Shaw, D.A., Holland, D.M.P., Macdonald, M.A., Hopkirk, A., Hayes, M.A., McSweeney, S.M., 1992. A study of the absolute photoabsorption cross section and the photoionization quantum efficiency of nitrogen from the ionization threshold to $485 \AA$ A. Chem. Phys. 166, 379-391.

Shebanits, O., Wahlund, J.E., Edberg, N., Crary, F., Wellbrock, A., Andrews, D., Vigren, E., Desai, R., Coates, A., Mandt, K., Waite Jr., J., 2016. Ion and aerosol precursor densities in Titan's ionosphere: A multi-instrument case study. J. Geophys. Res. Space Physics 121, 10,075-10,090.

Shebanits, O., Wahlund, J.E., Mandt, K., Ågren, K., Edberg, N., Waite, J.J., 2013. Negative ion densities in the ionosphere of Titan-Cassini RPWS/LP results. Planet. Space Sci. 84, 153-162.

Sheehan, C., Le Padellec, A., Lennard, W., Talbi, D., Mitchell, J., 1999. Merged beam measurement of the dissociative recombination of $\mathrm{HCN}^{+}$and $\mathrm{HNC}^{+}$. J. Phys. B At. Mol. Opt. Phys. 32, 3347-3360.

Sheehan, C., St.-Maurice, J.P., 2004a. Dissociative recombination of $\mathrm{N}_{2}{ }^{+}, \mathrm{O}_{2}{ }^{+}$, and $\mathrm{NO}^{+}$: Rate coefficients for ground state and vibrationally excited ions. J. Geophys. Res. 109, A03302.

Sheehan, C., St.-Maurice, J.P., 2004b. Dissociative recombination of the methane family ions: Rate coefficients and implications. Adv. Space Res. $33,216-220$.

Shemansky, D., 1972. $\mathrm{CO}_{2}$ extinction coefficient 1700 - 3000 Å. J. Chem. Phys. $56,1582-1587$. 
Shemansky, D., Liu, X., 2005. Evaluation of electron impact excitation of $\mathrm{N}_{2} \mathrm{X}$ ${ }^{1} \Sigma_{g}^{+}(0)$ into the $\mathrm{N}_{2}^{+} \mathrm{X}^{2} \Sigma_{g}^{+}(\nu), \mathrm{A}^{2} \Pi_{u}^{+}(\nu)$, and B ${ }^{2} \Sigma_{u}^{+}(\nu)$ states. J. Geophys. Res. 110, \#A07307.

Shiba, Y., Hirano, T., Nagashima, U., Ishii, K., 1998. Potential energy surfaces and branching ratio of the dissociative recombination reaction $\mathrm{HCNH}^{+}+\mathrm{e}^{-}$: An ab initio molecular orbital study. J. Chem. Phys. 108, 698-705.

Shindo, F., Bénilan, Y., Guillemin, J.C., Chaquin, P., Jolly, A., Raulin, F., 2003. Ultraviolet and infrared spectrum of $\mathrm{C}_{6} \mathrm{H}_{2}$ revisited and vapor pressure curve in Titan's atmosphere. Planet. Space Sci. 51, 9-17.

Shul, R., Passarella, R., DiFazio, L.T., J., Keesee, R., Castleman, A.W., J., 1988. Ion-molecule reactions involving $\mathrm{H}_{3} \mathrm{O}^{+}, \mathrm{H}_{2} \mathrm{O}^{+}$, and $\mathrm{OH}^{+}$at thermal energy. J. Phys. Chem. 92, 4947-4951.

Sillanpää, I., Johnson, R., 2015. The role of ion-neutral collisions in Titan's magnetospheric interaction. Planet. Space Sci. 108, 73-86.

Silva, R., Gichuhi, W., Huang, C., Doyle, M., Kislov, V., Mebel, A., Suits, A., 2008. H elimination and metastable lifetimes in the UV photoexcitation of diacetylene. P. Natl. Acad. Sci. USA 105, 12713-12718.

Silva, R., Gichuhi, W., Kislov, V., Landera, A., Mebel, A., Suits, A., 2009. UV photodissociation of cyanoacetylene: A combined ion imaging and theoretical investigation. J. Phys. Chem. A 113, 11182-11186.

Sims, I., Queffelec, J.L., Travers, D., Rowe, B., Herbert, L., Karthauser, J., Smith, I., 1993. Rate constants for the reactions of CN with hydrocarbons at low and ultra-low temperatures. Chem. Phys. Lett. 211, 461-468.

Sivaramakrishnan, R., Su, M.C., Michael, J., Klippenstein, S., Harding, L., Ruscic, B., 2011. Shock tube and theoretical studies on the thermal decomposition of propane: Evidence for a roaming radical channel. J. Phys. Chem. A $115,3366-3379$.

Smith, D., Adams, N., 1978a. Binary and ternary reacions of $\mathrm{CH}_{3}{ }^{+}$ions with several molecules at thermal energies. Chem. Phys. Lett. 54, 535-540.

Smith, D., Adams, N., 1978b. Molecular synthesis in interstellar clouds: radiative association reactions of $\mathrm{CH}_{3}{ }^{+}$ions. Chem. Phys. Lett. 220, L87-L92.

Smith, D., Adams, N., Miller, T., 1978a. A laboratory study of the reactions of $\mathrm{N}^{+}, \mathrm{N}_{2}{ }^{+}, \mathrm{N}_{3}{ }^{+}, \mathrm{N}_{4}{ }^{+}, \mathrm{O}^{+}, \mathrm{O}_{2}{ }^{+}$, and $\mathrm{NO}^{+}$ions with several molecules at 300 K. J. Chem. Phys. 69, 308-318.

Smith, D., Church, M., Miller, T., 1978b. Mutual neutralization of simple and clustered positive and negative ions. J. Chem. Phys. 68, 1224-1229. 
Smith, D., Spanel, P., Mayhew, C., 1992. A selected ion-flow tube study of the reactions of $\mathrm{O}+, \mathrm{H}+$ and $\mathrm{HeH}+$ with several molecular gases at $300 \mathrm{~K}$. Int. J. Mass Spectrom. Ion Processes 117, 457-473.

Smith, I., Herbst, E., Chang, Q., 2004. Rapid neutral-neutral reactions at low temperatures: A new network and first results for TMC-1. Mon. Not. R. Astron. Soc. 350, 323-330.

Smith, I., Sage, A., Donahue, N., Herbst, E., Quan, D., 2006. The temperaturedependence of rapid low temperature reactions: experiment, understanding and prediction. Faraday Discuss. 133, 137-156.

Smith, N., Bénilan, Y., Bruston, P., 1998. The temperature dependant absorption cross sections of $\mathrm{C}_{4} \mathrm{H}_{2}$ at mid ultraviolet wavelengths. Planet. Space Sci. $46,1215-1220$.

Smith, N., Raulin, F., 1999. Modeling of methane photolysis in the reducing atmospheres of the outer solar system. J. Geophys. Res. 104, 1873-1876.

Smith, P., Yoshino, K., Parkinson, W., Ito, K., Stark, G., 1991. High resolution VUV (147-201 nm) photoabsorption cross sections for $\mathrm{C}_{2} \mathrm{H}_{2}$ at 195 and 295 K. J. Geophys. Res. 96, 17529-17533.

Smyth, K., Lias, S., Ausloos, P., 1981. Can $\mathrm{C}_{3} \mathrm{H}_{3}{ }^{+}$be an important soot precursor, in: Chemical and Physical Processes in Combustion, pp. 187-190.

Smyth, K., Lias, S., Ausloos, P., 1982. The ion-molecule chemistry of $\mathrm{C}_{3} \mathrm{H}_{3}{ }^{+}$ and the implications for soot formation. Combust. Sci. Technol. 28, 147-154.

Snow, M., McClintock, W.E., Rottman, G., Woods, T., 2005. Solar stellar irradiance comparison experiment II (SOLSTICE II): Examination of the solar stellar comparison technique. Solar Phys. 203, 295-324.

Snow, T., Stepanovic, M., Betts, N., Eichelberger, B., Martinez, O., Bierbaum, V., 2009. Formation of gas-phase glycine and cyanoacetylene via associative detachment reactions. Astrobiology 9, 1001-1005.

Snowden, D., Smith, M., Jimson, T., Higgins, A., 2018. Energy deposition and ion production from thermal oxygen ion precipitation during Cassini's T57 flyby. Icarus submitted.

Snowden, D., Yelle, R., Galand, M., Coates, A., Wellbrock, A., Jones, G., Lavvas, P., 2013. Auroral electron precipitation and flux tube erosion in Titan's upper atmosphere. Icarus 226, 186-204.

Song, Y., Lucas, M., Alcaraz, M., Zhang, J., Brazier, C., 2012. Ultraviolet photodissociation dynamics of the phenyl radical. J. Chem. Phys. 136, \#044308.

Song, Y., Zheng, X., Lucas, M., Zhang, J., 2011. Ultraviolet photodissociation dynamics of the benzyl radical. Phys. Chem. Chem. Phys. 13, 8296-8305. 
Spanel, P., Smith, D., Henchman, M., 1995. The reactions of some interstellar ions with benzene, cyclopropane and cyclohexane. Int. J. Mass Spectrom. Ion Processes 141, 117-126.

Speller, C., Vacher, J., Duc, E., Fitaire, M., 1995. Mass spectrometric study of three-body ion-molecule clustering reactions of $\mathrm{C}_{p} \mathrm{H}_{q}^{+}$ions with $\mathrm{N}_{2}$ or $\mathrm{CH}_{4}$ in $\mathrm{N}_{2}-\mathrm{CH}_{4}$ mixtures. Planet. Space Sci. 43, 33-39.

Stamatovic, A., Schulz, G., 1970. Dissociative attachment in CO and formation of $\mathrm{C}^{-}$. J. Chem. Phys. 53, 2663-2667.

Stancil, P., Dalgarno, A., 1998. Chemical processes in astrophysical radiation fields. Faraday Discuss. 109, 61-69.

Stanton, J.F., Gauss, J., Harding, M.E., Szalay, P.G., 2010. with contributions from Auer, A. A., Bartlett, R. J., Benedikt, U., Berger, C., Bernholdt, D. E., Bomble, Y. J., et al. and the integral packages MOLECULE (Almlöf, J., Taylor, P. R.), PROPS (Taylor, P. R.), ABACUS (Helgaker, T., Jensen, H. J., Jørgensen, P., Olsen, J.), and ECP routines by Mitin, A. V., van Wüllen, C. For the current version, see http://www.cfour.de.

Stavish, L., Fondren, L., Adams, N., 2009. Reactions of $\mathrm{N}^{+}$and $\mathrm{N}_{2}^{+}$with several cyclic molecules studied using a selected ion flow tube. Int. J. Mass Spectrom. 281, 103-107.

Stearns, J., Zwier, T., Kraka, E., Cremer, D., 2006. Experimental and computational study of the ultraviolet photolysis of vinylacetylene. Part II. Phys. Chem. Chem. Phys. 8, 5317-5327.

Steinbauer, M., Giegerich, J., Fischer, K., Fischer, I., 2012. The photodissociation dynamics of the ethyl radical, $\mathrm{C}_{2} \mathrm{H}_{5}$, investigated by velocity map imaging. J. Chem. Phys. 137, \#014303.

Stevens, M., Evans, J., Lumpe, J., Westlake, J., Ajello, J., Bradley, E., Esposito, L., 2015. Molecular nitrogen and methane density retrievals from Cassini UVIS dayglow observations of Titan's upper atmosphere. Icarus 247, 301312 .

Stief, L., Nesbitt, F., Payne, W., Kuo, S., Tao, W., Klemm, R., 1995. Rate constant and reaction channels for the reaction of atomic nitrogen with the ethyl radical. J. Chem. Phys. 102, 5309-5316.

Stief, L., Payne, W., Klemm, R., 1975. A flash photolysis-resonance fluorescence study of the formation of $\mathrm{O}\left({ }^{1} \mathrm{D}\right)$ in the photolysis of water and the reaction of $\mathrm{O}\left({ }^{1} \mathrm{D}\right)$ with $\mathrm{H}_{2}$, Ar, and He. J. Chem. Phys. 62, 4000-4008.

Stoliarov, S., Knyazev, V., Slagle, I., 2000. Experimental study of the reaction between vinyl and methyl radicals in the gas phase. Temperature and pressure dependence of overall rate constants and product yields. J. Phys. Chem. A 104, 9687-9697. 
Stolte, W., He, Z., Cutler, J., Lu, Y., Samson, J., 1998. Dissociative photoionization cross sections of $\mathrm{N}_{2}$ and $\mathrm{O}_{2}$ from 100 to $800 \mathrm{eV}$. At. Data Nucl. Data Tables $69,171-179$.

Stranges, D., Stemmler, M., Yang, X., Chesko, J., Suits, A., Lee, Y., 1998. UV photodissociation dynamics of allyl radical by photofragment translational spectroscopy. J. Chem. Phys. 109, 5372-5382.

Strobel, D., 1974. The photochemistry of hydrocarbons in the atmosphere of Titan. Icarus 21, 466-470.

Strobel, D., 2010. Molecular hydrogen in Titan's atmosphere: Implications of the measured tropospheric and thermospheric mole fractions. Icarus 208, $878-886$.

Strobel, D.F., 2008. Titan's hydrodynamically escaping atmosphere. Icarus 193, $588-594$.

Su, T., Bowers, M., 1973. Ion-polar molecule collisions: the effect of ion size on ion-polar molecule rate constants; the parameterization of the average-dipoleorientation theory. Int. J. Mass Spectrom. Ion Processes 12, 347-356.

Suto, M., Lee, L., 1985. Photoabsorption cross section of $\mathrm{CH}_{3} \mathrm{CN}$ : Photodissociation rates by solar flux and interstellar radiation. J. Geophys. Res. 90, 13,037-13,040.

Talbi, D., Ellinger, Y., 1998. Potential energy surface for the electronic dissociative recombination reaction of $\mathrm{HCNH}^{+}$: Astrophysical implications on the HCN/HNC abundance ratio. Chem. Phys. Lett. 288, 155-164.

Teanby, N., Irwin, P., de Kok, R., Jolly, A., Bézard, B., Nixon, C., Calcutt, S., 2009. Titan's stratospheric $\mathrm{C}_{2} \mathrm{~N}_{2}, \mathrm{C}_{3} \mathrm{H}_{4}$, and $\mathrm{C}_{4} \mathrm{H}_{2}$ abundances from Cassini/CIRS far-infrared spectra. Icarus 202, 620-631.

Teanby, N., Irwin, P., de Kok, R., Nixon, C., 2010. Mapping Titan's HCN in the far infra-red: implications for photochemistry. Faraday Discuss. 147, 51-64.

Teanby, N., Irwin, P., de Kok, R., Nixon, C., Coustenis, A., Royer, E., Calcutt, S., Bowles, N., Fletcher, L., Howett, C., Taylor, F., 2008a. Global and temporal variations in hydrocarbons and nitriles in Titan's stratosphere for northern winter observed by Cassini/CIRS. Icarus 193, 595-611.

Teanby, N., Irwin, P., Nixon, C., Courtin, R., Swinyard, B., Moreno, R., Lellouch, E., Rengel, M., Hartogh, P., 2013. Constraints on Titan's middle atmosphere ammonia abundance from Herschel/SPIRE sub-millimetre spectra. Planet. Space Sci. 75, 136-147.

Teanby, N., Irwin, P., Nixon, C., de Kok, R., Vinatier, S., Coustenis, A., SeftonNash, E., Calcutt, S., Flasar, F., 2012. Active upper-atmosphere chemistry and dynamics from polar circulation reversal on Titan. Nature 491, 732-735. 
Teanby, N., de Kok, R., Irwin, P., Osprey, S., Vinatier, S., Gierasch, P., Read, P., Flasar, F., Conrath, B., Achterberg, R., Bézard, B., Nixon, C., Calcutt, S., 2008b. Titan's winter polar vortex structure revealed by chemical tracers. J. Geophys. Res. 113, \#E12003.

Teanby, N.A., Irwin, P.G.J., de Kok, R., Nixon, C.A., Coustenis, A., Bézard, B., Calcutt, S.B., Bowles, N.E., Flasar, F.M., Fletcher, L., Howett, C., Taylor, F.W., 2006. Latitudinal variations of $\mathrm{HCN}, \mathrm{HC}_{3} \mathrm{~N}$, and $\mathrm{C}_{2} \mathrm{~N}_{2}$ in Titan's stratosphere derived from Cassini CIRS data. Icarus 181, 243-255.

Teolis, B., Niemann, H., Waite, H., Gell, D., Perryman, R., Kasprzak, W., Mandt, K., Yelle, R., Lee, A., Pelletier, F., Miller, G., Young, D., Bell, J., Magee, B., Patrick, E., Grimes, J., Fletcher, G., Vuitton, V., 2015. A revised sensitivity model for Cassini INMS: Results at Titan. Space Sci. Rev. 190, $47-84$.

Terzieva, R., Herbst, E., 2000. The possibility of nitrogen isotopic fractionation in interstellar clouds. Mon. Not. R. Astron. Soc. 317, 563-568.

Teslja, A., Dagdigian, P., Banck, M., Eisfeld, W., 2006. Experimental and theoretical study of the electronic spectrum of the methylene amidogen radical $\left(\mathrm{H}_{2} \mathrm{CN}\right)$ : Verification of the ${ }^{2} \mathrm{~A}_{1} \leftarrow{ }^{2} \mathrm{~B}_{2}$ assignment. J. Phys. Chem. A 110, $7826-7834$.

Teslja, A., Nizamov, B., Dagdigian, P., 2004. The electronic spectrum of methyleneimine. J. Phys. Chem. A 108, 4433-4439.

Thomas, R., Hellberg, F., Neau, A., Rosén, S., Larsson, M., Vane, C., Bannister, M., Datz, S., Petrignani, A., van der Zande, W., 2005. Three-body fragmentation dynamics of amidogen and methylene radicals via dissociative recombination. Phys. Rev. A 71, 032711.

Thomas, R., Kashperka, I., Vigren, E., Geppert, W., Hamberg, M., Larsson, M., af Ugglas, M., Zhaunerchyk, V., 2013. Dissociative recombination of $\mathrm{CH}_{4}{ }^{+}$. J. Phys. Chem. A 117, 9999-10005.

Tichý, M., Rakshit, A., Lister, D., Twiddy, N., Adams, N., Smith, D., 1979. A study of the reactions of the ground and metastable states of $\mathrm{C}^{+}, \mathrm{N}^{+}, \mathrm{S}^{+}$ and $\mathrm{N}_{2}{ }^{+}$at 300 K. Int. J. Mass Spectrom. Ion Processes 29, 231-247.

Tickner, A., Lossing, F., 1951. The measurement of low vapor pressures by means of a mass spectrometer. J. Phys. Colloid Chem. 55, 733-740.

Tobie, G., Lunine, J., Sotin, C., 2006. Episodic outgassing as the origin of atmospheric methane on Titan. Nature 440, 61-64.

Tomasko, M., Doose, L., Engel, S., Dafoe, L., West, R., Lemmon, M., Karkoschka, E., See, C., 2008. A model of Titan's aerosols based on measurements made inside the atmosphere. Planet. Space Sci. 56, 669-707. 
Tomasko, M.G., Archinal, B., Becker, T., Bézard, B., Bushroe, M., Combes, M., Cook, D., Coustenis, A., de Bergh, C., Dafoe, L.E., Doose, L., Douté, S., Eibl, A., Engel, S., Gliem, F., Grieger, B., Holso, K., Howington-Kraus, E., Karkoschka, E., Keller, H.U., Kirk, R., Kramm, R., Küppers, M., Lanagan, P., Lellouch, P., Lemmon, M., Lunine, J., McFarlane, E., Moores, J., Prout, G.M., Rizk, B., Rosiek, M., Rueffer, P., Schröder, S.E., Schmitt, B., See, C., Smith, P., Soderblom, L., Thomas, N., West, R., 2005. Rain, winds and haze during the Huygens probe's descent to Titan's surface. Nature 438, 765-778.

Tonokura, K., Koshi, M., 2000. Absorption spectrum and cross sections of the allyl radical measured using cavity ring-down spectroscopy: The $\mathrm{A} \leftarrow \mathrm{X}$ band. J. Phys. Chem. A 104, 8456-8461.

Toublanc, D., Parisot, J., Brillet, J., Gautier, D., Raulin, F., McKay, C., 1995. Photochemical modeling of Titan's atmosphere. Icarus 113, 2-26.

Trevitt, A., Prendergast, M., Goulay, F., Savee, J., Osborn, D., Taatjes, C., Leone, S., 2013. Product branching fractions of the $\mathrm{CH}+$ propene reaction from synchrotron photoionization mass spectrometry. J. Phys. Chem. A 117, 6450-6457.

Trevitt, A., Soorkia, S., Savee, J., Selby, T., Osborn, D., Taatjes, C., Leone, S., 2011. Branching fractions of the $\mathrm{CN}+\mathrm{C}_{3} \mathrm{H}_{6}$ reaction using synchrotron photoionization mass spectrometry: Evidence for the 3-cyanopropene product. J. Phys. Chem. A 115, 13467-13473.

Troe, J., 1979. Predictive possibilities of unimolecular rate theory. J. Phys. Chem. 83, 114-126.

Troe, J., 1983. Theory of thermal unimolecular reactions in the fall-off range. I. Strong collision rate constants. Ber. Bunsenges. Phys. Chem. 87, 161-169.

Truhlar, D., Garrett, B., Klippenstein, S., 1996. Current status of transitionstate theory. J. Phys. Chem. 100, 12771-12800.

Tsai, S.T., Lin, C.K., Lee, Y., Ni, C.K., 2000. Dissociation rate of hot benzene. J. Chem. Phys. 113, \#67.

Tsang, W., 1987. Chemical kinetic database for combustion chemistry part II: methanol. J. Phys. Chem. Ref. Data 16, 471.

Tsang, W., 1988. Chemical kinetic database for combustion chemistry part III: propane. J. Phys. Chem. Ref. Data 17, 887.

Tsang, W., 1991. Chemical kinetic database for combustion chemistry part V: propene. J. Phys. Chem. Ref. Data 20, 221-274.

Tsang, W., 1992. Chemical kinetic database for propellant combustion. II. Reactions involving CN, NCO, and HNCO. J. Phys. Chem. Ref. Data 21, 753-791. 
Tsang, W., Hampson, R., 1986. Chemical kinetic data base for combustion chemistry. Part I. Methane and related compounds. J. Phys. Chem. Ref. Data $15,1087-1279$.

Tsang, W., Herron, J., 1991. Chemical kinetic database for propellant combustion. I. Reactions involving $\mathrm{NO}, \mathrm{NO}_{2}, \mathrm{HNO}, \mathrm{HNO}_{2}, \mathrm{HCN}$ and $\mathrm{N}_{2} \mathrm{O}$. J. Phys. Chem. Ref. Data 20, 609-663.

Tsuda, S., Yokohata, A., Umaba, T., 1973. Measurement of negative ions formed by electron impact. IX. Negative ion mass spectra and ionization efficiency curves of negative ions of $\mathrm{m} / \mathrm{e} 25,26,27,38,39,40$ and 50 from acrylonitrile. Bull. Chem. Soc. Jpn 46, 2273-2277.

Tsuji, M., Matsumura, K., Funatsu, T., Nishimura, Y., Obase, H., 1994. Ion/molecule reactions of $\mathrm{CO}_{2}{ }^{+}$with simple aliphatic hydrocarbons at thermal energy. Int. J. Mass Spectrom. Ion Processes 135, 165-180.

Tsuji, M., Matsumura, K., Funatsu, T., Nishimura, Y., Obase, H., Kagawa, S., Kanetaka, Y., 1993. Ion-molecule reactions of $\mathrm{CO}_{2}{ }^{+}$with butane and isobutane at thermal energy. B. Chem. Soc. Jpn. 66, 2864-2870.

Tucker, O.J., Waalkes, W., Tenishev, V.M., Johnson, R.E., Bieler, A., Combi, M.R., Nagy, A.F., 2016. Examining the exobase approximation: DSMC models of Titan's upper atmosphere. Icarus 272, 290-300.

Tully, J., 1975. Reactions of $\mathrm{O}\left({ }^{1} \mathrm{D}\right)$ with atmospheric molecules. J. Chem. Phys. $62,1893-1898$.

Vacher, J., Le Duc, E., Fitaire, M., 1997. Ion-molecule clustering reactions of $\mathrm{HCNH}^{+}$ion with $\mathrm{N}_{2}$ and/or $\mathrm{CH}_{4}$ in $\mathrm{N}_{2}-\mathrm{CH}_{4}$ mixtures. Planet. Space Sci. 45, $1407-1416$.

Vacher, J., Le Duc, E., Fitaire, M., 1999. Titan's atmosphere: possible clustering reactions of $\mathrm{HCNH}^{+}, \mathrm{HCNH}^{+}\left(\mathrm{N}_{2}\right)$ and $\mathrm{HCNH}^{+}\left(\mathrm{CH}_{4}\right)$ ions with acetylene. Planet. Space Sci. 47, 151-162.

Vacher, J., Le Duc, E., Fitaire, M., 2000. Clustering reactions of $\mathrm{HCNH}^{+}$, $\mathrm{HCNH}^{+}\left(\mathrm{N}_{2}\right)$ and $\mathrm{HCNH}^{+}\left(\mathrm{CH}_{4}\right)$ with ethane: application to Titan atmosphere. Planet. Space Sci. 48, 237-247.

Vaghjiani, G., 1993. Ultraviolet absorption cross sections for $\mathrm{N}_{2} \mathrm{H}_{4}$ vapor between 191-291 nm and $\mathrm{H}\left({ }^{2} \mathrm{~S}\right)$ quantum yield in $248 \mathrm{~nm}$ photodissociation at 296 K. J. Chem. Phys. 98, 2123-2131.

Vaghjiani, G., 1995. Laser photolysis studies of hydrazine vapor: 193 and 222 $\mathrm{nm}$ H-atom primary quantum yields at $296 \mathrm{~K}$, and the kinetics of $\mathrm{H}+\mathrm{N}_{2} \mathrm{H}_{4}$ reaction over the temperature range 222-657 K. Int. J. Chem. Kin. 27, 777790. 
Vakhtin, A., Heard, D., Smith, I., Leone, S., 2001a. Kinetics of $\mathrm{C}_{2} \mathrm{H}$ radical reactions with ethene, propene and 1-butene measured in a pulsed Laval nozzle apparatus at $\mathrm{T}=103$ and $296 \mathrm{~K}$. Chem. Phys. Lett. 348, 21-26.

Vakhtin, A., Heard, D., Smith, I., Leone, S., 2001b. Kinetics of reactions of $\mathrm{C}_{2} \mathrm{H}$ radical with acetylene, $\mathrm{O}_{2}$, methylacetylene, and allene in a pulsed Laval nozzle apparatus at $\mathrm{T}=103 \mathrm{~K}$. Chem. Phys. Lett. 344, 317-324.

Vejby-Christensen, L., Andersen, L., Heber, O., Kella, D., Pedersen, H., Schmidt, H., Zajfman, D., 1997. Complete branching ratios for the dissociative recombination of $\mathrm{H}_{2} \mathrm{O}^{+}, \mathrm{H}_{3} \mathrm{O}^{+}$, and $\mathrm{CH}_{3}{ }^{+}$. Astrophys. J. 483, 531-540.

Vereecken, L., Peeters, J., 1999. Detailed microvariational RRKM master equation analysis of the product distribution of the $\mathrm{C}_{2} \mathrm{H}_{2}+\mathrm{CH}\left(\mathrm{X}^{2} \Pi\right)$ reaction over extended temperature and pressure ranges. J. Phys. Chem. A 103, 55235533 .

Viggiano, A., Ehlerding, A., Arnold, S., Larsson, M., 2005. Dissociative recombination of hydrocarbon ions. J. Phys. Conf. Ser. 4, 191-197.

Viggiano, A., Paulson, J., 1983. Temperature dependence of associative detachment reactions. J. Chem. Phys. 79, 2241-2245.

Vigren, E., Galand, M., Shebanits, O., Wahlund, J.E., Geppert, W., Lavvas, P., Vuitton, V., Yelle, R., 2014. Increasing positive ion number densities below the peak of ion-electron pair production in Titan's ionosphere. Astrophys. J. 786, \#69.

Vigren, E., Galand, M., Yelle, R., Cui, J., Wahlund, J.E., Ågren, K., Lavvas, P., Müller-Wodarg, I., Strobel, D., Vuitton, V., Bazin, A., 2013. On the thermal electron balance in Titan's sunlit upper atmosphere. Icarus 223, 234-251.

Vigren, E., Galand, M., Yelle, R., Wellbrock, A., Coates, A., Snowden, D., Cui, J., Lavvas, P., Edberg, N., Shebanits, O., Wahlund, J.E., Vuitton, V., Mandt, K., 2015. Ionization balance in Titan's nightside ionosphere. Icarus $248,539-546$.

Vigren, E., Hamberg, M., Zhaunerchyk, V., Kamińska, M., Thomas, R., Larsson, M., Millar, T., Walsh, C., Geppert, W., 2009. The dissociative recombination of protonated acrylonitrile, $\mathrm{CH}_{2} \mathrm{CHCNH}^{+}$, with implications for the nitrile chemistry in dark molecular clouds and the upper atmosphere of Titan. Astrophys. J. 695, 317-324.

Vigren, E., Hamberg, M., Zhaunerchyk, V., Kamińska, M., Thomas, R., Trippel, S., Wester, R., Zhang, M., Kashperka, I., af Ugglas, M., Semaniak, J., Larsson, M., Geppert, W., 2010a. Dissociative recombination of protonated propionitrile, $\mathrm{CH}_{3} \mathrm{CH}_{2} \mathrm{CNH}^{+}$: Implications for Titan's upper atmosphere. Astrophys. J. 722, 847-850. 
Vigren, E., Hamberg, M., Zhaunerchyk, V., Kamińska, M., Thomas, R., Trippel, S., Zhang, M., Kashperka, I., af Ugglas, M., Walsh, C., Wester, R., Semaniak, J., Larsson, M., Geppert, W., 2010b. Dissociative recombination of the acetaldehyde cation, $\mathrm{CH}_{3} \mathrm{CHO}^{+}$. Phys. Chem. Chem. Phys. 12, 11670-11673.

Vigren, E., Kamińska, M., Hamberg, M., Zhaunerchyk, V., Thomas, R., Danielsson, M., Semaniak, J., Andersson, P., Larsson, M., Geppert, W., 2008. Dissociative recombination of fully deuterated protonated acetonitrile, $\mathrm{CD}_{3} \mathrm{CND}^{+}$: product branching fractions, absolute cross section and thermal rate coefficient. Phys. Chem. Chem. Phys. 10, 4014-4019.

Vigren, E., Semaniak, J., Hamberg, M., Zhaunerchyk, V., Kamińska, M., Thomas, R., af Ugglas, M., Larsson, M., Geppert, W., 2012a. Dissociative recombination of nitrile ions with implications for Titan's upper atmosphere. Planet. Space Sci. 60, 102-106.

Vigren, E., Zhaunerchyk, V., Hamberg, M., Kamińska, M., Semaniak, J., af Ugglas, M., Larsson, M., Thomas, R., Geppert, W., 2012b. Reassessment of the dissociative recombination of $\mathrm{N}_{2} \mathrm{H}^{+}$at CRYRING. Astrophys. J. 757, \#34.

Vinatier, S., Bézard, B., Fouchet, T., Teanby, N., de Kok, R., Irwin, P., Conrath, B., Nixon, C., Romani, P., Flasar, F., Coustenis, A., 2007a. Vertical abundance profiles of hydrocarbons in Titan's atmosphere at $15^{\circ} \mathrm{S}$ and $80^{\circ}$ $\mathrm{N}$ retrieved from Cassini/CIRS spectra. Icarus 188, 120-138.

Vinatier, S., Bézard, B., Nixon, C., 2007b. The Titan ${ }^{14} \mathrm{~N} /{ }^{15} \mathrm{~N}$ and ${ }^{12} \mathrm{C} /{ }^{13} \mathrm{C}$ isotopic ratios in HCN from Cassini/CIRS. Icarus 191, 712-721.

Vinatier, S., Bézard, B., Nixon, C., Mamoutkine, A., Carlson, R., Jennings, D., Guandique, E., Teanby, N., Bjoraker, G., Flasar, F., Kunde, V., 2010. Analysis of Cassini/CIRS limb spectra of Titan acquired during the nominal mission I. Hydrocarbons, nitriles and $\mathrm{CO}_{2}$ vertical mixing ratio profiles. Icarus 205, 559-570.

Vuitton, V., Doussin, J.F., Bénilan, Y., Raulin, F., Gazeau, M.C., 2006a. Experimental and theoretical study of hydrocarbon photochemistry applied to Titan stratosphere. Icarus 185, 287-300.

Vuitton, V., Dutuit, O., Smith, M., Balucani, N., 2014. Chemistry of Titan's atmosphere, in: Müller-Wodarg, I., Griffith, C., Lellouch, E., Cravens, T. (Eds.), Titan: Interior, Surface, Atmosphere and Space Environment. Cambridge University Press. chapter 7, pp. 224-284.

Vuitton, V., Lavvas, P., Yelle, R., Galand, M., Wellbrock, A., Lewis, G., Coates, A., Wahlund, J.E., 2009. Negative ion chemistry in Titan's upper atmosphere. Planet. Space Sci. 57, 1558-1572.

Vuitton, V., Yelle, R., Anicich, V., 2006b. The nitrogen chemistry of Titan's upper atmosphere revealed. Astrophys. J. 647, L175-L178. 
Vuitton, V., Yelle, R., Cui, J., 2008. Formation and distribution of benzene on Titan. J. Geophys. Res. 113, \#E05007.

Vuitton, V., Yelle, R., Lavvas, P., Klippenstein, S., 2012. Rapid association reactions at low pressure: Impact on the formation of hydrocarbons on Titan. Astrophys. J. 744, \#11.

Vuitton, V., Yelle, R., McEwan, M., 2007. Ion chemistry and N-containing molecules in Titan's upper atmosphere. Icarus 191, 722-742.

Wagener, R., 1990. Influence of temperature on the removal rates of methylene $\left(\mathrm{a}^{1} \mathrm{~A}_{1}\right)$ by inert gases and hydrocarbons. Z. Naturforsch. A 45, 649-56.

Wahlund, J.E., Boström, R., Gustafsson, G., Gurnett, D., Kurth, W., Pedersen, A., Averkamp, T., Hospodarsky, G., Persoon, A., Canu, P., Neubauer, F., Dougherty, M., Eriksson, A., Morooka, M., Gill, R., André, M., Eliasson, L., Müller-Wodarg, I., 2005. Cassini measurements of cold plasma in the ionosphere of Titan. Science 308, 986-989.

Waite, J.J., Niemann, H., Yelle, R., Kasprzak, W., Cravens, T., Luhmann, J., McNutt, R., Ip, W., Gell, D., De La Haye, V., Müller-Wodarg, I., Magee, B., Borggren, N., Ledvina, S., Fletcher, G., Walter, E., Miller, R., Scherer, S., Thorpe, R., Xu, J., Block, B., Arnett, K., 2005. Ion Neutral Mass Spectrometer results from the first flyby of Titan. Science 308, 982-986.

Waite, J.J., Young, D., Cravens, T., Coates, A., Crary, F., Magee, B., Westlake, J., 2007. The process of tholin formation in Titan's upper atmosphere. Science $316,870-875$.

Wakelam, V., Herbst, E., Loison, J.C., Smith, I., Chandrasekaran, V., Pavone, B., Adams, N., Bacchus-Montabonel, M.C., Bergeat, A., Béroff, K., Bierbaum, V., Chabot, M., Dalgarno, A., van Dishoeck, E., Faure, A., Geppert, W., Gerlich, D., Galli, D., Hébrard, E., Hersant, F., Hickson, K., Honvault, P., Klippenstein, S., Le Picard, S., Nyman, G., Pernot, P., Schlemmer, S., Selsis, F., Sims, I., Talbi, D., Tennyson, J., Troe, J., Wester, R., Wiesenfeld, L., 2012. A kinetic database for astrochemistry (KIDA). Astrophys. J. Suppl. Ser. 199, \#21.

Wakelam, V., Loison, J.C., Herbst, E., Pavone, B., Bergeat, A., Béroff, K., Chabot, M., Faure, A., Galli, D., Geppert, W., Gerlich, D., Gratier, P., Harada, N., Hickson, K., Honvault, P., Klippenstein, S., Le Picard, S., Nyman, G., Ruaud, M., Schlemmer, S., Sims, I., Talbi, D., Tennyson, J., Wester, R., 2015. The 2014 KIDA network for interstellar chemistry. Astrophys. J. Suppl. Ser. 217, \#20.

Wallington, T., Egsgaard, H., Nielsen, O., Platz, J., Sehested, J., Stein, T., 1998. UV-visible spectrum of the phenyl radical and kinetics of its reaction with NO in the gas phase. Chem. Phys. Lett. 290, 363-370. 
Walsh, C., Harada, N., Herbst, E., Millar, T., 2009. The effects of molecular anions on the chemistry of dark clouds. Astrophys. J. 700, 752-761.

Walter, C.W., Cosby, P.C., Helm, H., 1993. $\mathrm{N}\left({ }^{4} \mathrm{~S}_{0}\right), \mathrm{N}\left({ }^{2} \mathrm{D}_{0}\right)$ and $\mathrm{N}\left({ }^{2} \mathrm{P}_{0}\right)$ yields in predissociation of exacted singlet state of $\mathrm{N}_{2}$. J. Chem. Phys. 99, 3553-3561.

Wang, H., Frenklach, M., 1994. Calculations of rate coefficients for the chemically activated reactions of acetylene with vinylic and aromatic radicals. J. Phys. Chem. 98, 11465-11489.

Wellbrock, A., Coates, A., Jones, G., Lewis, G., Waite, J., 2013. Cassini CAPSELS observations of negative ions in Titan's ionosphere: Trends of density with altitude. Geophys. Res. Lett. 40, 4481-4485.

Wendt, H., Hunziker, H., 1984. The UV spectra of primary, secondary, and tertiary radicals. J. Chem. Phys. 81, 717-723.

Werner, H.J., Knowles, P., Knizia, G., Manby, F., Schutz, M., Celani, P., Korona, T., Lindh, R., Mitrushenkov, A., Rauhut, G., et al., 2012. MOLPRO, version 2012.1, A package of ab initio programs. see http://www.molpro.net.

Westlake, J., Waite, J., Bell, J., Perryman, R., 2014. Observed decline in Titan's thermospheric methane due to solar cycle drivers. J. Geophys. Res. Space Physics 119, 8586-8599.

Westlake, J., Waite Jr., J., Mandt, K., Carrasco, N., Bell, J., Magee, B., Wahlund, J.E., 2012. Titan's ionospheric composition and structure: Photochemical modeling of Cassini INMS data. J. Geophys. Res. 117, \#E01003.

Wiese, W.L., Fuhr, J.R., 2007. Improved critical compilations of selected atomic transition probabilities for neutral and singly ionized carbon and nitrogen. J. Phys. Chem. Ref. Data 36, 1287-1345.

Wilhelm, M., Nikow, M., Letendre, L., Dai, H.L., 2009. Photodissociation of vinyl cyanide at $193 \mathrm{~nm}$ : Nascent product distributions of the molecular elimination channels. J. Chem. Phys. 130, \#044307.

Willacy, K., Allen, M., Yung, Y., 2016. A new astrobiological model of the atmosphere of Titan. Astrophys. J. 829, \#79.

Wilson, E., Atreya, S., 2000. Sensitivity studies of methane photolysis and its impact on hydrocarbon chemistry in the atmosphere of Titan. J. Geophys. Res. 105, 20263-20273.

Wilson, E., Atreya, S., 2003. Chemical sources of haze formation in Titan's atmosphere. Planet. Space Sci. 51, 1017-1033.

Wilson, E., Atreya, S., 2004. Current state of modeling the photochemistry of Titan's mutually dependent atmosphere and ionosphere. J. Geophys. Res. 109, \#E06002. 
Wilson, E., Atreya, S., Coustenis, A., 2003. Mechanisms for the formation of benzene in the atmosphere of Titan. J. Geophys. Res. 108, \#5014.

Wilson, P., McEwan, M., Meot-Ner, M., 1994a. Reactions of CH3OCH2+ with nitrogen bases: a mechanism for the formation of protonated imines. Int. J. Mass Spectrom. Ion Processes 132, 149-152.

Wilson, S., Ashfold, M., Dixon, R., 1994b. The dissociation dynamics of the methyl radical following excitation to the $\mathrm{B}^{2} \mathrm{~A}_{1}^{\prime}$ state. Chem. Phys. Lett. $222,457-464$

Wilson, S., Howe, J., Rosser, K., Ashfold, M., Dixon, R., 1994c. A reinvestigation of the near-ultraviolet photodissociation dynamics of the methyl radical. Chem. Phys. Lett. 227, 456-460.

Wincel, H., Fokkens, R., Nibbering, N., 1989. On the structure and isomerization/dissociation reactions of $\mathrm{C}_{3} \mathrm{H}_{6} \mathrm{~N}^{+}$generated by methylation of acetonitrile in the gas phase. Int. J. Mass Spectrom. Ion Processes 91, 339-355.

Wincel, H., Wlodek, S., Bohme, D., 1988. Acetonitrile in gas-phase ion/molecule chemistry. Int. J. Mass Spectrom. Ion Processes 84, 69-87.

Wong, A., Morgan, C., Yung, Y., Owen, T., 2002. Evolution of CO on Titan. Icarus $155,382-392$.

Woodall, J., Agúndez, M., Markwick-Kemper, A., Millar, T., 2007. The UMIST database for astrochemistry 2006. Astron. Astrophys. 466, 1197.

Woods, T., Eparvier, F., Bailey, S., Chamberlin, P., Lean, J., Rottman, G., Solomon, S., Tobiska, W., Woodraska, D., 2005. Solar EUV Experiment (SEE): Mission overview and first results. J. Geophys. Res. 110, \#A01312.

Woon, D., Herbst, E., 2009. Quantum chemical predictions of the properties of known and postulated neutral interstellar molecules. Astrophys. J. Suppl. Ser. 185, 273-288.

Woon, D., Park, J.Y., 2009. Modeling chemical growth processes in Titan's atmosphere. 2. Theoretical study of reactions between $\mathrm{C}_{2} \mathrm{H}$ and ethene, propene, 1-butene, 2-butene, isobutene, trimethylethene, and tetramethylethene. Icarus 202, 642-655.

Wu, G., Jiang, B., Ran, Q., Zhang, J., Harich, S., Yang, X., 2004. Photodissociation dynamics of the methyl radical at $212.5 \mathrm{~nm}$ : Effect of parent internal excitation. J. Chem. Phys. 120, 2193-2198.

Wu, R.Y., Chien, T.S., Liu, G.S., Judge, D.L., Caldwell, J.J., 1989. Photoabsorption and direct dissociation cross sections of $\mathrm{C}_{2} \mathrm{H}_{2}$ in the 1530-1930 $\AA$ region: A temperature dependant study. J. Chem. Phys. 91, 272-280. 
Xu, Y., Chang, Y., Lu, Z., Ng, C., 2013. Absolute integral cross sections and product branching ratios for the vibrationally selected ion-molecule reactions: $\mathrm{N}_{2}{ }^{+}\left(X^{2} \Sigma_{g}^{+} ; \nu^{+}=0-2\right)+\mathrm{CH}_{4}$. Astrophys. J. 769, \#72.

Xu, Z.F., Li, S.M., Yu, Y.X., Li, Z.S., Sun, C.C., 1999. Theoretical studies on the reaction path dynamics and variational transition-state theory rate constants of the hydrogen-abstraction reactions of the $\mathrm{NH}\left(\mathrm{X}^{3} \Sigma^{-}\right)$radical with methane and ethane. J. Phys. Chem. A 103, 4910-4917.

Yang, D., Yu, T., Lin, M., Melius, C., 1992a. Cyanogen (CN) radical reactions with hydrogen cyanide and ethanedinitrile $\left(\mathrm{C}_{2} \mathrm{~N}_{2}\right)$ : comparison of theory and experiment. J. Chem. Phys. 97, 222-226.

Yang, D., Yu, T., Wang, N., Lin, M., 1992b. CN radical reactions with selected olefins in the temperature range 174-740 K. Chem. Phys. 160, 317-325.

Yang, D., Yu, T., Wang, N., Lin, M., 1992c. Temperature dependence of cyanogen radical reactions with selected alkanes: CN reactivities towards primary, secondary and tertiary C-H bonds. Chem. Phys. 160, 307-315.

Yang, Z., Cole, C., Martinez, O.J., Carpenter, M., Snow, T., Bierbaum, V., 2011. Experimental and theoretical studies of reactions between $\mathrm{H}$ atoms and nitrogen-containing carbanions. Astrophys. J. 739, \#19.

Ye, L., Georgievskii, Y., Klippenstein, S., 2015. Pressure-dependent branching in the reaction of ${ }^{1} \mathrm{CH}_{2}$ with $\mathrm{C}_{2} \mathrm{H}_{4}$ and other reactions on the $\mathrm{C}_{3} \mathrm{H}_{6}$ potential energy surface. Proc. Comb. Inst. 35, 223-230.

Yelle, R., Cui, J., Müller-Wodarg, I., 2008. Methane escape from Titan's atmosphere. J. Geophys. Res. 113, \#E10003.

Yelle, R., Vuitton, V., Lavvas, P., Klippenstein, S.J., Smith, M.A., Hörst, S.M., Cui, J., 2010. Formation of $\mathrm{NH}_{3}$ and $\mathrm{CH}_{2} \mathrm{NH}$ in Titan's upper atmosphere. Faraday Discuss. 147, 31-49.

Yen, T., Garand, E., Shreve, A., Neumark, D., 2010. Anion photoelectron spectroscopy of $\mathrm{C}_{3} \mathrm{~N}^{-}$and $\mathrm{C}_{5} \mathrm{~N}^{-}$. J. Phys. Chem. A 114, 3215-3220.

Yoshino, K., Esmond, J., Sun, Y., Parkinson, W., Ito, K., Matsui, T., 1996. Absorption cross section measurements of carbon dioxide in the wavelength region 118.7 - $175.5 \mathrm{~nm}$ and the temperature dependence. J. Quant. Spectrosc. Radiat. Transfer 55, 53-60.

Yu, T., Lin, M., 1993. Kinetics of phenyl radical reactions studied by the "cavity-ring-down" method. J. Am. Chem. Soc. 115, 4371-4372.

Yung, Y.L., Allen, M., Pinto, J.P., 1984. Photochemistry of the atmosphere of Titan: Comparison between model and observations. Astrophys. J. Suppl. Ser. 55, 465-506. 
Zabarnick, S., Fleming, J., Lin, M., 1989. Kinetics of methylidyne ( $\left.\mathrm{CH} \mathrm{X}{ }^{2} \Pi\right)$ radical reactions with ammonia and methylamines. Chem. Phys. 132, 407411.

Zabarnick, S., Fleming, J., Lin, M., 1991. Direct measurement of rate constants for the reactions of $\mathrm{CH}$ and $\mathrm{CD}$ with $\mathrm{HCN}$ and DCN. Chem. Phys. 150, $109-115$.

Zabarnick, S., Lin, M., 1989. Kinetics of $\mathrm{CN}\left(\mathrm{X}^{2} \Sigma^{+}\right)$radical reactions with $\mathrm{HCN}, \mathrm{BrCN}$ and $\mathrm{CH}_{3} \mathrm{CN}$. Chem. Phys. 134, 185-191.

Žabka, J., Polášek, M., Ascenzi, D., Tosi, P., Roithová, J., Schröder, D., 2009. Reactivity of $\mathrm{C}_{2} \mathrm{H}_{5}{ }^{+}$with benzene: Formation of ethylbenzenium ions and implications for Titan's ionospheric chemistry. J. Phys. Chem. A 113, 1115311160.

Žabka, J., Roithová, J., Španěl, P., Herman, Z., 2010. Dynamics of formation of products $\mathrm{D}_{2} \mathrm{CN}^{+}, \mathrm{DCN}^{+}$, and $\mathrm{CD}_{3}{ }^{+}$in the reaction of $\mathrm{N}^{+}$with $\mathrm{CD}_{4}$ : $\mathrm{A}$ crossed-beam and theoretical study. J. Phys. Chem. A 114, 1384-1391.

Zhang, F., Kim, S., Kaiser, R., 2009. A crossed molecular beams study of the reaction of the ethynyl radical $\left(\mathrm{C}_{2} \mathrm{H}\left(\mathrm{X}^{2} \Sigma^{+}\right)\right)$with allene $\left(\mathrm{H}_{2} \mathrm{CCCH}_{2}\left(\mathrm{X}^{1} \mathrm{~A}_{1}\right)\right)$. Phys. Chem. Chem. Phys. 11, 4707-4714.

Zhang, F., Parker, D., Kim, Y., Kaiser, R., Mebel, A., 2011. On the formation of ortho-benzyne $\left(\mathrm{o}-\mathrm{C}_{6} \mathrm{H}_{4}\right)$ under single collision conditions and its role in interstellar chemistry. Astrophys. J. 728, 141-150.

Zhang, Q., Zhang, R., Chan, K., Bello, I., 2005. Ab initio and variational transition state approach to $\beta-\mathrm{C}_{3} \mathrm{~N}_{4}$ formation: Kinetics for the reaction of $\mathrm{CH}_{3} \mathrm{NH}_{2}$ with H. J. Phys. Chem. A 109, 9112-9117.

Zhao, Z.X., Liu, J.Y., Wang, L., Zhang, H., Hou, C.Y., Sun, C.C., 2008. A study of the hydrogen abstraction reactions of $\mathrm{C}_{2} \mathrm{H}$ radical with $\mathrm{CH}_{3} \mathrm{CN}, \mathrm{C}_{2} \mathrm{H}_{5} \mathrm{CN}$, and $\mathrm{C}_{3} \mathrm{H}_{7} \mathrm{CN}$ by dual-level generalized transition state theory. J. Phys. Chem. A $112,8455-8463$.

Zhu, Z., Zhang, Z., Huang, C., Pei, L., Chen, C., Chen, Y., 2003. Kinetics of CCN radical reactions with a series of normal alkanes. J. Phys. Chem. A 107, 10288-10291.

Zielinska, T., Wincel, H., 1970. Ion-molecule reactions in methylamine and methylamine-ammonia systems. Nukleonika 15, 26-36.

Zymak, I., Hejduk, M., Mulin, D., Plašil, R., Glosík, J., Gerlich, D., 2013. Lowtemperature ion trap studies of $\mathrm{N}^{+}\left({ }^{3} P_{j a}\right)+\mathrm{H}_{2}(j) \rightarrow \mathrm{NH}^{+}+\mathrm{H}$. Astrophys J. $768, \# 86$. 